\title{
Floods of March 1964 Along the Ohio River
}

GEOLOGICAL SURVEY WATER-SUPPLY PAPER 1840-A

Prepared in cooperation with the

States of Kentucky, Ohio, Indiana, Pennsylvania, and West Virginia, and with agencies of the Federal Government

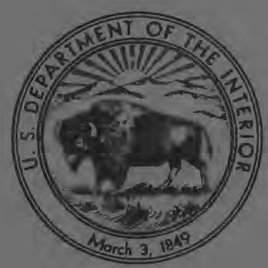




\section{Floods of March 1964}

Along the Ohio River

By H. C. BEABER and J. O. ROSTVEDT

FLOODS OF 1964 IN THE UNITED STATES

GEOLOGICAL SURVEY WATER-SUPPLY PAPER 1840-A

Prepared in cooperation with the

States of Kentucky, Ohio, Indiana,

Pennsylvania, and $W$ est Virginia, and with agencies of the Federal Government

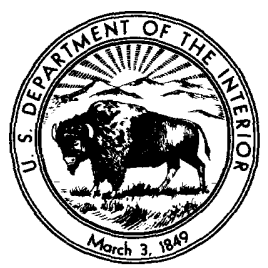

UNITED STATES GOVERNMENT PRINTING OFFICE, WASHINGTON : 1965 
UNITED STATES DEPARTMENT OF THE INTERIOR

STEWART L. UDALL, Secretary

\section{GEOLOGICAL SURVEY}

William T. Pecora, Director

For sale by the Superintendent of Documents, U.S. Government Printing Office Washington, D.C. 20402 - Price 65 cents (paper cover) 


\section{CONTENTS}

$\begin{array}{lr}\text { Abstract.ren } & \text { Page } \\ \text { Introduction } & \text { A1 } \\ \text { The storms. } & 6 \\ \text { The floods } \\ \text { Pennsylvania }\end{array}$

\section{ILLUSTRATIONS}

FIgure 1. Map showing area covered by this report_....... A2

2. Map showing location of flood-determination sites......... 4

3-5. Isohyetal maps:

3. March 2-5

4. March 8-10

5. March 2-10

6. Discharge hydrographs for stations in Ohio ........ 12

7. Flooding in Athens, Ohio 
FiguRe 8. Discharge hydrographs for stations in Kentucky

9. Flooding in Shepherdsville, $\mathrm{Ky}$

10. Flooding in Jasper, Ind

11. Discharge hydrographs for stations in Indiana . . . . . . .

12. Comparative crest stages on Ohio River.....

13. Discharge hydrographs for Ohio River......................

14. Recurrence intervals of peak discharges.

\section{TABLES}

Table 1. Flood damage along the Ohio River

2. Expenditures by American Red Cross

3. Recurrence intervals of peak discharges on the Ohio River ...

4. Summary of flood stages and discharges 


\title{
FLOODS OF 1964 IN THE UNITED STATES
}

\section{FLOODS OF MARGH 1964 ALONG THE OHIO RIVER}

\author{
By H. C. Beaber and J. O. Rostvedt
}

\section{ABSTRACT}

The floods of March 1964 in the Ohio River basin caused widespread damage in six States adjacent to the Ohio River main stem. Flood damage was estimated at over $\$ 100$ million, of which about 75 percent was along the Ohio River main stem. Over 21,000 homes were damaged or destroyed, and more than 29,000 families suffered losses. Eighteen lives were reported lost.

Floods were caused by two storms; the first occurred March 2-5, and the other March 8-10. Both storms approximately paralleled the Ohio River in a belt extending from western Kentucky through northern Kentucky, southern Indiana, and central Ohio, to western Pennsylvania. In most localities the storm of March 8-10 was the more severe. Total rainfall from the storms exceeded 14 inches in western Kentucky. Greatest 24-hour precipitation was 8.00 inches at Paducah, Ky., on March 4.

Maximum discharges previously known were exceeded at many points in Ohio, Kentucky, and Indiana. Peak discharges of the March 1964 floods exceeded the 50-year flood at many localities. The Licking River at Catawba, Ky., reached the highest stage since 1888. The Ohio River in Kentucky reached stages which were second or third highest since the maximum known flood in 1937.

This report describes the 1964 floods and gives detailed streamflow records and information on precipitation and on damage incurred.

\section{INTRODUCTION}

Severe floods occurred along the entire reach of the Ohio River during March 1964, and inflicted heavy damage in western Pennsylvania, northwestern West Virginia, southern Ohio, and Indiana, and northern Kentucky (fig. 1).

Heavy rains in two periods, March 2-5 and 8-10, caused extreme floods on many tributaries to the Ohio River. Floods during the first period were heaviest on Ohio River tributaries in southern Indiana and northern Kentucky. The extreme flooding in the second period was on the Ohio River tributaries in southern Indiana, northern Kentucky, 


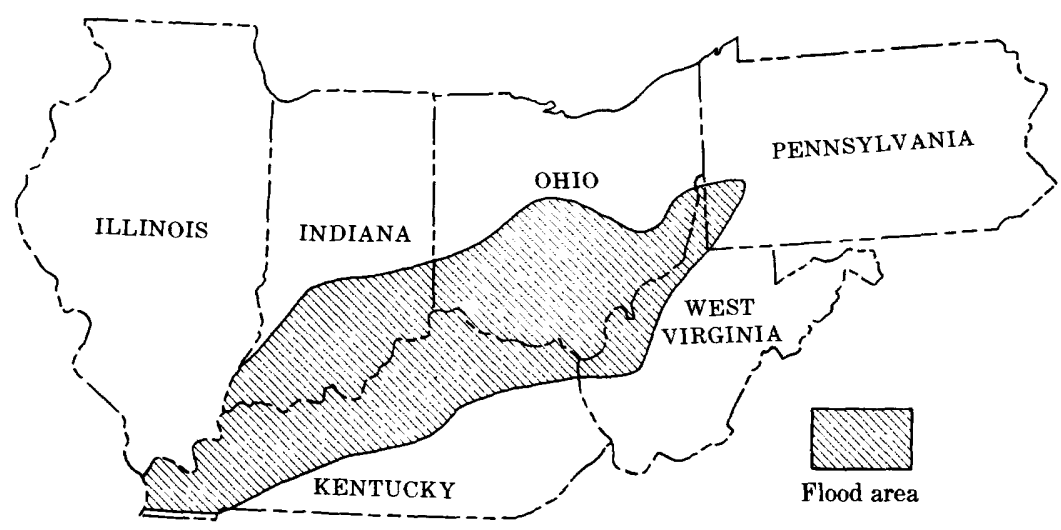

Frgore 1.-Area covered by this report.

and southwestern and central Ohio. The floods of March 8-10 were, in general, more severe than those of March 2-5.

The severe tributary floods combined with lesser floods on tributaries in eastern Ohio, northwestern West Virginia, and western Pennsylvania and caused the Ohio River to rise above flood stage from Pittsburgh, Pa., to Cairo, Ill. Melted snow in western Pennsylvania added to the volume of flood runoff.

Prior to March 1964, soil moisture was seriously deficient in Ohio, Kentucky, and Indiana. Rainfall in March 1964 marked the first time since the summer of 1963 that monthly precipitation in these States was near normal.

The entire length of the Ohio River was at or near pool stage on March 1. Flow in tributary streams was generally deficient and many reservoirs were at low levels.

Peak stages and discharges during March in Kentucky were the greatest of record on the lower reaches of the Licking River and Eagle Creek in the Kentucky River basin. Maximum discharges for the period of record occurred on many streams in Beargrass Creek, Salt River, Nolin River, and Rough River basins. Recurrence intervals of the March 1964 floods were 50 years or more at 15 gaging stations in Kentucky.

Floods in extreme southern Indiana were the greatest since 1913. Heaviest flooding occurred in the White River and the Patoka River basins and along streams that are directly tributary to the Ohio River. The recurrence interval of the flood was more than 50 years at several places in the above areas.

Floods were maximum of record in the East Fork Little Miami River, Paint Creek, and Hocking River basins in Ohio. Damage was severe, particularly in the Hocking River basin in the of vicinity of 
Athens, Ohio, but was not as great as along the Ohio River where the stages were the highest since 1945 .

Flood stages on the Ohio River from Maysville, Ky., to Golconda, Ill., were second or third highest since 1937.

The purpose of this report is to supplement, in a more detailed form, data of stage and discharge published in the annual streamflow reports of the Geological Survey. This report discusses precipitation, floods, and flood damage, and relates the magnitude of the March 1964 floods to large known floods of the past. Figure 2 shows locations of sites for which flood data are available in this report. Reference in the text to the flood-determination points shown in figure 2 gives the identifying station number in parenthesis, as follows: Beaver River at Beaver Falls, Pa. (No. 4),---.

The terms and abbreviations of streamflow and other hydrologic data used in this report are defined as follows:

1. Cubic foot per second (cfs) is the rate of discharge of a stream whose channel is 1 square foot in cross-sectional area and whose average velocity is 1 foot per second.

2. Runoff in inches (in.) shows the depth to which the drainage area would be covered if the runoff for a given time period were uniformly distributed on the surface.

3. Acre-foot (acre-ft) is the quantity of water required to cover an acre to the depth of 1 foot and is equivalent to 43,560 cubic feet.

4. Stage-discharge relation is the relation between gage height, in feet, and the amount of water flowing in a channel expressed as volume per unit of time.

5. Contents is the volume of water in a reservoir or lake. Unless otherwise indicated, volume is computed on the basis of a level pool and does not include bank storage.

6. Drainage area of a stream at a specified location is that area, measured in a horizontal plane, which is so enclosed by a topographic divide that direct surface runoff from precipitation normally would drain by gravity into the river above the specified point. Figures of drainage area given herein include all closed basins, or noncontributing areas, within the area unless otherwise noted.

7. A partial-record station is a site where limited streamflow data are collected systematically over a period of years for use in hydrologic analyses.

Records of discharge in the area covered by this report were collected as part of cooperative programs between the U.S. Geological Survey and the States of Pennsylvania, Ohio, West Virginia, Kentucky, Indiana, and Tennessee, the U.S. Army Corps of Engineers, and other Federal or local agencies. The U.S. Weather Bureau and 


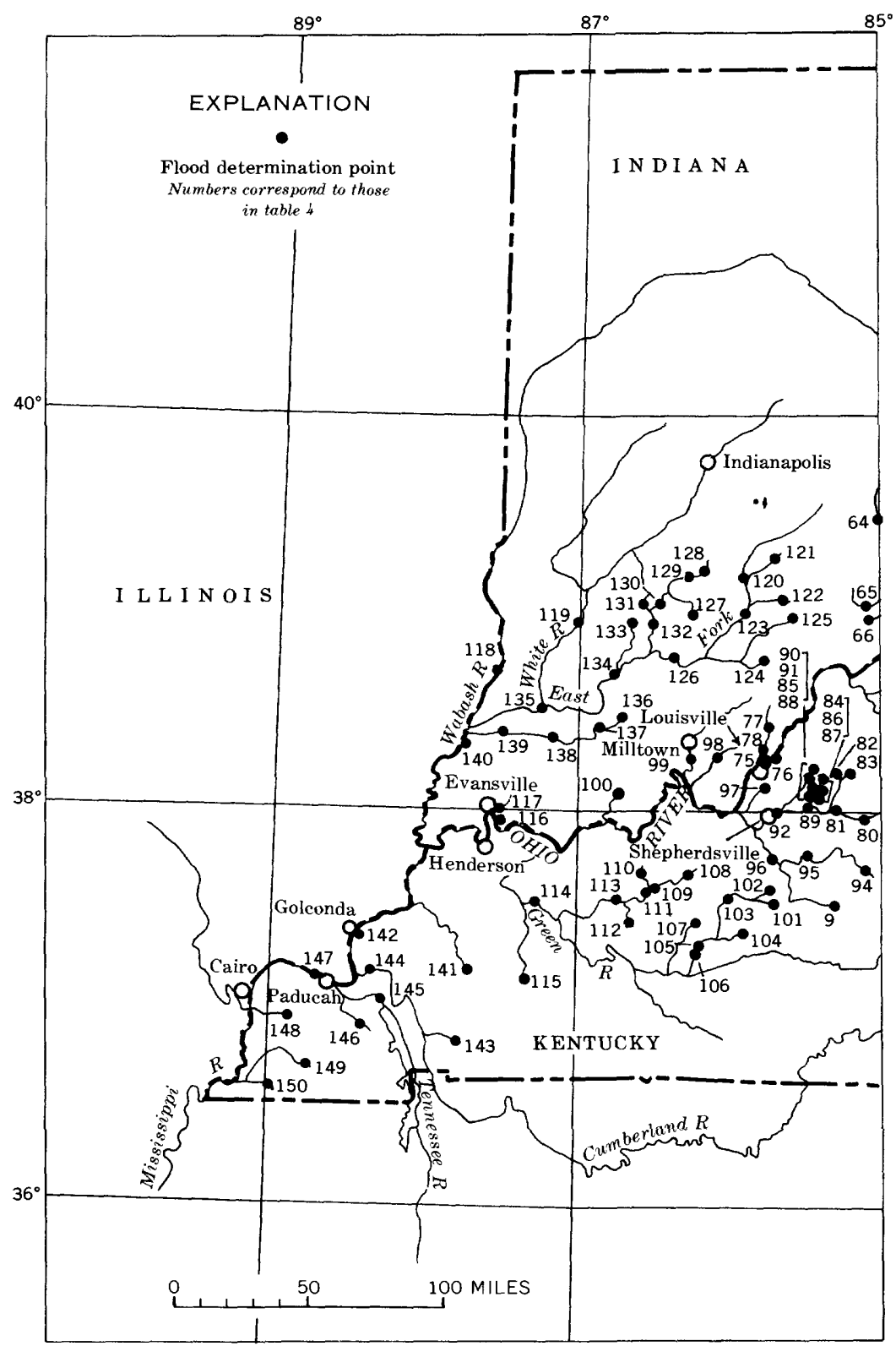

Figure 2.-Flood area showing 


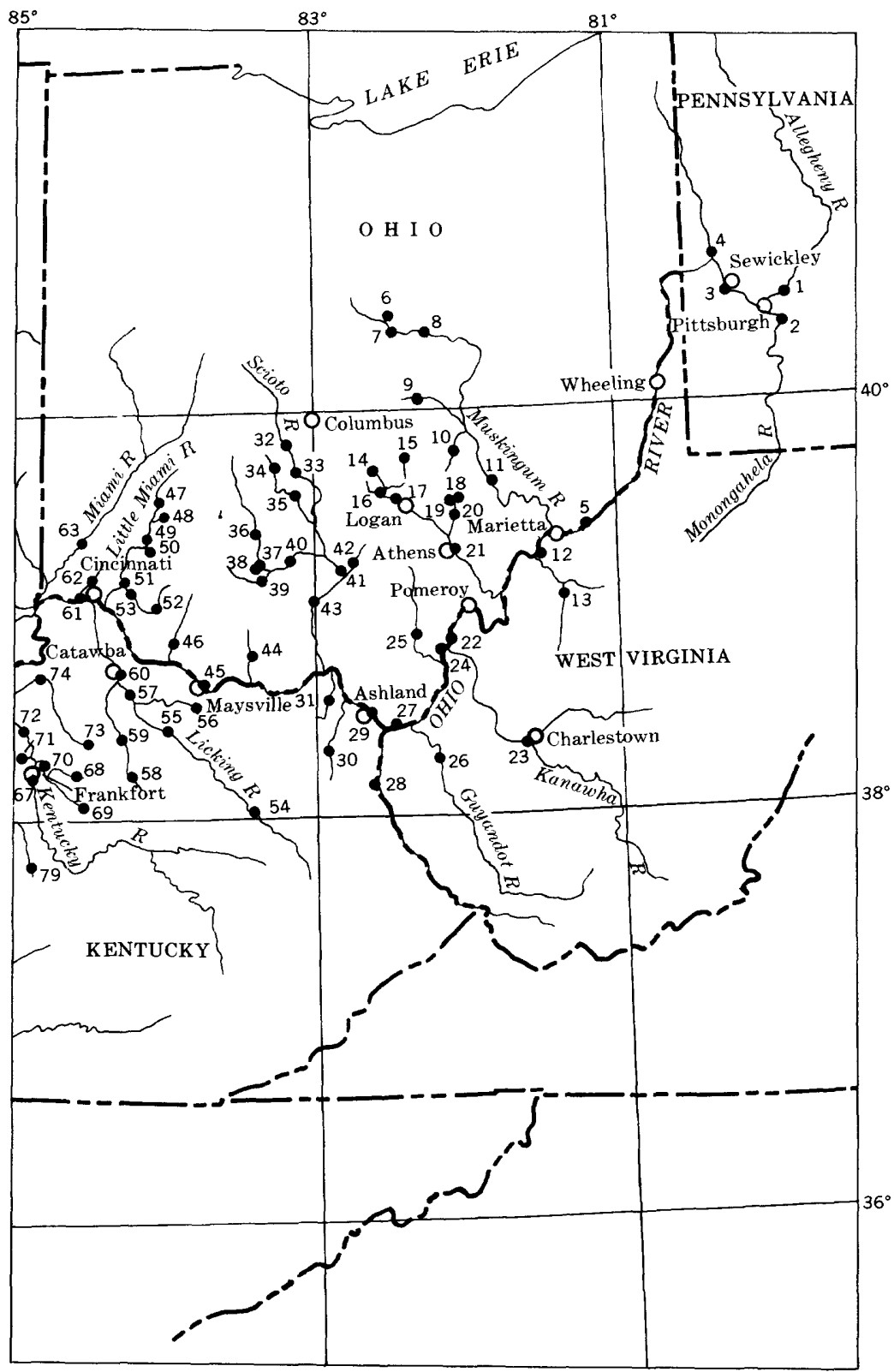

location of flood-determination sites. 
several State, municipal, and private organizations furnished some data or information included in this report, and appropriate acknowledgment is made in the text.

Data were collected and compiled by personnel in the U.S. Geological Survey district offices under supervision of the following: Robert E. Steacy, district engineer, Pennsylvania; W. C. Griffin, district engineer, West Virginia; J. J. Molloy, district engineer, Ohio; F. F. Schrader, district engineer, Kentucky; Malcolm D. Hale, district engineer, Indiana; and J. S. Cragwall, Jr., district hydrologist, Tennessee. The text material describing the floods in Ohio was obtained from the State report "Floods of March 1964 in Ohio," (Bulletin 39) by William P. Cross, hydraulic engineer, Columbus, Ohio.

\section{THE STORMS}

The flood-producing rains of early March 1964 marked the end of several months of below-normal precipitation in most of the flood area. This below-normal precipitation had caused soil moisture to be seriously deficient in Kentucky, Ohio, and Indiana for many months prior to the March floods. The period September-December 1963 was the driest comparable period on record in Ohio. Precipitation in Kentucky during March 1964 was near normal to above normal for the first time since July 1963. Monthly rainfall in Indiana had been below normal since late summer of 1963 and had caused soil moisture to be deficient.

Snow depths in the flood area on March 1-2, preceding the rains, were appreciable only in western Pennsylvania and eastern Ohio and averaged 2-4 inches. Lesser amounts were recorded in northwestern West Virginia, and no snow was on the ground in southern Ohio and northern Kentucky.

The flood-producing rains of March 1964 along the Ohio River fell in two principal periods, March 2-5 and 8-10. Heavy rains during the first period extended from southeastern Ohio to extreme western Kentucky in a broad belt approximately paralleling the Ohio River (fig. 3). Heaviest concentrations of rain occurred in northwestern Kentucky and southern Indiana. Greatest 1-day precipitation was 8.00 inches at Paducah, in western Kentucky, on March 4. According to the U.S. Weather Bureau, the probability of rainfalls of this intensity recurring in any month is less than once in 100 years. The March 2-5 rains were accompanied by strong thunderstorm-wind activity in Ohio and Indiana and by damaging tornadoes which killed three persons in western Kentucky.

In less than a week a second series of storms hit the flood area and produced floods that were generally greater in magnitude than those 


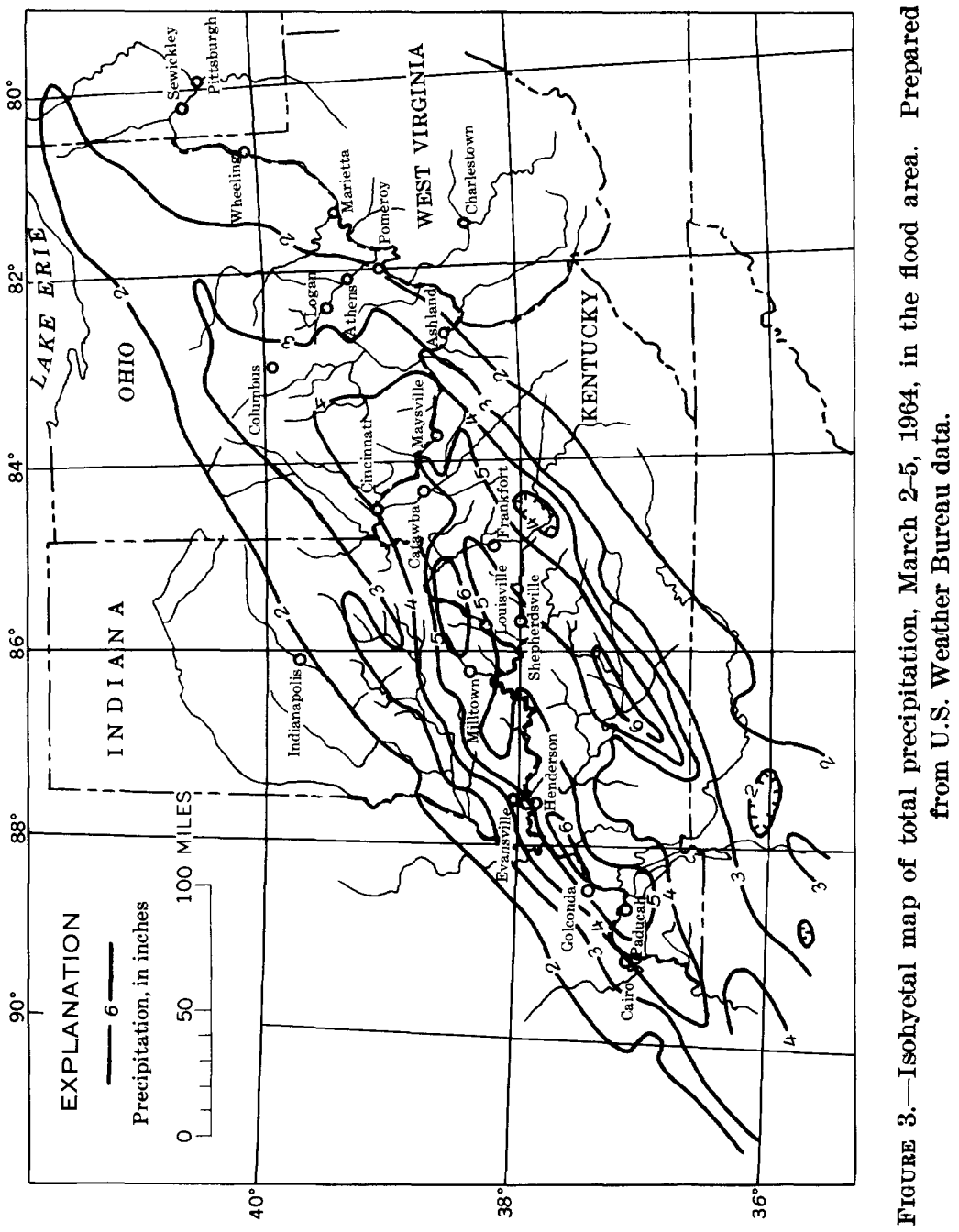


of the first storm. Heaviest. concentrations again were along the Ohio River in northern Kentucky and southern Indiana. However, unlike the first storm, the rains extended into southern and central Ohio and western Pennsylvania (fig. 4). Greatest 24-hour precipitation in the March 8-10 period was 5.32 inches in south-central Ohio, 5.63 inches in southern Indiana, and 6.97 inches at Louisville, $\mathrm{Ky}$. The U.S. Weather Bureau reported that the 24-hour precipitation of 6.97 inches at Louisville on March 9 was the greatest total for a 24hour period recorded since records began in 1871 at that site.

Total precipitation of the two storms ranged from 4 inches in western Pennsylvania to over 14 inches in western Kentucky (fig. 5). The rainfall together with snowmelt in Pennsylvania produced damaging floods on the Ohio River from Pittsburgh, Pa., to river's mouth. Additional scattered rainfall from March 11 to 31 made March 1964 the wettest March on record in some localities. Rains in Kentucky exceeded previous 24-hour records for March and at several locations exceeded previous 24 -hour records for any month.

Temperatures for March 1964 in the flood area were above normal except in southeastern Ohio and north-central Kentucky, where they were slightly below normal.

\section{THE FLOODS}

The damaging floods of March 2-5 occurred on Ohio River tributaries in southwestern Ohio, southern Indiana, and northwestern Kentucky. These floods hardly had subsided when the March 8-10 floods hit most of the same tributaries plus additional tributaries in southern and central Ohio. Floods exceeded previous records in Ohio, Kentucky, and Indiana. Tributary floodflows that resulted from both series of storms produced damaging floods on the Ohio River main stem which increased in magnitude as the flood wave progressed downstream. Recurrence intervals of peak discharges upstream from Louisville, Ky., were 12 years or less, increasing to more than 50 years downstream at Evansville, Ind.

\section{PENNSYLVANIA}

Below-normal precipitation in January and February was followed by two storms that occurred over western Pennsylvania on March 4-5 and $9-11$; these storms were accompanied by snowmelt and caused damaging floods in the Ohio River basin in Pennsylvania. Previous maximum stages or discharges were not exceeded. The Allegheny River was above flood stage, and heavy ice flows from ice jams inflicted considerable damage to barges and boats. The Ohio River at Pittsburgh was above flood stage for 4 days in March. The Corps of 


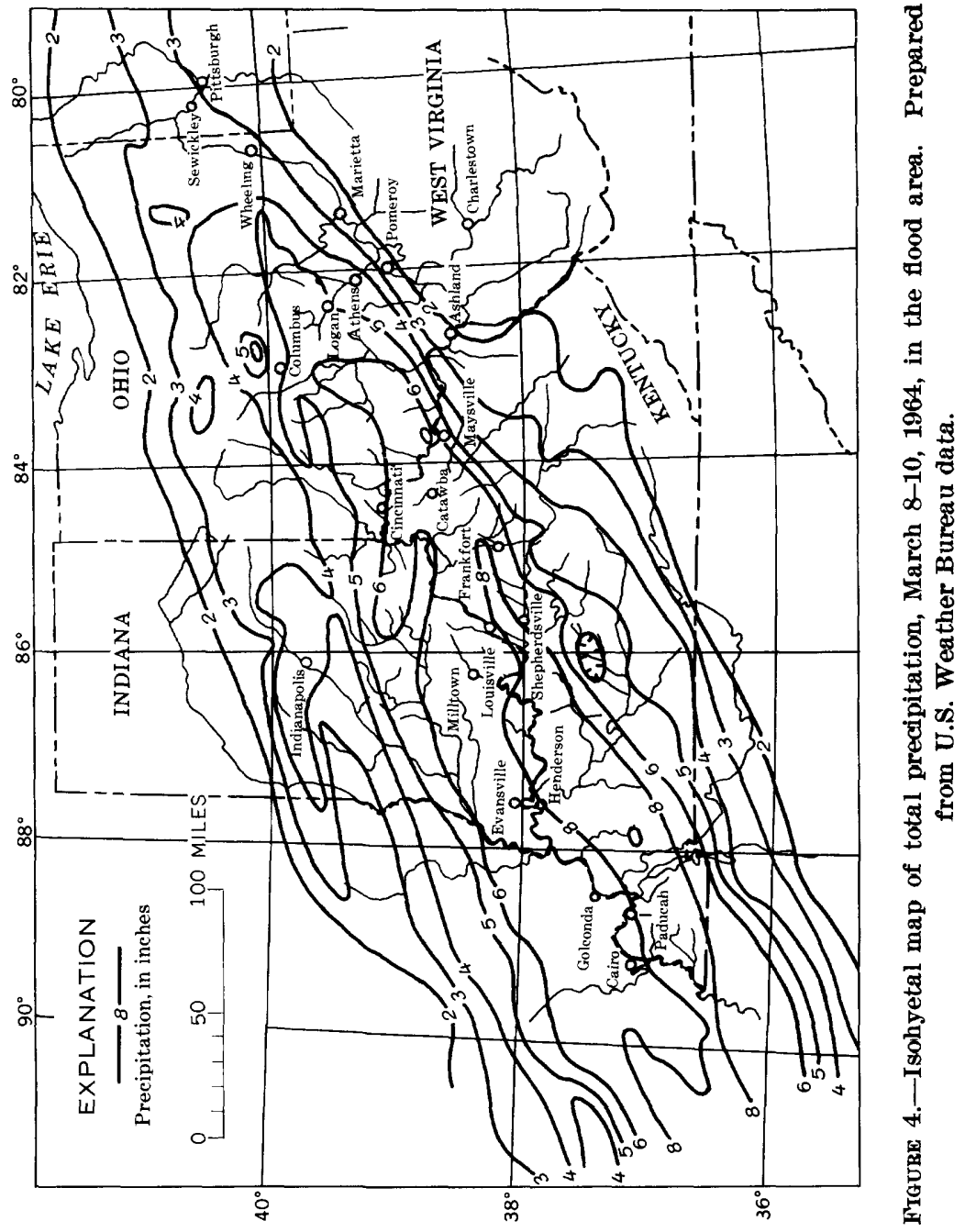




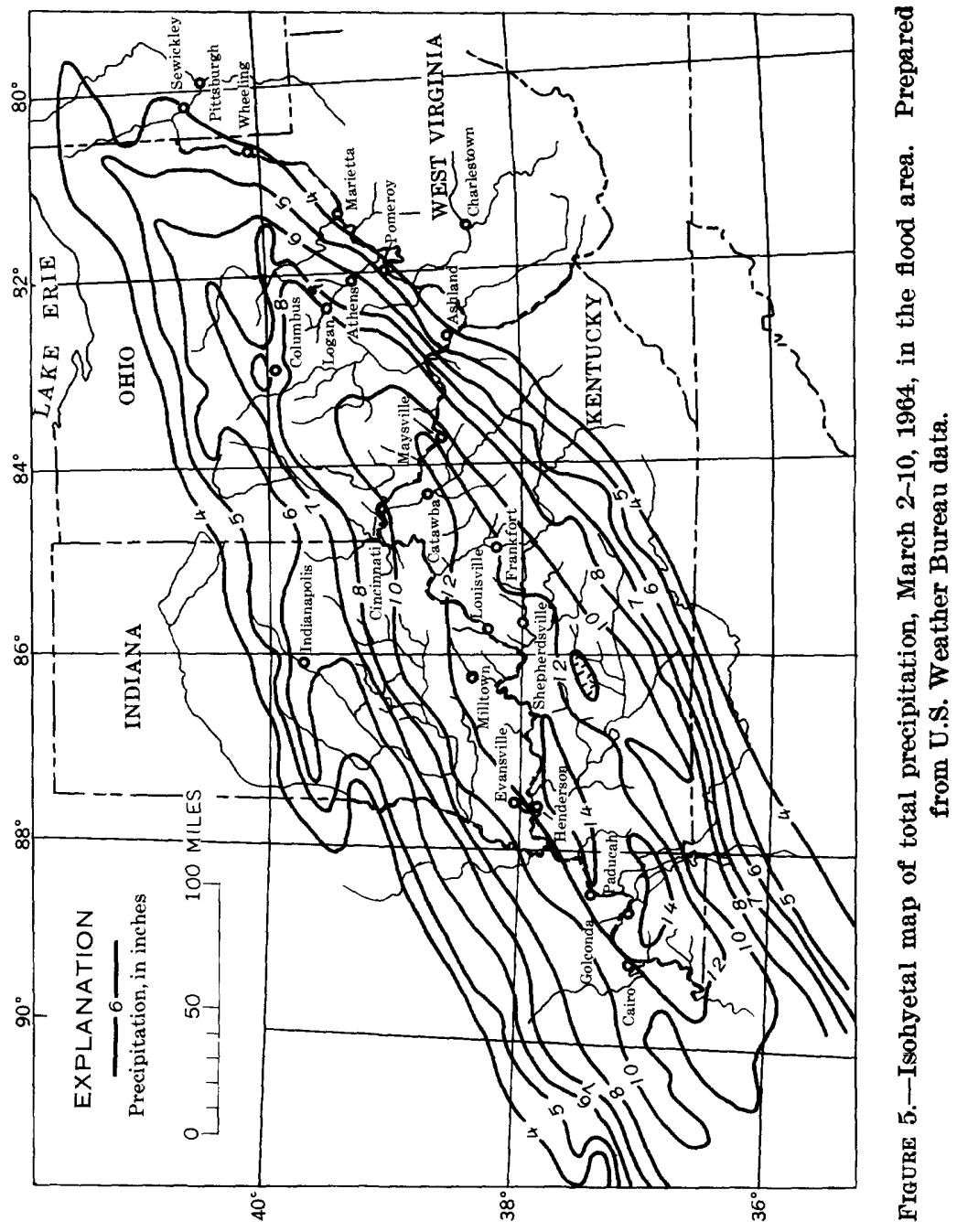


Engineers estimated that damage in the Ohio River basin in Pennsylvania was $\$ 11,740,000$. The Corps of Engineers flood-control system on Ohio River headwater streams reduced flood stages on the Ohio River by several feet and prevented approximately $\$ 38$ million damage. According to the American Red Cross, 2,604 dwellings received major or minor damage and 4,026 families had losses. One death was attributed to the flood.

\section{WEST VIRGINIA}

Total precipitation for most of northwestern West Virginia was less than 3 inches during the storms of March 2-10, but about 4 inches fell in the northern panhandle. Flooding in the State was confined mostly to low-lying lands adjacent to the Ohio River. This river was above flood stage along the entire reach within the State, but other streams and rivers in the State were not subject to major flooding. Twelve counties along the Ohio River were declared disaster areas. Damage amounted to millions of dollars, and the American Red Cross reported that 3,850 families had losses and that 3,629 dwellings received major or minor damage. No deaths were attributed to the floods.

\section{OHIO}

Heavy rains on March 4-6 and 8-12, 1964, broke the drought of 1963-64, which followed the March 1963 flood period. The rains caused a major Ohio River flood and exceptionally high stages on tributaries in Ohio. Floods were the worst in the Hocking River basin since 1907, and they exceeded all previous records on East Fork Little Miami River and on Paint Creek in the Scioto River basin.

Total damage in Ohio probably exceeded $\$ 30$ million. The American Red Cross reported 8 lives lost, 84 dwellings destroyed, major damage to 1,026 dwellings, and minor damage to 7,225. From 3,000 to 5,000 persons were evacuated from their homes and more than 3,800 families were given aid.

Discharges at selected gaging stations in Ohio on March 4-13 are shown in figure 6.

\section{MUSKINGUM RIVER BASTN}

Fifteen flood-control reservoirs of the Muskingum Conservancy District, operated by the Corps of Engineers, reduced potential flooding by the Muskingum River. Uncontrolled tributaries in the area of heavy rainfall had severe flooding, but not as great as in 1959 . In general, the uncontrolled flood peaks were about the same order of magnitude as those in 1963. Wills Creek Reservoir stored more water from this flood than from any other since its construction in 1938. 


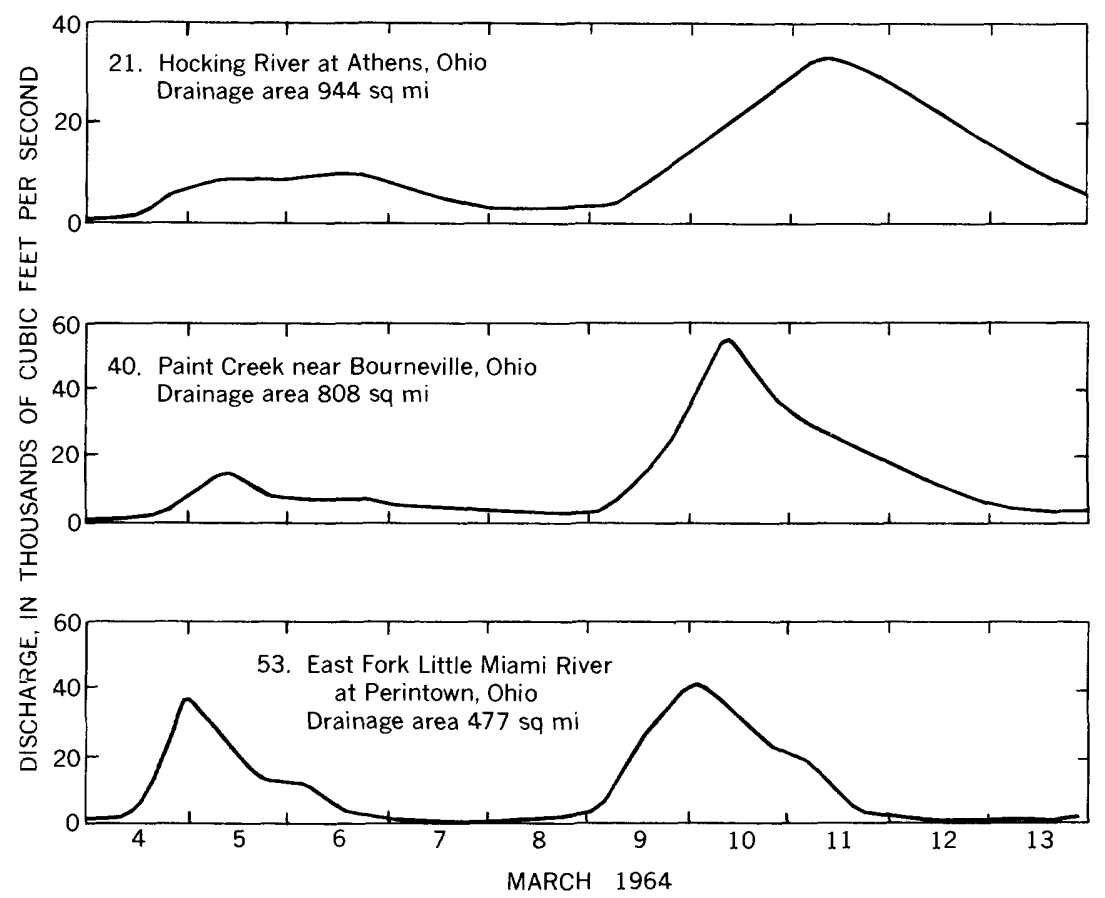

FigURE 6.-Discharge hydrographs at selected gaging stations, March 4-13, 1964, in Ohio.

Local heavy rains caused floods on Beaverdam Creek at New Philadelphia, Ohio, and on Laurel Creek at Uhrichsville, Ohio. Damage in the Muskingum River basin was widespread and estimates of loss exceeded $\$ 1$ million. The Corps of Engineers estimated that the stages of the Muskingum River were lower than they would have been had the reservoir not existed; the level was 6.2 feet lower at Coshocton; 11.4, at Dresden; 11.8, at Zanesville; and 6.7, at McConnelsville, Ohio.

\section{HOCKING RIVER BASIN}

Athens, on the Hocking River, was the only city in the interior of Ohio that incurred appreciable flood damage. The 1964 peak stage exceeded that estimated for 1913 and was higher than all other known floods except that in 1907 .

March 1964 peak stages on the Hocking River and on tributaries that were gaged upstream from Enterprise, Ohio, were lower than they were in 1963, as was the peak stage on Little Rush Creek. The stages on Sunday Creek were also lower in 1964, which suggests that extremely high stages on the Hocking River from Enterprise to Athens, Ohio, were caused by high runoff from Rush Creek and from downstream tributaries above Sunday Creek. 
Breman, Ohio, was almost submerged and was isolated by floodwaters. Fourteen hundred students were evacuated from Ohio University dormitories at Athens, Ohio (fig. 7 ), and 200 families were evacuated at Logan, Ohio. Total damage in the Hocking River basin was estimated at $\$ 2,137,000$.

\section{SCIOTO RIVER BASIN}

The floods on the upper Scioto River above Big Darby Creek were not unusually high. The greatest floods were in the Paint Creek area, where in some places stages were higher than they had been in 1913 and in all subsequent. floods. Much of the damage, estimated at $\$ 1,351,000$, was concentrated in the Paint Creek area in and west of Chillicothe, Ohio. Floods on the Scioto River main stem were not unusual. The Corps of Engineers estimated a reduction of stage of 1.1 feet at Columbus and Chillicothe, Ohio; this reduction of stage is attributable to the Delaware Reservoir.

\section{LITTLE MIAMI RIVER BASIN}

Flooding in the Little Miami River basin was not appreciable upstream from Caesar Creek. Except at Milford, Ohio, and in the East Fork area, the flood was lower than in 1963. East Fork Little Miami River at Perintown, Ohio (No. 53), reached a stage of 23.84 feet on March 10, exceeding all records since 1916 and also the estimated stage of 22 feet in 1913. Damage in the Little Miami River basin was estimated at $\$ 4,220,000$, of which $\$ 500,000$ was in South Lebanon, Ohio. In Batavia, Ohio, a bottled gas company's bulk plant and office building were destroyed, and other buildings were damaged.

\section{KENTUCKY}

Thousands of families were eracuated from low-lying areas along the Ohio River and its tributaries, and 47 counties were declared disaster areas. Hundreds of roads and highways were closed to traffic, and many schools were closed in the severely flooded areas. Communications were interrupted, preventing many people from receiving ample flood warnings. Water supplies were contaminated in some towns, thus necessitating the importing of water. Evacuation centers were established in churches, armories, schools, and private buildings. Total damage in Kentucky was estimated at about $\$ 30$ million. The death toll in Kentucky was seven persons. The American Red Cross reported that 59 dwellings were destroyed and that 4,277 sustained major or minor damage; 109 farm buildings were destroyed and 190 suffered major damage. Almost 6,000 families suffered losses.

Discharges at selected gaging stations in Kentucky on March 4-13 are shown in figure 8 .

$782-0010-65-2$ 


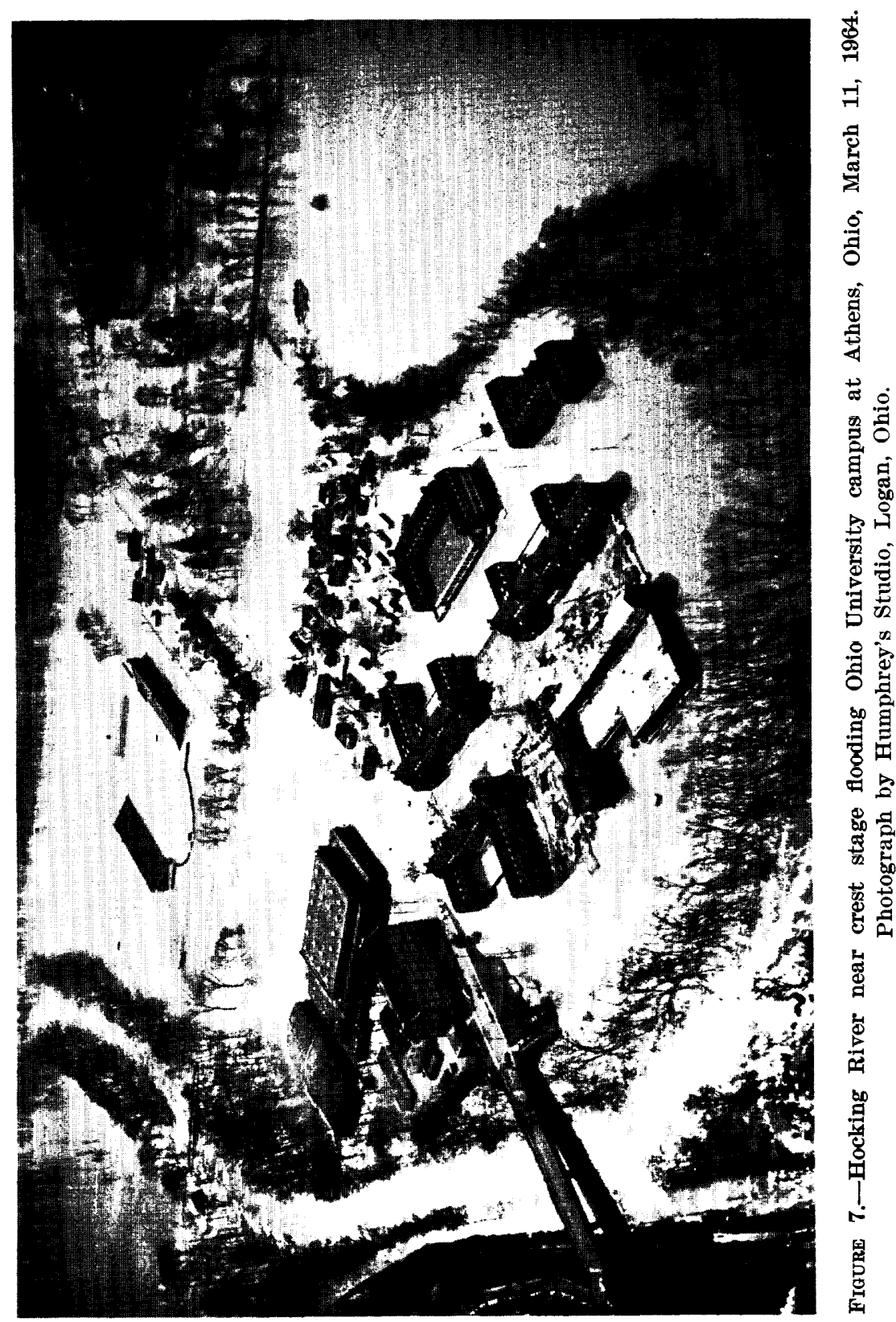



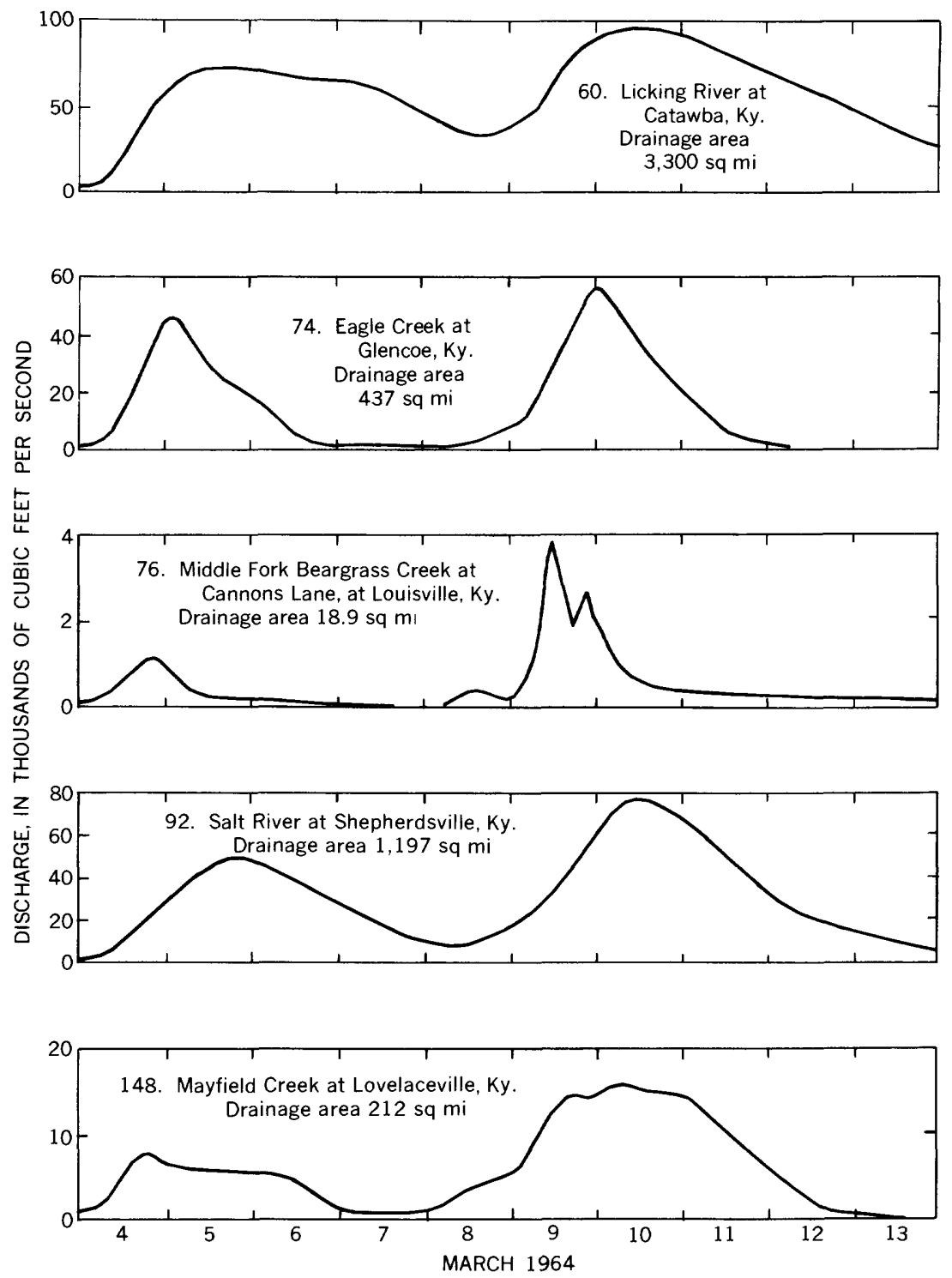

FIGURE 8.-Discharge hydrographs at selected gaging stations, March 4-13, 1964, in Kentucky.

\section{LICKING RIVER BASIN}

Record-breaking floods occurred on the lower reaches of the Licking River. The town of Falmouth, Ky., was the most extensively damaged town on the Licking River. The rapidly rising water prevented families from removing household goods. Telephone services were 
disrupted and prevented the receiving of flood warnings. About two-thirds of the residents were evacuated and floodwaters reached rooftop level in many sections. The cities of Paris, Ky., on Stoner Creek, and Cynthiana, Ky., on South Fork Licking River also sustained considerable damage.

Stages in the Licking River basin exceeded previously known maximum stages at three gaging stations. The stage on the Licking River at McKinneysburg (No. 57), upstream from Falmouth, exceeded that of the flood of 1937 by 2.5 feet. The Licking River at Catawba (No. $60)$ reached a stage of 52.60 feet, the highest since 1888, and exceeded by 5.6 feet the flood stage of 1948. A maximum stage of 19.59 feet occurred on Stoner Creek at Paris, Ky. (No. 58).

Total damage in the Licking River basin was estimated by the Corps of Engineers to be $\$ 2,630,000$, of which $\$ 2,530,000$ occurred on the main stem and $\$ 100,000$ occurred on South Fork Licking River.

\section{KENTUCKY RIVER BASIN}

Severe flooding occurred in the lower reaches of the Kentucky River basin, and as a result, Frankfort, Lexington, and Georgetown, Ky., were heavily damaged. Peak flow for a gaging station on North Elkhorn Creek (No. 68) had a recurrence interval of more than 50 years. Eagle Creek at Glencoe, Ky. (No. 74), reached a peak stage of 26.05 feet compared with the previous crest of 23.60 feet in 1943 .

The Corps of Engineers estimated damage to be $\$ 1,500,000$ in the Kentucky River basin. Of this total, $\$ 1,333,000$ was on the main stem, $\$ 2,000$ on the Red River, and $\$ 165,000$ on Eagle Creek.

\section{BEARGRASS CREEK BASIN}

Beargrass Creek, whose drainage area consists mostly of highly urbanized areas adjacent to Louisville, $\mathrm{Ky}$., was the highest since 1943 . The entire basin was in the area of intense rainfall. According to the U.S. Weather Bureau, the 24-hour precipitation of 6.97 inches recorded at Louisville on March 9 was the greatest 24-hour total recorded since 1871. Considerable damage was inflicted on business and residential property.

\section{SALT RIVER BASIN}

Severe floods which occurred on the lower reaches of the Salt River and its tributaries incurred $\$ 2,280,000$ damage as estimated by the Corps of Engineers. The greatest impact was at Shepherdsville, Ky., where water covered the entire business district and most of the residential area (fig. 9). Flooding was caused by backwater from the Ohio River and Rolling Fork in conjunction with headwater 


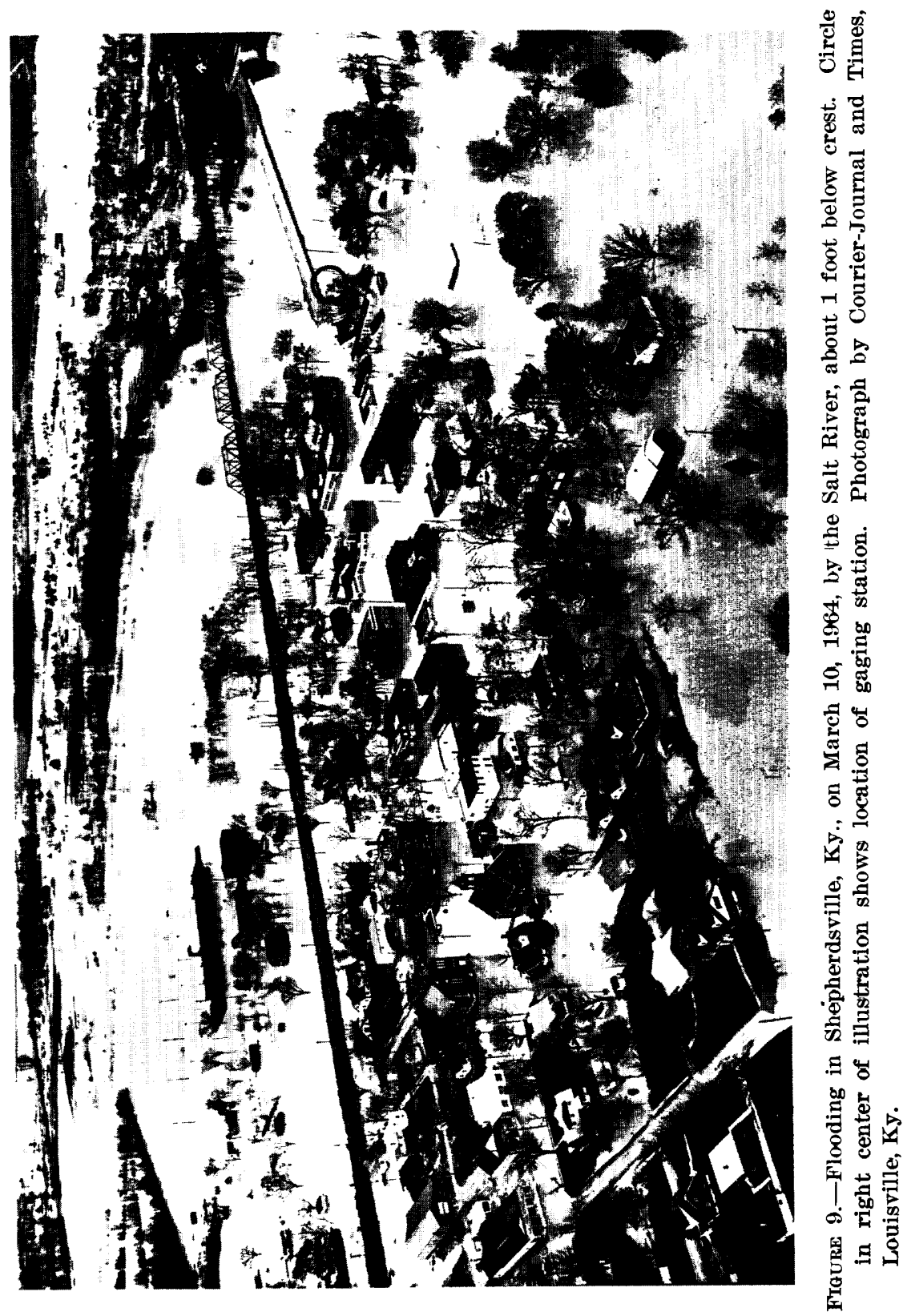


floods on the Salt River. Damage occurred in Taylorsville, Ky., from surface water which accumulated behind floodwalls built to protect the city from floodwaters of the Salt River and Brashears Creek. The Corps of Engineers estimated that $\$ 288,000$ potential damage was prevented by the floodwall.

At the gaging station on Salt River at Shepherdsville, Ky. (No. 92), the stage exceeded that of the 1961 flood by 0.7 foot but did not exceed that of the 1937 flood. The maximum discharge during the period of gaging-station operation occurred on Floyds Fork at Fisherville, $\mathrm{Ky}$. (No. 91), and the stage was 1.1 feet lower than that of the 1937 flood. Rolling Fork near Boston, Ky. (No. 96), reached a stage of 51.0 feet, the highest since 1938 but about 4.2 feet below that of the 1937 flood. Potential floods in the Plum Creek basin were reduced to some extent by 11 small detention reservoirs on Plum Creek.tributaries.

\section{GREEN RIVER BASIN}

Although most of the Green River basin was outside the area of intense rainfall, severe flooding occurred in the Nolin and Rough River basins. The maximum for the period of record occurred on North Fork Nolin River at Hodgenville, Ky. (No. 102). In the Rough River basin the maximum for the period of record occurred on North Fork Rough River near Westview, Ky. (No. 108).

Potential flooding in the Green River basin was reduced by Corps of Engineers flood-control reservoirs on the Rough, Barren, and Nolin Rivers. According to the Corps of Engineers, $\$ 2,390,000$ in damage was prevented by operation of these reservoirs. Total damage in the Green River basin amounted to $\$ 2,107,000$, of which about 75 percent occurred on the Green River main stem.

\section{WESTERN KENTUCKY}

The entire Tradewater River basin in western Kentucky received heary concentrations of rainfall during March. The Tradewater River at Olney, Ky. (No. 141), reached a stage of 18.68 feet, which was 0.6 foot less than the maximum known flood in 1937.

Stages on the Cumberland River in Kentucky were not particularly high, as most of the drainage area was outside the area of heavy rainfall and the flow was partly regulated by reservoirs. The flood on Little River, the major Cumberland River tributary in Kentucky, did not exceed the flood of 1951, which was the highest for the tributary's period of record.

Damage due to floodwaters in the Tennessee River basin in Kentucky was confined to Clarks River, which empties into the Tennessee River downstream from Kentucky Lake. McCracken, Marshall, and Graves Counties were especially hard hit by overflow from West and 
East Forks of Clarks River. The peak discharge of East Fork Clarks River Near Benton, Ky. (No. 146), did not exceed the floods of either 1937 or 1957; however, the recurrence interval of the March flood was more than 50 years. Flow of the Tennessee River, excluding the Clarks River, was regulated by Kentucky Lake and other upstream reservoirs in the Tennessee River basin. Elevation of Kentucky Lake reached 363.86 feet, fourth highest since storage began, as a result of water impounded in the lake to reduce flood stages on the Ohio River.

Moderate flooding occurred in extreme western Kentucky on tributaries to the Mississippi River; however, peak discharges did not exceed previous maximum stages or discharges.

\section{INDIANA}

The floods of March 1964 were the greatest since 1913 in the extreme southern counties of Indiana. In several places the recurrence intervals of the 1964 flood were more than 50 years. In counties farther north, the 1964 flood was less severe than the 1959 flood. Flooding was greatest in the White River and the Patoka River basins and along streams that flow directly into the Ohio River. In the Patoka River basin some stages and discharges exceeded those in 1913 (fig. 10).

Both floods were heaviest in the southern third of the State. Nearly one-third of the average annual rainfall for the area fell during the week of March 3-10. Total precipitation for the two storms exceeded 13 inches at Louisville, $\mathrm{Ky}$., and 10 inches at Evansville, Ind. Unofficial rainfall reports of more than 12 inches were common throughout the flood area. The magnitude of the flood discharges during the period March 3-5 indicates that much greater rainfall must have fallen than was recorded by official rain gages.

Flood damage was estimated to be several million dollars. In 25 southern counties the total damage to publicly owned facilities alone exceeded $\$ 700,000$, as reported by the State of Indiana Office of Emergency Planning. Indiana highways were closed by floodwaters at more than 40 places on March 10,1964.

Hundreds of families were evacuated in Evansville, Ind., when storm runoff from Pigeon Creek, augmented by backwater from the Ohio River, flooded residential areas. Milltown and other cities in the Blue River valley were inundated by a flood nearly identical in stage to that in 1959 .

The American Red Cross reported that 2 deaths were caused by the floods; 38 dwellings were destroyed and 2,714 received major or minor damage. Families suffering losses totaled 3,687.

Discharges at selected gaging stations in Indiana on March 4-13 are shown in figure 11. 


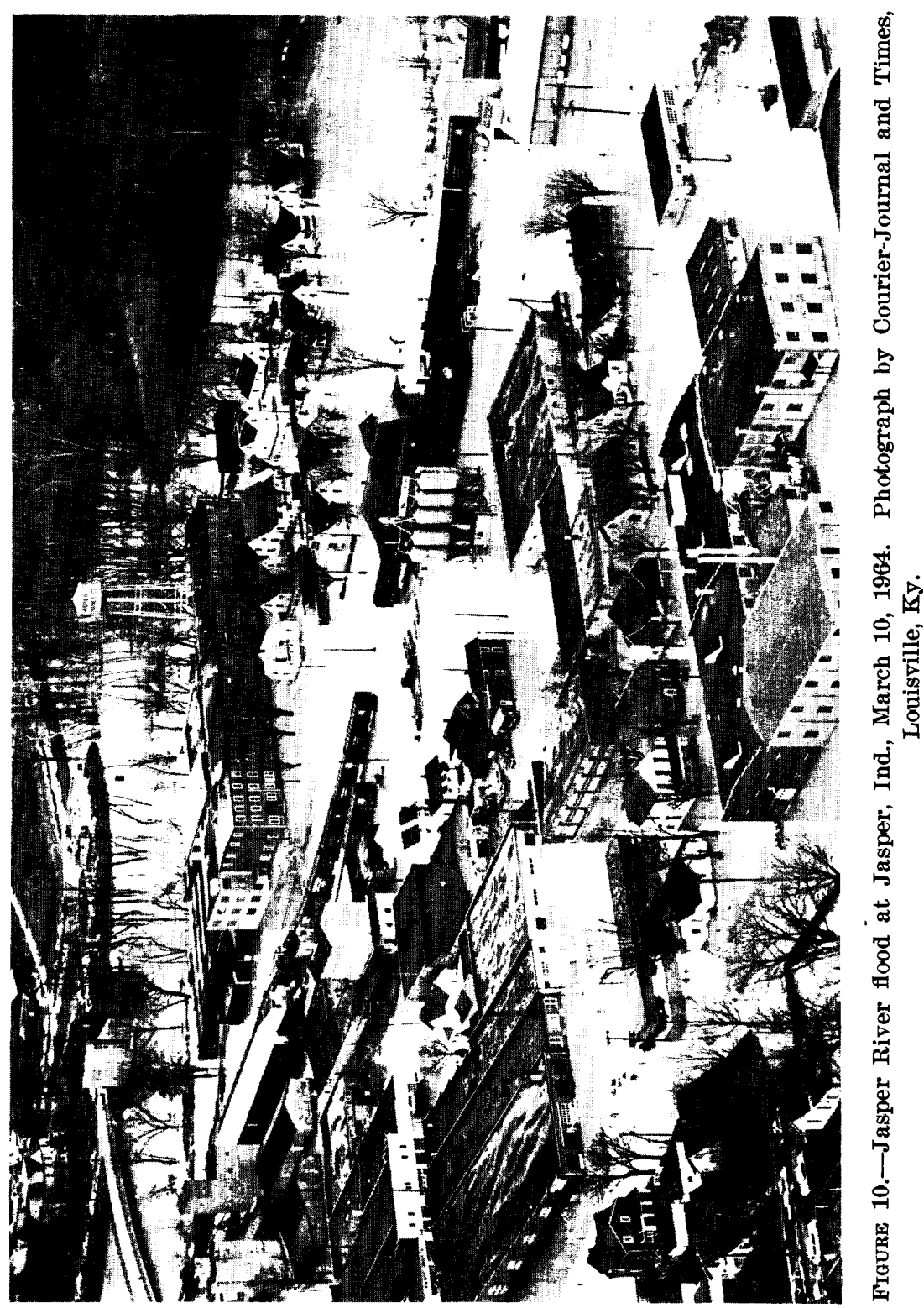




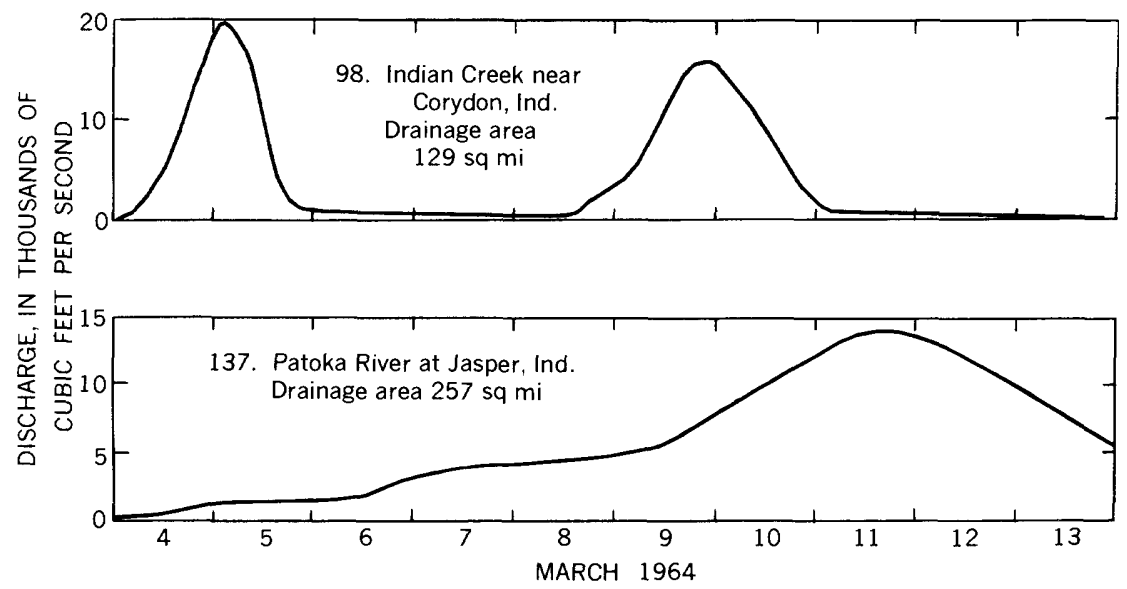

Frouke 11.-Discharge hydrographs at selected gaging stations, March 4-13, 1964 , in Indiana.

\section{OHIO RIVER MAIN STEM}

The Ohio River and most of its tributaries received heavy concentrations of rainfall during March 1964. Flood stages were exceeded throughout the entire reach of the main stem from Pittsburgh, $\mathrm{Pa}$, to Cairo, Ill. Many cities, towns, railroads, and highways are situated on the riverbank or along the relatively narrow confines of the flood plains of the Ohio River. Since the floods of 1913, 1936, and 1937, local flood-protection projects, which have prevented damage totaling millions of dollars, have been completed in many cities along the river. The amount of flood damage in March 1964 along the Ohio River main stem was estimated by the Corps of Engineers at $\$ 80$ million.

Figure 12 shows comparative stages for gaging stations on the Ohio River for March 1964 and the maximum stages previously known. From Sewickley, Pa., to Ashland, Ky., the March 1964 floods were exceeded by several previous floods. At Maysville, Ky., and Golconda, Ill., the crest stages for March were third highest since 1937, and those at Cincinnati, Ohio, Louisville, Ky., and Evansville, Ind., were second highest since 1937.

The March floods closed innumerable roads and highways, inundated bridges, tied up traffic, and interrupted train and bus services at many points. Many cities were isolated by floodwaters. Thousands of persons were evacuated prior to the floods. Floodwaters ran through downtown sections of dozens of towns and cities, encroached upon industrial plants and business houses, and inundated croplands. 


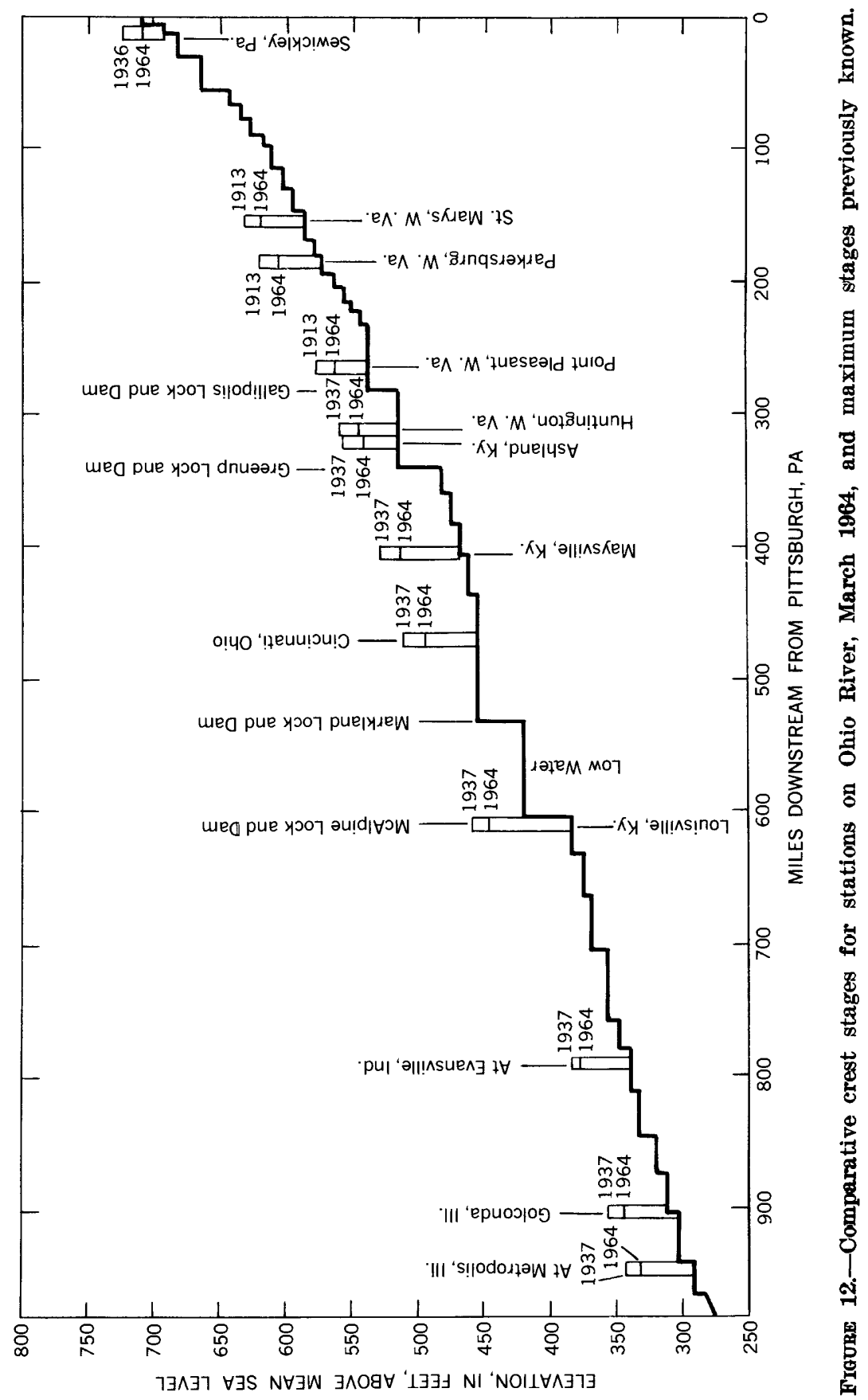


Stages on the Ohio River in Pennsylvania were not particularly high. The peak discharge for the March flood at Sewickley, Pa. (No. 3), was the sixth highest since records began in 1933. The peak 1964 stage of 21.19 feet was far below that of 34.75 feet in 1936 .

The business districts of New Cumberland, Wheeling, and New Martinsville in the northern part of West Virginia were inundated by floodwaters and thousands of homes were damaged. More than 15,000 persons were displaced in the Wheeling region. New Martinsville, W. Va., was virtually isolated. Families were evacuated at Steubenville, Martins Ferry, and Bellaire, Ohio. At Bellaire, just across the river from Wheeling, W. Va., some industrial plants suspended operation.

At Ohio River at St. Marys, W. Va. (No. 5), the maximum stage of 42.75 feet during March was second highest since 1938 but was well under the maximum known stage of 54.2 feet in 1913 .

Approximately 1,000 persons were forced from their homes at Marietta, Ohio. Streets were flooded, part of the business district was covered by several feet of water, and major highways into the city were blocked.

The peak discharge on Ohio River at Parkersburg, W. Va. (No. 12), was the largest since the completion of floodwall protection in 1950 but slightly less than that during the floods of 1943 and 1945 . During March 1964 many families were forced to move from the low-lying unprotected areas. Highways were closed and passenger train and bus services were disrupted.

The main-street business section at Pomeroy, Ohio, was flooded with several feet of water. Downstream at Point Pleasant, W. Va., which was protected by a floodwall, the peak discharge was fifth highest since beginning of gaging-station operations in 1940 but was far below the flood of 1913. Low-lying areas and streets were flooded and schools closed at Gallipolis, Ohio.

The cities of Huntington, W. Va., Ashland, Ky., and Ironton and Portsmouth, Ohio, were protected from the March flood by floodwalls. Flood stages at gaging stations at Huntington (No. 27) and Ashland (No. 29) were considerably below those of numerous floods in previous years.

Manchester, Ripley, and New Richmond, Ohio, were flooded and many persons were evacuated. New Richmond was especially hard hit by several feet of floodwaters in the business district. Families were evacuated at Vanceburg and Augusta, Ky., and the business district was covered in Augusta.

The peak stage of 62.65 feet at the gaging station near Maysville, Ky. (No. 45), was third highest since the maximum known flood in 
1937 ; however, the city of Maysville, protected by a floodwall, received no serious flooding.

In the Cincinnati, Ohio, metropolitan area, including Covington and Newport, Ky., flooding was confined almost entirely to lowlands unprotected by floodwalls. In these areas streets and basements were flooded, minor landslides occurred, and thousands of persons were evacuated. Disaster headquarters was established in Cincinnati to assist more than 100,000 persons in five States. The peak stage of 66.20 feet at the Cincinnati gaging station (No.61) was second highest since the maximum known flood in 1937. Six previous floods exceeded the flood of March 1964: those in 1937, 1773, 1884, 1913, 1945, and 1883 (in order of magnitude).

Businesses and homes in Aurora and Madison, Ind., were flooded. U.S. Highway 421 across the Ohio River at Madison was closed to traffic. Foodwaters inundated those parts of the Louisville, $\mathrm{Ky}$., and New Albany and Jeffersonville, Ind., metropolitan areas that were not protected by floodwalls. Basements, roads, streets, and viaducts were flooded in many places. Numerous schools in the area were closed. Evacuees from hundreds of homes were lodged in schools and armories manned by Civil Defense and Red Cross personnel.

In the Louisville, $\mathrm{Ky}$., area fire alarms were the most numerous in years, and the Metropolitan Sewer District received many telephone calls. The Southern Bell Telephone Co. received 3,800 more requests than normal for repair service. Electric power was disrupted in Jefferson and Bullitt Counties, in at least 1,200 homes, if interruption of the power can be based on the number of meters reported inundated by the Louisville Gas and Electric Co. Financial assistance by the American Red Cross to flood victims in Jefferson County was slightly over $\$ 231,000$.

The peak stage of 73.46 feet on Ohio River at Louisville (No.78) was the third highest known. This flood was exceeded only by the floods in 1884 and 1945 (each 74.4 feet) and the greatest flood known, in 1937 ( 85.44 feet).

Numerous towns and cities along the Ohio River between Louisville, Ky., and Evansville, Ind., sustained major damage. About 80 percent of West Point, Ky., was evacuated. Evacuations were also made at Brandenburg, Ky., Lewisport, $\mathrm{Ky}$., and Grandview, Ind., as well as at other communities along the river. Owing to floodwall protection, only minor flooding occurred at Tell City, Ind., Cannelton, Ind., and Hawesville, Ky.

The flood of March 1964 at Evansville, Ind., was sixth highest since 1874. Ohio River at Evansville (No. 116) reached a stage of 47.72 feet, which was 0.6 to 1.1 feet lower than the floods of $1883,1884,1913$, and 
1945 , and 6.0 feet lower than the flood of 1937. Many families were displaced in low-lying areas outside the protection of the city's floodwall.

Henderson, Ky., Uniontown, Ky., and Golconda, Ill, had flooding in low-lying areas adjacent to the Ohio River. Both Uniontown and Golconda were protected by floodwalls. The Ohio River reached a peak stage of 52.40 feet at Golconda, Ill. (No. 142), as compared with the highest previous crest of 62.6 feet in 1937 . Since 1937 only the 1945 and 1950 floods have exceeded the March 1964 flood at this point.

Flood damage at Paducah, Ky., was extremely high, and although the city was protected by a floodwall, hundreds of homes were inundated in suburban areas owing to local surface runoff and to flow from small tributaries. Barge traffic was tied up at Paducah because of high water on the Ohio River. Hundreds of families were evacuated from low-lying lands in McCracken County in the vicinity of Paducah.

The peak discharge of $1,100,000 \mathrm{cfs}$ at the gaging station at Metropolis, Ill. (No. 147), was not particularly high in comparison with discharges for floods in other years. Flow of the March 1964 flood was considerably reduced by storage in Kentucky Lake and other reservoirs in the Tennessee and the Cumberland River basins.

Downstream from Metropolis, Ill., thousands of acres of land were inundated by the Ohio River and by backwater from tributaries and ditches. Severe flooding, due in part to backwater from the Ohio River, occurred in the Saline River basin in southern Illinois, and, on March 18 near Junction, Ill., 15,200 cfs was measured flowing upstream.

Discharge at gaging stations on the Ohio River on March 3-31 is shown in figure 13 .

\section{FLOOD DAMAGE}

The floods of March 1964 along the Ohio River caused heavy damage in Pennsylvania, West Virginia, Ohio, Kentucky, Indiana, and Illinois. Most of the damage occurred along the Ohio River main stem and was extensive along the entire reach from Pittsburgh, $\mathrm{Pa}$., to Cairo, Ill.

Flood-damage figures furnished by the Corps of Engineers and the Indiana Flood Control and Water Resources Comm. totaled about $\$ 99$ million in the six-State area. This total does not include some minor damage from tributaries in various States or damage in the White River basin in Indiana; therefore, total damage is estimated to be slightly over $\$ 100$ million of which about 75 percent occurred along Ohio River main stem. Estimated figures furnished by the Corps of Engineers were based on stage-damage curves and do not represent detailed damage investigations. 

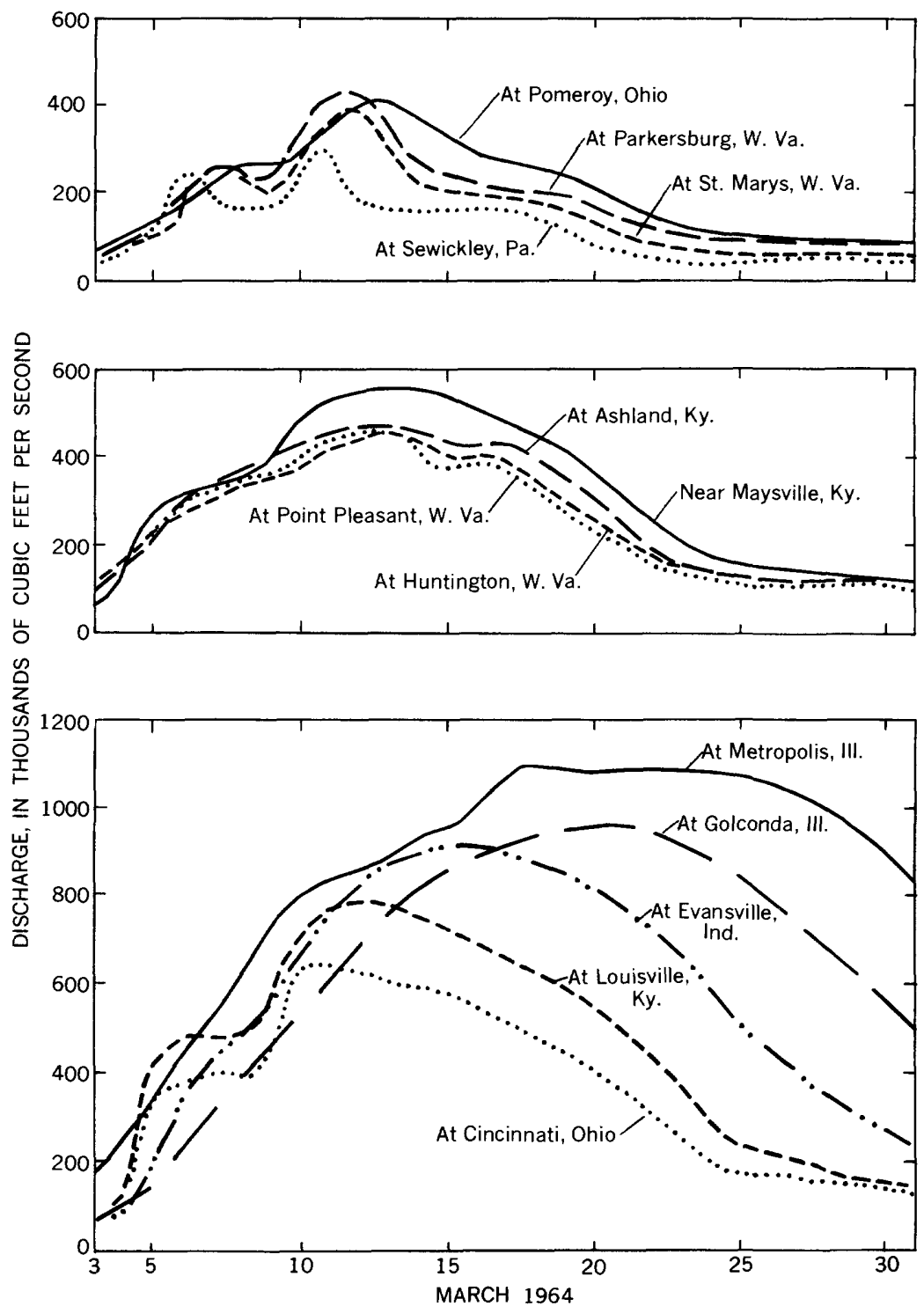

Figure 13.-Comparative hydrographs of discharge for stations on the Ohio River, March 3-31, 1964. 
Table 1 lists damage by reaches of the Ohio River and by States. A breakdown of main-stem damage by States is not available as stagedamage curves for the Ohio River main stem refer to both sides of the river.

TABLE 1.-Flood damage along the Ohio River, March 1964

[Furnished by the Corps of Engineers except where noted]

\begin{tabular}{|c|c|}
\hline River reach and State & $\begin{array}{l}\text { Flood damage, } \\
\text { in thousands } \\
\text { of dollars }\end{array}$ \\
\hline $\begin{array}{l}\text { Ohio River main stem: } \\
\text { Pennsylvania (including tributaries) } \\
\text { Ohio and West Virginia } \\
\text { Ohio and Kentucky } \\
\text { Indiana and Kentucky. } \\
\text { Illinois and Kentucky } \\
\text { Ohio (excluding Ohio River main stem) } \\
\text { Kentucky (excluding Ohio River main stem) } \\
\text { Indiana (Patoka River only) }{ }^{1}\end{array}$ & $\begin{array}{r}\$ 11,740 \\
21,346 \\
25,154 \\
18,806 \\
2,618 \\
9,991 \\
8,517 \\
840\end{array}$ \\
\hline Total. & 299,012 \\
\hline
\end{tabular}

1 Furnished by Indiana Flood Control and Water Resources Comm.

2 Does not include numerous small tributaries to the Ohio River in various States or the White River basin in Indiana.

According to the American Red Cross, 21,745 homes were damaged and 181 were destroyed in Pennsylvania, Ohio, Kentucky, West Virginia, and Indiana. More than 29,000 families suffered losses of various kinds. Eighteen lives were lost in the March 1964 floods. Expenditures by the Red Cross for relief to flood victims are tabulated by States in table 2.

TABLE 2.-Expenditures by the American Red Cross, by States, as a result of the floods of March 1964 along the Ohio River

\begin{tabular}{|c|c|c|c|c|c|c|}
\hline Expenditure classification & Indiana & Kentucky & Ohio & $\begin{array}{l}\text { Pennsyl- } \\
\text { vania }\end{array}$ & $\begin{array}{l}\text { West } \\
\text { Virginia }\end{array}$ & Total \\
\hline Emergency mass care & $\$ 11,152$ & $\$ 38,885$ & $\$ 96,734$ & $\$ 5,797$ & $\$ 7,343$ & $\$ 159,911$ \\
\hline $\begin{array}{l}\text { Individual family care: } \\
\text { Food, clothing, and mainte- }\end{array}$ & 21.757 & 70.174 & 191.911 & 7,987 & 10,485 & 302,314 \\
\hline Building and repair of homes.- & 76,266 & 214,171 & 169,401 & 13,244 & 15,149 & 488,231 \\
\hline Household furnishings & 86,110 & 333,483 & 501,364 & 31,649 & 41,623 & 994,229 \\
\hline $\begin{array}{l}\text { Medical and nursing care } \\
\text { Occupational supplies and }\end{array}$ & 2500 & 6,068 & 9,827 & 324 & 423 & 19,142 \\
\hline $\begin{array}{l}\text { equipment... } \\
\text { Other assistance and costs }\end{array}$ & $\begin{array}{r}2,417 \\
15,899\end{array}$ & $\begin{array}{r}9,015 \\
53,350\end{array}$ & $\begin{array}{r}9,032 \\
77,688\end{array}$ & $\begin{array}{r}101 \\
4,693\end{array}$ & $\begin{array}{l}1,269 \\
6,059\end{array}$ & $\begin{array}{r}21,834 \\
157,689\end{array}$ \\
\hline Total relief costs & 216,101 & 725,146 & $1,055,957$ & 63,795 & 82,351 & $2,143,350$ \\
\hline
\end{tabular}

\section{RECURRENCE INTERVALS OF PEAK DISCHARGES}

The recurrence interval, or return period, of a flood of a given magnitude is the average interval of time within which the given flood will be equaled or exceeded once. The recurrence intervals of the peak discharges of the floods of March 1964, shown in the last column of table 4, were mostly computed from Water-Supply Paper 1675, 
"Magnitude and frequency of floods in the United States, Part 3-A, Ohio River basin except Cumberland and Tennessee River basins."

Figure 14 shows recurrence intervals at the points of peak determination where the data can be computed. The data cannot be computed for streams that are controlled or appreciably regulated or for streams of drainage areas less than about 30 square miles. The frequency curves in WSP 1675 are defined to 50 years; extrapolation of recurrence intervals beyond 50 years is not recommended. A peak discharge having a recurrence interval greater than 50 years is described as its ratio to the 50-year flood.

Figure 14 also shows the distribution of recurrence intevals of the floods of March 1964. The storm moved in a narrow belt up the Ohio River valley and did not affect the upper ends of the medium and large tributaries that lie, in general, perpendicular to the Ohio River. The short tributaries to the Ohio River and the reaches of streams lying parallel and near to the Ohio River mostly had peaks of high recurrence intervals.

No flood-determination points lying outside the area bounded by the 7-inch isohyet (fig. 5) had peaks of high recurrence intervals. Within the 7-inch isohyet the recurrence interval of 50 years or greater was principally on small streams; however, recurrence intervals of peaks were high on some medium and large streams in which appreciable parts of the drainage basins lie within the area bounded by the 10- and 12-inch isohyets. For example, the peak discharge on Licking River at Catawba, Ky. (No. 60), and on Eagle Creek at Glencoe, Ky. (No. 74), each had recurrence intervals that were greater than 50 years.

The effect of the accumulating discharges from tributary streams on the recurrence interval of the discharge in the Ohio River main stem is shown by table 3 .

TABLE 3.-Recurrence intervals of peak discharges of March 1964 at gaging stations on the Ohio River

\begin{tabular}{|c|c|c|c|}
\hline Location of gaging station & $\begin{array}{l}\text { Miles } \\
\text { downstream } \\
\text { from Pitts- } \\
\text { burgh, Pa. }\end{array}$ & $\begin{array}{l}\text { Drainage } \\
\text { area } \\
\text { (sq mi) }\end{array}$ & $\begin{array}{c}\text { Recurrence } \\
\text { interval } \\
\text { (years) }\end{array}$ \\
\hline $\begin{array}{l}\text { At Huntington, W. Va } \\
\text { At Ashland, Ky } \\
\text { Near Maysville, } \overline{\mathbf{K}}_{\mathrm{y}} \\
\text { At Cincinnati, Ohio } \\
\text { At Louisville, Ky } \\
\text { At E vansville, Ind, } \\
\text { At Golconda, IIl }\end{array}$ & $\begin{array}{l}311.6 \\
322.5 \\
405.1 \\
470.5 \\
607.3 \\
792.3 \\
903.1\end{array}$ & $\begin{array}{r}\mathbf{5 5}, \mathbf{9 0 0} \\
60,750 \\
70,130 \\
76,580 \\
91,170 \\
107,000 \\
143,900\end{array}$ & $\begin{array}{l}2 \\
3 \\
5 \\
12 \\
44 \\
50+ \\
14\end{array}$ \\
\hline
\end{tabular}

As the flood wave moved downstream the recurrence interval continued to increase until the peak discharge at Evansville, Ind. (No. 116), became 1.08 times as large as the 50 -year flood. The Wabash 
River enters the Ohio River below Evansville. The peak discharge on the farthest downstream gaging station on the Wabash River, at Mount Carmel, Ill. (No. 140)-which has a drainage area, 28,600 square miles-had a recurrence interval of about 2 years. The amount of flow contributed by the Wabash River to the Ohio River main stem was insignificant, and by the time the flood wave on the Ohio River reached Golconda, Ill. (No. 142), the recurrence interval had been reduced to 14 years.

\section{DETERMINATION OF FLOOD DISCHARGES}

The operation of a stream-gaging station is principally for the purpose of establishing a relation between stage and discharge from which the discharge can be calculated when the stage is known. The stagedischarge relation (rating curve) is based upon current-meter measurements throughout the range of stage experienced or through a sufficient part of the range so that discharge corresponding to maximum stage can be obtained by a reasonable extension of the rating curve. Short extensions of rating curves are usually made by logarithmic plotting or from velocity-area studies and other measurable hydraulic factors.

During major floods current-meter measurements are often impossible to obtain because of insufficient notice of flood stages on rapidly changing streams and because of impassable roads, heavy floating debris, or destruction of structures from which flood measurements are made. Where necessary, the stage-discharge relation at many gaging stations was extended to peak flow by means of indirect measurements such as computation of flow through contracted openings, slope-area measurements, or a combination of these methods. At several miscellaneous sites where high runoff occurred, the peak flow was determined by indirect measurement.

For a number of gaging stations in this report, fall (or slope) in the reach between two gages was a factor in establishing a relation between stage and discharge and was also a factor in computing discharge. At a few stations, the stage-discharge relation was affected by changing stage. For such stations, rate of change in stage was a factor in determining discharge.

\section{STREAMFLOW DATA AT GAGING STATIONS}

\section{EXPLANATION OF DATA}

The following section gives detailed information on stage and discharge during the floods of March 1964. Much of the information is in addition to records usually published in annual streamflow reports of the Geological Survey. 
The systematic collection of basic data at a stream-gaging station includes a record of stage, measurements of discharge, and any other general information pertinent to determination of daily flow at the station. Record of stage is determined from periodic readings of some type of nonrecording gage, from an automatic water-stage recorder which gives a continuous graph of stage, or from a digital recorder which provides a tape punched at $15-, 30$-, or 60 -minute intervals. Measurements of discharge generally are made by current meter.

In general, station data consist of a description of the station, a table showing daily mean discharge at gaging stations for March 1964, and a table of stages and discharges at indicated times for many of the gaging stations.

\section{STATION DESCRIPTIONS AND DISCHARGE TABLES}

The description of the gaging station gives information concerning location, datum, type of gage, area of drainage basin, details of gageheight and discharge records, and miscellaneous remarks. The paragraph on discharge record briefly explains methods used to define stagedischarge relation over the range of stage that occurred during the floods; use of auxiliary gages or other methods used to obtain discharge; and conditions that may have affected the stage-discharge relation.

Maximum stage and discharge are given for the March 1964 period and for the period of previous record. Information regarding floods outside the period of gaging-station operation also is given in as much detail as possible. Remarks on cooperation, regulation and diversion, and other pertinent information are included where applicable.

The table of daily mean discharges gives data for March 1964; however, for some stations discharges are given for the first few days in April 1964. Periods of daily discharge were chosen to show the relation of flood discharges to discharges of the preceding and the following periods. The table also shows monthly mean discharge and generally shows the volume of runoff, in inches, from the drainage area. The volume was not computed if flow at a station was affected by more than an estimated 10 percent by regulation or if it was known that the storm did not cover the entire drainage basin.

The table of stages and discharges at indicated times generally is presented for the period starting March 2 and ending when the recession reached the point where sufficient definition for a hydrograph is furnished by the table of daily mean discharges. These data are not given for most stations outside of the flooded areas.

The stages at most stations were obtained from records of digital or continuous water-stage recorders. For a station with an incomplete 
stage record, the gage height at a specific time may be selected from a graph which has been reconstructed from supplemental gage readings, high-water marks, and other pertinent evidence. Description of the methods used in defining the stage are given in the section of the description concerning gage-height record.

The stations are numbered and arranged in downstream order from headwater to mouth, with stations on tributaries inserted in corresponding order following the order in which the tributaries enter the main stream. The reference number preceding the name of the gaging station is the same as that used in figure 2 and will aid in identifying the site.

\section{SUMMARY OF PEAK STAGES AND DISChaRges}

Maximum floodflows at stream-gaging stations and other sites on streams in the area covered by this report are summarized in table 4. The reference number is the same as that designating the site in figure 2 and will aid in identifying the location at which discharge was determined. As an added means of identification, each gaging station and partial-record station is listed with its permanent station number in the same downstream order used in the annual streamflow reports of the Geological Survey. No distinction is made between partialrecord stations and continuous-record gaging stations. Numbers are not consecutive as gaps were left in the number series to allow for new stations that might be established.

In table 4 the first column under maximum floods shows the period of known floods prior to March 1964. This period does not necessarily correspond to that in which continuous records of discharge were obtained, and for some stations the record extends back to an earlier date. More than one period of record is shown for some stations. Time periods are shown when they can be associated with a maximum stage even though the corresponding discharge is not known; a second period of known floods is then given in which maximum discharge and stage are both known.

The last column shows the average interval of time in which the peak discharge of March 1964 can be expected to be equaled or exceeded once. Whenever the peak discharge exceeds that of the 50-year recurrence interval (the maximum defined by frequency curves), the ratio of the peak discharge to the 50-year flood is shown. 


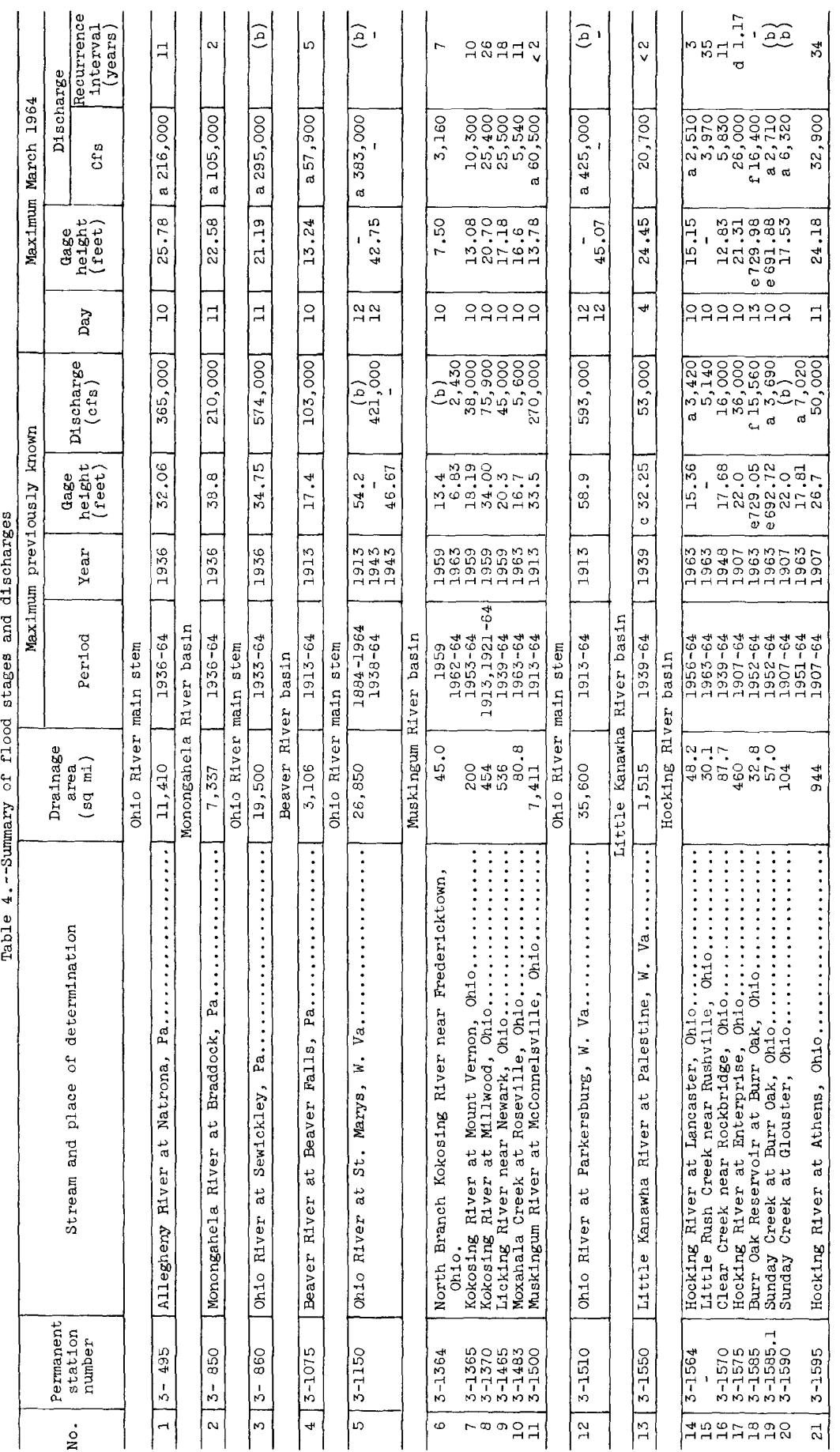




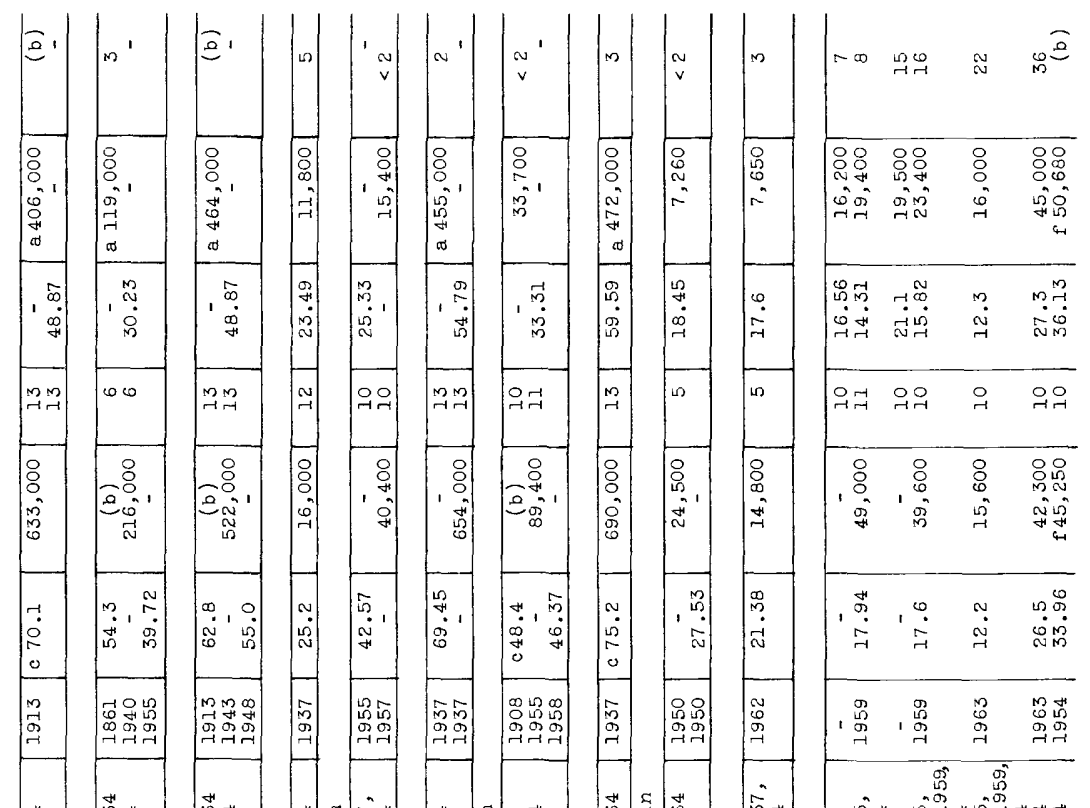

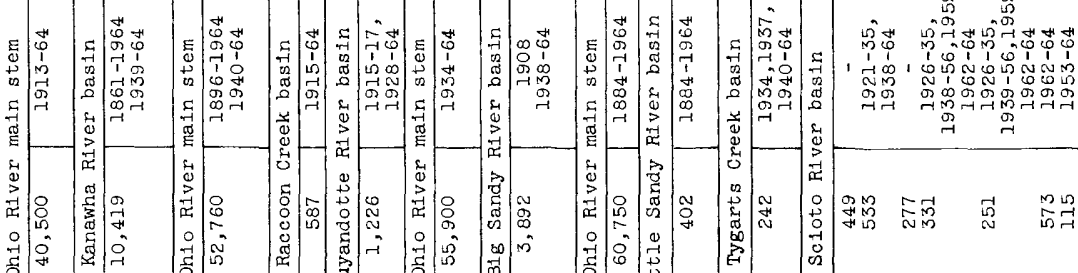

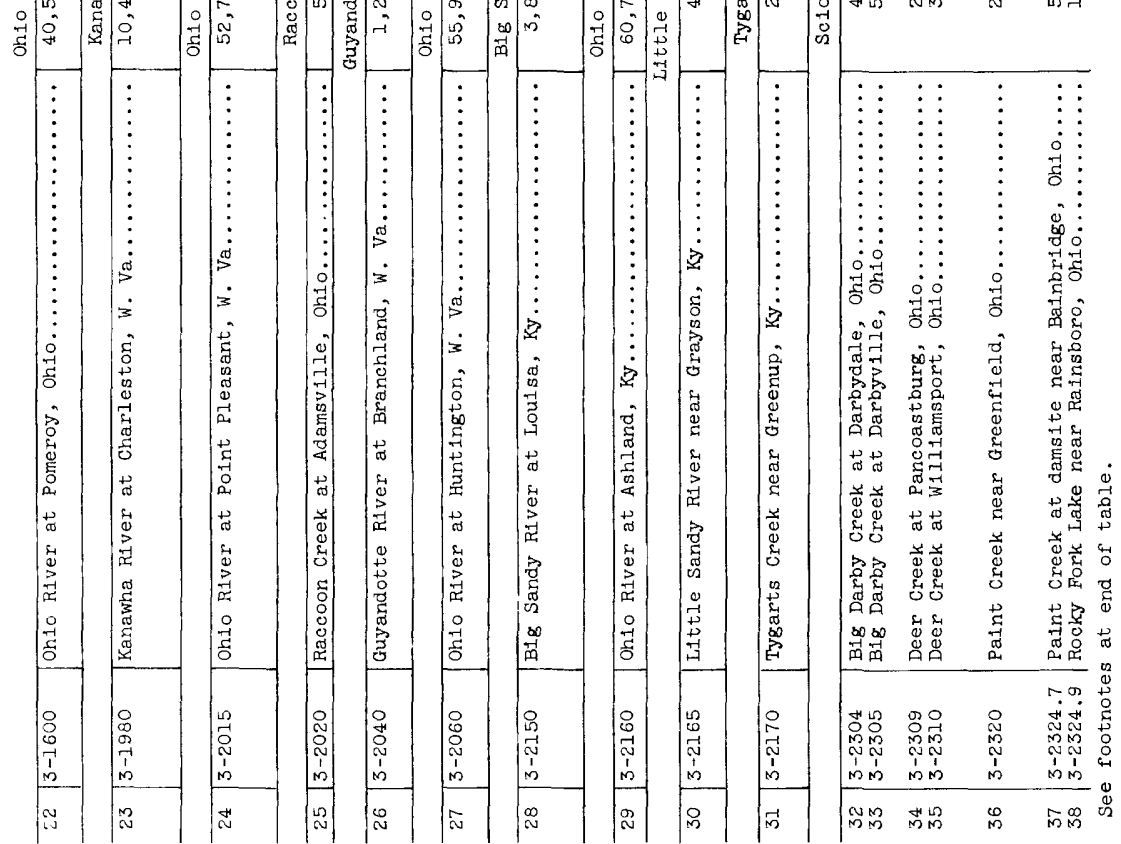




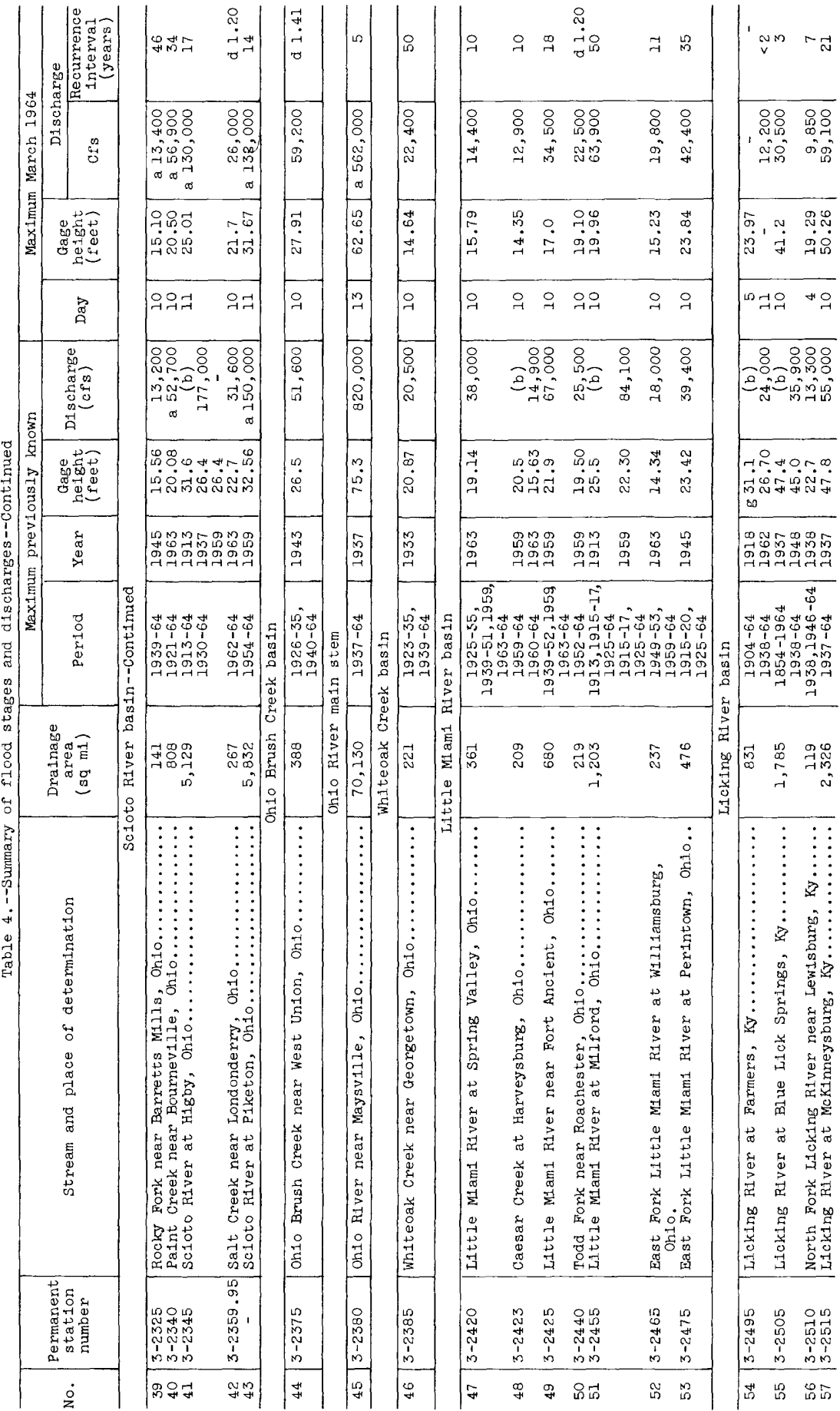




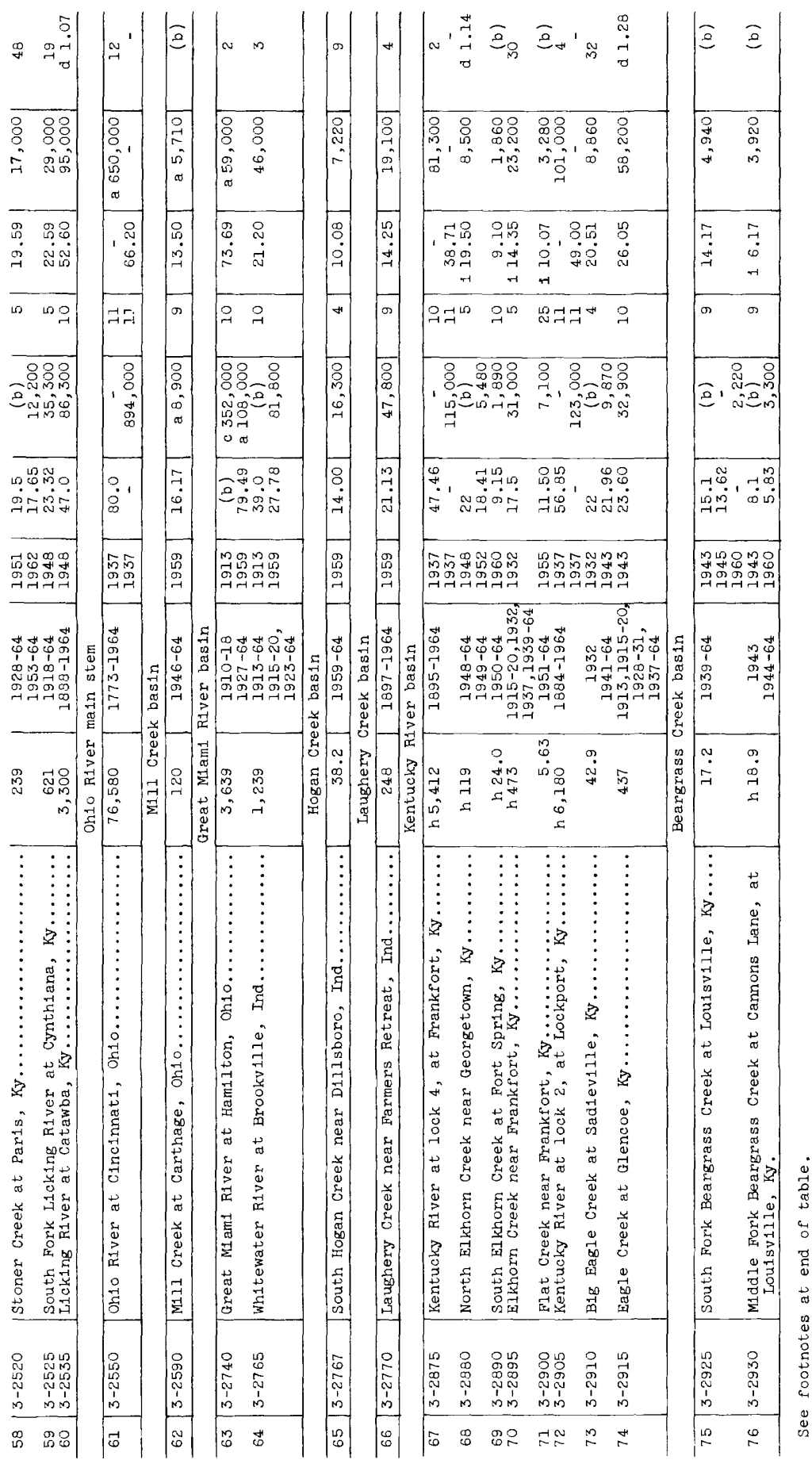




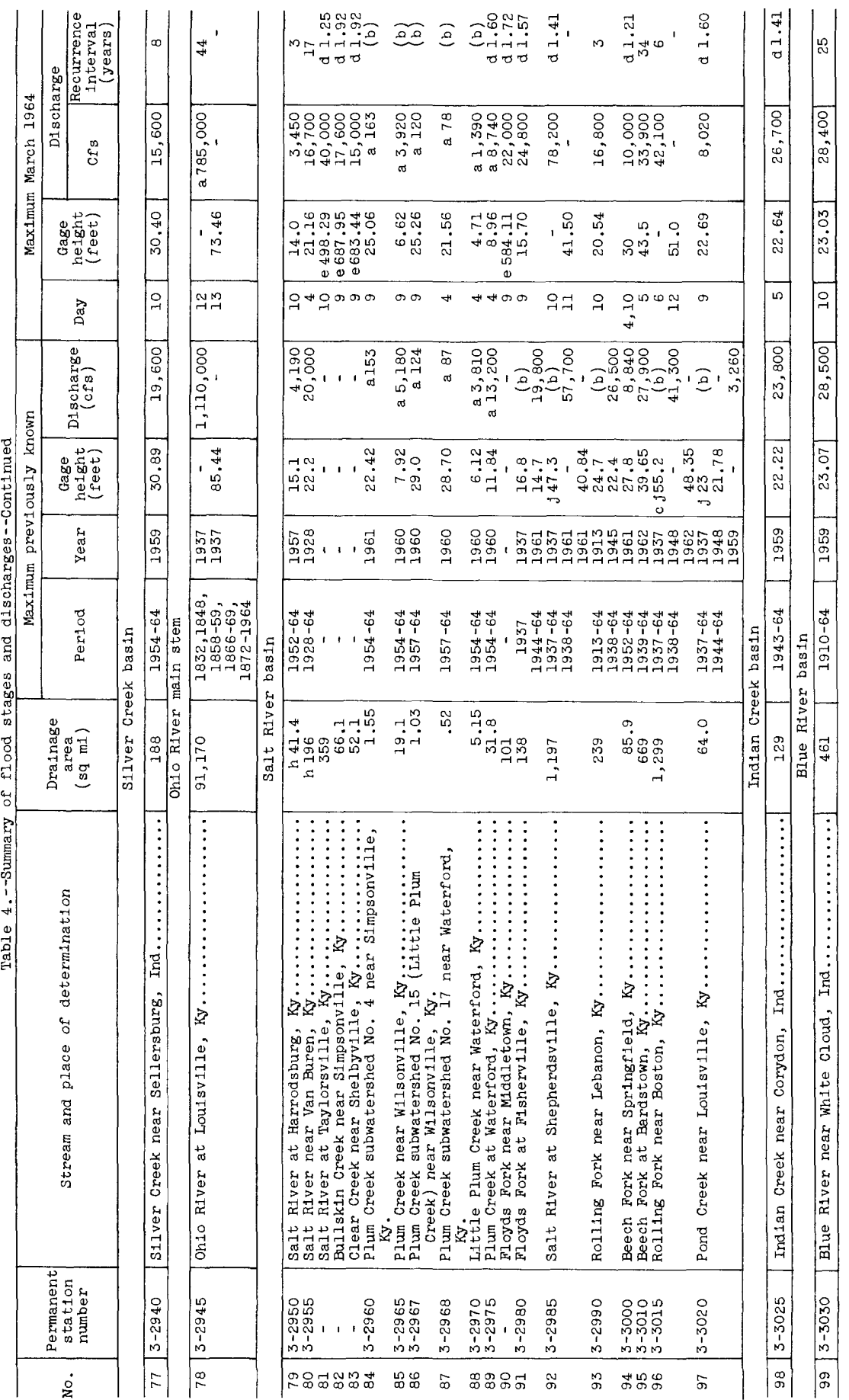




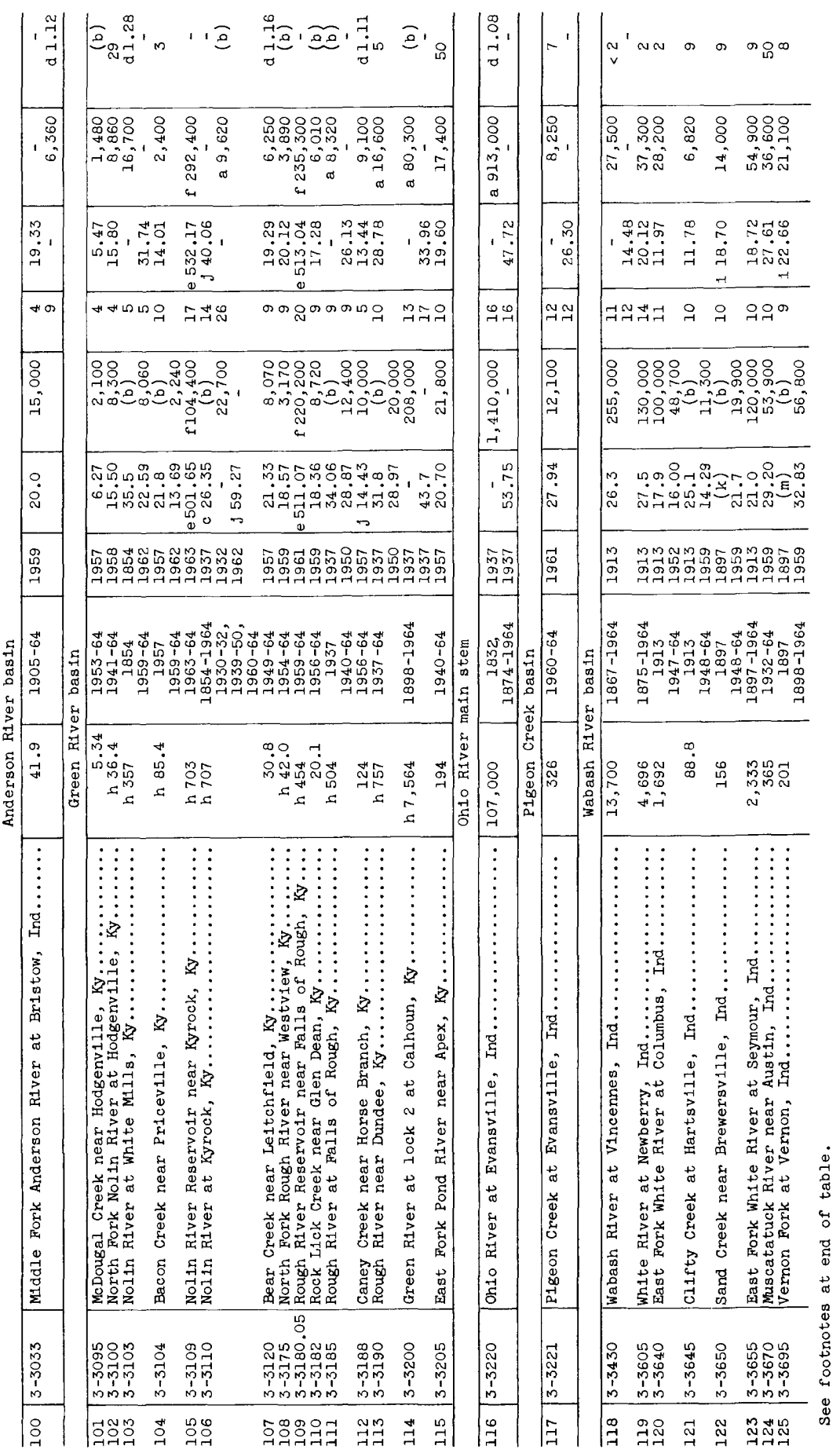




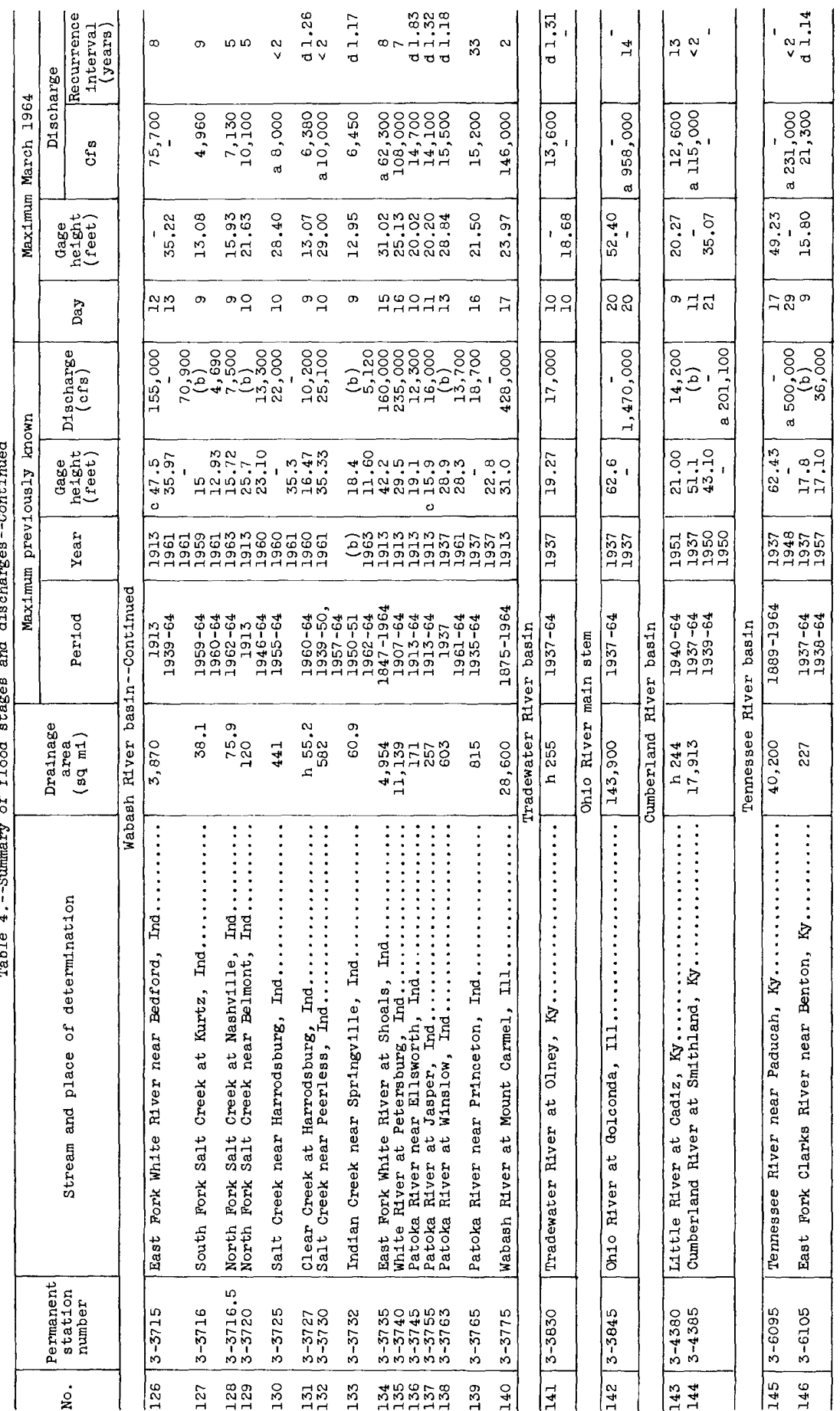




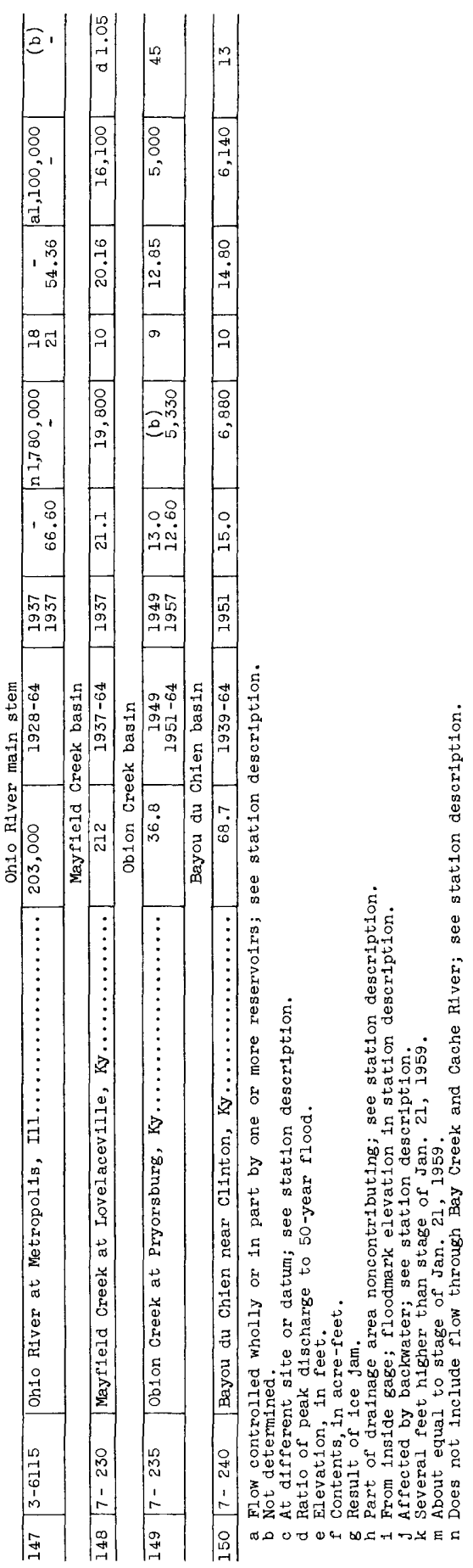




\section{STATION DATA}

\section{OHIO RIVER MAIN STEM}

(1) 3-495. Allegheny River at Natrona, $\mathrm{Pa}$.

Location.--Lat $40^{\circ} 36^{\prime} 55^{\prime \prime}$, long $79^{\circ} 43^{\prime} 10^{\prime \prime}$, on right bank 550 ft upstream from dam at lock 4 at Natrona, Allegheny County, 6 miles downstream from Kiskiminetas River, and at mile 24.2 .

Drainage area.--11,410 sq $\mathrm{mi}$, approximately.

Gage-helght record.--Water-stage recorder graph. Datum of gage is $736.74 \mathrm{ft}$ above mean sea level, adjustment of 1912 (Corps of Engineers bench mark).

Discharge record.--Stage-discharge relation defined by current-meter measurements .

Maxima --March 1964: Discharge, 216,000 cfs Mar. 10, 2200 hours (gage he1ght, $25.78 \mathrm{ft}$ ).

1938 to February 1964: D1scharge, 238,000 efs Dec. 30, 1942 (gage height, $27.46 \mathrm{ft}^{\mathrm{t}}$ ).

Maximum stage known, $32.06 \mathrm{ft}$ Mar. 18, 1936 (discharge, 365,000 ofs, determined by Corps of Engineers).

Remarks:--Flow regulated by Chautauqua Lake and by Tionesta Creek, East Branch Clarion River, Mahoning Creek, Crooked Creek, Conemaugh River, and Loyalhanna Creek Reservoirs and by 12 smaller reservoirs (combined capacity, excluding that of Chautauqua Lake, 864,600 acre-ft). Slight diversion since 1952 from Beaver Run Reservolr into the Monongahela River basin.

Mean discharge, in cubic feet per second, March 1964

\begin{tabular}{|c|c|c|c|c|c|c|c|c|c|}
\hline Day & Discharge & Day & Discharge & Day & Discharge & Day & Discharge & Day & Discharge \\
\hline $\begin{array}{l}1 \ldots \ldots \\
2 \ldots \ldots \\
3 \ldots \ldots \\
4 \ldots \\
5 \ldots \ldots \\
6 \ldots\end{array}$ & $\begin{array}{r}5,800 \\
6,450 \\
10,100 \\
17,300 \\
66,700 \\
159,000\end{array}$ & $\begin{array}{r}7 \ldots \ldots \\
8 \ldots \ldots \\
9 \ldots \\
10 \ldots \\
11 \ldots \\
12 \ldots\end{array}$ & $\begin{array}{l}131,000 \\
131,000 \\
130,000 \\
188,000 \\
186,000 \\
134,000\end{array}$ & $\begin{array}{l}13 \ldots \ldots \\
14 \ldots \\
15 \ldots \\
16 \ldots \\
17 \ldots \\
18 \ldots\end{array}$ & $\begin{array}{r}127,000 \\
117,000 \\
104,000 \\
104,000 \\
100,000 \\
84,000\end{array}$ & $\begin{array}{l}19 \ldots \ldots \\
20 \ldots \ldots \\
21 \ldots \ldots \\
22 \ldots \ldots \\
23 \ldots \\
24 \ldots \ldots\end{array}$ & $\begin{array}{l}61,300 \\
42,200 \\
32,800 \\
30,000 \\
28,000 \\
27,000\end{array}$ & $\begin{array}{l}25 \ldots \ldots \\
26 \ldots \ldots \\
27 \ldots \ldots \\
28 \ldots \ldots \\
29 \ldots \ldots \\
30 \ldots \ldots \\
31 \ldots \ldots\end{array}$ & $\begin{array}{l}26,900 \\
27,200 \\
33,600 \\
37,400 \\
34,000 \\
31,400 \\
30,500\end{array}$ \\
\hline
\end{tabular}

Gage height, in feet, and discharge, in cubic feet per second, at indicated time, 1964

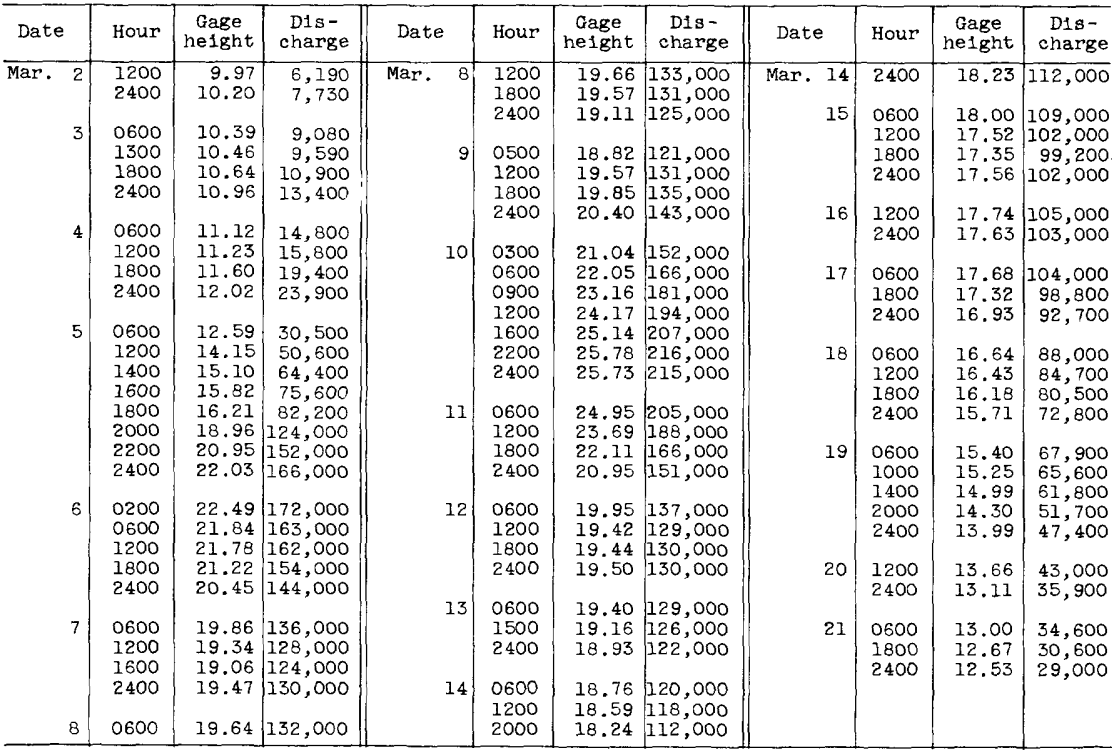

Note.--Da1ly means for some days computed from data in addition to flgures shown. 


\section{MONONGAHELA RIVER BASIN}

(2) 3-850. Monongahela River at Braddock, Pa.

Location.--Lat $40^{\circ} 23^{\prime} 30^{\prime \prime}$, long $79^{\circ} 51^{\prime} 30^{\prime \prime}$, near right bank on river gulde wall $380 \mathrm{ft}^{\prime}$ upstream from dam at lock 2 , at Braddock, Allegheny County, 1,700 ft downstream from Turtle creek, and 11.2 miles upstream from confluence with Allegheny River.

Drainage area. $--7,337 \mathrm{sq} \mathrm{mi}$.

Gage-height record.--Water-stage recorder graph. Datum of gage is $707.16 \mathrm{ft}$ above mean sea level, adjustment of 1929.

Discharge record.--Stage-discharge relation defined by current-meter measurements.

Maxima.--March 1964: Discharge, 105,000 cfs Mar. 11, 0500-0600 hours (gage height, $22.58 \mathrm{ft}$ ).

1938 to February 1964: Discharge, 201,000 cfs June 5, 1941 (gage height,

$31.20 \mathrm{ft})$.

Maximum stage known, $38.8 \mathrm{ft}$ Mar. 18, 1936, from floodmarks (discharge, $210,000 \mathrm{cf}$ ).

Remarks.--Flow regulated by locks and hydroelectric plants, since 1938 by Tygart Reservolr and since 1926 by Lake Lynn, since 1925 by Deep Creek Reservoir and since 1943 by Youghiogheny River Reservolr, combined capacity, 718,960 acre-ft. Figures of daily discharge include slight diversion from Beaver Run Reservoir and Thorn Run, in the Kiskiminetas River basin, to the Borough of Jeannette and into Turtle Creek, respectively.

Mean discharge, in cub1c feet per second, March 1964

\begin{tabular}{|c|c|c|c|c|c|c|c|c|c|}
\hline Day & Discharge & Day & D1scharge & Day & Discharge & Day & Discharge & Day & D1scharge \\
\hline $\begin{array}{l}1 \ldots \ldots \\
2 \ldots \ldots \\
3 \ldots \ldots \\
4 \ldots \\
5 \ldots \ldots \\
6 \ldots \ldots\end{array}$ & $\begin{array}{r}6,600 \\
6,750 \\
20,000 \\
38,100 \\
69,100 \\
90,800\end{array}$ & $\begin{array}{r}7 \ldots \ldots \\
8 \ldots \ldots \\
9 \ldots \ldots \\
10 \ldots \\
11 \ldots \ldots \\
12 \ldots\end{array}$ & $\begin{array}{l}45,600 \\
34,700 \\
41,700 \\
73,100 \\
90,200 \\
45,800\end{array}$ & $\mid \begin{array}{l}13 \ldots \ldots \\
14 \ldots \ldots \\
15 \ldots \ldots \\
16 \ldots \ldots \\
17 \ldots \ldots \\
18 \ldots \ldots\end{array}$ & $\begin{array}{l}39,200 \\
41,000 \\
52,800 \\
55,300 \\
47,300 \\
43,800\end{array}$ & $\begin{array}{l}19 \ldots \ldots \\
20 \ldots \ldots \\
21 \ldots \ldots \\
22 \ldots \ldots \\
23 \ldots \ldots \\
24 \ldots \ldots\end{array}$ & $\begin{array}{l}39,300 \\
32,100 \\
25,600 \\
17,300 \\
12,000 \\
13,000\end{array}$ & $\begin{array}{l}25 \ldots \ldots \\
26 \ldots \ldots \\
27 \ldots \ldots \\
28 \ldots \ldots \\
29 \ldots \ldots \\
30 \ldots \ldots \\
31 \ldots \ldots\end{array}$ & $\begin{array}{r}14,000 \\
14,900 \\
13,200 \\
12,900 \\
11,400 \\
9,020 \\
10,100\end{array}$ \\
\hline
\end{tabular}

Gage height, in feet, and discharge, in cubic feet per second, at indicated t1me, 1964

\begin{tabular}{|c|c|c|c|c|c|c|c|c|c|c|c|}
\hline Date & Hour & $\begin{array}{c}\text { Gage } \\
\text { he1ght }\end{array}$ & $\begin{array}{l}\text { D1s - } \\
\text { charge }\end{array}$ & Date & Hour & $\begin{array}{c}\text { Gage } \\
\text { he1ght }\end{array}$ & $\begin{array}{l}\text { D1s- } \\
\text { charge }\end{array}$ & Date & our & $\begin{array}{c}\text { Gage } \\
\text { he1ght }\end{array}$ & $\begin{array}{c}\text { D1s- } \\
\text { charge }\end{array}$ \\
\hline Mar. I & 2400 & 13.48 & 6,400 & Mar. 8 & 2400 & 17.62 & 38,700 & Mar. 15 & 0600 & 18 & 51,000 \\
\hline & & & & & & & & & 1200 & 18 & 53,900 \\
\hline 2 & 0600 & 13.47 & 6,350 & 9 & 0600 & 17.80 & 40,700 & & 1500 & 19.02 & 54,700 \\
\hline & 1200 & 13.50 & 6,500 & & 1200 & 17.91 & 41,900 & & 2400 & 18.96 & 54,000 \\
\hline & 1800 & 13.54 & 6,700 & & 1500 & 17.87 & 41,500 & & & & \\
\hline & 2000 & 13.65 & 7,250 & & 1800 & 17.94 & 42,200 & 16 & 0600 & 19.10 & 55,700 \\
\hline & 2400 & 14.03 & 9,380 & & 2400 & 18.43 & 47,700 & & $\begin{array}{l}1100 \\
1900\end{array}$ & .20 & $\begin{array}{l}56,900 \\
54,700\end{array}$ \\
\hline 3 & 0600 & 14.83 & 14,400 & 10 & 0600 & 19.36 & 58,800 & & 2400 & 18.79 & 52,000 \\
\hline & 1200 & 15.64 & 20,300 & & 1200 & 20.25 & 70,700 & & & & \\
\hline & 1800 & 16.22 & 25,200 & & 1800 & 21.35 & 87,300 & 17 & 0600 & 18.51 & 48,600 \\
\hline & & 16.78 & 30,200 & & 2400 & 22.45 & 104,000 & & 1700 & .26 & 45,800 \\
\hline 4 & 0600 & 17.13 & 33,500 & 11 & 0500 & 22.58 & 105,000 & & 0 & 20 & 00 \\
\hline & 1200 & 17.54 & 37,800 & & 0600 & 22.58 & 105,000 & 18 & 0900 & 18.11 & 44,100 \\
\hline & 1800 & 17.97 & 42,600 & & 1200 & 21.96 & 96,600 & & 2000 & 18.01 & 43,000 \\
\hline & 2400 & 18.44 & 47,800 & & $\begin{array}{l}1800 \\
2400\end{array}$ & $\begin{array}{l}20.71 \\
19.56\end{array}$ & $\begin{array}{l}77,400 \\
61,400\end{array}$ & & 2400 & 17.95 & 42,400 \\
\hline 5 & 0600 & 18.95 & 53,900 & & & & 01,400 & 19 & 0600 & 17.84 & 41,100 \\
\hline & 1200 & 20.11 & 68,700 & 12 & 0600 & 18.66 & 50,400 & & 1800 & 17.47 & 37,100 \\
\hline & 1800 & 21.13 & 83,800 & & 1200 & 18.06 & 43,600 & & 2400 & 17.39 & 36,200 \\
\hline & 2400 & 21.78 & 93,900 & & 1800 & 17.78 & 40,500 & & & & \\
\hline & & & & & 2400 & 17.65 & 39,000 & 20 & 0600 & 17.28 & 35,000 \\
\hline 6 & 0700 & 22.18 & 99,700 & & & & & & 1500 & 16.77 & 30,100 \\
\hline & 1200 & 22.02 & 97,500 & 13 & 0600 & 17.68 & 39,400 & & 1900 & 16.61 & 28,700 \\
\hline & 1800 & 21.18 & 84,600 & & 1200 & 17.70 & 39,600 & & 2400 & 16.72 & 29,700 \\
\hline & 2400 & 20.02 & 67,500 & & 1800 & 17.65 & 39,000 & & & & \\
\hline & & & & & 2200 & 17.59 & 00 & 21 & 0300 & 16.73 & 29,800 \\
\hline 7 & 0600 & 18.89 & 53,200 & & 2400 & 17.60 & 38,500 & & 0900 & 16.62 & 28,800 \\
\hline & 1200 & 18.07 & 43,700 & & & & & & 1200 & 16.37 & 26,500 \\
\hline & 2400 & 17.15 & 33,700 & 14 & 0600 & 17.72 & 39,800 & & 1500 & 16.06 & 23,700 \\
\hline & & & & & 1200 & 17.77 & 40,400 & & 2400 & 15.58 & 19,800 \\
\hline 8 & 0500 & 17.00 & 32,200 & & 1800 & 17.93 & 42,100 & & & & \\
\hline & 1400 & 17.28 & 35,000 & & 2400 & 18.36 & 46,900 & & & & \\
\hline
\end{tabular}

Note.--Daily means for some days computed from data in addition to figures shown. 


\section{OHIO RIVER MAIN STEM}

(3) 3-860. Ohio River at Sewickley, $\mathrm{Pa}$.

Location.--Lat $40^{\circ} 31^{\prime} 50^{\prime \prime}$, long $80^{\circ} 11^{\prime} 20^{\prime \prime}$, on left bank 200 ft upstream from highway bridge at Sewickley, Allegheny County, $0.5 \mathrm{mile}$ upstream from Narrows Run, 1.5 miles upstream from Dashields Dam, and 11.8 miles downstream from confluence of Allegheny and Monongahela Rivers.

Drainage area. $--19,500 \mathrm{sq} \mathrm{ml}$, approximately.

Gage-he1ght record.--Water-stage recorder graph. Datum of gage is $690.00 \mathrm{ft}$ above mean sea leve1, adjustment of 1912 .

Discharge record.--Stage-discharge relation defined by current-meter measurements.

Maxima.--March 1964: Discharge, 295,000 efs Mar. 11,0500 hours (gage height, $21.19 \mathrm{ft}$.

1933 to February 1964: D1scharge, 574,000 cfs Mar. 18, 1936 (gage he1ght, $34.75 \mathrm{ft}$, from floodmark in gage house).

Remarks.--Some regulation by locks, and by many reservolrs above station. Combined capacity of reservoirs excluding that of Chautauqua Lake but including Lake Lynn and Deep Creek Reservolrs and 12 smaller reservolrs, 1,584,000 acre-ft.

Mean discharge, in cubic feet per second, March 1964

\begin{tabular}{|c|c|c|c|c|c|c|c|c|c|}
\hline Day & Discharge & Day & D1scharge & Day & Discharge & Day & I1scharge & Day & Discharge \\
\hline $\begin{array}{l}1 \ldots \ldots \\
2 \ldots \ldots \\
3 \ldots \ldots \\
4 \ldots \ldots \\
5 \ldots \ldots \\
6 \ldots \ldots\end{array}$ & $\begin{array}{r}12,500 \\
14,100 \\
33,400 \\
59,400 \\
116,000 \\
227,000\end{array}$ & $\begin{array}{r}7 \ldots \ldots \\
8 \ldots \ldots \\
9 \ldots \\
10 \ldots \\
11 \ldots \ldots \\
12 \ldots\end{array}$ & $\begin{array}{l}186,000 \\
162,000 \\
170,000 \\
240,000 \\
276,000 \\
192,000\end{array}$ & $\mid \begin{array}{l}13 \ldots \ldots \\
14 \ldots \ldots \\
15 \ldots \ldots \\
16 \ldots \ldots \\
17 \ldots \ldots \\
18 \ldots \ldots\end{array}$ & $\begin{array}{l}164,000 \\
158,000 \\
159,000 \\
162,000 \\
156,000 \\
143,000\end{array}$ & $\begin{array}{l}19 \ldots \ldots \\
20 \ldots \ldots \\
21 \ldots \ldots \\
22 \ldots \ldots \\
23 \ldots \ldots \\
24 \ldots \ldots\end{array}$ & $\begin{array}{r}116,000 \\
84,000 \\
67,100 \\
53,900 \\
44,300 \\
43,300\end{array}$ & $\begin{array}{l}25 \ldots \ldots \\
26 \ldots \ldots \\
27 \ldots \ldots \\
28 \ldots \ldots \\
29 \ldots \ldots \\
30 \ldots \ldots \\
31 \ldots \ldots\end{array}$ & $\begin{array}{l}44,400 \\
44,400 \\
49,200 \\
53,300 \\
47,600 \\
41,300 \\
42,800\end{array}$ \\
\hline
\end{tabular}

Gage helght, in feet, and discharge, in cuble feet per second, at indicated time, 1964

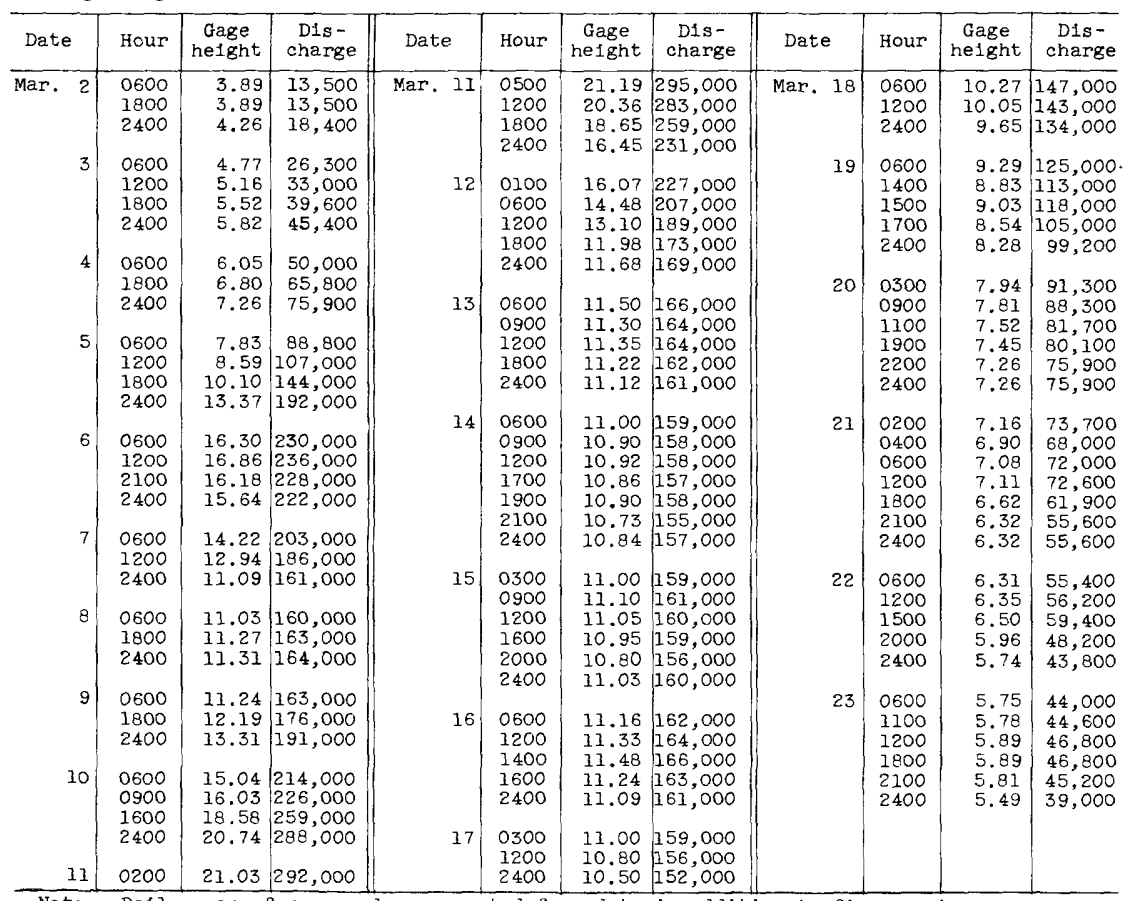

Note.--Dally means for some days computed from data in addition to flgures shown. 


\section{BEAVER RIVER BASIN}

(4) 3-1075. Beaver River at Beaver Falls, Pa.

Location.--Lat $40^{\circ} 45^{\prime} 45^{\prime \prime}$, long $80^{\circ} 18^{\prime} 55^{\prime \prime}$, on left bank at Beaver Falls, Beaver County, $200 \mathrm{ft}$ upstream from pumping plant of Beaver Valley Water Co., 5.5 miles upstream from mouth, and 7 miles downstream from Connoquenessing Creek.

Drainage area. $--3,106 \mathrm{sq} \mathrm{m} 1$.

Gage-height record.--D1gital recorder tape punched at 60-minute intervals. Datum of gage is $727.48 \mathrm{ft}$ above mean sea level, datum of 1929 (Corps of Engineers bench mark).

Discharge record.--Stage-discharge relation defined by current-meter measurements below 57,000 cfs and extended above by logarithmic plotting.

Maxima.--March 1964: Discharge, 57,900 cf's Mar. 10, 2200 hours (gage height, $13.24 \mathrm{ft}$ ).

1935 to February 1964: Discharge, 69,900 cfs Jan. 22, 1959 (gage he1ght, $14.42 \mathrm{ft}$ ).

Maxímum stage known, 17.4 ft Mar. 27, 1913 (discharge, 103,000 cfs).

Remarks.--Flow regulated since 1942 by Berlin, since 1916 by Milton, since 1943 by Mosquito Creek, since 1929 by Meander Creek, and since 1933 by Pymatuning Reservoirs.

Mean discharge, in cublc feet per second, March 1964

\begin{tabular}{|c|c|c|c|c|c|c|c|c|c|}
\hline Day & Discharge & Day & Discharge & Day & Discharge & Day & Discharge & Day & Discharge \\
\hline $\begin{array}{l}1 \ldots \ldots \\
2 \ldots \ldots \\
3 \ldots \ldots \\
4 \ldots \\
5 \ldots \\
6 \ldots\end{array}$ & $\begin{array}{r}860 \\
1,050 \\
2,980 \\
9,060 \\
28,400 \\
31,100\end{array}$ & $\begin{array}{r}7 \ldots \ldots \\
8 \ldots \ldots \\
9 \ldots \ldots \\
10 \ldots \ldots \\
11 \ldots \ldots \\
12 \ldots \ldots\end{array}$ & $\begin{array}{l}26,400 \\
15,200 \\
20,000 \\
50,300 \\
49,000 \\
30,400\end{array}$ & $\mid \begin{array}{l}13 \ldots \ldots \\
14 \ldots \ldots \\
15 \ldots \ldots \\
16 \ldots \ldots \\
17 \ldots \ldots \\
18 \ldots \ldots\end{array}$ & $\begin{array}{r}16,200 \\
10,100 \\
10,100 \\
8,480 \\
7,230 \\
7,070\end{array}$ & $\begin{array}{l}19 \ldots \ldots \\
20 \ldots \ldots \\
21 \ldots \ldots \\
22 \ldots \ldots \\
23 \ldots \ldots \\
24 \ldots \ldots\end{array}$ & $\begin{array}{l}6,340 \\
5,070 \\
5,090 \\
6,810 \\
6,330 \\
5,120\end{array}$ & $\mid \begin{array}{l}25 \ldots \ldots \\
26 \ldots \ldots \\
27 \ldots \ldots \\
28 \ldots \ldots \\
29 \ldots \ldots \\
30 \ldots \ldots \\
31 \ldots \ldots\end{array}$ & $\begin{array}{l}4,510 \\
4,540 \\
4,670 \\
4,160 \\
3,770 \\
3,230 \\
2,890\end{array}$ \\
\hline
\end{tabular}

Monthly mean discharge, in cub1c feet per second................... Note. - Mean da11y discharges computed on basis of 60 -minute intervals.

Gage height, in feet, and discharge, in cublc feet per second, at indicated time, 1964

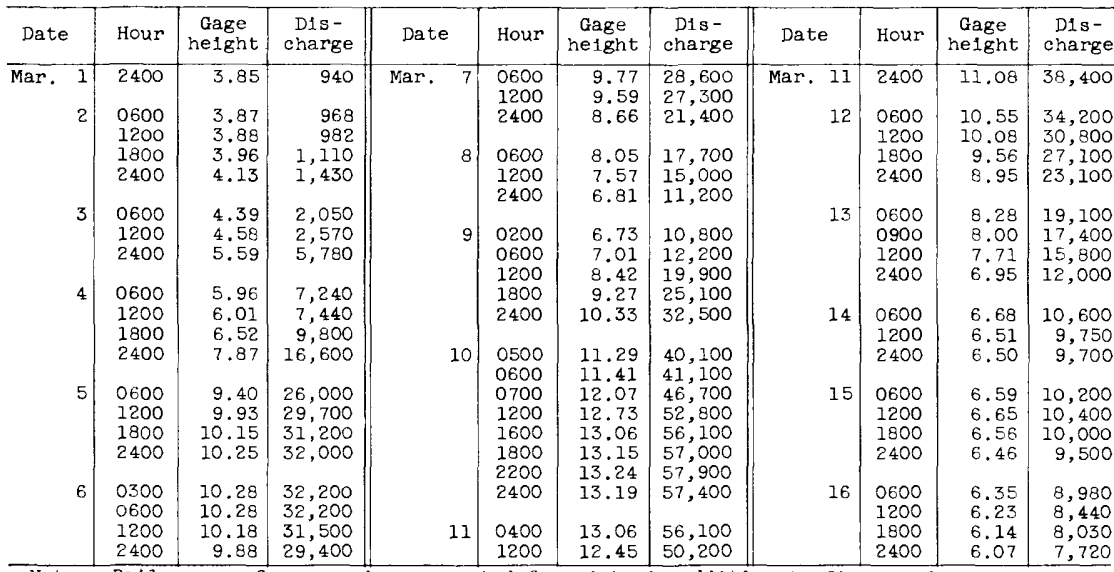

Note.--Dally means for some days computed from data in addition to flgures shown. 


\section{OHIO RIVER MAIN STEM}

(5) 3-1150. Ohio River at St. Marys, W. Va.

Location.--Lat $39^{\circ} 23^{\prime} 25^{\prime \prime}$, long $81^{\circ} 12^{\prime} 30^{\prime \prime}$, on left bank at downstream side of bridge on U.S. Highway 50 (alternate) at St. Marys, Pleasants County, 0.9 mile downstream from Middle Island Creek at mile 155.0.

Drainage area.--26,850 sq $\mathrm{m} 1$, approximately.

Gage-height record.--Water-stage recorder graphs. Auxillary water-stage recorder graph $1.5 \mathrm{miles}$ upstream from lock and dam 17, and $11 \mathrm{mlles}$ downstream from base gage. Datum of gage is $577.30 \mathrm{ft}$ above mean sea level, Sandy Hook datum.

Discharge record.--Stage-fall-discharge relation defined by current-meter measurements below 378,000 efs. Discharge computed by using fall as a factor.

Maxima.--March 1964: Discharge, 383,000 cfs Mar. 12, 0600-0800 hours; gage he1ght, $42.75 \mathrm{ft}$ Mar. 12,1000 hours.

1938 to February 1964: Discharge, $421,000 \mathrm{cfs} J a n .1$, 1943: gage he1ght, $46.67 \mathrm{ft}$ Jan. 1, 1943

Maximum stage known since at least 1884, $54.2 \mathrm{ft}$ in Mar. 1913.

Remarks.--Flow partly regulated by locks, dams, and reservoirs upstream.

Mean discharge, in cublc feet per second, March 1964

\begin{tabular}{|c|c|c|c|c|c|c|c|c|c|}
\hline Day & Discharge & Day & Discharge & Day & D1scharge & Day & Discharge & Day & Discharge \\
\hline $\begin{array}{l}1 \ldots \ldots \\
2 \ldots \ldots \\
3 \ldots \ldots \\
4 \ldots \ldots \\
5 \ldots \ldots \\
6 \ldots \ldots\end{array}$ & $\begin{array}{r}15,700 \\
17,400 \\
37,600 \\
62,800 \\
93,900 \\
164,000\end{array}$ & $\begin{array}{r}7 \ldots \ldots \\
8 \ldots \ldots \\
9 \ldots \\
10 \ldots \\
11 \ldots \\
12 \ldots\end{array}$ & $\begin{array}{l}248,000 \\
233,000 \\
208,000 \\
269,000 \\
345,000 \\
374,000\end{array}$ & $\begin{array}{l}13 \ldots \ldots \\
14 \ldots \ldots \\
15 \ldots \ldots \\
16 \ldots \\
17 \ldots \ldots \\
18 \ldots \ldots\end{array}$ & $\begin{array}{l}306,000 \\
226,000 \\
200,000 \\
191,000 \\
182,000 \\
170,000\end{array}$ & $\begin{array}{l}19 \ldots \ldots \\
20 \ldots \ldots \\
21 \ldots \ldots \\
22 \ldots \ldots \\
23 \ldots \ldots \\
24 \ldots \ldots\end{array}$ & $\begin{array}{r}153,000 \\
129,000 \\
99,000 \\
81,400 \\
68,100 \\
58,300\end{array}$ & $\begin{array}{l}25 \ldots \ldots \\
26 \ldots \ldots \\
27 \ldots \ldots \\
28 \ldots \ldots \\
29 \ldots \ldots \\
30 \ldots \ldots \\
31 \ldots \ldots\end{array}$ & $\begin{array}{l}55,800 \\
54,100 \\
54,100 \\
56,100 \\
59,600 \\
56,200 \\
53,500\end{array}$ \\
\hline
\end{tabular}

Gage height, in feet, and discharge, in cublc feet per second, at indicated time, 1964

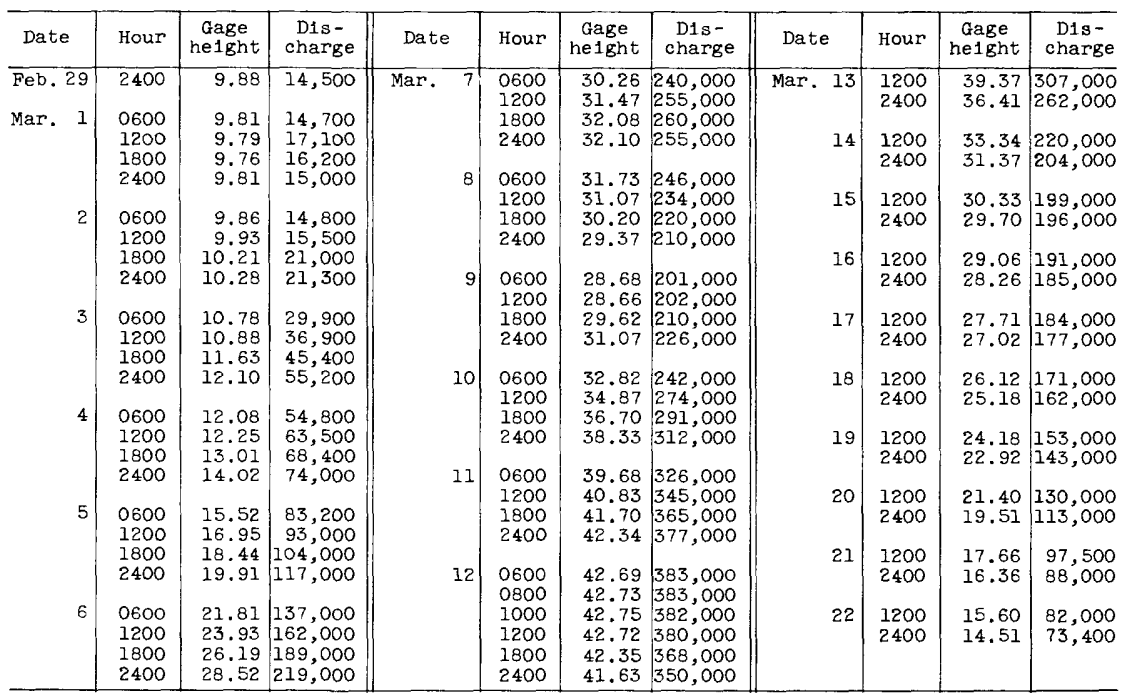




\section{MUSKINGUM RIVER BASIN}

(6) 3-1364. North Branch Kokosing R1ver near Fredericktown, Oh10

(Crest-stage station)

Location.--Lat $40^{\circ} 30^{\prime} 10^{\prime \prime}$, long $82^{\circ} 34^{\prime} 15^{\prime \prime}$, at bridge on county road 2 miles northwest of Fredericktown, Knox County, and 2.7 miles above East Branch.

Drainage area $--45.0 \mathrm{sq} \mathrm{mi}$.

Gage-height record.-W1re-welght gage read twice dally, and crest-stage gage. Datum of gage is 1,092.05 ft above mean sea level, datum of 1929, supplementary adjustment of 1960 .

Discharge record.--Stage-discharge relation defined by current-meter measurements.

Maxima --March 1964: D1scharge, 3,160 cfs Mar. 10 (gage he1ght, 7.50 ft).

1959: Gage helght, $13.4 \mathrm{ft}$, from floodmarks (discharge unknown).

June 1962 to February 1964: Discharge, 2,430 cfs Mar. 4, 1963 (gage height, $6.83 \mathrm{ft})$.

(7) 3-1365. Kokosing River at Mount Vernon, Oh1o

Location.--Lat $40^{\circ} 24^{\prime} 25^{\prime \prime}$, long $82^{\circ} 30^{\prime} 00^{\prime \prime}$, on right bank at downstream side of Tilden Avenue Bridge at Mount Vermon, Knox County, 0.8 mile downstream from North Branch and 2.7 miles upstream from Dry Run.

Drainage area. $--200 \mathrm{sq} \mathrm{ml}$.

Gage-he1ght record.--Water-stage recorder graph. Datum of gage is $984.16 \mathrm{ft}$ above mean sea level, datum of 1929, supplementary adjustment of 1944 (levels by Corps of Engineers).

Discharge record.--Stage-discharge relation defined by current-meter measurements below $6,400 \mathrm{cfs}$ and by indirect measurements at $7,300 \mathrm{cf}$ 's and $38,000 \mathrm{cfs}$.

Maxima.--March 1964: Discharge, 10,300 cfs Mar. 10, 0930 hours (gage height, $13.08 \mathrm{ft})$.

1953 to February 1964: D1scharge, 38,000 cfs Jan. 21, 1959 (gage he1ght, $18.19 \mathrm{ft})$.

Remarks.--Some regulation by Knox Lake on East Branch of North Branch (30.3 sq $\mathrm{ml}$, 3,750 acre-ft).

Mean discharge, in cuble feet per second, March 1964

\begin{tabular}{|c|c|c|c|c|c|c|c|c|c|}
\hline Day & Discharge & Day & Discharge & Day & Discharge & Day & Discharge & Day & Discharge \\
\hline $\begin{array}{l}1 \ldots \ldots \\
2 \ldots \ldots \\
3 \ldots \ldots \\
4 \ldots \ldots \\
5 \ldots \ldots \\
6 \ldots \ldots\end{array}$ & $\begin{array}{r}30 \\
41 \\
82 \\
309 \\
3,190 \\
1,160\end{array}$ & $\begin{array}{r}7 \ldots \ldots \\
8 \ldots \\
9 \ldots \\
10 \ldots \\
11 \ldots \\
12 \ldots\end{array}$ & $\begin{array}{r}465 \\
286 \\
2,820 \\
7,390 \\
2,110 \\
1,070\end{array}$ & $\begin{array}{l}13 \ldots \ldots \\
14 \ldots \ldots \\
15 \ldots \ldots \\
16 \ldots \ldots \\
17 \ldots \ldots \\
18 \ldots \ldots\end{array}$ & $\begin{array}{r}925 \\
935 \\
1,720 \\
860 \\
541 \\
384\end{array}$ & $\begin{array}{l}19 \ldots \ldots \\
20 \ldots \ldots \\
21 \ldots \ldots \\
22 \ldots \ldots \\
23 \ldots \ldots \\
24 \ldots \ldots\end{array}$ & $\begin{array}{l}302 \\
266 \\
458 \\
546 \\
354 \\
276\end{array}$ & $\begin{array}{l}25 \ldots \ldots \\
26 \ldots \ldots \\
27 \ldots \ldots \\
28 \ldots \ldots \\
29 \ldots \ldots \\
30 \ldots \ldots \\
31 \ldots \ldots\end{array}$ & $\begin{array}{l}241 \\
294 \\
272 \\
224 \\
199 \\
184 \\
181\end{array}$ \\
\hline
\end{tabular}

Gage height, In feet, and discharge, in cub1c feet per second, at indicated time, 1964

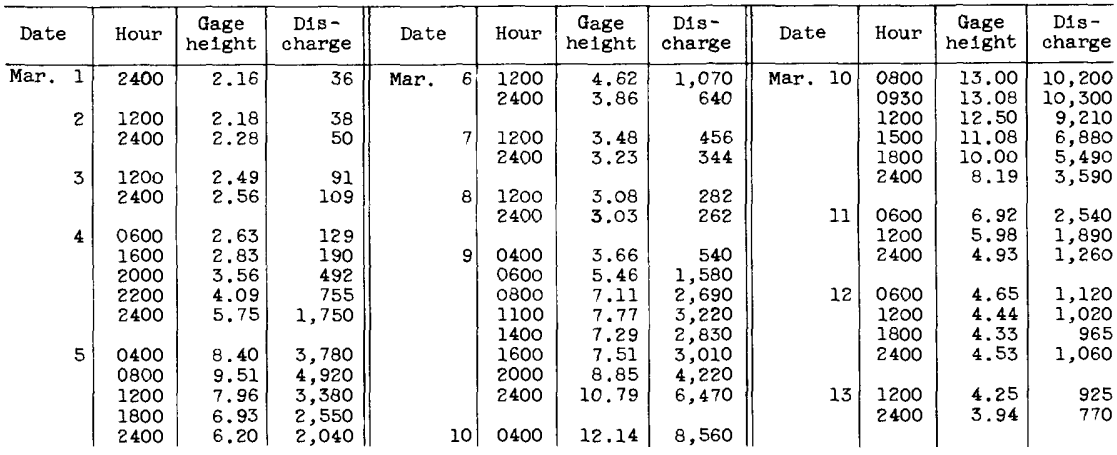


Gage height, in feet, and discharge, in cublc feet per second, at indicated time, 1964, of Kokosing River at Mount Vernon, Ohio--Continued

\begin{tabular}{|c|c|c|c|c|c|c|c|c|c|c|c|}
\hline Date & Hour & $\begin{array}{l}\text { Gage } \\
\text { helght }\end{array}$ & $\begin{array}{l}\text { D1s- } \\
\text { charge }\end{array}$ & Date & Hour & $\begin{array}{c}\text { Gage } \\
\text { helght }\end{array}$ & $\begin{array}{l}\text { Dis- } \\
\text { charge }\end{array}$ & Date & Hour & $\begin{array}{c}\text { Gage } \\
\text { helght }\end{array}$ & $\begin{array}{l}\text { D1s- } \\
\text { charge }\end{array}$ \\
\hline \multirow[t]{3}{*}{ Mar. 14} & \multirow{3}{*}{$\begin{array}{l}0600 \\
1200 \\
1800 \\
2200 \\
2400\end{array}$} & \multirow{3}{*}{$\begin{array}{l}3.85 \\
3.88 \\
4.54 \\
5.12 \\
5.97\end{array}$} & \multirow{3}{*}{$\begin{array}{r}725 \\
740 \\
1,070 \\
1,370 \\
1,880\end{array}$} & \multirow[t]{3}{*}{ Mar. 15} & \multirow{3}{*}{$\begin{array}{l}1200 \\
2400 \\
\\
1200 \\
2400\end{array}$} & \multirow{3}{*}{$\begin{array}{l}5.72 \\
4.77 \\
4.06 \\
3.69\end{array}$} & \multirow{3}{*}{$\begin{array}{r}1,730 \\
1,180 \\
\\
830 \\
645\end{array}$} & \multirow{3}{*}{$\begin{aligned} \text { Mar. } & 17 \\
& 18\end{aligned}$} & 2400 & 3.30 & 46 \\
\hline & & & & & & & & & $\begin{array}{l}1200 \\
2400\end{array}$ & $\begin{array}{l}3.12 \\
3.02\end{array}$ & 379 \\
\hline & & & & & & & & & 2400 & 2.87 & \\
\hline 15 & 0300 & 6.73 & 2,410 & 17 & 1200 & 3.48 & 541 & 20 & 2400 & 2.79 & 252 \\
\hline
\end{tabular}

Note.--Daily means for some days computed from data in addition to figures shown.

\section{(8) 3-1370. Kokosing River at Millwood, Ohio}

Location.--Lat $40^{\circ} 23^{\prime} 55^{\prime \prime}$, long $82^{\circ} 17^{\prime} 10^{\prime \prime}$, on left bank 0.4 mile west of M1llwood, Knox County, $1 \frac{1}{2}$ miles upstream from Honey Run, 2 miles downstream from Jelloway Creek, and $3 \frac{1}{2}$ miles upstream from Brush Run.

Drainage area. $--454 \mathrm{sq} \mathrm{mi}$.

Gage-height record.--Water-stage recorder graph, except 1330 hours Mar. 13 to 1100 hours Mar. 15, for which graph was estimated on basis of record before and after this period and record for station at Mount Vemon. Datum of gage is $865.00 \mathrm{ft}$ above mean sea level.

Discharge record.--Stage-discharge relation defined by current-meter measurements below 19,000 cf's and by indirect measurement at $75,900 \mathrm{cfs}$.

Maxima.--March 1964: Discharge, 25,400 cfs Mar. 10, 1200 hours (gage he1ght, $20.70 \mathrm{ft}$ )

1913, 1921 to February 1964: Discharge, 75,900 cfs Jan. 21, 1959 (gage height, $34.00 \mathrm{ft}$, from high-water mark in well).

Mean discharge, in cublc feet per second, March 1964

\begin{tabular}{|c|c|c|c|c|c|c|c|c|c|}
\hline Day & Discharge & Day & Discharge & Day & D1scharge & Day & D1scharge & Day & Discharge \\
\hline $\begin{array}{l}1 \ldots \\
2 \ldots \\
3 \ldots \\
4 \ldots \\
5 \ldots \\
6 \ldots \\
\end{array}$ & $\begin{array}{r}62 \\
121 \\
202 \\
889 \\
7,140 \\
2,700\end{array}$ & $\begin{array}{r}7 \ldots \ldots \\
8 \ldots \ldots \\
9 \ldots \ldots \\
10 \ldots \\
11 \ldots \ldots \\
12 \ldots\end{array}$ & $\begin{array}{r}1,170 \\
748 \\
6,440 \\
20,100 \\
6,570 \\
2,650\end{array}$ & $\begin{array}{l}13 \ldots \ldots \\
14 \ldots \ldots \\
15 \ldots \ldots \\
16 \ldots \\
17 \ldots \\
18 \ldots\end{array}$ & $\begin{array}{l}2,080 \\
1,700 \\
3,540 \\
2,290 \\
1,740 \\
1,200\end{array}$ & $\begin{array}{l}19 \ldots \ldots \\
20 \ldots \ldots \\
21 \ldots \ldots \\
22 \ldots \ldots \\
23 \ldots \ldots \\
24 \ldots \ldots\end{array}$ & $\begin{array}{r}868 \\
772 \\
1,010 \\
1,180 \\
892 \\
802\end{array}$ & $\mid \begin{array}{l}25 \ldots \ldots \\
26 \ldots \ldots \\
27 \ldots \ldots \\
28 \ldots \ldots \\
29 \ldots \ldots \\
30 \ldots \ldots \\
31 \ldots \ldots\end{array}$ & $\begin{array}{l}754 \\
790 \\
766 \\
692 \\
648 \\
626 \\
615\end{array}$ \\
\hline
\end{tabular}

Gage height, in feet, and discharge, in cublc feet per second, at indicated time, 1964

\begin{tabular}{|c|c|c|c|c|c|c|c|c|c|c|c|}
\hline Date & Hour & $\begin{array}{c}\text { Gage } \\
\text { he1ght }\end{array}$ & $\begin{array}{l}\text { Dis- } \\
\text { charge }\end{array}$ & Date & Hour & $\begin{array}{c}\text { Gage } \\
\text { helght }\end{array}$ & $\begin{array}{l}\text { Dis- } \\
\text { charge }\end{array}$ & Date & Hour & $\begin{array}{c}\text { Gage } \\
\text { helght }\end{array}$ & $\begin{array}{c}\text { Dis- } \\
\text { charge }\end{array}$ \\
\hline $\begin{array}{r}\text { Mar. } 1 \\
2\end{array}$ & $\begin{array}{l}2400 \\
1200\end{array}$ & $\begin{array}{l}4.35 \\
4.55\end{array}$ & 79 & Mar. 8 & $\begin{array}{l}1200 \\
2400\end{array}$ & $\begin{array}{l}5.99 \\
5.95\end{array}$ & $\begin{array}{l}724 \\
702\end{array}$ & Mar. 14 & $\begin{array}{l}1200 \\
1800 \\
2400\end{array}$ & $\begin{array}{l}6.95 \\
7.25 \\
8.9\end{array}$ & $\begin{array}{l}1,360 \\
1,590 \\
3,120\end{array}$ \\
\hline & 2400 & 4.78 & 189 & 9 & $\begin{array}{l}0400 \\
0600\end{array}$ & $\begin{array}{l}7.50 \\
9.02\end{array}$ & $\begin{array}{l}7,790 \\
3,240\end{array}$ & 15 & 0400 & 10.05 & 4,400 \\
\hline 3 & $\begin{array}{l}0300 \\
1800 \\
2400\end{array}$ & $\begin{array}{l}4.86 \\
4.73 \\
4.98\end{array}$ & $\begin{array}{l}215 \\
174 \\
257\end{array}$ & & $\begin{array}{l}0800 \\
1200 \\
1800\end{array}$ & $\begin{array}{l}10.36 \\
12.16 \\
13.25\end{array}$ & $\begin{array}{l}4,770 \\
7,270 \\
9,020\end{array}$ & & $\begin{array}{l}1200 \\
2400\end{array}$ & $\begin{array}{l}9.30 \\
8.58\end{array}$ & $\begin{array}{l}3,540 \\
2,800\end{array}$ \\
\hline & & & & & 2400 & 15.02 & 12,200 & 16 & 1200 & 8.00 & 2,240 \\
\hline 4 & $\begin{array}{l}0600 \\
1200\end{array}$ & $\begin{array}{l}4.98 \\
5.28\end{array}$ & $\begin{array}{l}257 \\
376\end{array}$ & 10 & 0400 & 17.06 & 16,400 & & 2400 & 7.71 & 1,980 \\
\hline & & $\begin{array}{l}6.06 \\
7.02\end{array}$ & & & & & & 17 & $\begin{array}{l}1200 \\
2400\end{array}$ & $\begin{array}{l}7.44 \\
7.10\end{array}$ & $\begin{array}{l}1,740 \\
1,470\end{array}$ \\
\hline & $\begin{array}{l}2200 \\
2400\end{array}$ & $\begin{array}{l}8.61 \\
9.88\end{array}$ & $\begin{array}{l}2,830 \\
4,200\end{array}$ & & $\begin{array}{l}1500 \\
1800 \\
2400\end{array}$ & $\begin{array}{l}20.19 \\
19.20 \\
16.11\end{array}$ & $\begin{array}{l}24,000 \\
21,500 \\
14,400\end{array}$ & 18 & $\begin{array}{l}1200 \\
2400\end{array}$ & $\begin{array}{l}6.70 \\
6.43\end{array}$ & $\begin{array}{r}1,780 \\
994\end{array}$ \\
\hline 5 & $\begin{array}{l}0400 \\
0830\end{array}$ & $\begin{array}{l}12.39 \\
13.36\end{array}$ & $\begin{array}{l}7,620 \\
9,210\end{array}$ & 11 & 0600 & 12.63 & 8,010 & 19 & 2400 & 6.12 & 802 \\
\hline & 1200 & 13.07 & 8,720 & & $\begin{array}{l}1200 \\
2400\end{array}$ & $\begin{array}{r}10.69 \\
9.05\end{array}$ & $\begin{array}{l}5,200 \\
3,270\end{array}$ & 20 & 2400 & 6.03 & 748 \\
\hline & 2400 & 9.92 & 4,240 & & & & & 21 & 0600 & 6.05 & 760 \\
\hline 6 & $\begin{array}{l}1200 \\
2400\end{array}$ & $\begin{array}{l}8.26 \\
7.27\end{array}$ & $\begin{array}{l}2,480 \\
1,610\end{array}$ & 12 & $\begin{array}{l}1200 \\
2400\end{array}$ & $\begin{array}{l}8.36 \\
8.17\end{array}$ & $\begin{array}{l}2,580 \\
2,390\end{array}$ & & $\begin{array}{l}1200 \\
1800 \\
2400\end{array}$ & $\begin{array}{l}6.38 \\
6.86 \\
6.98\end{array}$ & $\begin{array}{r}962 \\
1,290 \\
1,380\end{array}$ \\
\hline 7 & $\begin{array}{l}1200 \\
2400\end{array}$ & $\begin{array}{l}6.66 \\
6.24\end{array}$ & $\begin{array}{r}1,150 \\
874\end{array}$ & 13 & $\begin{array}{l}1200 \\
2400\end{array}$ & $\begin{array}{l}7.91 \\
7.3\end{array}$ & $\begin{array}{l}2,160 \\
1,630\end{array}$ & 22 & $\begin{array}{l}1200 \\
2400\end{array}$ & $\begin{array}{l}6.68 \\
6.43\end{array}$ & $\begin{array}{r}1,170 \\
994\end{array}$ \\
\hline
\end{tabular}

Note.--Da1lv means for some days computed from data in addition to figures shown. 
(9) 3-1465. Licking River near Newark, Ohio

Location.--Lat $40^{\circ} 03^{1} 33^{\prime \prime}$, long $82^{\circ} 20^{\prime} 23^{\prime \prime}$, on right bank at downstream side of Stadden Bridge, I mile downstream from Shawnee Run, $1 \frac{1}{2}$ miles upstream from Equality Run, and $3 \frac{1}{2}$ miles east of Newark, Licking County.

Drainage area. $-536 \mathrm{sq} \mathrm{mi}$.

Gage-height record.--Water-stage recorder graph except 0900 hours to 1930 hours Mar. 5, for which graph was estimated on basis of recorded range in stage and record before and after period of clock stoppage. Datum of gage is $779.02 \mathrm{ft}$ above mean sea level, datum of 1929, supplementary adjustment of 1944 .

Discharge record.--Stage-discharge relation defined by current-meter measurements below $24,000 \mathrm{cfs}$ and extended on basis of flood-routing studies for station at Toboso for January 1959 peak flow.

Maxima --March 1964: Discharge, 25,500 cfs Mar. 10, 0830 hours (gage height, $17.18 \mathrm{ft})$

1939 to February 1964: Discharge, 45,000 cfs Jan. 21, 1959 (gage height, $20.3 \mathrm{ft}$, from high-water mark).

Remarks.--Flow slightly regulated by Buckeye Lake on South Fork $(27,300$ acre-ft; $3,140$ acres surface area, $46.2 \mathrm{sq} \mathrm{mi})$. Gates operated intermittently to maintain normal lake level.

Mean d1scharge, in cub1c feet per second, March 1964

\begin{tabular}{|c|c|c|c|c|c|c|c|c|c|}
\hline Day & Discharge & Day & D1scharge & Day & D1scharge & Day & D1 scharge & Day & Discharge \\
\hline $\begin{array}{l}1 \ldots \ldots \\
2 \ldots \ldots \\
3 \ldots \ldots \\
4 \ldots \ldots \\
5 \ldots \ldots \\
6 \ldots \ldots\end{array}$ & $\begin{array}{r}55 \\
100 \\
154 \\
699 \\
6,180 \\
2,890\end{array}$ & $\begin{array}{r}7 \ldots \ldots \\
8 \ldots \ldots \\
9 \ldots \ldots \\
10 \ldots \\
11 \ldots \ldots \\
12 \ldots \ldots\end{array}$ & $\begin{array}{r}1,270 \\
845 \\
7,070 \\
21,300 \\
9,090 \\
4,700\end{array}$ & $\begin{array}{l}13 \ldots \ldots \\
14 \ldots \ldots \\
15 \ldots \ldots \\
16 \ldots \ldots \\
17 \ldots \ldots \\
18 \ldots \ldots\end{array}$ & $\begin{array}{r}3,240 \\
2,700 \\
3,090 \\
2,080 \\
1,460 \\
800\end{array}$ & $\begin{array}{l}19 \ldots \ldots \\
20 \ldots \ldots \\
21 \ldots \ldots \\
22 \ldots \ldots \\
23 \ldots \ldots \\
24 \ldots \ldots\end{array}$ & $\begin{array}{r}618 \\
546 \\
919 \\
1,210 \\
785 \\
610\end{array}$ & $\begin{array}{l}25 \ldots \ldots \\
26 \ldots \ldots \\
27 \ldots \ldots \\
28 \ldots \ldots \\
29 \ldots \ldots \\
30 \ldots \ldots \\
31 \ldots \ldots\end{array}$ & $\begin{array}{l}526 \\
626 \\
646 \\
506 \\
438 \\
398 \\
374\end{array}$ \\
\hline
\end{tabular}

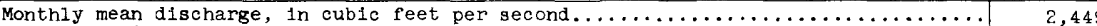

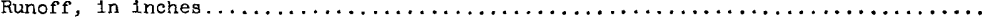

Gage helght, in feet, and discharge, in cublc feet per second, at indlcated t1me, 1964

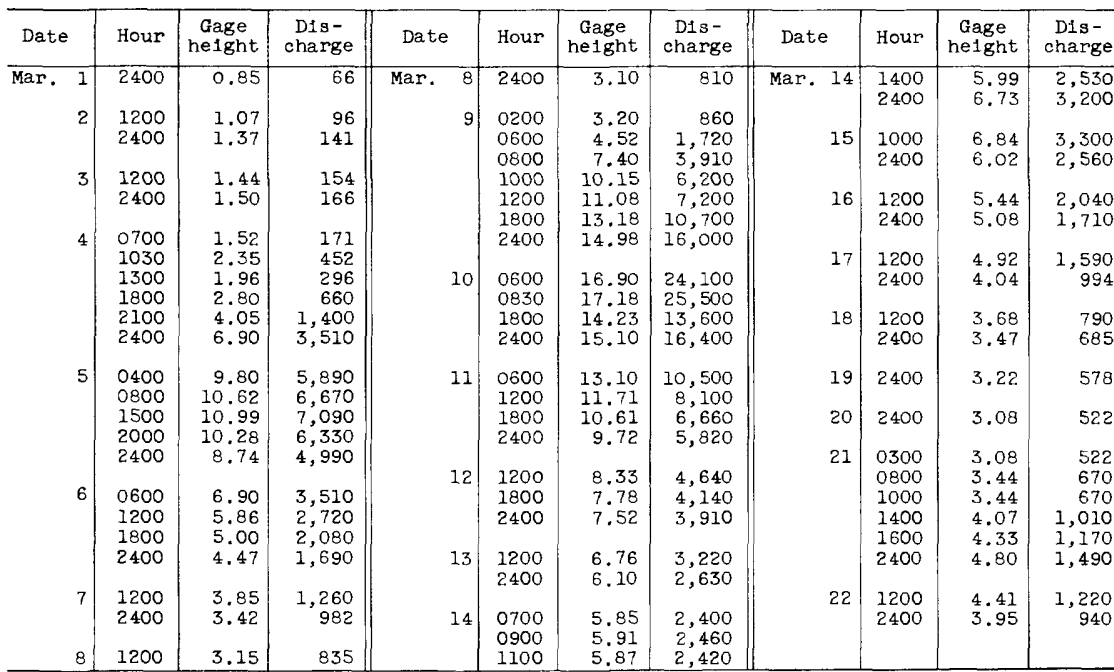

Note.--Dally means for some days computed from data in addition to figures shown. 
(10) 3-1483. Moxahala Creek at Roseville, Oh1o

(Recording crest-stage station)

Location.--Lat $39^{\circ} 48^{\prime} 40^{\prime \prime}$, long $82^{\circ} 04^{\prime} 10^{\prime \prime}$, at pumping station about $2,500 \mathrm{ft}$ below First Street Bridge in Roseville, Nuskingum County.

Drainage area. $--80.8 \mathrm{sq} \mathrm{ml}$.

Gage-helght record.--Crest stage only, from floodmarks, because of faulty operation of bubble gage. Datum of gage is $717.38 \mathrm{ft}$ above mean sea level, datum of 1929 , unadjusted.

Discharge record. --Stage-discharge relation deflined by current-meter measurements.

Maxima.--March 1964: D1scharge, 5,540 cfs Mar. 10, 1100 hours (gage he1ght, $16.6 \mathrm{ft}$, from floodmarks).

1962 to February 1964: D1scharge, 5,600 cfs Mar. 5, 1963 (gage he1ght, $16.7 \mathrm{ft}$, from floodmarks).

(11) 3-1500. Muskingum River at McConnelsville, Ohio

Locat1on.--Lat $39^{\circ} 38^{\prime} 40^{\prime \prime}$, long $81^{\circ} 51^{\prime} 00^{\prime \prime}$, on left bank just upstream from dam 7 , at McConnelsville, Morgan County, and $3 \frac{1}{2}$ miles downstream from 01lspring Run.

Drainage area $.-7,411 \mathrm{sq} \mathrm{mi}$.

Gage-helght record.--Water-stage recorder graph. Datum of gage is $650.31 \mathrm{ft}$ above mean sea level, adjustment of 1912.

Discharge record.--Stage-discharge relation defined by current-meter measurements below 35,000 cfs. Maxima - -March 1964: Discharge, $60,500 \mathrm{cfs}$ Mar. 10, 1100 hours (gage height,

1921 to Febmuary 1964: Discharge, 126,000 cfs Jan. 26, 1937 (gage he1ght, $21.14 \mathrm{ft}$ )

Maximum stage known, $33.5 \mathrm{ft}$ Mar. 27, 1913 (discharge, 270,000 cfs, computed by Corps of Engineers).

Remarks.--Flow regulated by 15 flood-control reservolrs (total capacity, 1,813,200 acre-ft, 5,014.5 sq mi controlled) since 1959. Flood stage, $11 \mathrm{ft}$.

Mean discharge, in cub1c feet per second, March 1964

\begin{tabular}{|c|c|c|c|c|c|c|c|c|c|}
\hline Day & D1scharge & Day & D1scharge & Day & D1scharge & Day & D1scharge & Day & D1scharge \\
\hline $\begin{array}{l}1 \ldots \ldots \\
2 \ldots \ldots \\
3 \ldots \ldots \\
4 \ldots \\
5 \ldots \\
6 \ldots\end{array}$ & $\begin{array}{r}1,280 \\
1,710 \\
2,590 \\
7,320 \\
28,000 \\
30,100\end{array}$ & $\begin{array}{r}7 \ldots \ldots \\
8 \ldots \ldots \\
9 \ldots \\
10 \ldots \\
11 \ldots \\
12 \ldots\end{array}$ & $\begin{array}{l}19,100 \\
15,800 \\
31,000 \\
56,600 \\
47,200 \\
33,100\end{array}$ & $\begin{array}{l}13 \ldots \ldots \\
14 \ldots \ldots \\
15 \ldots \ldots \\
16 \ldots \ldots \\
17 \ldots \ldots \\
18 \ldots \ldots\end{array}$ & $\begin{array}{l}22,800 \\
28,600 \\
33,600 \\
34,300 \\
34,000 \\
34,800\end{array}$ & $\begin{array}{l}19 \ldots \ldots \\
20 \ldots \ldots \\
21 \ldots \ldots \\
22 \ldots \ldots \\
23 \ldots \ldots \\
24 \ldots \ldots\end{array}$ & $\begin{array}{l}34,400 \\
34,000 \\
33,900 \\
33,700 \\
33,500 \\
33,000\end{array}$ & $\mid \begin{array}{l}25 \ldots \ldots \\
26 \ldots \ldots \\
27 \ldots \ldots \\
28 \ldots \ldots \\
29 \ldots \ldots \\
30 \ldots \ldots \\
31 \ldots \ldots\end{array}$ & $\begin{array}{l}32,500 \\
32,800 \\
32,700 \\
31,500 \\
30,000 \\
28,600 \\
24,900\end{array}$ \\
\hline
\end{tabular}

Gage helght, in feet, and discharge, in cub1c feet per second, at 1nd1cated t1me, 1964

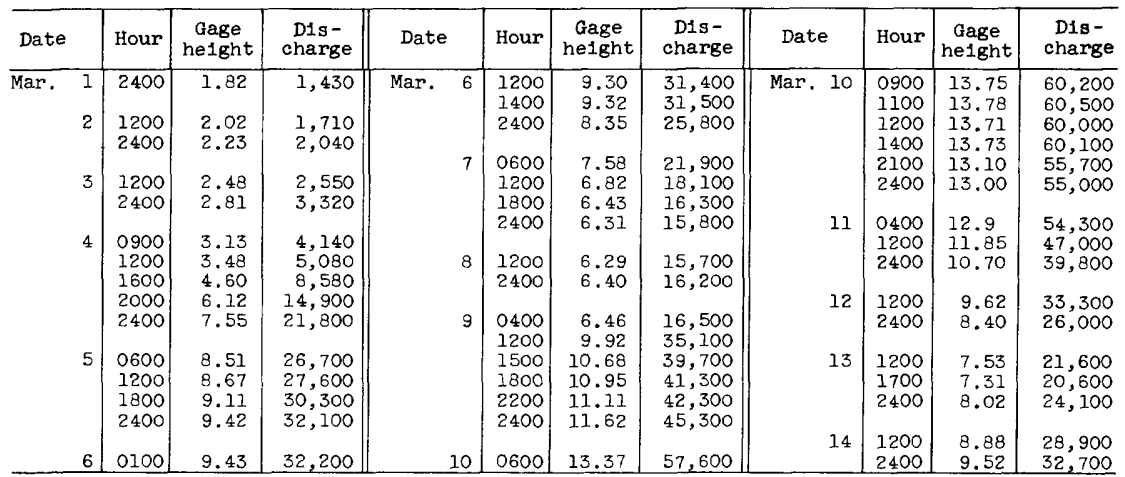

Note.--Dally means for some days computed from data in addition to flgures shown. 


\section{OHIO RIVER MAIN STEM}

(12) 3-1510. Ohio River at Parkersburg, W. Va.

Location. --Lat $39^{\circ} 16^{\prime} 05^{\prime \prime}$, long $81^{\circ} 33^{\prime} 50^{\prime \prime}$, on left bank on downstream side of bridge on U.S. Highway 50 at Parkersburg, Wood County, 0.3 mile upstream from Little Kanawha River, and at mile 184.4.

Drainage area. $--35,600 \mathrm{sq} \mathrm{mi}$, approximately.

Gage-helght record.--Water-stage recorder graphs. Auxillary water-stage recorder graph 0.4 mile downstream from lock and dam 18 and 4.0 miles upstream from base gage. Datum of gage is $562.01 \mathrm{ft}$ above mean sea level, Sandy Hook datum.

Discharge record. - Stage-fall-discharge relation defined by current-meter measurements. Discharge computed by using fall as a factor.

Maxima --March 1964: Discharge, 425,000 cfs Mar. 12, 0500 hours: gage height, 45.07 ft Mar. 12 , 1700 hours.

January 1940 to February 1964: Discharge, 440,000 cfs Jan. 1, 1943; gage height, $49.03 \mathrm{ft}$ Jan. 1, 1943.

Maximum stage known, 58.9 ft Mar. 29, 1913 (discharge, 593,000 cf's).

Remarks.--Flow partly regulated by locks, dams, and reservoirs upstream.

Mean discharge, In cubic feet per second, March 1964

\begin{tabular}{|c|c|c|c|c|c|c|c|c|c|}
\hline Day & Discharge & Day & Discharge & Day & Discharge & Day & Discharge & Day & Discharge \\
\hline $\begin{array}{l}1 \ldots \ldots \\
2 \ldots \ldots \\
3 \ldots \ldots \\
4 \ldots \ldots \\
5 \ldots \ldots \\
6 \ldots \ldots\end{array}$ & $\begin{array}{r}16,900 \\
20,800 \\
46,000 \\
71,400 \\
122,000 \\
180,000\end{array}$ & $\begin{array}{r}7 \ldots \ldots \\
8 \ldots \ldots \\
9 \ldots \ldots \\
10 \ldots \\
11 \ldots \ldots \\
12 \ldots\end{array}$ & $\begin{array}{l}246,000 \\
240,000 \\
235,000 \\
331,000 \\
410,000 \\
416,000\end{array}$ & $\begin{array}{l}13 \ldots \ldots \\
14 \ldots \ldots \\
15 \ldots \ldots \\
16 \ldots \ldots \\
17 \ldots \ldots \\
18 \ldots \ldots\end{array}$ & $\begin{array}{l}348,000 \\
263,000 \\
238,000 \\
221,000 \\
204,000 \\
202,000\end{array}$ & $\begin{array}{l}19 \ldots \ldots \\
20 \ldots \ldots \\
21 \ldots \ldots \\
22 \ldots \ldots \\
23 \ldots \ldots \\
24 \ldots \ldots\end{array}$ & $\begin{array}{r}190,000 \\
166,000 \\
139,000 \\
119,000 \\
105,000 \\
93,800\end{array}$ & $\begin{array}{l}25 \ldots \ldots \\
26 \ldots \ldots \\
27 \ldots \ldots \\
28 \ldots \ldots \\
29 \ldots \ldots \\
30 \ldots \ldots \\
31 \ldots \ldots\end{array}$ & $\begin{array}{l}88,700 \\
85,400 \\
85,200 \\
85,900 \\
89,400 \\
85,100 \\
78,400\end{array}$ \\
\hline
\end{tabular}

Gage height, in feet, and discharge, in cublc feet per second, at indicated time, 1964

\begin{tabular}{|c|c|c|c|c|c|c|c|c|c|c|c|c|}
\hline Date & Hour & $\begin{array}{c}\text { Gage } \\
\text { helght }\end{array}$ & $\begin{array}{c}\text { D1s- } \\
\text { charge }\end{array}$ & \multicolumn{2}{|l|}{ Date } & Hour & $\begin{array}{c}\text { Gage } \\
\text { height }\end{array}$ & $\begin{array}{l}\text { Dis- } \\
\text { charge }\end{array}$ & Date & Hour & $\begin{array}{c}\text { Gage } \\
\text { height }\end{array}$ & $\begin{array}{c}\text { Dis- } \\
\text { charge }\end{array}$ \\
\hline Feb. 29 & 2400 & 11.05 & 16,500 & \multirow[t]{18}{*}{ Mar. } & & $\begin{array}{l}0600 \\
1200\end{array}$ & $\begin{array}{l}29.66 \\
30.89\end{array}$ & $\begin{array}{l}234,000 \\
252,000\end{array}$ & \multirow[t]{2}{*}{ Mar. 13} & $\begin{array}{l}1200 \\
2400\end{array}$ & $\begin{array}{l}43.60 \\
41.40\end{array}$ & $\begin{array}{l}353,000 \\
292,000\end{array}$ \\
\hline Mar. I & 0600 & 10.97 & 17,300 & & & 1800 & 31.82 & 260,000 & & & & \\
\hline & $\begin{array}{l}1200 \\
1800\end{array}$ & $\begin{array}{l}10.88 \\
10.83\end{array}$ & $\begin{array}{l}17,400 \\
16,600\end{array}$ & & & 2400 & 32.40 & 259,000 & \multirow[t]{2}{*}{14} & $\begin{array}{l}1200 \\
2400\end{array}$ & $\begin{array}{l}38.72 \\
36.40\end{array}$ & $\begin{array}{l}261,000 \\
247,000\end{array}$ \\
\hline & 2400 & & 16,400 & & 8 & 0600 & 32.61 & 252,000 & & & & \\
\hline & & & & & & 1200 & 32.46 & 242,000 & \multirow[t]{2}{*}{15} & 1200 & 34.95 & 238,000 \\
\hline 2 & $\begin{array}{l}0600 \\
1200\end{array}$ & $\begin{array}{r}10.98 \\
11.38\end{array}$ & $\begin{array}{l}17,400 \\
21,600\end{array}$ & & & $\begin{array}{l}1800 \\
2400\end{array}$ & $\begin{array}{l}32.10 \\
31.58\end{array}$ & $\begin{array}{l}227,000 \\
216,000\end{array}$ & & 2400 & .04 & 231,000 \\
\hline & 1800 & 11.43 & 21,700 & & & & & & \multirow[t]{2}{*}{16} & 1200 & 33.20 & 220,000 \\
\hline & 2400 & 11.75 & 28,500 & & 9 & $\begin{array}{l}0600 \\
1200\end{array}$ & $\begin{array}{l}31.05 \\
30.86\end{array}$ & $\begin{array}{l}211,000 \\
225,000\end{array}$ & & 2400 & 18 & 000 \\
\hline 3 & $\begin{array}{l}0600 \\
1200 \\
1800\end{array}$ & $\begin{array}{l}12.00 \\
12.22 \\
12.65\end{array}$ & $\begin{array}{l}35,000 \\
46,700 \\
58,100\end{array}$ & & & $\begin{array}{l}1800 \\
2400\end{array}$ & $\begin{array}{l}31.66 \\
33.02\end{array}$ & $\begin{array}{l}256,000 \\
282,000\end{array}$ & 17 & $\begin{array}{l}1200 \\
2400\end{array}$ & $\begin{array}{l}31.12 \\
30.11\end{array}$ & $\begin{array}{l}203,000 \\
201,000\end{array}$ \\
\hline & 2400 & 12.71 & 60,000 & & 10 & $\begin{array}{l}0600 \\
1200\end{array}$ & $\begin{array}{l}35.02 \\
36.92\end{array}$ & $\begin{array}{l}302,000 \\
329,000\end{array}$ & 18 & $\begin{array}{l}1200 \\
2400\end{array}$ & $\begin{array}{l}28.84 \\
27.80\end{array}$ & $\begin{array}{l}205,000 \\
199,000\end{array}$ \\
\hline 4 & 0600 & 13.08 & 60,700 & & & 1800 & 38.68 & 359,000 & \multirow[b]{2}{*}{19} & & & \\
\hline & $\begin{array}{l}1200 \\
1800\end{array}$ & & $\begin{array}{l}71,000 \\
77,900\end{array}$ & & & 2400 & 40.30 & 38 & & $\begin{array}{l}1200 \\
2400\end{array}$ & $\begin{array}{l}26.71 \\
25.52\end{array}$ & $\begin{array}{l}191,000 \\
178,000\end{array}$ \\
\hline & 2400 & .10 & & & 11 & $\begin{array}{l}0600 \\
1200\end{array}$ & $\begin{array}{l}41.67 \\
42.74\end{array}$ & $\begin{array}{l}404,000 \\
416,000\end{array}$ & \multirow[t]{2}{*}{20} & 1200 & & 000 \\
\hline 5 & $\begin{array}{l}0600 \\
1200\end{array}$ & $\begin{array}{l}18.83 \\
20.48\end{array}$ & $\begin{array}{l}110,000 \\
123,000\end{array}$ & & & $\begin{array}{l}1800 \\
2400\end{array}$ & $\begin{array}{l}42.14 \\
43.57 \\
44.19\end{array}$ & $\begin{array}{l}419,000 \\
422,000\end{array}$ & & 2400 & .100 & 000 \\
\hline & 1800 & 21.73 & 135,000 & & & & & & \multirow[t]{2}{*}{21} & 1200 & 20.72 & 000 \\
\hline & 2400 & 22.72 & 150,000 & & 12 & $\begin{array}{l}0500 \\
0600\end{array}$ & $\begin{array}{l}44.58 \\
44.65\end{array}$ & $\begin{array}{l}425,000 \\
423,000\end{array}$ & & 2400 & 19.21 & 000 \\
\hline 6 & $\begin{array}{l}0500 \\
1200\end{array}$ & $\begin{array}{l}23.82 \\
24.97\end{array}$ & $\begin{array}{l}162,000 \\
178,000\end{array}$ & & & $\begin{array}{l}1200 \\
1700\end{array}$ & $\begin{array}{l}44.96 \\
45.07\end{array}$ & $\begin{array}{l}421,000 \\
412,000\end{array}$ & \multirow[t]{2}{*}{22} & $\begin{array}{l}1200 \\
2400\end{array}$ & $\begin{array}{l}18.28 \\
17.42\end{array}$ & $\begin{array}{l}120,000 \\
112,000\end{array}$ \\
\hline & $\begin{array}{l}2800 \\
2400\end{array}$ & $\begin{array}{l}26.42 \\
28.13\end{array}$ & $\begin{array}{l}196,000 \\
215,000\end{array}$ & & & $\begin{array}{l}1800 \\
2400\end{array}$ & $\begin{array}{l}45.06 \\
44.87\end{array}$ & $\begin{array}{l}408,000 \\
395,000\end{array}$ & & & & \\
\hline
\end{tabular}




\section{LITTLE KANAWHA RIVER BASIN}

(13) 3-1550. Little Kanawha River at Palestine, W. Va.

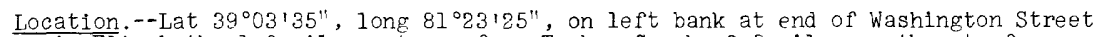
In Elizabeth, 1.0 mile upstream from Tucker Creek, 2.3 miles northeast of Palestine, Wirt county, and 2.4 miles upstream from old lock 3.

Drainage area. $--1,515 \mathrm{sq} \mathrm{mi}$.

Gage-height record.--Water-stage recorder graphs. Auxiliary water-stage recorder graph 3 miles upstream at old lock 4. Datum of gage is $585.51 \mathrm{ft}$ above mean sea level, datum of 1929, Parkersburg-Uniontown supplementary adjustment of 1944 .

Discharge record.--Stage-fall-discharge relation or stage-discharge relation defined by current-meter measurements below 39,000 cfs. Fall used as a factor Mar. 11-13.

Maxima --Narch 1964: Discharge, 20,700 cfs Mar. 4, 1800-1900 hours (gage helght, $24.45 \mathrm{ft}$ ).

1939 to February 1964: Discharge, 46,300 cfs Apr. 14, 1948 (gage height, $30.4 \mathrm{ft}$ at datum about $5 \mathrm{ft}$ higher).

Flood of Apr. 17, 1939, reached a stage of $32.25 \mathrm{ft}$, from floodmarks at

lock 4, present datum (discharge, about $53,000 \mathrm{cfs}$ ).

Mean discharge, in cubic feet per second, March 1964

\begin{tabular}{|c|c|c|c|c|c|c|c|c|c|}
\hline Day & Discharge & Day & Discharge & Day & Discharge & Day & Discharge & Day & Discharge \\
\hline $\begin{array}{l}1 \ldots \ldots \\
2 \ldots \ldots \\
3 \ldots \ldots \\
4 \ldots \ldots \\
5 \ldots \\
6 \ldots\end{array}$ & $\begin{array}{r}1,580 \\
2,770 \\
11,500 \\
19,600 \\
16,000 \\
10,500\end{array}$ & $\begin{array}{r}7 \ldots \ldots \\
8 \ldots \ldots \\
9 \ldots \ldots \\
10 \ldots \\
11 \ldots \ldots \\
12 \ldots \ldots\end{array}$ & $\begin{array}{r}5, E 20 \\
3,480 \\
6,650 \\
10,200 \\
8,240 \\
5,440\end{array}$ & $\mid \begin{array}{l}13 \ldots \ldots \\
14 \ldots \ldots \\
15 \ldots \ldots \\
16 \ldots \ldots \\
17 \ldots \ldots \\
18 \ldots \ldots\end{array}$ & $\begin{array}{r}3,680 \\
2,750 \\
10,800 \\
13,300 \\
7,750 \\
3,800\end{array}$ & $\mid \begin{array}{l}19 \ldots \ldots \\
20 \ldots \ldots \\
21 \ldots \ldots \\
22 \ldots \ldots \\
23 \ldots \ldots \\
24 \ldots\end{array}$ & $\begin{array}{l}2,340 \\
1,660 \\
1,360 \\
1,300 \\
1,380 \\
1,350\end{array}$ & $\begin{array}{l}25 \ldots \ldots \\
26 \ldots \ldots \\
27 \ldots \ldots \\
28 \ldots \ldots \\
29 \ldots \ldots \\
30 \ldots \ldots \\
31 \ldots \ldots\end{array}$ & $\begin{array}{r}1,160 \\
1,040 \\
970 \\
890 \\
784 \\
736 \\
728\end{array}$ \\
\hline $\begin{array}{l}\text { thly } \\
\text { aof }\end{array}$ & $\begin{array}{l}\text { mean d } \\
\text { in inc }\end{array}$ & cge, in & dofc feet & per se & & & & & $\begin{array}{r}5,141 \\
3.91\end{array}$ \\
\hline
\end{tabular}

\section{HOCKING RIVER BASIN}

(14) 3-1564. Hocking River at Lancaster, Ohio

Location.--Lat $39^{\circ} 42^{\prime} 2^{\prime \prime}$, long $82^{\circ} 36^{\prime} 05^{\prime \prime}$, on right bank $25 \mathrm{ft}$ upstream from columbus Street Bridge in Lancaster, Falrfield County, and half a mile downstream from Hunters Run.

Drainage area,$-48.2 \mathrm{sq} \mathrm{mi}$.

Gage-height record.--Water-stage recorder graph. Datum of gage is $797.9 \mathrm{ft}$ above mean sea level, datum of 1929.

Discharge record.--Stage-discharge relation defined by current-meter measurements below $1,500 \mathrm{cf}$ 's by indrect measurement at 2,510 ef's.

Maxima --March 1964: Discharge, 2,510 cfs Mar. 10, 0300 hours (gage height, $15.15 \mathrm{ft}^{\circ}$.

1956 to February 1964: D1scharge, 3,420 cfs Mar. 4, 1963 (gage height, $15.36 \mathrm{ft}$ ).

Remarks.--Floodflow affected by temporary retention in eight retarding basins (combined capacity, 6,245 acre-ft) above station. Controlled area is $24.4 \mathrm{sq} \mathrm{m}$.

\begin{tabular}{|c|c|c|c|c|c|c|c|c|c|}
\hline Day & Discharge & Day & Discharge & Day & Discharge & Day & Discharge & Day & Discharge \\
\hline $\begin{array}{l}1 \ldots \ldots \\
2 \ldots \\
3 \ldots \\
4 \ldots \\
5 \ldots \\
6 \ldots\end{array}$ & $\begin{array}{r}20 \\
27 \\
29 \\
213 \\
308 \\
104\end{array}$ & $\begin{array}{r}7 \ldots \ldots \\
8 \ldots \ldots \\
9 \ldots \\
10 \ldots \\
11 \ldots \\
12 \ldots\end{array}$ & $\begin{array}{r}62 \\
71 \\
1,220 \\
1,830 \\
542 \\
204\end{array}$ & $\begin{array}{l}13 \ldots \ldots \\
14 \ldots \ldots \\
15 \ldots \ldots \\
16 \ldots \\
17 \ldots \ldots \\
18 \ldots \ldots\end{array}$ & $\begin{array}{r}137 \\
129 \\
129 \\
85 \\
68 \\
56\end{array}$ & $\begin{array}{l}19 \ldots \ldots \\
20 \ldots \ldots \\
21 \ldots \ldots \\
22 \ldots \ldots \\
23 \ldots \ldots \\
24 \ldots \ldots\end{array}$ & $\begin{array}{l}50 \\
47 \\
62 \\
54 \\
46 \\
42\end{array}$ & $\begin{array}{l}25 \ldots \ldots \\
26 \ldots \ldots \\
27 \ldots \ldots \\
28 \ldots \ldots \\
29 \ldots \ldots \\
30 \ldots \ldots \\
31 \ldots \ldots\end{array}$ & $\begin{array}{l}42 \\
93 \\
64 \\
51 \\
45 \\
41 \\
39\end{array}$ \\
\hline thly & mean discl & ge, in & cublc feet & per se & ond....... & $\ldots$ & - & & 191 \\
\hline
\end{tabular}


(15) Little Rush Creek near Rushville, Ohio

(Miscellaneous site)

Location.--Lat $39^{\circ} 48^{\prime} 01^{\prime \prime}$, long $82^{\circ} 25^{\prime} 41^{\prime \prime}$, at bridge on State Highway 664, $1 \frac{1}{4}$ miles upstream from Indian Creek and $2 \frac{1}{2}$ miles north of Rushville, Fairfield county.

Drainage area. $--30.1 \mathrm{sq} \mathrm{ml}$.

Discharge record.--Peak discharge by indirect measurement, at same site used for 1963 flood measurement.

Maxima.--March 1964: Discharge, 3,970 cfs Mar. 10. March 1963: Discharge, 5,140 cf's Mar. 4.

(16) 3-1570. Clear Creek near Rockbridge, Onio

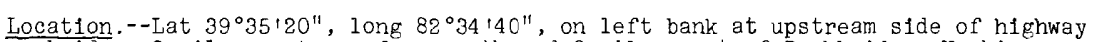
bridge, 2 miles upstream from mouth and 3 miles west of Rockbridge, Hocking County.

Drainage area. $--89.0 \mathrm{sq} \mathrm{m}$.

Gage-helght record.--Water-stage recorder graph. Datum of gage is $760.13 \mathrm{ft}$ above mean sea level, adjustment of 1912 .

Discharge record.--Stage-discharge relation defined by current-meter measurements below $4,300 \mathrm{c}$ fs and by indirect measurements at 16,000 and $8,480 \mathrm{cfs}$.

Maxima.--March 1964: Discharge, 5,830 cfs Mar. 10, 0530 hours (gage height, $12.83 \mathrm{ft}^{\circ} \mathrm{t}$.

1939 to February 1964: Discharge, 16,000 cf's July 22, 1948 (gage height, $17.68 \mathrm{ft}$, from high-water mark in well).

Mean discharge, in cublc feet per second, March 1964

\begin{tabular}{|c|c|c|c|c|c|c|c|c|c|}
\hline Day & Discharge & Day & Discharge & Day & Discharge & Day & Discharge & Day & Discharge \\
\hline $\begin{array}{l}1 \ldots \ldots \\
2 \ldots \\
3 \ldots \ldots \\
4 \ldots \ldots \\
5 \ldots \ldots \\
6 \ldots \ldots\end{array}$ & $\begin{array}{r}21 \\
29 \\
32 \\
305 \\
671 \\
172\end{array}$ & $\begin{array}{r}7 \ldots \\
8 \ldots \\
9 \ldots \\
10 \ldots \\
11 \ldots \\
12 \ldots\end{array}$ & $\begin{array}{r}104 \\
103 \\
2,180 \\
3,540 \\
770 \\
448\end{array}$ & $\mid \begin{array}{l}13 \ldots \ldots \\
14 \ldots \ldots \\
15 \ldots \ldots \\
16 \ldots \ldots \\
17 \ldots \ldots \\
18 \ldots \ldots\end{array}$ & $\begin{array}{l}345 \\
370 \\
415 \\
289 \\
229 \\
155\end{array}$ & $\begin{array}{l}19 \ldots \ldots \\
20 \ldots \ldots \\
21 \ldots \ldots \\
22 \ldots \ldots \\
23 \ldots \ldots \\
24 \ldots \ldots\end{array}$ & $\begin{array}{r}136 \\
149 \\
180 \\
157 \\
112 \\
97\end{array}$ & $\mid \begin{array}{l}25 \ldots \ldots \\
26 \ldots \ldots \\
27 \ldots \ldots \\
28 \ldots \ldots \\
29 \ldots \ldots \\
30 \ldots \ldots \\
31 \ldots \ldots\end{array}$ & $\begin{array}{r}91 \\
178 \\
128 \\
108 \\
97 \\
90 \\
85\end{array}$ \\
\hline hly & 0 & & & & & & & & $\begin{array}{r}380 \\
4.92 \\
\end{array}$ \\
\hline
\end{tabular}


(17) 3-1575. Hocking River at Enterprise, Ohio

Location.--Lat $39^{\circ} 33^{\prime} 54^{\prime \prime}$, long $82^{\circ} 28^{\prime} 30^{\prime \prime}$, on right bank at upstream side of highway bridge at Enterprise, Hocking County, 4 miles downstream from Buck Rur.

Drainage area. $--459 \mathrm{sq} \mathrm{mi}$.

Gage-height record.--Digital recorder tape punched at 30-minute intervals except 0800 hours to 2400 hours Mar. 9 when float was hung up on frost floor. Waterstage recorder graph is avaliable 0800 hours to 1130 hours Mar. 9 , and estimates for remainder of missing record are based on record before and after period.

Datum of gage is $723.58 \mathrm{ft}$ above mean sea level, datum of 1929 , supplementary adjustment of 1944 .

Discharge record.--Stage-discharge relation defined by current-meter measurements below $17,000 \mathrm{cfs}$ and by indirect measurements at $25,200 \mathrm{cfs}$ and $26,000 \mathrm{cfs}$.

Maxima.--March 1964: Discharge, 26,000 efs Mar. 10, 1230 hours (gage height, $21.31 \mathrm{ft}$ ).

1930 to February 1964: Discharge, 25,200 cfs Apr. 20, 1940 (gage height, $19.94 \mathrm{ft}$ ).

Maximum stage known, $22.0 \mathrm{ft}$ in March 1907, from floodmark (discharge, 36,000 cfs), from reports of Corps of Engineers.

Mean discharge, in cubic feet per second, March 1964

\begin{tabular}{|c|c|c|c|c|c|c|c|c|c|}
\hline Day & Discharge & Day & D1scharge & Day & D1scharge & Day & D1scharge & Day & D1scharge \\
\hline $\begin{array}{l}1 \ldots \ldots \\
2 \ldots \ldots \\
3 \ldots \ldots \\
4 \ldots \ldots \\
5 \ldots \ldots \\
6 \ldots \ldots\end{array}$ & $\begin{array}{r}102 \\
156 \\
239 \\
943 \\
3,260 \\
2,860\end{array}$ & $\begin{array}{r}7 \ldots \ldots \\
8 \ldots \ldots \\
9 \ldots \ldots \\
10 \ldots \\
11 \ldots \ldots \\
12 \ldots \ldots\end{array}$ & $\begin{array}{r}1,240 \\
762 \\
4,440 \\
20,600 \\
14,300 \\
5,520\end{array}$ & $\mid \begin{array}{l}13 \ldots \ldots \\
14 \ldots \ldots \\
15 \ldots \ldots \\
16 \ldots \ldots \\
17 \ldots \ldots \\
18 \ldots \ldots\end{array}$ & $\begin{array}{r}2,350 \\
1,910 \\
1,990 \\
1,610 \\
1,180 \\
912\end{array}$ & $\begin{array}{l}19 \ldots \ldots \\
20 \ldots \ldots \\
21 \ldots \ldots \\
22 \ldots \ldots \\
23 \ldots \ldots \\
24 \ldots \ldots\end{array}$ & $\begin{array}{l}783 \\
744 \\
853 \\
937 \\
755 \\
667\end{array}$ & $\left(\begin{array}{l}25 \ldots \ldots \\
26 \ldots \ldots \\
27 \ldots \ldots \\
28 \ldots \ldots \\
29 \ldots \ldots \\
30 \ldots \ldots \\
31 \ldots \ldots\end{array}\right.$ & $\begin{array}{l}615 \\
835 \\
797 \\
660 \\
611 \\
566 \\
538\end{array}$ \\
\hline
\end{tabular}

Note.--Da1ly mean discharge computed on basis of 30 -minute intervals.

Gage he1ght, In feet, and discharge, in cub1c feet per second, at indicated time, 1964

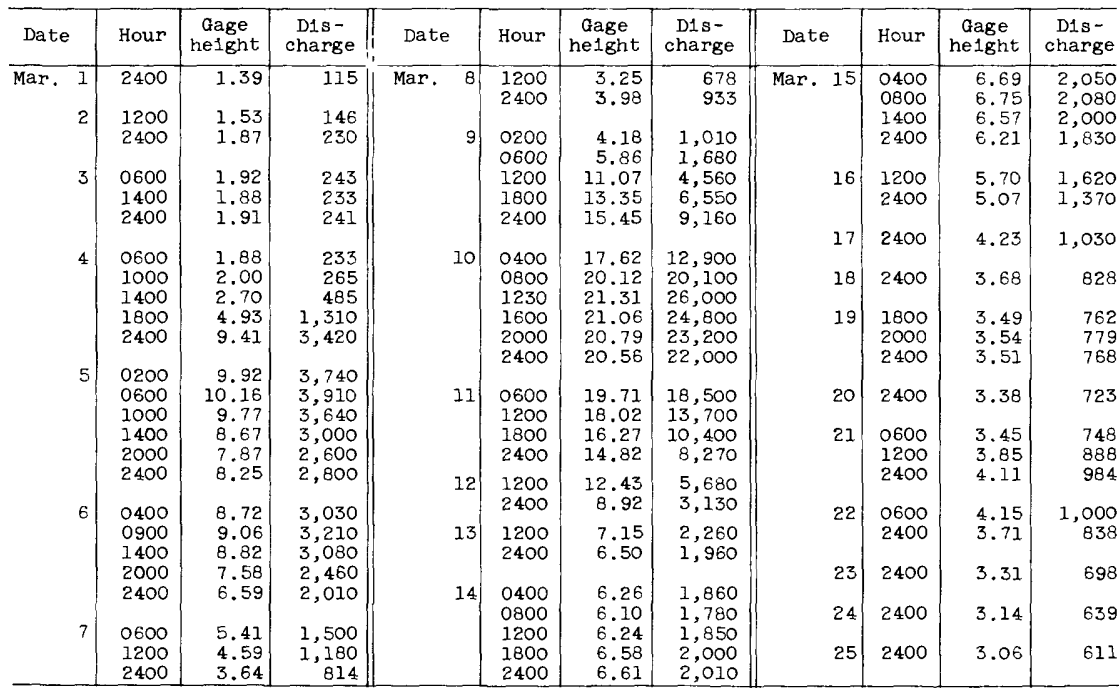


(18) 3-1585. Burr Oak Reservolr at Burr Oak, Ohio

Location.--Lat $39^{\circ} 32^{\prime} 35^{\prime \prime}$, long $82^{\circ} 03^{\prime} 30^{\prime \prime}$, at Tom Jenkins dam on East Branch Sunday Creek, 0.2 mile upstream from mouth, 0.4 mile southeast of Burr Oak, Athens County, and 3 miles northeast of Glouster.

Drainage area. $--33.1 \mathrm{sq} \mathrm{mi}$.

Gage-helght record.--Water-stage recorder graph. Datum of gage is at mean sea level (levels by Corps of Engineers).

Maxima.--March 1964: Contents, 16,370 acre-ft Mar. 13, 2100 hours (elevation, $729.98 \mathrm{ft}$.

1952 to February 1964: Contents, 15,560 acre-ft Mar. 8, 1963 (elevation, $729.05 \mathrm{ft}$ ).

Remarks.--Reservoir is formed by earth dam with emergency spillway; storage began $\overline{\mathrm{Feb}}$ 2, 1952 . Capacity at spillway level (elevation, $740 \mathrm{ft}$ ), 26,900 acre-ft, of which 9,300 acre-ft is in conservation pool. Dead storage, 35 acre-ft. Reservoir is used for flood control, with conservation pool operated for increased low flow for water supply and for recreation and conservation of fish and wildlife. outflow is controlled by operation of gates in conduit through dam.

Cooperation.--Gage-height record and capacity curve furnished by Corps of Engineers.

Elevation and contents, February and March 1964

\begin{tabular}{l|c|c|c||c|c|c|c}
\hline Date & Hour & $\begin{array}{c}\text { Elevation } \\
\text { (feet) }\end{array}$ & $\begin{array}{c}\text { Contents } \\
\text { (acre-feet) }\end{array}$ & Date & Hour & $\begin{array}{c}\text { Elevation } \\
\text { (feet) }\end{array}$ & $\begin{array}{c}\text { Contents } \\
\text { (acre-feet) }\end{array}$ \\
\hline Feb. $29 \ldots \ldots$ & 2400 & 721.12 & 9,340 & Mar. $13 \ldots \ldots$ & 2100 & 729.98 & 16,400 \\
Mar. $7 \ldots \ldots$ & 1000 & 725.27 & 12,380 & Mar. $31 \ldots \ldots$ & 2400 & 721.20 & 9,400 \\
Mar. $9 \ldots \ldots$ & 0500 & 722.49 & 10,290 & & \\
\hline
\end{tabular}

(19) 3-1585.1. Sunday Creek at Burr Oak, Oh10

(Unpublished gaging station)

Location.--Lat $39^{\circ} 32^{\prime} 15^{\prime \prime}$, long $82^{\circ} 04^{\prime} 00^{\prime \prime}, 400 \mathrm{ft}$ upstream from New York Central rallroad bridge, $0.4 \mathrm{mile}$ downstream from East Branch Sunday Creek, and $0.7 \mathrm{mile}$ south of Burr Dak, Athens County.

Drainage area. $--57.5 \mathrm{sq} \mathrm{mi}$.

Gage-height record.--Water-stage recorder graph. Datum of gage is $679.07 \mathrm{ft}$ above mean sea level, datum of 1929. Gage helghts have been computed as elevations above mean sea level.

Discharge record. - Stage-discharge relation defined by current-meter measurements below $900 \mathrm{crs}$ and by indirect measurement at $3,690 \mathrm{cfs}$.

Maxima.--March 1964: Discharge, 2,710 cfs Mar. 10, 0730 hours (elevation, $691.88 \mathrm{ft})$. i 952 to February 1964: Discharge, 3,690 cfs Mar. 5, 1963 (elevation, $692.72 \mathrm{f} t)$.

Remarks.--Affected by storage in Burr Oak Reservolr (see station 3-1585). 
(20) 3-1590. Sunday Creek at Glouster, onfo

Location. --Lat $39^{\circ} 30^{\prime} 00^{\prime \prime}$, long $82^{\circ} 05^{\prime} 05^{\prime \prime}$, on left bank $150 \mathrm{ft}$ downstream from West Branch Sunday Creek and $200 \mathrm{ft}$ upstream from bridge on State Highway 78 at GIouster, Athens County.

Drainage area.--104 sq $\mathrm{ms}$.

Gage-he1ght record.--Water-stage recorder graph. Datum of page is $665.23 \mathrm{ft}$ above mean sea level, adjustment of 1912.

Discharge record.--Stage-discharge relation deflned by current-meter measurements below $3,600 \mathrm{c}$ s and extended on basis of flow-over-road and velocity-area study of Narch 1963 peak flow.

Maxima.--March 1964: D1scharge, 6,320 cfs Mar. 10, 0600 hours (gage he1ght, $17.53 \mathrm{ft}$ ).

1951 to February 1964: D1scharge, 7,020 cfs Mar. 5, 1963 (gage height, $17.81 \mathrm{ft})$.

Maximum stage known, $22.0 \mathrm{ft}$ in March 1907. Flood in March 1913 reached a stage of $18.9 \mathrm{ft}$ and flood in April 1940 a stage of $18.3 \mathrm{ft}$, from information by the Corps of Engineers.

Remarks.--Flow regulated by Burr Oak Reservoir (see station 3-1585).

Mean discharge, in cubic feet per second, March 1964

\begin{tabular}{|c|c|c|c|c|c|c|c|c|c|}
\hline Day & Discharge & Day & Discharge & Day & Discharge & Day & Discharge & Day & D1scharge \\
\hline $\begin{array}{l}1 \ldots \ldots \\
2 \ldots \ldots \\
3 \ldots \ldots \\
4 \ldots \\
5 \ldots \\
6 \ldots\end{array}$ & $\begin{array}{r}29 \\
65 \\
111 \\
607 \\
2,010 \\
511\end{array}$ & $\begin{array}{r}7 \ldots \ldots \\
8 \ldots \ldots \\
9 \ldots \ldots \\
10 \ldots \\
11 \ldots \ldots \\
12 \ldots \ldots\end{array}$ & $\begin{array}{r}436 \\
968 \\
2,160 \\
4,880 \\
823 \\
249\end{array}$ & $\begin{array}{l}13 \ldots \ldots \\
14 \ldots \ldots \\
15 \ldots \ldots \\
16 \ldots \ldots \\
17 \ldots \ldots \\
18 \ldots \ldots\end{array}$ & $\begin{array}{r}210 \\
806 \\
1,140 \\
1,070 \\
909 \\
931\end{array}$ & $\begin{array}{l}19 \ldots \ldots \\
20 \ldots \ldots \\
21 \ldots \ldots \\
22 \ldots \ldots \\
23 \ldots \ldots \\
24 \ldots \ldots\end{array}$ & $\begin{array}{r}755 \\
220 \\
110 \\
89 \\
74 \\
68\end{array}$ & $\begin{array}{l}25 \ldots \ldots \\
26 \ldots \ldots \\
27 \ldots \ldots \\
28 \ldots \ldots \\
29 \ldots \ldots \\
30 \ldots \ldots \\
31 \ldots \ldots\end{array}$ & $\begin{array}{l}69 \\
67 \\
57 \\
52 \\
52 \\
51 \\
49\end{array}$ \\
\hline
\end{tabular}

Gage height, in feet, and discharge, in cubic feet per second, at indicated time, 1964

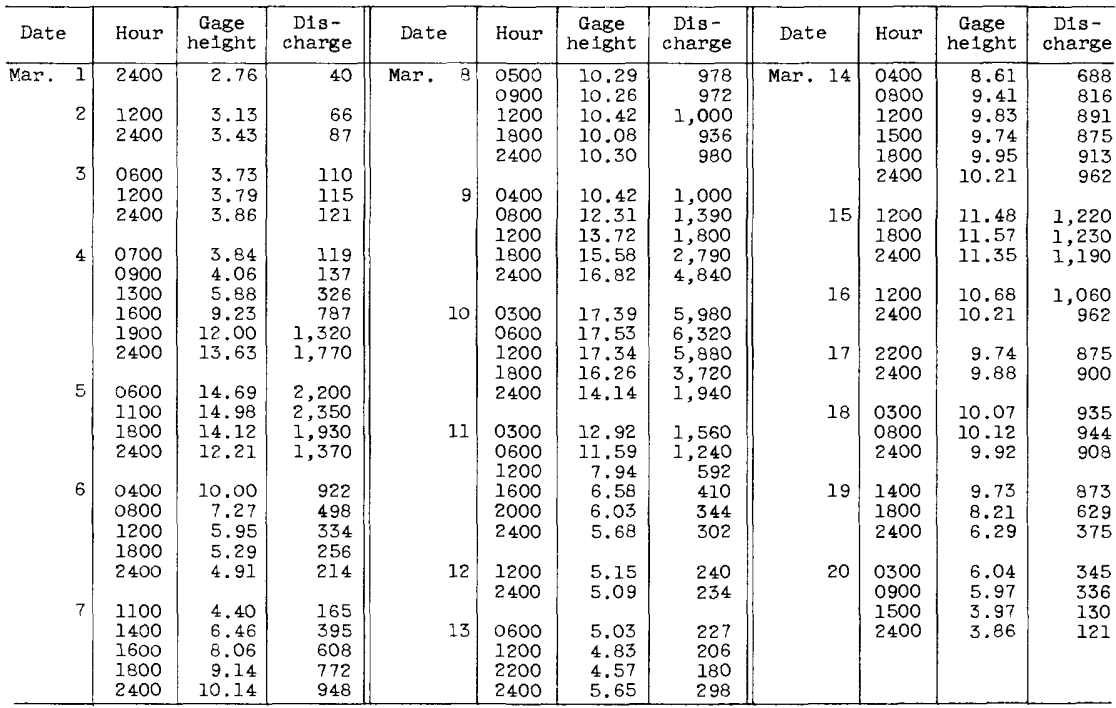

Note.--Dally means for some days computed from data in addition to figures shown. 
(21) 3-1595. Hocking River at Athens, Ohio

Location.--Lat $39^{\circ} 19^{\prime} 45^{\prime \prime}$, long $82^{\circ} 05^{\prime} 17^{\prime \prime}$, on left bank at upstream side of Mill Street Bridge, three-quarters of a mile east of business section of Athens, Athens County, and $3 \frac{1}{2}$ miles downstream from Margaret Creek.

Dra1nage area,$-943 \mathrm{sq} \mathrm{mi}$.

Gage-height record.--Water-stage recorder graph. Datum of gage is $614.81 \mathrm{ft}$ above mean sea lever, datum of 1929 , supplementary adjustment of 1944 .

Discharge record.--Stage-discharge relation defined by current-meter measurements.

Maxima --March 1964: Discharge, 32,900 efs Mar. 11, 0830 hours (gage helght, $24.18 \mathrm{ft}^{\prime} \mathrm{t}$.

1915 to February 1964: Discharge, 30,400 cfs Mar. 7, 1945 (gage height, $23.0 \mathrm{ft}$ ).

Maximum stage known, 26.7 ft in March 1907, from information by local resident

(discharge, 50,000 cfs, computed by Corps of Engineers).

Remarks.--Some regulation by Burr Oak Reservolr (see station 3-1585).

Mean discharge, in cubic feet per second, March 1964

\begin{tabular}{l|r||r|r|r|r||r|r|r|r}
\hline \multicolumn{1}{c|}{ Day } & Discharge & \multicolumn{1}{c|}{ Day } & Discharge & Day & Discharge & Day & Discharge & Day & Discharge \\
\hline $1 \ldots \ldots$ & 178 & $7 \ldots \ldots$ & 4,960 & $13 \ldots \ldots$ & 10,800 & $19 \ldots \ldots$ & 2,340 & $25 \ldots \ldots$ & 1,050 \\
$2 \ldots \ldots$ & 254 & $8 \ldots \ldots$ & 2,830 & $14 \ldots \ldots$ & 4,940 & $20 \ldots \ldots$ & 1,690 & $26 \ldots \ldots$ & 1,120 \\
$3 \ldots \ldots$ & 436 & $9 \ldots \ldots$ & 7,260 & $15 \ldots \ldots$ & 5,790 & $21 \ldots \ldots$ & 1,340 & $27 \ldots \ldots$ & 1,250 \\
$4 \ldots$ & 2,220 & $10 \ldots \ldots$ & 20,100 & $16 \ldots \ldots$ & 5,130 & $22 \ldots \ldots$ & 1,520 & $28 \ldots \ldots$ & 1,080 \\
$5 \ldots$ & 7,740 & $11 \ldots \ldots$ & 31,200 & $17 \ldots \ldots$ & 3,550 & $23 \ldots \ldots$ & 1,350 & $29 \ldots \ldots$ & 958 \\
$6 \ldots$ & 8,830 & $12 \ldots \ldots$ & 21,900 & $18 \ldots \ldots$ & 2,740 & $24 \ldots \ldots$ & 1,150 & $30 \ldots \ldots$ & 888 \\
\end{tabular}

Gage he1ght, in feet, and discharge, in cubic feet per second, at indicated time, 1964

\begin{tabular}{|c|c|c|c|c|c|c|c|c|c|c|c|}
\hline Date & Hour & $\begin{array}{c}\text { Gage } \\
\text { helght }\end{array}$ & $\begin{array}{l}\text { D1s- } \\
\text { charge }\end{array}$ & Date & Hour & $\begin{array}{c}\text { Gage } \\
\text { height }\end{array}$ & $\begin{array}{l}\text { Dis- } \\
\text { charge }\end{array}$ & Date & Hour & $\begin{array}{c}\text { Gage } \\
\text { helght }\end{array}$ & $\begin{array}{l}\text { Dis- } \\
\text { charge }\end{array}$ \\
\hline \multirow[t]{20}{*}{ Mar. } & 2400 & 2.88 & 192 & \multirow[t]{4}{*}{ Mar. } & 0600 & 9.20 & 3,630 & Mar. 16 & 2400 & 10.19 & 4,220 \\
\hline & 2400 & 3.26 & 316 & & 1800 & 16.82 & 10,100 & 17 & 1200 & 9.02 & 3,520 \\
\hline & & & & & 2400 & 18.37 & 13,100 & & 2400 & 8.18 & 3,020 \\
\hline & 2400 & 3.87 & 556 & & & & & \multirow[b]{2}{*}{18} & & & \\
\hline & & & & \multirow[t]{2}{*}{10} & 1200 & 21.02 & 20,400 & & 2400 & 7.26 & 2,510 \\
\hline & $\begin{array}{l}0600 \\
1200\end{array}$ & $\begin{array}{l}3.88 \\
4.93\end{array}$ & $\begin{array}{r}561 \\
1,160\end{array}$ & & 2400 & 23.37 & 28,800 & \multirow[t]{2}{*}{19} & 2400 & 6.55 & 2,160 \\
\hline & 1800 & 9.50 & 3,810 & \multirow[t]{2}{*}{11} & 0500 & 24.00 & 31,800 & & & & \\
\hline & 2400 & 13.23 & 6,180 & & $\begin{array}{l}0830 \\
1200\end{array}$ & $\begin{array}{l}24.18 \\
24.13\end{array}$ & $\begin{array}{l}32,900 \\
32,600\end{array}$ & 20 & $\begin{array}{l}1200 \\
2400\end{array}$ & $\begin{array}{l}5.52 \\
5.08\end{array}$ & $\begin{array}{l}1,620 \\
1,380\end{array}$ \\
\hline & 0600 & 14.87 & 7,650 & \multirow{3}{*}{12} & 2400 & 23.26 & 28,300 & \multirow{2}{*}{21} & 1200 & 4.96 & 1310 \\
\hline & $\begin{array}{l}1200 \\
2400\end{array}$ & $\begin{array}{l}15.20 \\
15.40\end{array}$ & $\begin{array}{l}7,990 \\
8,210\end{array}$ & & 1200 & 21.58 & 22,200 & & 2400 & 5.19 & 1,440 \\
\hline & & & & & 2400 & 19.34 & 15,600 & 22 & 1200 & 5.37 & 1,540 \\
\hline & 0600 & 16.00 & 8,910 & \multirow{3}{*}{13} & & & & & 2400 & 5.29 & 1,490 \\
\hline & $\begin{array}{l}1300 \\
2400\end{array}$ & $\begin{array}{l}16.30 \\
15.04\end{array}$ & $\begin{array}{l}9,300 \\
7,820\end{array}$ & & $\begin{array}{l}1200 \\
2400\end{array}$ & $\begin{array}{l}17.08 \\
13.86\end{array}$ & $\begin{array}{r}10,500 \\
6,690\end{array}$ & 23 & 2400 & 4.79 & 1,220 \\
\hline & & & & & & & & & & 7.10 & I, the \\
\hline & $\begin{array}{l}0600 \\
1200\end{array}$ & $\begin{array}{l}13.34 \\
11.29\end{array}$ & $\begin{array}{l}6,270 \\
4,880\end{array}$ & \multirow[t]{2}{*}{14} & $\begin{array}{l}0800 \\
1200\end{array}$ & $\begin{array}{l}10.76 \\
10.44\end{array}$ & $\begin{array}{l}4,570 \\
4,370\end{array}$ & 24 & 2400 & 4.55 & 1,090 \\
\hline & 1800 & $\begin{array}{r}11.29 \\
8.86\end{array}$ & 3,430 & & 2400 & 11.52 & 5,020 & 25 & 2400 & 4.48 & 1,050 \\
\hline & 2400 & 7.60 & 2,700 & \multirow[t]{3}{*}{15} & 1200 & 12.98 & 6,000 & \multirow{2}{*}{26} & 00 & & \\
\hline & 1200 & 7.56 & 2,670 & & $\begin{array}{l}1800 \\
2400\end{array}$ & $\begin{array}{l}13.05 \\
12.94\end{array}$ & $\begin{array}{l}6,040 \\
5,970\end{array}$ & & & 10 & 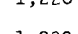 \\
\hline & 1800 & 8.15 & 3,000 & & & & 5,510 & \multirow[t]{2}{*}{27} & 0900 & 4.90 & 1,280 \\
\hline & 2400 & 8.75 & 360 & 16 & 1200 & 11.78 & 5,180 & & 2400 & 4.71 & 1,180 \\
\hline
\end{tabular}

Note.--Dally means for some days computed from data in addition to f'lgures shown. 


\section{OHIO RIVER MAIN STEM}

(22) 3-1600. On10 River at Pomeroy, Ohio

Location.--Lat $38^{\circ} 50^{\prime} 25^{\prime \prime}$, long $82^{\circ} 08^{\prime} 30^{\prime \prime}$, on left bank at Point Pleasant, Mason County, W. Va., I,200 ft upstream from Kanawha R1ver and at mile 265.4 .

Drainage area. $--40,500 \mathrm{sq} \mathrm{ml}$, approximately.

Gage-helght record.--Water-stage recorder graphs except Mar. 1-6. Auxil1ary waterstage recorder graph for gage $13.8 \mathrm{mlles}$ upstream on downstream side of pler of bridge on U.S. H1ghway 33 to Pomeroy. Datum of gage is $514.10 \mathrm{ft}$ above mean sea level, Sandy Hook datum. Datum of auxillary gage is $517.50 \mathrm{f} t$ above mean sea leve1, Sandy Hook datum.

Discharge record.--Stage-fall-discharge relation defined by current-meter measurements below 305,000 cfs. Discharge computed by using fall as a factor. Discharge for period of no gage-helght record estimated on basis of records for other Oh1o River stations.

Maxima.--March 1964: D1scharge, 406,000 cfs Mar. 13, 0200-1000 hours; gage he1ght, 48.87 ft Mar. 13, 0700 hours.

1936-37, 1940 to February 1964: Discharge, 554,000 cf's Jan. 27, 1937 (gage helght, $67.7 \mathrm{ft}$, pass s1ll gage, lock 24 ).

Maximum discharge known, 633,000 cf's Mar. 30, 1913 (gage helght, $70.1 \mathrm{ft}$, pass sil1 gage, lock 24 ).

Remarks.--Flow partiy regulated by locks, dams, and reservolrs upstream.

Mean discharge, in cubic feet per second, March 1964

\begin{tabular}{|c|c|c|c|c|c|c|c|c|c|}
\hline Day & Discharge & Day & Discharge & Day & D1scharge & Day & Discharge & Day & Dlscharge \\
\hline $\begin{array}{l}1 \ldots \ldots \\
2 \ldots \ldots \\
3 \ldots \ldots \\
4 \ldots \ldots \\
5 \ldots \ldots \\
6 \ldots \ldots\end{array}$ & $\begin{array}{r}23,000 \\
26,000 \\
66,000 \\
88,000 \\
119,000 \\
171,000\end{array}$ & $\begin{array}{r}7 \ldots \ldots \\
8 \ldots \ldots \\
9 \ldots \ldots \\
10 \ldots \ldots \\
11 \ldots \ldots \\
12 \ldots \ldots\end{array}$ & $\begin{array}{l}221,000 \\
259,000 \\
260,000 \\
288,000 \\
347,000 \\
394,000\end{array}$ & $\begin{array}{l}13 \ldots \ldots \\
14 \ldots \ldots \\
15 \ldots \ldots \\
16 \ldots \ldots \\
17 \ldots \ldots \\
18 \ldots \ldots\end{array}$ & $\begin{array}{l}403,000 \\
370,000 \\
324,000 \\
288,000 \\
268,000 \\
252,000\end{array}$ & $\begin{array}{l}19 \ldots \ldots \\
20 \ldots \ldots \\
21 \ldots \ldots \\
22 \ldots \ldots \\
23 \ldots \ldots \\
24 \ldots \ldots\end{array}$ & $\begin{array}{l}232,000 \\
204,000 \\
169,000 \\
137,000 \\
119,000 \\
106,000\end{array}$ & $\begin{array}{l}25 \ldots \ldots \\
26 \ldots \ldots \\
27 \ldots \ldots \\
28 \ldots \ldots \\
29 \ldots \ldots \\
30 \ldots \ldots \\
31 \ldots \ldots\end{array}$ & $\begin{array}{l}96,200 \\
93,900 \\
89,800 \\
92,400 \\
93,300 \\
93,200 \\
87,800\end{array}$ \\
\hline
\end{tabular}

Gage height, in feet, and discharge, in cubic feet per second, at indicated time, 1964

\begin{tabular}{|c|c|c|c|c|c|c|c|c|c|c|c|}
\hline Date & Hour & $\begin{array}{c}\text { Gage } \\
\text { helght }\end{array}$ & $\begin{array}{c}\text { D1s- } \\
\text { charge }\end{array}$ & Date & Hour & $\begin{array}{c}\text { Gage } \\
\text { helght }\end{array}$ & $\begin{array}{c}\text { Dis- } \\
\text { charge }\end{array}$ & Date & Hour & $\begin{array}{c}\text { Gage } \\
\text { helght }\end{array}$ & $\begin{array}{c}\text { D1s- } \\
\text { charge }\end{array}$ \\
\hline Mar. 6 & 2400 & 35.43 & 193,000 & Mar. 11 & $\begin{array}{l}1200 \\
1800\end{array}$ & $\begin{array}{l}46.73 \\
47.20\end{array}$ & $\begin{array}{l}348,000 \\
360,000\end{array}$ & Mar. 16 & $\begin{array}{l}1200 \\
2400\end{array}$ & $\begin{array}{l}43.93 \\
43.50\end{array}$ & $\begin{array}{l}286,000 \\
277,000\end{array}$ \\
\hline 7 & 0600 & $\begin{array}{l}35.92 \\
36.57\end{array}$ & 208,000 & & 2400 & 47.52 & 376,000 & 7 & 700 & & \\
\hline & $\begin{array}{l}1800 \\
2400\end{array}$ & $\begin{array}{l}37.18 \\
37.79\end{array}$ & $\begin{array}{l}235,000 \\
246,000\end{array}$ & 12 & $\begin{array}{l}0600 \\
1200\end{array}$ & $\begin{array}{l}47.93 \\
48.24\end{array}$ & $\begin{array}{l}389,000 \\
397,000\end{array}$ & 17 & 2400 & .07 & 258,000 \\
\hline & & & & & 1800 & & 401,000 & 18 & 1200 & 39.47 & 254,000 \\
\hline 8 & $\begin{array}{l}0600 \\
1200\end{array}$ & $\begin{array}{l}38.24 \\
38.58\end{array}$ & $\begin{array}{l}256,000 \\
262,000\end{array}$ & & 2400 & 48,71 & 405,000 & & 2400 & .29 & 244,000 \\
\hline & $\begin{array}{l}1800 \\
2400\end{array}$ & $\begin{array}{l}39.07 \\
39.57\end{array}$ & $\begin{array}{l}264,000 \\
264,000\end{array}$ & 13 & $\begin{array}{l}0200 \\
0700 \\
1000\end{array}$ & $\begin{array}{l}48.78 \\
48.87 \\
48.85\end{array}$ & $\begin{array}{l}406,000 \\
406,000 \\
406,000\end{array}$ & 19 & $\begin{array}{l}1200 \\
2400\end{array}$ & $\begin{array}{l}35.27 \\
33.40\end{array}$ & $\begin{array}{l}232,000 \\
218,000\end{array}$ \\
\hline 9 & $\begin{array}{l}0600 \\
1200 \\
1800\end{array}$ & $\begin{array}{l}39.86 \\
40.37 \\
40.85\end{array}$ & $\begin{array}{l}262,000 \\
258,000 \\
256,000\end{array}$ & & $\begin{array}{l}1200 \\
1800 \\
2400\end{array}$ & $\begin{array}{l}48.85 \\
48.73 \\
48.47\end{array}$ & $\begin{array}{l}405,000 \\
402,000 \\
391,000\end{array}$ & 20 & $\begin{array}{l}1200 \\
2400\end{array}$ & $\begin{array}{l}31.60 \\
29.77\end{array}$ & $\begin{array}{l}204,000 \\
188,000\end{array}$ \\
\hline & 2400 & 41.41 & 260,000 & 14 & 0600 & 48.11 & 381,000 & 21 & $\begin{array}{l}1200 \\
2400\end{array}$ & $\begin{array}{l}28.29 \\
27.60\end{array}$ & $\begin{array}{l}169,000 \\
150,000\end{array}$ \\
\hline 10 & $\begin{array}{l}0600 \\
1200 \\
1800 \\
2400\end{array}$ & $\begin{array}{l}42.25 \\
43.36 \\
44.32 \\
45.24\end{array}$ & $\begin{array}{l}272,000 \\
290,000 \\
303,000 \\
317,000\end{array}$ & & $\begin{array}{l}1200 \\
1800 \\
2400\end{array}$ & $\begin{array}{l}47.65 \\
47.00 \\
46.24\end{array}$ & $\begin{array}{l}370,000 \\
358,000 \\
348,000\end{array}$ & 22 & $\begin{array}{l}1200 \\
2400\end{array}$ & $\begin{array}{l}27.07 \\
27.12\end{array}$ & $\begin{array}{l}136,000 \\
127,000\end{array}$ \\
\hline 11 & 0600 & 45.98 & 335,000 & 15 & $\begin{array}{l}1200 \\
2400\end{array}$ & $\begin{array}{l}45.01 \\
44.21\end{array}$ & $\begin{array}{l}322,000 \\
302,000\end{array}$ & & & & \\
\hline
\end{tabular}




\section{KANAWHA RIVER BASIN}

(23) 3-1980. Kanawha River at Charleston, W. Va.

Location. --Lat $38^{\circ} 22^{\prime} 10^{\prime \prime}$, long $81^{\circ} 42^{\prime} 05^{\prime \prime}$, on left bank at old lock $6,1.0 \mathrm{mile}$ upstream from Davis Creek, $1 \frac{1}{2}$ miles downstream from Twomile Creek, 2.0 miles downstream from Patrick Street Bridge at Charleston, Kanawha County, and 3.5 miles downstream from Elk River.

Drainage area. $--10,419 \mathrm{sq} \mathrm{m} 1$.

Gage-he1ght record.--Water-stage recorder graphs. Auxiliary water-stage recorder graph for gage 2.3 miles upstream. Datum of gage $15548.00 \mathrm{f}^{\prime} t$ above mean sea level, datum of 1929 (levels by Corps of Engineers).

Discharge record.--Stage-fall-discharge relation defined by current-meter measurements. Discharge computed by using fall as a factor.

Maxima.--March 1964: D1scharge, 119,000 cfs Mar. 6, 0100 hours: gage he1ght, $30.23 \mathrm{ft}$ Mar. 6, 0400-0500 hours.

1939 to February 1964: Discharge, 216,000 cf's Aug. 15, 1940; gage height, $39.72 \mathrm{ft}$ Mar. $7,1955$.

Maximum stage known, about $54.3 \mathrm{ft}$ Sept. 29, 1861

Remarks.--Some regulation since 1939 by Claytor Reservoir, since 1949 by Bluestone Reservolr and since 1960 by Sutton Reservoir (combined usable capacity, 982,000 acre-ft).

\begin{tabular}{|c|c|c|c|c|c|c|c|c|c|}
\hline Day & Discharge & Day & Discharge & Day & D1scharge & Day & Discharge & Day & D1scharge \\
\hline $\begin{array}{l}1 \ldots \ldots \\
2 \ldots \ldots \\
3 \ldots \ldots \\
4 \ldots \\
5 \ldots \ldots \\
6 \ldots\end{array}$ & $\begin{array}{r}12,200 \\
14,400 \\
37,400 \\
72,400 \\
111,000 \\
106,000\end{array}$ & $\begin{array}{r}7 \ldots \ldots \\
8 \ldots \ldots \\
9 \ldots \ldots \\
10 \ldots \ldots \\
11 \ldots \ldots \\
12 \ldots \ldots\end{array}$ & $\begin{array}{l}80,500 \\
71,800 \\
91,800 \\
93,400 \\
61,600 \\
35,400\end{array}$ & $\begin{array}{l}13 \ldots \ldots \\
14 \ldots \ldots \\
15 \ldots \ldots \\
16 \ldots \ldots \\
17 \ldots \ldots \\
18 \ldots \ldots\end{array}$ & $\begin{array}{l}21,500 \\
16,400 \\
31,500 \\
73,500 \\
68,500 \\
39,900\end{array}$ & $\begin{array}{l}19 \ldots \ldots \\
20 \ldots \ldots \\
21 \ldots \ldots \\
22 \ldots \ldots \\
23 \ldots \ldots \\
24 \ldots \ldots\end{array}$ & $\begin{array}{l}24,900 \\
19,400 \\
18,000 \\
17,800 \\
15,000 \\
14,900\end{array}$ & $\begin{array}{l}25 \ldots \ldots \\
26 \ldots \ldots \\
27 \ldots \ldots \\
28 \ldots \ldots \\
29 \ldots \ldots \\
30 \ldots \ldots \\
31 \ldots \ldots\end{array}$ & $\begin{array}{l}16,000 \\
15,800 \\
15,400 \\
15,000 \\
14,100 \\
13,900 \\
12,700\end{array}$ \\
\hline
\end{tabular}

\section{OHIO RIVER MAIN STEM}

(24) 3-2015. Oh10 River at Point Pleasant, W. Va.

Location.--Lat $38^{\circ} 50^{\prime} 25^{\prime \prime}$, long $82^{\circ} 08^{\prime} 30^{\prime \prime}$, on left bank at Point Pleasant, Mason County, 1,200 ft upstream from Kanawha River and at mile 265.4. Records include flow of Kanawha River.

Drainage area.--52,760 $\mathrm{sq} \mathrm{mi}$, approximately (Includes that of Kanawha River).

Gage-height record. --Water-stage recorder graphs except Mar. 4-6. Auxillary waterstage recorder graph for gage $0.7 \mathrm{mlle}$ upstream from Gallipolis lock and dam and 13.1 miles downstream from the base gage. Datum of gage is $514.10 \mathrm{ft}$ above mean sea level, Sandy Hook datum. Datum of auxillary gage is $505.22 \mathrm{ft}$ above mean sea level, Sandy Hook datum.

Discharge record.--Stage-fall-discharge relation defined by current-meter measurements below 330,000 cfs. Discharge for Mar. 1, 2, 4-6 estimated on basis of records for other Ohio River stations (stage-fall-discharge relation indefinite Mar. 1, 2). Discharge computed by using fall as a factor.

Maxima.--March 1964: D1scharge, 464,000 efs Mar. 13, 0500-1300 hours; gage height, $48.87 \mathrm{ft}$ Mar. 13, 0700 hours.

1940 to February 1964: Discharge, 522,000 efs Jan. 1, 1943; gage he1ght, 55.00 ft Apr. 16, 1948 .

Maximum stage known since at least 1896, 62.8 ft Mar. 30, 1913. Flood of

Jan. 27, 1937, reached a stage of $62.7 \mathrm{ft}$.

Remarks.--Flow partly regulated by locks, dams, and reservoirs upstream.

Mean discharge, in cuble feet per second, March 1964

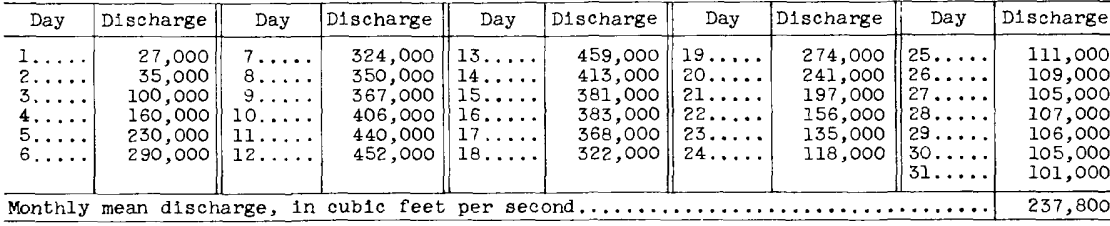


Gage he1ght, in feet, and discharge, in cublc feet per second, at indlcated time, 1964, of

\begin{tabular}{|c|c|c|c|c|c|c|c|c|c|c|c|}
\hline Date & Hour & $\begin{array}{c}\text { Gage } \\
\text { helght }\end{array}$ & $\begin{array}{c}\text { D1s- } \\
\text { charge }\end{array}$ & Date & Hour & $\begin{array}{c}\text { Gage } \\
\text { he1ght }\end{array}$ & $\begin{array}{c}\text { D1s- } \\
\text { charge }\end{array}$ & Date & Hour & $\begin{array}{c}\text { Gage } \\
\text { he1ght }\end{array}$ & $\begin{array}{c}\text { D1s- } \\
\text { charge }\end{array}$ \\
\hline $\begin{array}{l}\text { Feb. } 29 \\
\text { Mer. }\end{array}$ & 2400 & 24.39 & - & Mar. 9 & $\begin{array}{l}1200 \\
1800\end{array}$ & $\begin{array}{l}40.37 \\
40.85\end{array}$ & $\begin{array}{l}368,000 \\
373,000\end{array}$ & Mar. 14 & $\begin{array}{l}1800 \\
2400\end{array}$ & $\begin{array}{l}47.00 \\
46.24\end{array}$ & $\begin{array}{l}398,000 \\
384,000\end{array}$ \\
\hline Mar. 1 & $\begin{array}{l}1200 \\
2400\end{array}$ & $\begin{array}{l}24.46 \\
24.55\end{array}$ & - & & 2400 & 41.41 & 378,000 & 15 & 1200 & 45.01 & 380,000 \\
\hline 2 & $\begin{array}{l}1200 \\
1800 \\
2400\end{array}$ & $\begin{array}{l}24.71 \\
24.73 \\
24.94\end{array}$ & - & 10 & $\begin{array}{l}0600 \\
1200 \\
1800 \\
2400\end{array}$ & $\begin{array}{l}42.25 \\
43.36 \\
44.32 \\
45.24\end{array}$ & $\begin{array}{l}392,000 \\
411,000 \\
417,000 \\
427,000\end{array}$ & 16 & $\begin{array}{l}2400 \\
1200 \\
2400\end{array}$ & $\begin{array}{l}44.21 \\
43.93 \\
43.50\end{array}$ & $\begin{array}{l}380,000 \\
385,000 \\
381,000\end{array}$ \\
\hline 3 & $\begin{array}{l}0600 \\
1100 \\
1700\end{array}$ & $\begin{array}{l}25.11 \\
25.48 \\
25.84\end{array}$ & $\begin{array}{r}79,900 \\
97,100 \\
117,000\end{array}$ & 11. & $\begin{array}{l}0600 \\
1200 \\
1800\end{array}$ & $\begin{array}{l}45.98 \\
46.73 \\
47.20\end{array}$ & $\begin{array}{l}432,000 \\
446,000 \\
447,000\end{array}$ & 17 & $\begin{array}{l}1200 \\
2400\end{array}$ & $\begin{array}{l}42.67 \\
41.37\end{array}$ & $\begin{array}{l}371,000 \\
350,000\end{array}$ \\
\hline & 2400 & $\begin{array}{l}26.34 \\
35.43\end{array}$ & 126,000 & & 2400 & 47.52 & 446,000 & 18 & $\begin{array}{l}1200 \\
2400\end{array}$ & $\begin{array}{l}39.47 \\
37.29\end{array}$ & $\begin{array}{l}323,000 \\
294,000\end{array}$ \\
\hline 6 & 2400 & 35.43 & 309,000 & 12 & $\begin{array}{l}0600 \\
1200\end{array}$ & $\begin{array}{l}47.93 \\
48.24\end{array}$ & $\begin{array}{l}452,000 \\
452,000\end{array}$ & 19 & 1200 & 35.27 & 272,000 \\
\hline 7 & $\begin{array}{l}0600 \\
1200 \\
1800\end{array}$ & $\begin{array}{l}35.92 \\
36.51 \\
37.18\end{array}$ & $\begin{array}{l}315,000 \\
323,000 \\
332,000\end{array}$ & & $\begin{array}{l}1800 \\
2400\end{array}$ & $\begin{array}{l}48.53 \\
48.71\end{array}$ & $\begin{array}{l}454,000 \\
457,000\end{array}$ & 20 & 2400 & $\begin{array}{r}33.40 \\
31.60\end{array}$ & 258,000 \\
\hline & 2400 & 37.79 & 341,000 & 13 & $\begin{array}{l}0500 \\
0700\end{array}$ & $\begin{array}{l}48.85 \\
48.87\end{array}$ & $\begin{array}{l}464,000 \\
464,000\end{array}$ & 20 & 2400 & $\begin{array}{l}31.60 \\
29.77\end{array}$ & 223,000 \\
\hline 8 & $\begin{array}{l}0600 \\
1200 \\
1800\end{array}$ & $\begin{array}{l}38.24 \\
38.58 \\
39.07\end{array}$ & $\begin{array}{l}346,000 \\
348,000 \\
356,000\end{array}$ & & $\begin{array}{l}1300 \\
1800 \\
2400\end{array}$ & $\begin{array}{l}48.85 \\
48.73 \\
48.47\end{array}$ & $\begin{array}{l}464,000 \\
457,000 \\
442,000\end{array}$ & 21 & $\begin{array}{l}1200 \\
2400\end{array}$ & $\begin{array}{l}28.29 \\
27.60\end{array}$ & $\begin{array}{l}195,000 \\
176,000\end{array}$ \\
\hline 9 & $\begin{array}{l}2400 \\
0600\end{array}$ & $\begin{array}{l}39.57 \\
39.86\end{array}$ & $\begin{array}{l}361,000 \\
359,000\end{array}$ & 14 & $\begin{array}{l}0600 \\
1200\end{array}$ & $\begin{array}{l}48.11 \\
47.65\end{array}$ & $\begin{array}{l}426,000 \\
415,000\end{array}$ & 22 & $\begin{array}{l}1200 \\
2400\end{array}$ & $\begin{array}{l}27.07 \\
27.12\end{array}$ & $\begin{array}{l}152,000 \\
146,000\end{array}$ \\
\hline
\end{tabular}

\section{RACCOON CREEK BASIN}

(25) 3-2020. Raccoon Creek at Adamsville, Ohio

Location.--Lat $38^{\circ} 52^{\prime} 31^{\prime \prime}$, long $82^{\circ} 21^{\prime} 18^{\prime \prime}$, on right bank at downstream side of highway bridge, $480 \mathrm{ft}$ upstream from U.S. H1ghway 35 , at Adamsville, Gallia County, and $1.3 \mathrm{~m} 1$ les downstream from Indian Creek.

Drainage area. $--585 \mathrm{sq} \mathrm{m} 1$.

Gage-helght record.--Water-stage recorder graph. Datum of gage is $570.85 \mathrm{ft}$ above mean sea level, adjustment of 1912 .

D1scharge record.--Stage-discharge relation defined by current-meter measurements below $11,000 \mathrm{cfs}$ and extended on basis of veloc1ty-area studies.

Maxima.--March 1964: D1scharge, 11,800 cf's Mar. 12, 1300 hours (gage he1ght, $23.49 \mathrm{ft})$

1915-35, 1938 to February 1964: Discharge, 15,500 cf's Apr. 15, 1948 (gage height, $24.92 \mathrm{ft}$ ).

Maximum stage known, 25.2 ft in January 1937, from floodmark (discharge 16,000 cfs).

Mean discharge, in cubic feet per second, March 1964

\begin{tabular}{|c|c|c|c|c|c|c|c|c|c|}
\hline Day & D1scharge & Day & D1scharge & Day & D1scharge & Day & D1scharge & Day & $\longdiv { \text { D1scharge } }$ \\
\hline $\begin{array}{l}1 \ldots \ldots \\
2 \ldots \ldots \\
3 \ldots \ldots \\
4 \ldots \\
5 \ldots \ldots \\
6 \ldots \ldots\end{array}$ & $\begin{array}{r}90 \\
113 \\
358 \\
1,180 \\
3,040 \\
3,510\end{array}$ & $\begin{array}{r}7 \ldots \\
8 \ldots \\
9 \ldots \\
10 \ldots \\
11 \ldots \\
12 \ldots\end{array}$ & $\begin{array}{r}4,010 \\
4,170 \\
4,000 \\
5,910 \\
9,570 \\
11,600\end{array}$ & $\begin{array}{l}13 \ldots \ldots \\
14 \ldots \ldots \\
15 \ldots \ldots \\
16 \ldots \ldots \\
17 \ldots \ldots \\
18 \ldots \ldots\end{array}$ & $\begin{array}{r}10,600 \\
7,770 \\
5,710 \\
4,060 \\
2,900 \\
2,070\end{array}$ & $\begin{array}{l}19 \ldots \ldots \\
20 \ldots \ldots \\
21 \ldots \ldots \\
22 \ldots \ldots \\
23 \ldots \ldots \\
24 \ldots \ldots\end{array}$ & $\begin{array}{r}1,290 \\
840 \\
755 \\
815 \\
815 \\
742\end{array}$ & $\mid \begin{array}{l}25 \ldots \ldots \\
26 \ldots \ldots \\
27 \ldots \ldots \\
28 \ldots \ldots \\
29 \ldots \ldots \\
30 \ldots \ldots \\
31 \ldots \ldots\end{array}$ & $\begin{array}{l}636 \\
627 \\
935 \\
788 \\
640 \\
541 \\
480\end{array}$ \\
\hline
\end{tabular}

Gage he1ght, in feet, and discharge, in cuble feet per second, at indicated t1me, 1964

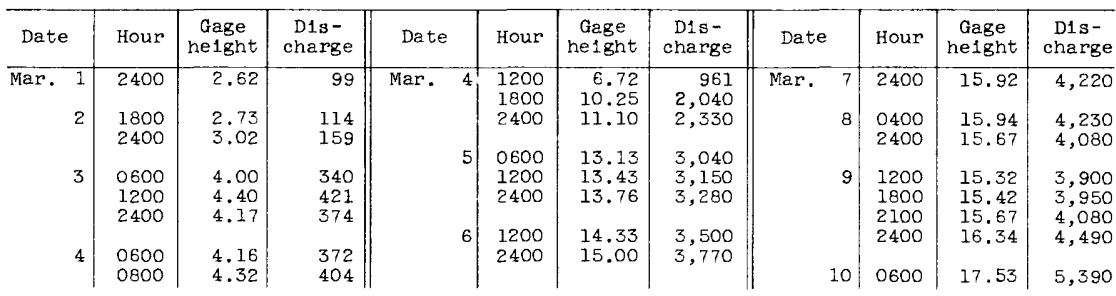


Gage he1ght, in feet, and discharge, in cub1c feet per second, at ind1cated t1me, 1964 , of Raccoon Creek at Adamsville, Oh10--Continued

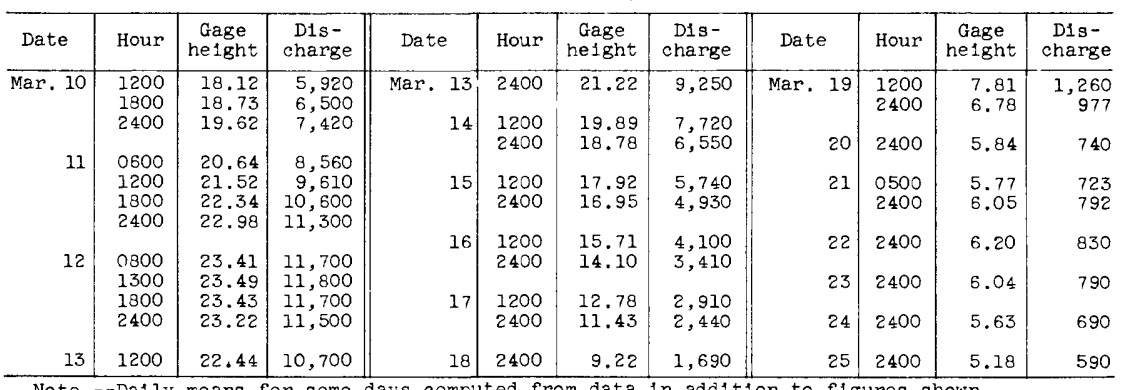

Note.--Dally means for some days computed from data in addition to figures shown.

\section{GUYANDOTYE RIVER BASIN}

(26) 3-2040. Guyandotte River at Branchland, W. Va.

Location.--Lat $38^{\circ} 13^{\prime} 15^{\prime \prime}$, long $82^{\circ} 12^{\prime} 10^{\prime \prime}$, on right bank at downstream side of highway bridge at Branchland, Lincoln County, $20 \mathrm{ft}$ downstream from Fourmile Creek.

Drainage area. $--1,226 \mathrm{sq} \mathrm{mi}$.

Gage-he1ght record.--Water-stage recorder graph except 1900 hours Mar. 8 to

1300 hours Mar. 13, for which graph was constructed from twice-daily U.S. Weather

Bureau readings. Auxiliary gage graph was constructed from once-daily U.S. Corps

of Engineers readings. Datum of gage is $547.91 \mathrm{ft}$ above mean sea level.

Discharge record.--Stage-fall-discharge relation defined by current-meter measurements below $32,000 \mathrm{cfs}$. Fall used as a factor Mar. 9-11.

Maxima.--March 1964: Discharge, 15,400 cfs Mar. 10, 1000 hours; gage height, 25.33 ft Mar. 10, 0800 hours.

1915-17, 1928 to February 1964: Discharge, 40,400 cfs Jan. 30, 1957: gage height, $42.57 \mathrm{ft}$ Mar. 1, 1955.

Mean discharge, in cublc feet per second, March 1964

\begin{tabular}{|c|c|c|c|c|c|c|c|c|c|}
\hline Day & D1scharge & Day & D1scharge & Day & D1scharge & Day & D1scharge & Day & D1scharge \\
\hline $\begin{array}{l}1 \ldots \ldots \\
2 \ldots \ldots \\
3 \ldots \ldots \\
4 \ldots \ldots \\
5 \ldots \ldots \\
6 \ldots \ldots\end{array}$ & $\begin{array}{l}1,500 \\
2,080 \\
4,860 \\
7,130 \\
7,780 \\
7,150\end{array}$ & $\begin{array}{r}7 \ldots \ldots \\
8 \ldots \ldots \\
9 \ldots \\
10 \ldots \\
11 \ldots \ldots \\
12 \ldots \ldots\end{array}$ & $\begin{array}{r}5,000 \\
4,670 \\
12,200 \\
14,000 \\
8,070 \\
5,440\end{array}$ & $\begin{array}{l}13 \ldots \ldots \\
14 \ldots \ldots \\
15 \ldots \ldots \\
16 \ldots \ldots \\
17 \ldots \ldots \\
18 \ldots \ldots\end{array}$ & $\begin{array}{l}4,220 \\
3,270 \\
4,370 \\
7,800 \\
6,730 \\
4,630\end{array}$ & $\begin{array}{l}19 \ldots \ldots \\
20 \ldots \ldots \\
21 \ldots \ldots \\
22 \ldots \ldots \\
23 \ldots \ldots \\
24 \ldots \ldots\end{array}$ & $\begin{array}{l}3,430 \\
2,690 \\
2,660 \\
3,020 \\
3,260 \\
3,010\end{array}$ & $\begin{array}{l}25 \ldots \ldots \\
26 \ldots \ldots \\
27 \ldots \ldots \\
28 \ldots \ldots \\
29 \ldots \ldots \\
30 \ldots \ldots \\
31 \ldots \ldots\end{array}$ & $\begin{array}{l}2,590 \\
2,260 \\
2,100 \\
1,850 \\
1,670 \\
1,610 \\
1,480 \\
\end{array}$ \\
\hline $\begin{array}{l}\text { Monthly } \\
\text { Runoff, }\end{array}$ & $\begin{array}{l}\text { mean disch } \\
\text { in inches. }\end{array}$ & $\begin{array}{l}\text { rge, in } \\
\ldots . . .\end{array}$ & cublc fee & per & 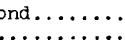 & . & $\ldots . .$. & $\cdots$ & $\begin{array}{r}4,598 \\
4.32\end{array}$ \\
\hline
\end{tabular}


(27) 3-2060. Ohio River at Huntington, $W$. Va.

Location.--Eat $38^{\circ} 24^{\prime} 48^{\prime \prime}$, long $82^{\circ} 30^{\prime} 02^{\prime \prime}$, on right bank at old lock 28 at Sybene, Lawrence County, onio, 0.1 mile upstream from Fourpole Creek, 3.0 miles downstream from Symmes Creek, and at mile 311.6.

Drainage area. $--55,900 \mathrm{sq} \mathrm{mi}$, approximately.

Gage-height record.--Water-stage recorder graphs except for doubtful or no gagehelght record at auxiliary gage Mar. 6 to 0700 hours Mar. 10, Mar. 16, 17, 21-23. Water-stage recorder graph for auxiliary gage 4.7 miles upstream and 1.5 miles downstream from Guyandotte River. Datum of gage is 490.26 ft above mean sea level, Sandy Hook datum.

Discharge record. --Stage-fall-discharge relation defined by current-meter measurements. Discharge for periods of doubtful or no gage-height record estimated from partly sketched graph and records for other ohio River stations. Discharge computed by using fall as a factor. The gage height used to compute discharge is the mean of the gage heights at base and auxiliary gages.

Maxima.--March 1964: Discharge, 455,000 cf's Mar. 13, 0800-1700 hours; gage height. $54.79 \mathrm{ft}$ Mar. 13, 1100-1400 hours.

1934 to February 1964: Discharge, 654,000 cfs Jan. 28, 1937; gage height, 69.45 ft Jan. 27, 1937 .

Remarks.--Flow partly regulated by locks, dams, and reservolrs upstream.

Mean discharge, in cubic feet per second, March 1964

\begin{tabular}{|c|c|c|c|c|c|c|c|c|c|}
\hline Day & Discharge & Day & Discharge & Day & Discharge & Day & D1scharge & Day & Discharge \\
\hline $\begin{array}{l}1 \ldots \ldots \\
2 \ldots \ldots \\
3 \ldots \ldots \\
4 \ldots \\
5 \ldots \\
6 \ldots\end{array}$ & $\begin{array}{r}28,000 \\
36,700 \\
95,100 \\
160,000 \\
229,000 \\
270,000\end{array}$ & $\begin{array}{r}7 \ldots \ldots \\
8 \ldots \ldots \\
9 \ldots \ldots \\
10 \ldots \\
11 \ldots \ldots \\
12 \ldots \ldots\end{array}$ & $\begin{array}{l}300,000 \\
340,000 \\
360,000 \\
379,000 \\
418,000 \\
441,000\end{array}$ & $\begin{array}{l}13 \ldots \ldots \\
14 \ldots \ldots \\
15 \ldots \ldots \\
16 \ldots \ldots \\
17 \ldots \ldots \\
18 \ldots \ldots\end{array}$ & $\begin{array}{l}453,000 \\
438,000 \\
404,000 \\
400,000 \\
391,000 \\
346,000\end{array}$ & $\begin{array}{l}19 \ldots \ldots \\
20 \ldots \ldots \\
21 \ldots \ldots \\
22 \ldots \ldots \\
23 \ldots \ldots \\
24 \ldots \ldots\end{array}$ & $\begin{array}{l}297,000 \\
259,000 \\
215,000 \\
174,000 \\
155,000 \\
136,000\end{array}$ & $\mid \begin{array}{l}25 \ldots \\
26 \ldots \\
27 \ldots \ldots \\
28 \ldots \\
29 \ldots \\
30 \ldots \ldots \\
31 \ldots \ldots\end{array}$ & $\begin{array}{l}125,000 \\
122,000 \\
109,000 \\
112,000 \\
113,000 \\
111,000 \\
106,000\end{array}$ \\
\hline
\end{tabular}

Gage height, in feet, and discharge, in cubic feet per second, at 1ndicated time, 1964

\begin{tabular}{|c|c|c|c|c|c|c|c|c|c|c|c|c|}
\hline Date & Hour & $\begin{array}{c}\text { Gage } \\
\text { helght }\end{array}$ & $\begin{array}{c}\text { Dis- } \\
\text { charge }\end{array}$ & \multicolumn{2}{|l|}{ Date } & Hour & $\begin{array}{c}\text { Gage } \\
\text { height }\end{array}$ & $\begin{array}{l}\text { Dis- } \\
\text { charge }\end{array}$ & Date & Hour & $\begin{array}{c}\text { Gage } \\
\text { height }\end{array}$ & $\begin{array}{c}\text { Dis- } \\
\text { charge }\end{array}$ \\
\hline Feb. 29 & 2400 & 25.33 & 26,100 & Mar. & & $\begin{array}{l}0600 \\
1200\end{array}$ & $\begin{array}{l}33.36 \\
34.26\end{array}$ & $\begin{array}{l}212,000 \\
234,000\end{array}$ & \multirow[t]{3}{*}{ Mar. 13} & 0600 & 54.74 & $\begin{array}{l}450,000 \\
455,000\end{array}$ \\
\hline Mar. 1 & 0600 & 25.40 & 24,200 & & & 1800 & 35.34 & 245,000 & & 1800 & 54.74 & 454,000 \\
\hline & $\begin{array}{l}1200 \\
1800\end{array}$ & $\begin{array}{l}25.51 \\
25.57\end{array}$ & $\begin{array}{l}26,400 \\
34,000\end{array}$ & & & 2400 & 36.43 & 252,000 & & 2400 & 54.62 & 449,000 \\
\hline & 2400 & 25.59 & 28,700 & & 9 & 2400 & 46.55 & 288,000 & \multirow[t]{3}{*}{14} & $\begin{array}{l}0600 \\
1200\end{array}$ & $\begin{array}{l}54.38 \\
54.10\end{array}$ & $\mid \begin{array}{l}445,000 \\
441,000\end{array}$ \\
\hline 2 & 0600 & 25.59 & 26,600 & & $10^{\prime}$ & 0600 & 47.78 & 366,000 & & 1800 & 53.72 & 432,000 \\
\hline & $\begin{array}{l}1200 \\
1800\end{array}$ & $\begin{array}{l}25.52 \\
25.71\end{array}$ & $\begin{array}{l}33,900 \\
45,200\end{array}$ & & & $\begin{array}{l}1200 \\
1800\end{array}$ & $\begin{array}{l}48.94 \\
50.11\end{array}$ & $\begin{array}{l}382,000 \\
390,000\end{array}$ & & 2400 & 53.28 & 418,000 \\
\hline & 2400 & 25.85 & 53,600 & & & 2400 & 51.13 & 404,000 & 25 & $\begin{array}{l}1200 \\
2400\end{array}$ & $\begin{array}{l}52.31 \\
51.37\end{array}$ & $\begin{array}{l}401,000 \\
393,000\end{array}$ \\
\hline 3 & 0600 & 26.60 & 75,700 & & 11 & 0600 & 51.98 & 408,000 & \multirow{4}{*}{28} & & & \\
\hline & 1200 & 27.23 & 96,400 & & & 1200 & 52.61 & 420,000 & & 1200 & 47.00 & 351,000 \\
\hline & 1800 & 28.12 & 116,000 & & & 1800 & 53.19 & 428,000 & & 2400 & 45.11 & 317,000 \\
\hline & 2400 & 28.86 & 131,000 & & & 2400 & 53.67 & 431,000 & & 1200 & 42.88 & 296,000 \\
\hline 4 & 0600 & 29.55 & 143,000 & & 12 & 0600 & 54.02 & 436,000 & 19 & 2400 & 40.74 & 276,000 \\
\hline & 1800 & 31.33 & $\begin{array}{l}155,000 \\
179,000\end{array}$ & & & 1800 & $\begin{array}{l}54.31 \\
54.49\end{array}$ & 447,000 & \multirow[t]{2}{*}{20} & 1200 & 38.58 & 000 \\
\hline & 2400 & 32.35 & 198,000 & & & 2400 & 54.64 & 449,000 & & 2400 & 36.52 & 240,000 \\
\hline
\end{tabular}




\section{BIG SANDY RIVER BASIN}

(28) 3-2150. B1g Sandy River at Louisa, Ky.

Location.--Lat $38^{\circ} 10^{\prime} 16^{\prime \prime}$, long $82^{\circ} 38^{\prime} 05^{\prime \prime}$, on left bank 1.6 miles upstream from Bla1ne Creek, $4.3 \mathrm{miles}$ northwest of Loulsa, Lawrence County, and at mile 21.2 .

Drainage area $--3,892 \mathrm{sq} \mathrm{ml}$.

Gage-helght record.--Water-stage recorder graphs. Auxiliary gage at site 5.0 miles upstream. Datum of gage is $512.81 \mathrm{ft}$ above mean sea level, datum of 1929 .

Discharge record.--Stage-discharge relation or stage-fall-discharge relation defined by current-meter measurements. Fali used as a factor Mar. 3-11, 17-26. Stagefall-discharge relation indefinite Mar. 12-16; discharge estimated on basis of records for Tug Fork near Kermit, W. Va. and Levisa Fork at Paintsville, Ky.

Maxima --March 1964: Discharge, 33,700 cfs Mar. 10, 1830 hours; gage height, 33.31 ft Mar. 11, 0100-0200 hours.

1938 to February 1964: Discharge, 89,400 efs Mar. 2, 1955; gage helght, 46.37 ft Miay 8, 1958.

Flood of Apr. 3, 1908, reached a stage of $48.4 \mathrm{ft}$, at site 5 miles upstream at datum $3.65 \mathrm{ft}$ higher.

Cooperation.--Gage-helght record furnished by Corps of Engineers.

\begin{tabular}{|c|c|c|c|c|c|c|c|c|c|}
\hline Day & D1scharge & Day & Discharge & Day & D1scharge & Day & D1scharge & Day & D1scharge \\
\hline $\begin{array}{l}1 \ldots \ldots \\
2 \ldots \ldots \\
3 \ldots \ldots \\
4 \ldots \ldots \\
5 \ldots \ldots \\
6 \ldots \ldots\end{array}$ & $\begin{array}{r}2,960 \\
3,360 \\
6,190 \\
9,660 \\
14,300 \\
15,500\end{array}$ & $\begin{array}{r}7 \ldots \ldots \\
8 \ldots \ldots \\
9 \ldots \ldots \\
10 \ldots \ldots \\
11 \ldots \ldots \\
12 \ldots \ldots\end{array}$ & $\begin{array}{l}15,200 \\
12,600 \\
24,200 \\
33,300 \\
27,000 \\
16,000\end{array}$ & $\mid \begin{array}{l}13 \ldots \\
14 \ldots \\
15 \ldots \\
16 \ldots \\
17 \ldots \\
18 \ldots\end{array}$ & $\begin{array}{r}11,000 \\
8,000 \\
6,600 \\
16,000 \\
20,000 \\
16,200\end{array}$ & $\begin{array}{l}19 \ldots \ldots \\
20 \ldots \ldots \\
21 \ldots \ldots \\
22 \ldots \ldots \\
23 \ldots \ldots \\
24 \ldots \ldots\end{array}$ & $\begin{array}{r}10,300 \\
7,100 \\
6,740 \\
8,170 \\
8,440 \\
7,480\end{array}$ & $\mid \begin{array}{l}25 \ldots \ldots \\
26 \ldots \ldots \\
27 \ldots \ldots \\
28 \ldots \ldots \\
29 \ldots \ldots \\
30 \ldots \ldots \\
31 \ldots \ldots\end{array}$ & $\begin{array}{l}6,180 \\
5,200 \\
5,010 \\
4,700 \\
4,190 \\
3,830 \\
3,560\end{array}$ \\
\hline onthiy & mean disc & ge & cublc fee & se & & & & & 10,930 \\
\hline
\end{tabular}

\section{OHIO RIVER MAIN STEM}

(29) 3-2160. Ohio River at Ashland, Ky.

Location.--Lat 38 $28^{\prime} 52^{\prime \prime}$, long $82^{\circ} 38^{\prime} 12^{\prime \prime}$, on left bank at Ashland, Boyd County, $1,700 \mathrm{ft}$ upstream from Ashland-Coal Grove highway bridge, 1.5 miles upstream from Hood Creek, 5.4 miles downstream from Blg Sandy River and at mile 322.5 .

Drainage area. $--60,750 \mathrm{sq} \mathrm{mi}$, approximately.

Gage-he1ght record.-Water-stage recorder graphs. Datum of gage is $481.50 \mathrm{ft}$ above mean sea leve1, Ohio River datum (levels by Corps of Englneers); $481.03 \mathrm{ft}$ above mean sea level, datum of 1929 . Auxillary water-stage recorder graph $18.5 \mathrm{miles}$ downstream.

Discharge record.--Stage-fall-discharge relation defined by current-meter measurements. Fall used as a factor in computing discharge. Stage-fal1-discharge relation indefinite Mar. 1, 2; discharge estimated on basis of other ohio River main-stem stations.

Maxima.--March 1964: Discharge, 472,000 cfs Mar. 13, 1200 hours (gage helght, $59.59 \mathrm{ft})$.

1884 , 1907, 1913, 1917 to February 1964: Discharge, 690,000 cfs Jan. 27, 1937

(gage helght, $75.2 \mathrm{ft}$, at site 1.6 miles upstream).

Flood of Jan. 27, 1937, is maximum known.

Remarks.--Partly regulated by locks, dams, and reservolrs.

Cooperation.--Gage-helght record furnished by Corps of Engineers.

\begin{tabular}{|c|c|c|c|c|c|c|c|c|c|}
\hline Day & Discharge & Day & Discharge & Day & D1scharge & Day & D1scharge & Day & D1scharge \\
\hline $\begin{array}{l}1 \ldots \ldots \\
2 \ldots \ldots \\
3 \ldots \ldots \\
4 \ldots \ldots \\
5 \ldots \ldots \\
6 \ldots \ldots\end{array}$ & $\begin{array}{r}32,000 \\
40,000 \\
94,900 \\
147,000 \\
225,000 \\
296,000\end{array}$ & $\begin{array}{r}7 \ldots \ldots \\
8 \ldots \ldots \\
9 \ldots \ldots \\
10 \ldots \\
12 \ldots \ldots\end{array}$ & $\begin{array}{l}334,000 \\
367,000 \\
403,000 \\
424,000 \\
450,000 \\
463,000\end{array}$ & $\begin{array}{l}13 \ldots \ldots \\
14 \ldots \ldots \\
15 \ldots \ldots \\
16 \ldots \ldots \\
17 \ldots \ldots \\
18 \ldots \ldots\end{array}$ & $\begin{array}{l}470,000 \\
458,000 \\
439,000 \\
432,000 \\
429,000 \\
398,000\end{array}$ & $\begin{array}{l}19 \ldots \ldots \\
20 \ldots \ldots \\
21 \ldots \ldots \\
22 \ldots \ldots \\
23 \ldots \ldots \\
24 \ldots \ldots\end{array}$ & $\begin{array}{l}346,000 \\
302,000 \\
244,000 \\
184,000 \\
158,000 \\
138,000\end{array}$ & $\begin{array}{l}25 \ldots \ldots \\
26 \ldots \ldots \\
27 \ldots \ldots \\
28 \ldots \ldots \\
29 \ldots \ldots \\
30 \ldots \ldots\end{array}$ & $\begin{array}{l}124,000 \\
120,000 \\
111,000 \\
114,000 \\
114,000 \\
114,000 \\
110,000\end{array}$ \\
\hline
\end{tabular}

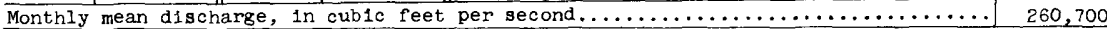


Gage height, in feet, and discharge, in cublo feet per second, at indicated t1me, 1964, of Ohio River at Ashland, Ky.

\begin{tabular}{|c|c|c|c|c|c|c|c|c|c|c|c|c|}
\hline Date & Hour & $\begin{array}{c}\text { Gage } \\
\text { he1ght }\end{array}$ & $\begin{array}{l}\text { Dis- } \\
\text { charge }\end{array}$ & Date & Hour & $\begin{array}{c}\text { Gage } \\
\text { he1ght }\end{array}$ & $\begin{array}{l}\text { Dis- } \\
\text { charge }\end{array}$ & \multicolumn{2}{|l|}{ Date } & Hour & $\begin{array}{c}\text { Gage } \\
\text { helght }\end{array}$ & $\begin{array}{l}\text { Dis- } \\
\text { charge }\end{array}$ \\
\hline Mar. 2 & 2400 & 34.12 & 52,900 & Mar. 9 & 2400 & 50.90 & 414,000 & Mar. & & 2400 & 53.27 & 422,000 \\
\hline 3 & $\begin{array}{l}0600 \\
1200 \\
2400\end{array}$ & $\begin{array}{l}34.49 \\
34.59 \\
35.22\end{array}$ & $\begin{array}{r}83,700 \\
101,000 \\
118,000\end{array}$ & 10 & $\begin{array}{l}0600 \\
1200 \\
1800\end{array}$ & $\begin{array}{l}52.27 \\
53.51 \\
54.79\end{array}$ & $\begin{array}{l}423,000 \\
420,000 \\
427,000\end{array}$ & & 18 & $\begin{array}{l}1200 \\
2400\end{array}$ & $\begin{array}{l}51.87 \\
50.03\end{array}$ & $\begin{array}{l}400,000 \\
370,000\end{array}$ \\
\hline 4 & 0600 & 35.55 & 134,000 & & 2400 & 55.86 & 439,000 & & 19 & $\begin{array}{l}1200 \\
2400\end{array}$ & $\begin{array}{l}47.87 \\
45.70\end{array}$ & $\begin{array}{l}345,000 \\
325,000\end{array}$ \\
\hline & $\begin{array}{l}1200 \\
1800 \\
2400\end{array}$ & $\begin{array}{l}35.88 \\
36.37 \\
37.09\end{array}$ & $\begin{array}{l}143,000 \\
160,000 \\
187,000\end{array}$ & 11 & $\begin{array}{l}1200 \\
1800 \\
2400\end{array}$ & $\begin{array}{l}57.39 \\
58.01 \\
58.51\end{array}$ & $\begin{array}{l}452,000 \\
455,000 \\
454,000\end{array}$ & & 20 & $\begin{array}{l}1200 \\
2400\end{array}$ & $\begin{array}{l}43.48 \\
41.32\end{array}$ & $\begin{array}{l}302,000 \\
278,000\end{array}$ \\
\hline 5 & $\begin{array}{l}0600 \\
1200 \\
1800\end{array}$ & $\begin{array}{l}37.84 \\
38.24 \\
39.39\end{array}$ & $\begin{array}{l}206,000 \\
226,000 \\
242,000\end{array}$ & 12 & $\begin{array}{l}0600 \\
1200 \\
1800\end{array}$ & $\begin{array}{l}58.84 \\
59.16 \\
59.33\end{array}$ & $\begin{array}{l}458,000 \\
466,000 \\
467,000\end{array}$ & & 21 & $\begin{array}{l}1200 \\
2400\end{array}$ & $\begin{array}{l}38.93 \\
37.55\end{array}$ & $\begin{array}{l}245,000 \\
209,000\end{array}$ \\
\hline & 2400 & 40.51 & 262,000 & & 2400 & 59.46 & 469,000 & & 22. & $\begin{array}{l}1200 \\
2400\end{array}$ & $\begin{array}{l}36.86 \\
36.25\end{array}$ & $\begin{array}{l}182,000 \\
162,000\end{array}$ \\
\hline 6 & 0600 & 41.65 & 285,000 & 13 & 0600 & 59.54 & 470,000 & & & & & \\
\hline & $\begin{array}{l}1200 \\
2400\end{array}$ & $\begin{array}{l}42.45 \\
43.80\end{array}$ & $\begin{array}{l}298,000 \\
319,000\end{array}$ & & $\begin{array}{l}1200 \\
1800 \\
2400\end{array}$ & $\begin{array}{l}59.59 \\
59.55 \\
59.40\end{array}$ & $\begin{array}{l}472,000 \\
471,000 \\
466,000\end{array}$ & & 23 & $\begin{array}{l}1200 \\
2400\end{array}$ & $\begin{array}{l}36.47 \\
36.06\end{array}$ & $\begin{array}{l}161,000 \\
146,000\end{array}$ \\
\hline 7 & $\begin{array}{l}0600 \\
1800 \\
2400\end{array}$ & $\begin{array}{l}44.26 \\
45.05 \\
45.45\end{array}$ & $\begin{array}{l}325,000 \\
343,000 \\
351,000\end{array}$ & 14 & $\begin{array}{l}1200 \\
2400\end{array}$ & $\begin{array}{l}58.91 \\
58.11\end{array}$ & $\begin{array}{l}4.58,000 \\
449,000\end{array}$ & & 24 & $\begin{array}{l}1200 \\
2400\end{array}$ & $\begin{array}{l}35.45 \\
35.28\end{array}$ & $\begin{array}{l}136,000 \\
132,000\end{array}$ \\
\hline 8 & $\begin{array}{l}0600 \\
1800\end{array}$ & $\begin{array}{l}45.83 \\
46.84\end{array}$ & $\begin{array}{l}357,000 \\
376,000\end{array}$ & 15 & $\begin{array}{l}1200 \\
2400\end{array}$ & $\begin{array}{l}57.21 \\
56.29\end{array}$ & $\begin{array}{l}437,000 \\
432,000\end{array}$ & & 25 & $\begin{array}{l}1200 \\
2400\end{array}$ & $\begin{array}{l}34.82 \\
34.90\end{array}$ & $\begin{array}{l}121,000 \\
121,000\end{array}$ \\
\hline & 2400 & 47.54 & 386,000 & 16 & 1200 & 55.54 & 00 & & 26 & $\begin{array}{l}1200 \\
2400\end{array}$ & $\begin{array}{l}34.90 \\
34.79\end{array}$ & $\begin{array}{l}121,000 \\
116,000\end{array}$ \\
\hline 9 & 1200 & 48.97 & 40 & & 2400 & 54.93 & 434,000 & & & & & \\
\hline & 1800 & & 00 & 17 & 1200 & 54.19 & 430,000 & & 27 & $\begin{array}{l}1200 \\
2400 \\
\end{array}$ & $\begin{array}{l}34.84 \\
35.06 \\
\end{array}$ & $\begin{array}{l}110,000 \\
109,000\end{array}$ \\
\hline
\end{tabular}

\section{LITTLE SANDY RIVER BASIN}

(30) 3-2165. Little Sandy River near Grayson, Ky.

Location.--Lat $38^{\circ} 20^{\prime} 25^{\prime \prime}$, long $82^{\circ} 55^{\prime} 10^{\prime \prime}$, on downstream side of center pier of highway bridge, $0.3 \mathrm{mile}$ upstream from Lower Stinson Creek, $1.3 \mathrm{miles}$ downstream from U.S. H1ghway 60 , and 1.7 miles northeast of Grayson, Carter county.

Drainage area. $-402 \mathrm{sq} \mathrm{mi}$.

Gage-height record.--Water-stage recorder graph. Datum of gage is $557.95 \mathrm{ft}$ above mean sea level, datum of 1929.

D1scharge record.--Stage-discharge relation defined by current-meter measurements.

Maxima.--Given in the following table.

March 1964:

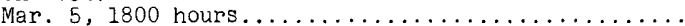

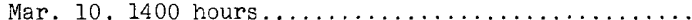

1884 to February 1964:

Sept. 22, 1950 .

$\begin{array}{cc}\text { Discharge } & \text { Gage height } \\ \text { (cfs) } & \text { (feet) } \\ 7,260 & 18.45 \\ 7,020 & 18.10\end{array}$

24,500

18.10

Sept. $22,1950 \ldots \ldots \ldots \ldots \ldots \ldots \ldots \ldots \ldots \ldots \ldots \ldots \ldots \ldots$

Mean discharge, in cubic feet per second, March 1964

\begin{tabular}{|c|c|c|c|c|c|c|c|c|c|}
\hline Day & Discharge & Day & D1scharge & Day & Discharge & Day & D1scharge & Day & Discharge \\
\hline $\begin{array}{l}1 \ldots \ldots \\
2 \ldots \ldots \\
3 \ldots \ldots \\
4 \ldots \ldots \\
5 \ldots \ldots \\
6 \ldots \ldots\end{array}$ & $\begin{array}{r}157 \\
292 \\
1,600 \\
3,220 \\
6,780 \\
5,110\end{array}$ & $\begin{array}{r}7 \ldots \ldots \\
8 \ldots \ldots \\
9 \ldots \\
10 \ldots \\
11 \ldots \\
12 \ldots\end{array}$ & $\begin{array}{l}1,320 \\
1,990 \\
5,820 \\
6,800 \\
5,060 \\
1,650\end{array}$ & $\begin{array}{l}13 \ldots \ldots \\
14 \ldots \ldots \\
15 \ldots \ldots \\
16 \ldots \ldots \\
17 \ldots \ldots \\
18 \ldots \ldots\end{array}$ & $\begin{array}{r}994 \\
781 \\
1,430 \\
1,620 \\
1,050 \\
748\end{array}$ & $\begin{array}{l}19 \ldots \ldots \\
20 \ldots \ldots \\
21 \ldots \ldots \\
22 \ldots \ldots \\
23 \ldots \ldots \\
24 \ldots \ldots\end{array}$ & $\begin{array}{l}548 \\
488 \\
667 \\
919 \\
799 \\
667\end{array}$ & $\begin{array}{l}25 \ldots \ldots \\
26 \ldots \ldots \\
27 \ldots \ldots \\
28 \ldots \ldots \\
29 \ldots \ldots \\
30 \ldots \ldots \\
31 \ldots \ldots\end{array}$ & $\begin{array}{l}552 \\
495 \\
410 \\
328 \\
290 \\
255 \\
225\end{array}$ \\
\hline
\end{tabular}


TYGARTS CREEK BASIN

(31) 3-2170. Tygarts Creek near Greenup, Ky .

Location.--Lat $38^{\circ} 33^{\prime} 51^{\prime \prime}$, 1ong $82^{\circ} 57^{\prime} 08^{\prime \prime}$, on downstream side of center pler of bridge on State H1ghway $7,100 \mathrm{ft}$ downstream from Lick Run, 0.4 mile upstream from White Oak Creek, and $6 \frac{1}{2}$ miles west of Greenup, Greenup county.

Drainage area,$-242 \mathrm{sq} \mathrm{ml}$.

Gage-helght record.--Water-stage recorder graph except Mar. 1-17, for which graph was reconstructed on basis of numerous wire-we1ght-gage readings. Datum of gage is $547.14 \mathrm{ft}$ above mean sea level, datum of 1929.

Discharge record.--Stage-discharge relation defined by current-meter measurements. Naxima.--Given in the following table.

\section{March 1964:}

Mar. 5, 1700 hours. 1934 :

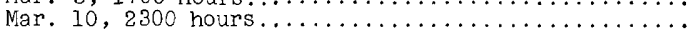

Maximum stage for year.

1940 to February 1964:

Feb. 28,1962 .

\section{Discharge Gage he1ght (cf's) \\ 7,650 5,400 \\ (feet) \\ 17.6}

- 20
21.38

Mean discharge, In cubic feet per second, March 1964

\begin{tabular}{|c|c|c|c|c|c|c|c|c|c|}
\hline Day & Discharge & Day & Discharge & Day & Discharge & Day & Discharge & Day & Discharge \\
\hline $\begin{array}{l}1 \ldots \ldots \\
2 \ldots \ldots \\
3 \ldots \ldots \\
4 \ldots \ldots \\
5 \ldots \ldots \\
6 \ldots \ldots\end{array}$ & $\begin{array}{r}68 \\
135 \\
1,100 \\
3,450 \\
6,750 \\
2,700\end{array}$ & $\begin{array}{r}7 \ldots \ldots \\
8 \ldots \ldots \\
9 \ldots \ldots \\
10 \ldots \\
11 \ldots \ldots \\
12 \ldots \ldots\end{array}$ & $\begin{array}{r}685 \\
1,080 \\
3,500 \\
4,620 \\
3,300 \\
895\end{array}$ & $\begin{array}{l}13 \ldots \ldots \\
14 \ldots \ldots \\
15 \ldots \ldots \\
16 \ldots \ldots \\
17 \ldots \ldots \\
18 \ldots \ldots\end{array}$ & $\begin{array}{r}525 \\
525 \\
1,050 \\
1,020 \\
525 \\
493\end{array}$ & $\begin{array}{l}19 \ldots \ldots \\
20 \ldots \ldots \\
21 \ldots \ldots \\
22 \ldots \ldots \\
23 \ldots \ldots \\
24 \ldots \ldots\end{array}$ & $\begin{array}{l}373 \\
318 \\
385 \\
665 \\
541 \\
433\end{array}$ & $\begin{array}{l}25 \ldots \ldots \\
26 \ldots \ldots \\
27 \ldots \ldots \\
28 \ldots \ldots \\
29 \ldots \ldots \\
30 \ldots \ldots \\
31 \ldots \ldots\end{array}$ & $\begin{array}{l}365 \\
361 \\
308 \\
244 \\
216 \\
192 \\
168\end{array}$ \\
\hline $1 y$ & , & & 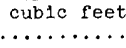 & & & & & & $\begin{array}{r}1,193 \\
5.68\end{array}$ \\
\hline
\end{tabular}

\section{SCIOTO RIVER BASIN}

(32) 3-2304. Big Darby Creek at Darbydale, Oh10

(Crest-stage station)

Location.--Lat $39^{\circ} 50^{\prime} 55^{\prime \prime}$, long $83^{\circ} 11^{\prime} 20^{\prime \prime}$, near left abutment at downstream side of McKinley Bridge at Darbydale, Franklin County.

Drainage area. $--449 \mathrm{sq} \mathrm{mi}$.

Gage-height record.--Water-stage recorder graph. Datum of gage is $790.00 \mathrm{ft}$ above mean sea level (levels by Corps of Englneers).

D1scharge record.--Stage-discharge relation defined by current-meter measurements.

Maxima --March 1964: D1scharge, 16,200 cfs Mar. 10, 0800 hours (gage height, $16.56 \mathrm{ft})$. 
(33) 3-2305. Big Darby Creek at Darbyville, Ohio

Location, --Lat $39^{\circ} 42^{\prime} 05^{\prime \prime}$, long $83^{\circ} 06^{\prime} 35^{\prime \prime}$, near right bank on downstream side of pler of briage on State Highway 316, three-elghths of a mile northeast of Darbyville, Pickaway County, and 3 miles downstream from Greenbrier Creek.

Drainage area.-- $534 \mathrm{sq} \mathrm{mi}$.

Gage-height record.--Water-stage recorder graph. Datum of gage is $713.6 \mathrm{ft}$ above mean sea level, adjustment of 1912 .

Discharge record.--Stage-discharge relation defined by current-meter measurements below 22,000 ofs and by indirect measurement of $49,000 \mathrm{cfs}$.

Maxima.--March 1964: Discharge, 19,400 cfs Mar. 11, 0230 hours (gage height, $14.31 \mathrm{ft})$.

1921-35, 1938 to February 1964: Discharge, 49,000 cf's Jan. 22, 1959 (gage height, $17.94 \mathrm{ft}$ ).

\begin{tabular}{|c|c|c|c|c|c|c|c|c|c|}
\hline Day & Discharge & Day & D1scharge & Day & Discharge & Day & Discharge & Day & Discharge \\
\hline $\begin{array}{l}1 \ldots \ldots \\
2 \ldots \ldots \\
3 \ldots \ldots \\
4 \ldots \ldots \\
5 \ldots \\
6 \ldots\end{array}$ & $\begin{array}{r}34 \\
35 \\
38 \\
363 \\
3,740 \\
5,440\end{array}$ & $\begin{array}{r}7 \ldots \ldots \\
8 \ldots \ldots \\
y \ldots \\
10 \ldots \\
11 \ldots \ldots \\
12 \ldots \ldots\end{array}$ & $\begin{array}{r}2,040 \\
1,040 \\
5,030 \\
13,100 \\
15,000 \\
5,800\end{array}$ & $\mid \begin{array}{l}13 \ldots \ldots \\
14 \ldots \ldots \\
15 \ldots \ldots \\
16 \ldots \ldots \\
17 \ldots \ldots \\
18 \ldots \ldots\end{array}$ & $\begin{array}{r}z, 140 \\
2,570 \\
2,720 \\
2,060 \\
1,360 \\
984\end{array}$ & $\begin{array}{l}19 \ldots \ldots \\
20 \ldots \ldots \\
21 \ldots \ldots \\
22 \ldots \ldots \\
23 \ldots \ldots \\
24 \ldots \ldots\end{array}$ & $\begin{array}{l}780 \\
680 \\
796 \\
948 \\
763 \\
630\end{array}$ & $\mid \begin{array}{l}25 \ldots \ldots \\
26 \ldots \ldots \\
27 \ldots \ldots \\
28 \ldots \ldots \\
29 \ldots \ldots \\
30 \ldots \ldots \\
31 \ldots \ldots\end{array}$ & $\begin{array}{l}571 \\
600 \\
508 \\
454 \\
399 \\
370 \\
338\end{array}$ \\
\hline $\begin{array}{l}\text { onthly } \\
\text { unoff, }\end{array}$ & $\begin{array}{l}\text { mean disc } \\
\text { In inches }\end{array}$ & ge, & bic & per & & & & & $\begin{array}{r}2,333 \\
5.04\end{array}$ \\
\hline
\end{tabular}

(34) 3-2309. Deer Creek at Pancoastburg, Onio

(Crest-stage station)

Location.--Lat $39^{\circ} 377^{\prime} 14^{\prime \prime}$, long $83^{\circ} 12^{\prime} 47^{\prime \prime}$, on left bank at downstream side of bridge on Crownover Mill Road, 1.2 miles downstream from Clark Run, 2.8 miles east of Pancoastburg, Fayette County, and 5.2 miles northwest of wililamsport.

Drainage area. $--277 \mathrm{sq} \mathrm{mi}$.

Gage-height record.--Water-stage recorder graph. Datum of gage is $760.00 \mathrm{ft}$ above mean sea level (levels by Corps of Engineers).

Discharge record.--Stage-discharge relation defined by current-meter measurements below 4,700 of's and extended above by logarithmic plotting.

Maxima.--March 1964: Discharge, 19,500 cfs Mar. 10, 0330 hours (gage height, $21.1 \mathrm{ft}$.

(35) 3-2310. Deer Creek at Williamsport, Ohio

Location.--Lat $39^{\circ} 35^{\prime} 09^{\prime \prime}$, long $83^{\circ} 07^{\prime} 22^{\prime \prime}$, on left bank at downstream side of bridge on U.S. Highway 22 at west edge of Williamsport, Pickaway County, 2 miles downstream from Dry Run.

Drainage area. $--333 \mathrm{sq} \mathrm{mi}$.

Gage-height record.--Water-stage recorder graph from bubble gage. Datum of gage is 718.7 ft above niean sea level, adjustment of 1912 .

Discharge record.--Stage-discharge relation defined by current-meter measurements below $25,000 \mathrm{cs}$, and by ind1rect measurements at 37,600 and $39,600 \mathrm{cfs}$.

Maxima.--March 1964: Discharge, 23,400 cf's Mar. 10, 0600 hours (gage height, $15.82 \mathrm{ft}$ )

1926-35, 1938-56, 1962 to Pebruary 1964: Discharge, 37,600 ofs Mar. 5, 1963

(gage height, $17.5 \mathrm{ft}$, from floodmarks).

Flood of January 1959 reached a stage of $17.6 \mathrm{ft}$, from floodmarks (discharge, 39,600 ofs). 
Mean discharge, in cubic feet per second, March 1964, of Deer Creek at Williamsport, Ohio

\begin{tabular}{|c|c|c|c|c|c|c|c|c|c|}
\hline Day & Discharge & Day & Discharge & $\overline{\text { Day }}$ & D1scharge & Day & Discharge & Day & $\longdiv { \text { Discharge } }$ \\
\hline $\begin{array}{l}1 \ldots \ldots \\
2 \ldots \ldots \\
3 \ldots \ldots \\
4 \ldots \\
5 \ldots \\
6 \ldots\end{array}$ & $\begin{array}{r}30 \\
31 \\
34 \\
679 \\
4,620 \\
3,250\end{array}$ & $\begin{array}{r}7 \ldots \ldots \\
8 \ldots \ldots \\
9 \ldots \\
10 \ldots \\
11 \\
12 \ldots \\
\end{array}$ & $\begin{array}{r}919 \\
718 \\
6,790 \\
19,600 \\
9,690 \\
2,140\end{array}$ & $\begin{array}{l}13 \ldots \ldots \\
14 \ldots \ldots \\
15 \ldots \ldots \\
16 \ldots \ldots \\
17 \ldots \ldots \\
18 \ldots \ldots\end{array}$ & $\begin{array}{r}1,540 \\
1,310 \\
1,600 \\
1,060 \\
772 \\
610\end{array}$ & $\begin{array}{l}19 \ldots \ldots \\
20 \ldots \ldots \\
21 \ldots \ldots \\
22 \ldots \ldots \\
23 \ldots \ldots \\
24 \ldots \ldots\end{array}$ & $\begin{array}{l}493 \\
444 \\
548 \\
644 \\
532 \\
444\end{array}$ & $\begin{array}{l}25 \ldots \ldots \\
26 \ldots \ldots \\
27 \ldots \ldots \\
28 \ldots \ldots \\
29 \ldots \ldots \\
30 \ldots \ldots \\
31 \ldots \ldots\end{array}$ & $\begin{array}{l}389 \\
567 \\
500 \\
395 \\
337 \\
294 \\
270\end{array}$ \\
\hline $1 y$ & ea & & & & & & & & $\begin{array}{r}1,976 \\
6.84\end{array}$ \\
\hline
\end{tabular}

Gage height, in feet, and discharge, in cuble feet per second, at indicated time, 1964

\begin{tabular}{|c|c|c|c|c|c|c|c|c|c|c|c|c|}
\hline Date & \multirow{2}{*}{$\begin{array}{l}\text { Hour } \\
2400\end{array}$} & \multirow{2}{*}{$\begin{array}{r}\begin{array}{c}\text { Gage } \\
\text { helght }\end{array} \\
2.41\end{array}$} & \multirow{2}{*}{$\begin{array}{r}\begin{array}{c}\text { Dis- } \\
\text { charge }\end{array} \\
37\end{array}$} & \multicolumn{2}{|l|}{ Date } & \multirow{2}{*}{$\begin{array}{l}\text { Hour } \\
1200 \\
2400\end{array}$} & \multirow{2}{*}{$\begin{array}{r}\begin{array}{c}\text { Gage } \\
\text { height }\end{array} \\
5.92 \\
5.50\end{array}$} & \multirow{2}{*}{$\begin{array}{r}\begin{array}{c}\text { Dis- } \\
\text { charge }\end{array} \\
893 \\
728\end{array}$} & Date & \multirow{3}{*}{$\begin{array}{l}\text { Hour } \\
0600 \\
1200 \\
2400\end{array}$} & \multirow{3}{*}{$\begin{array}{r}\begin{array}{c}\text { Gage } \\
\text { he ight }\end{array} \\
8.12 \\
7.80 \\
7.38\end{array}$} & \multirow{3}{*}{$\begin{array}{r}\begin{array}{c}\text { Dis- } \\
\text { charge }\end{array} \\
2,340 \\
2,060 \\
1,730\end{array}$} \\
\hline Mar. 3 & & & & Mar. & 7 & & & & Mar. 12 & & & \\
\hline 4 & 0600 & 2.47 & 42 & & 8 & 1200 & 5.28 & 656 & & & & \\
\hline & 1600 & 4.07 & 318 & & & & & 8 & 13 & 2400 & 6.77 & 1,32 \\
\hline & 2000 & 6.85 & 1,370 & & 9 & 0300 & 6.00 & 928 & & & & \\
\hline & 2200 & 8.88 & 3,140 & & & 0600 & 9.50 & 3,920 & 14 & 0700 & 6.56 & 1,200 \\
\hline & 2400 & 9.48 & 3,890 & & & $\begin{array}{l}1200 \\
1800\end{array}$ & $\begin{array}{l}11.30 \\
12.15\end{array}$ & & & $\begin{array}{l}1900 \\
2400\end{array}$ & $\begin{array}{l}6.85 \\
7.15\end{array}$ & $\begin{array}{l}1,370 \\
1,560\end{array}$ \\
\hline 5 & 0500 & 10.12 & 4,870 & & & 2400 & 14.00 & 14,800 & & & & \\
\hline & 1000 & 9.90 & 4,520 & & & & & & 15 & 1100 & 7.35 & 1,700 \\
\hline & 1400 & 9.85 & 4,440 & & 10 & 0600 & 15.82 & 23,400 & & 1800 & 7.16 & 1,570 \\
\hline & 2400 & 10.18 & 4,970 & & & $\begin{array}{l}1200 \\
2400\end{array}$ & $\begin{array}{l}15.38 \\
14.42\end{array}$ & $\begin{array}{l}21,000 \\
16,500\end{array}$ & & 2400 & 38 & 1,390 \\
\hline 6 & 0030 & 10.20 & 5,000 & & & & & & 16 & 1200 & 6.23 & 1,04 \\
\hline & $\begin{array}{l}0600 \\
1200\end{array}$ & $\begin{array}{r}10.00 \\
9.23\end{array}$ & $\begin{array}{l}4,680 \\
3,560\end{array}$ & & 11 . & $\begin{array}{l}0600 \\
1200\end{array}$ & $\begin{array}{l}13.68 \\
12.45\end{array}$ & $\begin{array}{r}13,700 \\
9,850\end{array}$ & & 2400 & & \\
\hline & 1800 & 7.26 & 40 & & & 1800 & 10.40 & 5,340 & 17 & 1200 & & 772 \\
\hline & 2400 & 6.59 & & & & 2400 & 8.94 & & & 2400 & 5.42 & 697 \\
\hline
\end{tabular}

Note.--Daily means for some days computed from data in addition to figures shown.

(36) 3-2320. Paint Creek near Greenfleld, Ohio

(Crest-stage station)

Location.--Lat $39^{\circ} 22^{\prime} 45^{\prime \prime}$, long $83^{\circ} 22^{\prime} 30^{\prime \prime}$, at bridge on State Highway 753 in Fayette County, a quarter of a mile north of county line, 0.6 mile above Stone Run, and 2 miles north of Greenfield, Highland County.

Drainage area. $--249 \mathrm{sq} \mathrm{ml}$.

Gage-helght record.--Crest stages only. Datum of gage 1s $845.30 \mathrm{ft}$ above mean sea level, datum of 1929.

Discharge record.--Stage-discharge relation defined by current-meter measurements below 6,000 cfs, and by indirect measurement at 15,600 cfs.

Maxima.- March 1964: Discharge, 16,000 cfs Mar. 10 (gage height, 12.3 ft, from floodmarks).

1926-35, 1939-56, 1959, 1962 to February 1964: Discharge, 15,600 ofs Mar. 5, 1963 (gage helght, $12.2 \mathrm{ft}$, from floodmarks).

Remarks.--Crest-stage gage is at discontinued station site 1926-35, 1939-56. 
(37) 3-2324.7. Paint Creek at damsite near Balnbridge, Ohio

(Recording crest-stage station)

Location.--Lat $39^{\circ} 15^{\prime} 05^{\prime \prime}$, long $83^{\circ} 20^{\prime} 45^{\prime \prime}, 4.5$ miles west of Bainbridge, Ross County, and 1.5 miles upstream from Rocky Fork.

Drainage area. $--573 \mathrm{sq} \mathrm{mi}$.

Gage-helght record.--Crest stages from floodmarks, because of faulty operation of bubble gage. Datum of gage is $742.6 \mathrm{ft}$ above mean sea level, datum of 1929 .

D1scharge record.--Stage-discharge relation defined by current-meter measurements below $1,900 \mathrm{c}$ 's and extended above on basis of current-meter measurement made Mar. 10, 1964, below the mouth of Rocky Fork, at gage he1ght $20.78 \mathrm{ft}$, adjusted for storage changes and Rocky Fork flow, and estimated flow for the March 1963 peak.

Maxima --Narch 1964: Discharge, 45,000 cfs (estimated) Mar. 10 (gage height, $27.3 \mathrm{ft}$, from floodmarks).

August 1962 to February 1964: Discharge, 42,300 efs (estimated) Mar. 5, 1963 (gage helght, $26.5 \mathrm{ft}$, from floodmarks).

(38) 3-2324.9. Rocky Fork Lake near Rainsboro, Ohio

Location. --Lat $39^{\circ} 11^{\prime} 0^{\prime \prime}$, long $83^{\circ} 26^{\prime} 20^{\prime \prime}$, at dam on Rocky Fork, $2 \frac{1}{2}$ miles southwest of Rainsboro, Highland County.

Drainage area. $-114 \mathrm{sq} \mathrm{ml}$.

Gage-helght record.--Water-stage recorder graph. Datum of gage is $851.32 \mathrm{ft}$ above mean sea level.

Max1ma --March 1964: Contents, 50,680 acre-ft Nar. 10, 0630 hours (gage he1ght, $36.13 \mathrm{ft}$ ).

1953 to February 1964: Contents, 45,250 acre-ft July 21, 1954 (gage helght, $33.96 \mathrm{ft})$.

Remarks.--Reservolr formed by earth dam with concrete splllway; lake fllled for recreational use in 1952. Capacity at spillway level (elevation, $29.2 \mathrm{ft}$ gage datum), 34,100 acre-ft.

Cooperation.--Capacity curve fumished by Ohio Department of Natural Resources, Division of Parks.

Gage he1ght and contents, February and March 1964

\begin{tabular}{c|c|c|c||c|c|c|c}
\hline Date & Hour & $\begin{array}{c}\text { Gage helght } \\
\text { (feet) }\end{array}$ & $\begin{array}{c}\text { Contents } \\
\text { (acre-feet) }\end{array}$ & Date & Hour & $\begin{array}{c}\text { Gage height } \\
\text { (feet) }\end{array}$ & $\begin{array}{c}\text { Contents } \\
\text { (acre-feet) }\end{array}$ \\
\hline Feb. $29 \ldots$ & 2400 & 25.72 & 26,400 & Mar. 10... & 0630 & 36.13 & 50,680 \\
Mar. $5 \ldots$ & 0900 & 30.49 & 36,640 & Mar. 31... & 2400 & 29.63 & 34,630 \\
Mar. $9 \ldots$ & 0300 & 29.83 & 35,090 & & \\
\hline
\end{tabular}

(39) 3-2325. Rocky Fork near Barretts Mills, Ohio

Location.--Lat $39^{\circ} 13^{105^{\prime \prime}}$, long $83^{\circ} 23^{\prime} 05^{\prime \prime}$, on left bank at downstream side of highway bridge, 1.1 miles north of Barretts Mills, Highland County, 2 miles east of Rainsboro, $2 \frac{3}{4}$ miles upstream from mouth, and 6 miles downstream from Rocky Fork Lake.

Drainage area. - - $140 \mathrm{sq} \mathrm{mi}$.

Gage-height record --Water-stage recorder graph; Datum of gage is $770.7 \mathrm{ft}$ above mean sea level (levels by Corps of Englneers).

Discharge record.--Stage-discharge relation defined by current-meter measurements below $8,800 \mathrm{c} f \mathrm{~s}$ and extended above on basis of velocity-area studies.

Maxima --March 1964: Discharge, 13,400 cfs Mar. 10, 0230 hours (gage height, 15. $10 \mathrm{ft}$ ).

1939 to February 1964: Discharge, 13,200 cfs Mar. 6, 1945 (gage height, $15.56 \mathrm{ft})$.

Remarks.--Flow regulated by Rocky Fork Lake (see station 3-2324.9). 
Mean discharge, in cublc feet per second, March 1964, of Rocky Fork near Barretts M1lis, Oh1.0

\begin{tabular}{|c|c|c|c|c|c|c|c|c|c|}
\hline Day & Discharge & Day & D1scharge & Day & D1.scharge & Day & D1scharge & Day & D1scharge \\
\hline $\begin{array}{l}1 \ldots \ldots \\
2 \ldots \\
3 \ldots \\
4 \ldots \\
5 \ldots \\
6 \ldots\end{array}$ & $\begin{array}{r}65 \\
67 \\
70 \\
592 \\
1,030 \\
635\end{array}$ & $\begin{array}{r}7 \ldots \ldots \\
8 \ldots \ldots \\
9 \ldots \\
10 \ldots \\
11 \ldots \ldots \\
12 \ldots \ldots\end{array}$ & $\begin{array}{r}409 \\
318 \\
4,470 \\
9,520 \\
3,160 \\
1,390\end{array}$ & $\begin{array}{l}13 \ldots \ldots \\
14 \ldots \ldots \\
15 \ldots \ldots \\
16 \ldots \ldots \\
17 \ldots \ldots \\
18 \ldots \ldots\end{array}$ & $\begin{array}{l}780 \\
700 \\
695 \\
445 \\
312 \\
206\end{array}$ & $\begin{array}{l}19 \ldots \ldots \\
20 \ldots \ldots \\
21 \ldots \ldots \\
22 \ldots \ldots \\
23 \ldots \ldots \\
24 \ldots \ldots\end{array}$ & $\begin{array}{l}162 \\
139 \\
219 \\
214 \\
182 \\
155\end{array}$ & $\mid \begin{array}{l}25 \ldots \ldots \\
26 \ldots \ldots \\
27 \ldots \ldots \\
28 \ldots \ldots \\
29 \ldots \ldots \\
30 \ldots \ldots \\
31 \ldots \ldots\end{array}$ & $\begin{array}{l}143 \\
241 \\
196 \\
166 \\
145 \\
126 \\
110\end{array}$ \\
\hline
\end{tabular}

Gage helght, in feet, and discharge, in cublc feet per second, at 1ndicated time, 1964

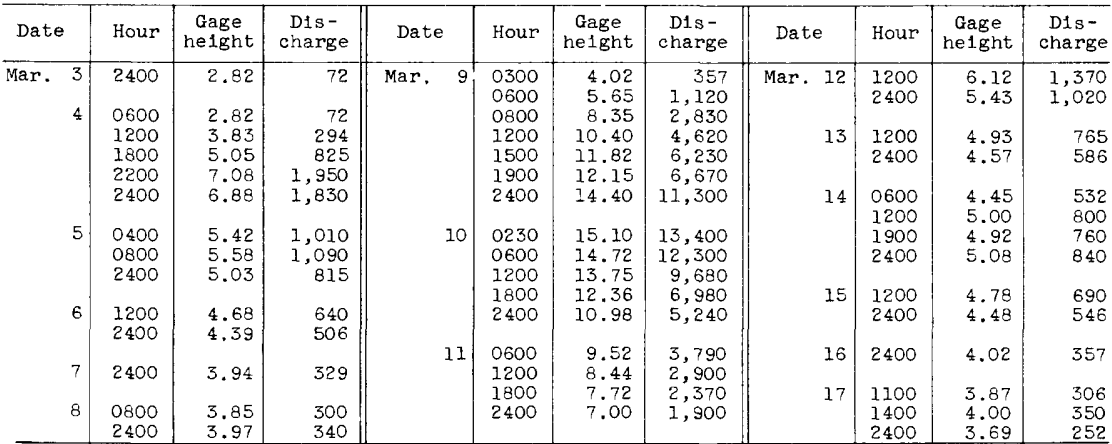

Note.--Dally means for some days computed from data in addition to figures shown.

(40) 3-2340. Paint Creek near Boumeville, Ohio

Location.--Lat $39^{\circ} 15^{\prime} 49^{\prime \prime}$, long $83^{\circ} 10^{\prime} 01^{\prime \prime}$, on downstream side of left pier of highway bridge, $1 \frac{1}{4}$ miles southwest of Boumeville, Ross County, and $1 \frac{1}{4}$ miles upstream

from Upper Twin Creek.

Drainage area. $--807 \mathrm{sq} \mathrm{mi}$.

Gage-height record.--Water-stage recorder graph. Datum of gage is $665.2 \mathrm{ft}$ above mean sea level, adjustment of 1912 .

Discharge record.--Stage-discharge relation defined by current-meter measurements below 27,000 cfs and by indirect measurement at $52,700 \mathrm{cfs}$.

Maxima.--March 1964: Discharge, 56,900 efs Mar. 10, 0900 hours (gage height, $20.50 \mathrm{ft}$ ).

1921 to February 1964: Discharge, 52,700 cfs Mar. 5, 1963 (gage height, $20.08 \mathrm{ft}$ ).

Remarks.--Flow slightly regulated by Rocky Fork Lake (see station 3-2324.9).

Mean discharge, in cubic feet per second, March 1964

\begin{tabular}{|c|c|c|c|c|c|c|c|c|c|}
\hline Day & D1scharge & Day & D1scharge & Day & Discharge & Day & Discharge & Day & Discharge \\
\hline $\begin{array}{l}1 \ldots \ldots \\
2 \ldots \ldots \\
3 \ldots \\
4 \ldots \\
5 \ldots \\
6 \ldots\end{array}$ & $\begin{array}{r}130 \\
132 \\
144 \\
1,450 \\
11,900 \\
7,880\end{array}$ & $\begin{array}{r}7 \ldots \ldots \\
8 \ldots \ldots \\
9 \ldots \ldots \\
10 \ldots \ldots \\
11 \ldots \ldots \\
12 \ldots \ldots\end{array}$ & $\begin{array}{r}5,480 \\
3,490 \\
10,400 \\
44,600 \\
25,300 \\
11,500\end{array}$ & $\begin{array}{l}13 \ldots \ldots \\
14 \ldots \ldots \\
15 \ldots \ldots \\
16 \ldots \ldots \\
17 \ldots \ldots \\
18 \ldots \ldots\end{array}$ & $\begin{array}{l}5,370 \\
4,290 \\
4,410 \\
3,340 \\
2,500 \\
1,970\end{array}$ & $\begin{array}{l}19 \ldots \ldots \\
20 \ldots \ldots \\
21 \ldots \ldots \\
22 \ldots \ldots \\
23 \ldots \ldots \\
24 \ldots \ldots\end{array}$ & $\begin{array}{l}1,590 \\
1,390 \\
1,550 \\
1,710 \\
1,550 \\
1,380\end{array}$ & $\mid \begin{array}{l}25 \ldots \ldots \\
26 \ldots \ldots \\
27 \ldots \ldots \\
28 \ldots \ldots \\
29 \ldots \ldots \\
30 \ldots \ldots \\
31 \ldots \ldots\end{array}$ & $\begin{array}{l}1,230 \\
1,830 \\
1,810 \\
1,510 \\
1,280 \\
1,140 \\
1,030\end{array}$ \\
\hline onthly & ean d & ge, & & & & & & & $\begin{array}{r}5,267 \\
7.53\end{array}$ \\
\hline
\end{tabular}


Gage helght, in feet, and discharge, in cub1c feet per second, at indicated time, 1964 , of Palnt Creek near Bournevilie, Ohio

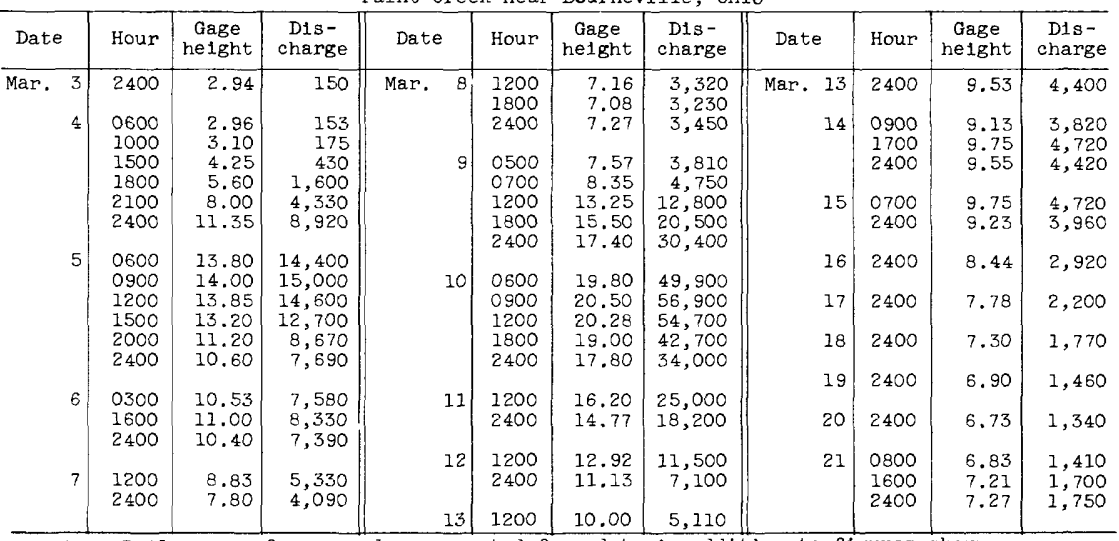

Note. - Dally means for some days computed from data in addition to figures shown.

(41) 3-2345. Scloto River at Higby, Onio

Location.--Lat $39^{\circ} 12^{\prime} 44^{\prime \prime}$, long $82^{\circ} 51^{\prime} 35^{\prime \prime}$, on left bank at downstream side of highway bridge, three-quarters of a mile downstream from Walnut Creek and $1 \frac{1}{4}$ miles north of Higby, Ross County.

Drainage area. $--5,131 \mathrm{sq} \mathrm{mi}$.

Gage-height record.--Digital recorder tape punched at hourly intervals. Datum of gage is $567.6 \mathrm{ft}$ above mean sea level, adjustment of 1912 .

Discharge record.--Stage-discharge relation defined by current-meter measurements below 120,000 efs.

Maxima.--March 1964: D1scharge, 130,000 efs Mar. 11, 0500 hours (gage he1ght, $25.01 \mathrm{ft}^{\mathrm{t}}$.

1930 to February 1964: Discharge, 177,000 ofs Jan. 23, 1937; gage he1ght,

$26.4 \mathrm{ft}$ Jan. 23, 1937, from floodmarks, and Jan. 23, 1959.

Maximum stage known, $31.6 \mathrm{ft}$ Mar. 26, 1913.

Remarks.--Flow slightly regulated by O'Shaughnessy Reservolr (16,940 acre-ft, $987 \mathrm{sq} \mathrm{ml}$ ), Griggs Reservolr (4,415 acre-ft, $1,052 \mathrm{sq} \mathrm{m1}, 65 \mathrm{sq} \mathrm{ml}$ plus

0 'Shaughnessy Reservoir) Delaware Reservoir (132,000 acre-ft, $381 \mathrm{sq} \mathrm{ml}$ ), Hoover Reservoir $(60,340$ acre-ft, $190 \mathrm{sq} \mathrm{m1})$ and Rocky Fork Lake (34,100 acre-ft, $115 \mathrm{sq} \mathrm{mi}$ ).

Mean discharge, in cuble feet per second, March 1964

\begin{tabular}{|c|c|c|c|c|c|c|c|c|c|}
\hline Day & Discharge & Day & D1scharge & Day & Discharge & Day & Discharge & Day & Discharge \\
\hline $\begin{array}{l}1 \ldots \ldots \\
2 \ldots \ldots \\
3 \ldots \ldots \\
4 \ldots \\
5 \ldots \\
6 \ldots\end{array}$ & $\begin{array}{r}569 \\
571 \\
612 \\
2,980 \\
24,700 \\
27,600\end{array}$ & $\begin{array}{r}7 \ldots \ldots \\
8 \ldots \ldots \\
9 \ldots \\
10 \ldots \\
11 \ldots \\
12 \ldots\end{array}$ & $\begin{array}{r}24,700 \\
19,100 \\
30,700 \\
86,100 \\
122,000 \\
96,800\end{array}$ & $\begin{array}{l}13 \ldots \ldots \\
14 \ldots \ldots \\
15 \ldots \ldots \\
16 \ldots \ldots \\
17 \ldots \ldots \\
18 \ldots \ldots\end{array}$ & $\begin{array}{l}58,200 \\
35,700 \\
29,100 \\
23,900 \\
22,200 \\
18,400\end{array}$ & $\begin{array}{l}19 \ldots \ldots \\
20 \ldots \ldots \\
21 \ldots \ldots \\
22 \ldots \ldots \\
23 \ldots \ldots \\
24 \ldots \ldots\end{array}$ & $\begin{array}{r}14,300 \\
11,800 \\
10,800 \\
11,200 \\
11,400 \\
9,180\end{array}$ & $\begin{array}{l}25 \ldots \ldots \\
26 \ldots \ldots \\
27 \ldots \ldots \\
28 \ldots \ldots \\
29 \ldots \ldots \\
30 \ldots \ldots \\
31 \ldots \ldots\end{array}$ & $\begin{array}{r}8,910 \\
10,000 \\
8,350 \\
7,130 \\
6,240 \\
5,450 \\
4,860\end{array}$ \\
\hline
\end{tabular}

Note.--Da1ly mean discharges computed on basis of 60 -minute intervals.

Gage height, in feet, and discharge, in cubic feet per second, at indicated time, 1964

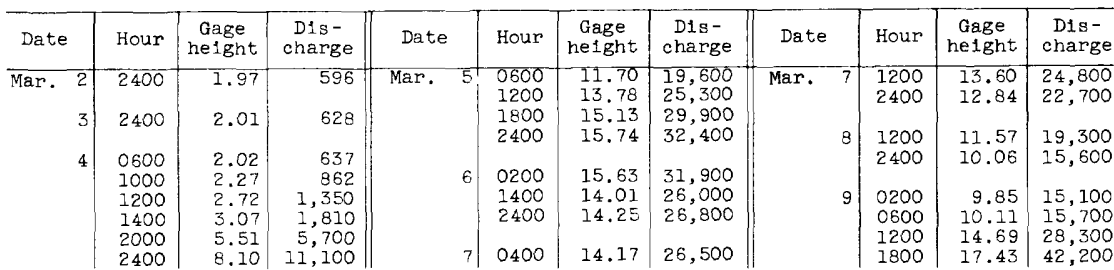


Gage height, in feet, and discharge, in cublc feet per second, at 1ndicated t1me, 1964, of Scloto River at Higby, Ohio--Continued

\begin{tabular}{|c|c|c|c|c|c|c|c|c|c|c|c|}
\hline Date & Hour & $\begin{array}{c}\text { Gage } \\
\text { helght }\end{array}$ & $\begin{array}{l}\text { Dis- } \\
\text { charge }\end{array}$ & Date & Hour & $\begin{array}{c}\text { Gage } \\
\text { helght }\end{array}$ & $\begin{array}{l}\text { Dis- } \\
\text { charge }\end{array}$ & Date & Hour & $\begin{array}{c}\text { Gage } \\
\text { helght }\end{array}$ & $\begin{array}{l}\text { Dis- } \\
\text { charge }\end{array}$ \\
\hline$\overline{\text { Mar. }} 9$ & 2400 & 18.76 & 52,700 & Mar. 14 & 2400 & 15.53 & 31,500 & Mar. 21 & 2400 & 8.08 & 11,000 \\
\hline 10 & $\begin{array}{l}0600 \\
1200\end{array}$ & $\begin{array}{l}20.15 \\
21.47\end{array}$ & $\begin{array}{l}65,500 \\
79,200\end{array}$ & 15 & $\begin{array}{l}1200 \\
2400\end{array}$ & $\begin{array}{l}15.02 \\
13.99\end{array}$ & $\begin{array}{l}29,500 \\
26,000\end{array}$ & 22 & 2400 & 8.23 & 11,400 \\
\hline & $\begin{array}{l}1800 \\
2400\end{array}$ & $\begin{array}{l}23.50 \\
24.81\end{array}$ & $\begin{array}{l}104,000 \\
126,000\end{array}$ & 16 & $\begin{array}{l}1200 \\
2400\end{array}$ & $\begin{array}{l}13.21 \\
12.94\end{array}$ & $\begin{array}{l}23,700 \\
23,000\end{array}$ & 23 & $\begin{array}{l}0800 \\
1800 \\
2400\end{array}$ & $\begin{array}{l}8.26 \\
8.22 \\
8.12\end{array}$ & $\begin{array}{l}11,400 \\
11,300 \\
11,100\end{array}$ \\
\hline 12 & $\begin{array}{l}0200 \\
0500 \\
1200 \\
2400\end{array}$ & $\begin{array}{l}24.95 \\
25.01 \\
24.69 \\
24.03\end{array}$ & $\begin{array}{l}128,000 \\
130,000 \\
124,000 \\
112,000\end{array}$ & 17 & $\begin{array}{l}1200 \\
2400\end{array}$ & $\begin{array}{l}12.74 \\
12.17\end{array}$ & $\begin{array}{l}22,400 \\
20,900\end{array}$ & 24 & $\begin{array}{l}0600 \\
2400\end{array}$ & $\begin{array}{l}7.74 \\
6.57\end{array}$ & $\begin{array}{r}10,300 \\
7,780\end{array}$ \\
\hline 12 & $\begin{array}{l}0600 \\
1200\end{array}$ & $\begin{array}{l}23.70 \\
23.11\end{array}$ & $\begin{array}{r}107,000 \\
98,700\end{array}$ & 18 & $\begin{array}{l}2400 \\
2400\end{array}$ & $\begin{array}{r}10.25 \\
8.88\end{array}$ & $\begin{array}{l}16,100 \\
12,900\end{array}$ & 25 & $\begin{array}{l}1200 \\
2400\end{array}$ & $\begin{array}{l}7.21 \\
7.33\end{array}$ & $\begin{array}{l}9,120 \\
9,390\end{array}$ \\
\hline 13 & $\begin{array}{l}1200 \\
2400\end{array}$ & $\begin{array}{l}19.36 \\
17.69\end{array}$ & $\begin{array}{l}77,400 \\
58,100 \\
44,000\end{array}$ & 20 & $\begin{array}{l}1200 \\
2400\end{array}$ & $\begin{array}{l}8.41 \\
8.06\end{array}$ & $\begin{array}{l}11,800 \\
11,000\end{array}$ & 26 & $\begin{array}{l}0800 \\
1600 \\
2400\end{array}$ & $\begin{array}{l}7.42 \\
7.93 \\
7.57\end{array}$ & $\begin{array}{r}9,580 \\
10,700 \\
9,910\end{array}$ \\
\hline 14 & 1200 & 16.30 & 35,100 & 21 & $\begin{array}{l}1400 \\
2000\end{array}$ & $\begin{array}{l}7.89 \\
7.99\end{array}$ & $\begin{array}{l}10,600 \\
10,800\end{array}$ & 27 & $\begin{array}{l}1200 \\
2400\end{array}$ & $\begin{array}{l}6.75 \\
6.49\end{array}$ & $\begin{array}{l}8,160 \\
7,610\end{array}$ \\
\hline
\end{tabular}

(42) 3-2359.95. Salt Creek near Londonderry, Ohio

(Crest-stage station)

Location.--Lat $39^{\circ} 17125^{\prime \prime}$, long $82^{\circ} 44145^{\prime \prime}$, at bridge on State Highway 671 in Vinton County, half a mile east of county line, 2.8 miles northeast of Londonderry, Ross County.

Drainage area. $--268 \mathrm{sq} \mathrm{mi}$.

Gage-height record.--Twice-dally wire-welght-gage readings and crest-stage gage. Datum of gage is $600.14 \mathrm{ft}$ above mean sea level, unadjusted.

Discharge record.--Stage-discharge relation defined by current-meter measurements below $12,000 \mathrm{cfs}$ and by indirect measurement at $31,600 \mathrm{cfs}$.

Maxima.--March 1964: Discharge, 26,000 cfs Mar. 10 (gage height, $21.7 \mathrm{ft}$, from floodmarks ).

August 1962 to February 1964: Discharge, 31,600 efs Mar. 5, 1963 (gage helght, $22.7 \mathrm{ft}$, from floodmarks).

(43) Scioto River at P1keton, Ohio

(Weather Bureau stage station)

Location. - Lat $39^{\circ} 04^{\prime} 15^{\prime \prime}$, long $83^{\circ} 01^{\prime} 00^{\prime \prime}$, at pumping station of Atomic Energy Commission plant west of Piketon, Pike County.

Drainage area. $--5,836 \mathrm{sq} \mathrm{ml}$.

Gage-helght record.--Twice-daily wire-weight-gage readings, supplemented by additional readings during high-water periods. Datum of gage is $531.39 \mathrm{ft}$ above mean sea level (U.S. Weather Bureau).

Discharge record.--Stage-discharge relation defined by current-meter measurements below 128,000 efs.

Maxima.--March 1964: Discharge, $132,000 \mathrm{cfs}$ Mar. 11, 1100 hours (gage height, $31.67 \mathrm{ft}$ ).

June 1954 to February 1964: Discharge, 150,000 ofs Jan. 24, 1959 (gage height, $32.56 \mathrm{ft}$ ).

Remarks.--Flow slightly regulated (see station 3-2345). 


\section{OHIO BRUSH CREEK BASIN}

(44) 3-2375. Ohio Brush Creek near West Union, ohio

Location.--Lat $38^{\circ} 48^{\prime} 13^{\prime \prime}$, long $83^{\circ} 25^{\prime} 16^{\prime \prime}$, on right bank at downstream side of bridge on State Highway $348,0.3$ mile downstream from Cedar Run, 2 miles southwest of Cedar Mills, and 7 miles east of West Union, Adams County.

Drainage area. $--381 \mathrm{sq} \mathrm{mi}$.

Gage-helght record.--Water-stage recorder graph. Datum of gage is $510.6 \mathrm{ft}$ above mean sea level, adjustment of 1912 .

Discharge record.--Stage-discharge relation defined by current-meter measurements below $22,000 \mathrm{cfs}$ and by indirect measurements at 35,500 ofs, $51,600 \mathrm{cfs}$, and $59,200 \mathrm{cfs}$. Backwater from Ohio River Mar. 11-15: discharge estimated on basis of records for Whiteoak Creek and East Fork Little Miami River.

Maxima.--March 1964: Discharge, 59,200 ef's Mar. 10, 0630 hours (gage height, $27.91 \mathrm{ft}$ )

1926-35, 1940 to February 1964: Discharge, 51,600 cfs Mar. 19, 1943 (gage height, $26.5 \mathrm{ft}$ ).

Mean discharge, in cubic feet per second, March 1964

\begin{tabular}{|c|c|c|c|c|c|c|c|c|c|}
\hline Day & Discharge & Day & Dlscharge & Day & Discharge & Day & Discharge & Day & D1scharge \\
\hline $\begin{array}{l}1 \ldots \ldots \\
2 \ldots \ldots \\
3 \ldots \ldots \\
4 \ldots \ldots \\
5 \ldots \ldots \\
6 \ldots\end{array}$ & $\begin{array}{r}67 \\
75 \\
698 \\
8,950 \\
17,300 \\
1,550\end{array}$ & $\begin{array}{r}7 \ldots \ldots \\
8 \ldots \ldots \\
9 \ldots \ldots \\
10 \ldots \\
11 \ldots \ldots \\
12 \ldots \ldots\end{array}$ & $\begin{array}{r}730 \\
1,480 \\
26,400 \\
40,500 \\
2,400 \\
1,400\end{array}$ & $\begin{array}{l}13 \ldots \ldots \\
14 \ldots \ldots \\
15 \ldots \ldots \\
16 \ldots \\
17 \ldots \ldots \\
18 \ldots \ldots\end{array}$ & $\begin{array}{r}1,200 \\
2,500 \\
5,000 \\
2,120 \\
799 \\
444\end{array}$ & $\begin{array}{l}19 \ldots \ldots \\
20 \ldots \ldots \\
21 \ldots \ldots \\
22 \ldots \ldots \\
23 \ldots \ldots \\
24 \ldots \ldots\end{array}$ & $\begin{array}{r}345 \\
311 \\
1,230 \\
883 \\
508 \\
371\end{array}$ & $\begin{array}{l}25 \ldots \ldots \\
26 \ldots \ldots \\
27 \ldots \ldots \\
28 \ldots \ldots \\
29 \ldots \ldots \\
30 \ldots \ldots \\
31 \ldots \ldots\end{array}$ & $\begin{array}{r}315 \\
1,680 \\
656 \\
413 \\
340 \\
274 \\
240\end{array}$ \\
\hline
\end{tabular}

Gage height, in feet, and discharge, in cubic feet per second, at indicated time, 1964

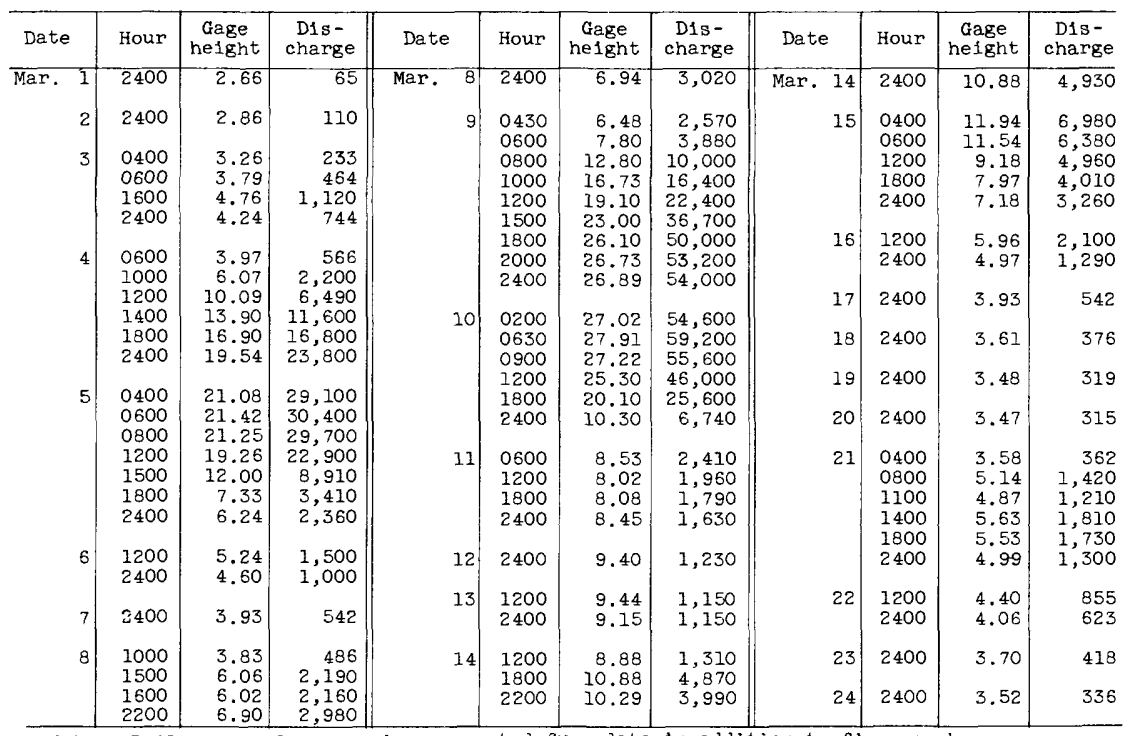

Note.--Daily means for some days computed from data in addition to flgures shown. 


\section{OHIO RIVER MAIN STEM}

(45) 3-2380. Oh1o River near Maysv1lle, Ky.

Location.--Lat $38^{\circ} 38^{\prime} 05^{\prime \prime}$, Iong $83^{\circ} 42^{\prime} 11^{\prime \prime}$, on left bank at lock and dam $33,1.8 \mathrm{mlles}$ upstream from c1ty limits of Maysville, Mason County, 2.2 miles downstream from Cabin Creek, and at mile 405.1.

Drainage area. $--70,130 \mathrm{sq} \mathrm{mi}$, approximately.

Gage-he1ght record.--Graph drawn from hourly staff-gage readings. Graph drawn for auxlliary staff gage, 29.0 miles downstream, from once-dally or more frequent readings, except Mar. 21, 24, 26, Mar. 31 to Apr. 2, when no readings were made. Graph for Mar. 21, 24, 26 drawn on basis of recession graph at base gage and readings on adjacent days. Datum of gage is $452.57 \mathrm{ft}$ above mean sea level, Ohio River datum.

Discharge record.--Stage-fall-discharge relation defined by current-meter measurements. Fall used as a factor in computing discharge. Discharge for periods of no gage-helght record Mar. 30 to Apr. 2 and Indefinite stage-fall-d1scharge relation Mar. 1-3 estimated on basis of records for other OhIo River ma1n-stem stations. $\frac{\text { Maxima }}{62.65 \mathrm{ft} \text { ) }}$-March 1964: D1scharge, 562,000 cfs Mar. 13, 1200 hours (gage helght,

1937, 1939 to February 1964: D1scharge, 820,000 cfs Jan. 27, 1937 (gage height, $75.3 \mathrm{ft}$ ).

Remarks.-- Partly regulated by locks, dams, and reservoirs.

Cooperation.--Gage-height record furnished by Corps of Engineers.

\begin{tabular}{|c|c|c|c|c|c|c|c|c|c|}
\hline Day & Discharge & Day & Discharge & Day & D1scharge & Day & D1 scharge & Day & Discharge \\
\hline $\begin{array}{l}1 \ldots \ldots \\
2 \ldots \\
3 \ldots \\
4 \ldots \\
5 \ldots \\
6 \ldots\end{array}$ & $\begin{array}{r}34,000 \\
42,000 \\
60,000 \\
148,000 \\
280,000 \\
313,000\end{array}$ & $\begin{array}{r}7 \ldots \ldots \\
8 \ldots \ldots \\
9 \ldots \ldots \\
10 \ldots \\
11 \ldots \ldots \\
12 \ldots \ldots\end{array}$ & $\begin{array}{l}332,000 \\
352,000 \\
403,000 \\
491,000 \\
527,000 \\
547,000\end{array}$ & $\begin{array}{l}13 \ldots \ldots \\
14 \ldots \ldots \\
15 \ldots \ldots \\
16 \ldots \ldots \\
17 \ldots \ldots \\
18 \ldots \ldots\end{array}$ & $\begin{array}{l}559,000 \\
548,000 \\
533,000 \\
504,000 \\
478,000 \\
448,000\end{array}$ & $\begin{array}{l}19 \ldots \ldots \\
20 \ldots \ldots \\
21 \ldots \ldots \\
22 \ldots \ldots \\
23 \ldots \ldots \\
24 \ldots \ldots\end{array}$ & $\begin{array}{l}414,000 \\
358,000 \\
307,000 \\
253,000 \\
207,000 \\
183,000\end{array}$ & $\begin{array}{l}25 \ldots \ldots \\
26 \ldots \ldots \\
27 \ldots \ldots \\
28 \ldots \ldots \\
29 \ldots \ldots \\
30 \ldots \ldots \\
31 \ldots \ldots\end{array}$ & $\begin{array}{l}164,000 \\
149,000 \\
144,000 \\
137,000 \\
133,000 \\
125,000 \\
120,000\end{array}$ \\
\hline lonthly & mean disch & rge, in & cub1c & er se & & & & & 299,800 \\
\hline
\end{tabular}

Mean discharge, in cubic feet per second, April 1964

Apr. $1 \ldots \ldots \ldots \ldots \ldots 115,000$

Gage height, in feet, and discharge, in cublc feet per second, at indicated t1me, 1964

\begin{tabular}{|c|c|c|c|c|c|c|c|c|c|c|c|}
\hline Date & \multirow{2}{*}{$\begin{array}{l}\text { Hour } \\
2400\end{array}$} & \multirow{2}{*}{$\frac{\begin{array}{c}\text { Gage } \\
\text { he1ght }\end{array}}{18.50}$} & \multirow{2}{*}{$\begin{array}{c}\begin{array}{c}\text { D1s- } \\
\text { charge }\end{array} \\
33,300\end{array}$} & Date & \multirow{3}{*}{$\begin{array}{l}\text { Hour } \\
0600 \\
1200 \\
2400\end{array}$} & \multirow{3}{*}{\begin{tabular}{|r}
\multicolumn{1}{c}{ Gage } \\
helght
\end{tabular}} & \multirow{3}{*}{$\begin{array}{c}\begin{array}{c}\text { Dis- } \\
\text { charge }\end{array} \\
518,000 \\
530,000 \\
540,000\end{array}$} & Date & \multirow{2}{*}{$\begin{array}{c}\text { Hour } \\
2400\end{array}$} & \multirow{2}{*}{$\frac{\begin{array}{c}\text { Gage } \\
\text { he1ght }\end{array}}{50.55}$} & \multirow{2}{*}{$\frac{\begin{array}{c}\text { Dis- } \\
\text { charge }\end{array}}{389,000}$} \\
\hline Mar. 3 & & & & Mar. 11 & & & & Mar. 19 & & & \\
\hline 4 & $\begin{array}{l}0600 \\
1200\end{array}$ & $\begin{array}{l}21.35 \\
24.85\end{array}$ & $\begin{array}{l}103,000 \\
141,000\end{array}$ & & & & & 20 & $\begin{array}{l}1200 \\
2400\end{array}$ & $\begin{array}{l}48.60 \\
46.40\end{array}$ & $\begin{array}{l}358,000 \\
326,000\end{array}$ \\
\hline & 2400 & 34.45 & 238,000 & 12 & 1200 & 62.25 & 54 & & & & \\
\hline & & & & & 1800 & 62. & & 21 & 1200 & 45 & 309,000 \\
\hline 5 & $\begin{array}{l}0600 \\
1200\end{array}$ & $\begin{array}{l}37.25 \\
39.30\end{array}$ & $\begin{array}{l}259,000 \\
285,000\end{array}$ & & 2400 & 62.50 & 554, & & 2400 & 25 & 283 \\
\hline & $\begin{array}{l}1800 \\
2400\end{array}$ & $\begin{array}{l}41.10 \\
42.05\end{array}$ & $\begin{array}{l}304,000 \\
306,000\end{array}$ & 13 & $\begin{array}{l}0600 \\
1200 \\
1800\end{array}$ & $\begin{array}{l}62.60 \\
62.65 \\
62.55\end{array}$ & $\begin{array}{l}561,000 \\
562,000 \\
561,000\end{array}$ & 22 & $\begin{array}{l}1200 \\
2400\end{array}$ & $\begin{array}{l}39.55 \\
36.75\end{array}$ & $\begin{array}{l}251,000 \\
228,000\end{array}$ \\
\hline 6 & $\begin{array}{l}0600 \\
1200\end{array}$ & $\begin{array}{l}42.70 \\
43.25\end{array}$ & $\begin{array}{l}306,000 \\
312,000\end{array}$ & & 2400 & 62.40 & 553,000 & 23 & $\begin{array}{l}1200 \\
2400\end{array}$ & $\begin{array}{l}34.10 \\
32.65\end{array}$ & $\begin{array}{l}202,000 \\
198,000\end{array}$ \\
\hline & 2400 & 44.30 & 324,000 & 14 & $\begin{array}{l}0600 \\
1200\end{array}$ & $\begin{array}{l}62.25 \\
62.05\end{array}$ & $\begin{array}{l}551,000 \\
548,000\end{array}$ & 24 & 1200 & 75 & 182 \\
\hline 7 & $\begin{array}{l}1200 \\
2400\end{array}$ & $\begin{array}{l}45.05 \\
45.60\end{array}$ & $\begin{array}{l}332,000 \\
341,000\end{array}$ & & 2400 & 61.70 & 543,000 & & 2400 & 30 & 171,000 \\
\hline 8 & $\begin{array}{l}0600 \\
1200\end{array}$ & $\begin{array}{l}45.90 \\
46.15\end{array}$ & $\begin{array}{l}348,000 \\
351,000\end{array}$ & 15 & $\begin{array}{l}0600 \\
1200 \\
2400\end{array}$ & $\begin{array}{l}61.45 \\
61.10 \\
60.05\end{array}$ & $\begin{array}{l}539,000 \\
534,000 \\
519,000\end{array}$ & 25 & $\begin{array}{l}1200 \\
2400\end{array}$ & $\begin{array}{l}28.40 \\
27.20\end{array}$ & $\begin{array}{l}165,000 \\
154,000\end{array}$ \\
\hline & 2400 & 47.50 & 363,000 & 16 & 1200 & 59.00 & 504,000 & 26 & $\begin{array}{l}1200 \\
2400\end{array}$ & $\begin{array}{l}26.55 \\
26.40\end{array}$ & $\begin{array}{l}148,000 \\
146,000\end{array}$ \\
\hline 9 & 0600 & 48.15 & 371 & & 2400 & 58.05 & 491,000 & & & & \\
\hline & $\begin{array}{l}1200 \\
1800 \\
2400\end{array}$ & $\begin{array}{l}50.80 \\
52.50 \\
55.10\end{array}$ & $\begin{array}{l}404,000 \\
426,000 \\
461,000\end{array}$ & 17 & $\begin{array}{l}1200 \\
2400\end{array}$ & $\begin{array}{l}57.10 \\
56.05\end{array}$ & $\begin{array}{l}473,000 \\
464,000\end{array}$ & 27 & $\begin{array}{l}1200 \\
2400\end{array}$ & $\begin{array}{l}26.15 \\
25.20\end{array}$ & $\begin{array}{l}145,000 \\
241,000\end{array}$ \\
\hline 10 & $\begin{array}{l}0600 \\
1200\end{array}$ & $\begin{array}{l}57.15 \\
58.00\end{array}$ & $\begin{array}{l}474,000 \\
496,000\end{array}$ & 18 & $\begin{array}{l}1200 \\
2400\end{array}$ & $\begin{array}{l}55.10 \\
53.90\end{array}$ & $\begin{array}{l}447,000 \\
436,000\end{array}$ & 28 & $\begin{array}{l}1200 \\
2400\end{array}$ & $\begin{array}{l}24.55 \\
24.50\end{array}$ & $\begin{array}{l}137,000 \\
134,000\end{array}$ \\
\hline & 1800 & 58.90 & 508,000 & & & & & 29 & 1200 & 45 & 133,000 \\
\hline & 2400 & 59.60 & 513,000 & 19 & 1200 & 52.35 & 416,000 & & 2400 & 24.40 & 133,000 \\
\hline
\end{tabular}




\section{WHITEOAK CREEK BASIN}

(46) 3-2385. Whiteoak Creek near Georgetown, Ohio

Location.--Lat $38^{\circ} 50^{\prime} 42^{\prime \prime}$, long $83^{\circ} 55^{\prime} 16^{\prime \prime}$, on left bank at upstream side of bridge on State Highway $221,600 \mathrm{f}^{\prime}$ downstream from Opossum Run, $1 \frac{3}{14}$ miles southwest of Georgetown, Brown County, and $6 \frac{1}{2}$ miles upstream from mouth.

Drainage area. $--222 \mathrm{sq} \mathrm{ml}$

Gage-he1ght record.--Water-stage recorder graph above a stage of $6.9 \mathrm{ft}$ and graph based on twice-daily wire-weight-gage readings below this stage. Datum of gage is $569.21 \mathrm{ft}$ above mean sea level, adjustment of 1912 .

Discharge record.--Stage-discharge relation defined by current-meter measurements below 20,000 cfs.

Maxima.--March 1964: Discharge, 22,400 cfs Mar. 10, 0500 hours (gage height, $14.64 \mathrm{ft})$.

1923-35, 1939 to February 1964: Discharge, 20,500 cfs May 14, 1933 (gage height, $20.87 \mathrm{ft}$ ), from rating curve extended above 10,000 cfs by logarithmic plotting.

Mean discharge, in cubic feet per second, March 1964

\begin{tabular}{|c|c|c|c|c|c|c|c|c|c|}
\hline Day & Discharge & Day & Discharge & Day & Discharge & Day & Discharge & Day & Discharge \\
\hline $\begin{array}{l}1 \ldots \ldots \\
2 \ldots \ldots \\
3 \ldots \ldots \\
4 \ldots \\
5 \ldots \ldots \\
6 \ldots \ldots\end{array}$ & $\begin{array}{r}18 \\
17 \\
72 \\
3,680 \\
10,100 \\
1,120\end{array}$ & $\begin{array}{r}7 \ldots \ldots \\
8 \ldots \ldots \\
9 \ldots \ldots \\
10 \ldots \\
11 \ldots \ldots \\
12 \ldots \ldots\end{array}$ & $\begin{array}{r}304 \\
474 \\
10,900 \\
19,400 \\
2,530 \\
446\end{array}$ & $\begin{array}{l}13 \ldots \ldots \\
14 \ldots \ldots \\
15 \ldots \ldots \\
16 \ldots \ldots \\
17 \ldots \ldots \\
18 \ldots \ldots\end{array}$ & $\begin{array}{r}351 \\
781 \\
1,470 \\
417 \\
212 \\
130\end{array}$ & {$\left[\begin{array}{l}19 \ldots \ldots \\
20 \ldots \ldots \\
21 \ldots \ldots \\
22 \ldots \ldots \\
23 \ldots \ldots \\
24 \ldots \ldots\end{array}\right.$} & $\begin{array}{r}98 \\
98 \\
431 \\
450 \\
238 \\
144\end{array}$ & $\mid \begin{array}{l}25 \ldots \ldots \\
26 \ldots \ldots \\
27 \ldots \ldots \\
28 \ldots \ldots \\
29 \ldots \ldots \\
30 \ldots \ldots \\
31 \ldots \ldots\end{array}$ & $\begin{array}{r}105 \\
537 \\
302 \\
144 \\
98 \\
85 \\
69\end{array}$ \\
\hline hl & ean $d$ & ge, & 4 & & & & & & $\begin{array}{r}1,781 \\
9.25\end{array}$ \\
\hline
\end{tabular}

Gage height, in feet, and discharge, in cubic feet per second, at indicated time, 1964

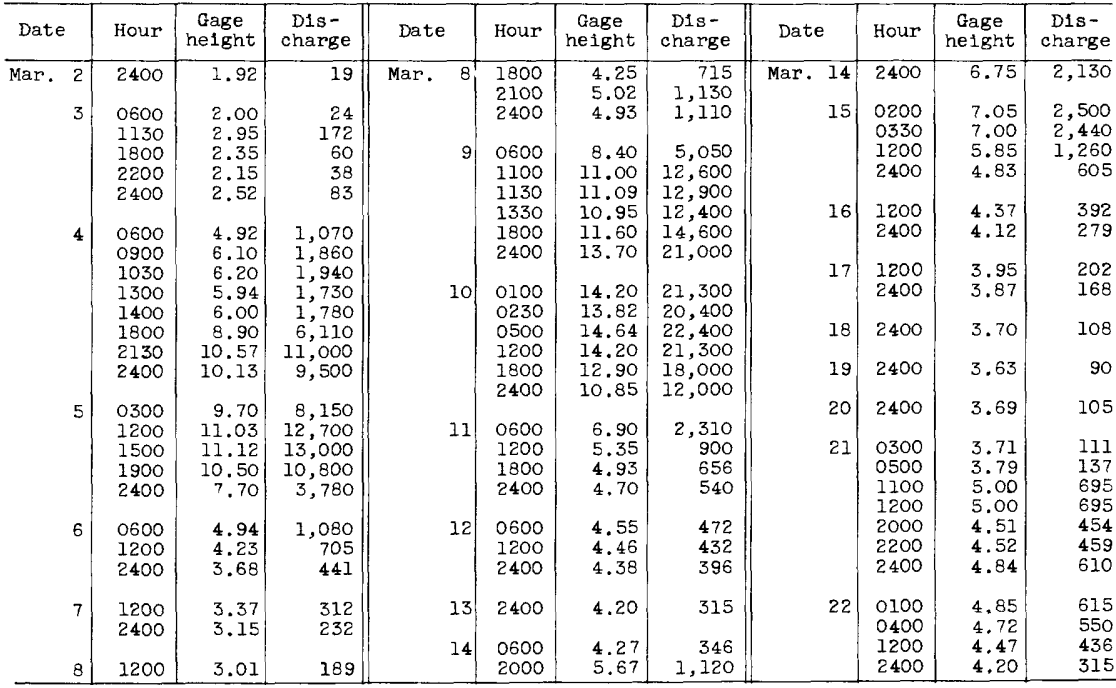

Note.--Daily means for some days computed from data in addition to figures shown. 


\section{LITTLE MIAMI RIVER BASIN}

(47) 3-2420. Little Miami River at Spring Valley, Ohio

(Discontinued gaging station)

Location.--Lat $39^{\circ} 36^{\prime} 20^{\prime \prime}$, long $84^{\circ} 00^{\prime} 50^{\prime \prime}$, at site of bridge, now destroyed, on U.S. Highway 42, three-eighths of a mile southwest of Spring Valley, Greene County, and $2 \frac{1}{2}$ miles downstream from Sugar Creek.

Drainage area. $--361 \mathrm{sq} \mathrm{m} 1$.

Gage-height record.--Floodmark. Datum of gage is $737.9 \mathrm{ft}$ above mean sea level, adjustment of 1912 .

Discharge record. - Stage-discharge relation defined by current-meter measurements below $12,400 \mathrm{css}$, prior to 1952 , and indirect measurement of 1959 peak flow of $36,400 \mathrm{cfs}$.

Maxima.--March 1964: Discharge, 14,400 cfs Mar. 10 (gage height, $15.79 \mathrm{ft}$, from floodmark).

1925-35, 1939-51, 1959, 1963: Discharge, 38,000 ef's Mar. 5, 1963 (gage he1ght, $19.14 \mathrm{ft}$, from floodmark).

\section{(48) 3-2423. Caesar Creek at Harveysburg, Ohio}

Location. --Lat $39^{\circ} 30127^{\prime \prime}$, Iong $84^{\circ} 00^{\prime} 42^{\prime \prime}$, at downstream side of bridge on state Highway 73, a quarter of a mile north of Harveysburg, Warren County.

Drainage area. $--209 \mathrm{sq} \mathrm{ml}$.

Gage-height record.--Water-stage recorder graph. Datum of gage is $781.90 \mathrm{ft}$ above mean sea level, datum of 1929 .

Discharge record.--Stage-discharge relation defined by current-meter measurements below 12,000 efs.

Maxima - -March 1964: Discharge, 12,900 efs Mar. 10, 1000 hours (gage height, $14.35 \mathrm{ft})$.

1960 to February 1964: Discharge, 14,900 cfs Mar. 5, 1963 (gage height, $15.63 \mathrm{ft}$.

Maximum stage known, $20.5 \mathrm{ft}$ Jan. 21, 1959, from floodmark.

Mean discharge, in cubic feet per second, March 1964

\begin{tabular}{|c|c|c|c|c|c|c|c|c|c|}
\hline Day & Discharge & Day & Discharge & Day & D1scharge & Day & Discharge & Day & Discharge \\
\hline $\begin{array}{l}1 \ldots \ldots \\
2 \ldots \ldots \\
3 \ldots \ldots \\
4 \ldots \\
5 \ldots \ldots \\
6 \ldots \ldots\end{array}$ & $\begin{array}{r}18 \\
19 \\
20 \\
1,030 \\
3,400 \\
1,010\end{array}$ & $\begin{array}{r}7 \ldots \ldots \\
8 \ldots \ldots \\
9 \ldots \\
10 \ldots \\
11 \ldots \ldots \\
12 \ldots\end{array}$ & $\begin{array}{r}526 \\
449 \\
7,260 \\
11,500 \\
2,720 \\
1,330\end{array}$ & $\begin{array}{l}13 \ldots \ldots \\
14 \ldots \ldots \\
15 \ldots \ldots \\
16 \ldots \ldots \\
17 \ldots \ldots \\
18 \ldots \ldots\end{array}$ & $\begin{array}{l}823 \\
990 \\
939 \\
557 \\
407 \\
319\end{array}$ & $\begin{array}{l}19 \ldots \ldots \\
20 \ldots \ldots \\
21 \ldots \ldots \\
22 \ldots \ldots \\
23 \ldots \ldots \\
24 \ldots \ldots\end{array}$ & $\begin{array}{l}267 \\
227 \\
275 \\
299 \\
255 \\
216\end{array}$ & $\mid \begin{array}{l}25 \ldots \ldots \\
26 \ldots \ldots \\
27 \ldots \ldots \\
28 \ldots \ldots \\
29 \ldots \ldots \\
30 \ldots \ldots \\
31 \ldots \ldots\end{array}$ & $\begin{array}{l}204 \\
521 \\
387 \\
287 \\
227 \\
189 \\
174\end{array}$ \\
\hline $1 \mathrm{y}$ & ean & & & & & & & & $\begin{array}{r}1,189 \\
6.56\end{array}$ \\
\hline
\end{tabular}

(49) 3-2425. Little Miami River near Fort Ancient, Ohio

(Discontinued gaging station)

Location.--Lat $39^{\circ} 22^{\prime} 42^{\prime \prime}$, long $84^{\circ} 05^{\prime} 32^{\prime \prime}$, at highway bridge 2 miles south of Fort Ancient, Warren County, $2 \frac{1}{2}$ miles northeast of Morrow, and $2 \frac{3}{4}$ miles upstream from Todd Fork.

Drainage area. $--680 \mathrm{sq} \mathrm{mi}$.

Gage-height record.--Floodmark. Datum of gage is $643.65 \mathrm{ft}$ above mean sea level, adjustment of 1912 .

Discharge record.--Stage-discharge relation defined by current-meter measurements; prior to 1952 , below $25,700 \mathrm{cfs}$.

Maxima --March 1964: Discharge, $34,500 \mathrm{efs}$ (estimated) Mar. 10 (gage height,

$17.0 \mathrm{ft}$, from floodmark).

1939-52, 1959, 1963: Discharge, 67,000 cfs Jan. 21, 1959 (gage height,

21.9 ft from floodmark). 


\section{(50) 3-2440. Todd Fork near Roachester, Ohio}

Location.--Lat $39^{\circ} 20^{\prime} 0^{\prime \prime}$, long $84^{\circ} 05^{\prime} 10^{\prime \prime}$, on right bank at downstream side of bridge on State Highway $123,0.3 \mathrm{mlle}$ downstream from Lick Run, 1.6 miles southeast of Roachester, Warren County, $2 \frac{3}{4}$ miles southeast of Morrow, and 4 miles upstream from mouth.

Drainage area. $--219 \mathrm{sq} \mathrm{ml}$.

Gage-helght record.--Water-stage recorder graph. Datum of gage is $679.40 \mathrm{ft}$ above mean sea level, datum of 1929.

Discharge record.--Stage-discharge relation defined by current-meter measurements below $12,000 \mathrm{cfs}$, and by indrect measurement at $25,500 \mathrm{cfs}$.

Maxima.--March 1964: D1scharge, $22,500 \mathrm{cfs}$ Mar. 10, 0200 hours (gage height, $19.10 \mathrm{ft}$ ).

1952 to February 1964: Discharge, 25,500 cf's Jan. 21, 1959 (gage he1ght, $19.50 \mathrm{ft})$.

Remarks.--Flow slightly regulated by Cowan Lake on Cowan Creek (capacity, 12,000 acre-ft, $51 \mathrm{sq} \mathrm{ml}$ ).

Mean discharge, in cubic feet per second, March 1964

\begin{tabular}{|c|c|c|c|c|c|c|c|c|c|}
\hline Day & Discharge & Day & Discharge & Day & Discharge & Day & Discharge & Day & Discharge \\
\hline $\begin{array}{l}1 \ldots \ldots \\
2 \ldots \ldots \\
3 \ldots \ldots \\
4 \ldots \ldots \\
5 \ldots \ldots \\
6 \ldots \ldots\end{array}$ & $\begin{array}{r}18 \\
19 \\
22 \\
2,950 \\
6,100 \\
1,120\end{array}$ & $\begin{array}{r}7 \ldots \ldots \\
8 \ldots \ldots \\
9 \ldots \\
10 \ldots \\
11 \ldots \ldots \\
12 \ldots\end{array}$ & $\begin{array}{r}595 \\
846 \\
13,500 \\
14,100 \\
2,040 \\
1,110\end{array}$ & $\begin{array}{l}13 \ldots \ldots \\
14 \ldots \ldots \\
15 \ldots \ldots \\
16 \ldots \ldots \\
17 \ldots \ldots \\
18 \ldots \ldots\end{array}$ & $\begin{array}{r}735 \\
980 \\
1,110 \\
585 \\
378 \\
266\end{array}$ & $\begin{array}{l}19 \ldots \ldots \\
20 \ldots \ldots \\
21 \ldots \ldots \\
22 \ldots \ldots \\
23 \ldots \ldots \\
24 \ldots \ldots\end{array}$ & $\begin{array}{l}204 \\
182 \\
364 \\
365 \\
263 \\
207\end{array}$ & $\mid \begin{array}{l}25 \ldots \ldots \\
26 \ldots \ldots \\
27 \ldots \ldots \\
28 \ldots \ldots \\
29 \ldots \ldots \\
30 \ldots \ldots \\
31 \ldots \ldots\end{array}$ & $\begin{array}{r}173 \\
1,030 \\
469 \\
310 \\
231 \\
180 \\
155\end{array}$ \\
\hline
\end{tabular}

Gage height, in feet, and discharge, in cubic feet per second, at indicated time, 1964

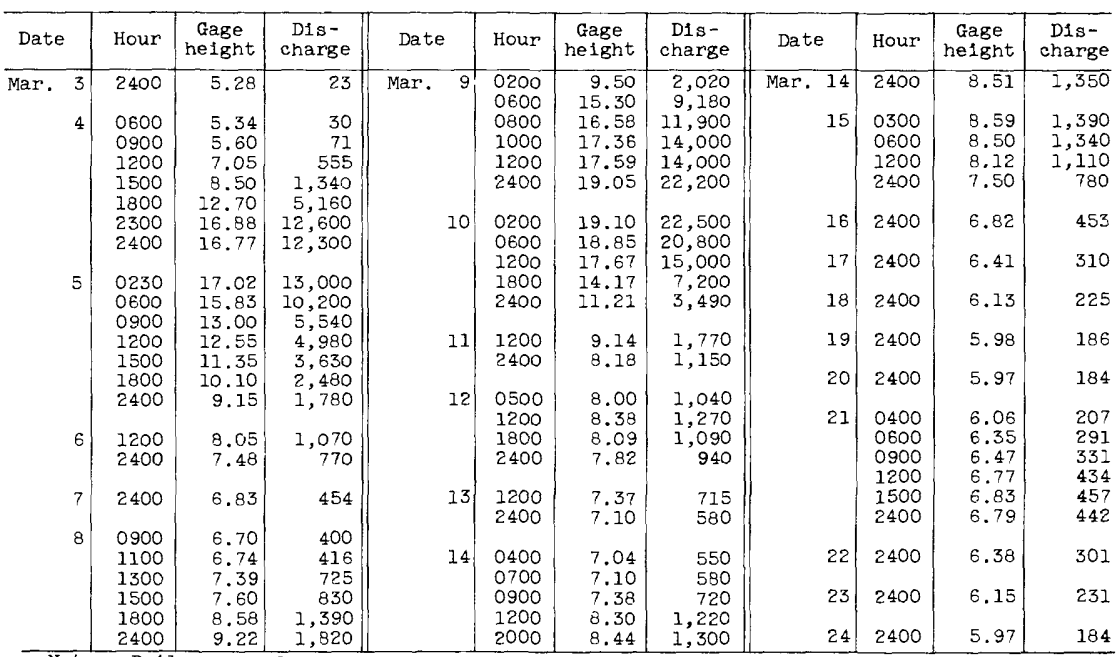

Note.--Da1ly means for some days computed from data in addition to figures shown. 
(51) 3-2455. Littie Miami River at Milford, Ohio

Location.--Lat $39^{\circ} 10^{\prime} 17^{\prime \prime}$, long $84^{\circ} 17^{\prime} 53^{\prime \prime}$, on right bank $500 \mathrm{ft}^{\prime}$ downstream from Wooster Pike Bridge (U.S. Highway 50) in Milford, Clermont County, and $1 \frac{1}{4}$ miles upstream from East Fork.

Drainage area. $--1,203$ sq mi.

Gage-height record.--Water-stage recorder graph. Datum of gage is 499.35 ft above mean sea level, adjustment of 1912 .

Discharge record.--Stage-discharge relation defined by current-meter measurements below $60,000 \mathrm{cfs}$ and by indirect measurement at $84,100 \mathrm{cfs}$.

Maxima.--March 1964: Discharge, 63,900 cfs Mar. 10, 0300 hours (gage height, $19.96 \mathrm{ft}$ )

1915-17, 1925-36, 1938 to February 1964: Discharge, 84,100 cfs Jan. 22, 1959 (gage he1ght, $22.30 \mathrm{ft}$ ).

Flood in March 1913 reached a stage of $25.5 \mathrm{ft}$, from information by Corps of Engineers.

Remarks.--Some regulation by Cowan Lake (capac1ty, 12,000 acre-ft, 51 sq mi).

Mean discharge, in cubic feet per second, March 1964

\begin{tabular}{|c|c|c|c|c|c|c|c|c|c|}
\hline Day & Discharge & Day & D1scharge & Day & Discharge & Day & Discharge & Day & Discharge \\
\hline $\begin{array}{l}1 \ldots \ldots \\
2 \ldots \ldots \\
3 \ldots \ldots \\
4 \ldots \ldots \\
5 \ldots \ldots \\
6 \ldots \ldots\end{array}$ & $\begin{array}{r}155 \\
155 \\
155 \\
5,750 \\
23,600 \\
6,300\end{array}$ & $\begin{array}{r}7 \ldots \ldots \\
8 \ldots \ldots \\
9 \ldots \\
10 \ldots \\
11 \ldots \\
12 \ldots\end{array}$ & $\begin{array}{r}3,400 \\
2,570 \\
31,200 \\
55,400 \\
26,200 \\
10,100\end{array}$ & $\begin{array}{l}13 \ldots \ldots \\
14 \ldots \ldots \\
15 \ldots \ldots \\
16 \ldots \ldots \\
17 \ldots \ldots \\
18 \ldots \ldots\end{array}$ & $\begin{array}{l}4,870 \\
4,430 \\
5,430 \\
3,490 \\
2,480 \\
1,910\end{array}$ & $\begin{array}{l}19 \ldots \ldots \\
20 \ldots \ldots \\
21 \ldots \ldots \\
22 \ldots \ldots \\
23 \ldots \ldots \\
24 \ldots \ldots\end{array}$ & $\begin{array}{l}1,550 \\
1,360 \\
1,710 \\
1,950 \\
1,520 \\
1,290\end{array}$ & $\mid \begin{array}{l}25 \ldots \ldots \\
26 \ldots \ldots \\
27 \ldots \ldots \\
28 \ldots \ldots \\
29 \ldots \ldots \\
30 \ldots \ldots \\
31 \ldots \ldots\end{array}$ & $\begin{array}{r}1,130 \\
2,980 \\
2,300 \\
1,550 \\
1,250 \\
1,070 \\
957\end{array}$ \\
\hline
\end{tabular}

Monthly mean discharge, in cuble feet per second....................

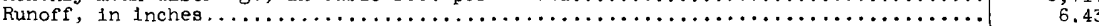

Gage helght, in feet, and discharge, in cubic feet per second, at ind1cated time, 1964

\begin{tabular}{|c|c|c|c|c|c|c|c|c|c|c|c|}
\hline Date & Hour & $\begin{array}{c}\text { Gage } \\
\text { he1ght }\end{array}$ & $\begin{array}{l}\text { D1s- } \\
\text { charge }\end{array}$ & Date & Hour & $\begin{array}{c}\text { Gage } \\
\text { he1ght }\end{array}$ & $\begin{array}{l}\text { Dis- } \\
\text { charge }\end{array}$ & Date & Hour & $\begin{array}{c}\text { Gage } \\
\text { he1 ght }\end{array}$ & $\begin{array}{l}\text { Dis- } \\
\text { charge }\end{array}$ \\
\hline $\operatorname{Mar} .3$ & 2400 & 0.15 & 155 & Mar. 9 & 0900 & 12.15 & 29,200 & Mar. 15 & 1200 & 4.66 & 5,220 \\
\hline 4 & 0600 & .08 & 177 & & 1800 & 16.95 & 47,500 & & & & \\
\hline & $\begin{array}{l}1200 \\
1800\end{array}$ & $\begin{array}{r}.90 \\
5.24\end{array}$ & $\begin{array}{r}600 \\
6,470\end{array}$ & & 2400 & 19.50 & 60,900 & 16 & $\begin{array}{l}1200 \\
2400\end{array}$ & $\begin{array}{l}3.65 \\
3.27\end{array}$ & $\begin{array}{l}3,460 \\
2,900\end{array}$ \\
\hline & 2100 & 9.30 & 18,900 & 10 & 0300 & 19.96 & 63,900 & & & & \\
\hline & 2400 & 12.68 & 31,100 & & $\begin{array}{l}0600 \\
1200\end{array}$ & $\begin{array}{l}19.75 \\
18.78\end{array}$ & $\begin{array}{l}62,100 \\
56,900\end{array}$ & 17 & $\begin{array}{l}1200 \\
2400\end{array}$ & $\begin{array}{l}2.96 \\
2.78\end{array}$ & $\begin{array}{l}2,470 \\
2,260\end{array}$ \\
\hline 5 & 0330 & 14.06 & 36,300 & & 1800 & 17.40 & 50,800 & & & & \\
\hline & $\begin{array}{l}0600 \\
1200\end{array}$ & $\begin{array}{l}13.48 \\
11.18\end{array}$ & $\begin{array}{l}34,100 \\
25,700\end{array}$ & & 2400 & 15.87 & 44,700 & 18 & 2400 & 2.24 & 1,680 \\
\hline & $\begin{array}{l}1800 \\
2400\end{array}$ & $\begin{array}{l}7.55 \\
6.14\end{array}$ & $\begin{array}{r}13,000 \\
8,710\end{array}$ & 11 & $\begin{array}{l}0600 \\
1200\end{array}$ & $\begin{array}{l}13.43 \\
10.90\end{array}$ & $\begin{array}{l}34,900 \\
25,700\end{array}$ & 19 & 2400 & 1.96 & 1,410 \\
\hline & & & & & 1800 & 8.49 & 19,200 & 20 & 2400 & 1.85 & 1,320 \\
\hline 6 & $\begin{array}{l}1200 \\
2400\end{array}$ & $\begin{array}{l}5.11 \\
4.61\end{array}$ & $\begin{array}{l}6,170 \\
5,110\end{array}$ & & 2400 & 7.50 & 14,900 & 21 & 1200 & 2.28 & 1,720 \\
\hline 7 & $\begin{array}{l}1200 \\
2400\end{array}$ & $\begin{array}{l}3.49 \\
2.89\end{array}$ & $\begin{array}{l}3,190 \\
2,390\end{array}$ & 12 & $\begin{array}{l}1200 \\
2400\end{array}$ & $\begin{array}{l}6.17 \\
5.25\end{array}$ & $\begin{array}{l}9,800 \\
6,750\end{array}$ & 22 & 2400 & $? 28$ & 2,160 \\
\hline 8 & $\begin{array}{l}1200 \\
1800 \\
2400\end{array}$ & $\begin{array}{l}2.62 \\
3.16 \\
4.40\end{array}$ & $\begin{array}{l}2,070 \\
2,730 \\
4,700\end{array}$ & 13 & $\begin{array}{l}1200 \\
2400\end{array}$ & $\begin{array}{l}4.40 \\
3.97\end{array}$ & $\begin{array}{l}4,740 \\
3,970\end{array}$ & 23 & 2400 & 1.92 & 1,380 \\
\hline & & & & 14 & 0800 & 3.78 & 3,670 & 24 & 2400 & 1.72 & 1,210 \\
\hline 9 & $\begin{array}{l}0300 \\
0600\end{array}$ & $\begin{array}{l}4.80 \\
8.02\end{array}$ & $\begin{array}{r}5,490 \\
14,600\end{array}$ & & $\begin{array}{l}1600 \\
2400\end{array}$ & $\begin{array}{l}4.40 \\
5.02\end{array}$ & $\begin{array}{l}4,740 \\
6,080\end{array}$ & 25 & 2400 & 1.63 & 1,130 \\
\hline
\end{tabular}

Note.--Dally means for some days computed from data in addition to figures shown. 
(52) 3-2465. East Fork Little Miaml River at W1lliamsburg, Ohio

Location.--Lat $39^{\circ} 03^{\prime} 10^{\prime \prime}$, long $84^{\circ} 03^{\prime} 05^{\prime \prime}$, on right bank at downstream side of Main

Street Bridge in Williamsburg, Clermont County, 1.1 miles upstream from Todd Run.

Drainage area. $-237 \mathrm{sq} \mathrm{mi}$.

Gage-height record. -Water-stage recorder graph. Datum of gage is $784.09 \mathrm{ft}$ above mean sea level, datum of 1929, supplementary adjustment of 1944 .

Discharge record.--Stage-discharge relation defined by current-meter measurements below $14,000 \mathrm{cfs}$ and by indirect measurement at 19,800 cfs.

Maxima.--Given in the following table.

March 1964 :

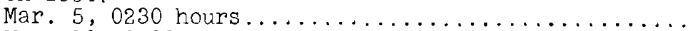

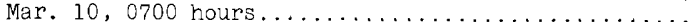

$1949-53,1959$ to February 1964 :

$\begin{array}{cc}\begin{array}{c}\text { Discharge } \\ \text { (cfs) }\end{array} & \text { Gage height } \\ 13,700 & \text { feet) } \\ 19,800 & 12.18 \\ 18,000 & 15.23 \\ & 14.34\end{array}$

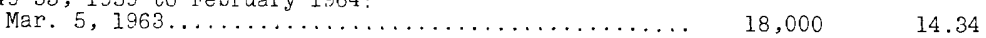

Mean discharge, In cubic feet per second, March 1964

\begin{tabular}{|c|c|c|c|c|c|c|c|c|c|}
\hline Day & Discharge & Day & Discharge & Day & D1scharge & Day & D1scharge & Day & D1scharge \\
\hline $\begin{array}{l}1 \ldots \\
2 \ldots \\
3 \ldots \\
4 \ldots \\
5 \ldots \\
6 \ldots \\
\ldots\end{array}$ & $\begin{array}{r}17 \\
18 \\
38 \\
3,030 \\
12,600 \\
2,360\end{array}$ & $\begin{array}{r}7 \ldots \\
8 \ldots \\
9 \ldots \\
10 \ldots \\
11 \ldots \\
12 \ldots\end{array}$ & $\begin{array}{r}345 \\
410 \\
9,490 \\
18,200 \\
4,640 \\
561\end{array}$ & $\begin{array}{l}13 \ldots \ldots \\
14 \ldots \ldots \\
15 \ldots \ldots \\
16 \ldots \ldots \\
17 \ldots \ldots \\
18 \ldots \ldots\end{array}$ & $\begin{array}{r}400 \\
923 \\
1,440 \\
420 \\
216 \\
150\end{array}$ & $\begin{array}{l}19 \ldots \ldots \\
20 \ldots \ldots \\
21 \ldots \ldots \\
22 \ldots \ldots \\
23 \ldots \ldots \\
24 \ldots \ldots\end{array}$ & $\begin{array}{l}111 \\
101 \\
297 \\
395 \\
206 \\
141\end{array}$ & $\begin{array}{l}25 \ldots \ldots \\
26 \ldots \ldots \\
27 \ldots \ldots \\
28 \ldots \ldots \\
29 \ldots \ldots \\
30 \ldots \ldots \\
31 \ldots \ldots\end{array}$ & $\begin{array}{r}119 \\
1,180 \\
454 \\
195 \\
141 \\
111 \\
91\end{array}$ \\
\hline $\begin{array}{l}\text { onthly } \\
\text { unoff, }\end{array}$ & $\begin{array}{l}\text { mean d } \\
\text { In incl }\end{array}$ & 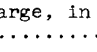 & & & & & & & $\begin{array}{r}1,897 \\
9.22\end{array}$ \\
\hline
\end{tabular}

Gage he1ght, in feet, and discharge, in cub1c feet per second, at 1ndicated time, 1964

\begin{tabular}{|c|c|c|c|c|c|c|c|c|c|c|c|}
\hline Date & Hour & $\begin{array}{c}\text { Gage } \\
\text { helght }\end{array}$ & $\begin{array}{c}\text { D1s- } \\
\text { charge }\end{array}$ & Date & Hour & $\begin{array}{c}\text { Gage } \\
\text { height }\end{array}$ & $\begin{array}{c}\text { D1s- } \\
\text { charge }\end{array}$ & Date & Hour & $\begin{array}{c}\text { Gage } \\
\text { helght }\end{array}$ & $\begin{array}{c}\text { Dis- } \\
\text { charge }\end{array}$ \\
\hline Mar. I & 2400 & 1.21 & 17 & Mar. & 1800 & 2.80 & 505 & Mar. 14 & 1000 & 2.74 & 492 \\
\hline & & & & & 2400 & 3.40 & 910 & & 1400 & 3.65 & 1,110 \\
\hline 2 & 1800 & 1.20 & 1.7 & & & & & & 2400 & 4.67 & 2,020 \\
\hline & 2400 & 1.32 & 34 & 9 & $\begin{array}{l}0300 \\
0600\end{array}$ & $\begin{array}{l}4.00 \\
7.10\end{array}$ & 1,420 & 75 & 0200 & & 3720 \\
\hline 3 & $\begin{array}{l}1200 \\
2400\end{array}$ & $\begin{array}{l}1.35 \\
1.65\end{array}$ & $\begin{array}{l}27 \\
74\end{array}$ & & $\begin{array}{l}0900 \\
1200 \\
1800\end{array}$ & $\begin{array}{r}9.60 \\
11.10 \\
12.25\end{array}$ & $\begin{array}{r}7,820 \\
11,000 \\
13,800\end{array}$ & & $\begin{array}{l}1200 \\
2400\end{array}$ & $\begin{array}{l}4.78 \\
4.05 \\
3.10\end{array}$ & $\begin{array}{r}1,460 \\
705\end{array}$ \\
\hline 4 & $\begin{array}{l}0600 \\
0900\end{array}$ & $\begin{array}{l}1.73 \\
1.97\end{array}$ & $\begin{array}{r}91 \\
155\end{array}$ & & 2400 & 14.18 & 17,700 & 16 & $\begin{array}{l}1200 \\
2400\end{array}$ & $\begin{array}{l}2.58 \\
2.30\end{array}$ & $\begin{array}{l}405 \\
270\end{array}$ \\
\hline & 1200 & 2.43 & 330 & 10 & 0300 & 14.93 & 19,200 & & & & \\
\hline & & $\begin{array}{l}4.18 \\
8.00\end{array}$ & $\begin{array}{l}1,580 \\
5,580\end{array}$ & & $\begin{array}{l}0700 \\
1200\end{array}$ & $\begin{array}{l}15.23 \\
14.82\end{array}$ & $\begin{array}{l}19,800 \\
18,900\end{array}$ & 17 & 2400 & 2.03 & 173 \\
\hline & $\begin{array}{l}2100 \\
2400\end{array}$ & $\begin{array}{l}10.68 \\
11.91\end{array}$ & $\begin{array}{r}9,950 \\
13,000\end{array}$ & & $\begin{array}{l}1800 \\
2400\end{array}$ & $\begin{array}{l}14.13 \\
12.95\end{array}$ & $\begin{array}{l}17,600 \\
15,200\end{array}$ & 18 & 2400 & 1.87 & 127 \\
\hline & & & & & & & & 19 & 2400 & 1.76 & 98 \\
\hline 5 & $\begin{array}{l}0230 \\
1200 \\
1800\end{array}$ & $\begin{array}{l}12.18 \\
11.58 \\
11.58\end{array}$ & $\begin{array}{l}13,700 \\
12,200 \\
12,200\end{array}$ & 11 & $\begin{array}{l}0300 \\
0600 \\
0900\end{array}$ & $\begin{array}{r}11.95 \\
10.00 \\
5.75\end{array}$ & $\begin{array}{r}13,100 \\
8,520 \\
3,050\end{array}$ & 20 & 2400 & 1.82 & 113 \\
\hline & 2400 & 11.02 & 10,800 & & 1200 & 4.07 & 1,480 & 21 & 0600 & 2.09 & 192 \\
\hline 6 & 0300 & 9.00 & 6,900 & & 2400 & $\begin{array}{l}3.44 \\
3.12\end{array}$ & $\begin{array}{l}942 \\
718\end{array}$ & & $\begin{array}{l}1200 \\
2400\end{array}$ & $\begin{array}{l}2.47 \\
2.64\end{array}$ & $\begin{array}{l}350 \\
437\end{array}$ \\
\hline & $\begin{array}{l}0600 \\
1200 \\
2400\end{array}$ & $\begin{array}{l}4.70 \\
3.33 \\
2.72\end{array}$ & $\begin{array}{r}2,050 \\
861 \\
481\end{array}$ & 12 & $\begin{array}{l}1200 \\
2400\end{array}$ & $\begin{array}{l}2.82 \\
2.76\end{array}$ & $\begin{array}{l}537 \\
503\end{array}$ & 22 & $\begin{array}{l}0600 \\
2400\end{array}$ & $\begin{array}{l}2.71 \\
2.32\end{array}$ & $\begin{array}{l}476 \\
279\end{array}$ \\
\hline 7 & 2400 & 2.27 & 258 & 13 & $\begin{array}{l}1200 \\
2400\end{array}$ & $\begin{array}{l}2.57 \\
2.34\end{array}$ & $\begin{array}{l}400 \\
288\end{array}$ & 23 & 2400 & 1.99 & 161 \\
\hline 8 & 0900 & 2.18 & 223 & 14 & 0530 & 2.32 & 279 & 24 & 2400 & 1.86 & 124 \\
\hline
\end{tabular}

Note.--Daliy means for some days computed from data in addition to f1gures shown. 
(53) 3-2475. East Fork Little Miami River at Perintown, Ohio

Location.--Lat $39^{\circ} 08^{\prime} 13^{\prime \prime}$, long $84^{\circ} 14^{\prime} 17^{\prime \prime}$, on left bank at downstream side of highway bridge at Perintown, Clermont County, 5 miles upstream from mouth.

Drainage area. $--476 \mathrm{sq} \mathrm{ml}$.

Gage-height record.--Water-stage recorder graph. Datum of gage is $507.28 \mathrm{ft}$ above mean sea level, adjustment of 1912 .

Discharge record.--Stage-discharge relation defined by current-meter measurements.

Maxima.--Given in the following table.

March 1964:

Mar. 5, 0030 hours.

$\begin{array}{cc}\begin{array}{c}\text { Discharge } \\ \text { (cfs) }\end{array} & \begin{array}{c}\text { Gage height } \\ \text { (feet) }\end{array} \\ 38,200 & 23.00 \\ 42,400 & 23.84 \\ 39,400 & 23.42\end{array}$

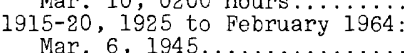

Mean discharge, In cubic feet per second, March 1964

\begin{tabular}{|c|c|c|c|c|c|c|c|c|c|}
\hline Day & Discharge & Day & Discharge & Day & Discharge & Day & Discharge & Day & Discharge \\
\hline $\begin{array}{l}1 \ldots \ldots \\
2 \ldots \\
3 \ldots \\
4 \ldots \\
5 \ldots \\
6 \ldots\end{array}$ & $\begin{array}{r}41 \\
42 \\
72 \\
9,910 \\
22,300 \\
5,220\end{array}$ & $\begin{array}{r}7 \ldots \ldots \\
8 \ldots \ldots \\
9 \ldots \\
10 \ldots \\
11 \ldots \ldots \\
12 \ldots \ldots\end{array}$ & $\begin{array}{r}800 \\
890 \\
21,700 \\
33,000 \\
10,200 \\
1,290\end{array}$ & $\begin{array}{l}13 \ldots \ldots \\
14 \ldots \ldots \\
15 \ldots \ldots \\
16 \ldots \ldots \\
17 \ldots \ldots \\
18 \ldots \ldots\end{array}$ & $\begin{array}{r}945 \\
1,810 \\
2,930 \\
1,160 \\
622 \\
422\end{array}$ & $\begin{array}{l}19 \ldots \ldots \\
20 \ldots \\
21 \ldots \ldots \\
22 \ldots \ldots \\
23 \ldots \ldots \\
24 \ldots \ldots\end{array}$ & $\begin{array}{r}328 \\
307 \\
1,160 \\
1,090 \\
645 \\
419\end{array}$ & $\begin{array}{l}25 \ldots \ldots \\
26 \ldots \ldots \\
27 \ldots \ldots \\
28 \ldots \ldots \\
29 \ldots \ldots \\
30 \ldots \ldots \\
31 \ldots \ldots\end{array}$ & $\begin{array}{r}335 \\
1,790 \\
1,160 \\
548 \\
374 \\
291 \\
240\end{array}$ \\
\hline $\begin{array}{l}\text { onthly } \\
\text { unoff, }\end{array}$ & $\begin{array}{l}\text { mean dis } \\
\text { in inche }\end{array}$ & ge, & abic $f$ & per seco & - & & & & $\begin{array}{r}3,937 \\
9.53\end{array}$ \\
\hline
\end{tabular}

Gage height, in feet, and discharge, in cubic feet per second, at 1ndicated time, 1964

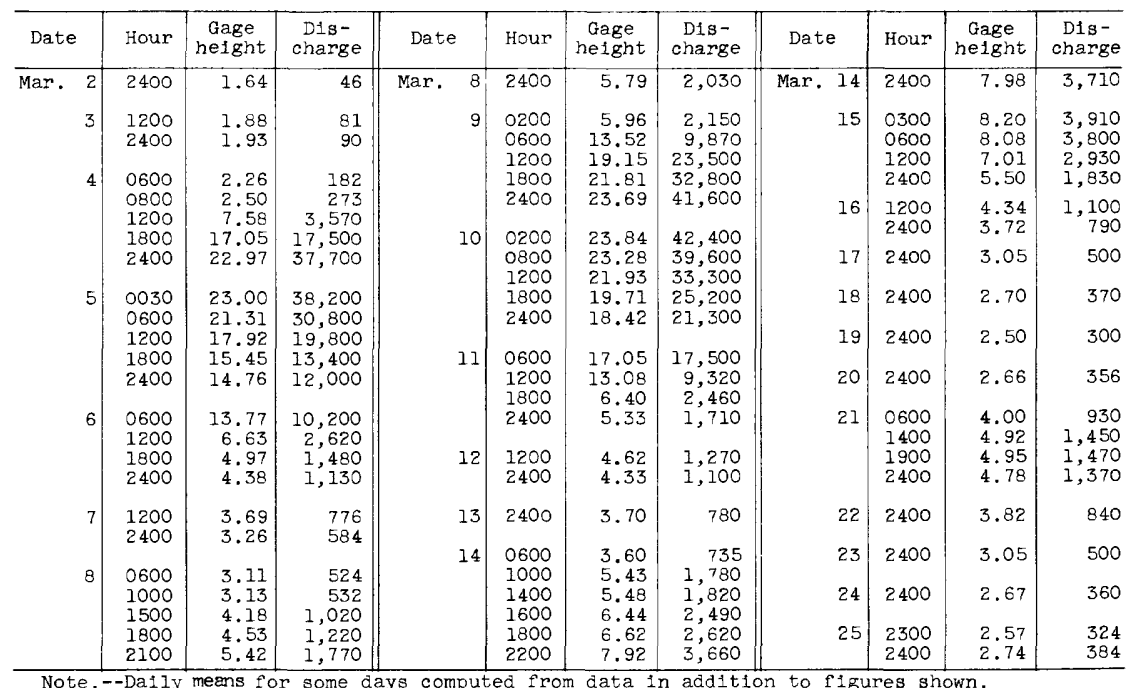

Note.--Da1ly means for some days computed from data in addition to figures shown. 


\section{LICKING RIVER BASIN}

(54) 3-2495. Licking River at Farmers, Ky.

Location. - Lat $38^{\circ} 08^{\prime} 25^{\prime \prime}$, long $83^{\circ} 33130^{\prime \prime}$, near right bank on downstream side of bridge on U.S. Highway $60,300 \mathrm{ft}$ upstream from Che sapeake and Oh1o Railway bridge, three-quarters of a mile west of Farmers, Rowan County, and 1.1 miles upstream from Triplett Creek.

Drainage area. $--831 \mathrm{sq} \mathrm{mi}$.

Gage-helght record. - Water-stage recorder graph. Graph drawn for auxillary staff gage 4.1 miles upstream, from twice-dally readings. Datum of gage is $646.55 \mathrm{ft}$ above mean sea level, datum of 1929.

Discharge record. - Stage-fall-discharge relation or stage-discharge relation defined by current-meter measurements. Fall used as a factor Mar. 4-18, 21, 22. Maxima.--Given in the following table.

March 1964:

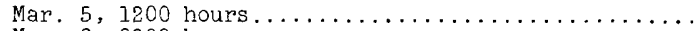

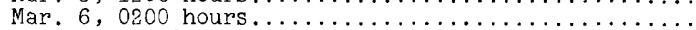

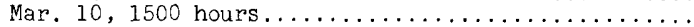

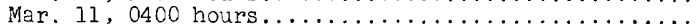

1938 to February 1964:

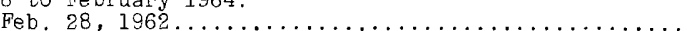

$\begin{array}{cc}\begin{array}{c}\text { Discharge } \\ \text { (cfs) }\end{array} & \begin{array}{c}\text { Gage height } \\ \text { feet) } \\ -\end{array} \\ 11,800 & - \\ - & 23.47 \\ 12,200 & - \\ 24,000 & 26.70\end{array}$

Maximum stage known since at least 1904 was $31.1 \mathrm{ft}$ on Feb. 9, 1918 (1ce Jam), from reports of U.S. Weather Bureau.

Mean discharge, in cublc feet per second, March 1964

\begin{tabular}{|c|c|c|c|c|c|c|c|c|c|}
\hline Day & Discharge & Day & Discharge & Day & Discharge & Day & DIscharge & Day & D1scharge \\
\hline $\begin{array}{l}1 \ldots \ldots \\
2 \ldots \ldots \\
3 \ldots \ldots \\
4 \ldots \\
5 \ldots \\
6 \ldots\end{array}$ & $\begin{array}{r}518 \\
675 \\
3,070 \\
5,580 \\
8,990 \\
10,300\end{array}$ & $\begin{array}{r}7 \ldots \ldots \\
8 \ldots \ldots \\
9 \ldots \ldots \\
10 \ldots \\
11 \ldots \ldots \\
12 \ldots \ldots\end{array}$ & $\begin{array}{r}5,380 \\
4,800 \\
9,100 \\
11,300 \\
11,600 \\
9,410\end{array}$ & $\begin{array}{l}13 \ldots \ldots \\
14 \ldots \ldots \\
15 \ldots \ldots \\
16 \ldots \ldots \\
17 \ldots \ldots \\
18 \ldots \ldots\end{array}$ & $\begin{array}{l}5,260 \\
2,610 \\
2,820 \\
4,060 \\
3,750 \\
2,780\end{array}$ & $\begin{array}{l}19 \ldots \ldots \\
20 \ldots \ldots \\
21 \ldots \ldots \\
22 \ldots \ldots \\
23 \ldots \ldots \\
24 \ldots \ldots\end{array}$ & $\begin{array}{l}1,860 \\
1,480 \\
1,750 \\
2,510 \\
2,500 \\
2,020\end{array}$ & $\begin{array}{l}25 \ldots \ldots \\
26 \ldots \ldots \\
27 \ldots \ldots \\
28 \ldots \ldots \\
29 \ldots \ldots \\
30 \ldots \ldots \\
31 \ldots \ldots\end{array}$ & $\begin{array}{r}1,640 \\
1,420 \\
1,230 \\
1,040 \\
885 \\
785 \\
710\end{array}$ \\
\hline thly & $\begin{array}{l}\text { mean } \mathrm{d} \\
\text { In inc. }\end{array}$ & & & & & & & & $\begin{array}{r}3,930 \\
5.45\end{array}$ \\
\hline
\end{tabular}

(55) 3-2505. Licking River at Blue Lick Springs, Ky .

(Gaging station discontinued 1959; crest-stage station)

Location.--Lat $38^{\circ} 25^{\prime} 19^{\prime \prime}$, long $83^{\circ} 59^{\prime} 57^{\prime \prime}$, near center of span on downstream side of bridge on U.S. Highway 68 , at Blue Lick Springs, Nicholas County, I miles upstream from Indian Run, 10 miles upstream from Johnson Creek, and 10 miles downstream from Fleming Creek.

Drainage area. $--1,785 \mathrm{sq} \mathrm{mi}$.

Gage-helght record.--Graph drawn on basis of floodmark and twice-dally wire-weightgage readings. Datum of gage is $560.99 \mathrm{ft}$ above mean sea level, datum of 1929 .

Discharge record.--Stage-discharge relation defined by current-meter measurements. Maxima.--Given in the following table.

March 1964 :

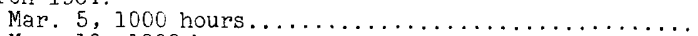

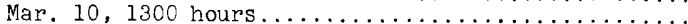

1938 to February 1964:

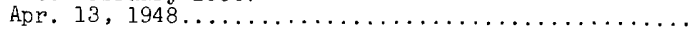

$\begin{array}{cc}\begin{array}{c}\text { Discharge } \\ \text { (cfs) }\end{array} & \begin{array}{c}\text { Gage height } \\ \text { (feet) }\end{array} \\ 30,700 & 39.9 \\ 30,500 & 41.2 \\ 35,900 & 45.0\end{array}$

Flood of 1937 reached a stage of $47.4 \mathrm{ft}$, exceeding all known floods since at least 1854 
Mean discharge, In cublc feet per second, March 1964, of LIcking River at Blue Lick Springs, Ky.

\begin{tabular}{|c|c|c|c|c|c|c|c|c|c|}
\hline Day & Discharge & Day & Discharge & Day & Discharge & Day & D1scharge & Day & Discharge \\
\hline $\begin{array}{l}1 \ldots \ldots \\
2 \ldots \ldots \\
3 \ldots \ldots \\
4 \ldots \ldots \\
5 \ldots \ldots \\
6 \ldots \ldots\end{array}$ & $\begin{array}{r}880 \\
910 \\
2,280 \\
11,800 \\
27,300 \\
23,500\end{array}$ & $\begin{array}{r}7 \ldots \ldots \\
8 \ldots \ldots \\
9 \ldots \ldots \\
10 \ldots \ldots \\
11 \ldots \ldots \\
12 \ldots \ldots\end{array}$ & $\begin{array}{l}21,000 \\
19,700 \\
23,200 \\
29,300 \\
26,600 \\
23,200\end{array}$ & $\mid \begin{array}{l}13 \ldots \ldots \\
14 \ldots \ldots \\
15 \ldots \ldots \\
16 \ldots \ldots \\
17 \ldots \ldots \\
18 \ldots \ldots\end{array}$ & $\begin{array}{r}20,000 \\
16,400 \\
13,300 \\
8,370 \\
6,670 \\
5,530\end{array}$ & $\begin{array}{l}19 \ldots \ldots \\
20 \ldots \ldots \\
21 \ldots \ldots \\
22 \ldots \ldots \\
23 \ldots \ldots \\
24 \ldots \ldots\end{array}$ & $\begin{array}{l}4,240 \\
3,900 \\
3,480 \\
3,090 \\
3,160 \\
4,240\end{array}$ & $\mid \begin{array}{l}25 \ldots \ldots \\
26 \ldots \ldots \\
27 \ldots \ldots \\
28 \ldots \ldots \\
29 \ldots \ldots \\
30 \ldots \ldots \\
31 \ldots \ldots\end{array}$ & $\begin{array}{l}3,620 \\
3,400 \\
3,120 \\
2,280 \\
1,640 \\
1,420 \\
1,280\end{array}$ \\
\hline & an d & & & & & & & & $\begin{array}{r}10,280 \\
6.64\end{array}$ \\
\hline
\end{tabular}

Gage height, in feet, and discharge, in cubic feet per second, at Indicated t1me, 1964

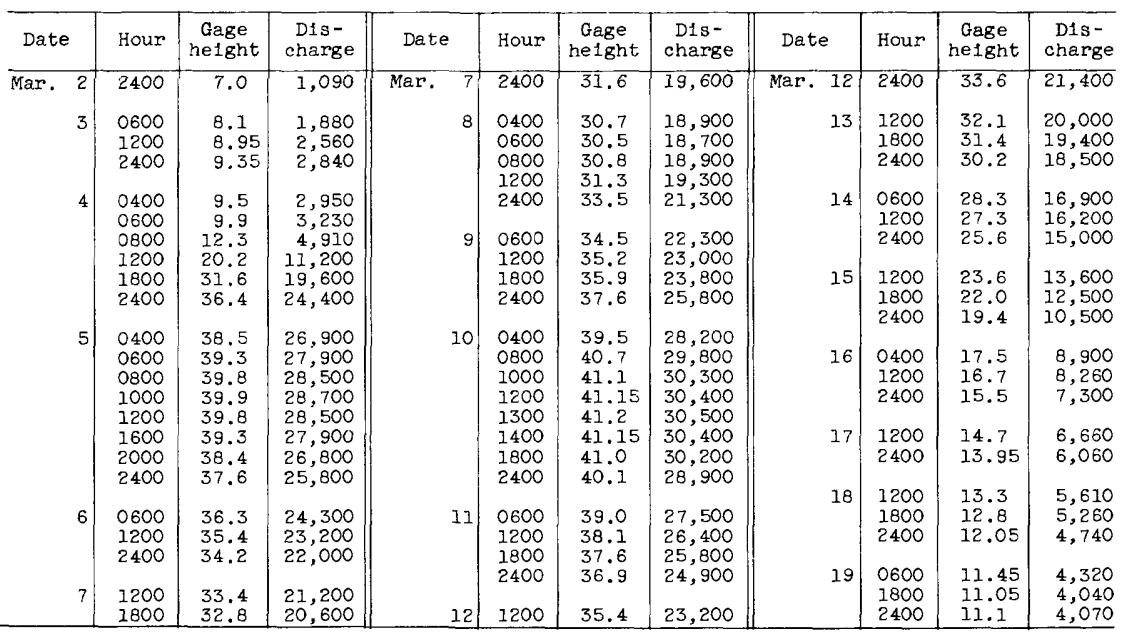

(56) 3-2510. North Fork Licking River near Lewisburg, Ky.

Location.--Lat $38^{\circ} 25^{\prime} 5^{\prime \prime}$, long $83^{\circ} 47^{\prime} 30^{\prime \prime}$, on left bank at downstream side of bridge on State Hlghway $419,1 \frac{1}{4}$ miles west of Lewisburg, Mason County, and $1 \frac{1}{2}$ miles downstream from Mill creek.

Drainage area. $--119 \mathrm{sq} \mathrm{ml}$.

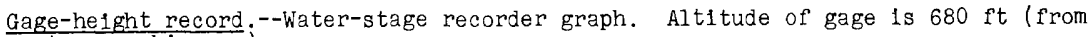
topographic map).

Discharge record.--Stage-discharge relation defined by current-meter measurements. Maxima.--Given in the following table.

\section{March 1964:}

Mar. 4, 2330 hours.

Nar 10, 0200 hours.

1938,1946 to February 1964 :
July $1938 . \ldots \ldots \ldots \ldots \ldots$

$\ldots \ldots$

$\begin{array}{cc}\begin{array}{c}\text { Discharge } \\ \text { (cfs) }\end{array} & \text { Gage height } \\ 9,850 & 19.29 \\ 7,620 & 16.83\end{array}$

Mean discharge, In cub1c feet per second, March 1964

\begin{tabular}{|c|c|c|c|c|c|c|c|c|c|}
\hline Day & Discharge & Day & Discharge & Day & Discharge & Day & Discharge & Day & Discharge \\
\hline $\begin{array}{l}1 \ldots \ldots \\
2 \ldots \ldots \\
3 \ldots \ldots \\
4 \ldots \ldots \\
5 \ldots \ldots \\
6 \ldots\end{array}$ & $\begin{array}{r}34 \\
42 \\
389 \\
4,260 \\
6,060 \\
820\end{array}$ & $\begin{array}{r}7 \ldots \ldots \\
8 \ldots \ldots \\
9 \ldots \ldots \\
10 \ldots . \\
11 \ldots . . \\
12 \ldots\end{array}$ & $\begin{array}{r}270 \\
1,490 \\
3,670 \\
4,420 \\
800 \\
310\end{array}$ & $\begin{array}{l}13 \ldots \ldots \\
14 \ldots \ldots \\
15 \ldots \ldots \\
16 \ldots \ldots \\
17 \ldots \ldots \\
18 \ldots \ldots\end{array}$ & $\begin{array}{r}203 \\
404 \\
1,280 \\
416 \\
246 \\
164\end{array}$ & $\begin{array}{l}19 \ldots \ldots \\
20 \ldots \ldots \\
21 \ldots \ldots \\
22 \ldots \ldots \\
23 \ldots \ldots \\
24 \ldots \ldots\end{array}$ & $\begin{array}{l}125 \\
123 \\
388 \\
313 \\
218 \\
162\end{array}$ & $\mid \begin{array}{l}25 \ldots \\
26 \ldots \\
27 \ldots \\
28 \ldots \\
29 \ldots \\
30 \ldots \\
31 \ldots \ldots\end{array}$ & $\begin{array}{r}151 \\
567 \\
243 \\
166 \\
130 \\
103 \\
87\end{array}$ \\
\hline$t$ & $\mathrm{an}$ & & & & & & & & $\begin{array}{r}905 \\
8.77\end{array}$ \\
\hline
\end{tabular}


Gage helght, in feet, and discharge, in cublc feet per second, at indicated time, 1964, of

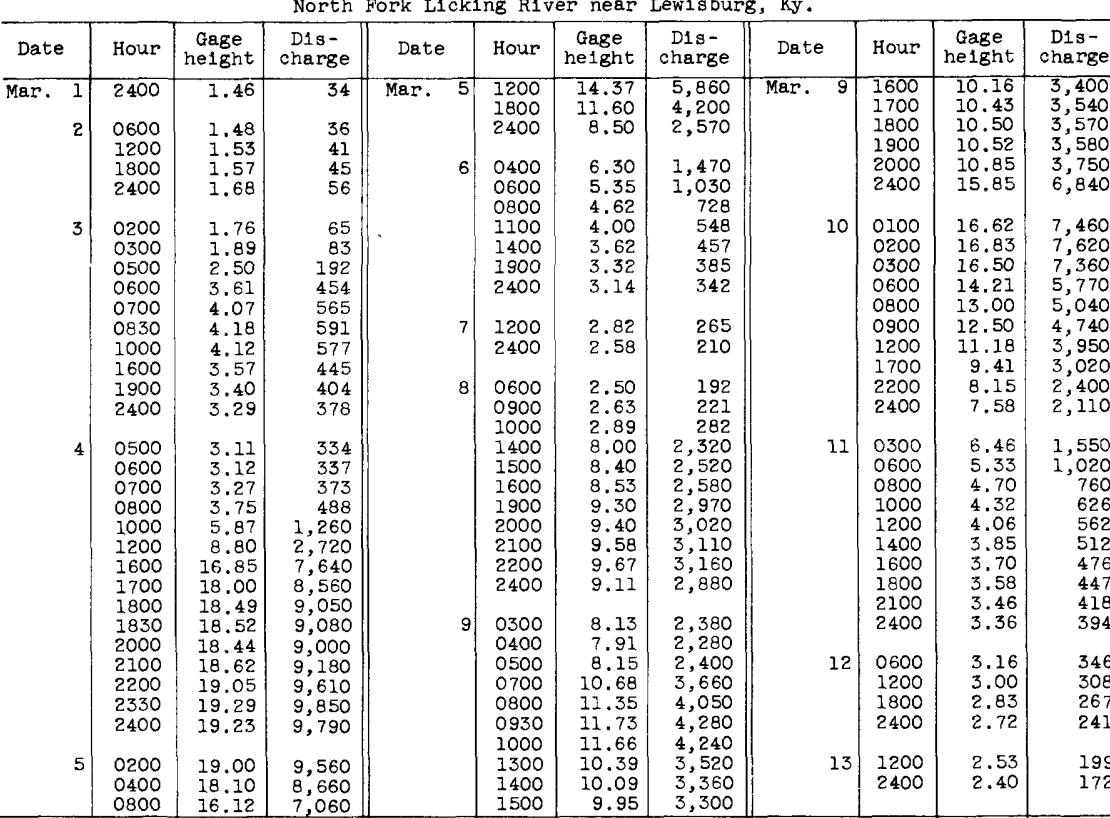

(57) 3-2515. Licking River at McKinneysburg, Ky.

Location.--Lat $38^{\circ} 35^{\prime} 52^{\prime \prime}$, long $84^{\circ} 16^{\prime} 00^{\prime \prime}$, on right bank at downstream side of highway bridge at McKinneysburg, Pendleton County, 6.5 miles southeast of Falmouth, 9.0 miles upstream from Blanket Creek, and 12.8 miles upstream from South Fork.

Drainage area. $--2,326 \mathrm{sq} \mathrm{mi}$.

Gage-helght record. --Water-stage recorder graph. Datum of gage is $520.83 \mathrm{ft}$ above mean sea level, datum of 1929 .

Discharge record.--Stage-discharge relation defined by current-meter measurements below 42,000 cis.

Maxima.--Given in the following table.

March 1964:

Mar. 5,2200 hours . . . . . . . . . . . . . . .

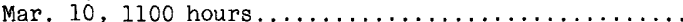

1937 to February 1964:

January $1937 \ldots \ldots \ldots$. .

$\begin{array}{cc}\text { Discharge } & \text { Gage he1ght } \\ \text { (cfs) } & \text { (feet) } \\ 43,000 & 42.14 \\ 59,100 & 50.26 \\ 55,000 & 47.8\end{array}$

Mean d1scharge, in cub1c feet per second, March 1964

\begin{tabular}{|c|c|c|c|c|c|c|c|c|c|}
\hline Day & D1scharge & Day & Discharge & Day & D1 scharge & Day & D1scharge & Day & D1scharge \\
\hline $\begin{array}{l}1 \ldots \ldots \\
2 \ldots \ldots \\
3 \ldots \ldots \\
4 \ldots \ldots \\
5 \ldots \ldots \\
6 \ldots\end{array}$ & $\begin{array}{r}1,100 \\
1,110 \\
3,220 \\
17,600 \\
41,300 \\
40,800\end{array}$ & $\begin{array}{r}7 \ldots \ldots \\
8 \ldots \ldots \\
9 \ldots \ldots \\
10 \ldots \ldots \\
11 \ldots \ldots \\
12 \ldots \ldots\end{array}$ & $\begin{array}{l}32,900 \\
25,200 \\
38,000 \\
57,400 \\
49,100 \\
35,300\end{array}$ & $\mid \begin{array}{c}13 \ldots \ldots \\
14 \ldots \ldots \\
15 \ldots \ldots \\
16 \ldots \ldots \\
17 \ldots \ldots \\
18 \ldots \ldots\end{array}$ & $\begin{array}{r}24,700 \\
19,800 \\
18,600 \\
12,700 \\
9,040 \\
6,910\end{array}$ & $\begin{array}{l}19 \ldots \ldots \\
20 \ldots \ldots \\
21 \ldots \ldots \\
22 \ldots \ldots \\
23 \ldots \ldots \\
24 \ldots \ldots\end{array}$ & $\begin{array}{l}5,570 \\
4,200 \\
6,080 \\
5,710 \\
6,430 \\
5,510\end{array}$ & $\mid \begin{array}{l}25 \ldots \\
26 \ldots \\
27 \ldots \ldots \\
28 \ldots \\
29 \ldots \\
30 \ldots \\
31 \ldots \ldots \\
.\end{array}$ & $\begin{array}{l}4,470 \\
7,600 \\
4,230 \\
3,140 \\
2,410 \\
1,990 \\
1,700\end{array}$ \\
\hline thly & & & & & & & & & $\begin{array}{r}15,930 \\
7.90\end{array}$ \\
\hline
\end{tabular}


Gage height, in feet, and discharge, in cublc feet per second, at indicated t1me, 1964, of L1ck1ng River at McKinneysburg, Ky.

\begin{tabular}{|c|c|c|c|c|c|c|c|c|c|}
\hline Day & Discharge & Day & Discharge & Day & Discharge & Day & Discharge & Day & Discharge \\
\hline $\begin{array}{l}1 \ldots \ldots \\
2 \ldots \\
3 \ldots \\
4 \ldots \\
5 \ldots \\
6 \ldots\end{array}$ & $\begin{array}{r}1,100 \\
1,110 \\
3,220 \\
17,600 \\
41,300 \\
40,800\end{array}$ & $\begin{array}{r}7 \ldots \ldots \\
8 \ldots \\
9 \ldots \\
10 \ldots \\
11 \ldots \\
12 \ldots\end{array}$ & $\begin{array}{l}32,900 \\
25,200 \\
38,000 \\
57,400 \\
49,100 \\
35,300\end{array}$ & $\mid \begin{array}{l}13 \ldots \ldots \\
14 \ldots \ldots \\
15 \ldots \ldots \\
16 \ldots \ldots \\
17 \ldots \ldots \\
18 \ldots \ldots\end{array}$ & $\begin{array}{r}24,700 \\
19,800 \\
18,600 \\
12,700 \\
9,040 \\
6,910\end{array}$ & $\begin{array}{l}19 \ldots \ldots \\
20 \ldots \ldots \\
21 \ldots \ldots \\
22 \ldots \ldots \\
23 \ldots \ldots \\
24 \ldots \ldots\end{array}$ & $\begin{array}{l}5,570 \\
4,200 \\
6,080 \\
5,710 \\
6,430 \\
5,510\end{array}$ & $\mid \begin{array}{l}25 \ldots \ldots \\
26 \ldots \ldots \\
27 \ldots \ldots \\
28 \ldots \ldots \\
29 \ldots \ldots \\
30 \ldots \ldots \\
31 \ldots \ldots\end{array}$ & $\begin{array}{l}4,47 \\
7,60 \\
4,23 \\
3,14 \\
2,41 \\
1,990 \\
1,700\end{array}$ \\
\hline
\end{tabular}

Gage helght, in feet, and discharge, in cublo feet per second, at indicated time, 1964

\begin{tabular}{|c|c|c|c|c|c|c|c|c|c|c|c|c|}
\hline Date & \multirow{3}{*}{$\begin{array}{l}\text { Hour } \\
2400\end{array}$} & \multirow{3}{*}{$\frac{\begin{array}{c}\text { Gage } \\
\text { he } 1 \text { ght }\end{array}}{6.57}$} & \multirow{3}{*}{$\begin{array}{l}\begin{array}{c}\text { Dis- } \\
\text { charge }\end{array} \\
\stackrel{1,070}{ }\end{array}$} & \multicolumn{2}{|l|}{ Date } & \multirow{3}{*}{$\begin{array}{l}\text { Hour } \\
1200 \\
2400\end{array}$} & \multirow{3}{*}{$\begin{array}{c}\begin{array}{c}\text { Gage } \\
\text { he1ght }\end{array} \\
35.15 \\
30.52\end{array}$} & \multirow{3}{*}{$\begin{array}{c}\begin{array}{c}\text { Dis - } \\
\text { charge }\end{array} \\
33,200 \\
27,200\end{array}$} & Date & Hour & $\begin{array}{c}\text { Gage } \\
\text { he1ght }\end{array}$ & $\begin{array}{c}\text { Dis- } \\
\text { charge }\end{array}$ \\
\hline Mar. & & & & Mar. & 7 & & & & Mar. 1 & 0600 & 29.87 & 26,300 \\
\hline & & & & & & & & & & 1200 & 28.32 & 24,500 \\
\hline 2 & 0800 & 6.56 & 1,060 & & & & & & & 1800 & 26.95 & 2,800 \\
\hline & 1800 & 6.69 & 1,130 & & 8 & 0800 & 27.87 & 23,900 & & 2400 & 25.89 & 21,600 \\
\hline & 2400 & 6.85 & 1,230 & & & 1000 & 27.48 & 23,500 & & & & \\
\hline & & & & & & 1100 & 27.50 & 23,500 & 1 & 0600 & 24.97 & 20,500 \\
\hline 3 & 0200 & 6.98 & 1,310 & & & 1200 & 27.73 & 23,800 & & 1200 & 24.16 & \\
\hline & 0300 & 7.17 & 1,440 & & & 2200 & 30.04 & 26,600 & & 1900 & 23.59 & 800 \\
\hline & 0800 & 9.32 & 3,190 & & & 2400 & 30.35 & 27,000 & & 2400 & 24.03 & 19,300 \\
\hline & $\begin{array}{l}1000 \\
1200\end{array}$ & $\begin{array}{l}10.02 \\
10.28\end{array}$ & 4,080 & & 9 & 0300 & 30.55 & 27,200 & & 0400 & 24.29 & 19,60 \\
\hline & 1400 & 10.29 & 4,090 & & & 0400 & 30.80 & 27,500 & & 0700 & 24.53 & 10, \\
\hline & 2200 & 9.89 & 3,700 & & & 0600 & 32.30 & 29,500 & & 1000 & 24.37 & 19 \\
\hline & 2400 & 9.96 & 3,760 & & & 0800 & 34.85 & 32,800 & & 1200 & 24.07 & 19,400 \\
\hline & & & & & & 1200 & 39.83 & 39,400 & & 1800 & 22.37 & 17,300 \\
\hline s & 0400 & 10.25 & 4,050 & & & 1600 & 41.95 & 42,600 & & 2400 & 21.21 & 16,00 \\
\hline & 0500 & 10.45 & 4,250 & & & 2000 & 44.10 & 46,500 & & & & \\
\hline & 0600 & 11.50 & 5,300 & & & 2400 & 46.97 & 52,200 & 1 & 0600 & 19.51 & 13,90 \\
\hline & 0800 & 16.40 & 10,400 & & & & & & & 1200 & 18.12 & 12,30 \\
\hline & 1200 & 21.43 & 16,200 & & 10 & 0400 & 49.10 & 56,500 & & 1800 & 17.17 & 11,30 \\
\hline & 1600 & 27.20 & 23,100 & & & 0600 & 49.70 & 57,800 & & 2400 & 16.48 & 10,500 \\
\hline & 2000 & 34.45 & 32,300 & & & 0800 & 50.05 & 58,600 & & & & \\
\hline & 2400 & 38.35 & 37,400 & & & 1000 & 50.23 & 59,000 & 1 & 1200 & 15.13 & \\
\hline & & & & & & 1100 & 50.26 & 59,100 & & 1800 & 14.43 & \\
\hline 5 & 0400 & 39.90 & 39,500 & & & 1200 & 50.23 & 59,000 & & 2400 & 13.89 & 7,690 \\
\hline & 0800 & 40.90 & 41,000 & & & 1600 & 49.97 & 58,400 & & & & \\
\hline & 1200 & 41.50 & 41,900 & & & 2000 & 49.45 & 57,300 & 1 & 0600 & 13.43 & 7,23 \\
\hline & 1600 & 41.88 & 42,300 & & & 2400 & 48.80 & 55,900 & & 1200 & 13.08 & 6,880 \\
\hline & 2000 & 42.12 & 42,900 & & & & & & & 2400 & 12.45 & 6,25 \\
\hline & 2200 & 42.14 & 43,000 & & 11 & 0600 & 47.15 & 52,600 & & & & \\
\hline & 2400 & 42.12 & 42,900 & & & 1200 & 45.48 & 49,300 & 1 & 1200 & 11.79 & 5,590 \\
\hline & & & & & & 1800 & 43.58 & 45,500 & & 2400 & 11.04 & 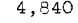 \\
\hline 6 & 0600 & 41.76 & 42,300 & & & 2400 & 41.43 & 41,800 & & & & \\
\hline & 1200 & 41.02 & 41,100 & & & & & & 2 & 1200 & 10.31 & 4,11 \\
\hline & 1800 & 39.98 & 39,600 & & 12 & 0600 & 39.23 & 38,500 & & 1800 & 10.08 & 7 \\
\hline & 2400 & 38.68 & 37,800 & & & 1200 & 36.84 & 35,400 & & 2400 & 10.06 & 860 \\
\hline 7 & 0600 & 37.15 & 35,800 & & & $\begin{array}{l}1800 \\
2400\end{array}$ & $\begin{array}{l}34.22 \\
31.87\end{array}$ & $\begin{array}{l}32,000 \\
28,900\end{array}$ & & & & \\
\hline
\end{tabular}


(58) 3-2520. Stoner Creek at Parls, Ky.

Location. --Lat $38^{\circ} 13^{1} 45^{\prime \prime}$, long $84^{\circ} 15^{\prime} 22^{\prime \prime}$, on left bank at upstream side of bridge on county road, $0.5 \mathrm{mile}$ north of Paris, Bourbon County, and 1.5 miles downstream

from Huston Creek.

Drainage area. $--239 \mathrm{sq} \mathrm{ml}$.

Gage-height record.--Digital recorder tape punched at 15-minute intervals. Datum of gage is 770.43 ft above mean sea leve1, datum of 1929

Discharge record.--Stage-discharge relation defined by current-meter measurements. Maxima.--Given in the following table.

March 1964:

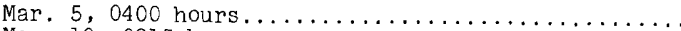

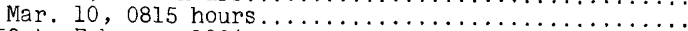

1953 to February 1964

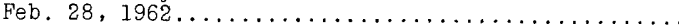

$\begin{array}{cc}\begin{array}{c}\text { Discharge } \\ \text { (cfs) }\end{array} & \begin{array}{c}\text { Gage helght } \\ \text { (feet) } \\ 17,000\end{array} \\ 12,000 & 17.59 \\ 12,200 & 17.65\end{array}$

Prior to 1964, highest stage known since about 1928 was about $19.5 \mathrm{ft}$ in winter of 1951, from information by local resident.

Mean discharge, in cubic feet per second, March 1964

\begin{tabular}{|c|c|c|c|c|c|c|c|c|c|}
\hline Day & Discharge & Day & Discharge & Day & Discharge & Day & Discharge & Day & Discharge \\
\hline $\begin{array}{l}1 \ldots \ldots \\
2 \ldots \ldots \\
3 \ldots \\
4 \ldots \\
5 \ldots \\
6 \ldots \\
\end{array}$ & $\begin{array}{r}138 \\
141 \\
274 \\
5,240 \\
14,200 \\
4,320\end{array}$ & $\begin{array}{r}7 \ldots \ldots \\
8 \ldots \ldots \\
9 \ldots \ldots \\
10 \ldots \\
11 \ldots \ldots \\
12 \ldots\end{array}$ & $\begin{array}{r}933 \\
1,940 \\
5,760 \\
9,700 \\
3,400 \\
1,020\end{array}$ & $\begin{array}{l}13 \ldots \ldots \\
14 \ldots \ldots \\
15 \ldots \ldots \\
16 \ldots \ldots \\
17 \ldots \ldots \\
18 \ldots \ldots\end{array}$ & $\begin{array}{r}645 \\
515 \\
1,190 \\
1,040 \\
597 \\
420\end{array}$ & $\begin{array}{l}19 \ldots \ldots \\
20 \ldots \ldots \\
21 \ldots \ldots \\
22 \ldots \ldots \\
23 \ldots \ldots \\
24 \ldots \ldots\end{array}$ & $\begin{array}{l}324 \\
304 \\
417 \\
535 \\
422 \\
350\end{array}$ & $\mid \begin{array}{l}25 \ldots \ldots \\
26 \ldots \ldots \\
27 \ldots \ldots \\
28 \ldots \ldots \\
29 \ldots \ldots \\
30 \ldots \ldots \\
31 \ldots \ldots\end{array}$ & $\begin{array}{l}296 \\
262 \\
211 \\
176 \\
156 \\
133 \\
115\end{array}$ \\
\hline
\end{tabular}

Note.--Daily mean discharges computed on basis of 15 -minute intervals.

Gage height, In feet, and discharge, in cublc feet per second, at indicated time, 1964

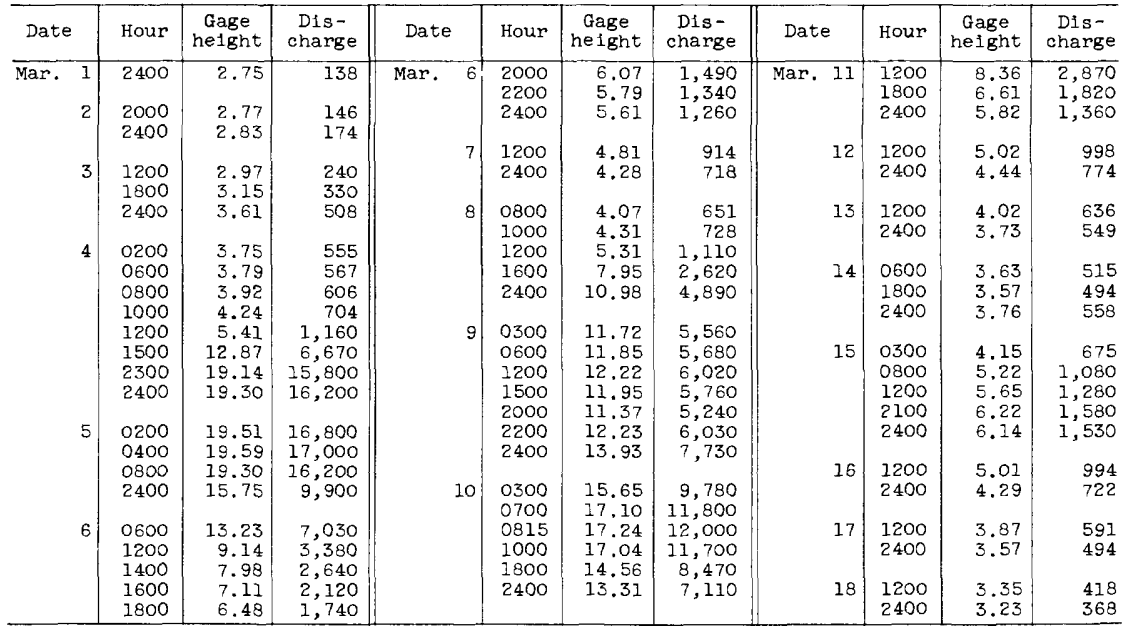


(59) 3-2525. South Fork Licking River at Cynthiana, Ky.

Location. - Lat $38^{\circ} 23^{\prime} 27^{\prime \prime}$, long $84^{\circ} 18^{\prime} 11^{\prime \prime}$, on left bank at downstream side of bridge on State H1ghways 356 and 36 , at Cynthiana, Harrison County, 0.4 mile downstream from Grays Run and in pool formed by old milldam 2.6 miles downstream.

Drainage area. $--621 \mathrm{sq} \mathrm{mi}$.

Gage-helght recora.--Water-stage recorder graph. Datum of gage is $688.52 \mathrm{ft}$ above mean sea level, datum of 1929, supplementary adjustment of 1944 .

Discharge record.--Stage-discharge relation defined by current-meter measurements. Maxima.--Given in the following table.

March 1964:

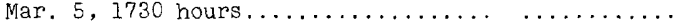

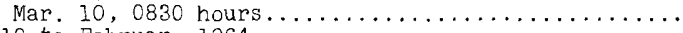

1918 to February 1964 :

$\begin{array}{cc}\begin{array}{c}\text { Discharge } \\ \text { (cfs) }\end{array} & \text { Gage height } \\ 29,000 & 22.59 \\ 28,100 & 22.33 \\ & \\ 35,300 & 23.32\end{array}$

Mean discharge, in cubic feet per second, March 1964

\begin{tabular}{|c|c|c|c|c|c|c|c|c|c|}
\hline Day & Discharge & Day & Discharge & Day & Discharge & Day & Discharge & Day & Discharge \\
\hline $\begin{array}{l}1 \ldots \ldots \\
2 \ldots \ldots \\
3 \ldots \ldots \\
4 \ldots \\
5 \ldots \ldots \\
6 \ldots\end{array}$ & $\begin{array}{r}284 \\
270 \\
440 \\
8,750 \\
28,000 \\
22,200\end{array}$ & $\begin{array}{r}7 \ldots \ldots \\
8 \ldots \ldots \\
9 \ldots \ldots \\
10 \ldots \\
11 \ldots \ldots \\
12 \ldots \ldots\end{array}$ & $\begin{array}{r}7,780 \\
4,450 \\
15,400 \\
26,000 \\
15,800 \\
6,080\end{array}$ & $\begin{array}{l}13 \ldots \ldots \\
14 \ldots \ldots \\
15 \ldots \ldots \\
16 \ldots \\
17 \ldots \ldots \\
18 \ldots \ldots\end{array}$ & $\begin{array}{l}2,380 \\
1,650 \\
3,780 \\
3,950 \\
2,150 \\
1,300\end{array}$ & $\begin{array}{l}19 \ldots \ldots \\
20 \ldots \ldots \\
21 \ldots \ldots \\
22 \ldots \ldots \\
23 \ldots \ldots \\
24 \ldots \ldots\end{array}$ & $\begin{array}{r}906 \\
746 \\
1,200 \\
1,640 \\
1,330 \\
1,010\end{array}$ & $\begin{array}{l}25 \ldots \ldots \\
26 \ldots \ldots \\
27 \ldots \ldots \\
28 \ldots \ldots \\
29 \ldots \ldots \\
30 \ldots \ldots \\
31 \ldots \ldots\end{array}$ & $\begin{array}{r}914 \\
1,180 \\
626 \\
500 \\
395 \\
353 \\
310\end{array}$ \\
\hline
\end{tabular}

Gage height, in feet, and discharge, in cublc feet per second, at indicated time, 1964

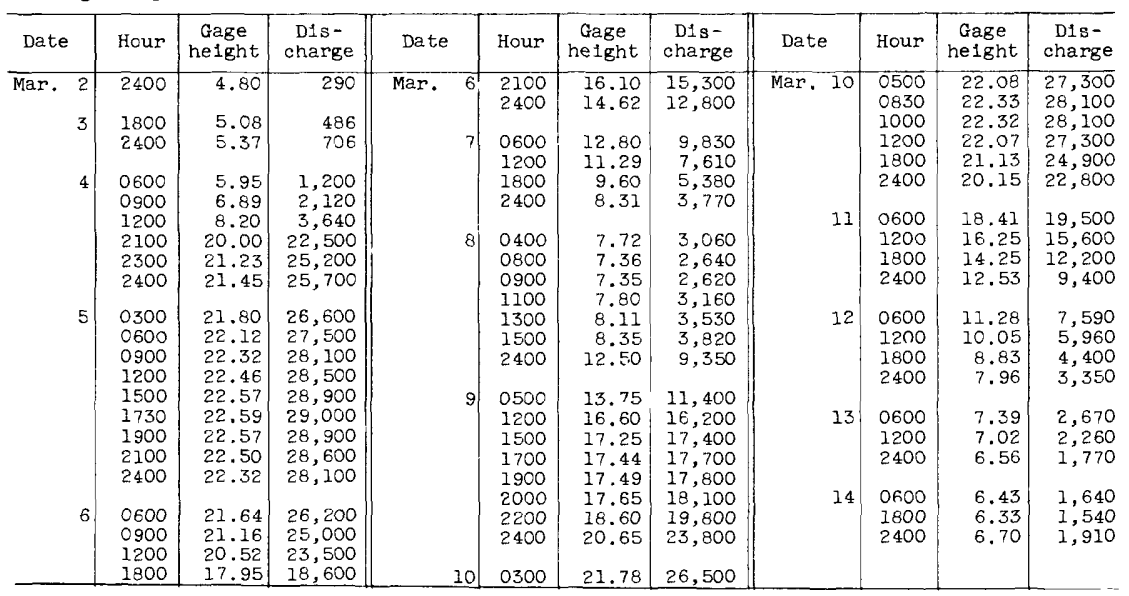


(60) 3-2535. Licking River at Catawba, Ky .

Location.--Lat $38^{\circ} 42^{\prime} 37^{\prime \prime}$, Iong $84^{\circ} 18^{\prime} 39^{\prime \prime}$, on left bank I mile southeast of Catawba, Pendleton County, 1.6 miles upstream from Kincald Creek, and 2.3 miles north of Falmouth.

Drainage area. $--3,300 \mathrm{sq} \mathrm{mi}$.

Gage-height record.--Water-stage recorder graph except 2400 hours Mar. 9 to

1400 hours Mar. 24 , for which graph was reconstructed on basis of high-water mark in gage house and inside gage readings. Datum of gage is $500.01 \mathrm{ft}$ above mean sea level, datum of 1929 (levels by Corps of Engineers).

Discharge record.--Stage-discharge relation defined by current-meter measurements. Maxima.--Given in the following table.

\section{March 1964 :}

Mar. 5,1700 hours $\ldots \ldots \ldots \ldots \ldots \ldots \ldots \ldots \ldots \ldots \ldots$

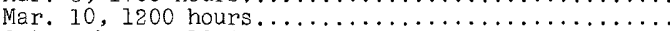

1888 to February 1964 .

Apr. 14, 1948

$\begin{array}{cc}\text { Discharge } & \text { Gage height } \\ \text { (cfs) } & \text { (feet) } \\ 72,100 & 43.05 \\ 95,000 & 52.60\end{array}$

86,300

Highest flood known prior to 1888 , in 1854 (gage height, $41.1 \mathrm{ft}$, discharge, $84,900 \mathrm{cfs}$ ), at Falmouth, at site $3.8 \mathrm{miles}$ upstream at datum $13.8 \mathrm{ft}$ higher.

Mean discharge, in cubic feet per second, March 1964

\begin{tabular}{|c|c|c|c|c|c|c|c|c|c|}
\hline Day & Discharge & Day & Discharge & Day & Discharge & Day & D1scharge & Day & Discharg \\
\hline $\begin{array}{l}1 \ldots \ldots \\
2 \ldots \ldots \\
3 \ldots \ldots \\
4 \ldots \ldots \\
5 \ldots \ldots \\
6 \ldots \ldots\end{array}$ & $\begin{array}{r}1,430 \\
1,370 \\
3,380 \\
25,300 \\
69,500 \\
69,200\end{array}$ & $\begin{array}{r}7 \ldots \\
8 \ldots \\
9 \ldots \\
10 \ldots \\
11 \ldots \\
12 \ldots\end{array}$ & $\begin{array}{l}57,500 \\
36,200 \\
62,000 \\
93,400 \\
81,300 \\
59,400\end{array}$ & $\begin{array}{l}13 \ldots \ldots \\
14 \ldots \ldots \\
15 \ldots \ldots \\
16 \ldots \ldots \\
17 \ldots \ldots \\
18 \ldots \ldots\end{array}$ & $\begin{array}{r}35,900 \\
24,900 \\
25,500 \\
18,700 \\
13,100 \\
9,310\end{array}$ & $\begin{array}{l}19 \ldots \ldots \\
20 \ldots \ldots \\
21 \ldots \ldots \\
22 \ldots \ldots \\
23 \ldots \ldots \\
24 \ldots \ldots\end{array}$ & $\begin{array}{l}7,160 \\
5,420 \\
7,750 \\
6,100 \\
6,870 \\
7,030\end{array}$ & $\begin{array}{l}25 \ldots \ldots \\
26 \ldots \ldots \\
27 \ldots \ldots \\
28 \ldots \ldots \\
29 \ldots \ldots \\
30 \ldots \ldots \\
31 \ldots \ldots\end{array}$ & $\begin{array}{r}5 \\
13 \\
6 \\
4 \\
3 \\
2 \\
2\end{array}$ \\
\hline & dart & & & & & & & & $\begin{array}{r}24, \\
8\end{array}$ \\
\hline
\end{tabular}

Gage height, In feet, and discharge, In cubic feet per second, at indicated t1me, 1964

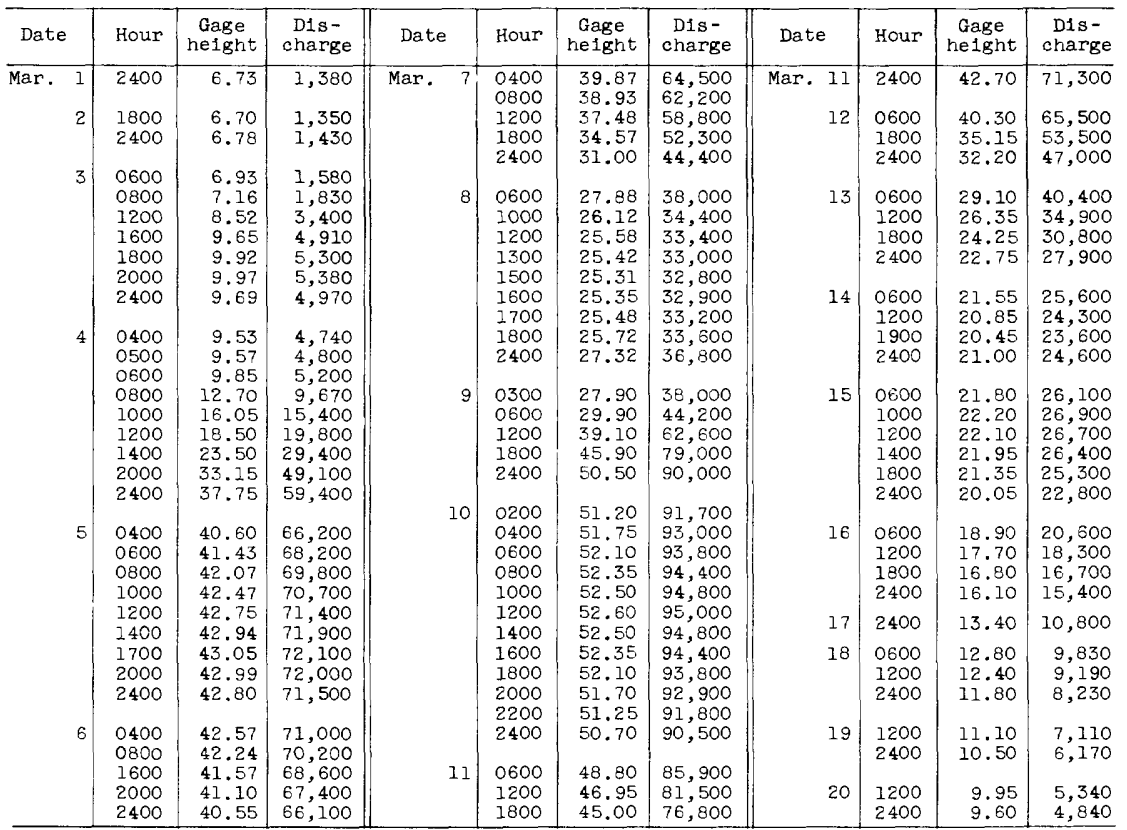




\section{OHIO RIVER MAIN STEM}

(61) 3-2550. Ohio River at Cincinnati, Ohio

Location.--Lat $39^{\circ} 05^{14} 40^{\prime \prime}$, long $84^{\circ} 30140^{\prime \prime}$, on right bank at downstream side of Covington-Cincinnati suspension bridge, Cincinnati, Hamilton County, $0.2 \mathrm{mile}$ downstream from Licking River, 1.9 miles upstream from Mill Creek, and at m1le 470.5 .

Drainage area. $--76,580 \mathrm{sq} \mathrm{ml}$, approximately.

Gage-height record.--Water-stage recorder graph. Graph drawn for auxillary staff gage, 12.7 miles downstream, from twice-dally readings, except Mar. 10, 13, when one reading was made and Mar. 11, 12, 14, when no readings were made. Graph for Mar. 11, 12, 14 was drawn on basis of shape of graph at base gage and readings on adjacent days. Datum of gage is $429.61 \mathrm{ft}$ above mean sea level, oh10 River datum.

D1scharge record.--Stage-fall-discharge relation defined by current-meter measurements. Fall used as a factor in computing discharge. Stage-fall-discharge relation Indefinite Mar. I to 0600 hours Mar. 4; discharge estimated on bas 1 s of records for other ohlo River main-stem stations.

Maxima.--March 1964: Discharge, 650,000 cf's Mar. 11, 0100 hours; gage he1ght, 66.20 ft Mar. 11,1200 hours.

$1773,1792-93,1832,1848,1858$ to February 1964: Discharge, $894,000 \mathrm{cfs}$

Jan. 26, 1937; gage helght, 80.0 ft Jan. 25, 26, 1937.

Flood in January 1937 is maximum known.

Remarks.--Partly regulated by locks, dams, and reservoirs.

Mean discharge, in cublc feet per second, March 1964

\begin{tabular}{|c|c|c|c|c|c|c|c|c|c|}
\hline Day & Discharge & Day & Discharge & Day & Discharge & Day & Discharge & Day & Discharge \\
\hline $\begin{array}{l}1 \ldots \ldots \\
2 \ldots \ldots \\
3 \ldots \ldots \\
4 \ldots \\
5 \ldots \ldots \\
6 \ldots \ldots\end{array}$ & $\begin{array}{r}36,000 \\
42,000 \\
50,000 \\
119,000 \\
323,000 \\
373,000\end{array}$ & $\begin{array}{r}7 \ldots \ldots \\
8 \ldots \ldots \\
9 \ldots \\
10 \ldots \\
11 \ldots \\
12 \ldots\end{array}$ & $\begin{array}{l}394,000 \\
392,000 \\
476,000 \\
625,000 \\
643,000 \\
626,000\end{array}$ & $\begin{array}{l}13 \ldots \ldots \\
14 \ldots \ldots \\
15 \ldots \ldots \\
16 \ldots \ldots \\
17 \ldots \ldots \\
18 \ldots \ldots\end{array}$ & $\begin{array}{l}606,000 \\
588,000 \\
573,000 \\
542,000 \\
518,000 \\
487,000\end{array}$ & $\begin{array}{l}19 \ldots \ldots \\
20 \ldots \ldots \\
21 \ldots \ldots \\
22 \ldots \ldots \\
23 \ldots \ldots \\
24 \ldots \ldots\end{array}$ & $\begin{array}{l}444,000 \\
404,000 \\
359,000 \\
306,000 \\
252,000 \\
206,000\end{array}$ & $\begin{array}{l}25 \ldots \ldots \\
26 \ldots \ldots \\
27 \ldots \ldots \\
28 \ldots \ldots \\
29 \ldots \ldots \\
30 \ldots \ldots \\
31 \ldots \ldots\end{array}$ & $\begin{array}{l}174,000 \\
168,000 \\
156,000 \\
147,000 \\
147,000 \\
132,000 \\
126,000\end{array}$ \\
\hline $11 \mathrm{~J}$ & ean & ge, & & $J I^{2}$ & & & & & 336,600 \\
\hline
\end{tabular}

Mean discharge, in cublc feet per second, April 1964

$$
\text { Apr. } \begin{aligned}
& 1 \ldots \ldots \ldots \ldots \\
& 2 \ldots \ldots \ldots \ldots \\
& 2 \ldots \ldots, 000
\end{aligned}
$$

Gage height, in feet, and discharge, in cubic feet per second, at indicated time, 1964

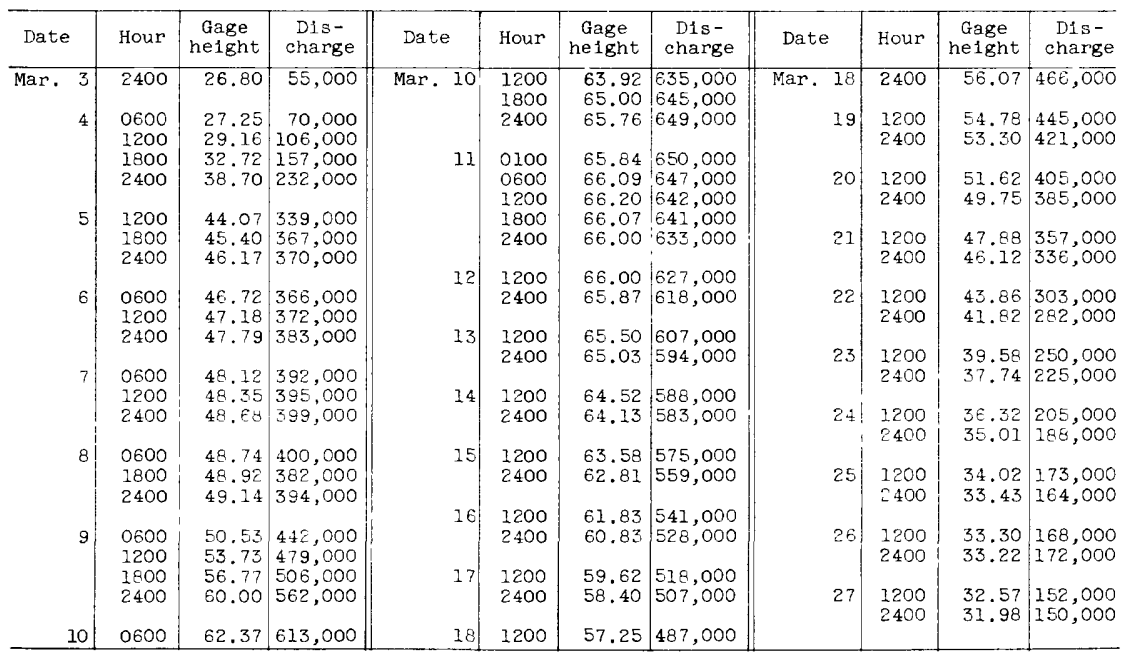




\section{MILL CREEK BASIN}

(62) 3-2590. Mill Creek at Carthage, Ohio

Location.--Lat $39^{\circ} 12^{\prime} 05^{\prime \prime}$, long $84^{\circ} 28^{\prime} 10^{\prime \prime}$, on right bank 100 ft downstream from Anthony Wayne Avenue Bridge in Carthage, Hamilton County, I mile downstream from West Fork Mill Creek, and 11 miles upstream from mouth.

Drainage area. $--115 \mathrm{sq} \mathrm{mi}$.

Gage-he1ght record.--Water-stage recorder graph. Datum of gage is $512.00 \mathrm{ft}$ above mean sea level, ohio River datum.

Discharge record.--Stage-discharge relation defined by current-meter measurements below 4,000 fs and by indirect measurement at $8,900 \mathrm{cfs}$.

Maxima.--March 1964: Discharge, 5,710 cfs Mar. 9, 2400 hours (gage height, $13.50 \mathrm{ft}$ ).

1946 to February 1964: Discharge, 8,900 cfs Jan. 21, 1959 (gage height, $16.17 \mathrm{ft})$.

Remarks.--Flow regulated by West Fork Mill Creek Reservoir (capacity, 1l,380 acre-ft, drainage area, $29.9 \mathrm{sq} \mathrm{mi}$ ).

Mean discharge, in cublc feet per second, March 1964

\begin{tabular}{|c|c|c|c|c|c|c|c|c|c|}
\hline Day & Discharge & Day & Discharge & Day & Discharge & Day & D1scharge & Day & D1scharge \\
\hline $\begin{array}{l}1 \ldots \ldots \\
2 \ldots \ldots \\
3 \ldots \ldots \\
4 \ldots \ldots \\
5 \ldots \ldots \\
6 \ldots \ldots\end{array}$ & $\begin{array}{r}4.1 \\
6.5 \\
11 \\
1,200 \\
1,290 \\
258\end{array}$ & $\begin{array}{r}7 \ldots \ldots \\
8 \ldots \ldots \\
9 \ldots \ldots \\
10 \ldots \ldots \\
11 \ldots \ldots \\
12 \ldots \ldots\end{array}$ & $\begin{array}{r}55 \\
163 \\
3,740 \\
3,080 \\
826 \\
345\end{array}$ & $\begin{array}{l}13 \ldots \ldots \\
14 \ldots \ldots \\
15 \ldots \ldots \\
16 \ldots \ldots \\
17 \ldots \ldots \\
18 \ldots \ldots\end{array}$ & $\begin{array}{r}754 \\
1,240 \\
1,200 \\
1,160 \\
186 \\
57\end{array}$ & $\begin{array}{l}19 \ldots \ldots \\
20 \ldots \ldots \\
21 \ldots \ldots \\
22 \ldots \ldots \\
23 \ldots \ldots \\
24 \ldots \ldots\end{array}$ & $\begin{array}{r}44 \\
63 \\
189 \\
90 \\
73 \\
57\end{array}$ & $\begin{array}{l}25 \ldots \ldots \\
26 \ldots \ldots \\
27 \ldots \ldots \\
28 \ldots \ldots \\
29 \ldots \ldots \\
30 \ldots \ldots \\
31 \ldots \ldots\end{array}$ & $\begin{array}{r}54 \\
463 \\
105\end{array}$ \\
\hline
\end{tabular}

GREAT MIAMI RIVER BASIN

(63) 3-2740. Great Miami River at Hamilton, Ohio

Location.--Lat $39^{\circ} 23^{\prime} 28^{\prime \prime}$, Iong $84^{\circ} 34^{\prime} 20^{\prime \prime}$, on right bank 1,000 ft downstream from Columbia Bridge at Hamilton, Butler County, and 3 miles downstream from Four Mile Creek.

Drainage area. $--3,639 \mathrm{sq} \mathrm{mi}$.

Gage-helght record.--Water-stage recorder graph. Datum of gage is $499.98 \mathrm{ft}$ above mean sea level, adjustment of 1912 .

Discharge record.--Stage-discharge relation defined by current-meter measurements below $74,000 \mathrm{cfs}$ and by indirect measurement at $108,000 \mathrm{cfs}$.

Maxima, - March 1964: Discharge, 59,000 cfs Mar. 10, 0730 hours (gage height, $73.69 \mathrm{ft}$.

1910-1918: Discharge, 352,000 cfs Mar. 26, 1913 (computed by Miami Conservancy District) at site 0.7 mile upstream.

1927 to February 1964: Discharge, 108,000 cfs Jan. 21, 1959 (gage height, $79.49 \mathrm{ft}$ ).

Remarks.--Floodflow regulated by flve retarding basins above station beginning in 1920. Base data furnished by Miami Conservancy District.

Mean discharge, in cubic feet per second, March 1964

\begin{tabular}{|c|c|c|c|c|c|c|c|c|c|}
\hline Day & Discharge & Day & Discharge & Day & Discharge & Day & Discharge & Day & D1scharge \\
\hline $\begin{array}{l}1 \ldots \ldots \\
2 \ldots \ldots \\
3 \ldots \ldots \\
4 \ldots \\
5 \ldots \ldots \\
6 \ldots \ldots\end{array}$ & $\begin{array}{r}414 \\
389 \\
468 \\
3,640 \\
18,600 \\
12,700\end{array}$ & $\begin{array}{r}7 \ldots \ldots \\
8 \ldots \ldots \\
9 \ldots \\
10 \ldots \\
11 \ldots \ldots \\
12 \ldots\end{array}$ & $\begin{array}{r}6,940 \\
4,660 \\
28,500 \\
52,600 \\
41,200 \\
31,700\end{array}$ & $\begin{array}{l}13 \ldots \ldots \\
14 \ldots \ldots \\
15 \ldots \ldots \\
16 \ldots \\
17 \ldots \ldots \\
18 \ldots \ldots\end{array}$ & $\begin{array}{r}21,500 \\
17,800 \\
16,500 \\
12,900 \\
8,830 \\
6,800\end{array}$ & $\begin{array}{l}19 \ldots \ldots \\
20 \ldots \ldots \\
21 \ldots \ldots \\
22 \ldots \ldots \\
23 \ldots \ldots \\
24 \ldots \ldots\end{array}$ & $\begin{array}{l}5,490 \\
4,640 \\
5,020 \\
4,980 \\
4,640 \\
4,130\end{array}$ & $\begin{array}{l}25 \ldots \ldots \\
26 \ldots \ldots \\
27 \ldots \ldots \\
28 \ldots \ldots \\
29 \ldots \ldots \\
30 \ldots \ldots \\
31 \ldots \ldots\end{array}$ & $\begin{array}{l}3,660 \\
4,170 \\
4,070 \\
3,790 \\
3,260 \\
2,960 \\
2,760\end{array}$ \\
\hline
\end{tabular}


(64) 3-2765. Whitewater River at Brookville, Ind.

Location. --Lat $39^{\circ} 24^{1} 24^{\prime \prime}$, long $85^{\circ} 00145^{\prime \prime}$, in NW $\frac{1}{4}$ sec. 32 , T.9 N., R.2 W., on right bank at downstream side of highway bridge, 0.3 mile downstream from East Fork and 1.1 miles south of Brookvilie.

Dra1nage area. $--1,239 \mathrm{sq} \mathrm{ml}$.

Gage-he1ght record.--Water-stage recorder graph. Datum of gage is $595.71 \mathrm{ft}$ above mean sea level, datum of 1929 .

Discharge record.--Stage-discharge relation defined by current-meter measurements below 46,000 cfs and by contracted-opening measurement at $81,800 \mathrm{cfs}$.

Maxima.--Given in the following table.

March 1964:

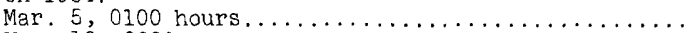

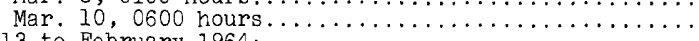

1913 to February 1964:

Mar. 25, 1973

$1915-20,1923$ to February $1964:$

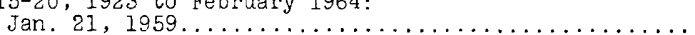

a Not determined.

$\begin{array}{cc}\begin{array}{c}\text { Discharge } \\ \text { (cfs) }\end{array} & \begin{array}{c}\text { Gage helght } \\ \text { (feet) }\end{array} \\ 12,700 & 10.44 \\ 46,000 & 21.20 \\ \text { (a) } & 39.0 \\ 81,800 & 27.78\end{array}$

Mean discharge, in cub1c feet per second, March 1964

\begin{tabular}{|c|c|c|c|c|c|c|c|c|c|}
\hline Day & Discharge & Day & Discharge & Day & Discharge & Day & Discharge & Day & Discharge \\
\hline $\begin{array}{l}1 \ldots \ldots \\
2 \ldots \ldots \\
3 \ldots \ldots \\
4 \ldots \ldots \\
5 \ldots \ldots \\
6 \ldots \ldots\end{array}$ & $\begin{array}{r}181 \\
194 \\
239 \\
2,900 \\
6,430 \\
2,050\end{array}$ & $\begin{array}{r}7 \ldots \ldots \\
8 \ldots \\
9 \ldots \\
10 \ldots \\
11 \ldots \ldots \\
12 \ldots\end{array}$ & $\begin{array}{r}1,280 \\
1,010 \\
19,900 \\
35,400 \\
10,800 \\
6,160\end{array}$ & $\begin{array}{l}13 \ldots \ldots \\
14 \ldots \ldots \\
15 \ldots \ldots \\
16 \ldots \ldots \\
17 \ldots \ldots \\
18 \ldots \ldots\end{array}$ & $\begin{array}{l}4,070 \\
3,290 \\
3,650 \\
2,560 \\
2,040 \\
1,670\end{array}$ & $\begin{array}{l}19 \ldots \ldots \\
20 \ldots \ldots \\
21 \ldots \ldots \\
22 \ldots \ldots \\
23 \ldots \ldots \\
24 \ldots \ldots\end{array}$ & $\begin{array}{l}1,450 \\
1,350 \\
1,530 \\
1,570 \\
1,380 \\
1,240\end{array}$ & $\left\{\begin{array}{l}25 \ldots \ldots \\
26 \ldots \ldots \\
27 \ldots \ldots \\
28 \ldots \ldots \\
29 \ldots \ldots \\
30 \ldots \ldots \\
31 \ldots \ldots\end{array}\right.$ & $\begin{array}{r}1,170 \\
1,880 \\
1,560 \\
1,310 \\
1,160 \\
1,030 \\
970 \\
\end{array}$ \\
\hline
\end{tabular}

\section{HOGAN CREEK BASIN}

(65) 3-2767. South Hogan Creek near Dillsboro, Ind.

Location.--Lat $38^{\circ} 01147^{\prime \prime}$, long $85^{\circ} 02^{\prime} 17^{\prime \prime}$, In NW $\frac{1}{4}$ sec.7, T.4 N., R.2 W. , on left downstream abutment of bridge on county road at Dillsboro station, $1 \frac{1}{4}$ miles northeast of Dilisboro, and $1 \frac{1}{2}$ miles downstream from Whitaker Creek.

Dra1nage area. $--38.2 \mathrm{sq} \mathrm{mi}$.

Gage-helght record.--Water-stage recorder graph. Datum of gage is $571.00 \mathrm{ft}$ above mean sea level, datum of 1929 .

Discharge record.--Stage-discharge relation defined by current-meter measurements below $3,300 \mathrm{c}$ 's and by contracted-opening measurement at $16,300 \mathrm{cf}$.

Maxima.--Given in the following table.

March 1964:

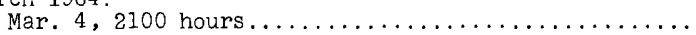

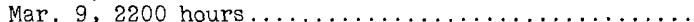

1959 to February 1964:

Jan. 21, 1959 .

$\begin{array}{cc}\text { Discharge } & \text { Gage height } \\ \text { (cfs) } & \text { (feet) } \\ 7,220 & 10.08 \\ 5,750 & 9.21\end{array}$

$16,300 \quad 14.00$

Mean discharge, in cubic feet per second, March 1964

\begin{tabular}{|c|c|c|c|c|c|c|c|c|c|}
\hline Day & Discharge & Day & Discharge & Day & D1scharge & Day & Discharge & Day & Discharge \\
\hline $\begin{array}{l}1 \ldots \ldots \\
2 \ldots \ldots \\
3 \ldots \ldots \\
4 \ldots \ldots \\
5 \ldots \ldots \\
6 \ldots \ldots\end{array}$ & $\begin{array}{r}2.6 \\
2.7 \\
10 \\
1,520 \\
566 \\
43\end{array}$ & $\begin{array}{r}7 \ldots \ldots \\
8 \ldots \ldots \\
9 \ldots \ldots \\
10 \ldots \ldots \\
11 \ldots \ldots \\
12 \ldots \ldots\end{array}$ & $\begin{array}{r}23 \\
33 \\
3,460 \\
944 \\
114 \\
62\end{array}$ & $\begin{array}{l}13 \ldots \ldots \\
14 \ldots \ldots \\
15 \ldots \ldots \\
16 \ldots \ldots \\
17 \ldots \ldots \\
18 \ldots \ldots\end{array}$ & $\begin{array}{r}35 \\
106 \\
104 \\
34 \\
22 \\
17\end{array}$ & $\begin{array}{l}19 \ldots \ldots \\
20 \ldots \ldots \\
21 \ldots \ldots \\
22 \ldots \ldots \\
23 \ldots \ldots \\
24 \ldots \ldots\end{array}$ & $\begin{array}{l}15 \\
15 \\
17 \\
16 \\
15 \\
12\end{array}$ & $\begin{array}{l}25 \ldots \ldots \\
26 \ldots \ldots \\
27 \ldots \ldots \\
28 \ldots \ldots \\
29 \ldots \ldots \\
30 \ldots \ldots \\
31 \ldots \ldots\end{array}$ & $\begin{array}{r}28 \\
104 \\
29 \\
22 \\
16 \\
14 \\
12 \\
\end{array}$ \\
\hline
\end{tabular}




\section{LAUGHERY CREEK BASIN}

(66) 3-2770. Laughery Creek near Farmers Retreat, Ind.

Location.--Lat $38^{\circ} 57^{105^{\prime \prime}}$, long $85^{\circ} 04^{\prime} 22^{\prime \prime}$, in sec.2, T.4 N., R.3 W., on right bank 2 miles southeast of Farmers Retreat and $3 \frac{3}{4}$ miles downstream from Bear Creek.

Drainage area $--248 \mathrm{sq} \mathrm{m}$.

Gage-he1ght record.--Water-stage recorder graph. Alt1tude of gage is $526 \mathrm{ft}$ (by barometer).

Discharge record.--Stage-discharge relation defined by current-meter measurements below $14,000 \mathrm{cf}$ 's and by slope-area measurement at $47,800 \mathrm{cfs}$.

Maxima.--Given in the following table.

March 1964:

Mar. 4, 2300 hours

Mar. 9,2300 hours

1897 to February 1964 :

Jan. 21,1959

$\begin{array}{cc}\begin{array}{c}\text { Discharge } \\ \text { (cfs) }\end{array} & \text { Gage he1ght } \\ 16,600 & \text { feet) } \\ 19,100 & 13.35 \\ 47,800 & 14.25 \\ & 21.13\end{array}$

Mean discharge, in cubic feet per second, March 1964

\begin{tabular}{|c|c|c|c|c|c|c|c|c|c|}
\hline Day & Discharge & Day & Discharge & Day & Discharge & Day & Discharge & Day & D1scharge \\
\hline $\begin{array}{l}1 \ldots \ldots \\
2 \ldots \ldots \\
3 \ldots \ldots \\
4 \ldots \\
5 \ldots \\
6 \ldots\end{array}$ & $\begin{array}{r}17 \\
16 \\
31 \\
4,060 \\
5,500 \\
1,250\end{array}$ & $\begin{array}{r}7 \ldots \ldots \\
8 \ldots \ldots \\
9 \ldots \\
10 \ldots \\
11 \ldots \\
12 \ldots\end{array}$ & $\begin{array}{r}335 \\
316 \\
11,200 \\
12,700 \\
5,680 \\
1,100\end{array}$ & $\begin{array}{l}13 \ldots \ldots \\
14 \ldots \ldots \\
15 \ldots \ldots \\
16 \ldots \\
17 \ldots \ldots \\
18 \ldots \ldots\end{array}$ & $\begin{array}{r}845 \\
785 \\
1,220 \\
626 \\
335 \\
226\end{array}$ & $\begin{array}{l}19 \ldots \ldots \\
20 \ldots \ldots \\
21 \ldots \ldots \\
22 \ldots \ldots \\
23 \ldots \ldots \\
24 \ldots \ldots\end{array}$ & $\begin{array}{l}172 \\
153 \\
191 \\
207 \\
218 \\
166\end{array}$ & $\begin{array}{l}25 \ldots \ldots \\
26 \ldots \ldots \\
27 \ldots \ldots \\
28 \ldots \ldots \\
29 \ldots \ldots \\
30 \ldots \ldots \\
31 \ldots \ldots\end{array}$ & $\begin{array}{l}143 \\
488 \\
466 \\
273 \\
191 \\
148 \\
116\end{array}$ \\
\hline thly & $\begin{array}{l}\text { an } d i \\
\text { inch }\end{array}$ & $\mathrm{ge}$ & & & & & & & $\begin{array}{r}1,586 \\
7.38\end{array}$ \\
\hline
\end{tabular}

\section{KENTUCKY RIVER BASIN}

(67) 3-2875. Kentucky River at lock 4, at Frankfort, Ky.

Location. - Lat $38^{\circ} 12^{\prime} 06^{\prime \prime}$, long $84^{\circ} 52^{\prime} 54^{\prime \prime}$, on left bank at downstream side of Broadway Street Bridge at Frankfort, Franklin County, $300 \mathrm{ft}$ upstream from Benson Creek, $0.9 \mathrm{mile}$ upstream from lock 4 , and at mile 65.9. Records include f'low of Benson Creek.

Drainage area.--5,412 sq $\mathrm{ml}$ (includes that of Benson Creek), of which about $120 \mathrm{sq} \mathrm{m}$ does not contribute directly to surface runoff.

Gage-helght record.--Water-stage recorder graph except Mar. 4, 1700 hours to Mar. 5, 1700 hours, for which graph was reconstructed on basis of twice-daily staff-gage readings at lock 4 and shape of recorder graph on adjacent days. Graph drawn for auxiliary staff gage, 16.3 miles upstream, from twice-dally readings. Datum of gage is $462.10 \mathrm{f}^{\prime} \mathrm{t}$ above mean sea level, datum of 1929 .

Discharge record.--Stage-fall-discharge relation or stage-discharge relation defined by current-meter measurements. Fall used as a factor Mar. 4-26.

Maxima.--Given in the following table.

March 1964:

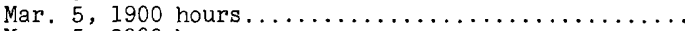

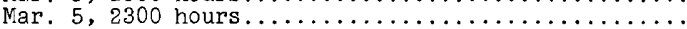

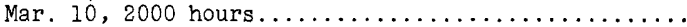

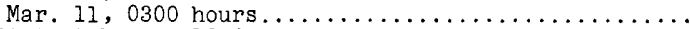

1895 to February 1964:

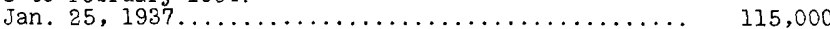

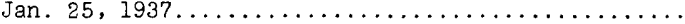

$\begin{array}{cc}\begin{array}{c}\text { Discharge } \\ \text { (cfs) } \\ 65,100\end{array} & \begin{array}{c}\text { Gage height } \\ \text { (feet) }\end{array} \\ 81,300 & - \\ - & - \\ 115,000 & 38.71 \\ - & -\end{array}$

Remarks.--Flow partly regulated by Buckhom Reservolr, Herrington Lake, and by hydroelectric plant at lock 7 .

Cooperation.--Auxillary gage readings furnished by Corps of Engineers. 
Mean discharge, in cubic feet per second, March 1964, of Kentucky River at lock 4 ,

\begin{tabular}{|c|c|c|c|c|c|c|c|c|c|}
\hline Day & Discharge & Day & Discharge & Day & Discharge & Day & Discharge & Day & Discharge \\
\hline $\begin{array}{l}1 \ldots \ldots \\
2 \ldots \ldots \\
3 \ldots \ldots \\
4 \ldots \ldots \\
5 \ldots \ldots \\
6 \ldots \ldots\end{array}$ & $\begin{array}{r}4,050 \\
4,250 \\
7,930 \\
33,300 \\
62,800 \\
55,700\end{array}$ & $\begin{array}{r}7 \ldots \ldots \\
8 \ldots \ldots \\
9 \ldots \\
10 \ldots \\
11 \ldots \\
12 \ldots\end{array}$ & $\begin{array}{l}41,700 \\
32,600 \\
55,900 \\
76,100 \\
76,800 \\
63,400\end{array}$ & $\begin{array}{l}13 \ldots \ldots \\
14 \ldots \ldots \\
15 \ldots \ldots \\
16 \ldots \ldots \\
17 \ldots \ldots \\
18 \ldots \ldots\end{array}$ & $\begin{array}{l}37,500 \\
20,100 \\
16,800 \\
23,900 \\
28,200 \\
24,200\end{array}$ & $\begin{array}{l}19 \ldots \ldots \\
20 \ldots \ldots \\
21 \ldots \ldots \\
22 \ldots \ldots \\
23 \ldots \ldots \\
24 \ldots \ldots\end{array}$ & $\begin{array}{l}18,300 \\
14,700 \\
14,400 \\
15,700 \\
16,400 \\
16,100\end{array}$ & $\begin{array}{l}25 \ldots \ldots \\
26 \ldots \ldots \\
27 \ldots \ldots \\
28 \ldots \ldots \\
29 \ldots \ldots \\
30 \ldots \ldots \\
31 \ldots \ldots\end{array}$ & $\begin{array}{r}14,900 \\
13,500 \\
12,400 \\
11,200 \\
10,600 \\
9,890 \\
8,300\end{array}$ \\
\hline
\end{tabular}

(68) 3-2880. North Elkhorn Creek near Georgetown, Ky.

Location.--Lat $38^{\circ} 12^{\prime} 20^{\prime \prime}$, long $84^{\circ} 30^{\prime} 4^{\prime \prime}$, on left bank at upstream side of bridge on Crumbull Pike, 1.7 miles downstream from Miller Run, $2 \frac{1}{2}$ miles east of Georgetown, Scott County, and 2.6 miles upstream from Lanes Run.

Drainage area. - - $119 \mathrm{sq} \mathrm{mi}$, of which about $8 \mathrm{sq} \mathrm{ml}$ does not contribute directly to surface runoff.

Gage-he1ght record.--Digital recorder tape punched at 30-minute intervals and creststage gage. Datum of gage is $796.49 \mathrm{ft}$ above mean sea level, unadjusted.

Discharge record.--Stage-discharge relation def'ined by current-meter measurements. Maxima.--Given in the following table.

March 1964:

Mar. 5, 0330 hours.....

1948:

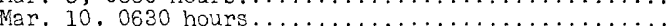

April

1949 to February 1964

Mar. 23,1952

$\begin{array}{cc}\text { Discharge } & \text { Gage height } \\ \text { (cfs) } & \text { (feet) } \\ 8,500 & 219.50 \\ 7,380 & \mathrm{~b} 18.75\end{array}$

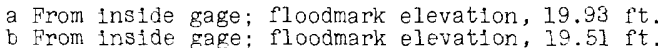

Unknown 22

$$
6,480
$$

Mean discharge, in cubic feet per second, March 1.964

\begin{tabular}{|c|c|c|c|c|c|c|c|c|c|}
\hline Day & Discharge & Day & Discharge & Day & Discharge & Lay & Diacharge & Day & Discharge \\
\hline $\begin{array}{l}1 \ldots \ldots \\
2 \ldots \ldots \\
3 \ldots \\
4 \ldots \\
5 \ldots \\
6 \ldots\end{array}$ & $\begin{array}{r}7 z \\
70 \\
117 \\
2,380 \\
E, 650 \\
1,540\end{array}$ & $\begin{array}{r}7 \ldots \ldots \\
8 \ldots \ldots \\
y \ldots \\
10 \ldots \\
11 \ldots \\
12 \ldots\end{array}$ & $\begin{array}{r}764 \\
1,200 \\
3,450 \\
5,990 \\
1,970 \\
0 \equiv 1\end{array}$ & $\begin{array}{l}13 \ldots \ldots \\
14 \ldots \ldots \\
15 \ldots \ldots \\
16 \ldots \\
17 \ldots \ldots \\
17 \ldots \ldots\end{array}$ & $\begin{array}{l}564 \\
476 \\
991 \\
640 \\
455 \\
8=9\end{array}$ & $\begin{array}{l}19 \ldots \ldots \\
20 \ldots \ldots \\
27 \ldots \ldots \\
22 \ldots \ldots \\
-3 \ldots \ldots \\
24 \ldots \ldots\end{array}$ & $\begin{array}{l}244 \\
210 \\
360 \\
324 \\
265 \\
218\end{array}$ & $\begin{array}{l}25 \ldots \ldots \\
26 \ldots \ldots \\
27 \ldots \ldots \\
28 \ldots \ldots \\
29 \ldots \ldots \\
30 \ldots \ldots \\
31 \ldots \ldots\end{array}$ & $\begin{array}{r}181 \\
162 \\
128 \\
109 \\
96 \\
82 \\
74\end{array}$ \\
\hline
\end{tabular}

Note. - Daily mean discharges computed on basis of 30-minute intervals.

Gage helght, in feet, and discharge, in cublc feet per second, at indicated t1me, 1964

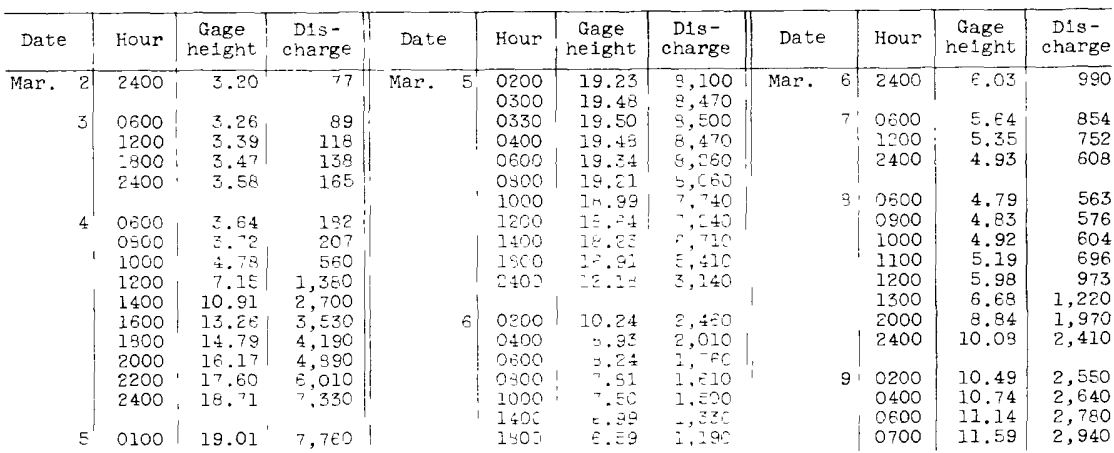


(71) 3-2900. Flat Creek near Frankfort, Ky.

Location.--Lat $38^{\circ} 17153^{\prime \prime}$, long $84^{\circ} 56^{\prime} 32^{\prime \prime}$, on left bank at downstream side of bridge on U.S. Highway $421,0.4$ mile downstream from confluence of Goose Creek and Bald Knob Branch and 7 miles northwest of Frankfort, Franklin County.

Drainage area. $--5.63 \mathrm{sq} \mathrm{mi}$.

Gage-height record.--Water-stage recorder graph and crest-stage gage. Recorder graph corrected for drawdown by relation curve. Datum of gage is 668.65 ft above mean sea level, unadjusted.

Discharge record.--Stage-discharge relation defined by current-meter measurements below $500 \mathrm{cfs}$ and by slope-area measurement at $1,840 \mathrm{cfs}$ and contracted-opening measurement at $7,100 \mathrm{cfs}$.

Maxima.--Given in the following table.

March 1964:

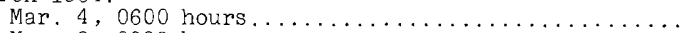

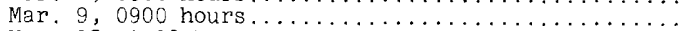

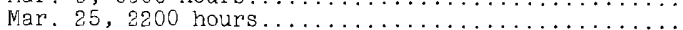

1951 to February 1964:

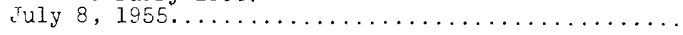

a From inside gage; floodmark elevation, $8.04 \mathrm{f}^{\prime} \mathrm{t}$.

b From inslde gage; floodmark elevation, $8.99 \mathrm{ft}$.

c From inside gage; floodmark elevation, $10.53 \mathrm{ft}$.

$\begin{array}{cc}\begin{array}{c}\text { Discharge } \\ \text { (cfs) }\end{array} & \begin{array}{c}\text { Gage height } \\ \text { (feet) }\end{array} \\ 1,740 & \text { a } 7.66 \\ 2,120 & \text { b8.64 } \\ 3,280 & \text { c10.07 }\end{array}$

$7,100 \quad 11.50$

Mean discharge, in cublc feet per second, March 1964

\begin{tabular}{|c|c|c|c|c|c|c|c|c|c|}
\hline Day & Discharge & Day & Discharge & Day & Discharge & Day & Discharge & Day & Discharge \\
\hline $\begin{array}{l}1 \ldots \ldots \\
2 \ldots \ldots \\
3 \ldots \ldots \\
4 \ldots \ldots \\
5 \ldots \ldots \\
6 \ldots \ldots\end{array}$ & $\begin{array}{r}0.4 \\
2.0 \\
17 \\
685 \\
33 \\
3.4\end{array}$ & $\begin{array}{r}7 \ldots \ldots \\
8 \ldots \ldots \\
9 \ldots \\
10 \ldots \\
11 \ldots \ldots \\
12 \ldots\end{array}$ & $\begin{array}{c}2.0 \\
130 \\
928 \\
75 \\
6.7 \\
3.1\end{array}$ & $\begin{array}{l}13 \ldots \ldots \\
14 \ldots \ldots \\
15 \ldots \ldots \\
16 \ldots \ldots \\
17 \ldots \ldots \\
18 \ldots \ldots\end{array}$ & $\begin{array}{c}2.2 \\
50 \\
16 \\
3.6 \\
2.2 \\
1.7\end{array}$ & $\mid \begin{array}{l}19 \ldots \ldots \\
20 \ldots \ldots \\
21 \ldots \ldots \\
22 \ldots \ldots \\
23 \ldots \ldots \\
24 \ldots \ldots\end{array}$ & $\begin{array}{r}1.4 \\
2.4 \\
22 \\
3.4 \\
2.2 \\
1.8\end{array}$ & $\begin{array}{l}25 \ldots \ldots \\
26 \ldots \ldots \\
27 \ldots \ldots \\
28 \ldots \ldots \\
29 \ldots \ldots \\
30 \ldots \ldots \\
31 \ldots \ldots\end{array}$ & $\begin{array}{r}178 \\
37 \\
4.7 \\
2.6 \\
2.0 \\
1.6 \\
1.6\end{array}$ \\
\hline ins & Inch & ge & 101 & 1 & & & & & $\begin{array}{r}71.7 \\
14.7\end{array}$ \\
\hline
\end{tabular}

(72) 3-2905. Kentucky River at lock 2, at Lockport, Ky .

Location.--Lat $38^{\circ} 26^{\prime} 20^{\prime \prime}$, long $84^{\circ} 57148^{\prime \prime}$, on left bank at lock 2 at Lockport, Henry County, 0.1 mile downstream from Sixmile Creek and at mile 31.0 . $\begin{aligned} & \text { Drainage area } \\ & \text { to surface }\end{aligned}--6,180 \mathrm{sq} \mathrm{mi}$, of which about $200 \mathrm{sq} \mathrm{ml}$ does not contribute directly

Gage-height record.--Graph drawn from twice-daily upper staff-gage readings, except Mar. 12 , when several readings were made. Graph drawn for auxiliary staff gage, 11.0 miles upstream, from twice-daily readings. Datum of gage is $433.36 \mathrm{ft}$ above mean sea level, datum of 1929.

Discharge record.--Stage-fall-discharge relation or stage-discharge relation defined by current-meter measurements below 96,500 cfs.

Maxima.--Given in the following table.

March 1964

Mar. 6,0600 hours. . . . . . . . . . . . . . . . . . .

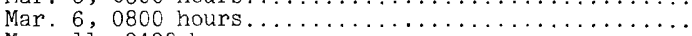

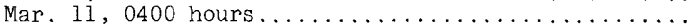

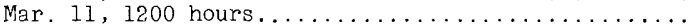

1884 to February 1964:

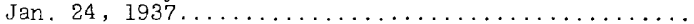

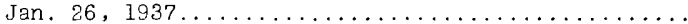

$\begin{array}{cc}\begin{array}{c}\text { Discharge } \\ \text { (cf's) } \\ 81,000\end{array} & \begin{array}{c}\text { Gage height } \\ \text { feet) }\end{array} \\ - & - \\ 101,000 & 41.05 \\ - & - \\ - & 49.00 \\ 123,000 & 56.85\end{array}$

Remarks.--Flow partly regulated by Buckhorn Reservoir, Herrington Lake, and hydroelectric plant at lock 7 .

Cooperation.--Gage-helght record furnished by Corps of Engineers. 
Mean discharge, in cub1c feet per second, March 1964, of Kentucky R1ver at lock 2 ,

\begin{tabular}{|c|c|c|c|c|c|c|c|c|c|}
\hline Day & Discharge & Day & Discharge & Day & D1scharge & Day & Discharge & Day & D1scharge \\
\hline $\begin{array}{l}1 \ldots \ldots \\
2 \ldots \ldots \\
3 \ldots \ldots \\
4 \ldots \ldots \\
5 \ldots \ldots \\
6 \ldots \ldots\end{array}$ & $\begin{array}{r}4,400 \\
4,100 \\
6,580 \\
34,200 \\
75,100 \\
76,400\end{array}$ & $\begin{array}{r}7 \ldots \\
8 \ldots \\
9 \ldots \\
10 \ldots \\
11 \ldots \\
12 \ldots\end{array}$ & $\begin{array}{l}58,900 \\
43,800 \\
66,400 \\
97,100 \\
99,000 \\
86,700\end{array}$ & $\begin{array}{l}13 \ldots \ldots \\
14 \ldots \ldots \\
15 \ldots \ldots \\
16 \ldots \ldots \\
17 \ldots \ldots \\
18 \ldots \ldots\end{array}$ & $\begin{array}{l}62,800 \\
35,600 \\
15,900 \\
25,600 \\
32,800 \\
31,400\end{array}$ & $\begin{array}{l}19 \ldots \ldots \\
20 \ldots \ldots \\
21 \ldots \ldots \\
22 \ldots \ldots \\
23 \ldots \ldots \\
24 \ldots \ldots\end{array}$ & $\begin{array}{l}23,800 \\
16,500 \\
17,900 \\
20,600 \\
19,600 \\
16,400\end{array}$ & $\begin{array}{l}25 \ldots \ldots \\
26 \ldots \ldots \\
27 \ldots \ldots \\
28 \ldots \ldots \\
29 \ldots \ldots \\
30 \ldots \ldots \\
31 \ldots \ldots\end{array}$ & $\begin{array}{r}15,000 \\
16,200 \\
13,000 \\
12,000 \\
11,200 \\
10,400 \\
9,000\end{array}$ \\
\hline
\end{tabular}

Gage helght, in feet, and discharge, in cubic feet per second, at indicated time, 1964

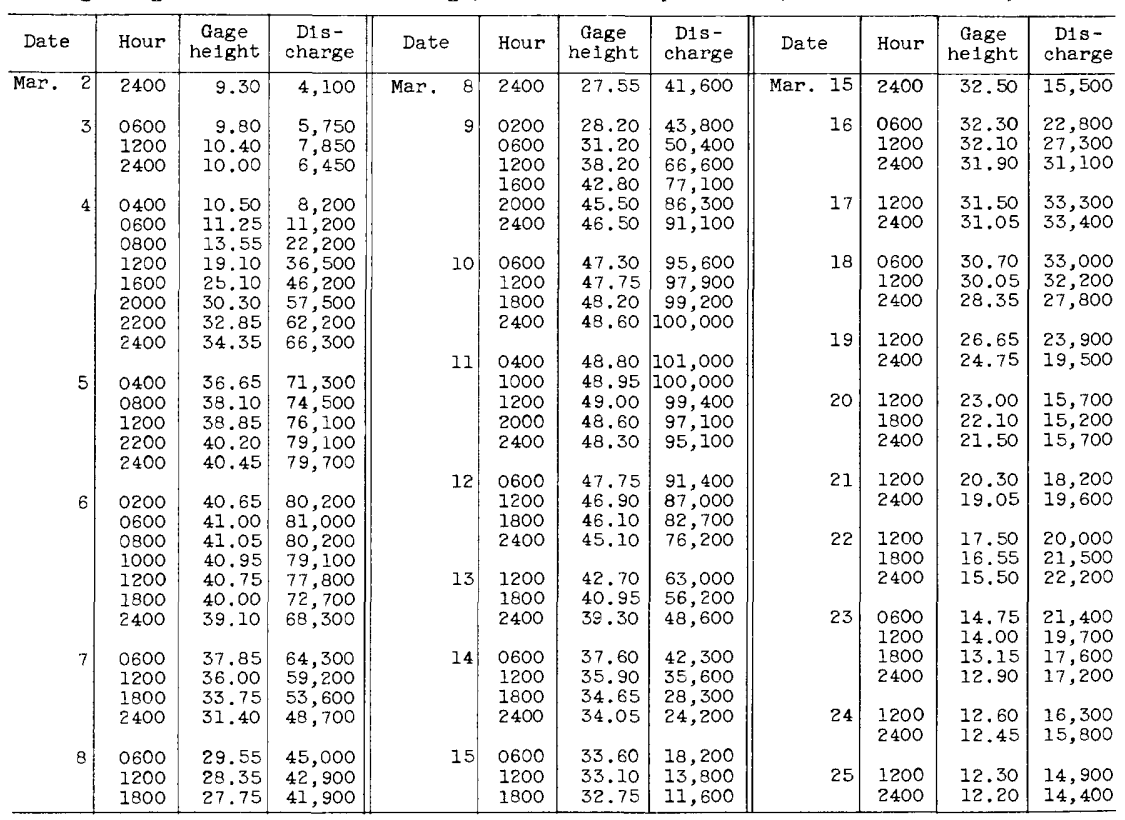

(73) 3-2910. Big Eagle Creek at Sadieville, Ky.

Location. - Iat $38^{\circ} 23^{\prime} 22^{\prime \prime}$, long $84^{\circ} 32^{\prime} 36^{\prime \prime}$, on left bank $15 \mathrm{ft}$ upstream from Spoon Branch, a quarter of a mile west of Sadieville, Scott County, and 5.8 miles upstream from Iittle Eagle Creek. Records include flow of Spoon Branch.

Drainage area. $--42.9 \mathrm{sq} \mathrm{mi}$, includes that of Spoon Branch.

Gage-height record.--Water-stage recorder graph. Datum of gage is $757.18 \mathrm{ft}$ above mean sea level, unadjusted.

Discharge record.--Stage-discharge relation defined by current-meter measurements below $4,100 \mathrm{c}$ s and extended above by logarithmic plotting.

Maxima.--Given in the following table.

March 1964:

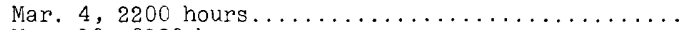

1932:

32:

$\begin{array}{cc}\begin{array}{c}\text { Discharge } \\ \text { (cfs) }\end{array} & \begin{array}{c}\text { Gage height } \\ \text { (feet) }\end{array} \\ 8,860 & 20.51 \\ 6,710 & 17.35 \\ \text { Unknown } & 22\end{array}$

1941 to February 1964 :

Unknown $\quad 22$

Mar. 19, 1943.

9,870

21.96 
Mean discharge, in cub1c feet per second, March 1964, of Big Eagle Creek at Sadiev1lie, Ky.

\begin{tabular}{|c|c|c|c|c|c|c|c|c|c|}
\hline Day & Discharge & Day & Discharge & Day & D1scharge & Day & Discharge & Day & Discharge \\
\hline $\begin{array}{l}1 \ldots \ldots \\
2 \ldots \ldots \\
3 \ldots \ldots \\
4 \ldots \\
5 \ldots \ldots \\
6 \ldots \ldots\end{array}$ & $\begin{array}{r}7.5 \\
18 \\
202 \\
4,180 \\
2,100 \\
96\end{array}$ & $\begin{array}{r}7 \ldots \ldots \\
8 \ldots \ldots \\
9 \ldots \ldots \\
10 \ldots \\
11 \ldots \ldots \\
12 \ldots \ldots\end{array}$ & $\begin{array}{r}57 \\
725 \\
3,610 \\
2,760 \\
134 \\
69\end{array}$ & $\begin{array}{l}13 \ldots \ldots \\
14 \ldots \ldots \\
15 \ldots \ldots \\
16 \ldots \ldots \\
17 \ldots \ldots \\
18 \ldots \ldots\end{array}$ & $\begin{array}{r}46 \\
165 \\
360 \\
77 \\
44 \\
28\end{array}$ & $\begin{array}{l}19 \ldots \ldots \\
20 \ldots \ldots \\
21 \ldots \ldots \\
22 \ldots \ldots \\
23 \ldots \ldots \\
24 \ldots \ldots\end{array}$ & $\begin{array}{r}20 \\
38 \\
449 \\
83 \\
48 \\
34\end{array}$ & $\begin{array}{l}25 \ldots \ldots \\
26 \ldots \ldots \\
27 \ldots \ldots \\
28 \ldots \ldots \\
29 \ldots \ldots \\
30 \ldots \ldots \\
31 \ldots \ldots\end{array}$ & $\begin{array}{r}236 \\
479 \\
57\end{array}$ \\
\hline
\end{tabular}

Gage height, in feet, and discharge, in cublc feet per second, at indicated t1me, 1964

\begin{tabular}{|c|c|c|c|c|c|c|c|c|c|c|c|}
\hline Date & Hour & $\begin{array}{c}\text { Gage } \\
\text { he1ght }\end{array}$ & $\begin{array}{l}\text { Dis- } \\
\text { charge }\end{array}$ & Date & Hour & $\begin{array}{c}\text { Gage } \\
\text { helght }\end{array}$ & $\begin{array}{c}\text { Dis- } \\
\text { charge }\end{array}$ & Date & Hour & $\begin{array}{c}\text { Gage } \\
\text { helght }\end{array}$ & $\begin{array}{c}\text { Dis- } \\
\text { charge }\end{array}$ \\
\hline \multirow[t]{33}{*}{ Mar. } & 2400 & 1.70 & 7.5 & \multirow[t]{8}{*}{ Mar. 5} & 0600 & 12.32 & 3,830 & \multirow[t]{10}{*}{ Mar. 9} & 1300 & 15.09 & 5,350 \\
\hline & & & & & 0800 & 8.20 & 2,030 & & 1400 & 14.76 & 5,160 \\
\hline & 0600 & 1.73 & 8.8 & & 1000 & 4.50 & 785 & & 1600 & 13.90 & 4,640 \\
\hline & 1200 & 1.79 & 12 & & 1200 & 3.43 & 410 & & 1800 & 13.10 & 4,220 \\
\hline & 1800 & 1.91 & 22 & & 1600 & 2.97 & 256 & & 1900 & 12.88 & 4,110 \\
\hline & 2400 & 2.08 & 48 & & 2000 & 2.74 & 192 & & 2000 & 13.35 & 4,340 \\
\hline & & & & & 2400 & 2.58 & 150 & & 2200 & 15.30 & 5,480 \\
\hline & 0200 & 2.48 & 125 & & & & & & 2300 & 15.90 & 5,840 \\
\hline & 0400 & 2.82 & 215 & \multirow[t]{5}{*}{6} & 0600 & 2.42 & 112 & & 2400 & 16.30 & 6,080 \\
\hline & 0600 & 2.89 & 232 & & 1200 & 2.30 & 87 & & & & \\
\hline & 0800 & 3.12 & 302 & & 1800 & 2.24 & 75 & \multirow[t]{12}{*}{10} & 0200 & 17.32 & 6,690 \\
\hline & 0900 & 3.19 & 326 & & 2400 & 2.20 & 67 & & 0230 & 17.35 & 6,710 \\
\hline & 1000 & 3.17 & 320 & & & & & & 0300 & 17.28 & 6,670 \\
\hline & 1200 & 3.05 & 280 & 7 & 1200 & 2.13 & 56 & & 0400 & 17.00 & 6,500 \\
\hline & 1800 & 2.62 & 160 & & 2400 & 2.08 & 48 & & 0600 & 15.85 & 5,810 \\
\hline & 2400 & 2.38 & 103 & & & & & & 0900 & 11.93 & 3,640 \\
\hline & & & & 8 & 0400 & 2.07 & 46 & & 1200 & 6.58 & 1,440 \\
\hline & 0500 & 2.30 & 87 & & 0900 & 2.09 & 50 & & 1400 & 4.36 & 736 \\
\hline & 0600 & 3.43 & 410 & & 1000 & 2.40 & 107 & & 1600 & 3.65 & 488 \\
\hline & 0700 & 8.57 & 2,180 & & 1200 & 3.80 & 540 & & 2000 & 3.15 & 312 \\
\hline & 0800 & 10.28 & 2,860 & & 1300 & 3.90 & 575 & & 2400 & 2.87 & 228 \\
\hline & 1000 & 11.12 & 3,230 & & 1400 & 4.15 & 662 & & & & \\
\hline & 1200 & 12.57 & 3,960 & & 1600 & 6.76 & 1,510 & \multirow[t]{5}{*}{11} & 0600 & 2.62 & 160 \\
\hline & 1500 & 15.36 & 5,520 & & 1700 & 7.28 & 1,690 & & 1200 & 2.46 & 121 \\
\hline & 1600 & 15.73 & 5,740 & & 1800 & 7.40 & 1,730 & & 1800 & 2.37 & 101 \\
\hline & 1700 & 16.33 & 6,100 & & 1900 & 7.42 & 1,740 & & 2400 & 2.30 & 87 \\
\hline & 2000 & 20.13 & 8,590 & & 2000 & 7.44 & 1,740 & & & & \\
\hline & 2100 & 20.30 & 8,710 & & 2100 & 7.21 & 1,660 & \multirow[t]{2}{*}{12} & 1200 & 2.20 & 67 \\
\hline & $\begin{array}{l}2200 \\
2300\end{array}$ & $\begin{array}{l}20.51 \\
20.25\end{array}$ & $\begin{array}{l}8,860 \\
8,680\end{array}$ & & 2400 & 6.15 & 1,290 & & 2400 & 2.12 & 54 \\
\hline & 2400 & 19.60 & 8,220 & 9 & 0200 & 5.11 & 973 & \multirow[t]{4}{*}{13} & 1200 & 2.06 & 45 \\
\hline & & & & & 0400 & 4.17 & 669 & & 2400 & 2.04 & 42 \\
\hline & 0200 & 18.00 & 7,100 & & 0600 & 7.66 & 1,820 & & & & \\
\hline & 0400 & 15.68 & 5,710 & & 1200 & 14.92 & 5,250 & & & & \\
\hline
\end{tabular}

(74) 3-2915. Eagle Creek at Glencoe, Ky.

Location.--Lat $38^{\circ} 42^{\prime} 18^{\prime \prime}$, long $84^{\circ} 49^{1} 26^{\prime \prime}$, on left bank $600 \mathrm{ft}$ upstream from bridge on State Highway $16,0.6$ mile south of Glencoe, Gallatin County, 5.8 miles downstream from Tenmile Creek, and 22 miles upstream from mouth.

Drainage area. $-437 \mathrm{sq} \mathrm{mi}$.

Gage-height record. - Water-stage recorder graph except Mar. 9, 2100 hours to Mar. 10, 0900 hours, for which graph was reconstructed on the basis of high-water mark on gage house. Datum of gage is $508.36 \mathrm{ft}$ above mean sea level, unadjusted.

Discharge record.--Stage-discharge relation defined by current-meter measurements below 40,000 cfs.

Maxima.--Given in the following table.

March 1964:

Mar. 5, 0100 hours.

Discharge

(cfs)

51,100

58,200

Gage height

$1913,1915-20,1988-31,1937$ to February 1964 :

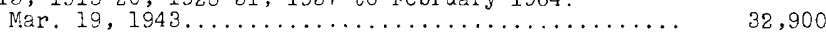

(feet)

24.95

26.05

23.60 
Mean discharge, in cub1c feet per second, March 1964, of Eagle Creek at Glencoe, Ky.

\begin{tabular}{|c|c|c|c|c|c|c|c|c|c|}
\hline Day & Discharge & Day & Discharge & Day & Discharge & Day & Discharge & Day & Discharge \\
\hline $\begin{array}{l}1 \ldots \ldots \\
2 \ldots \ldots \\
3 \ldots \ldots \\
4 \ldots \\
5 \ldots \ldots \\
6 \ldots \ldots\end{array}$ & $\begin{array}{r}50 \\
48 \\
196 \\
17,500 \\
32,000 \\
7,370\end{array}$ & $\begin{array}{r}7 \ldots \ldots \\
8 \ldots \ldots \\
9 \ldots \ldots \\
10 \ldots \\
11 \ldots \ldots \\
12 \ldots \ldots\end{array}$ & $\begin{array}{r}894 \\
2,630 \\
29,000 \\
39,300 \\
8,440 \\
1,290\end{array}$ & $\begin{array}{l}13 \ldots \ldots \\
14 \ldots \ldots \\
15 \ldots \ldots \\
16 \ldots \ldots \\
17 \ldots \ldots \\
18 \ldots \ldots\end{array}$ & $\begin{array}{r}737 \\
2,160 \\
3,610 \\
1,670 \\
739 \\
426\end{array}$ & $\begin{array}{l}19 \ldots \ldots \\
20 \ldots \ldots \\
21 \ldots \ldots \\
22 \ldots \ldots \\
23 \ldots \ldots \\
24 \ldots \ldots\end{array}$ & $\begin{array}{r}270 \\
220 \\
1,450 \\
1,930 \\
725 \\
400\end{array}$ & $\begin{array}{l}25 \ldots \ldots \\
26 \ldots \ldots \\
27 \ldots \ldots \\
28 \ldots \ldots \\
29 \ldots \ldots \\
30 \ldots \ldots \\
31 \ldots \ldots\end{array}$ & $\begin{array}{r}287 \\
4,410 \\
1,970 \\
614 \\
361 \\
248 \\
155\end{array}$ \\
\hline yy & 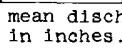 & & & & & & & & $\begin{array}{l}5,197 \\
13.71\end{array}$ \\
\hline
\end{tabular}

Gage height, in feet, and discharge, in cub1c feet per second, at ind1cated t1me, 1964

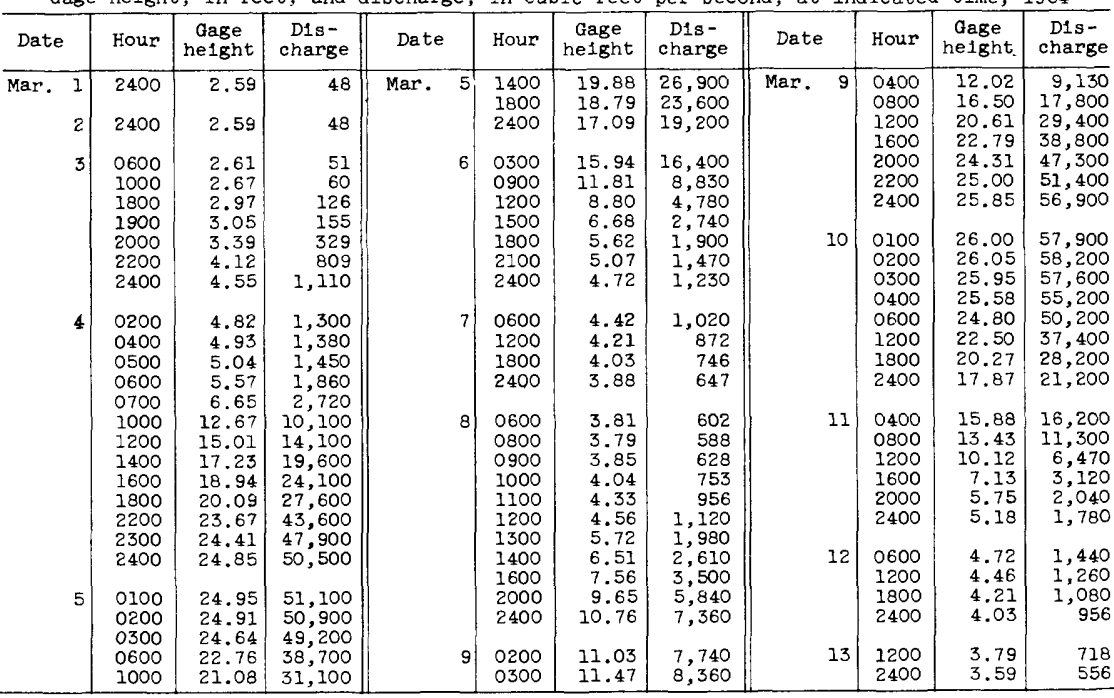

\section{BEARGRASS CREEK BASIN}

(75) 3-2925. South Fork Beargrass Creek at Louisville, Ky.

Location.--Lat $38^{\circ} 12^{\prime} 39^{\prime \prime}$, long $85^{\circ} 42^{\prime} 07^{\prime \prime}$, on upstream side of right abutment of Trevilian Way Bridge at Louisville, Jefferson County, 4.9 miles upstream from confluence with Middle Fork and 6.4 miles upstream from Onio River.

Drainage area. $--17.2 \mathrm{sq} \mathrm{ml}$.

Gage-height record. - Water-stage recorder graph (no low-water record). Datum of gage is 448.60 ft above mean sea level, Loulsville city datum, adfustment of 1912.

Discharge record.--Stage-discharge relation defined by current-meter measurements. Discharge for periods Mar. 3,2400 hours to Mar. 4, 0400 hours and Mar. 12, 1800 hours to 2400 hours, estimated on basis of records for Middle Fork Beargrass Creek.

Maxima.--Given in the following table.

March 1964

Mar. 4, 1900 hours. . . . . . . . . . . . . . .

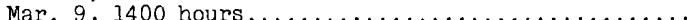

1939 to February 1964:

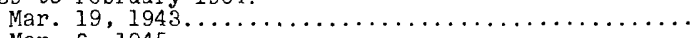

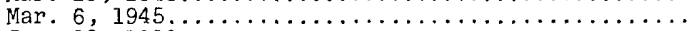

June $23,1960 \ldots \ldots \ldots \ldots \ldots \ldots \ldots \ldots \ldots \ldots \ldots \ldots \ldots$

$\begin{array}{cc}\begin{array}{c}\text { Discharge } \\ \text { (cfs) }\end{array} & \begin{array}{c}\text { Gage he fight } \\ \text { (feet) }\end{array} \\ 1,370 & 8.67 \\ 4,940 & 14.17 \\ & 15.1 \\ \text { Jnknown } & 13.62 \\ - & -\end{array}$


Mean discharge, in cublc feet per second, March 1964, of Middle Fork Beargrass Creek at Cannons Lane, at Loulsvilie, $\mathrm{Ky}$.

\begin{tabular}{|c|c|c|c|c|c|c|c|c|c|}
\hline Day & Discharge & Day & Discharge & Day & Discharge & Day & Discharge & Day & D1scharge \\
\hline $\begin{array}{l}1 \ldots \ldots \\
2 \ldots \ldots \\
3 \ldots \ldots \\
4 \ldots \\
5 \ldots \ldots \\
6 \ldots \ldots\end{array}$ & $\begin{array}{r}8.0 \\
9.1 \\
15 \\
612 \\
304 \\
137\end{array}$ & $\begin{array}{r}7 \ldots \ldots \\
8 \ldots \ldots \\
9 \ldots \\
10 \ldots \\
11 \ldots \\
12 \ldots\end{array}$ & $\begin{array}{r}92 \\
196 \\
2,000 \\
745 \\
322 \\
245\end{array}$ & $\begin{array}{l}13 \ldots \ldots \\
14 \ldots \ldots \\
15 \ldots \ldots \\
16 \ldots \ldots \\
17 \ldots \ldots \\
18 \ldots \ldots\end{array}$ & $\begin{array}{r}183 \\
191 \\
144 \\
97 \\
66 \\
47\end{array}$ & $\begin{array}{l}19 \ldots \ldots \\
20 \ldots \ldots \\
21 \ldots \ldots \\
22 \ldots \ldots \\
23 \ldots \ldots \\
24 \ldots \ldots\end{array}$ & $\begin{array}{l}38 \\
37 \\
38 \\
27 \\
24 \\
22\end{array}$ & $\mid \begin{array}{l}25 \ldots \ldots \\
26 \ldots \ldots \\
27 \ldots \ldots \\
28 \ldots \ldots \\
29 \ldots \ldots \\
30 \ldots \ldots \\
31 \ldots \ldots\end{array}$ & $\begin{array}{r}79 \\
168 \\
59 \\
43 \\
36 \\
28 \\
25\end{array}$ \\
\hline
\end{tabular}

Note. - Da1ly mean discharges computed on basis of 15-minute intervals.

Gage helght, in feet, and discharge, in cub1c feet per second, at ind1cated t1me, 1964

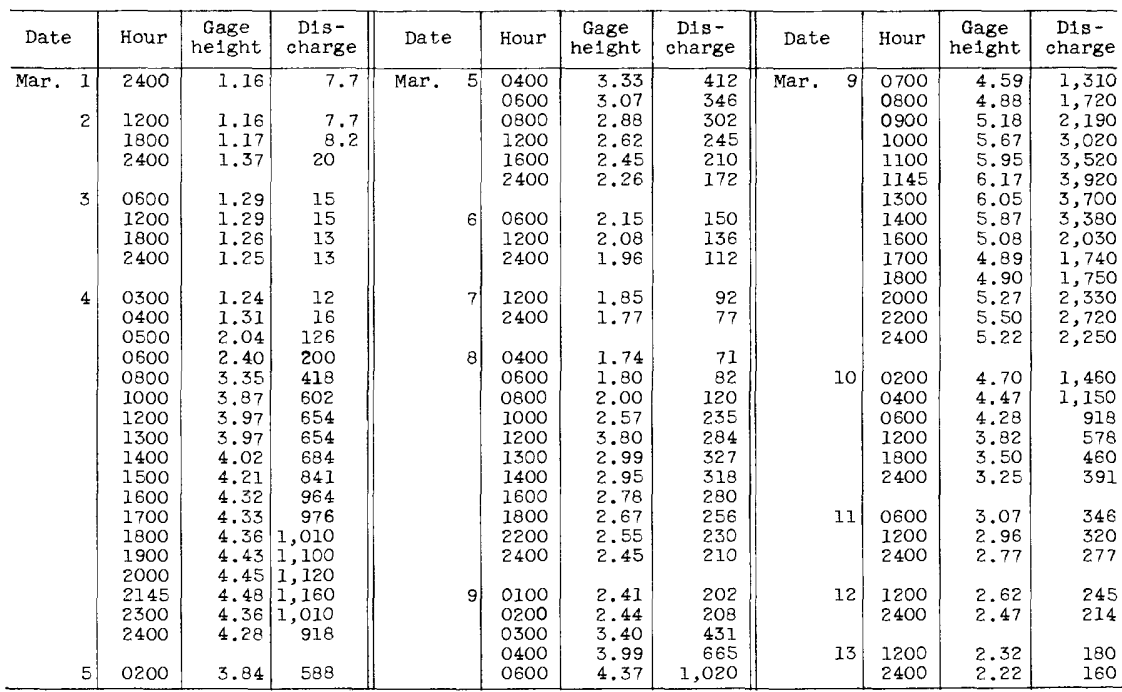

\section{SILVER CREEK BASIN}

(77) 3-2940. Silver Creek near Sellersburg, Ind.

Location.--Lat $38^{\circ} 22^{\prime} 15^{\prime \prime}$, long $85^{\circ} 43^{1} 35^{\prime \prime}$, in SW $\frac{1}{4}$ lot 68 , Clark Milltary Grant, on upstream side of Straws Mill bridge on Watson Road, $0.3 \mathrm{mile}$ downstream from Pleasant Run, 2.4 miles southeast of Sellersburg, and 11.9 miles upstream from mouth.

Drainage area. $--188 \mathrm{sq} \mathrm{ml}$.

Gage-helght record.--Graph drawn on basis of wire-weight-gage readings usually made twice dally. Peak stage of Mar. 10 was determined from high-water marks. Alt1tude of gage is $430 \mathrm{ft}$ (from topographic map).

Discharge record.--Stage-discharge relation defined by current-meter measurements below 15,000 cfs and by contracted-opening measurements at 16,600 and $19,500 \mathrm{cfs}$. Backwater from Oh10 R1ver Mar. 12-20; d1scharge estimated on basis of records for stations on nearby streams.

Maxima.--Given in the following table.

March 1964:

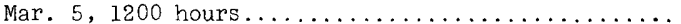

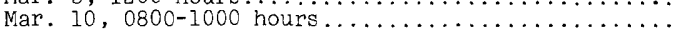
1954 to February 1964 :

Jan. $22,1959$.

$\begin{array}{cc}\begin{array}{c}\text { Discharge } \\ \text { (cfs) }\end{array} & \begin{array}{c}\text { Gage height } \\ \text { feet) }\end{array} \\ 14,800 & 29.76 \\ 15,600 & 30.40 \\ \text { a19,600 } & 30.89\end{array}$

a From rating curve extended above 6,300 cfs on basis of contracted-opening measurements of peak flow, at site 5.2 miles upstream (drainage area, $164 \mathrm{sq} \mathrm{ml}$ ), adjusted to gage site. 
Mean discharge, in cublc feet per second, March 1964, of S11ver Creek near Sellersburg, Ind.

\begin{tabular}{|c|c|c|c|c|c|c|c|c|c|}
\hline Day & Discharge & Day & Discharge & Day & Discharge & Day & Discharge & Day & Discharge \\
\hline $\begin{array}{l}1 \ldots \ldots \\
2 \ldots \\
3 \ldots \\
4 \ldots \\
5 \ldots \\
6 \ldots\end{array}$ & $\begin{array}{r}20 \\
23 \\
56 \\
3,410 \\
13,800 \\
8,070\end{array}$ & $\begin{array}{r}7 \ldots \ldots \\
8 \ldots \ldots \\
9 \ldots \ldots \\
10 \ldots \\
11 \ldots \ldots \\
12 \ldots \ldots\end{array}$ & $\begin{array}{r}1,100 \\
1,010 \\
7,490 \\
15,100 \\
9,480 \\
3,040\end{array}$ & $\begin{array}{l}13 \ldots \ldots \\
14 \ldots \ldots \\
15 \ldots \ldots \\
16 \ldots \ldots \\
17 \ldots \ldots \\
18 \ldots \ldots\end{array}$ & $\begin{array}{r}1,290 \\
844 \\
1,120 \\
688 \\
410 \\
304\end{array}$ & $\begin{array}{l}19 \ldots \ldots \\
20 \ldots \ldots \\
21 \ldots \ldots \\
22 \ldots \ldots \\
23 \ldots \ldots \\
24 \ldots \ldots\end{array}$ & $\begin{array}{l}258 \\
225 \\
227 \\
166 \\
140 \\
126\end{array}$ & $\begin{array}{l}25 \ldots \ldots \\
26 \ldots \ldots \\
27 \ldots \ldots \\
28 \ldots \ldots \\
29 \ldots \ldots \\
30 \ldots \ldots \\
31 \ldots \ldots\end{array}$ & $\begin{array}{r}182 \\
513 \\
240 \\
157 \\
126 \\
106 \\
90\end{array}$ \\
\hline $\begin{array}{l}\text { nthly } \\
\text { noff', }\end{array}$ & ean dis & ge, & & per & & & & & $\begin{array}{l}2,252 \\
13.81\end{array}$ \\
\hline
\end{tabular}

Gage height, in feet, and discharge, in cublc feet per second, at indicated time, 1964

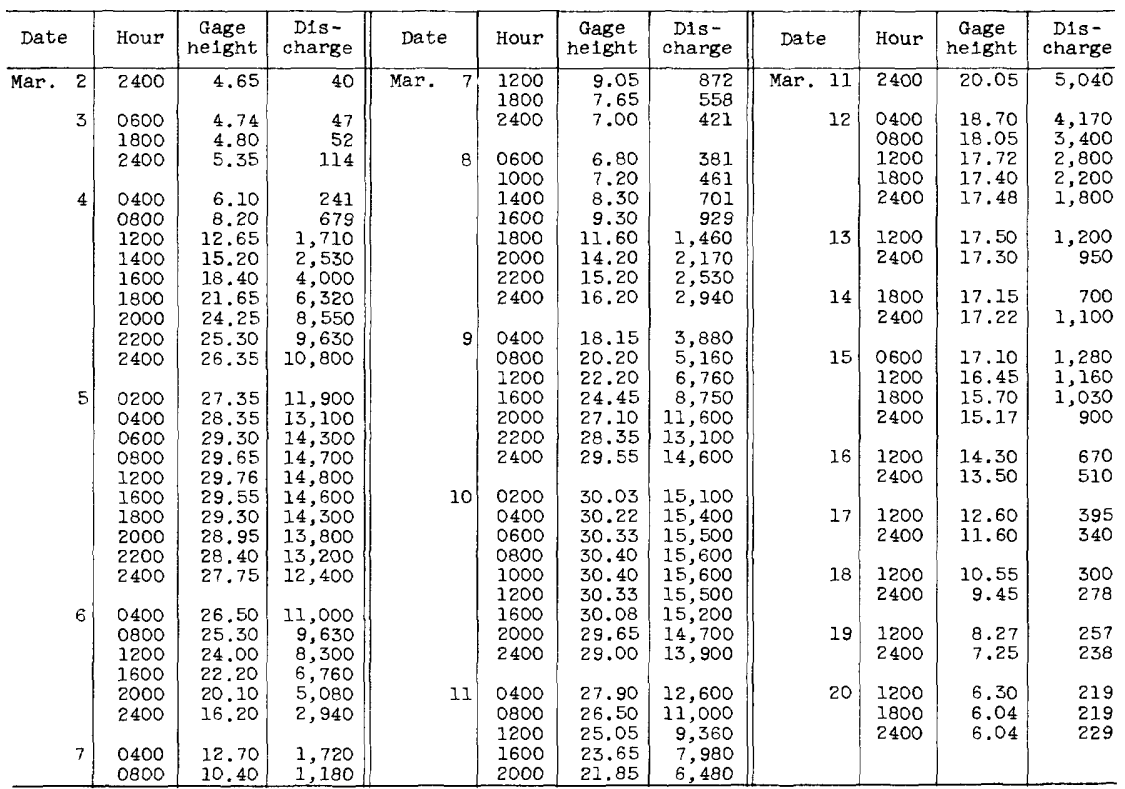




\section{OHIO RIVER MAIN STEM}

(78) 3-2945. Ohio River at Loulsville, Ky.

Location.--Lat $38^{\circ} 16^{\prime} 49^{\prime \prime}$, long $85^{\circ} 47^{\prime} 57^{\prime \prime}$, on left bank at downstream end of lock guide wall in lower pool at McAlpine locks at Louisville, Jefferson County,

5.3 miles downstream from Beargrass Creek and at mile 607.3.

Dra1nage area. $--91,170 \mathrm{sq} \mathrm{mi}$, approximately.

Gage-height record. - Water-stage recorder graph. Graph drawn for auxiliary upper staff gage, 26.4 miles downstream, from hourly readings. Datum of gage is 374.00 ft above mean sea level, onlo River datum.

Discharge record.--Stage-fall-discharge relation or stage-discharge relation defined by current-meter measurements. Fall used as a factor in computing discharge

Mar. I to 0600 hours Mar. 4; rate of change in stage used as a factor thereafter.

Max1ma.--March 1964: Discharge, 785,000 cfs Mar. 12, 0900 hours; gage he1ght, 73.46 f't Mar. 13,0500 hours.

$1832,1848,1858-59,1866-69,1872$ to February 1964: Discharge, 1,110,000 cf's Jan. 26, 27, 1937; gage he1ght, 85.44 ft Jan. 27, 1937 .

Remarks.--Partly regulated by locks, dams and reservoirs.

Cooperation.--Auxiliary gage-helght record and 5 discharge measurements furntshed by Corps of Engineers.

Mean discharge, in cuble feet per second, March 1964

\begin{tabular}{|c|c|c|c|c|c|c|c|c|c|}
\hline Day & Discharge & Day & Discharge & Day & D1scharge & Day & Discharge & Day & Discharge \\
\hline $\begin{array}{l}1 \ldots \\
2 \ldots \\
3 \ldots \\
4 \ldots \\
5 \ldots \\
6 \ldots \\
\ldots\end{array}$ & $\begin{array}{r}38,900 \\
42,000 \\
54,400 \\
151,000 \\
411,000 \\
475,000\end{array}$ & $\begin{array}{r}7 \ldots \ldots \\
8 \ldots \\
9 \ldots \\
10 \ldots \\
11 \ldots \\
12 \ldots\end{array}$ & $\begin{array}{l}477,000 \\
482,000 \\
587,000 \\
706,000 \\
770,000 \\
780,000\end{array}$ & $\begin{array}{l}13 \ldots \ldots \\
14 \ldots \ldots \\
15 \ldots \ldots \\
16 \ldots \\
17 \ldots \ldots \\
18 \ldots \ldots\end{array}$ & $\begin{array}{l}770,000 \\
747,000 \\
717,000 \\
688,000 \\
654,000 \\
619,000\end{array}$ & $\mid \begin{array}{l}19 \ldots \ldots \\
20 \ldots \ldots \\
21 \ldots \ldots \\
22 \ldots \ldots \\
23 \ldots \ldots \\
24 \ldots \ldots\end{array}$ & $\begin{array}{l}584,000 \\
539,000 \\
487,000 \\
435,000 \\
362,000 \\
290,000\end{array}$ & $\mid \begin{array}{l}25 \ldots \ldots \\
26 \ldots \ldots \\
27 \ldots \ldots \\
28 \ldots \ldots \\
29 \ldots \ldots \\
30 \ldots \ldots \\
31 \ldots \ldots\end{array}$ & $\begin{array}{l}236,000 \\
218,000 \\
200,000 \\
177,000 \\
160,000 \\
154,000 \\
148,000\end{array}$ \\
\hline onthly & mean disc & rge, in & $6 \cos 100$ & $501-2$ & & 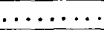 & & & 424,500 \\
\hline
\end{tabular}

Mean discharge, in cublc feet per second, April 1964

Apr. $1 \ldots \ldots \ldots \ldots 142,000$

$2 \ldots \ldots \ldots \ldots 136,000$

Gage height, in feet, and discharge, in cub1c feet per second, at indicated time, 1964

\begin{tabular}{|c|c|c|c|c|c|c|c|c|c|c|c|c|}
\hline Date & Hour & $\begin{array}{c}\text { Gage } \\
\text { helght }\end{array}$ & $\begin{array}{c}\text { D1s- } \\
\text { charge }\end{array}$ & Date & \multirow{2}{*}{$\begin{array}{l}\text { Hour } \\
1200 \\
1800\end{array}$} & \multirow{2}{*}{$\begin{array}{r}\begin{array}{c}\text { Gage } \\
\text { he1ght }\end{array} \\
70.95 \\
71.82\end{array}$} & \multirow{3}{*}{ 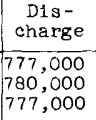 } & \multicolumn{2}{|l|}{ Date } & \multirow{2}{*}{$\frac{\text { Hour }}{2400}$} & \multirow{2}{*}{$\frac{\begin{array}{c}\text { Gage } \\
\text { helght }\end{array}}{56.75}$} & \multirow{2}{*}{$\frac{\begin{array}{c}\text { Dis- } \\
\text { charge }\end{array}}{465,000}$} \\
\hline Mar. I & 2400 & 11.52 & 34,700 & Mar. 11 & & & & Mar. & 21 & & & \\
\hline 2 & $\begin{array}{l}1200 \\
2400\end{array}$ & $\begin{array}{l}11.64 \\
12.34\end{array}$ & $\begin{array}{l}43,200 \\
47,400\end{array}$ & \multirow[b]{2}{*}{12} & & 72.41 & & \multirow{2}{*}{\multicolumn{2}{|c|}{22}} & \multirow[t]{2}{*}{$\begin{array}{l}1200 \\
2400\end{array}$} & \multirow[t]{2}{*}{$\begin{array}{l}54.81 \\
52.45\end{array}$} & \multirow[t]{2}{*}{$\begin{array}{l}437,000 \\
402,000\end{array}$} \\
\hline & & & & & 0600 & 72.84 & 778,000 & & & & & \\
\hline 3 & $\begin{array}{l}1200 \\
2400\end{array}$ & $\begin{array}{l}13.61 \\
14.12\end{array}$ & $\begin{array}{l}55,300 \\
59,400\end{array}$ & & $\begin{array}{l}0900 \\
1200 \\
1800\end{array}$ & $\begin{array}{l}73.00 \\
73.16 \\
73.33\end{array}$ & $\begin{array}{l}785,000 \\
782,000 \\
780,000\end{array}$ & \multicolumn{2}{|r|}{23} & $\begin{array}{l}1200 \\
2400\end{array}$ & $\begin{array}{l}49.57 \\
46.33\end{array}$ & $\begin{array}{l}363,000 \\
321,000\end{array}$ \\
\hline 4 & $\begin{array}{l}0600 \\
1200 \\
1800\end{array}$ & $\begin{array}{l}14.62 \\
18.50 \\
24.85\end{array}$ & $\begin{array}{r}74,600 \\
122,000 \\
213,000\end{array}$ & 13 & $\begin{array}{l}2400 \\
0500\end{array}$ & $\begin{array}{r}73.41 \\
73.46\end{array}$ & $\begin{array}{l}774,000 \\
775,000\end{array}$ & \multicolumn{2}{|r|}{24} & $\begin{array}{l}1200 \\
2400\end{array}$ & $\begin{array}{l}43.07 \\
39.85\end{array}$ & $\begin{array}{l}292,000 \\
257,000\end{array}$ \\
\hline & 2400 & .60 & 330,000 & & $\begin{array}{l}1200 \\
1800\end{array}$ & $\begin{array}{l}73.40 \\
73.33\end{array}$ & $\begin{array}{l}774,000 \\
765,000\end{array}$ & \multirow{2}{*}{\multicolumn{2}{|c|}{25}} & $\begin{array}{l}1200 \\
2400\end{array}$ & $\begin{array}{l}35.68 \\
34.24\end{array}$ & $\begin{array}{l}228,000 \\
229,000\end{array}$ \\
\hline 5 & 0600 & 50 & 392,000 & & 2400 & 73.18 & 762,000 & & & & & \\
\hline & $\begin{array}{l}1200 \\
1800 \\
2400\end{array}$ & $\begin{array}{l}.43 \\
.23 \\
.82\end{array}$ & $\begin{array}{l}417,000 \\
438,000 \\
463,000\end{array}$ & 14 & $\begin{array}{l}1200 \\
2400\end{array}$ & $\begin{array}{l}72.76 \\
72.22\end{array}$ & $\begin{array}{l}746 \\
735\end{array}$ & \multicolumn{2}{|r|}{26} & $\begin{array}{l}1200 \\
2400\end{array}$ & $\begin{array}{l}32.89 \\
32.90\end{array}$ & $\begin{array}{l}217,000 \\
210,000\end{array}$ \\
\hline & & & & & & & & \multirow{2}{*}{\multicolumn{2}{|c|}{27}} & 12 & & 201 \\
\hline 6 & $\begin{array}{l}0600 \\
1200\end{array}$ & $\begin{array}{l}49.70 \\
51.07\end{array}$ & $\begin{array}{l}473,000 \\
478,000\end{array}$ & 15 & $\begin{array}{l}1200 \\
2400\end{array}$ & $\begin{array}{l}71.57 \\
70.83\end{array}$ & $\begin{array}{l}716,000 \\
702,000\end{array}$ & & & & & \\
\hline & 2400 & & & 16 & 1200 & & & \multirow{2}{*}{\multicolumn{2}{|c|}{28}} & $\begin{array}{l}1200 \\
2400\end{array}$ & $\begin{array}{l}28.36 \\
27.00\end{array}$ & $\begin{array}{l}177,000 \\
166,000\end{array}$ \\
\hline 7 & 0600 & 30 & 480 & & 2400 & 27 & 67 & & & & & \\
\hline & $\begin{array}{l}1200 \\
2400\end{array}$ & $\begin{array}{l}53.62 \\
54.00\end{array}$ & $\begin{array}{l}475,000 \\
475,000\end{array}$ & 17 & $\begin{array}{l}1200 \\
2400\end{array}$ & & $\begin{array}{l}65 \\
63\end{array}$ & \multicolumn{2}{|r|}{29} & & & $\left\{\begin{array}{l}159,000 \\
155,000\end{array}\right.$ \\
\hline 8 & 1200 & 27 & & & & & & \multirow{2}{*}{\multicolumn{2}{|c|}{30}} & 1200 & & 000 \\
\hline & 2400 & & & 18 & $\begin{array}{l}1200 \\
2400\end{array}$ & $\begin{array}{l}66.40 \\
65.40\end{array}$ & $\begin{array}{l}619,000 \\
603,000\end{array}$ & & & 0 & & 00 \\
\hline 9 & 0600 & 28 & 540 & & & & & \multirow{2}{*}{\multicolumn{2}{|c|}{31}} & 1200 & & 000 \\
\hline & $\begin{array}{l}1200 \\
2400\end{array}$ & & $\begin{array}{l}596,000 \\
669,000\end{array}$ & 19 & $\begin{array}{l}1200 \\
2400\end{array}$ & $\begin{array}{l}64.34 \\
63.10\end{array}$ & $\begin{array}{l}581 \\
570\end{array}$ & & & & & \\
\hline 10 & $\begin{array}{l}1200 \\
1800\end{array}$ & $\begin{array}{l}65.57 \\
67.24\end{array}$ & $\begin{array}{l}701,000 \\
732,000\end{array}$ & 20 & $\begin{array}{l}1200 \\
2400\end{array}$ & $\begin{array}{l}61.74 \\
60.26\end{array}$ & $\begin{array}{l}539,000 \\
508,000\end{array}$ & \multirow{2}{*}{\multicolumn{2}{|c|}{ Apr. I }} & $\begin{array}{l}1200 \\
2400\end{array}$ & $\begin{array}{l}22.90 \\
22.72\end{array}$ & $\begin{array}{l}141,000 \\
139,000\end{array}$ \\
\hline & 2400 & 68.70 & 746,000 & 21 & 1200 & 58.60 & 487,000 & & & $\begin{array}{l}1200 \\
2400\end{array}$ & $\begin{array}{l}22.38 \\
21.80\end{array}$ & $\begin{array}{l}137,000 \\
132,000\end{array}$ \\
\hline
\end{tabular}




\section{SALT RIVER BASIN}

(79) 3-2950. Salt River near Harrodsburg, Ky.

Location.--Lat $37^{\circ} 45^{\prime} 26^{\prime \prime}$, long $84^{\circ} 52^{\prime} 23^{\prime \prime}$, near center of span on downstream side of bridge on State Highway $152,1 \frac{1}{2}$ miles west of Harrodsburg, Mercer County, and 11 miles downstream from Quirks Run.

Drainage area. $--41.4 \mathrm{sq} \mathrm{ml}$, of which about $2 \mathrm{sq} \mathrm{ml}$ does not contribute directly to surface runoff.

Gage-helght record.--Graph drawn from twlce-dally wire-weight-gage readings and peak from crest-stage gage, except Mar. 1, 15, when no readings were made. Datum of gage is $810.60 \mathrm{ft}$ above mean sea level, unadjusted.

D1scharge record.--Stage-discharge relation defined by current-meter measurements below 2,400 cfs. Discharge for Mar. 1, 15. estimated on basis of records for stations on nearby streams.

Naxima.--Given in the following table.

Narch 1964:

Mar. 4, 1600 hours.

Mar 10 . 0800 hours.

1952 to February 1964:

Nov. $18,1957$.

$\begin{array}{cc}\begin{array}{c}\text { Discharge } \\ \text { (cfs) }\end{array} & \text { Gage helght } \\ 3,400 & \text { (feet) } \\ 3,450 & 13.9 \\ & 14.0\end{array}$

4,190

15.1

Mean discharge, in cubic feet per second, March 1964

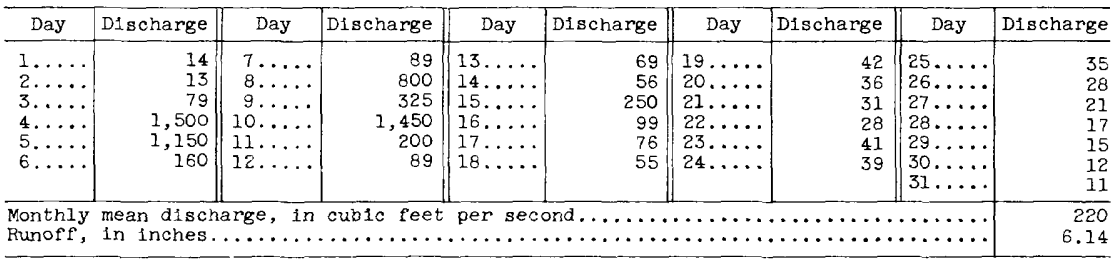

(80) 3-2955. Salt River near Van Buren, Ky.

Location.--Lat $37^{\circ} 58^{1} 06^{\prime \prime}$, long $85^{\circ} 08^{103^{\prime \prime}}$, on right bank at downstream side of Goodnight Bridge, $1.8 \mathrm{miles}$ east of Van Buren, Anderson County, and 2.3 miles upstream from Crooked Creek.

Drainage area. $-196 \mathrm{sq} \mathrm{ml}$, of which about $4 \mathrm{sq} \mathrm{ml}$ does not contribute directly to surface runoff.

Gage-height record.--Digital recorder tape punched at 30-minute intervals. Datum of gage is $535.38 \mathrm{ft}$ above mean sea level, datum of 1929 .

Discharge record.--Stage-discharge relation defined by current-meter measurements below 9,000 ifs and extended above by logarithmic plotting.

Maxima.--Given in the following table.

March 1964:

Mar. 4, 2000 hours

D1scharge

(cfs)

16,700

Gage helght

Mar 9, 2200 hours.

11,100

(feet)

1928 , 1937 to February 1964 :

20,000

21.16

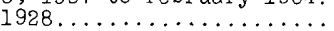

22.2

Flood in 1928 is maximum known. 
Mean discharge, in cuble feet per second, March 1964, of Salt River near Van Buren, Ky.

\begin{tabular}{|c|c|c|c|c|c|c|c|c|c|}
\hline Day & D1 scharge & Day & Discharge & Day & Discharge & Day & D1scharge & Day & D1scharge \\
\hline $\begin{array}{l}1 \ldots \ldots \\
2 \ldots \ldots \\
3 \ldots \ldots \\
4 \ldots \ldots \\
5 \ldots \ldots \\
6 \ldots \ldots\end{array}$ & $\begin{array}{r}74 \\
84 \\
237 \\
9,090 \\
8,130 \\
2,470\end{array}$ & $\begin{array}{r}7 \ldots \ldots \\
8 \ldots \ldots \\
9 \ldots \ldots \\
10 \ldots \\
11 \ldots \ldots \\
12 \ldots \ldots\end{array}$ & $\begin{array}{r}707 \\
2,810 \\
7,620 \\
7,170 \\
3,100 \\
757\end{array}$ & $\begin{array}{l}13 \ldots \ldots \\
14 \ldots \ldots \\
15 \ldots \ldots \\
16 \ldots \ldots \\
17 \ldots \ldots \\
18 \ldots \ldots\end{array}$ & $\begin{array}{r}438 \\
420 \\
1,310 \\
706 \\
368 \\
267\end{array}$ & $\begin{array}{l}19 \ldots \ldots \\
20 \ldots \ldots \\
21 \ldots \ldots \\
22 \ldots \ldots \\
23 \ldots \ldots \\
24 \ldots \ldots\end{array}$ & $\begin{array}{l}211 \\
198 \\
666 \\
379 \\
255 \\
209\end{array}$ & $\begin{array}{l}25 \ldots \ldots \\
26 \ldots \ldots \\
27 \ldots \ldots \\
28 \ldots \ldots \\
29 \ldots \ldots \\
30 \ldots \ldots \\
31 \ldots \ldots\end{array}$ & $\begin{array}{r}180 \\
165 \\
133 \\
111 \\
99 \\
85 \\
74\end{array}$ \\
\hline
\end{tabular}

Note.-- Dally mean discharges computed on basis of 30-minute intervals.

Gage he1ght, in feet, and discharge, in cubic feet per second, at indicated t1me, 1964

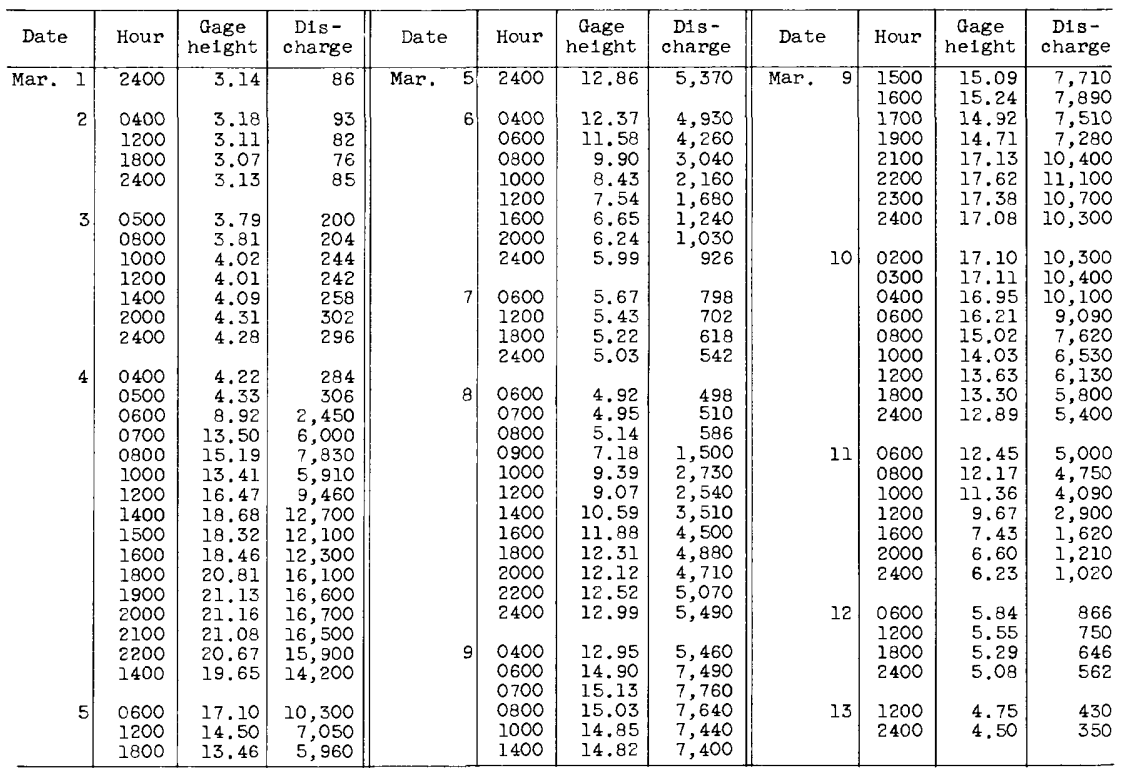

(81) Salt River at Taylorsville, Ky.

(Miscellaneous site)

Iocation.--Iat $38^{\circ} 01^{\prime} 41^{\prime \prime}$, long $85^{\circ} 20^{\prime} 34^{\prime \prime}$, at State Highway 55 bridge at Taylorsville, Spencer County, and $0.3 \mathrm{mile}$ upstream from Brashears Creek.

Drainage area. $--359 \mathrm{sq} \mathrm{mi}$.

Discharge record.--Stage-discharge relation defined by two current-meter measurements at 18,000 and $21,700 \mathrm{cfs}$.

Maxima.--Given in the following table.

March 1964:

$\begin{array}{cc}\text { Discharge } & \text { Elevation } \\ \text { (cfs) } & \text { (feet) } \\ 27,000 & 495.84 \\ 40,000 & 498.29\end{array}$

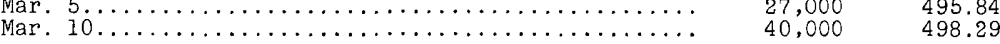

Cooperation.--Two discharge measurements and elevations from high-water marks furnished by Corps of Engineers. 
(82) Bullskin Creek near Simpsonville, Ky.

(M1scellaneous site)

Location.--Lat $38^{\circ} 12^{\prime} 10^{\prime \prime}$, long $85^{\circ} 18^{\prime} 58^{\prime \prime}$, at bridge on Interstate Highway $64,200 \mathrm{ft}$ downstream from L1ttle Buliskin Creek, and 2.5 miles southeast of Simpsonville, Shelby County, Ky.

Drainage area. $--66.1 \mathrm{sq} \mathrm{m} 1$.

Discharge record.--Peak discharge by contracted-opening measurement.

Maximum.--March 1964: Discharge, 17,600 cfs Mar. 9, 1964 (elevation, $687.95 \mathrm{ft}$ above mean sea level, datum of 1929 ).

(83) Clear Creek near Shelbyville, Ky.

(Miscellaneous site)

Location --Lat $38^{\circ} 11^{\prime} 07^{\prime \prime}$, long $85^{\circ} 14147^{\prime \prime}$, at bridge on Interstate Highway 64 , 2.3 miles southwest of Shelbyvilie, Shelby County, $\mathrm{Ky}$.

Drainage area. $--52.1 \mathrm{sq} \mathrm{ml}$.

Discharge record.--Peak discharge by contracted-opening measurement.

Maximum.--March 1964: Discharge, 15,000 cfs Mar. 9, 1964 (elevation, $683.44 \mathrm{ft}$ above mean sea level, datum of 1929).

(84) 3-2960. Plum Creek subwatershed No. 4 near Simpsonville, Ky.

Location.--Lat $38^{\circ} 10^{\prime} 27^{\prime \prime}$, long $85^{\circ} 22^{\prime} 05^{\prime \prime}$, near center of upstream side of Tucker Dam on tributary to Plum Creek, a quarter of a mile upstream from mouth and 3.5 miles south of Simpsonville, Shelby County.

Dra1nage area. $-1.55 \mathrm{sq} \mathrm{m} 1$.

Gage-he1ght record.--Water-stage recorder graph. Datum of gage is $687.99 \mathrm{ft}$ above mean sea level (levels by Soll Conservation Service).

Discharge record.--Stage-discharge relation defined by current-meter measurements.

Maxima.--Given in the following table.

March 1964:

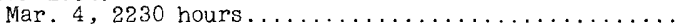

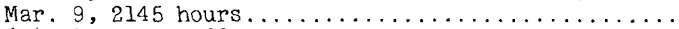

1954 to February 1964:

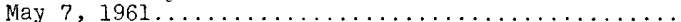

$\begin{array}{cc}\begin{array}{c}\text { Discharge } \\ \text { (cfs) }\end{array} & \begin{array}{c}\text { Gage height } \\ \text { (feet) }\end{array} \\ 152 & 22.0 \\ 163 & 25.06 \\ & \\ 153 & 22.42\end{array}$

Remarks.--Records of discharge are outflow from reservolr, determined from stagedischarge relation for outlet structure. Reservolr is formed by earth dam; dam completed and storage began in October 1954. Outlet structure is a 4-foot square concrete drop inlet connected to a 36 -inch concrete outlet pipe. A $120^{\circ} \mathrm{V}$-notch weir with notch at gage height $15.18 \mathrm{ft}$ is set in upstream side of drop inlet. Top of drop inlet is at gage helght $15.96 \mathrm{ft}$, emergency spillway at gage helght $27.0 \mathrm{ft}$. Reservolr capacity at top of drop inlet, 90.0 acre-ft. Capacity at emergency splilway level, 365 acre-ft.

Mean discharge, in cubic feet per second, March 1964

\begin{tabular}{|c|c|c|c|c|c|c|c|c|c|}
\hline Day & Discharge & Day & Discharge & Day & Discharge & Day & Discharge & Day & Discharge \\
\hline $\begin{array}{l}1 \ldots \ldots \\
2 \ldots \ldots \\
3 \ldots \ldots \\
4 \ldots \ldots \\
5 \ldots \ldots \\
6 \ldots \ldots\end{array}$ & $\begin{array}{c}0.20 \\
.25 \\
78 \\
.35 \\
44 \\
2.9\end{array}$ & $\begin{array}{r}7 \ldots \ldots \\
8 \ldots \ldots \\
9 \ldots \\
10 \ldots \\
11 \ldots \ldots \\
12 \ldots \ldots\end{array}$ & $\begin{array}{c}2.1 \\
18 \\
132 \\
63 \\
4.3 \\
2.5\end{array}$ & $\begin{array}{l}13 \ldots \ldots \\
14 \ldots \ldots \\
15 \ldots \ldots \\
16 \ldots \ldots \\
17 \ldots \ldots \\
18 \ldots \ldots\end{array}$ & $\begin{array}{l}1.9 \\
4.4 \\
3.4 \\
2.0 \\
1.5 \\
1.1\end{array}$ & $\begin{array}{l}19 \ldots \ldots \\
20 \ldots \ldots \\
21 \ldots \ldots \\
22 \ldots \ldots \\
23 \ldots \ldots \\
24 \ldots \ldots\end{array}$ & $\begin{array}{c}0.80 \\
.80 \\
1.6 \\
1.3 \\
.95 \\
.70\end{array}$ & $\begin{array}{l}25 \ldots \ldots \\
26 \ldots \ldots \\
27 \ldots \ldots \\
28 \ldots \ldots \\
29 \ldots \ldots \\
30 \ldots \ldots \\
31 \ldots \ldots\end{array}$ & $\begin{array}{l}3.9 \\
4.9 \\
1.8 \\
1.5 \\
1.1 \\
.75 \\
.55\end{array}$ \\
\hline
\end{tabular}


(85) 3-2965. Plum Creek near Wilsonville, Ky.

(Gaging station, discontinued 1961; crest-stage station)

Location.--Lat $38^{\circ} 06^{\prime} 20^{\prime \prime}$, long $85^{\circ} 26^{\prime} 14^{\prime \prime}$, on left bank 1.0 mile downstream from Drakes Branch, 2.2 miles southwest of wilsonville, Spencer County, and 3.7 miles upstream from Little Plum Creek.

Drainage area.--19.1 sq $\mathrm{mi}$

Gage-height record.--Water-stage recorder graph. Datum of gage is $582.99 \mathrm{ft}$ above mean sea level (levels by Soll Conservation Service).

Discharge record.--Stage-discharge relation defined by current-meter measurements below $2,400 \mathrm{c}$ 's and by contracted-opening measurement at $5,180 \mathrm{cfs}$.

Maxima.--Given in the following table.

March 1964:

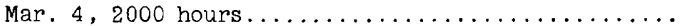

Mar. 9, 1000 hours $\ldots \ldots \ldots \ldots \ldots \ldots \ldots \ldots \ldots \ldots \ldots \ldots$
1954 to February 1964:

$\begin{array}{cc}\text { Discharge } & \text { Gage height } \\ \text { (cfs) } & \text { (feet) } \\ 3,890 & 6.59 \\ 3,920 & 6.62\end{array}$

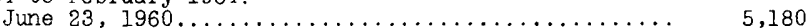

7.92

Remarks.--Flow appreciably regulated by seven small detention reservoirs.

(86) 3-2967. Plum Creek subwatershed No. 15 (Little Plum Creek) near W1lsonville, Ky.

(Gaging station, discontinued 1961; stage station)

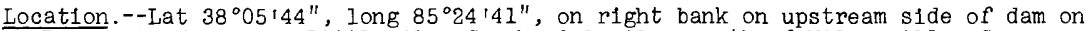
Reservolr No. 15 on Little Plum Creek, 2.1 miles south of W1lsonville, Spencer County, and 2.8 miles upstream from mouth.

Drainage area. $--1.03 \mathrm{sq} \mathrm{ml}$.

Gage-height record.--Water-stage recorder graph except Mar. 9. Crest-stage obtained from high-water mark.

Discharge record.--Stage-discharge relation defined by current-meter measurements.

Maxima. --Given in the following table.

March 1964.

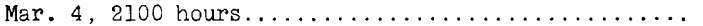

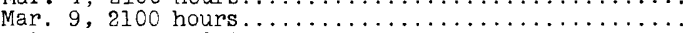

1957 to February 1964 :

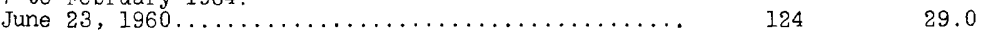

Remarks.--Records of discharge are outflow from reservolr, determined from stagedischarge relation for outlet structure. Reservoir is formed by earth dam; dam completed and storage began in February 1957. Outlet structure 1s a 4-foot square concrete drop inlet connected to a $30-1$ inch concrete outlet pipe. A $120^{\circ}$ $\mathrm{V}$-notch weir with notch at gage height $16.39 \mathrm{ft}$ is set in downstream side of drop inlet. Top of drop inlet is at gage helght $17.14 \mathrm{ft}$, emergency sp1llway at gage he1ght $29.6 \mathrm{ft}$. Reservo1r capac1ty at top of drop inlet, 63.5 acre-ft. Capac1ty at emergency spillway level, 225 acre-ft. 
(87) 3-2968. Plum Creek subwatershed No. 17 near Waterford, Ky.

(Gaging station discontinued 1961; stage station)

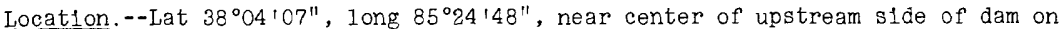
Reservolr No. $17,0.3 \mathrm{mile}$ upstream from confluence with unnamed tributary to Little Plum Creek, and $2.6 \mathrm{miles}$ northeast of waterford, Spencer county.

Drainage area. $--0.52 \mathrm{sq} \mathrm{mi}$.

Gage-he1ght record.--Water-stage recorder graph. Datum of gage is $577.20 \mathrm{ft}$ above mean sea leve1 (levels by Soll Conservation Service).

Discharge record.--Stage-discharge relation defined by current-meter measurements.

Maxima.--Given in the following table.

March 1964:

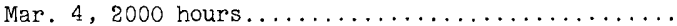

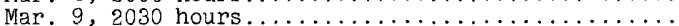

1957 to February 1964:

June $23,1960$.

$$
\text { : }
$$

$\begin{array}{cc}\begin{array}{c}\text { Discharge } \\ \text { (cfs) }\end{array} & \begin{array}{c}\text { Gage height } \\ \text { (feet) }\end{array} \\ 78 & 21.56 \\ 76 & 20.54 \\ 87 & 28.70\end{array}$

Remarks.--Records of discharge are outflow from reservolr, determined from stagedischarge relation for outlet structure. Reservolr is formed by earth dam; dam completed and storage began in May 1957. Outlet structure is a 4-foot square concrete drop inlet connected to a $24-1$ inch concrete outlet pipe. A $120^{\circ} \mathrm{V}$-notch weir with notch at gage height $15.13 \mathrm{ft}$ is set in downstream side of drop inlet. Top of drop inlet 1s at gage height $15.84 \mathrm{ft}$, emergency spi11way at gage height $30.8 \mathrm{ft}$. Reservoir capacity at top of drop inlet, 28.7 acre-ft. Capacity at emergency spillway level, 119 acre-ft.

(88) 3-2970. Little Plum Creek near Waterford, Ky.

(Gaging station, discontinued 1961; crest-stage station)

Location.--Lat $38^{\circ} 03^{1} 44^{\prime \prime}$, long $85^{\circ} 25^{1} 45^{\prime \prime}$, on right bank $800 \mathrm{ft}$ upstream from mouth and 1.7 miles north of Waterford, Spencer County.

Drainage area. $--5.15 \mathrm{sq} \mathrm{mi}$.

Gage-height record.-Water-stage recorder graph. Datum of gage is $506.37 \mathrm{ft}$ above mean sea level (levels by Soll Conservation Service).

Discharge record.--Stage-discharge relation defined by current-meter measurements below $900 \mathrm{cfs}$ and by slope-area measurement at $3,810 \mathrm{cfs}$.

Maxima.--Given in the following table.

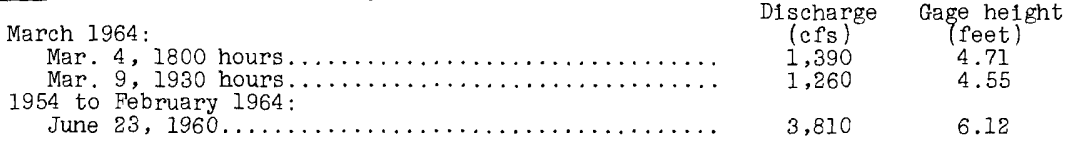

Remarks.--Flow appreciably regulated by three small detention reservolrs. 
(89) 3-2975. Plum Creek at Waterford, Ky.

Location.--Lat $38^{\circ} 03^{\prime} 05^{\prime \prime}$, long $85^{\circ} 25^{\prime} 5^{\prime \prime}$, on right bank $0.7 \mathrm{mile}$ downstream from Little Plum Creek, $1.0 \mathrm{mile}$ north of Waterford, Spencer County, and $3.2 \mathrm{miles}$ upstream from mouth.

Drainage area. $--31.8 \mathrm{sq} \mathrm{mi}$.

Gage-helght record.--Digital recorder tape punched at 15-minute intervals. Datum of gage is $479.70 \mathrm{ft}$ above mean sea level (levels by Soll Conservation Service).

Discharge record.--Stage-discharge relation defined by current-meter measurements below 3,200 cfs and by contracted-opening measurement at 13,200 cfs.

Maxima.--Given in the following table.

March 1964:

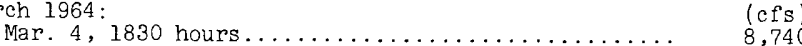

D1scharge Gage height

8,740

(feet)

Mar. 9, 1945 hours.

7,940

1954 to February 1964

13,200

11.84

Remarks.--Flow slightly regulated by 11 small detention reservoirs.

Mean discharge, in cubic feet per second, March 1964

\begin{tabular}{l} 
Day \\
\hline $1 \ldots \ldots$
\end{tabular}

Note.-Daily mean discharges computed on basis of 15 -minute intervals.

Gage height, in feet, and discharge, in cubic feet per second, at indicated time, 1964

\begin{tabular}{|c|c|c|c|c|c|c|c|c|c|c|c|}
\hline Date & Hour & $\begin{array}{c}\text { Gage } \\
\text { height }\end{array}$ & $\begin{array}{c}\text { Dis- } \\
\text { charge }\end{array}$ & Date & Hour & $\begin{array}{c}\text { Gage } \\
\text { height }\end{array}$ & $\begin{array}{c}\text { D1s- } \\
\text { charge }\end{array}$ & Date & Hour & $\begin{array}{c}\text { Gage } \\
\text { height }\end{array}$ & $\begin{array}{c}\text { Dis- } \\
\text { charge }\end{array}$ \\
\hline Mar. 1 & 2400 & 1.08 & 8.7 & Mar. 5 & 1900 & 2.04 & 177 & Mar. & 1200 & 7.64 & 6,760 \\
\hline & & & & & 2400 & 1.78 & 111 & & 1300 & 7.14 & 6,010 \\
\hline 2 & 1600 & 1.08 & 8.7 & & & & & & 1400 & 6.34 & 4,810 \\
\hline & 2400 & 1.17 & 15 & 6 & 0600 & 1.68 & 91 & & 1500 & 6.15 & 4,520 \\
\hline & & & & & 1800 & 1.59 & 73 & & 1600 & 5.47 & 3,500 \\
\hline 3 & 1200 & 1.19 & 16 & & 2400 & 1.57 & 70 & & 1700 & 5.05 & 2,880 \\
\hline & 2400 & 1.18 & 16 & & & & & & 1800 & 5.74 & 3,910 \\
\hline & & & & $?$ & 1200 & 1.50 & 57 & & 1900 & 7.54 & 6,610 \\
\hline 4 & 0300 & 1.18 & 16 & & 2400 & 1.47 & 52 & & 1945 & 8.43 & 7,940 \\
\hline & 0400 & 1.24 & 22 & & & & & & 2100 & 7.61 & 6,720 \\
\hline & 0500 & 1.64 & 83 & 8 & 0400 & 1.46 & 51 & & 2200 & 7.11 & 5,960 \\
\hline & 0600 & 3.26 & 818 & & 0500 & 1.49 & $5 \overline{5}$ & & 2300 & 6.24 & 4,660 \\
\hline & 0700 & 3.92 & 1,420 & & 0700 & 1.68 & 91 & & 2400 & 5.17 & 3,060 \\
\hline & 0800 & 4.34 & 1,910 & & 0800 & 1.96 & 155 & & & & \\
\hline & 0900 & 4.82 & 2,540 & & 0900 & 3.27 & 826 & 10 & 0100 & 4.58 & 2,210 \\
\hline & 1000 & 5.33 & 3,300 & & 1000 & 3.88 & 1,380 & & 0200 & 4.70 & 2,370 \\
\hline & 1100 & 5.88 & 4,120 & & 1100 & 3.90 & 1,400 & & 0300 & 4.61 & 2,250 \\
\hline & 1200 & 5.75 & 3,920 & & 1200 & 3.62 & 1,120 & & 0400 & 4.33 & 1,900 \\
\hline & 1300 & 5.65 & 3,780 & & 1300 & 3.35 & 890 & & 0600 & 3.97 & 1,470 \\
\hline & 1400 & 5.45 & 3,480 & & 1400 & 3.44 & 962 & & 0900 & 3.71 & 1,210 \\
\hline & 1500 & 5.13 & 3,000 & & 1500 & 3.81 & 1,310 & & 1200 & 3.34 & 882 \\
\hline & 1600 & 5.30 & 3,250 & & 1600 & 3.79 & 1,290 & & 1500 & 3.20 & 770 \\
\hline & 1700 & 6.11 & 4,460 & & 1700 & 3.69 & 1,190 & & 1800 & 3.11 & 707 \\
\hline & 1800 & 8.21 & 7,620 & & 1800 & 3.51 & 1,020 & & 2100 & 2.92 & 574 \\
\hline & 1830 & 8.96 & 8,740 & & 2100 & 2.96 & 602 & & 2400 & 2.54 & 368 \\
\hline & 1900 & 8.56 & 8,140 & & 2400 & 2.54 & 368 & & & & \\
\hline & 2000 & 8.43 & 7,940 & & & & & 11 & 0300 & 2.15 & 212 \\
\hline & 2100 & 7.95 & 7,220 & 9 & 0200 & 2.37 & 298 & & 0600 & 1.91 & 142 \\
\hline & 2200 & 6.35 & 4,820 & & 0300 & 2.58 & 386 & & 0900 & 1.77 & 109 \\
\hline & 2300 & 5.04 & 2,860 & & 0400 & 4.54 & 2,160 & & 1800 & 1.62 & 79 \\
\hline & 2400 & 4.27 & 1,820 & & 0500 & 6.59 & 5,180 & & 2400 & 1.57 & 70 \\
\hline & & & & & 0600 & & 5,280 & & & & \\
\hline 5 & $\begin{array}{l}0100 \\
0200\end{array}$ & $\begin{array}{l}3.97 \\
3.78\end{array}$ & $\begin{array}{l}1,470 \\
1,280\end{array}$ & & $\begin{array}{l}0700 \\
0800\end{array}$ & $\begin{array}{l}6.27 \\
6.74\end{array}$ & $\begin{array}{l}4,700 \\
5,410\end{array}$ & 12 & $\begin{array}{l}1200 \\
2400\end{array}$ & $\begin{array}{l}1.51 \\
1.44\end{array}$ & $\begin{array}{l}59 \\
47\end{array}$ \\
\hline & 0600 & 3.43 & $\begin{array}{r}1,254 \\
954\end{array}$ & & 0900 & 7.29 & 6,240 & & & & \\
\hline & 1200 & 2.79 & 494 & & 1000 & 7.51 & 6,560 & 13 & 1200 & 1.41 & 43 \\
\hline & 1800 & 2.21 & 234 & & 1100 & 7.90 & 7,150 & & 2400 & 1.36 & 35 \\
\hline
\end{tabular}


(90) Floyds Fork near Middletown, Ky .

(M1scellaneous site)

Location.--Lat $38^{\circ} 17117^{\prime \prime}$, long $85^{\circ} 28^{\prime} 18^{\prime \prime}$, at bridge on Interstate Highway 64 , $1.1 \mathrm{miles}$ southwest of Eastwood and 4.0 miles southeast of M1ddletown, Jefferson County, Ky.

Drainage area. --101 sq $\mathrm{mi}$.

Discharge record.--Peak discharge by contracted-opening measurement.

Maximum.--March 1964: Discharge, 22,000 cfs Mar. 9, 1964 (elevation, 584.11 ft above mean sea level, datum of 1929).

(91) 3-2980. Floyds Fork at F1sherville, Ky.

Location.--Lat $38^{\circ} 11^{\prime} 18^{\prime \prime}$, long $85^{\circ} 27^{\prime} 37^{\prime \prime}$, on left bank on downstream side of bridge on former State Highway 155, at Fisherville, Jefferson County, 0.2 mile downstream from Brush Run and 1.5 miles upstream from Poke Lick.

Drainage area.--138 sq $\mathrm{m1}$.

Gage-he1ght record. --Water-stage recorder graph except Mar. 9, 0700 hours to Mar. 10, 1000 hours for wh1ch graph was constructed on bas1s of high-water marks and outside gage readings. Datum of gage is $542.60 \mathrm{ft}$ above mean sea level, datum of 1929.

Discharge record.--Stage-discharge relation defined by current-meter measurements. Maxima.--Given in the following table.

March 1964:

Mar. 4, 2100 hours.

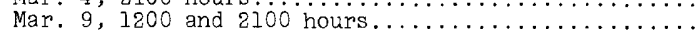
1937:

January 1937.

$\begin{array}{cc}\text { Discharge } & \text { Gage he1ght } \\ \text { (cfs) } & \text { (feet) } \\ 17,800 & \text { al4.03 } \\ 24,800 & \text { b15.25 }\end{array}$

1944 to February 1964 :

Unknown

c16.8

May 8,1961 .

19,800

d14.45

a From 1nside gage; floodmark gage he1ght $14.30 \mathrm{ft}^{\mathrm{t}}$.

b From inside gage; floodmark gage helght $15.70 \mathrm{ft}$.

c From floodmark.

d From inside gage; floodmark gage helght $14.7 \mathrm{ft}$.

Mean discharge, in cub1c feet per second, March 1964

\begin{tabular}{l}
\hline Day \\
\hline $1 \ldots \ldots$
\end{tabular}


Gage height, in feet, and discharge, in cublc feet per second, at 1nd1cated t1me, 1964, of Floyds Fork at Fisherville, Ky.

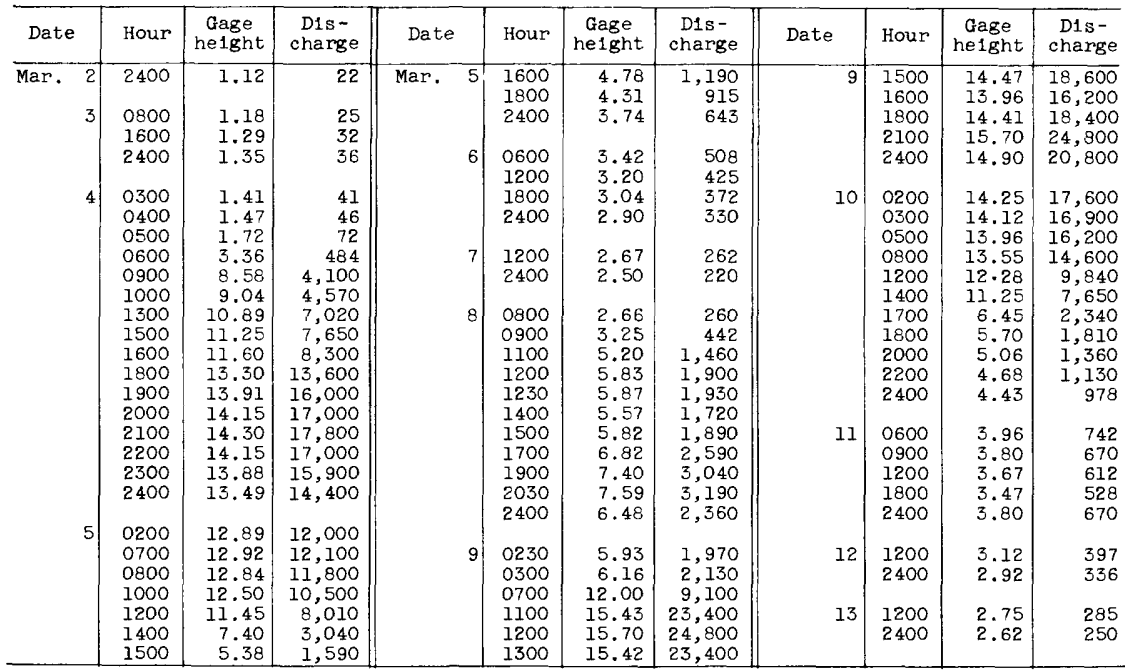

(92) 3-2985. Salt River at Shepherdsville, Ky.

Location.--Lat $37^{\circ} 59^{106^{\prime \prime}}$, long $85^{\circ} 43^{103^{\prime \prime}}$, near right bank on downstream side of pier of bridge on State Highway 61 at Shepherdsville, Bullitt County, 500 ft down-

stream from Louisville \& Nashville Railroad bridge and 2.5 miles downstream from Floyds Fork.

Drainage area $--1,197 \mathrm{sq} \mathrm{m} 1$.

Gage-he1ght record.--Water-stage recorder graph. Datum of gage is $406.62 \mathrm{ft}$ above mean sea level, datum of 1929.

Discharge record.--Stage-fall-discharge relation or stage-discharge relation defined by current-meter measurements. Fall used as a factor Mar. 4 to 25.

Maxima.--Given in the following table.

March 1964

Mar. 5, 2100 hours.

Mar. 6, 0030 hours.......

$\ldots \ldots \ldots \ldots \ldots \ldots \ldots$

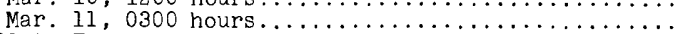

1938 to February 1964:

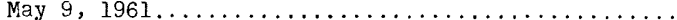

$\begin{array}{cc}\begin{array}{c}\text { Discharge } \\ \text { (cfs) }\end{array} & \text { Gage height } \\ 50,900 & - \\ \text { feet) } & - \\ 78,200 & 33.40 \\ - & - \\ & 41.50\end{array}$

May $9,1961 \ldots \ldots \ldots \ldots \ldots \ldots \ldots \ldots \ldots \ldots \ldots \ldots \ldots \ldots, 40_{-}^{-}$

Maximum stage known, $47.3 \mathrm{ft}$ Jan. 26,1937 , from floodmark (backwater from Ohio River.

Mean d1scharge, in cub1c feet per second, March 1964

\begin{tabular}{|c|c|c|c|c|c|c|c|c|c|}
\hline Day & D1scharge & Day & Discharge & Day & D1scharge & Day & Discharge & Day & D1scharge \\
\hline $\begin{array}{l}1 \ldots \ldots \\
2 \ldots \ldots \\
3 \ldots \ldots \\
4 \ldots \ldots \\
5 \ldots \\
6 \ldots\end{array}$ & $\begin{array}{r}365 \\
342 \\
417 \\
11,200 \\
43,800 \\
39,100\end{array}$ & $\begin{array}{r}7 \ldots \ldots \\
8 \ldots \ldots \\
9 \ldots \ldots \\
10 \ldots \\
11 \ldots \\
12 \ldots\end{array}$ & $\begin{array}{l}18,500 \\
10,800 \\
34,900 \\
73,500 \\
50,500 \\
22,600\end{array}$ & $\mid \begin{array}{l}13 \ldots \ldots \\
14 \ldots \ldots \\
15 \ldots \ldots \\
16 \ldots \ldots \\
17 \ldots \ldots \\
18 \ldots \ldots\end{array}$ & $\begin{array}{r}11,500 \\
8,410 \\
9,810 \\
9,600 \\
7,190 \\
6,100\end{array}$ & $\begin{array}{l}19 \ldots \ldots \\
20 \ldots \ldots \\
21 \ldots \ldots \\
22 \ldots \ldots \\
23 \ldots \ldots \\
24 \ldots \ldots\end{array}$ & $\begin{array}{l}4,470 \\
4,050 \\
3,540 \\
3,920 \\
3,150 \\
2,190\end{array}$ & $\mid \begin{array}{c}25 \ldots \ldots \\
26 \ldots \ldots \\
27 \ldots \ldots \\
28 \ldots \\
29 \ldots \\
30 \ldots \ldots \\
31 \ldots \ldots\end{array}$ & $\begin{array}{r}1,210 \\
4,330 \\
3,260 \\
1,640 \\
1,230 \\
960 \\
763\end{array}$ \\
\hline
\end{tabular}


Gage height, in feet, and discharge, in cublc feet per second, at indicated time, 1964 , of

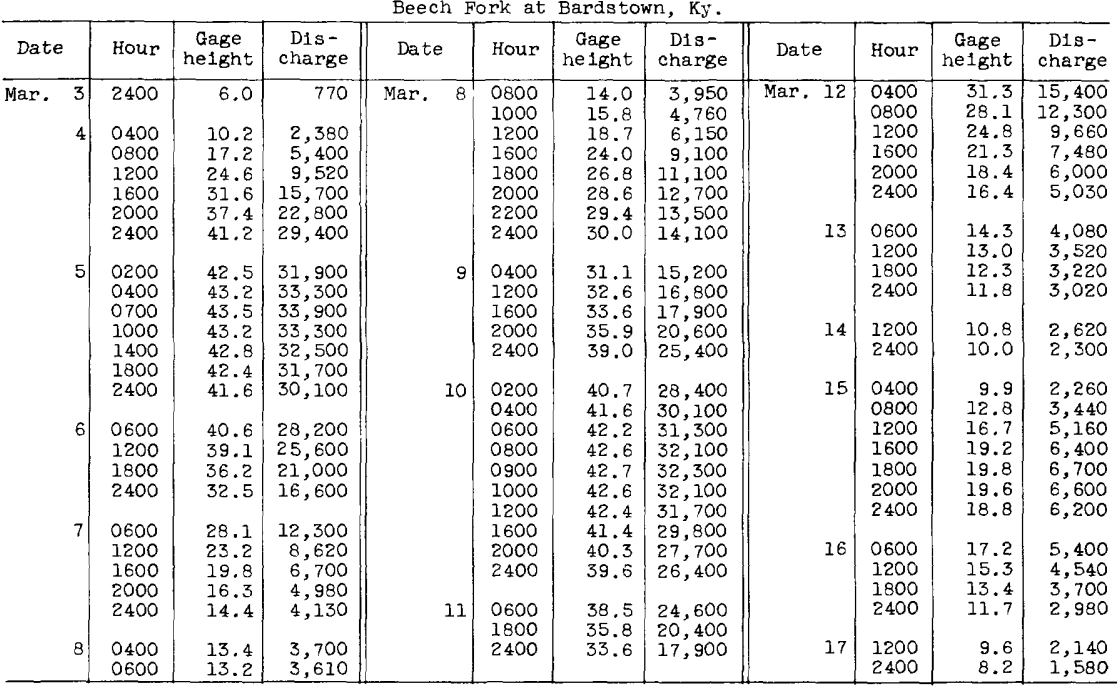

(96) 3-3015. Rolling Fork near Boston, Ky.

Location. - Lat $37^{\circ} 46^{\prime} 02^{\prime \prime}$, long $85^{\circ} 42^{\prime} 14^{\prime \prime}$, on downstream side near center of span of bridge on U.S. H1ghway 62 and State Highway $61,0.4$ mile downstream from Beech Fork and 2.3 miles southwest of Boston, Nelson County.

Drainage area $--1,299 \mathrm{sq} \mathrm{ml}$.

Gage-helght record.--Graph drawn on basis of floodmarks and twice-dally wire-welghtgage readings. Graph drawn for auxillary wire-welght gage, 7.6 miles downstream, from floodmark and once- or twice-dally gage readings. Datum of gage is $400.42 \mathrm{ft}$ above mean sea level, datum of 1929 .

D1scharge record.--Stage-fall-discharge or stage-discharge relation defined by current-meter measurements. Fall used as a factor Mar. 4-25.

Maxima.--Given in the following table.

March 1964

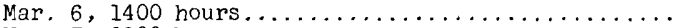

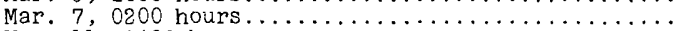

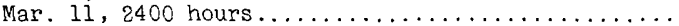

Mar. 12, 0900 hours......................

1938 to February 1964:

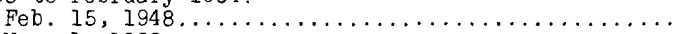

$\begin{array}{cc}\begin{array}{c}\text { Discharge } \\ \text { (cfs) }\end{array} & \text { Gage helght } \\ 42,100 & \text { feet) } \\ 35,900 & 48.0 \\ - & 51.0\end{array}$

41,300

Maximum stage known, $55.2 \mathrm{ft}$ in January 1937, former site, 1,400 ft upstream, from floodmarks (backwater from Ohlo Rlver).

Mean discharge, in cubic feet per second, March 1964

\begin{tabular}{|c|c|c|c|c|c|c|c|c|c|}
\hline Day & D1scharge & Day & D1scharge & Day & Discharge & Day & D1scharge & Day & D1scharge \\
\hline $\begin{array}{l}1 \ldots \ldots \\
2 \ldots \ldots \\
3 \ldots \ldots \\
4 \ldots \ldots \\
5 \ldots \ldots \\
6 \ldots \ldots\end{array}$ & $\begin{array}{r}472 \\
440 \\
681 \\
7,580 \\
31,300 \\
41,600\end{array}$ & $\begin{array}{r}7 \ldots \ldots \\
8 \ldots \ldots \\
9 \ldots \\
10 \ldots \\
11 \ldots \ldots \\
12 \ldots\end{array}$ & $\begin{array}{l}31,200 \\
18,000 \\
18,500 \\
24,700 \\
33,100 \\
29,000\end{array}$ & $\begin{array}{l}13 \ldots \ldots \\
14 \ldots \ldots \\
15 \ldots \ldots \\
16 \ldots \ldots \\
17 \ldots \ldots \\
18 \ldots \ldots\end{array}$ & $\begin{array}{r}8,720 \\
4,420 \\
6,390 \\
10,200 \\
9,530 \\
6,550\end{array}$ & $\begin{array}{l}19 \ldots \ldots \\
20 \ldots \ldots \\
21 \ldots \ldots \\
22 \ldots \ldots \\
23 \ldots \ldots \\
24 \ldots \ldots\end{array}$ & $\begin{array}{l}4,720 \\
3,570 \\
3,600 \\
4,090 \\
3,820 \\
2,630\end{array}$ & $\begin{array}{l}25 \ldots \ldots \\
26 \ldots \ldots \\
27 \ldots \ldots \\
28 \ldots \ldots \\
29 \ldots \ldots \\
30 \ldots \ldots \\
31 \ldots \ldots\end{array}$ & $\begin{array}{r}1,730 \\
1,330 \\
1,120 \\
968 \\
823 \\
718 \\
656\end{array}$ \\
\hline & 1 & & & & & & & & $\begin{array}{r}10,070 \\
8.94\end{array}$ \\
\hline
\end{tabular}


Gage helght, in feet, and discharge, In cub1e feet per second, at 1ndicated t1me, 1964, of Rolling Fork near Boston, Ky.

\begin{tabular}{|c|c|c|c|c|c|c|c|c|c|c|c|}
\hline Date & Hour & $\begin{array}{c}\text { Gage } \\
\text { he1ght }\end{array}$ & $\begin{array}{l}\text { D1s - } \\
\text { charge }\end{array}$ & Date & Hour & $\begin{array}{c}\text { Gage } \\
\text { height }\end{array}$ & $\begin{array}{l}\text { D1s- } \\
\text { charge }\end{array}$ & Date & Hour & $\begin{array}{c}\text { Gage } \\
\text { helght }\end{array}$ & $\begin{array}{c}\text { D1s- } \\
\text { charge }\end{array}$ \\
\hline Mar. 2 & 2400 & 7.45 & 432 & Mar. 9 & $\begin{array}{l}0600 \\
1200\end{array}$ & $\begin{array}{l}45.0 \\
45.3\end{array}$ & $\begin{array}{l}16,800 \\
18,400\end{array}$ & Mar. 16 & 2400 & 44.3 & 10,700 \\
\hline 3 & 1200 & 7.5 & 440 & & 1800 & 45.9 & 20,300 & 17 & 0600 & 43.9 & 10,60 \\
\hline & 1600 & 7.7 & 472 & & 2400 & 46.6 & 21,500 & & 1200 & 43.5 & $\begin{array}{l}9,720 \\
7,590\end{array}$ \\
\hline & 2400 & $\begin{array}{r}8.9 \\
14.9\end{array}$ & 2,040 & 10 & 0600 & 47.4 & 23,100 & & & & \\
\hline & & & & & 1200 & & 500 & 18 & 1200 & 41.1 & 6,47 \\
\hline 4 & $\begin{array}{l}1200 \\
1800\end{array}$ & $\begin{array}{l}26.8 \\
32.5\end{array}$ & $\begin{array}{r}5,800 \\
10,800\end{array}$ & & $\begin{array}{l}1800 \\
2400\end{array}$ & $\begin{array}{l}48.7 \\
49.4\end{array}$ & $\begin{array}{l}26,000 \\
28,800\end{array}$ & & 2400 & & 5.66 \\
\hline & 2400 & 37.4 & 17,600 & & & & & 19 & 1200 & 38.6 & 4,62 \\
\hline & & & & 11 & 0400 & 49.8 & 29,400 & & 2400 & .2 & 3,97 \\
\hline 5 & 0600 & 40.5 & 26,200 & & 1200 & 50.5 & 00 & & & & \\
\hline & 1200 & 42.4 & 32,600 & & 1800 & 50.8 & 600 & 20 & 1200 & 35.8 & 3,42 \\
\hline & $\begin{array}{l}1600 \\
2000\end{array}$ & $\begin{array}{l}43.3 \\
44.2\end{array}$ & $\begin{array}{l}35,900 \\
38,700\end{array}$ & & 2400 & 50.9 & 35,900 & & 2400 & 34.4 & 3,48 \\
\hline & 2400 & 44.9 & 00 & 12 & $\begin{array}{l}0600 \\
0900\end{array}$ & $\begin{array}{l}50.95 \\
51.0\end{array}$ & $\begin{array}{l}35,200 \\
33,100\end{array}$ & 21 & $\begin{array}{l}1200 \\
2400\end{array}$ & $\begin{array}{l}33.2 \\
32.0\end{array}$ & $\begin{array}{l}3,560 \\
3,810\end{array}$ \\
\hline 6 & 0200 & 45.3 & 41,300 & & 1200 & 50.9 & 30,400 & & & & \\
\hline & $\begin{array}{l}0600 \\
1000 \\
1400\end{array}$ & $\begin{array}{l}45.9 \\
46.5 \\
47.0\end{array}$ & $\begin{array}{l}41,700 \\
42,000 \\
42,100\end{array}$ & & $\begin{array}{l}1800 \\
2400\end{array}$ & $\begin{array}{l}50.7 \\
50.3\end{array}$ & $\begin{array}{l}24,100 \\
16,600\end{array}$ & 22 & $\begin{array}{l}1200 \\
2400\end{array}$ & $\begin{array}{l}30.7 \\
29.3\end{array}$ & $\begin{array}{l}4,23 \\
4,10\end{array}$ \\
\hline & 1800 & 47.4 & 41,700 & 13 & 0600 & 49.9 & 11,100 & 23 & 1200 & 27.5 & 3 \\
\hline & 2400 & 479 & 700 & & $\begin{array}{l}1200 \\
1800\end{array}$ & $\begin{array}{l}49.5 \\
49.0\end{array}$ & $\begin{array}{l}7,360 \\
5,820\end{array}$ & & 2400 & .1 & 3 \\
\hline 7 & $\begin{array}{l}0200 \\
0600\end{array}$ & $\begin{array}{l}48.0 \\
47.9\end{array}$ & $\begin{array}{l}39,900 \\
36,600\end{array}$ & & 2400 & 48.6 & 4,640 & 24 & $\begin{array}{l}0600 \\
1200\end{array}$ & $\begin{array}{l}23.4 \\
21.5\end{array}$ & $\begin{array}{l}2,98 \\
2,61\end{array}$ \\
\hline & 1200 & 47.6 & 31,700 & 14 & 1200 & 47 & & & 1800 & 19.4 & 2,22 \\
\hline & $\begin{array}{l}1800 \\
2400\end{array}$ & $\begin{array}{l}47.1 \\
46.5\end{array}$ & $\begin{array}{l}25,300 \\
21,300\end{array}$ & & 2400 & & & & 2400 & .1 & 0 \\
\hline & & & & 15 & 1200 & 46.3 & 6,170 & 25 & 1200 & 13.6 & 1,71 \\
\hline 8 & $\begin{array}{l}0600 \\
1200\end{array}$ & $\begin{array}{l}46.0 \\
45.5\end{array}$ & $\begin{array}{l}19,400 \\
17,800\end{array}$ & & 2400 & 45.7 & 8,350 & & 2400 & 12.6 & 1,46 \\
\hline & 2400 & 44.9 & 15,200 & 16 & $\begin{array}{l}0600 \\
1200\end{array}$ & $\begin{array}{l}45.4 \\
45.0\end{array}$ & $\begin{array}{l}10,100 \\
10,600\end{array}$ & 26 & $\begin{array}{l}1200 \\
2400\end{array}$ & $\begin{array}{l}12.0 \\
11.5\end{array}$ & $\begin{array}{l}1,320 \\
1,210\end{array}$ \\
\hline 9 & 0300 & 44.9 & 16,200 & & 1800 & 44.7 & 10,700 & & & & \\
\hline
\end{tabular}

(97) 3-3020. Pond Creek near Loulsville, Ky.

Location.--Lat $38^{\circ} 07^{\prime} I I^{\prime \prime}$, long $85^{\circ} 47145^{\prime \prime}$, on downstream side of bridge on Manslick Road, near center of span, $0.4 \mathrm{mlle}$ south of Third Street Road, 0.6 mile downstream from Bee Lick Creek, 1.5 miles downstream from confluence of Northem and Southern ditches, and 2.4 miles south of Loulsville clty limits, Jefferson County.

Drainage area,$-64.0 \mathrm{sq} \mathrm{ml}$.

Gage-he1ght record.--Digital recorder tape punched at 30-minute intervals. Datum of gage is 430.38 ft above mean sea level, datum of 1929.

Discharge record.--Stage-discharge relation defined by current-meter measurements. Backwater from Oh10 River Mar. 11-2I; discharge estimated on basis of records for Middle Fork Beargrass Creek at Louisville.

Maxima.--Given in the following table.

March 1964:

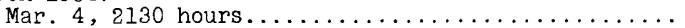

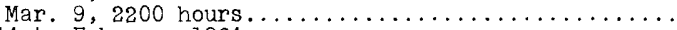

1944 to February 1964 :

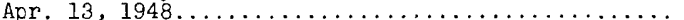

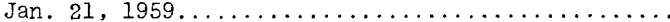

$\begin{array}{cc}\begin{array}{c}\text { Discharge } \\ \text { (cfs) }\end{array} & \begin{array}{c}\text { Gage height } \\ \text { (feet) }\end{array} \\ 5,460 & 19.52 \\ 8,020 & 22.69 \\ - & 21.78\end{array}$

Maximum stage known, about $23 \mathrm{ft}$ in January 1937 (backwater from Ohio River), from information by local residents. 


\begin{tabular}{|c|c|c|c|c|c|c|c|c|c|}
\hline Day & Discharge & Day & Discharge & Day & Discharge & Day & Discharge & Day & Discharge \\
\hline $\begin{array}{l}1 \ldots \\
2 \ldots \\
3 \ldots \\
4 \ldots \\
5 \ldots \\
6 \ldots \\
6 \ldots\end{array}$ & $\begin{array}{r}* 12 \\
* 14 \\
* 36 \\
* 3,030 \\
* 2,060 \\
* 219\end{array}$ & $\begin{array}{r}7 \ldots \ldots \\
8 \ldots \ldots \\
9 \ldots \\
10 \ldots \\
11 \ldots \ldots \\
12 \ldots \ldots\end{array}$ & $\begin{array}{r}* 125 \\
* 842 \\
* 5,530 \\
* 6,340 \\
1,370 \\
562\end{array}$ & $\begin{array}{l}13 \ldots \ldots \\
14 \ldots \ldots \\
15 \ldots \ldots \\
16 \ldots \ldots \\
17 \ldots \ldots \\
18 \ldots \ldots\end{array}$ & $\begin{array}{l}412 \\
392 \\
335 \\
220 \\
148 \\
101\end{array}$ & $\begin{array}{l}19 \ldots \ldots \\
20 \ldots \ldots \\
21 \ldots \ldots \\
22 \ldots \ldots \\
23 \ldots \ldots \\
24 \ldots \ldots\end{array}$ & $\begin{array}{r}76 \\
67 \\
74 \\
* 56 \\
* 43 \\
* 38\end{array}$ & $\begin{array}{l}25 \ldots \ldots \\
26 \ldots \ldots \\
27 \ldots \ldots \\
28 \ldots \ldots \\
29 \ldots \ldots \\
30 \ldots \ldots \\
31 \ldots \ldots\end{array}$ & $\begin{array}{l}* 150 \\
* 364 \\
* 95 \\
* 66 \\
* 46 \\
* 31 \\
* 29\end{array}$ \\
\hline
\end{tabular}

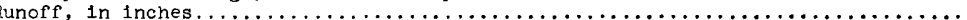

13.30

* Mean dally discharges computed on basis of 30-minute intervals.

Gage height, in feet, and discharge, in cuble feet per second, at indicated time, 1964

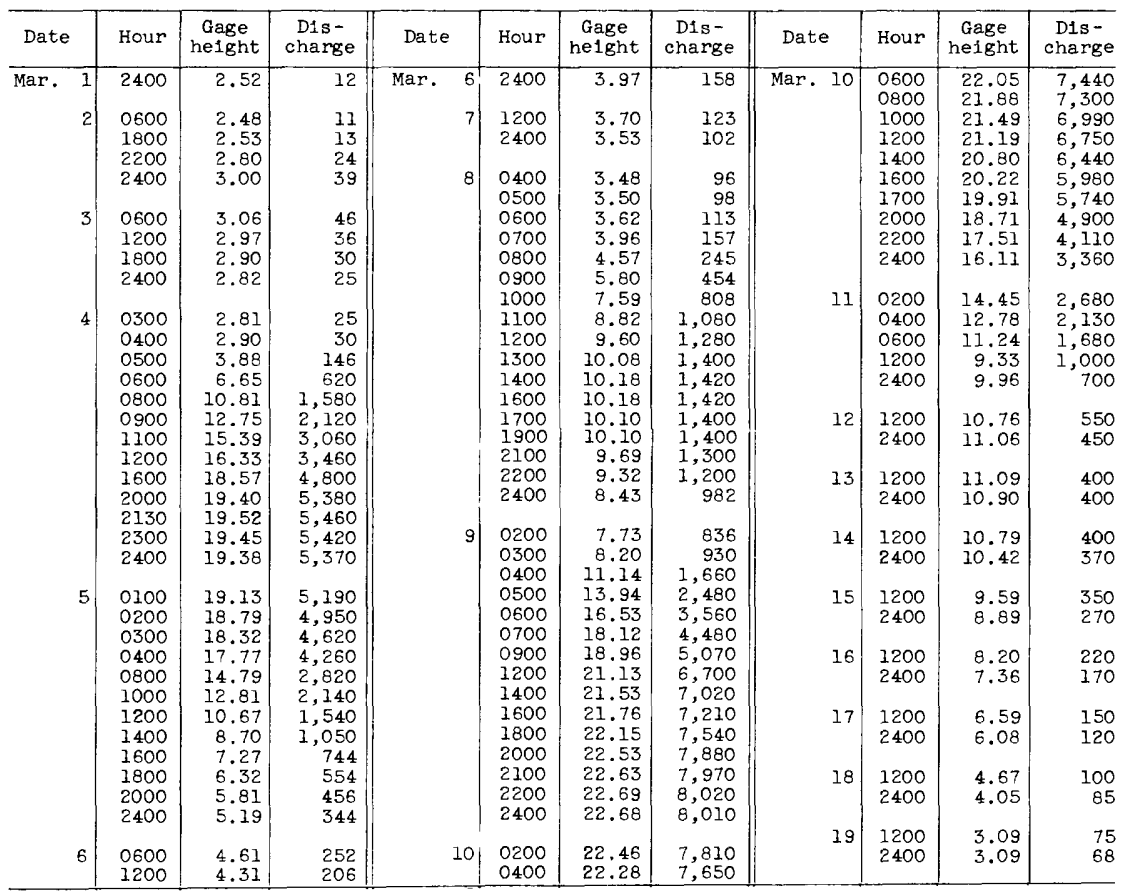




\section{INDIAN CREEK BASIN}

(98) 3-3025. Indian Creek near Corydon, Ind.

(Formerly published as Big Indian Creek near Corydon, Ind.)

Location. --Lat $38^{\circ} 16^{\prime} 35^{\prime \prime}$, long $86^{\circ} 06^{\prime} 35^{\prime \prime}$, in SE $\frac{1}{4}$ sec.6, T.3 S., R.4 E., on upstream side of bridge on State H1ghway 335, 0.6 mile upstream from Raccoon Branch and $4 \frac{1}{2}$ miles north of Corydon.

Drainage area. --129 sq $\mathrm{ml}$.

Gage-he1ght record. - Water-stage recorder graph. Datum of gage is $577.12 \mathrm{ft}$ above mean sea level, datum of 1929 .

Discharge record.--Stage-discharge relation defined by current-meter measurements. Maxima.--Given in the following table.

March 1964:

Mar. 5, 0230 hours.

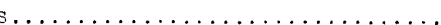

1943 to February 1964 :

Jan. $21,1959 . \ldots \ldots$. . .

$\begin{array}{cc}\begin{array}{c}\text { Discharge } \\ \text { (cfs) }\end{array} & \text { Gage height } \\ 26,700 & 22.64 \\ 17,600 & 21.13 \\ 23,800 & 22.22\end{array}$

Mean discharge, in cubic feet per second, March 1964

\begin{tabular}{|c|c|c|c|c|c|c|c|c|c|}
\hline Day & Discharge & Day & Discharge & Day & Discharge & Day & Discharge & Day & Discharge \\
\hline $\begin{array}{l}1 \ldots \ldots \\
2 \ldots \ldots \\
3 \ldots \ldots \\
4 \ldots \\
5 \ldots \ldots \\
6 \ldots \ldots\end{array}$ & $\begin{array}{r}25 \\
26 \\
46 \\
6,840 \\
11,500 \\
849\end{array}$ & $\begin{array}{r}7 \ldots \ldots \\
8 \ldots \ldots \\
9 \ldots \ldots \\
10 \ldots \\
11 \ldots \ldots \\
12 \ldots \ldots\end{array}$ & $\begin{array}{r}496 \\
1,210 \\
11,200 \\
9,450 \\
1,260 \\
737\end{array}$ & $\begin{array}{l}13 \ldots \ldots \\
14 \ldots \ldots \\
15 \ldots \ldots \\
16 \ldots \ldots \\
17 \ldots \ldots \\
18 \ldots \ldots\end{array}$ & $\begin{array}{l}482 \\
600 \\
598 \\
374 \\
273 \\
211\end{array}$ & $\begin{array}{l}19 \ldots \ldots \\
20 \ldots \ldots \\
21 \ldots \ldots \\
22 \ldots \ldots \\
23 \ldots \ldots \\
24 \ldots \ldots\end{array}$ & $\begin{array}{l}174 \\
162 \\
179 \\
152 \\
132 \\
121\end{array}$ & $\begin{array}{l}25 \ldots \ldots \\
26 \ldots \ldots \\
27 \ldots \ldots \\
28 \ldots \ldots \\
29 \ldots \ldots \\
30 \ldots \ldots \\
31 \ldots \ldots\end{array}$ & $\begin{array}{l}144 \\
700 \\
261 \\
188 \\
147 \\
122 \\
110\end{array}$ \\
\hline
\end{tabular}

Gage height, in feet, and discharge, in cubic feet per second, at indicated time, 1964

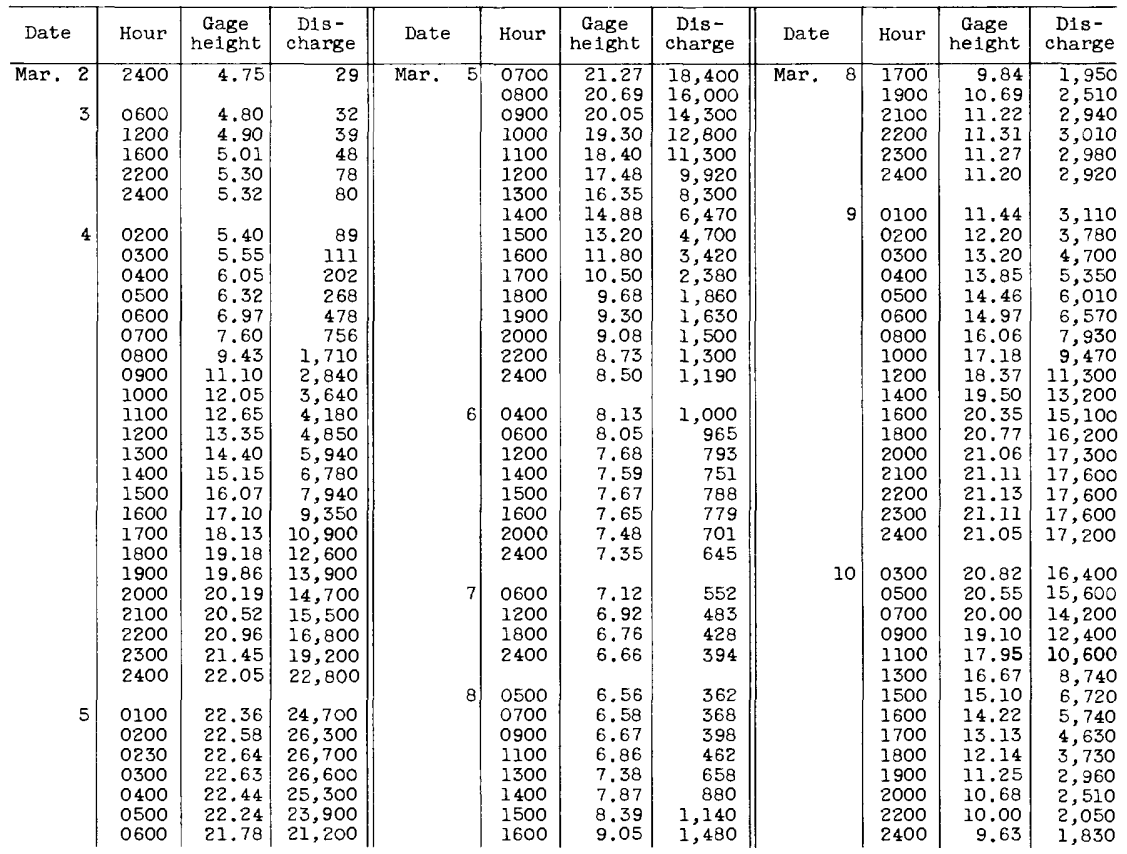


Gage height, in feet, and discharge, in cublc feet per second, at indlcated time, 1964, of Indian Creek near Corydon, Ind.--Continued

\begin{tabular}{|c|c|c|c|c|c|c|c|c|c|c|c|}
\hline Date & Hour & $\begin{array}{c}\text { Gage } \\
\text { helght }\end{array}$ & $\begin{array}{c}\text { Dis- } \\
\text { charge }\end{array}$ & Date & Hour & $\begin{array}{c}\text { Gage } \\
\text { height }\end{array}$ & $\begin{array}{c}\text { Dis- } \\
\text { charge }\end{array}$ & Date & Hour & $\begin{array}{c}\text { Gage } \\
\text { he1ght }\end{array}$ & $\begin{array}{c}\text { D1s- } \\
\text { charge }\end{array}$ \\
\hline Mar. 11 & $\begin{array}{l}0600 \\
1200 \\
1800 \\
2400\end{array}$ & $\begin{array}{l}9.06 \\
8.50 \\
8.14 \\
7.87\end{array}$ & $\begin{array}{r}1,490 \\
1,190 \\
1,010 \\
880\end{array}$ & Mar. 13 & $\begin{array}{l}1700 \\
2400 \\
\\
0600 \\
0900\end{array}$ & $\begin{array}{l}6.81 \\
6.70 \\
6.65 \\
6.74\end{array}$ & $\begin{array}{l}445 \\
408 \\
\\
391 \\
422\end{array}$ & Mar. 15 & $\begin{array}{l}0200 \\
0500 \\
1200 \\
1800 \\
2400\end{array}$ & $\begin{array}{l}7.54 \\
7.51 \\
7.19 \\
7.00 \\
6.85\end{array}$ & $\begin{array}{l}728 \\
715 \\
580 \\
510 \\
459\end{array}$ \\
\hline 12 & $\begin{array}{l}0600 \\
1400 \\
1700 \\
2400 \\
0900\end{array}$ & $\begin{array}{l}7.67 \\
7.56 \\
7.45 \\
7.19 \\
6.95\end{array}$ & $\begin{array}{l}788 \\
738 \\
688 \\
580 \\
493\end{array}$ & & $\begin{array}{l}1200 \\
1600 \\
1700 \\
1800 \\
2100 \\
2400\end{array}$ & $\begin{array}{l}7.14 \\
7.82 \\
7.86 \\
7.85 \\
7.62 \\
7.60\end{array}$ & $\begin{array}{l}560 \\
857 \\
876 \\
871 \\
765 \\
756\end{array}$ & 16 & $\begin{array}{l}0600 \\
1200 \\
1800 \\
2400\end{array}$ & $\begin{array}{l}6.69 \\
6.58 \\
6.48 \\
6.39\end{array}$ & $\begin{array}{l}405 \\
368 \\
338 \\
311\end{array}$ \\
\hline
\end{tabular}

BLUE RIVER BASIN

(99) 3-3030. Blue River near White Cloud, Ind.

Location.--Lat $38^{\circ} 14^{\prime} 15^{\prime \prime}$, 1ong $86^{\circ} 13^{\prime} 50^{\prime \prime}$, in NW $\frac{1}{4} \mathrm{SE} \frac{1}{4}$ sec.19, T.3 S., R.3 E., on left bank, $400 \mathrm{ft}$ downstream from Spring creek, $0.2 \mathrm{mile}$ upstream from bridge on State Highway 62 , and three-quarters of a mile north of White Cloud.

Drainage area $--461 \mathrm{sq} \mathrm{mi}$.

Gage-height record.--Water-stage recorder graph. Datum of gage is $434.30 \mathrm{ft}$ above mean sea level, datum of 1929.

Discharge record.--Stage-discharge relation defined by current-meter measurements below 22,000 cfs and by contracted-opening measurement at 28,500 cfs.

Maxima.--Given in the following table.

March 1964:

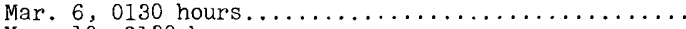

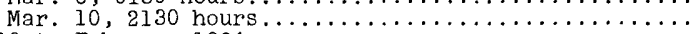

1910 to February 1964 :

Jan. $22,1959 \ldots \ldots$.

$\begin{array}{cc}\begin{array}{c}\text { Discharge } \\ \text { (cfs) }\end{array} & \begin{array}{c}\text { Gage helght } \\ \text { (feet) }\end{array} \\ 16,300 & 16.40 \\ 28,400 & 23.03 \\ 28,500 & 23.07\end{array}$

Mean discharge, in cubic feet per second, March 1964

\begin{tabular}{|c|c|c|c|c|c|c|c|c|c|}
\hline Day & D1scharge & Day & Discharge & Day & D1scharge & Day & Discharge & Day & Discharge \\
\hline $\begin{array}{l}1 \ldots \ldots \\
2 \ldots \ldots \\
3 \ldots \ldots \\
4 \ldots \\
5 \ldots \ldots \\
6 \ldots \ldots\end{array}$ & $\begin{array}{r}117 \\
110 \\
124 \\
4,040 \\
14,000 \\
9,600\end{array}$ & $\begin{array}{r}7 \ldots \ldots \\
8 \ldots \ldots \\
9 \ldots \\
10 \ldots \\
11 \ldots \\
12 \ldots\end{array}$ & $\begin{array}{r}2,430 \\
2,230 \\
14,000 \\
26,000 \\
20,400 \\
5,450\end{array}$ & $\begin{array}{l}13 \ldots \ldots \\
14 \ldots \ldots \\
15 \ldots \ldots \\
16 \ldots \\
17 \ldots \ldots \\
18 \ldots \ldots\end{array}$ & $\begin{array}{l}3,550 \\
3,020 \\
3,870 \\
2,630 \\
1,940 \\
1,540\end{array}$ & $\mid \begin{array}{l}19 \ldots \ldots \\
20 \ldots \ldots \\
21 \ldots \ldots \\
22 \ldots \ldots \\
23 \ldots \ldots \\
24 \ldots \ldots\end{array}$ & $\begin{array}{r}1,250 \\
1,100 \\
1,020 \\
903 \\
786 \\
708\end{array}$ & $\mid \begin{array}{l}25 \ldots \ldots \\
26 \ldots \ldots \\
27 \ldots \ldots \\
28 \ldots \ldots \\
29 \ldots \ldots \\
30 \ldots \ldots \\
31 \ldots \ldots\end{array}$ & $\begin{array}{l}664 \\
819 \\
960 \\
756 \\
670 \\
594 \\
531\end{array}$ \\
\hline & & & & & & & & & $\begin{array}{l}4,058 \\
10.14\end{array}$ \\
\hline
\end{tabular}

Gage height, in feet, and discharge, In cubic feet per second, at indicated time, 1964

\begin{tabular}{|c|c|c|c|c|c|c|c|c|c|c|c|}
\hline Date & Hour & $\begin{array}{c}\text { Gage } \\
\text { height }\end{array}$ & $\begin{array}{l}\text { Dis- } \\
\text { charge }\end{array}$ & Date & Hour & $\begin{array}{c}\text { Gage } \\
\text { helght }\end{array}$ & $\begin{array}{l}\text { D1s- } \\
\text { charge }\end{array}$ & Date & Hour & $\begin{array}{c}\text { Gage } \\
\text { helght }\end{array}$ & $\begin{array}{c}\text { Dis- } \\
\text { charge }\end{array}$ \\
\hline $\begin{array}{ll}\text { Mar. } & 2 \\
& 3\end{array}$ & $\begin{array}{l}2400 \\
1200 \\
2400 \\
0300 \\
0500 \\
0700 \\
1000 \\
1300 \\
1400 \\
1500 \\
1600 \\
1700 \\
1800 \\
1900 \\
2100\end{array}$ & $\begin{array}{r}2.15 \\
2.20 \\
2.24 \\
2.26 \\
2.34 \\
2.57 \\
3.44 \\
4.43 \\
5.01 \\
6.90 \\
7.77 \\
9.35 \\
9.98 \\
13.03 \\
14.09\end{array}$ & $\begin{array}{r}112 \\
125 \\
135 \\
140 \\
160 \\
230 \\
606 \\
1,220 \\
1,650 \\
3,300 \\
4,170 \\
6,060 \\
6,870 \\
11,100 \\
12,700\end{array}$ & Mar. 4 & $\begin{array}{l}2300 \\
2330 \\
2400 \\
\\
0200 \\
0300 \\
0400 \\
0500 \\
0700 \\
1000 \\
1300 \\
1600 \\
1900 \\
2100 \\
2400 \\
\\
0100\end{array}$ & $\begin{array}{l}14.67 \\
14.68 \\
14.64 \\
14.36 \\
14.24 \\
14.17 \\
14.16 \\
14.23 \\
14.41 \\
14.72 \\
15.13 \\
15.66 \\
16.00 \\
16.33 \\
16.39\end{array}$ & $\begin{array}{l}13,600 \\
13,600 \\
13,600 \\
13,100 \\
13,000 \\
12,900 \\
12,800 \\
12,900 \\
13,200 \\
13,700 \\
14,300 \\
15,200 \\
15,700 \\
16,200 \\
16,300\end{array}$ & Mar. $\overline{6}$ & $\begin{array}{l}0130 \\
0200 \\
0400 \\
0600 \\
0800 \\
0900 \\
1000 \\
1100 \\
1200 \\
1300 \\
1400 \\
1500 \\
1600 \\
1700 \\
1800 \\
2000 \\
2100\end{array}$ & $\begin{array}{r}16.40 \\
16.39 \\
16.22 \\
15.78 \\
15.02 \\
14.43 \\
13.63 \\
12.70 \\
11.63 \\
10.54 \\
9.66 \\
8.97 \\
8.45 \\
8.05 \\
7.75 \\
7.30 \\
7.14\end{array}$ & $\begin{array}{r}16,300 \\
16,300 \\
16,100 \\
15,300 \\
14,100 \\
13,200 \\
12,000 \\
10,600 \\
9,090 \\
7,600 \\
6,460 \\
5,580 \\
4,960 \\
4,480 \\
4,150 \\
3,700 \\
3,540\end{array}$ \\
\hline
\end{tabular}


Gage height, in feet, and discharge, in cublc feet per second, at indicated time, 1964 , of Blue River near White cloud, Ind.--Continued

\begin{tabular}{|c|c|c|c|c|c|c|c|c|c|c|c|}
\hline Date & Hour & $\begin{array}{c}\text { Gage } \\
\text { height }\end{array}$ & $\begin{array}{l}\text { Dis- } \\
\text { charge }\end{array}$ & Date & Hour & $\begin{array}{c}\text { Gage } \\
\text { helght }\end{array}$ & $\begin{array}{l}\text { Dis- } \\
\text { charge }\end{array}$ & Date & Hour & $\begin{array}{c}\text { Gage } \\
\text { helght }\end{array}$ & $\begin{array}{l}\text { Dis- } \\
\text { charge }\end{array}$ \\
\hline Mar. 6 & $\begin{array}{l}2200 \\
2400\end{array}$ & $\begin{array}{l}7.02 \\
6.79\end{array}$ & $\begin{array}{l}3,420 \\
3,190\end{array}$ & Mar. 10 & $\begin{array}{l}2130 \\
2200 \\
2300\end{array}$ & $\begin{array}{l}23.03 \\
23.02 \\
23.00\end{array}$ & $\begin{array}{l}28,400 \\
28,400 \\
28,300\end{array}$ & Mar. 15 & $\begin{array}{l}0200 \\
0300 \\
0600\end{array}$ & $\begin{array}{l}7.66 \\
7.65 \\
7.59\end{array}$ & $\begin{array}{l}4,060 \\
4,050 \\
3,990\end{array}$ \\
\hline 7 & 0300 & 6.52 & 2,930 & & 2400 & 22.95 & 28,200 & & 0700 & 7.58 & 3,980 \\
\hline & 0600 & 6.29 & 2,720 & & & & & & 0800 & $\begin{array}{l}7.58 \\
7.63\end{array}$ & 3,980 \\
\hline & $\begin{array}{l}1000 \\
1400\end{array}$ & $\begin{array}{l}6.03 \\
5.78\end{array}$ & $\begin{array}{l}2,490 \\
2,260\end{array}$ & 11 & $\begin{array}{l}0100 \\
0200\end{array}$ & $\begin{array}{l}22.87 \\
22.76\end{array}$ & $\begin{array}{l}27,900 \\
27,600\end{array}$ & & 1000 & $\begin{array}{l}7.63 \\
7.65\end{array}$ & $\begin{array}{l}4,030 \\
4,050\end{array}$ \\
\hline & $\begin{array}{l}1400 \\
1900\end{array}$ & $\begin{array}{l}5.78 \\
5.54\end{array}$ & $\begin{array}{l}2,260 \\
2,070\end{array}$ & & $\begin{array}{l}0200 \\
0400\end{array}$ & $\begin{array}{l}22.76 \\
22.50\end{array}$ & $\begin{array}{l}27,600 \\
27,000\end{array}$ & & $\begin{array}{l}1100 \\
1200\end{array}$ & $\begin{array}{l}7.65 \\
7.66\end{array}$ & $\begin{array}{l}4,050 \\
4,060\end{array}$ \\
\hline & 2400 & 5.31 & 1,890 & & 0700 & 21.87 & 25,800 & & 1300 & 7.65 & 4,050 \\
\hline & & & & & 1000 & 20.90 & 23,900 & & 1400 & 7.63 & 4,030 \\
\hline 8 & 0400 & 5.15 & 1,760 & & 1300 & 19.57 & 21,600 & & 1500 & 7.59 & 3,990 \\
\hline & 0700 & 5.07 & 1,700 & & 1500 & 18.25 & 19,300 & & 1700 & 7.45 & 3,850 \\
\hline & 1000 & 5.02 & 1,660 & & 1700 & 16.51 & 16,500 & & 2100 & 7.09 & 3,490 \\
\hline & $\begin{array}{l}1200 \\
1300\end{array}$ & $\begin{array}{l}5.06 \\
5.13\end{array}$ & $\begin{array}{l}1,690 \\
1,740\end{array}$ & & $\begin{array}{l}1800 \\
1900\end{array}$ & $\begin{array}{l}15.61 \\
14.55\end{array}$ & $\begin{array}{l}15,100 \\
13,400\end{array}$ & & 2400 & 6.84 & 3,240 \\
\hline & 1400 & 5.26 & $\begin{array}{l}1,140 \\
1,850\end{array}$ & & 2100 & 12.55 & 10,400 & 16 & 0400 & 6.56 & 2,960 \\
\hline & 1500 & 5.52 & 2,060 & & 2300 & 11.15 & 8,420 & & 0600 & 6.45 & 2,860 \\
\hline & 1700 & 6.25 & 2,680 & & 2400 & 10.64 & 7,730 & & 1200 & 6.15 & 2,600 \\
\hline & 1900 & 6.83 & 3,230 & & & & & & 1800 & 5.88 & 2,350 \\
\hline & 2000 & 6.93 & 3,330 & 12 & 0200 & 10.05 & 6,960 & & 2400 & 5.68 & 2,180 \\
\hline & 2100 & 6.95 & 3,350 & & 0400 & 9.65 & 6,440 & & & & \\
\hline & 2200 & 6.93 & 3,330 & & 0600 & 9.35 & 6,060 & 17 & 0800 & 5.46 & 2,010 \\
\hline & 2400 & 6.87 & 3,270 & & 0800 & 9.10 & 5,740 & & 1600 & 5.28 & 1,860 \\
\hline 9 & & & $=270$ & & 1100 & 8.80 & 5,380 & & 2400 & 5.11 & 1,730 \\
\hline & 0030 & 6.87 & 3,270 & & 1500 & 8.44 & 4,950 & & & & \\
\hline & $\begin{array}{l}0100 \\
0200\end{array}$ & $\begin{array}{l}6.89 \\
7.50\end{array}$ & $\begin{array}{l}3,290 \\
3,900\end{array}$ & & $\begin{array}{l}1900 \\
2400\end{array}$ & $\begin{array}{l}8.13 \\
7.83\end{array}$ & $\begin{array}{l}4,580 \\
4,230\end{array}$ & 18 & $\begin{array}{l}0800 \\
1600\end{array}$ & $\begin{array}{l}4.94 \\
4.79\end{array}$ & $\begin{array}{l}1,590 \\
1,470\end{array}$ \\
\hline & 0300 & 8.42 & 4,920 & & & & & & 2400 & 4.65 & 1,380 \\
\hline & 0400 & 9.18 & 5,840 & 13 & 0600 & 7.45 & 3,850 & & & & \\
\hline & 0500 & 10.42 & 7,450 & & 1200 & 7.12 & 3,520 & 19 & 0800 & 4.52 & 1,280 \\
\hline & 0600 & 11.36 & 8,710 & & 1800 & 6.83 & 3,230 & & 1600 & 4.42 & 1,210 \\
\hline & 0700 & 12.00 & 9,610 & & 2100 & 6.71 & 3,110 & & 2400 & 4.34 & 1,160 \\
\hline & 0800 & 13.15 & 11,300 & & 2400 & 6.59 & 2,990 & & & & \\
\hline & 0900 & 14.11 & 12,800 & & & & & 20 & 1200 & 4.26 & 1,100 \\
\hline & 1000 & 14.64 & 13,600 & 14 & 0300 & 6.49 & 2,900 & & 2400 & 4.20 & 1,060 \\
\hline & 1100 & 15.13 & 14,300 & & 0400 & 6.48 & 2,890 & 21 & 1200 & 44 & $10 ? 0$ \\
\hline & 1300 & 16.04 & 15,800 & & 0900 & $\begin{array}{l}6.42 \\
6.35\end{array}$ & $\begin{array}{l}2,840 \\
2,780\end{array}$ & & 1800 & $\begin{array}{l}4.14 \\
4.10\end{array}$ & 990 \\
\hline & 1500 & 17.03 & 17,300 & & 1200 & 6.32 & 2,750 & & 2400 & 4.09 & 984 \\
\hline & 1700 & 17.93 & 18,800 & & 1400 & 6.31 & 2,740 & & & & \\
\hline & 1900 & 18.66 & 20 & & 1500 & 6.33 & 2,760 & 22 & 0800 & 3.99 & 924 \\
\hline & 2200 & & 00 & & 1600 & 6.34 & 2,770 & & 1600 & 3.91 & 876 \\
\hline & 2400 & 20.17 & 22,600 & & 1700 & & 2,780 & & 2400 & 3.84 & 834 \\
\hline & & & & & 1800 & 49 & 2,900 & 23 & 1200 & 3.76 & 786 \\
\hline 10 & $\begin{array}{l}0500 \\
1000\end{array}$ & $\begin{array}{l}20.94 \\
21.75\end{array}$ & $\begin{array}{l}24,000 \\
25,500\end{array}$ & & $\begin{array}{l}1900 \\
2000\end{array}$ & $\begin{array}{l}6.82 \\
7.09\end{array}$ & $\begin{array}{l}3,220 \\
3,490\end{array}$ & & 2400 & 3.68 & 738 \\
\hline & 1400 & 22.42 & 26,800 & & 2100 & 7.29 & 3,690 & 24 & 1200 & 3.63 & 708 \\
\hline & 1700 & 22.81 & 27,700 & & 2200 & 7.44 & 3,840 & & 2400 & 3.58 & 678 \\
\hline & 1800 & 22.90 & 28,000 & & 2300 & 7.55 & 3,950 & & & & \\
\hline & 1900 & 22.95 & 28,200 & & 2400 & 7.62 & 4,020 & 25 & 1200 & 3.54 & 654 \\
\hline & 2000 & 23.00 & 28,300 & & & & & & & 3.53 & 648 \\
\hline & 2100 & 23.02 & 28,400 & 15 & 0100 & 7.65 & 4,050 & & 2400 & 3.61 & 696 \\
\hline
\end{tabular}

\section{ANDERSON RTVER BASIN}

(100) 3-3033. Middle Fork Anderson River at Bristow, Ind.

Location. --Lat $38^{\circ} 08^{\prime} 19^{\prime \prime}$, long $86^{\circ} 43^{\prime} 16^{\prime \prime}$, In E $\frac{1}{2}$ sec.27, T.4 S., R.3 W., at bridge on State Highway 145 at Bristow, 2.0 miles downstream from Coon Branch, and 6.0 miles upstream from Sulphur Fork Creek.

Drainage area. $--41.9 \mathrm{sq} \mathrm{ml}$.

Gage-height record.--Digital recorder tape punched at 15-minute intervals. Datum of gage is $395.00 \mathrm{ft}$ above mean sea level, datum of 1929.

Discharge record.--Stage-discharge relation defined by current-meter measurements below 4,000 $\mathrm{c}$ s and by contracted-opening measurements to $6,000 \mathrm{cf}$, and extended above on basis of logarithmic plotting.

Maxlma.--Given in the following table.

March 1964:

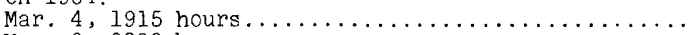

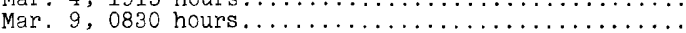

1905 to February 1964 :

Jan. 21,1959

$\begin{array}{cc}\begin{array}{c}\text { Discharge } \\ \text { (cfs) }\end{array} & \begin{array}{c}\text { Gage height } \\ \text { (feet) }\end{array} \\ 5,500 & 19.33 \\ 6,360 & 19.18\end{array}$

$15,000 \quad 20.0$ 
Mean discharge, in cublc feet per second, March 1964, of Middle Fork Anderson River at

\begin{tabular}{|c|c|c|c|c|c|c|c|c|c|}
\hline Day & Discharge & Day & Discharge & Day & Discharge & Day & Discharge & Day & Discharge \\
\hline $\begin{array}{l}1 \ldots \ldots \\
2 \ldots \ldots \\
3 \ldots \ldots \\
4 \ldots \\
5 \ldots \ldots \\
6 \ldots \ldots\end{array}$ & $\begin{array}{r}4.1 \\
4.5 \\
10 \\
2,050 \\
903 \\
120\end{array}$ & $\begin{array}{r}7 \ldots \ldots \\
8 \ldots \ldots \\
9 \ldots \\
10 \ldots \\
11 \ldots \ldots \\
12 \ldots\end{array}$ & $\begin{array}{r}64 \\
520 \\
4,870 \\
2,330 \\
226 \\
113\end{array}$ & $\begin{array}{l}13 \ldots \ldots \\
14 \ldots \ldots \\
15 \ldots \ldots \\
16 \ldots \ldots \\
17 \ldots \ldots \\
18 \ldots \ldots\end{array}$ & $\begin{array}{r}71 \\
118 \\
121 \\
64 \\
46 \\
34\end{array}$ & $\begin{array}{l}19 \ldots \ldots \\
20 \ldots \ldots \\
21 \ldots \ldots \\
22 \ldots \ldots \\
23 \ldots \ldots \\
24 \ldots \ldots\end{array}$ & $\begin{array}{l}28 \\
35 \\
34 \\
26 \\
22 \\
20\end{array}$ & $\begin{array}{l}25 \ldots \ldots \\
26 \ldots \ldots \\
27 \ldots \ldots \\
28 \ldots \ldots \\
29 \ldots \ldots \\
30 \ldots \ldots \\
31 \ldots \ldots\end{array}$ & $\begin{array}{r}33 \\
135 \\
55 \\
42 \\
32 \\
26 \\
23\end{array}$ \\
\hline $\begin{array}{l}\text { nthiy } \\
\text { inoff, }\end{array}$ & $\begin{array}{l}\text { mean disc } \\
\text { in inches }\end{array}$ & arge, in & $\begin{array}{l}\text { cubic feet } \\
\ldots \ldots \ldots \ldots\end{array}$ & $\begin{array}{l}\text { per seco } \\
\text {........ }\end{array}$ & ond $\ldots \ldots \ldots$ & …... & $\ldots \ldots \ldots$ & $\ldots \ldots \ldots$ & $\begin{array}{r}393 \\
10.81 \\
\end{array}$ \\
\hline
\end{tabular}

Note.--Da1ly mean discharges computed on basis of $15-\mathrm{m} 1$ nute intervals.

Gage height, in feet, and discharge, in cubic feet per second, at indicated time, 1964

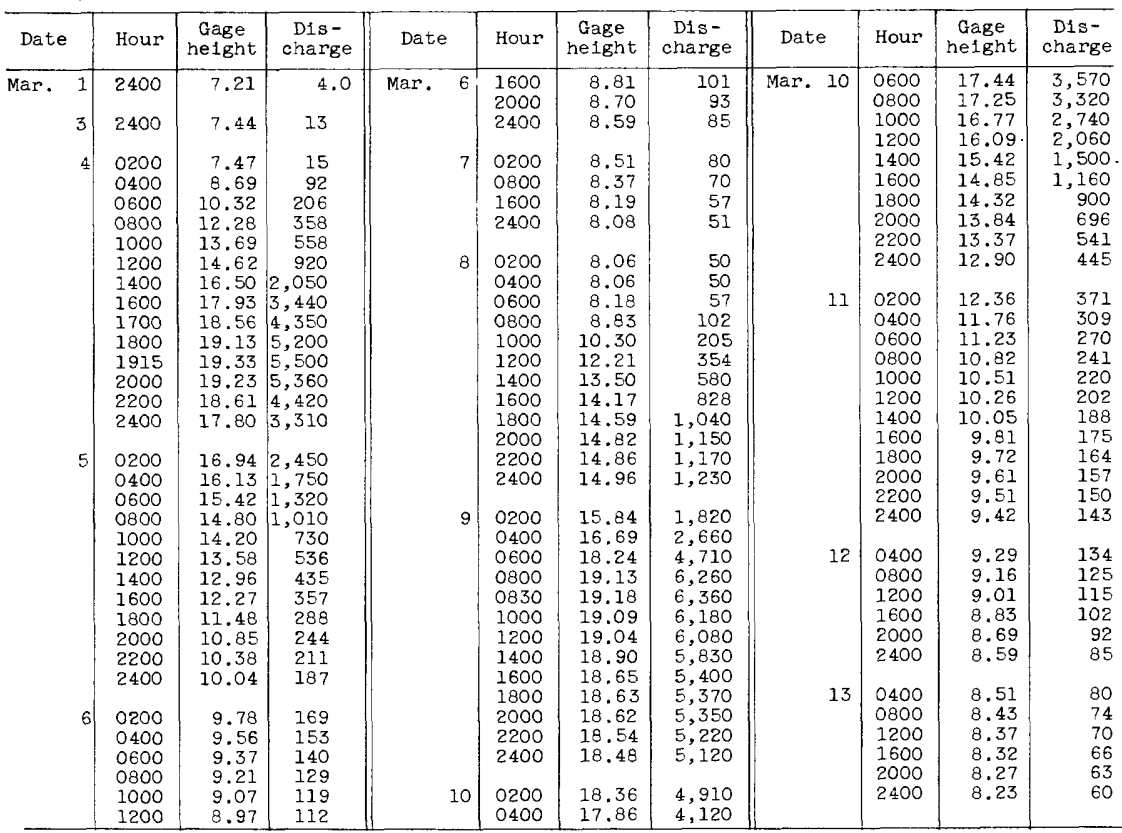

GREEN RIVER BASIN

(101) 3-3095. McDougal Creek near Hodgenville, Ky.

Location. - Lat $37^{\circ} 32^{\prime} 37^{\prime \prime}$, long $85^{\circ} 40^{\prime} 19^{\prime \prime}$, on left bank 0.3 mile upstream from bridge on State Highway $470,4 \frac{2}{4}$ miles southeast of Hodgenvilie, Larue County, and $4 \frac{3}{4}$ miles upstream from mouth.

Drainage area. $--5.34 \mathrm{sq} \mathrm{mi}$.

Gage-helght record.--Digital recorder tape punched at 15-minute intervals. Datum of gage is $774.34 \mathrm{ft}$ above mean sea level, datum of 1929 .

Discharge record.--Stage-discharge relation defined by current-meter measurements below $850 \mathrm{cfs}$.

Maxima.--Given in the following table.

March 1964:

Mar. 4, 1300 hours.

1953 to February 1964 .

Nov. 18,1957

$\begin{array}{cc}\begin{array}{c}\text { Discharge } \\ \text { (cfs) }\end{array} & \text { Gage helght } \\ 1,480 & 5.47 \\ 1,320 & 5.21 \\ 2,100 & 6.27\end{array}$


Mean discharge, in cublc feet per second, March 1964, of McDougal Creek near Hodgenville, Ky.

\begin{tabular}{l}
\hline Day \\
\hline $1 \ldots \ldots$
\end{tabular}

(102) 3-3100. North Fork Nolin River at Hodgenville, Ky.

Location. - Lat $37^{\circ} 34^{\prime} 33^{\prime \prime}$, long $85^{\circ} 44^{\prime} 23^{\prime \prime}$, on right bank $10 \mathrm{ft}$ upstream from bridge on State H1ghway 61 at Hodgenville, Larue County, and $0.6 \mathrm{mile}$ downstream from McDougal Creek.

Drainage area. $--36.4 \mathrm{sq} \mathrm{mi}$, of which about $0.8 \mathrm{sq} \mathrm{mi}$ does not contribute directly to surface runoff.

Gage-height record.--Water-stage recorder graph except Mar. 1-3, 6, 7, 11-31, for which graph was drawn on basis of twice-daily wire-weight-gage readings. Datum of gage is $701.64 \mathrm{ft}$ above mean sea level, unadjusted.

Discharge record.--Stage-discharge relation defined by current-meter measurements below 4,400 ifs.

Maxima.--Given in the following table.

March 1964 :

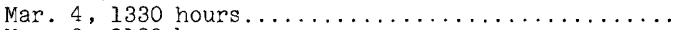

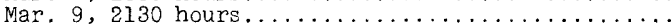

1941 to February 1964 :

July 11,1958

$\begin{array}{cc}\begin{array}{c}\text { Discharge } \\ \text { (cfs) }\end{array} & \text { Gage height } \\ 8,860 & 15.80 \\ 6,190 & 14.87 \\ 8,300 & 15.50\end{array}$

Mean discharge, in cublc feet per second, March 1964

\begin{tabular}{|c|c|c|c|c|c|c|c|c|c|}
\hline Day & Discharge & Day & Discharge & Day & Discharge & Day & Discharge & Day & Discharge \\
\hline $\begin{array}{l}1 \ldots \ldots \\
2 \ldots \ldots \\
3 \ldots \ldots \\
4 \ldots \ldots \\
5 \ldots \ldots \\
6 \ldots \ldots\end{array}$ & $\begin{array}{r}12 \\
12 \\
16 \\
2,560 \\
724 \\
249\end{array}$ & $\begin{array}{r}7 \ldots \ldots \\
8 \ldots \ldots \\
9 \ldots \ldots \\
10 \ldots \\
11 \ldots \ldots \\
12 \ldots \ldots\end{array}$ & $\begin{array}{r}162 \\
883 \\
1,550 \\
1,100 \\
306 \\
194\end{array}$ & $\begin{array}{l}13 \ldots \ldots \\
14 \ldots \ldots \\
15 \ldots \ldots \\
16 \ldots \ldots \\
17 \ldots \ldots \\
18 \ldots \ldots\end{array}$ & $\begin{array}{r}147 \\
142 \\
187 \\
130 \\
104 \\
88\end{array}$ & $\begin{array}{l}19 \ldots \ldots \\
20 \ldots \ldots \\
21 \ldots \ldots \\
22 \ldots \ldots \\
23 \ldots \ldots \\
24 \ldots \ldots\end{array}$ & $\begin{array}{l}66 \\
64 \\
81 \\
60 \\
50 \\
46\end{array}$ & $\mid \begin{array}{l}25 \ldots \ldots \\
26 \ldots \ldots \\
27 \ldots \ldots \\
28 \ldots \ldots \\
29 \ldots \ldots \\
30 \ldots \ldots \\
31 \ldots \ldots\end{array}$ & $\begin{array}{l}40 \\
40 \\
30 \\
27 \\
18 \\
16 \\
13\end{array}$ \\
\hline 8 & in & & & & & & & & $\begin{array}{r}294 \\
9.31\end{array}$ \\
\hline
\end{tabular}

Gage he1ght, in feet, and discharge, in cublc feet per second, at indicated time, 1964

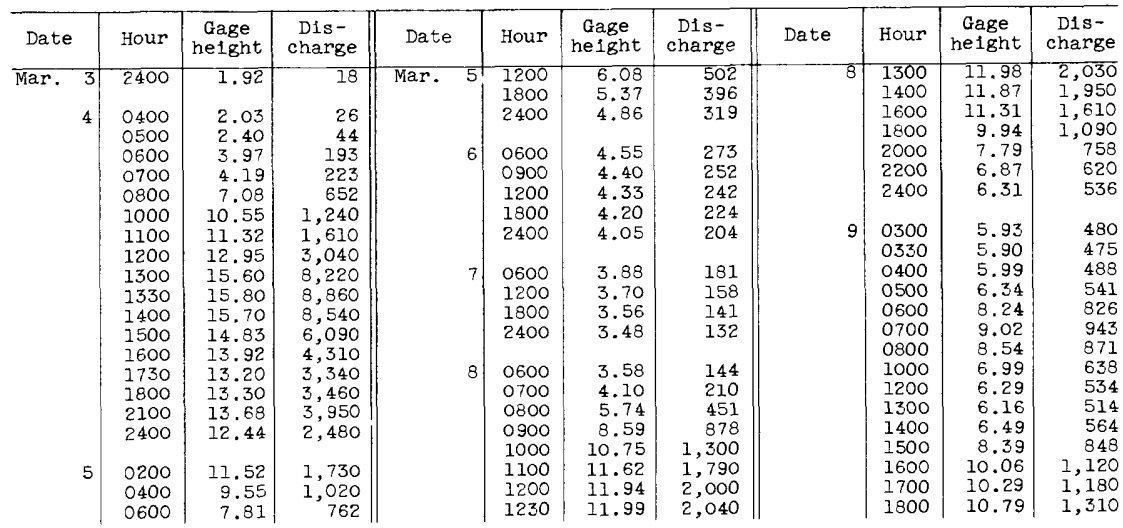


Mean discharge, in feet, and discharge, in cublc feet per second, at ind1cated time, 1964 , of North Fork Nolin RIver at Hodgenville, $\mathrm{Ky}$.--Continued

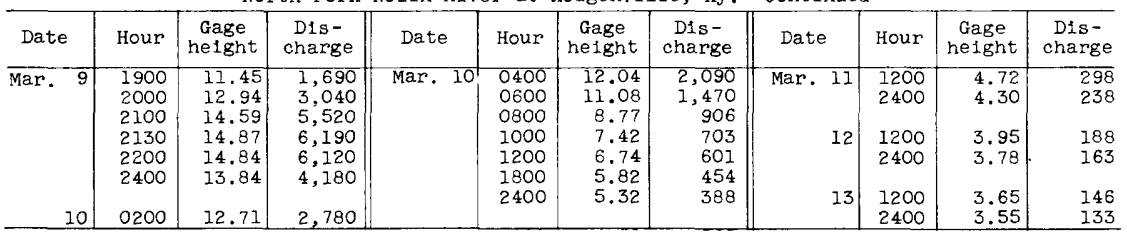

(103) 3-3103. Nolin River at White M11ls, Ky.

Location.--Lat $37^{\circ} 33^{1} 03^{\prime \prime}$, long $86^{\circ} 02^{14} 3^{\prime \prime}$, on right bank $0.8 \mathrm{mlle}$ southwest of White Mills, Hardin County, and 2.2 miles downstream from bridge on State Highway 84.

Drainage area. $--357 \mathrm{sq} \mathrm{ml}$, of which about $120 \mathrm{sq} \mathrm{m}$ does not contribute directly to surface runoff.

Gage-he1ght record.--Water-stage recorder graph. Datum of gage is 583.08 ft above mean sea level, datum of 1929 .

Discharge record.--Stage-discharge relation defined by current-meter measurements. Rate of change in stage used as a factor Mar. $5,6,9-11$.

Maxima.--Given in the following table.

March 1964:

Mar. 5, 1700 hours

Mar. 10, 2000 hours.

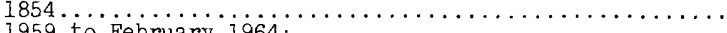

1959 to February 1964:

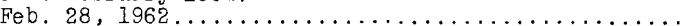

$\begin{array}{cc}\begin{array}{c}\text { Discharge } \\ \text { (cfs) }\end{array} & \begin{array}{c}\text { Gage height } \\ \text { (feet) }\end{array} \\ 16,700 & - \\ 15,200 & 31.74 \\ - & - \\ \text { Unknown } & 30.67 \\ 8,060 & 35.5 \\ & 22.59\end{array}$

Mean discharge, in cub1c feet per second, March 1964

\begin{tabular}{|c|c|c|c|c|c|c|c|c|c|}
\hline Day & Discharge & Day & D1scharge & Day & Discharge & Day & D1scharge & Day & D1scharge \\
\hline $\begin{array}{l}1 \ldots \ldots \\
2 \ldots \ldots \\
3 \ldots \ldots \\
4 \ldots \\
5 \ldots \ldots \\
6 \ldots\end{array}$ & $\begin{array}{r}127 \\
122 \\
129 \\
1,910 \\
12,800 \\
8,400\end{array}$ & $\begin{array}{r}7 \ldots \ldots \\
8 \ldots \ldots \\
9 \ldots \ldots \\
10 \ldots \\
11 \ldots \ldots \\
12 \ldots \ldots\end{array}$ & $\begin{array}{r}2,230 \\
2,730 \\
6,820 \\
11,900 \\
9,490 \\
2,990\end{array}$ & $\begin{array}{l}13 \ldots \ldots \\
14 \ldots \ldots \\
15 \ldots \ldots \\
16 \ldots \ldots \\
17 \ldots \ldots \\
18 \ldots \ldots\end{array}$ & $\begin{array}{l}1,970 \\
1,700 \\
2,070 \\
1,620 \\
1,370 \\
1,210\end{array}$ & $\begin{array}{l}19 \ldots \ldots \\
20 \ldots \ldots \\
21 \ldots \ldots \\
22 \ldots \ldots \\
23 \ldots \ldots \\
24 \ldots \ldots\end{array}$ & $\begin{array}{r}1,070 \\
1,020 \\
1,070 \\
964 \\
853 \\
793\end{array}$ & $\mid \begin{array}{l}25 \ldots \ldots \\
26 \ldots \ldots \\
27 \ldots \ldots \\
28 \ldots \ldots \\
29 \ldots \ldots \\
30 \ldots \ldots \\
31 \ldots \ldots\end{array}$ & $\begin{array}{l}745 \\
730 \\
667 \\
613 \\
571 \\
529 \\
496\end{array}$ \\
\hline $1 \mathrm{y}$ & $\operatorname{an}$ & & & & & & & & $\begin{array}{r}2,571 \\
8.30\end{array}$ \\
\hline
\end{tabular}


(104) 3-3104. Bacon Creek near Priceville, Ky.

Location.--Lat $37^{\circ} 21^{\prime} 31^{\prime \prime}$, Iong $85^{\circ} 59^{\prime} 53^{\prime \prime}$, on right bank 1.2 miles southwest of Priceville, Hart County, 1.3 miles downstream from small unnamed tributary, 2.1 miles upstream from unnamed tributary, and 7.2 miles upstream from mouth.

Drainage area. $--85.4 \mathrm{sq} \mathrm{ml}$, of which about $31 \mathrm{sq} \mathrm{ml}$ does not contribute directly to surface runoff.

Gage-height record.--Digital recorder tape punched at 15-minute intervals. Datum of gage is $568.13 \mathrm{ft}$ above mean sea level, datum of 1929.

Discharge record.--Stage-discharge relation defined by current-meter measurements below 1,600 cfs.

Maxima.--Given in the following table.

March 1964:

Mar. 5,0530 hours. . . . . . . . . . . . . . . .

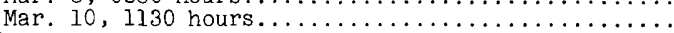
1957:

November...............

1959 to February 1964
Feb. $28,1962 . \ldots$

$\begin{array}{cc}\text { Discharge } & \text { Gage height } \\ \text { (cfs) } & \text { (feet) } \\ 2,380 & 13.96 \\ 2,400 & 14.01 \\ & \\ \text { Unknown } & 21.8\end{array}$

2,240

Mean discharge, in cubic feet per second, March 1964

\begin{tabular}{l}
\hline Day \\
\hline $1 \ldots \ldots$
\end{tabular}

(105) 3-3109. Nolin River Reservoir near Kyrock, Ky.

Location.--Lat $37^{\circ} 16^{\prime} 40^{\prime \prime}$, long $86^{\circ} 14^{\prime} 51^{\prime \prime}$, in intake structure of Nolin River Dam on Nolin River, 0.3 mile upstream from Dismal Creek, 1.1 miles northeast of Kyrock, Edmonson County, and at mile 7.8 .

Drainage area. $--703 \mathrm{sq} \mathrm{ml}$, of which about $223 \mathrm{sq} \mathrm{ml}$ does not contribute directly to surface runoff.

Gage-height record.--Water-stage recorder graph. Datum of gage is at mean sea level (levels by Corps of Engineers).

Maxima.--March 1964: Contents, 298,400 acre-ft Mar. 17, 1300 hours lelevation, $532.17 \mathrm{ft}$ ).

1963 to February 1964: Contents, 104,400 acre-ft Mar. 22, 1963 (elevation, $501.65 \mathrm{ft}$ ).

Remarks.--Reservoir is formed by rolled earthfill dam. Releases normally controlled by three gates, $7.25 \mathrm{ft}$ wide and $14 \mathrm{ft}$ high, in semi-elliptical concrete conduit through dam with inlet invert elevation at $422.00 \mathrm{ft}$, contents, 90 acre-ft.

Total capacity at ungated spillway level, elevation, $560.00 \mathrm{ft}$, is 609,400 acre-ft, of which a maximum of 570,100 acre-f't (contents between elevations 480.00 and $560.00 \mathrm{ft}$ ) or a minimum of 439,300 acre-ft (contents between elevations of 515.00 and $560.00 \mathrm{ft}^{\prime}$ ) is reserved for flood control depending on season. Contents of 130,800 acre-ft between minimum pool elevation of $480.00 \mathrm{ft}$ and seasonal pool elevation of $515.00 \mathrm{ft}$ is available for seasonal low-flow augmentation. Capacity at minimum pool elevation, $480.00 \mathrm{ft}$, is 39,280 acre-ft. Reservoir is used for flood control, low-flow augmentation, and recreation. Figures of contents include dead storage below invert of condult. Gates closed and storage began Mar. 4, 1963.

Cooperation.--Capacity table furnished by Corps of Engineers. 
Elevation and contents, February to April 1964, of Nolln River Reservolr near Kyrock, Ky.

\begin{tabular}{|c|c|c|c|c|c|c|c|}
\hline Date & Hour & $\begin{array}{c}\text { Elevation } \\
\text { (feet) }\end{array}$ & $\begin{array}{c}\text { Contents } \\
\text { (acre-feet) }\end{array}$ & Date & Hour & $\begin{array}{c}\text { Elevation } \\
\text { (feet) }\end{array}$ & $\begin{array}{c}\text { Contents } \\
\text { (acre-feet) }\end{array}$ \\
\hline $\begin{array}{l}\text { Feb. } 29 \ldots \\
\text { Mar. } 4 \ldots \\
\text { Mar. } 17 \ldots\end{array}$ & $\begin{array}{l}2400 \\
0400 \\
1300\end{array}$ & $\begin{array}{l}479.99 \\
480.08 \\
532.17\end{array}$ & $\begin{array}{r}39,260 \\
39,450 \\
292,400\end{array}$ & $\begin{array}{lr}\text { Mar. } & 31 \ldots \\
\text { Apr. } & 4 \ldots\end{array}$ & $\begin{array}{l}2400 \\
0700\end{array}$ & $\begin{array}{l}500.95 \\
488.65\end{array}$ & $\begin{array}{r}101,600 \\
60,020\end{array}$ \\
\hline
\end{tabular}

(106) 3-3110. Nolin River at Kyrock, Ky.

Location.--Lat $37^{\circ} 16^{1} 27^{\prime \prime}$, long $86^{\circ} 15^{\prime} 03^{\prime \prime}$, on right bank $470 \mathrm{ft}$ downstream from Dismai Creek, 0.3 mile downstream from Nolin River Dam, 0.9 mile northeast of Kyrock, Edmonson County, 1.2 miles upstream from Pigeon Creek, and 7.5 miles upstream from mouth.

Drainage area.--707 sq $\mathrm{mi}$ (Including that of Dismal Creek), of which about $223 \mathrm{sq} \mathrm{m}$ does not contribute directly to surface runoff.

Gage-height record.--Digital recorder tapes punched at 60-minute intervals. Auxil1ary gage at site 9.1 miles downstream. Datum of gage 1s $400.00 \mathrm{ft}$ above mean sea level, datum of 1929 (levels by Corps of Engineers).

Discharge record.--Stage-fall-discharge relation defined by current-meter measurements. Fall used as a factor Mar. 1-4, 8, 9, 17-31. Stage-fall-discharge relation indefinite Mar. 5-7, 10-16; discharge estimated on basis of releases from Nolin River Reservoir.

Maxima.--Given in the following table.

Discharge Gage height

March 1964 :

Mar. 14,1300 hours.

$\operatorname{Mar} .26,2000$ hours $\ldots \ldots \ldots \ldots \ldots \ldots \ldots$
$1930-32,1939-50,1960$ to February $1964: \ldots \ldots \ldots \ldots \ldots \ldots$

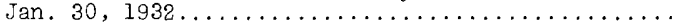

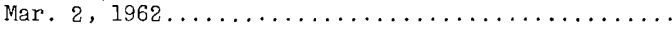

(cfs)

$9, \overline{6} 20$

22,700

a Backwater from Green River.

Maximum stage known since $1854,26.35 \mathrm{ft}$ in January 1937 at site 11.9 miles upstream at datum $48.36 \mathrm{ft}$ higher.

Remarks.--Flow regulated by Nolin River Reservo1r (see station 3109).

Mean discharge, in cublc feet per second, March 1964

\begin{tabular}{|c|c|c|c|c|c|c|c|c|c|}
\hline Day & Discharge & Day & Discharge & Day & D1scharge & Day & Discharge & Day & Dischange \\
\hline $\begin{array}{l}1 \ldots \ldots \\
2 \ldots \ldots \\
3 \ldots \ldots \\
4 \ldots \\
5 \ldots \ldots \\
6 \ldots \ldots\end{array}$ & $\begin{array}{r}283 \\
249 \\
298 \\
3,140 \\
300 \\
300\end{array}$ & $\begin{array}{r}7 \ldots \ldots \\
8 \ldots \ldots \\
9 \ldots \\
10 \ldots \\
11 \ldots \\
12 \ldots\end{array}$ & $\begin{array}{l}310 \\
318 \\
357 \\
310 \\
310 \\
320\end{array}$ & $\begin{array}{l}13 \ldots \ldots \\
14 \ldots \ldots \\
15 \ldots \ldots \\
16 \ldots \\
17 \ldots \ldots \\
18 \ldots \ldots\end{array}$ & $\begin{array}{r}320 \\
320 \\
320 \\
320 \\
2,180 \\
4,120\end{array}$ & $\mid \begin{array}{l}19 \ldots \ldots \\
20 \ldots \ldots \\
21 \ldots \ldots \\
22 \ldots \ldots \\
23 \ldots \ldots \\
24 \ldots \ldots\end{array}$ & $\begin{array}{l}4,530 \\
5,780 \\
8,030 \\
9,260 \\
9,420 \\
9,160\end{array}$ & $\mid \begin{array}{l}25 \ldots \ldots \\
26 \ldots \ldots \\
27 \ldots \ldots \\
28 \ldots \\
29 \ldots \\
30 \ldots \ldots \\
31 \ldots \ldots\end{array}$ & $\begin{array}{l}8,980 \\
9,210 \\
9,360 \\
9,110 \\
8,900 \\
8,600 \\
8,310\end{array}$ \\
\hline Monthly & mean disc & se, in & cublc fee & per sec & ond $\ldots \ldots$ & $\ldots \ldots$ & -1 & -2 & 3,959 \\
\hline
\end{tabular}


(107) 3-3120. Bear Creek near Le1tchfield, Ky.

Location. - - Lat $37^{\circ} 25^{\prime} 35^{\prime \prime}$, long $86^{\circ} 15^{\prime} 50^{\prime \prime}$, near center of span on upstream side of bridge on State H1ghway 65 , just downstream from Taylor Fork, 0.8 m.1le upstream from McClure Fork and 4 miles south of Le1tchfleld, Grayson County.

Drainage area. $--30.8 \mathrm{sq} \mathrm{m} 1$.

Gage-helght record.--Graph drawn on basis of twlce-dally wire-welght-gage readings and crests from crest-stage gage. Altitude of gage is $500 \mathrm{ft}$ (from topographic map).

D1scharge record.--Stage-discharge relation defined by current-meter measurements below $3,060 \mathrm{c}$ s and extended above by logarithmic plotting.

Maxima.--Given in the following table.

March 1964:

Mar. 4, 1100 hours.

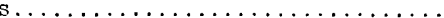

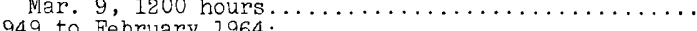

1949 to February 1964:

Nov. 18,1957

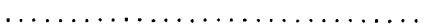

$\begin{array}{cc}\begin{array}{c}\text { Discharge } \\ \text { (cfs) }\end{array} & \begin{array}{c}\text { Gage height } \\ \text { (feet) }\end{array} \\ 6,060 & 19.05 \\ 6,250 & 19.29 \\ 8,070 & 21.33\end{array}$

Mean discharge, In cubic feet per second, March 1964

\begin{tabular}{|c|c|c|c|c|c|c|c|c|c|}
\hline Day & Discharge & Day & Discharge & Day & D1scharge & Day & Discharge & Day & D1scharge \\
\hline $\begin{array}{l}1 \ldots \ldots \\
2 \ldots \ldots \\
3 \ldots \ldots \\
4 \ldots \ldots \\
5 \ldots \ldots\end{array}$ & $\begin{array}{r}3.9 \\
4.9 \\
6.9 \\
2,350 \\
342 \\
151\end{array}$ & $\begin{array}{r}7 \ldots \ldots \\
8 \ldots \ldots \\
9 \ldots \ldots \\
10 \ldots \\
11 \ldots . \\
12 \ldots\end{array}$ & $\begin{array}{r}113 \\
855 \\
2,380 \\
378 \\
132 \\
80\end{array}$ & $\begin{array}{l}13 \ldots \ldots \\
14 \ldots \\
15 \ldots \\
16 \ldots \\
17 \ldots \\
18 \ldots \\
\end{array}$ & \begin{tabular}{r|}
49 \\
262 \\
220 \\
60 \\
40 \\
28
\end{tabular} & $\begin{array}{l}19 \ldots \ldots \\
20 \ldots \ldots \\
21 \ldots \ldots \\
22 \ldots \ldots \\
23 \ldots \ldots \\
24 \ldots \ldots\end{array}$ & \begin{tabular}{l||}
20 \\
24 \\
68 \\
31 \\
22 \\
14
\end{tabular} & $\begin{array}{l}25 \ldots \ldots \\
26 \ldots \ldots \\
27 \ldots \ldots \\
28 \ldots \ldots \\
29 \ldots \ldots \\
30 \ldots \ldots \\
31 \ldots \ldots\end{array}$ & $\begin{array}{l}20 \\
26 \\
18 \\
14 \\
12 \\
9.0 \\
9.4\end{array}$ \\
\hline $\begin{array}{l}\text { onthly } \\
\text { anoff, }\end{array}$ & $\begin{array}{l}\text { an disc } \\
\text { Inches }\end{array}$ & ge, & & & & & & & $\begin{array}{r}250 \\
9.35\end{array}$ \\
\hline
\end{tabular}

(108) 3-3175. North Fork Rough River near Westview, Ky.

Location. - Lat $37^{\circ} 41^{\prime} 32^{\prime \prime}$, long $86^{\circ} 23^{1} 9^{\prime \prime}$, on left bank $25 \mathrm{ft}$ downstream from bridge on State Highway $520,1.0 \mathrm{~m} 11 \mathrm{e}$ southeast of Westview, Breckinridge County, and 2.1 miles downstream from Buffalo Creek.

Drainage area. $-42.0 \mathrm{sq} \mathrm{m} 1$, of which about $19 \mathrm{sq} \mathrm{mi}$ does not contribute directly to surface runoff.

Gage-he1ght record.--Water-stage recorder graph. Datum of gage is $500.88 \mathrm{ft}$ above mean sea level, datum of 1929.

Discharge record.--Stage-discharge relation defined by current-meter measurements below 1,400 cfs. Backwater from Rough River Reservolr Mar. 11-31; discharge est1mated on basis of records for Rock L1ck Creek near Glen Dean and Bear Creek near Leitchfield.

Maxima.--Given in the following table.

March 1964:

Mar. 4, 1930 hours.

Mar. 9 , 2030 hours.

1954 to February 1964:

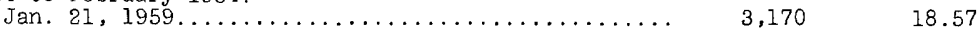

Mean discharge, in cubic feet per second, March 1964

\begin{tabular}{|c|c|c|c|c|c|c|c|c|c|}
\hline Day & Discharge & Day & Discharge & Day & Discharge & Day & Discharge & Day & Discharge \\
\hline $\begin{array}{l}1 \ldots \ldots \\
2 \ldots \ldots \\
3 \ldots \ldots \\
4 \ldots \ldots \\
5 \ldots \ldots \\
6 \ldots\end{array}$ & $\begin{array}{r}5.0 \\
5.2 \\
7.8 \\
800 \\
492 \\
123\end{array}$ & $\begin{array}{r}7 \ldots \ldots \\
8 \ldots \ldots \\
9 \ldots \ldots \\
10 \ldots \ldots \\
11 \ldots \ldots \\
12 \ldots \ldots\end{array}$ & $\begin{array}{r}79 \\
1,030 \\
2,700 \\
1,690 \\
392 \\
111\end{array}$ & $\mid \begin{array}{l}13 \ldots \ldots \\
14 \ldots \ldots \\
15 \ldots \ldots \\
16 \ldots \ldots \\
17 \ldots \ldots \\
18 \ldots \ldots\end{array}$ & $\begin{array}{r}75 \\
150 \\
60 \\
45 \\
35 \\
25\end{array}$ & $\begin{array}{l}19 \ldots \ldots \\
20 \ldots \ldots \\
21 \ldots \ldots \\
22 \ldots \ldots \\
23 \ldots \ldots \\
24 \ldots \ldots\end{array}$ & $\begin{array}{l}20 \\
50 \\
60 \\
35 \\
20 \\
15\end{array}$ & $\mid \begin{array}{l}25 \ldots \\
26 \ldots \ldots \\
27 \ldots \ldots \\
28 \ldots \ldots \\
29 \ldots \ldots \\
30 \ldots \ldots \\
31 \ldots \ldots\end{array}$ & $\begin{array}{l}25 \\
30 \\
20 \\
15 \\
12 \\
10 \\
10\end{array}$ \\
\hline & & & & & & & & & $\begin{array}{r}263 \\
7.21\end{array}$ \\
\hline
\end{tabular}


Gage helght, in feet, and discharge, in cublc feet per second, at ind1cated time, 1964, of North Fork Rough River near Westview, Ky.

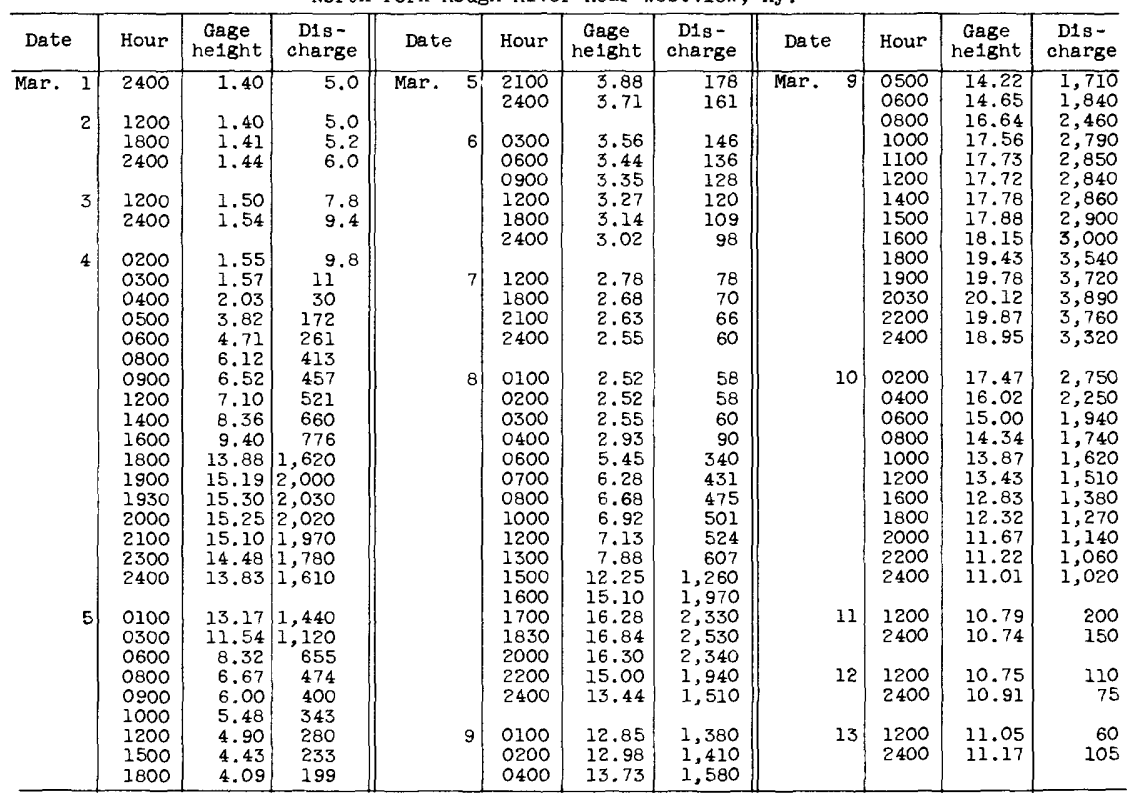


(109) 3-3180.05. Rough River Reservolr near Falls of Rough, Ky.

Location.--Lat $37^{\circ} 37^{\prime} 11^{\prime \prime}$, long $86^{\circ} 29^{\prime} 59^{\prime \prime}$, In intake structure of Rough River Dam on Rough River, Breckinridge County, 3.0 miles upstream from Cane Run, 3.1 miles northeast of Falls of Rough, Grayson County, and at mile 89.3.

Drainage area. $-454 \mathrm{sq} \mathrm{ml}$, of which about $110 \mathrm{sq} \mathrm{m} 1$ does not contribute directly to surface runoff.

Gage-he1ght record.-Water-stage recorder graph. Datum of gage is at mean sea level (1evels by Corps of Engineers).

Maxlma.--March 1964: Contents, 235,300 acre-ft Mar. 20, 1200 hours (elevation, $513.04 \mathrm{ft})$.

i959 to February 1964: Contents, 220,200 acre-ft May 16, 1961 (elevation, $511.07 \mathrm{ft}$ ).

Remarks.--Reservolr is formed by rolled earthfill dam. Releases controlled by three gates, $4.75 \mathrm{ft}$ wide and $9.5 \mathrm{ft}$ high, in semi-elliptical concrete conduit through dam with inlet invert elevation at $430.00 \mathrm{ft}$, contents 92 acre-ft. Total capacity at ungated splilway level, elevation, $524.00 \mathrm{ft}$, is 334,400 acre-ft, of which a maximum of 314,200 acre-ft (contents between elevations 465.00 and 524.00 $\mathrm{ft}$ ) or a minimum of 214,400 acre-ft (contents between elevations 495.00 and $524.00 \mathrm{ft}$ ) $1 \mathrm{~s}$ reserved for flood control depending on season. Contents of 99,840 acre-ft between minimum pool elevation of $465.00 \mathrm{ft}$ and seasonal pool elevation of $495.00 \mathrm{ft}$ is avallable for seasonal low-flow augmentation. Capacity at minimum pool elevation, $465.00 \mathrm{ft}$, is 20,170 acre- $\mathrm{ft}$. Reservoir is used for flood control, low-flow augmentation, and recreation. Figures of contents include dead storage below invert of conduit. Gates closed and storage began 0ct. 1, 1959.

Cooperation.--Capacity table furnished by Corps of Engineers.

Elevation and contents, Febmuary to April 1964

\begin{tabular}{|c|c|c|c|c|c|c|c|}
\hline Date & Hour & $\begin{array}{c}\text { Elevation } \\
\text { (feet) }\end{array}$ & $\begin{array}{c}\text { Contents } \\
(\text { acre-feet })\end{array}$ & Date & Hour & $\begin{array}{c}\text { Elevation } \\
\text { (feet) }\end{array}$ & $\begin{array}{c}\text { Contents } \\
\text { (acre-feet) }\end{array}$ \\
\hline $\begin{array}{l}\text { Feb. } 29 \ldots \\
\text { Mar. } 4 \ldots \\
\text { Mar. } 20 \ldots\end{array}$ & $\begin{array}{l}2400 \\
0300 \\
1200\end{array}$ & $\begin{array}{l}465.07 \\
465.08 \\
513.04\end{array}$ & $\begin{array}{r}20,290 \\
20,310 \\
235,300\end{array}$ & $\begin{array}{l}\text { Mar. } 31 \ldots \\
\text { Apr. } 30 . .\end{array}$ & $\begin{array}{l}2400 \\
2400\end{array}$ & $\begin{array}{l}509.02 \\
495.99\end{array}$ & $\begin{array}{l}205,000 \\
125,100\end{array}$ \\
\hline
\end{tabular}

(110) 3-3182. Rock Lick Creek near Glen Dean, Ky.

Location.--Lat $37^{\circ} 39^{\prime} 24^{\prime \prime}$, long $86^{\circ} 33^{\prime} 43^{\prime \prime}$, on left bank $8 \mathrm{ft}$ downstream from old bridge abutments on abandoned county road, 0.5 mile downstream from Black Lick Creek, 1.2 miles west of Glen Dean, Breckinridge County, and 7.0 miles upstream from mouth.

Dralnage area. $--20.1 \mathrm{sq} \mathrm{ml}$.

Gage-helght record.--Water-stage recorder graph. Datum of gage is $448.29 \mathrm{ft}$ above mean sea level, datum of 1929 .

Discharge record.--Stage-discharge relation defined by current-meter measurements below $2,400 \mathrm{c}$ 's and by slope-area measurement at $8,720 \mathrm{cfs}$.

Maxima.--Given in the following table.

March 1964:

Mar. 4, 1900 hours . . . . . . . . . . . . . . .

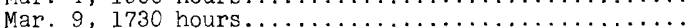

1956 to February 1964:

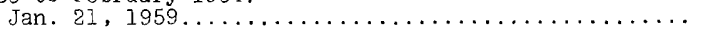

$\begin{array}{cc}\begin{array}{c}\text { Discharge } \\ \text { (cfs) }\end{array} & \begin{array}{c}\text { Gage height } \\ \text { (feet) }\end{array} \\ 2,330 & 14.63 \\ 6,010 & 17.28 \\ 8,720 & 18.36\end{array}$


Mean discharge, in cubic feet per second, March 1964, of Rock Lick Creek near Glen Dean, Ky.

\begin{tabular}{|c|c|c|c|c|c|c|c|c|c|}
\hline Day & D1scharge & Day & D1scharge & Day & Discharge & Day & Discharge & Day & Discharge \\
\hline $\begin{array}{l}1 \ldots \\
2 \ldots \\
3 \ldots \\
4 \ldots \\
5 \ldots \\
6 \ldots \\
\end{array}$ & $\begin{array}{r}2.0 \\
2.6 \\
8.5 \\
1,270 \\
190 \\
62\end{array}$ & $\begin{array}{r}7 \ldots \ldots \\
8 \ldots \ldots \\
9 \ldots \\
10 \ldots \ldots \\
11 \ldots \ldots \\
12 \ldots \ldots\end{array}$ & $\begin{array}{r}32 \\
1,350 \\
2,810 \\
458 \\
121 \\
95\end{array}$ & $\begin{array}{l}13 \ldots \ldots \\
14 \ldots \ldots \\
15 \ldots \ldots \\
16 \ldots \ldots \\
17 \ldots \ldots\end{array}$ & $\begin{array}{l}56 \\
88 \\
77 \\
43 \\
30 \\
22\end{array}$ & $\begin{array}{l}19 \ldots \ldots \\
20 \ldots \ldots \\
21 \ldots \ldots \\
22 \ldots \ldots \\
23 \ldots \ldots \\
24 \ldots \ldots\end{array}$ & $\begin{array}{l}18 \\
75 \\
67 \\
40 \\
22 \\
13\end{array}$ & $\left|\begin{array}{l}25 \ldots \\
26 \ldots \ldots \\
27 \ldots \ldots \\
28 \ldots \ldots \\
29 \ldots \ldots \\
30 \ldots \ldots \\
31 \ldots \ldots\end{array}\right|$ & $\begin{array}{l}22 \\
35 \\
17 \\
15 \\
11 \\
9.6 \\
9.2\end{array}$ \\
\hline
\end{tabular}

Gage height, in feet, and discharge, in cubic feet per second, at indicated time, 1964

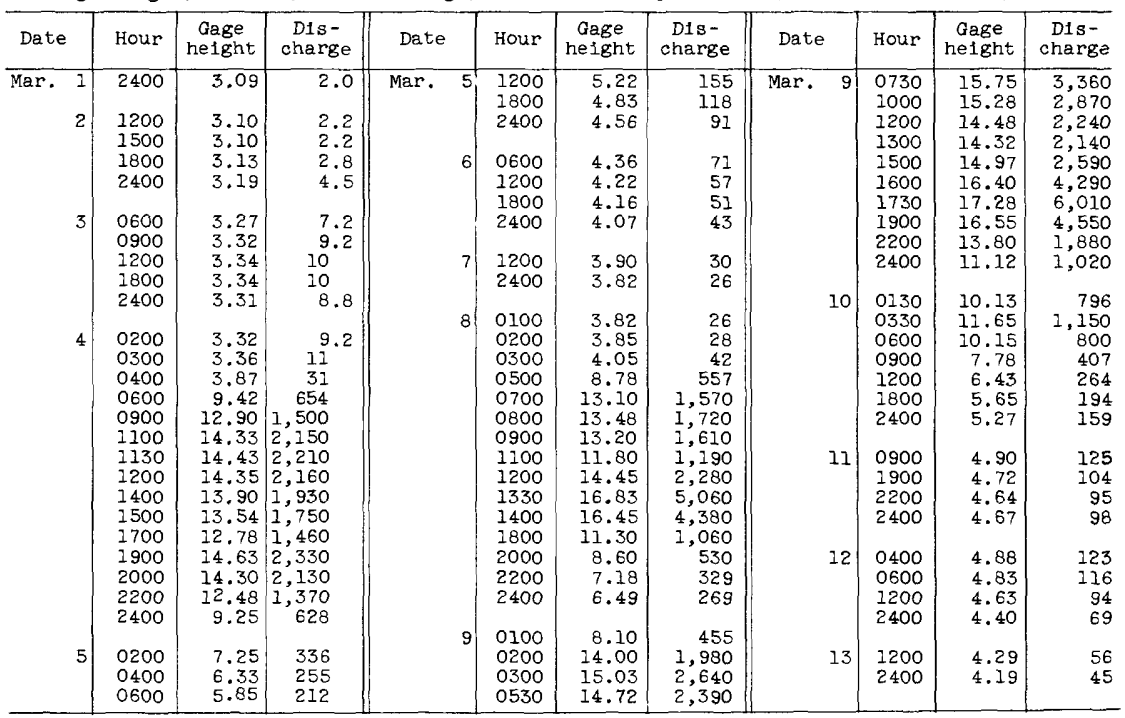


(111) 3-3185. Rough River at Falls of Rough, Ky.

Location. - Lat $37^{\circ} 35^{\prime} 20^{\prime \prime}$, long $86^{\circ} 33^{\prime} 05^{\prime \prime}$, on left bank $150 \mathrm{ft}$ downstream from bridge on State Highway 110 at Falls of Rough, Grayson County, 3.0 miles downstream from Rock Lick Creek, and $6 \frac{1}{2}$ miles downstream from Rough River Dam.

Drainage area. $-504 \mathrm{sq} \mathrm{mi}$, of which about $110 \mathrm{sq} \mathrm{mi}$ does not contribute directly to surface runoff.

Gage-he1ght record.--Water-stage recorder graph. Datum of gage, $420.94 \mathrm{ft}$ above mean sea level, datum of 1929.

Discharge record. - -Stage-discharge relation defined by current-meter measurements. Rate of change in stage used as a factor Mar. $4,5,8-11$.

Maxima.--Given in the following table.

March 1964

Mar. 4, 1700 hours.

Mar. 5, 0030 hours

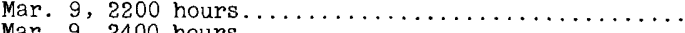

1940 to February 1964:

Jan. 14,1950 .

Discharge Gage helght (cfs) (feet)

4,590

$8, \overline{3} 20$

$-$

12,400

28.87

Floods of Jan. 12, 1913, 1915, March 1935, and Jan. 25, 1937, reached stages of $28.96,28.93,29.31$ and $34.06 \mathrm{ft}$, respectively, from floodmarks

Remarks.--Flow appreciably regulated by Rough River Reservo1r (see station 3180.05 ).

Mean discharge, in cubic feet per second, March 1964

\begin{tabular}{|c|c|c|c|c|c|c|c|c|c|}
\hline Day & Discharge & Day & Discharge & Day & Discharge & Day & Discharge & Day & Discharge \\
\hline $\begin{array}{l}1 \ldots \ldots \\
2 \ldots \ldots \\
3 \ldots \ldots \\
4 \ldots \ldots \\
5 \ldots \ldots \\
6 \ldots \ldots\end{array}$ & $\begin{array}{r}53 \\
116 \\
174 \\
2,730 \\
2,640 \\
897\end{array}$ & $\begin{array}{r}7 \ldots \ldots \\
8 \ldots \ldots \\
9 \ldots \\
10 \ldots \\
11 \ldots \\
12 \ldots\end{array}$ & $\begin{array}{r}229 \\
2,640 \\
6,100 \\
5,710 \\
2,120 \\
726\end{array}$ & $\begin{array}{l}13 \ldots \ldots \\
14 \ldots \ldots \\
15 \ldots \ldots \\
16 \ldots \ldots \\
17 \ldots \ldots \\
18 \ldots \ldots\end{array}$ & $\begin{array}{l}298 \\
310 \\
364 \\
255 \\
222 \\
200\end{array}$ & $\begin{array}{l}19 \ldots \ldots \\
20 \ldots \ldots \\
21 \ldots \ldots \\
22 \ldots \ldots \\
23 \ldots \ldots \\
24 \ldots \ldots\end{array}$ & $\begin{array}{r}185 \\
1,000 \\
2,050 \\
2,220 \\
2,050 \\
1,930\end{array}$ & $\left(\begin{array}{l}25 \ldots \ldots \\
26 \ldots \ldots \\
27 \ldots \ldots \\
28 \ldots \ldots \\
29 \ldots \ldots \\
30 \ldots \ldots \\
31 \ldots \ldots\end{array}\right.$ & $\begin{array}{l}1,920 \\
1,930 \\
1,900 \\
1,880 \\
1,880 \\
1,860 \\
1,940\end{array}$ \\
\hline
\end{tabular}

(112) 3-3188. Caney Creek near Horse Branch, ky.

Location. - Lat $37^{\circ} 27^{\prime} 50^{\prime \prime}$, Iong $86^{\circ} 39^{\prime} 20^{\prime \prime}$, on right bank $9 \mathrm{ft}$ downstream from bridge on U.S. Highway $62,0.6$ mile upstream from Horse Branch, 1.5 miles northeast of village of Horse Branch, Ohio County, and 11.2 miles upstream from mouth.

Drainage area.--124 sq $\mathrm{m}$, includes that of Horse Branch at U.S. H1ghway 62.

Gage-he1ght record.--Water-stage recorder graph. Datum of gage is $417.30 \mathrm{ft}$ above mean sea level, datum of 1929 .

D1scharge record.--Stage-discharge relation defined by current-meter measurements . Maxima.--Given in the following table.

March 1964 :

Mar. 5, 0530

(n)



$$
\begin{aligned}
& (\mathrm{cfs}) \\
& 9,100 \\
& 8,900
\end{aligned}
$$

Discharge
Gage height
13.40

13.44

1956 to February 1964

al0,000

b14. 43

a About.

b Backwater from Rough River or debris. 
Gage height, in feet, and discharge, in cublc feet per second, at indicated time, 1964, of

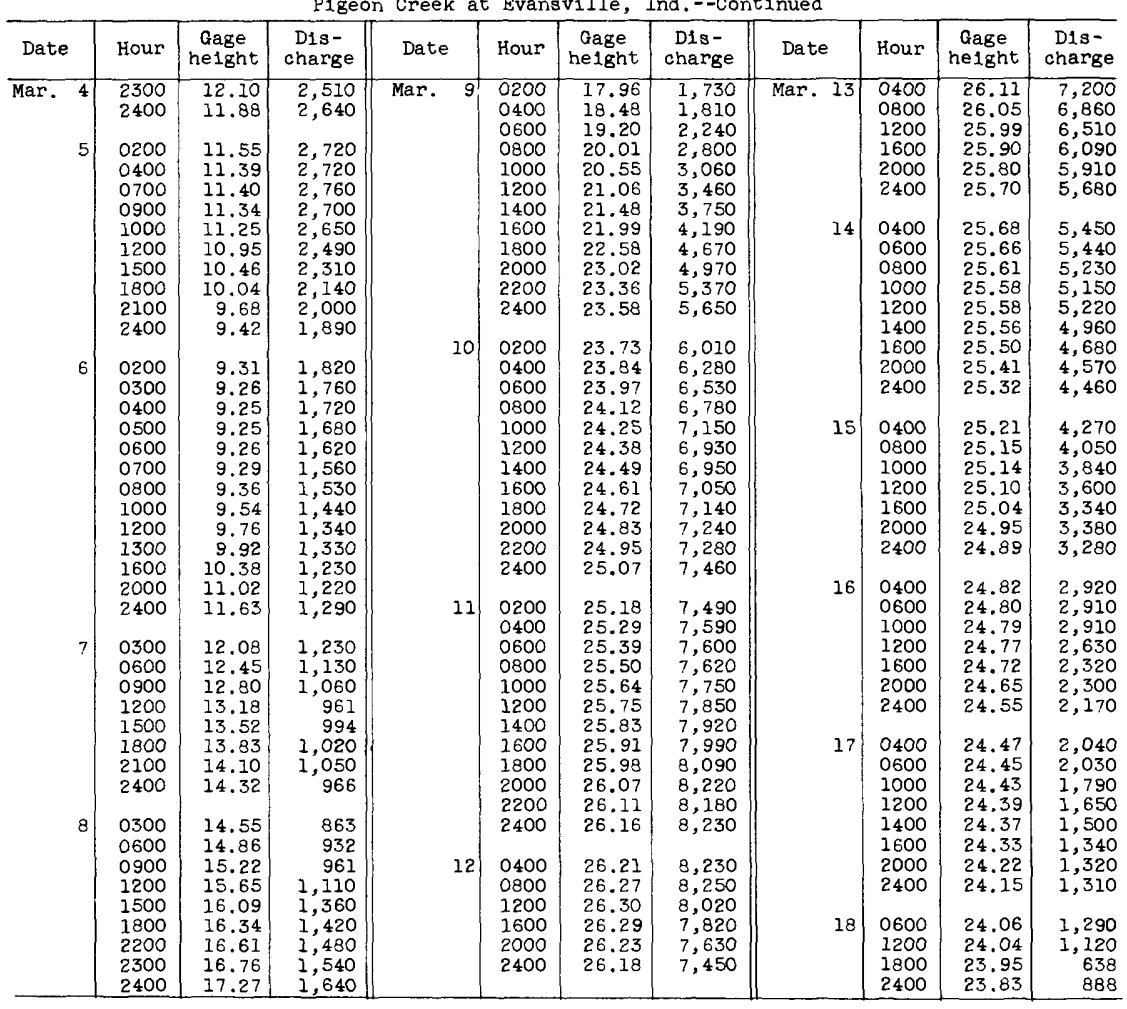




\section{WABASH RIVER BASIN}

(118) 3-3430. Wabash River at Vincennes, Ind.

Location.--Lat $38^{\circ} 40^{\prime} 52^{\prime \prime}$, long $87^{\circ} 32^{\prime} 04^{\prime \prime}$, near center of span on downstream side of bridge on U.S. H1ghway 50 at Vincennes, Knox County, 4.8 miles downstream from Maria Creek, 5.8 miles upstream from Embarrass River, and at mile 127.8.

Drainage area. $--13,700$ sq $\mathrm{mi}$, approximately.

Gage-helght record.--Graph drawn from twice-da1ly wire-weight-gage readings. Auxillary water-stage recorder graph, except Mar. 23-26, 4.7 miles upstream at datum

$0.80 \mathrm{ft}$ higher. Datum of gage is $394.43 \mathrm{ft}$ above mean sea level, datum of 1929 .

D1scharge record.--Stage-discharge or stage-fall-discharge relation defined by current-meter measurements below $106,000 \mathrm{cfs}$. Fall used as a factor Mar. 9-22, 27-31. Discharge for period of no gage-helght record at auxillary gage estimated on basis of records for nearby stations.

Maxima.--March 1964: D1scharge, 27,500 efs Mar. 11, 2400 hours; gage he1ght, $14.48 \mathrm{ft}$ Mar. 12,0800 hours. 1867 to February 1964: Discharge, 255,000 cfs (estimated) Mar. 29, 1913 (gage helght, $26.3 \mathrm{ft}$, from floodmarks, by Corps of Engineers).

Mean discharge, in cuble feet per second, March 1964

\begin{tabular}{|c|c|c|c|c|c|c|c|c|c|}
\hline Day & Discharge & Day & Discharge & Day & Discharge & Day & Discharge & Day & Discharge \\
\hline $\begin{array}{l}1 \ldots \ldots \\
2 \ldots \ldots \\
3 \ldots \ldots \\
4 \ldots \ldots \\
5 \ldots \ldots \\
6 \ldots \ldots\end{array}$ & $\begin{array}{l}2,150 \\
2,220 \\
2,260 \\
2,610 \\
4,490 \\
6,280\end{array}$ & $\begin{array}{r}7 \ldots \ldots \\
8 \ldots \ldots \\
9 \ldots \ldots \\
10 \ldots \\
11 \ldots \ldots \\
12 \ldots \ldots\end{array}$ & $\begin{array}{r}7,510 \\
8,700 \\
14,500 \\
25,200 \\
27,100 \\
27,100\end{array}$ & $\begin{array}{l}13 \ldots \ldots \\
14 \ldots \ldots \\
15 \ldots \ldots \\
16 \ldots \ldots \\
17 \ldots \ldots \\
18 \ldots \ldots\end{array}$ & $\begin{array}{l}26,300 \\
26,000 \\
25,500 \\
25,100 \\
24,400 \\
23,600\end{array}$ & $\begin{array}{l}19 \ldots \ldots \\
20 \ldots \ldots \\
21 \ldots \ldots \\
22 \ldots \ldots \\
23 \ldots \ldots \\
24 \ldots \ldots\end{array}$ & $\begin{array}{l}23,300 \\
22,700 \\
19,600 \\
16,200 \\
14,000 \\
13,000\end{array}$ & $\mid \begin{array}{l}25 \ldots \ldots \\
26 \ldots \ldots \\
27 \ldots \ldots \\
28 \ldots \ldots \\
29 \ldots \ldots \\
30 \ldots \ldots \\
31 \ldots \ldots\end{array}$ & $\begin{array}{l}12,00 \\
12,00 \\
14,00 \\
15,50 \\
17,60 \\
18,80 \\
17,40\end{array}$ \\
\hline
\end{tabular}

(119) 3-3605. White River at Newberry, Ind.

Location.--Lat $38^{\circ} 55^{\prime} 42^{\prime \prime}$, long $87^{\circ} 01^{\prime} 00^{\prime \prime}$, in sec.25, T.6 N., R.6 W., on right bank $500 \mathrm{ft}$ upstream from bridge on State Highway 57 at Newberry, $2 . \dot{3}$ miles downstream from Doans Creek, at mile 118.0 .

Drainage area. $--4,696 \mathrm{sq} \mathrm{mi}$.

Gage-he1ght record.--Water-stage recorder graph. Datum of gage is $465.59 \mathrm{ft}$ above mean sea leve1, datum of 1929 .

Discharge record.--Stage-discharge relation defined by current-meter measurements below 80,000 cis.

Maxima.--March 1964: Discharge, 37,300 cfs Mar. 14 (gage he1ght, $20.12 \mathrm{ft}$ ). i875 to February 1964: Discharge, 130,000 cfs (estimated) Mar. 27, 1913

(gage helght, $27.5 \mathrm{ft}$, from floodmarks, by State Highway Department of Indiana).

Mean discharge, in cublc feet per second, March 1964

\begin{tabular}{|c|c|c|c|c|c|c|c|c|c|}
\hline Day & Discharge & Day & D1scharge & Day & Discharge & Day & D1scharge & Day & Discharge \\
\hline $\begin{array}{l}1 \ldots \ldots \\
2 \ldots \ldots \\
3 \ldots \ldots \\
4 \ldots \ldots \\
5 \ldots \ldots \\
6 \ldots \ldots\end{array}$ & $\begin{array}{r}782 \\
796 \\
789 \\
1,210 \\
5,460 \\
8,460\end{array}$ & $\begin{array}{r}7 \ldots \ldots \\
8 \ldots \ldots \\
9 \ldots \ldots \\
10 \ldots \\
11 \ldots \ldots \\
12 \ldots \ldots\end{array}$ & $\begin{array}{r}6,950 \\
5,590 \\
11,700 \\
23,700 \\
26,200 \\
27,900\end{array}$ & $\begin{array}{l}13 \ldots \ldots \\
14 \ldots \ldots \\
15 \ldots \ldots \\
16 \ldots \\
17 \ldots \ldots \\
18 \ldots \ldots\end{array}$ & $\begin{array}{l}34,100 \\
36,200 \\
32,100 \\
27,300 \\
21,600 \\
15,900\end{array}$ & $\begin{array}{l}19 \ldots \ldots \\
20 \ldots \ldots \\
21 \ldots \ldots \\
22 \ldots \ldots \\
23 \ldots \ldots \\
24 \ldots \ldots\end{array}$ & $\begin{array}{r}11,300 \\
9,250 \\
8,300 \\
8,110 \\
7,920 \\
7,470\end{array}$ & $\mid \begin{array}{l}25 \ldots \ldots \\
26 \ldots \ldots \\
27 \ldots \ldots \\
28 \ldots \ldots \\
29 \ldots \ldots \\
30 \ldots \ldots \\
31 \ldots \ldots\end{array}$ & $\begin{array}{l}7,080 \\
7,210 \\
7,370 \\
7,340 \\
7,000 \\
6,470 \\
5,610\end{array}$ \\
\hline $\begin{array}{l}\text { onthiy } \\
\text { anof } \mathrm{f} \text {, }\end{array}$ & $\begin{array}{l}\text { mean dis } \\
\text { in inche }\end{array}$ & ge, & & per & & & & & $\begin{array}{r}12,490 \\
3.07\end{array}$ \\
\hline
\end{tabular}


(120) 3-3640. East Fork White River at Columbus, Ind.

Location.--Lat $39^{\circ} 12^{\prime}$, long $86^{\circ} 56^{\prime}$, in NW $\frac{1}{4}$ sec.25, T.9 N., R.5 E., on left bank at abutment of abandoned bridge at west end of Second Street in Cólumbus, 0.6 mile downstream from confluence of Driftwood River and Flatrock River, and 1.4 miles upstream from Haw Creek.

Drainage area. $--1,692 \mathrm{sq} \mathrm{ml}$.

Gage-height record.--Water-stage recorder graph. Datum of gage is $603.12 \mathrm{ft}$ above mean sea level, datum of 1929.

Discharge record.--Stage-discharge relation defined by current-meter measurements below 52,000 efs.

Maxima.--March 1964: Discharge, 28,200 cfs Mar. 11, 0800 hours.

1913: Gage helght, $17.9 \mathrm{ft}$, from floodmarks (discharge, about 100,000 cfs).

1947 to February 1964: Discharge, 48,700 cfs Jan. 28, 1952 (gage height, $16.00 \mathrm{ft}$ ).

Mean discharge, in cubic feet per second, March 1964

\begin{tabular}{|c|c|c|c|c|c|c|c|c|c|}
\hline Day & Discharge & Day & Discharge & Day & D1scharge & Day & Discharge & Day & Discharge \\
\hline $\begin{array}{l}1 \ldots \ldots \\
2 \ldots \ldots \\
3 \ldots \ldots \\
4 \ldots \\
5 \ldots \ldots \\
6 \ldots \ldots\end{array}$ & $\begin{array}{r}296 \\
290 \\
290 \\
1,400 \\
4,740 \\
4,890\end{array}$ & $\begin{array}{r}7 \ldots \ldots \\
8 \ldots \ldots \\
9 \ldots \\
10 \ldots \\
11 \ldots \\
12 \ldots\end{array}$ & $\begin{array}{r}3,620 \\
2,560 \\
7,820 \\
20,600 \\
27,800 \\
25,400\end{array}$ & $\begin{array}{l}13 \ldots \ldots \\
14 \ldots \ldots \\
15 \ldots \ldots \\
16 \ldots \ldots \\
17 \ldots \ldots \\
18 \ldots \ldots\end{array}$ & $\begin{array}{r}16,800 \\
10,600 \\
7,540 \\
5,840 \\
4,630 \\
3,650\end{array}$ & $\begin{array}{l}19 \ldots \ldots \\
20 \ldots \ldots \\
21 \ldots \ldots \\
22 \ldots \ldots \\
23 \ldots \ldots \\
24 \ldots \ldots\end{array}$ & $\begin{array}{l}2,930 \\
2,530 \\
2,280 \\
2,130 \\
1,960 \\
1,790\end{array}$ & $\left|\begin{array}{l}25 \ldots \\
26 \ldots \ldots \\
27 \ldots \ldots \\
28 \ldots \ldots \\
29 \ldots \ldots \\
30 \ldots \ldots \\
31 \ldots \ldots\end{array}\right|$ & $\begin{array}{l}1,680 \\
1,990 \\
2,220 \\
1,960 \\
1,710 \\
1,500 \\
1,370\end{array}$ \\
\hline $1 \mathrm{~J}$ & in & & & & & & & & $\begin{array}{r}5,639 \\
3.84\end{array}$ \\
\hline
\end{tabular}

(121) 3-3645. Clifty Creek at Hartsville, Ind.

Location.--Lat $39^{\circ} 16^{\prime} 25^{\prime \prime}$, long $85^{\circ} 42^{\prime} 10^{\prime \prime}$, in NW $\frac{1}{4}$ sec.36, T.10 N., R.7 E., at downstream side of left abutment of highway bridge, a quarter of a mile north of Hartsville and 5 miles upstream from Duck Creek.

Drainage area. $--88.8 \mathrm{sq} \mathrm{mi}$.

Gage-height record.--Water-stage recorder graph. Datum of gage is $677.34 \mathrm{ft}$ above mean sea level, datum of 1929 .

Discharge record.--Stage-discharge relation defined by current-meter measurements below 5,900 cis.

Maxima.--Given in the following table.

March 1964 :

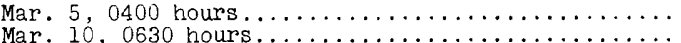

1913:

Gage helght

(cfs) (feet)

$1,420 \quad 5.27$

$6,820 \quad 11.78$

1948 to February 1964

Unknown 25.1

Jan. 21, 1959

11,300

14.29

Mean discharge, in cubic feet per second, March 1964

\begin{tabular}{|c|c|c|c|c|c|c|c|c|c|}
\hline Day & Discharge & Day & Discharge & Day & Discharge & Day & D1scharge & Day & Discharge \\
\hline $\begin{array}{l}1 \ldots \ldots \\
2 \ldots \ldots \\
3 \ldots \ldots \\
4 \ldots \\
5 \ldots \\
6 \ldots\end{array}$ & $\begin{array}{r}9.0 \\
9.0 \\
11 \\
314 \\
811 \\
237\end{array}$ & $\begin{array}{r}7 \ldots \ldots \\
8 \ldots \ldots \\
9 \ldots \\
10 \ldots \\
11 \ldots \ldots \\
12 \ldots \ldots\end{array}$ & $\begin{array}{r}161 \\
140 \\
3,340 \\
4,760 \\
804 \\
514\end{array}$ & $\begin{array}{l}13 \ldots \ldots \\
14 \ldots \ldots \\
15 \ldots \ldots \\
16 \ldots \ldots \\
17 \ldots \ldots \\
18 \ldots \ldots\end{array}$ & $\begin{array}{l}342 \\
310 \\
310 \\
208 \\
164 \\
128\end{array}$ & $\begin{array}{l}19 \ldots \ldots \\
20 \ldots \ldots \\
21 \ldots \ldots \\
22 \ldots \ldots \\
23 \ldots \ldots \\
24 \ldots \ldots\end{array}$ & $\begin{array}{r}106 \\
99 \\
146 \\
156 \\
128 \\
108\end{array}$ & $\begin{array}{l}25 \ldots \ldots \\
26 \ldots \ldots \\
27 \ldots \ldots \\
28 \ldots \ldots \\
29 \ldots \ldots \\
30 \ldots \ldots \\
31 \ldots \ldots\end{array}$ & $\begin{array}{l}10 \\
15 \\
13 \\
10\end{array}$ \\
\hline
\end{tabular}


(122) 3-3650. Sand Creek near Brewersv1lle, Ind.

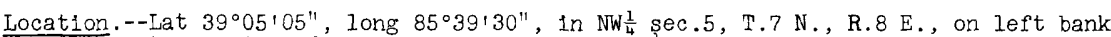
at downstream side of county highway bridge, $2 \frac{1}{2}$ miles west of Brewersville, and 5.2 miles upstream from Bear Creek.

Drainage area. --156 sq $\mathrm{ml}$.

Gage-helght record. - Water-stage recorder graph. Altitude of gage is $630 \mathrm{ft}$ (by barometer).

Discharge record.--Stage-discharge relation defined by current-meter measurements below $13,000 \mathrm{cfs}$ and by contracted-opening measurement at 19,900 cfs.

Maxima.--Given in the following table.

March 1964:

Mar. 5, 0300 hours.

1897:

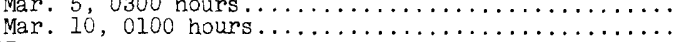

March 1897.

1948 to February 1964 :

Jan. 21, 1959.

$\begin{array}{cc}\begin{array}{c}\text { Discharge } \\ \text { (cfs) }\end{array} & \text { Gage helght } \\ 4,300 & \text { feet) } \\ 14,000 & 11.00 \\ & \text { al8.70 }\end{array}$

Unknown

(b)

19,900

21.7

a From inside gage; floodmark elevation, $19.3 \mathrm{ft}$.

b Several feet higher than stage of Jan. $21,1959$.

Mean discharge, in cubic feet per second, March 1964

\begin{tabular}{|c|c|c|c|c|c|c|c|c|c|}
\hline Day & Discharge & Day & Discharge & Day & Discharge & Day & Discharge & Day & Discharge \\
\hline $\begin{array}{l}1 \ldots \ldots \\
2 \ldots \\
3 \ldots \ldots \\
4 \ldots \ldots \\
5 \ldots \ldots \\
6 \ldots \ldots\end{array}$ & $\begin{array}{r}22 \\
22 \\
30 \\
1,070 \\
2,260 \\
471\end{array}$ & $\begin{array}{r}7 \ldots \ldots \\
8 \ldots \ldots \\
9 \ldots \\
10 \ldots \\
11 \ldots \ldots \\
12 \ldots\end{array}$ & $\begin{array}{r}246 \\
200 \\
8,700 \\
9,360 \\
1,180 \\
819\end{array}$ & $\begin{array}{l}13 \ldots \ldots \\
14 \ldots \ldots \\
15 \ldots \ldots \\
16 \ldots \ldots \\
17 \ldots \ldots \\
18 \ldots \ldots\end{array}$ & $\begin{array}{l}495 \\
489 \\
686 \\
312 \\
220 \\
168\end{array}$ & $\begin{array}{l}19 \ldots \ldots \\
20 \ldots \ldots \\
21 \ldots \ldots \\
22 \ldots \ldots \\
23 \ldots \ldots \\
24 \ldots \ldots\end{array}$ & $\begin{array}{l}121 \\
128 \\
204 \\
204 \\
162 \\
138\end{array}$ & $\begin{array}{l}25 \ldots \ldots \\
26 \ldots \ldots \\
27 \ldots \ldots \\
28 \ldots \ldots \\
29 \ldots \ldots \\
30 \ldots \ldots \\
31 \ldots \ldots\end{array}$ & $\begin{array}{r}126 \\
246 \\
189 \\
145 \\
124 \\
108 \\
92\end{array}$ \\
\hline inoff, & $\begin{array}{l}\text { mean disc } \\
\text { in inches }\end{array}$ & ge, & $a b 1 c$ & per & 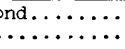 & & & & $\begin{array}{r}927 \\
6.85\end{array}$ \\
\hline
\end{tabular}

(123) 3-3655. East Fork White River at Seymour, Ind.

Location. - Lat $38^{\circ} 58157^{\prime \prime}$, long $85^{\circ} 53^{\prime} 57^{\prime \prime}$, in NW $\frac{1}{4}$ sec.7, T. 6 N., R.6 E., on left bank $1,700 \mathrm{ft}$ downstream from highway bridge, 1 mile north of Seymour, $9.6 \mathrm{mlles}$ downstream from Sand Creek, and at mile 219.2 .

Dra1nage area. $--2,333 \mathrm{sq} \mathrm{mi}$.

Gage-he1ght record.--Water-stage recorder graph. Datum of gage is $550.67 \mathrm{ft}$ above mean sea level, datum of 1929.

Discharge record.--Stage-discharge relation defined by current-meter measurements below 59,000 cis.

Maxima.--March 1964: Discharge, 54,900 cfs Mar. 10, 2000 hours (gage he1ght, $18.72 \mathrm{ft}$ ).

1897 to February 1964: Discharge, 120,000 cfs Mar. 26, 1913 (gage height, $21.0 \mathrm{ft}$, from Information by Corps of Engineers and State Highway Dept. of Indiana).

Mean discharge, in cubic feet per second, March 1964

\begin{tabular}{l} 
Day \\
\hline Discharge
\end{tabular}


(124) 3-3670. Muscatatuck River near Austin, Ind.

Location.--Iat $38^{\circ} 46^{\prime}$, long $85^{\circ} 49^{\prime}$, in sec.23, T.4 N., R.6 E., on right bank $15 \mathrm{ft}$ downstream from bridge on U.S. Highway 31, 2 miles north of Austin, and 4 miles upstream from stucker Fork.

Drainage area. $--365 \mathrm{sq} \mathrm{ml}$.

Gage-height record. - Water-stage recorder graph. Datum of gage is $513.96 \mathrm{ft}$ above mean sea level, datum of 1929.

Discharge record.--Stage-discharge relation defined by current-meter measurements below 34,000 cfs. Discharge not computed when gage helght is below $13.0 \mathrm{ft}$.

Maxima.--Given in the following table.

March 1964:

Mar. 5, 2100 hours.

.

1932 to February 1964:

Jan. 22, 1959

$\ldots$

$\ldots \ldots \ldots \ldots \ldots \ldots \ldots \ldots$

$\begin{array}{cc}\begin{array}{c}\text { Discharge } \\ \text { (cfs) }\end{array} & \begin{array}{c}\text { Gage height } \\ \text { (feet) }\end{array} \\ 13,200 & 23.71 \\ 36,600 & 27.61\end{array}$

53,900

29.20

Mean discharge, in cubic feet per second, March 1964

\begin{tabular}{|c|c|c|c|c|c|c|c|c|c|}
\hline Day & Discharge & Day & D1scharge & Day & Discharge & Day & Discharge & Day & D1scharge \\
\hline $\begin{array}{l}1 \ldots \ldots \\
2 \ldots \ldots \\
3 \ldots \ldots \\
4 \ldots \ldots \\
5 \ldots \ldots \\
6 \ldots \ldots\end{array}$ & $\begin{array}{c}- \\
\overline{-} \\
\overline{-} \\
6,860 \\
8,400\end{array}$ & $\begin{array}{r}7 \ldots \ldots \\
8 \ldots \ldots \\
9 \ldots \\
10 \ldots \\
11 \ldots \ldots \\
12 \ldots\end{array}$ & $\begin{array}{r}2,810 \\
1,120 \\
6,650 \\
30,400 \\
11,700 \\
4,120\end{array}$ & $\begin{array}{l}13 \ldots \ldots \\
14 \ldots \ldots \\
15 \ldots \ldots \\
16 \ldots \ldots \\
17 \ldots \ldots \\
18 \ldots \ldots\end{array}$ & $\begin{array}{c}1,820 \\
1,060 \\
1,380 \\
756 \\
- \\
-\end{array}$ & $\begin{array}{l}19 \ldots \ldots \\
20 \ldots \ldots \\
21 \ldots \ldots \\
22 \ldots \ldots \\
23 \ldots \ldots \\
24 \ldots \ldots\end{array}$ & $\begin{array}{l}- \\
\overline{-} \\
\overline{-} \\
-\end{array}$ & $\mid \begin{array}{l}25 \ldots \ldots \\
26 \ldots \ldots \\
27 \ldots \ldots \\
28 \ldots \ldots \\
29 \ldots \ldots \\
30 \ldots \ldots \\
31 \ldots \ldots\end{array}$ & $\begin{array}{l}- \\
- \\
- \\
- \\
-\end{array}$ \\
\hline
\end{tabular}

(125) 3-3695. Vernon Fork at Vernon, Ind.

Location.--Lat $38^{\circ} 57^{\prime}$, long $85^{\circ} 37^{\prime}$, In sec.10, T. 6 N., R. 8 E., on right bank just downstream from highway bridge, 1 mile southwest of Vernon and 2 miles downstream from confluence of Nurth and South Forks.

Dra1nage area. $--201 \mathrm{sq} \mathrm{ml}$.

Gage-height record. - Water-stage recorder graph. Datum of gage is $587.30 \mathrm{ft}$ above mean sea level, datum of 1929, supplementary adjustment of 1944 (levels by Ind1ana Flood Control and Water Resources Commission).

Discharge record.--Stage-discharge relation defined by current-meter measurements below $25,000 \mathrm{cfs}$ and by slope-area measurement at 56,800 $\mathrm{cfs}$.

Maxima.--Given in the following table.

March 1964:

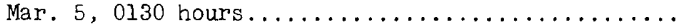

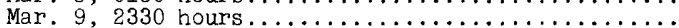

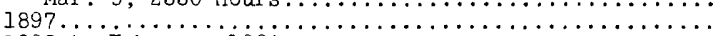

1898 to February 1964 :

Jan. 2l, 1959.

$\begin{array}{cc}\text { Discharge } & \text { Gage height } \\ \text { (cfs) } & \text { (feet) } \\ 7,500 & 13.07 \\ 21,100 & 222.66 \\ \text { Unknown } & \text { (b) }\end{array}$

56,800

a From inside gage; floodmark elevation, $22.8 \mathrm{ft}$.

b About equal to the stage of Jan. 21, 1959 .

Mean discharge, in cub1c feet per second, March 1964

\begin{tabular}{|c|c|c|c|c|c|c|c|c|c|}
\hline Day & D1scharge & Day & Discharge & Day & Discharge & Day & Discharge & Day & Discharge \\
\hline $\begin{array}{l}1 \ldots \ldots \\
2 \ldots \\
3 \ldots \\
4 \ldots \\
5 \ldots \\
6 \ldots \\
\ldots\end{array}$ & $\begin{array}{r}16 \\
16 \\
21 \\
1,620 \\
3,070 \\
428\end{array}$ & $\begin{array}{r}7 \ldots \ldots \\
8 \ldots \ldots \\
9 \ldots \\
10 \ldots \\
11 \ldots \\
12 \ldots\end{array}$ & $\begin{array}{r}233 \\
194 \\
13,700 \\
11,400 \\
1,350 \\
752\end{array}$ & $\begin{array}{l}13 \ldots \ldots \\
14 \ldots \ldots \\
15 \ldots \ldots \\
16 \ldots \ldots \\
17 \ldots \ldots \\
18 \ldots \ldots\end{array}$ & $\begin{array}{l}446 \\
472 \\
768 \\
335 \\
223 \\
161\end{array}$ & $\begin{array}{l}19 \ldots \ldots \\
20 \ldots \ldots \\
21 \ldots \ldots \\
22 \ldots \ldots \\
23 \ldots \ldots \\
24 \ldots \ldots\end{array}$ & $\begin{array}{l}125 \\
113 \\
159 \\
173 \\
130 \\
106\end{array}$ & $\mid \begin{array}{l}25 \ldots \ldots \\
26 \ldots \ldots \\
27 \ldots \ldots \\
28 \ldots \ldots \\
29 \ldots \ldots \\
30 \ldots \ldots \\
31 \ldots \ldots\end{array}$ & $\begin{array}{r}96 \\
242 \\
216 \\
139 \\
111 \\
90 \\
77\end{array}$ \\
\hline $\begin{array}{l}\text { onthly } \\
\text { inoff, }\end{array}$ & $\begin{array}{l}\text { mean disch } \\
\text { in inches. }\end{array}$ & ge, & $\mathrm{b} 1 \mathrm{c}$ & per & & & & & $\begin{array}{r}1,192 \\
6.84\end{array}$ \\
\hline
\end{tabular}


(126) 3-3715. East Fork White River near Bedford, Ind.

Location.--Lat $38^{\circ} 46^{\prime} 10^{\prime \prime}$, long $86^{\circ} 24^{\prime} 30^{\prime \prime}$, In NE $\frac{1}{2}$ sec.21, T.4 N., R.1 E., on downstream side of center pier of bridge on county road, 0.4 mile upstream from Mill Creek, 2.9 miles downstream from Sugar Creek, 3.9 miles northeast of Mitchell, and 7.8 miles southeast of Bedford.

Drainage area. $--3,870 \mathrm{sq} \mathrm{mi}$.

Gage-height record.--Water-stage recorder graphs. Datum of gage is $473.59 \mathrm{ft}$ above mean sea level, datum of 1929. Auxillary gage, 9.7 miles downstream, is at datum $4.39 \mathrm{ft}$ lower.

Discharge record. - -Stage-discharge or stage-fall-discharge relation defined by current-meter measurements. Fall used as a factor Mar. 9-20.

Maxima.--March 1964: Discharge, 75,700 cfs Mar. 12, 1400 hours; gage helght, 35.22 ft Mar. 13, 0200 hours.

1913: Gage height, $47.5 \mathrm{ft}^{\prime} \mathrm{f}$ from floodmarks determined by Corps of Englneers at site 9.7 miles downstream (discharge, $155,000 \mathrm{cfs}$ ).

1939 to February 1964: Discharge, 70,900 cfs May il, 1961; gage height, $35.97 \mathrm{ft}$ May 11, 1961 .

Mean discharge, in cubic feet per second, March 1964

\begin{tabular}{|c|c|c|c|c|c|c|c|c|c|}
\hline Day & Discharge & Day & Discharge & Day & Discharge & Day & Discharge & Day & Discharge \\
\hline $\begin{array}{l}1 \ldots . \\
2 \ldots \\
3 \ldots \\
4 \ldots \\
5 \ldots \\
6 \ldots \\
\end{array}$ & $\begin{array}{r}600 \\
581 \\
575 \\
1,440 \\
6,660 \\
7,670\end{array}$ & $\begin{array}{r}7 \ldots \ldots \\
8 \ldots \ldots \\
9 \ldots \ldots \\
10 \ldots \\
11 \ldots \ldots \\
12 \ldots \ldots\end{array}$ & $\begin{array}{r}8,900 \\
11,100 \\
17,800 \\
27,600 \\
40,700 \\
73,100\end{array}$ & $\begin{array}{l}13 \ldots \ldots \\
14 \ldots \ldots \\
15 \ldots \ldots \\
16 \ldots \ldots \\
17 \ldots \ldots \\
18 \ldots \ldots\end{array}$ & $\begin{array}{l}69,200 \\
60,200 \\
50,400 \\
39,700 \\
31,300 \\
25,800\end{array}$ & $\begin{array}{l}19 \ldots \ldots \\
20 \ldots \ldots \\
21 \ldots \ldots \\
22 \ldots \ldots \\
23 \ldots \ldots \\
24 \ldots \ldots\end{array}$ & $\begin{array}{r}21,000 \\
16,200 \\
12,200 \\
9,270 \\
7,010 \\
5,830\end{array}$ & $\begin{array}{l}25 \ldots \ldots \\
26 \ldots \ldots \\
27 \ldots \ldots \\
28 \ldots \ldots \\
29 \ldots \ldots \\
30 \ldots \ldots \\
31 \ldots \ldots\end{array}$ & $\begin{array}{l}5,180 \\
4,860 \\
5,080 \\
5,530 \\
5,370 \\
4,840 \\
4,310\end{array}$ \\
\hline & 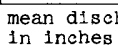 & & & & & & & & $\begin{array}{r}18,710 \\
5.57\end{array}$ \\
\hline
\end{tabular}

Gage he1ght, in feet, and discharge, in cublc feet per second, at indicated time, 1964

\begin{tabular}{|c|c|c|c|c|c|c|c|c|c|c|c|}
\hline Date & Hour & $\begin{array}{c}\text { Gage } \\
\text { helght }\end{array}$ & $\begin{array}{l}\text { Dis- } \\
\text { charge }\end{array}$ & Date & Hour & $\begin{array}{c}\text { Gage } \\
\text { height }\end{array}$ & $\begin{array}{c}\text { Dis- } \\
\text { charge }\end{array}$ & Date & Hour & $\begin{array}{c}\text { Gage } \\
\text { he1ght }\end{array}$ & $\begin{array}{l}\text { Dis- } \\
\text { charge }\end{array}$ \\
\hline Mar. 3 & 2400 & 2.98 & 572 & Mar. 9 & 2400 & 24.24 & 23,100 & Mar. 14 & 2400 & 33.17 & 55,500 \\
\hline 4 & $\begin{array}{l}1200 \\
1500 \\
1800 \\
2100 \\
2400\end{array}$ & $\begin{array}{l}3.22 \\
3.82 \\
5.08 \\
6.82 \\
7.84\end{array}$ & $\begin{array}{r}728 \\
1,120 \\
1,990 \\
3,340 \\
4,240\end{array}$ & 10 & $\begin{array}{l}0600 \\
1200 \\
1500 \\
1800 \\
2400\end{array}$ & $\begin{array}{l}25.16 \\
26.38 \\
26.58 \\
26.68 \\
26.82\end{array}$ & $\begin{array}{l}25,300 \\
29,200 \\
29,500 \\
29,600 \\
29,800\end{array}$ & 15 & $\begin{array}{l}1200 \\
2400 \\
1200 \\
2400\end{array}$ & $\begin{array}{l}32.20 \\
31.12 \\
29.92 \\
28.75\end{array}$ & $\begin{array}{l}50,500 \\
45,200 \\
39,400 \\
34,900\end{array}$ \\
\hline 5 & $\begin{array}{l}0600 \\
1000 \\
1300 \\
1700 \\
2400\end{array}$ & $\begin{array}{r}9.58 \\
10.84 \\
11.37 \\
11.54 \\
11.47\end{array}$ & $\begin{array}{l}5,800 \\
6,940 \\
7,420 \\
7,580 \\
7,510\end{array}$ & 11 & $\begin{array}{l}0300 \\
0800 \\
1200 \\
1800 \\
2400\end{array}$ & $\begin{array}{l}26.90 \\
27.23 \\
27.95 \\
30.07 \\
32.28\end{array}$ & $\begin{array}{l}30,000 \\
31,500 \\
35,200 \\
49,500 \\
65,600\end{array}$ & 17 & $\begin{array}{l}1200 \\
2400 \\
1200 \\
2400\end{array}$ & $\begin{array}{l}27.58 \\
26.45 \\
\\
25.28 \\
24.02\end{array}$ & $\begin{array}{l}30,900 \\
29,800 \\
25,800 \\
23,300\end{array}$ \\
\hline 6 & $\begin{array}{l}0400 \\
0900 \\
1800\end{array}$ & $\begin{array}{l}11.46 \\
11.52 \\
11.73\end{array}$ & $\begin{array}{l}7,500 \\
7,560 \\
7,760\end{array}$ & 12 & $\begin{array}{l}0600 \\
1100 \\
1200\end{array}$ & $\begin{array}{l}33.70 \\
34.43 \\
34.55\end{array}$ & $\begin{array}{l}72,400 \\
74,800 \\
74,800\end{array}$ & 19 & $\begin{array}{l}1200 \\
2400\end{array}$ & $\begin{array}{l}22.66 \\
21.11\end{array}$ & $\begin{array}{l}21,000 \\
18,700\end{array}$ \\
\hline & 2400 & 12.03 & 8,040 & & $\begin{array}{l}1300 \\
1400\end{array}$ & $\begin{array}{l}34.65 \\
34.76\end{array}$ & $\begin{array}{l}75,300 \\
75,700\end{array}$ & 20 & $\begin{array}{l}1200 \\
2400\end{array}$ & $\begin{array}{l}19.35 \\
17.57\end{array}$ & $\begin{array}{l}16,100 \\
13,800\end{array}$ \\
\hline 7 & $\begin{array}{l}1200 \\
2400\end{array}$ & $\begin{array}{l}12.87 \\
13.82\end{array}$ & $\begin{array}{l}8,870 \\
9,820\end{array}$ & & $\begin{array}{l}1500 \\
1800 \\
2400\end{array}$ & $\begin{array}{l}34.83 \\
35.00 \\
35.20\end{array}$ & $\begin{array}{l}75,400 \\
74,700 \\
73,800\end{array}$ & 21 & $\begin{array}{l}1200 \\
2400\end{array}$ & $\begin{array}{l}15.93 \\
14.56\end{array}$ & $\begin{array}{l}12,100 \\
10,600\end{array}$ \\
\hline 8 & $\begin{array}{l}0800 \\
1400 \\
2000 \\
2400\end{array}$ & $\begin{array}{l}14.57 \\
15.08 \\
15.88 \\
16.42\end{array}$ & $\begin{array}{l}10,600 \\
11,100 \\
12,000 \\
12,600\end{array}$ & 13 & $\begin{array}{l}0200 \\
0600 \\
1200\end{array}$ & $\begin{array}{l}35.22 \\
35.18 \\
35.03\end{array}$ & $\begin{array}{l}73,300 \\
71,600 \\
69,100\end{array}$ & 22 & $\begin{array}{l}1200 \\
2400\end{array}$ & $\begin{array}{l}13.27 \\
11.93\end{array}$ & $\begin{array}{l}9,270 \\
7,950\end{array}$ \\
\hline 9 & $\begin{array}{l}0600 \\
1200 \\
1800\end{array}$ & $\begin{array}{l}18.52 \\
20.55 \\
22.32\end{array}$ & $\begin{array}{l}15,300 \\
17,900 \\
20,800\end{array}$ & 14 & $\begin{array}{l}1800 \\
2400 \\
1200\end{array}$ & $\begin{array}{l}34.82 \\
34.53 \\
33.90\end{array}$ & $\begin{array}{l}66,900 \\
64,500 \\
60,300\end{array}$ & 23 & $\begin{array}{l}1200 \\
2400\end{array}$ & $\begin{array}{l}10.82 \\
10.08\end{array}$ & $\begin{array}{l}6,920 \\
6,250\end{array}$ \\
\hline
\end{tabular}


(127) 3-3716. South Fork Salt Creek at Kurtz, Ind.

Location. -Lat $38^{\circ} 57^{\prime} 46^{\prime \prime}$, long $86^{\circ} 12^{\prime} 12^{\prime \prime}$, in SW $\frac{1}{4}$ sec.9, T.6 N., R.3 E., on right bank at downstream side of county road bridge, at the north edge of Kurtz,

0.8 mile upstream from unnamed tributary from the right, and 6.1 miles upstream from Little Salt Creek.

Drainage area. $--38.1 \mathrm{sq} \mathrm{mi}$.

Gage-helght record.--Water-stage recorder graph. Datum of gage is $568.00 \mathrm{ft}$ above mean sea level, datum of 1929 (unadjusted).

D1scharge record.--Stage-discharge relation defined by current-meter measurements below $3,700 \mathrm{cf}$ s and extended above by logarithmic plotting.

Maxima.--Given in the following table.

March 1964:

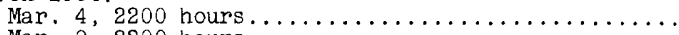

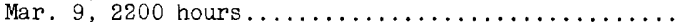

1959-1964:

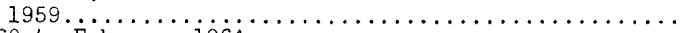

1960 to February 1964 :

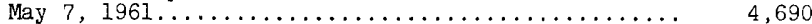

$\begin{array}{cc}\text { Discharge } & \text { Gage he ight } \\ \text { (cfs) } & \text { (feet) } \\ 2,470 & 10.44 \\ 4,960 & 13.08\end{array}$

Unknown al5

a About.

Mean discharge, in cubic feet per second, March 1964

\begin{tabular}{|c|c|c|c|c|c|c|c|c|c|}
\hline Day & D1scharge & Day & Discharge & Day & Discharge & Day & Discharge & Day & Discharge \\
\hline $\begin{array}{l}1 \ldots \ldots \\
2 \ldots \ldots \\
3 \ldots \ldots \\
4 \ldots \ldots \\
5 \ldots \ldots \\
6 \ldots \ldots\end{array}$ & $\begin{array}{r}2.2 \\
2.6 \\
5.8 \\
1,050 \\
220 \\
57\end{array}$ & $\begin{array}{r}7 \ldots \ldots \\
8 \ldots \\
9 \ldots \\
10 \ldots \\
11 \ldots \\
12 \ldots\end{array}$ & $\begin{array}{r}36 \\
92 \\
3,870 \\
1,910 \\
180 \\
137\end{array}$ & $\begin{array}{l}13 \ldots \ldots \\
14 \ldots \ldots \\
15 \ldots \ldots \\
16 \ldots \\
17 \ldots \ldots \\
18 \ldots \ldots\end{array}$ & $\begin{array}{r}76 \\
133 \\
101 \\
57 \\
39 \\
30\end{array}$ & $\begin{array}{l}19 \ldots \ldots \\
20 \ldots \ldots \\
21 \ldots \ldots \\
22 \ldots \ldots \\
23 \ldots \ldots \\
24 \ldots \ldots\end{array}$ & $\begin{array}{l}26 \\
40 \\
45 \\
36 \\
30 \\
28\end{array}$ & $\begin{array}{l}25 \ldots \ldots \\
26 \ldots \ldots \\
27 \ldots \ldots \\
28 \ldots \ldots \\
29 \ldots \ldots \\
30 \ldots \ldots \\
31 \ldots \ldots\end{array}$ & $\begin{array}{l}38 \\
71 \\
41 \\
34 \\
28 \\
24 \\
22\end{array}$ \\
\hline
\end{tabular}

Gage he1ght, in feet, and discharge, in cublc feet per second, at indicated t1me, 1964

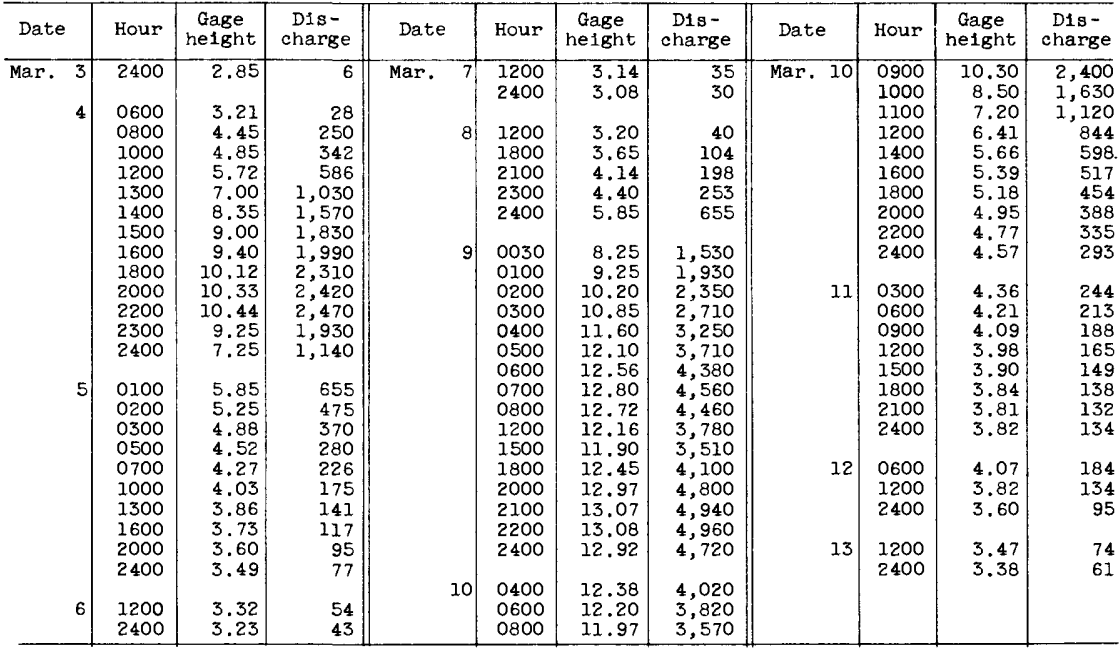


(128) 3-3716.5. North Fork Salt Creek at Nashville, Ind.

Location.--Lat $39^{\circ} 12^{\prime} 05^{\prime \prime}$, long $86^{\circ} 14^{\prime} 50^{\prime \prime}$, in SW $\frac{1}{4}$ sec.19, T.9 N., R.3 E., near center of stream at downstream side of bridge on State Highway $46,700 \mathrm{ft}$ downstream from Greasy Creek, and $0.4 \mathrm{mile}$ south of center of Nashvilie, Brown County.

Drainage area. $--75.9 \mathrm{sq} \mathrm{mi}$.

Gage-height record.--Graph drawn on basis of wire-weight-gage readings usually made twice dally. Peak stage of Mar. 9 was determined from high-water mark. Datum of gage is $579.576 \mathrm{ft}$ above mean sea level, datum of 1929.

Discharge record.--Stage-discharge relation defined by current-meter measurements below 2,000 cfs and extended above by logarithmic plotting.

Maxima.--March 1964: Discharge, 7,130 cfs Mar. 9, 2400 hours (gage height, $15.93 \mathrm{ft}$.

1962 to February 1964: Discharge, 7,500 cfs Mar. 4, 1963 (gage height, 15.72 ft, from floodmark).

Mean discharge, in cublc feet per second, March 1964

\begin{tabular}{l}
\hline Day \\
\hline$\ldots \ldots$
\end{tabular}

Gage height, in feet, and discharge, in cubic feet per second, at indicated time, 1964

\begin{tabular}{|c|c|c|c|c|c|c|c|c|c|c|c|}
\hline Date & Hour & $\begin{array}{c}\text { Gage } \\
\text { he1ght }\end{array}$ & $\begin{array}{l}\text { Dis- } \\
\text { charge }\end{array}$ & Date & Hour & $\begin{array}{c}\text { Gage } \\
\text { height }\end{array}$ & $\begin{array}{l}\text { Dis- } \\
\text { charge }\end{array}$ & Date & Hour & $\begin{array}{c}\text { Gage } \\
\text { helght }\end{array}$ & $\begin{array}{l}\text { Dis- } \\
\text { charge }\end{array}$ \\
\hline Mar. 2 & 2400 & 3.09 & 8 & Mar. & 1200 & 3.57 & 69 & Mar. 10 & 0300 & 15.81 & 7,010 \\
\hline & & & & & 1800 & 3.66 & 85 & & 0400 & 15.71 & 6,910 \\
\hline 3 & 1200 & 3.10 & 9 & & 2000 & 4.00 & 152 & & 0500 & 15.59 & 6,790 \\
\hline & 2400 & 3.43 & 44 & & 2100 & $\begin{array}{l}4.41 \\
5.28\end{array}$ & $\begin{array}{l}252 \\
462\end{array}$ & & 0600 & 15.38 & 6,580 \\
\hline 4 & 0600 & 3.73 & 92 & & 2300 & 6.48 & 772 & & 0900 & 13.58 & 4,820 \\
\hline & $\begin{array}{l}0900 \\
1200\end{array}$ & $\begin{array}{l}3.98 \\
4.57\end{array}$ & $\begin{array}{l}141 \\
285\end{array}$ & & 2400 & 7.75 & 1,270 & & $\begin{array}{l}1000 \\
1100\end{array}$ & $\begin{array}{l}12.08 \\
10.15\end{array}$ & $\begin{array}{l}3,560 \\
2,340\end{array}$ \\
\hline & 1500 & 5.26 & 498 & 9 & 0100 & 9.08 & 1,790 & & 1200 & 8.65 & 1,610 \\
\hline & $\begin{array}{l}1800 \\
2000\end{array}$ & $\begin{array}{l}5.90 \\
6.20\end{array}$ & $\begin{array}{l}720 \\
840\end{array}$ & & $\begin{array}{l}0200 \\
0300\end{array}$ & $\begin{array}{l}10.38 \\
11.68\end{array}$ & $\begin{array}{l}2,480 \\
3,280\end{array}$ & & $\begin{array}{l}1300 \\
1500\end{array}$ & $\begin{array}{l}8.13 \\
7.35\end{array}$ & $\begin{array}{l}1,400 \\
1,120\end{array}$ \\
\hline & 2100 & 6.26 & 864 & & 0400 & $\begin{array}{l}11.00 \\
12.78\end{array}$ & 4,120 & & 1700 & 6.93 & $\begin{array}{r}1,1<0 \\
976\end{array}$ \\
\hline & 2200 & 6.22 & 848 & & 0500 & 13.11 & 4,400 & & 2000 & 6.37 & 791 \\
\hline & 2400 & 6.11 & 804 & & 0600 & 13.30 & 4,570 & & 2200 & 6.12 & 716 \\
\hline & & & & & 0700 & 13.46 & 4,710 & & 2400 & 5.90 & 650 \\
\hline 5 & 0600 & 5.78 & 678 & & 0800 & 13.58 & 4,820 & & & & \\
\hline & 1200 & 5.45 & 562 & & 1200 & 13.77 & 4,990 & 11 & 0600 & 5.45 & 515 \\
\hline & 1800 & 5.11 & 453 & & 1600 & 14.00 & 5,200 & & 0900 & 5.31 & 478 \\
\hline & 2400 & 4.77 & 351 & & 1800 & 14.90 & 6,100 & & 1200 & 5.13 & 432 \\
\hline & & & & & 2000 & & 6,860 & & 1500 & 4.76 & 340 \\
\hline 6 & 0600 & 4.40 & 250 & & 2100 & 15.76 & 6,960 & & 1800 & 4.55 & 288 \\
\hline & 1200 & 4.11 & 177 & & 2200 & 15.85 & 7,050 & & 2400 & 4.37 & 245 \\
\hline & 1800 & 3.88 & 126 & & 2300 & 15.88 & 7,080 & & & & \\
\hline & 2400 & 3.79 & 108 & & 2400 & 15.93 & 7,130 & 12 & $\begin{array}{l}1200 \\
2400\end{array}$ & $\begin{array}{l}4.18 \\
4.08\end{array}$ & $\begin{array}{l}201 \\
178\end{array}$ \\
\hline 7 & $\begin{array}{l}1200 \\
2400\end{array}$ & $\begin{array}{l}3.61 \\
3.57\end{array}$ & $\begin{array}{l}76 \\
69\end{array}$ & 10 & $\begin{array}{l}0100 \\
0200\end{array}$ & $\begin{array}{l}15.89 \\
15.88\end{array}$ & $\begin{array}{l}7,090 \\
7,080\end{array}$ & & & & \\
\hline
\end{tabular}


(I29) 3-3720. North Fork Salt Creek near Belmont, Ind.

Location.--Lat $39^{\circ} 09^{100^{\prime \prime}}$, long $86^{\circ} 20^{\prime} 14^{\prime \prime}$, in NW $\frac{1}{4}$ sec.5, T. 8 N., R.2 E., on right bank $15 \mathrm{ft}$ downstream from bridge on State Highway $46,100 \mathrm{ft}$ upstream from Schooner Creek, 0.7 mile northeast of Belmont, $6 \frac{1}{2}$ miles upstream from Brummett Creek, and 20 miles upstream from mouth. Records include flow of Schooner Creek.

Drainage area.--120 sq mi, includes Schooner Creek.

Gage-he1ght record.--Water-stage recorder graph. Datum of gage is $543.62 \mathrm{ft}$ above mean sea level, datum of 1929 (levels by Indiana Flood Control and Water Resources Commission).

Discharge record.--Stage-discharge relation defined by current-meter measurements below $9,600 \mathrm{cf}$ 's and by contracted-opening measurement at $12,700 \mathrm{cfs}$. Rate of change in stage used as a factor Mar. 4-6, 9-13, 25-27.

Maxima.--Given in the following table.

March 1964 :

Mar. 5, 0600 hours.

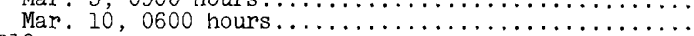

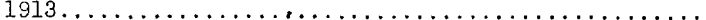

1946 to February 1964:

June 23,1960

Discharge

(cfs)

2,380

10,100

Unknown

13,300
Gage height (feet)

17.08

21.63

25.7

23.10

Mean discharge, in cubic feet per second, March 1964

\begin{tabular}{|c|c|c|c|c|c|c|c|c|c|}
\hline Day & D1scharge & Day & Discharge & Day & Discharge & Day & Discharge & Day & Discharge \\
\hline $\begin{array}{l}1 \ldots \ldots \\
2 \ldots \ldots \\
3 \ldots \ldots \\
4 \ldots \\
5 \ldots \\
6 \ldots\end{array}$ & $\begin{array}{r}11 \\
11 \\
12 \\
770 \\
1,620 \\
311\end{array}$ & $\begin{array}{r}7 \ldots \ldots \\
8 \ldots \ldots \\
9 \ldots \ldots \\
10 \ldots \\
11 \ldots \ldots \\
12 \ldots \ldots\end{array}$ & $\begin{array}{r}137 \\
117 \\
4,610 \\
8,080 \\
1,190 \\
508\end{array}$ & $\begin{array}{l}13 \ldots \ldots \\
14 \ldots \ldots \\
15 \ldots \ldots \\
16 \ldots \ldots \\
17 \ldots \ldots \\
18 \ldots \ldots\end{array}$ & $\begin{array}{r}309 \\
266 \\
274 \\
175 \\
124 \\
87\end{array}$ & $\begin{array}{l}19 \ldots \ldots \\
20 \ldots \ldots \\
21 \ldots \ldots \\
22 \ldots \ldots \\
23 \ldots \ldots \\
24 \ldots \ldots\end{array}$ & $\begin{array}{l}69 \\
75 \\
95 \\
85 \\
72 \\
64\end{array}$ & $\begin{array}{l}25 \ldots \ldots \\
26 \ldots \ldots \\
27 \ldots \ldots \\
28 \ldots \ldots \\
29 \ldots \ldots \\
30 \ldots \ldots \\
31 \ldots \ldots\end{array}$ & $\begin{array}{r}123 \\
616 \\
282 \\
168 \\
119 \\
87 \\
73\end{array}$ \\
\hline $\begin{array}{l}\text { Monthly } \\
\text { Runoff, }\end{array}$ & $\begin{array}{l}\text { lean di } \\
n \text { inch }\end{array}$ & $g e$, & h 10 fot & per & & & & & $\begin{array}{r}663 \\
6.36\end{array}$ \\
\hline
\end{tabular}

Gage height, in feet, and discharge, in cubic feet per second, at indicated time, 1964

\begin{tabular}{|c|c|c|c|c|c|c|c|c|c|c|c|}
\hline Date & Hour & $\begin{array}{c}\text { Gage } \\
\text { he1ght }\end{array}$ & $\begin{array}{c}\text { Dis- } \\
\text { charge }\end{array}$ & Date & Hour & $\begin{array}{c}\text { Gage } \\
\text { helght }\end{array}$ & $\begin{array}{c}\text { Dis- } \\
\text { charge }\end{array}$ & Date & Hour & $\begin{array}{c}\text { Gage } \\
\text { height }\end{array}$ & $\begin{array}{c}\text { Dis- } \\
\text { charge }\end{array}$ \\
\hline \multirow[t]{13}{*}{ Mar, 3} & 2400 & 2.99 & 13 & \multirow[t]{17}{*}{ Mar. } & 1200 & 4.38 & 103 & \multirow{4}{*}{ Mar. 10} & 2000 & 19.35 & 5,180 \\
\hline & & & & & 1800 & 4.52 & 119 & & 2200 & 18.77 & 4,030 \\
\hline & 0600 & 3.28 & $\begin{array}{l}24 \\
50\end{array}$ & & 2200 & 4.70 & 142 & & 2400 & 18.17 & 3,090 \\
\hline & 1000 & $\begin{array}{l}3.66 \\
4.35\end{array}$ & $\begin{array}{r}50 \\
12 ?\end{array}$ & & 2300 & 4.98 & 178 & & & & \\
\hline & 1300 & $\begin{array}{l}4.35 \\
5.20\end{array}$ & 231 & & 2400 & & 311 & \multirow[t]{10}{*}{11} & $\begin{array}{l}0200 \\
0400\end{array}$ & $\begin{array}{l}17.56 \\
16.76\end{array}$ & $\begin{array}{l}2,280 \\
1,560\end{array}$ \\
\hline & 1400 & 6.50 & 400 & & 0100 & 9.00 & 1,060 & & 0600 & 15.90 & 1,310 \\
\hline & 1500 & 8.10 & 803 & & 0200 & 12.00 & 1,680 & & 0800 & 15.00 & 1,230 \\
\hline & 1600 & 10.00 & 1,150 & & 0300 & 14.40 & 2,460 & & 1000 & 14.05 & 1,120 \\
\hline & 1700 & 12.00 & 1,510 & & 0400 & 15.50 & 2,410 & & 1200 & 12.97 & 1,060 \\
\hline & 1800 & 13.70 & 1,860 & & 0600 & 16.54 & 2,570 & & 1400 & 11.90 & 968 \\
\hline & $\begin{array}{l}2000 \\
2200\end{array}$ & 15.30 & $\begin{array}{l}2,250 \\
2,220\end{array}$ & & 0800 & 17.23 & 2,760 & & 1600 & 10.95 & $\begin{array}{l}863 \\
757\end{array}$ \\
\hline & $\begin{array}{l}2200 \\
2400\end{array}$ & $\begin{array}{l}16.00 \\
16.36\end{array}$ & $\begin{array}{l}2,220 \\
2,170\end{array}$ & & $\begin{array}{l}1000 \\
1200\end{array}$ & $\begin{array}{l}17.72 \\
18.15\end{array}$ & $\begin{array}{l}2,970 \\
3,300\end{array}$ & & $\begin{array}{l}1800 \\
2000\end{array}$ & $\begin{array}{r}10.03 \\
9.28\end{array}$ & $\begin{array}{l}757 \\
678\end{array}$ \\
\hline & חת & 76 & 380 & & $\begin{array}{l}1400 \\
1600\end{array}$ & $\frac{1}{1}$ & $\begin{array}{l}3,870 \\
5,080\end{array}$ & & 2200 & 65 & 617 \\
\hline \multirow{5}{*}{5} & 0800 & 17.07 & 2,370 & & 1800 & 20.10 & 6,720 & & 2400 & $8.2 c$ & 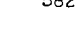 \\
\hline & 0900 & 17.08 & 2,280 & & 2000 & 21.03 & 8,770 & \multirow[t]{6}{*}{12} & 0300 & 7.97 & 561 \\
\hline & 1200 & 16.57 & 1,550 & & 2300 & 21.56 & 9,930 & & 0900 & 7.82 & 543 \\
\hline & $\begin{array}{l}1600 \\
2000\end{array}$ & $\begin{array}{l}14.66 \\
12.00\end{array}$ & $\begin{array}{r}1,090 \\
930\end{array}$ & & 2400 & 21.58 & 9,980 & & $\begin{array}{l}1200 \\
1500\end{array}$ & $\begin{array}{l}7.65 \\
7.45\end{array}$ & $\begin{array}{l}521 \\
495\end{array}$ \\
\hline & 2400 & 9.27 & 640 & 10 & $\begin{array}{l}0100 \\
0200\end{array}$ & $\begin{array}{r}21.59 \\
21.58\end{array}$ & 10,000 & & $\begin{array}{l}1800 \\
2100\end{array}$ & 7.22 & $\begin{array}{l}465 \\
427\end{array}$ \\
\hline 6 & 0400 & 7.30 & $45]$ & & 0300 & 21.59 & 10,000 & & 2400 & 6.69 & 401 \\
\hline & 0800 & 6.16 & 332 & & 0400 & 21.61 & 10,000 & & & & \\
\hline & 1200 & 5.60 & 259 & & 0500 & 21.62 & 10,100 & \multirow[t]{4}{*}{13} & 0600 & 6.27 & 346 \\
\hline & 1800 & 5.18 & 204 & & 0600 & 21.63 & 10,100 & & 1200 & 5.94 & 303 \\
\hline & 2400 & 4.95 & 174 & & 0700 & 21.62 & 10,100 & & 1800 & 5.66 & 267 \\
\hline & & & & & 0800 & 21.59 & 10,000 & & 2400 & 5.44 & 238 \\
\hline 7 & 0600 & 4.78 & 152 & & 0900 & 21.54 & 9,890 & \multirow{5}{*}{14} & & & \\
\hline & 1200 & 4.64 & 134 & & 1000 & 21.46 & 9,710 & & 0200 & 5.42 & 236 \\
\hline & 1800 & 4.55 & 123 & & 1200 & 21.22 & 9,180 & & 0800 & 5.48 & 243 \\
\hline & & & 106 & & $\begin{array}{l}1400 \\
1600\end{array}$ & $\begin{array}{l}20.88 \\
20.44\end{array}$ & $\begin{array}{l}8,440 \\
7,470\end{array}$ & & $\begin{array}{l}1400 \\
1600\end{array}$ & $\begin{array}{l}5.62 \\
5.76\end{array}$ & $\begin{array}{l}262 \\
280\end{array}$ \\
\hline 8 & 0600 & 4.35 & 100 & & 1800 & 19.92 & 6,320 & & 1800 & 5.89 & 297 \\
\hline
\end{tabular}


Gage height, in feet, and discharge, in cub1c feet per second, at ind1cated time, 1964, of North Fork Salt Creek near Belmont, Ind.--Continued

\begin{tabular}{|c|c|c|c|c|c|c|c|c|c|c|c|}
\hline Date & Hour & $\begin{array}{c}\text { Gage } \\
\text { height }\end{array}$ & $\begin{array}{l}\text { Dis- } \\
\text { charge }\end{array}$ & Date & Hour & $\begin{array}{c}\text { Gage } \\
\text { height }\end{array}$ & $\begin{array}{c}\text { Dis- } \\
\text { charge }\end{array}$ & Date & Hour & $\begin{array}{c}\text { Gage } \\
\text { height }\end{array}$ & $\begin{array}{c}\text { Dis- } \\
\text { charge }\end{array}$ \\
\hline Mar. 14 & $\begin{array}{l}2000 \\
2400 \\
\\
0400 \\
0800 \\
1200 \\
1800\end{array}$ & $\begin{array}{l}5.96 \\
6.00 \\
6.04 \\
5.94 \\
5.76 \\
5.47\end{array}$ & $\begin{array}{l}306 \\
311 \\
316 \\
303 \\
280 \\
242\end{array}$ & $\begin{array}{r}15 \\
16\end{array}$ & $\begin{array}{l}2400 \\
1200 \\
1800 \\
2100 \\
2200 \\
2400\end{array}$ & $\begin{array}{l}5.23 \\
4.93 \\
4.81 \\
4.76 \\
4.77 \\
4.76\end{array}$ & $\begin{array}{l}211 \\
172 \\
156 \\
150 \\
151 \\
150\end{array}$ & Mar. 17 & $\begin{array}{l}0400 \\
1000 \\
1200 \\
1400 \\
1800 \\
2100 \\
2400\end{array}$ & $\begin{array}{l}4.63 \\
4.56 \\
4.59 \\
4.57 \\
4.51 \\
4.42 \\
4.41\end{array}$ & $\begin{array}{l}133 \\
124 \\
128 \\
125 \\
118 \\
107 \\
106 \\
\end{array}$ \\
\hline
\end{tabular}

(130) 3-3725. Salt Creek near Harrodsburg, Ind.

Location.--Lat $39^{\circ} 00^{\prime} 16^{\prime \prime}$, long $86^{\circ} 30^{\prime} 31^{\prime \prime}$, in NW $\frac{1}{4} \sec .34$, T.7 N., R.1 W., on right bank $1,300 \mathrm{ft}$ downstream from Monroe Reservoir, 0.9 mile upstream from Clear Creek, 2.2 miles southeast of Harrodsburg, and 25.1 miles upstream from mouth.

Drainage area. $--441 \mathrm{sq} \mathrm{ml}$.

Gage-height record. - Water-stage recorder graph. Datum of gage is $480.00 \mathrm{ft}$ above mean sea leveI, datum of 1929 (levels by Corps of Engineers).

Discharge record.--Stage-discharge relation defined by current-meter measurements below 19,500 cfs and extended above by logarithmic plotting. Backwater from return of overbank flow or from the East Fork White River Mar. 10-18.

Maxima :--March 1964: Discharge, 8,000 cfs Mar. 10, 0300 hours (gage height, $28.40 \mathrm{ft}^{\mathrm{t}}$ ).

1955 to February 1964: Discharge, 22,000 cf's June 25, 1960 (gage height $32.76 \mathrm{ft}$, from graph based on gage readings at site $3,500 \mathrm{ft}^{\mathrm{t}}$ upstream and datum $2.41 \mathrm{ft}$ higher); gage helght at present site and datum, $35.35 \mathrm{ft}$ May 9, 1961 .

Remarks.--Flow regulated by Monroe Reservolr (flood control storage capacity, 258,800 acre-ft).

\begin{tabular}{|c|c|c|c|c|c|c|c|c|c|}
\hline Day & Dischance & Day & & & & & & & neschonte \\
\hline & & & & Day & & Day & 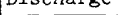 & Doy & Discnarb \\
\hline & 50 & & 20 & 13 & 384 & $19 \ldots$. & 2,5 & 25 & 3 \\
\hline & & & 2,210 & & 295 & 20. & 0 & & 2 \\
\hline & & 9 . & 5,200 & $15 \ldots \ldots$ & 303 & $21 \ldots \ldots$ & 3,020 & $27 \ldots \ldots$ & 3,410 \\
\hline $4 \ldots$. & 644 & 10. & 7,050 & $16 \ldots$ & 310 & $22 \ldots \ldots$ & 2,930 & $28 \ldots \ldots$ & 3,810 \\
\hline $5 \ldots$. & 2,270 & $11 \ldots$. & 4,880 & $17 \ldots \ldots$ & 316 & $23 \ldots \ldots$ & & $29 \ldots \ldots$ & 3,750 \\
\hline $6 \ldots \ldots$ & 1,910 & $12 .$. & 1,940 & $18 \ldots$ & 1,300 & $24 \ldots \ldots$ & 3,230 & $\begin{array}{l}30 \\
31\end{array} \ldots$ & $\begin{array}{l}3,470 \\
3,070\end{array}$ \\
\hline & & & & & & & & & 2,326 \\
\hline
\end{tabular}

Gage helght, in feet, and discharge, in cublc feet per second, at ind1cated t1me, 1964

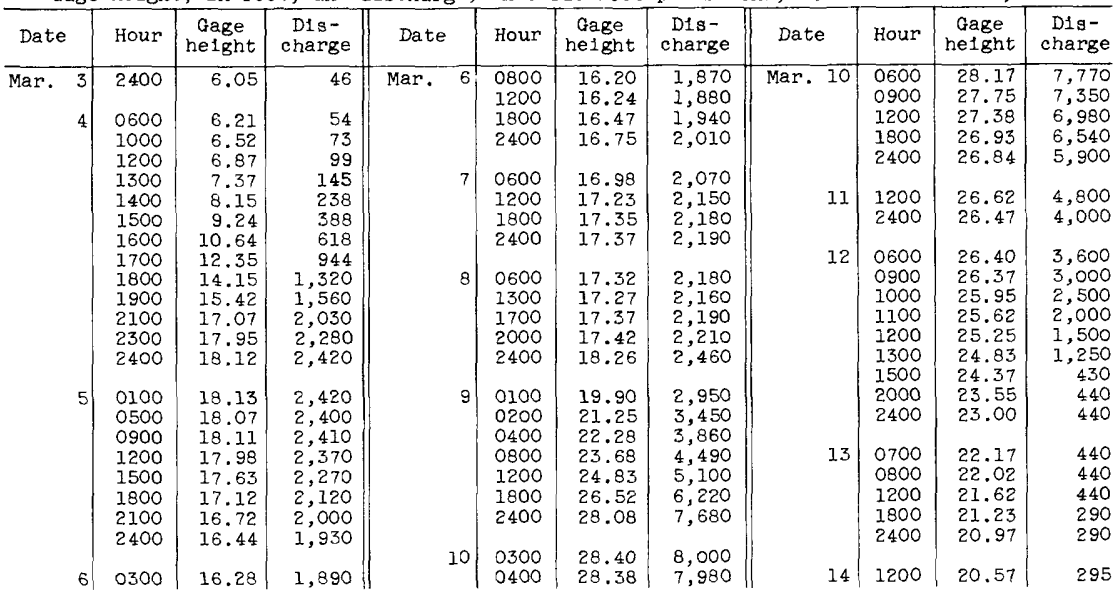


Gage helght, in feet, and discharge, In cublc feet per second, at indicated t1me, 1964, of

\begin{tabular}{|c|c|c|c|c|c|c|c|c|c|c|c|}
\hline Date & Hour & $\begin{array}{c}\text { Gage } \\
\text { helght }\end{array}$ & $\begin{array}{l}\text { D1s- } \\
\text { charge }\end{array}$ & Date & Hour & $\begin{array}{l}\text { Gage } \\
\text { he1ght }\end{array}$ & $\begin{array}{l}\text { D1s- } \\
\text { charge }\end{array}$ & Date & Hour & $\begin{array}{c}\text { Gage } \\
\text { helght }\end{array}$ & $\begin{array}{c}\text { D1s- } \\
\text { charge }\end{array}$ \\
\hline ar. 14 & 2400 & 20.18 & 300 & Mar. 18 & 1000 & 13.36 & 320 & 28 & 2400 & 22.16 & 3,81 \\
\hline 15 & $\begin{array}{l}0600 \\
1200\end{array}$ & $\begin{array}{l}19.95 \\
19.62\end{array}$ & $\begin{array}{l}300 \\
305\end{array}$ & & $\begin{array}{l}1200 \\
1300\end{array}$ & $\begin{array}{l}15.05 \\
15.10\end{array}$ & $\begin{array}{l}1,580 \\
1,600\end{array}$ & 29 & $\begin{array}{l}1200 \\
2400\end{array}$ & $\begin{array}{l}22.02 \\
21.77\end{array}$ & $\begin{array}{l}3,760 \\
3,660\end{array}$ \\
\hline & $\begin{array}{l}1800 \\
2400\end{array}$ & $\begin{array}{l}19.28 \\
18.89\end{array}$ & $\begin{array}{l}305 \\
305\end{array}$ & & $\begin{array}{l}1400 \\
1600\end{array}$ & $\begin{array}{l}15.95 \\
17.20\end{array}$ & $\begin{array}{l}1,810 \\
2,140\end{array}$ & 30 & $\begin{array}{l}1200 \\
2400\end{array}$ & $\begin{array}{l}21.38 \\
20.67\end{array}$ & $\begin{array}{l}3,500 \\
3,220\end{array}$ \\
\hline 16 & $\begin{array}{l}1200 \\
2400\end{array}$ & $\begin{array}{l}18.02 \\
16.98\end{array}$ & $\begin{array}{l}310 \\
315\end{array}$ & & 2400 & & $\begin{array}{l}2,400 \\
2,510\end{array}$ & 31 & $\begin{array}{l}0600 \\
1200\end{array}$ & $\begin{array}{l}20.36 \\
20.20\end{array}$ & $\begin{array}{l}3,110 \\
3,050\end{array}$ \\
\hline 17 . & $\begin{array}{l}1200 \\
2400\end{array}$ & $\begin{array}{l}15.80 \\
14.48\end{array}$ & $\begin{array}{l}315 \\
320\end{array}$ & 28 & $\begin{array}{l}0600 \\
1200 \\
1800\end{array}$ & $\begin{array}{l}22.15 \\
22.22 \\
22.20\end{array}$ & $\begin{array}{l}3,810 \\
3,840 \\
3,830\end{array}$ & & $\begin{array}{l}1800 \\
2100 \\
2400\end{array}$ & $\begin{array}{l}20.07 \\
20.20 \\
20.00\end{array}$ & $\begin{array}{l}3,000 \\
3,050 \\
2,980\end{array}$ \\
\hline
\end{tabular}

(131) 3-3727. Clear Creek at Harrodsburg, Ind.

Location. --Lat $39^{\circ} 02^{\prime} 03^{\prime \prime}$, long $86^{\circ} 34^{\prime} 01^{\prime \prime}$, in NW $\frac{1}{4}$ sec.19, T.7 N., R.I W., on left bank at downstream slde of county road bridge, 1.9 miles northwest of Harrodsburg, $3.9 \mathrm{mlles}$ upstream from Little Clear Creek, and 5.1 miles upstream from mouth.

Dra1nage area.--55.2 sq $\mathrm{mi}$, of which $6.4 \mathrm{sq} \mathrm{mi}$ does not contribute directly to surf'ace runoff.

Gage-height record.--Digital recorder tape punched at 15-minute intervals. Datum of gage is 517.00 ft above mean sea level, datum of 1929.

Discharge record.--Stage-discharge relation defined by current-meter measurements below 3,600 ffs and by contracted-opening measurement at 10,200 cfs.

Maxima.--Given in the following table.

March 1964:

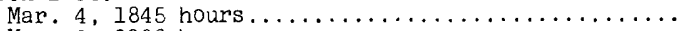

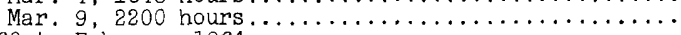

1960 to February 1964 :

June 23,1960

$\begin{array}{cc}\begin{array}{c}\text { Discharge } \\ \text { (cfs) }\end{array} & \begin{array}{c}\text { Gage helght } \\ \text { feet) }\end{array} \\ 1,900 & 8.12 \\ 6,380 & 13.07 \\ 10,200 & 16.47\end{array}$

Mean discharge, in cuble feet per second, March 1964

\begin{tabular}{|c|c|c|c|c|c|c|c|c|c|}
\hline Day & Discharge & Day & D1scharge & Day & Discharge & Day & Discharge & Day & D1scharge \\
\hline $\begin{array}{l}1 \ldots \\
2 \ldots \\
3 \ldots \\
4 \ldots \\
5 \ldots \\
6 \ldots\end{array}$ & $\begin{array}{r}13 \\
14 \\
16 \\
538 \\
335 \\
131\end{array}$ & $\begin{array}{r}7 \ldots \ldots \\
8 \ldots \ldots \\
9 \ldots \ldots \\
10 \ldots \\
11 \ldots \ldots \\
12 \ldots \ldots\end{array}$ & $\begin{array}{r}90 \\
117 \\
3,650 \\
1,430 \\
520 \\
381\end{array}$ & $\begin{array}{l}13 \ldots \ldots \\
14 \ldots \ldots \\
15 \ldots \ldots \\
16 \ldots \ldots \\
17 \ldots \ldots \\
18 \ldots \ldots\end{array}$ & $\begin{array}{r}268 \\
196 \\
143 \\
115 \\
95 \\
80\end{array}$ & $\begin{array}{l}19 \ldots \ldots \\
20 \ldots \ldots \\
21 \ldots \ldots \\
22 \ldots \ldots \\
23 \ldots \ldots \\
24 \ldots \ldots\end{array}$ & $\begin{array}{l}71 \\
95 \\
88 \\
76 \\
72 \\
65\end{array}$ & $\left|\begin{array}{l}25 \ldots \ldots \\
26 \ldots \ldots \\
27 \ldots \ldots \\
28 \ldots \ldots \\
29 \ldots \ldots \\
30 \ldots \ldots \\
31 \ldots \ldots\end{array}\right|$ & $\begin{array}{r}98 \\
171 \\
119 \\
100 \\
82 \\
69 \\
63\end{array}$ \\
\hline thl & $\begin{array}{l}\text { mean dis } \\
\text { In Inches }\end{array}$ & ge, & 126 & & & & & & $\begin{array}{r}300 \\
6.26\end{array}$ \\
\hline
\end{tabular}

Note.--Da1ly mean discharges computed on bas1s of 15-minute intervals.

Gage he1ght, in feet, and discharge, In cub1c feet per second, at indicated t1me, 1964

\begin{tabular}{|c|c|c|c|c|c|c|c|c|c|c|c|c|}
\hline Date & Hour & $\begin{array}{c}\text { Gage } \\
\text { helght }\end{array}$ & $\begin{array}{c}\text { Dis- } \\
\text { charge }\end{array}$ & Date & & Hour & $\begin{array}{c}\text { Gage } \\
\text { height }\end{array}$ & $\begin{array}{l}\text { D1s- } \\
\text { charge }\end{array}$ & Date & Hour & $\begin{array}{c}\text { Gage } \\
\text { helght }\end{array}$ & $\begin{array}{l}\text { D1s- } \\
\text { charge }\end{array}$ \\
\hline $\begin{array}{r}\text { Mar. } 3 \\
4\end{array}$ & $\begin{array}{l}2400 \\
0800 \\
1000 \\
1200 \\
1400 \\
1600 \\
1800 \\
1845 \\
2000 \\
2200 \\
2400 \\
\\
0600 \\
1200 \\
1800 \\
2400\end{array}$ & $\begin{array}{l}3.56 \\
3.81 \\
4.75 \\
4.57 \\
5.16 \\
6.98 \\
8.08 \\
8.12 \\
7.58 \\
6.75 \\
6.30 \\
5.66 \\
5.32 \\
5.01 \\
4.82\end{array}$ & $\begin{array}{r}17 \\
31 \\
160 \\
127 \\
258 \\
1,080 \\
1,860 \\
1,900 \\
1,500 \\
940 \\
710 \\
\\
424 \\
306 \\
218 \\
174\end{array}$ & Mar. & 6 & $\begin{array}{l}0600 \\
1200 \\
1800 \\
2400 \\
1200 \\
2400 \\
\\
0600 \\
1000 \\
1200 \\
1400 \\
1800 \\
2200 \\
2400 \\
0100\end{array}$ & $\begin{array}{l}4.66 \\
4.55 \\
4.52 \\
4.44 \\
4.30 \\
4.25 \\
4.23 \\
4.26 \\
4.49 \\
4.47 \\
4.54 \\
4.68 \\
6.24 \\
8.06\end{array}$ & $\begin{array}{r}143 \\
124 \\
119 \\
106 \\
\\
86 \\
79 \\
76 \\
80 \\
114 \\
111 \\
122 \\
146 \\
680 \\
1,850\end{array}$ & Mar. 9 & $\begin{array}{l}0200 \\
0300 \\
0400 \\
0500 \\
0600 \\
0700 \\
0800 \\
1000 \\
1200 \\
1400 \\
1600 \\
1800 \\
1900 \\
2000 \\
2100 \\
2200 \\
2300\end{array}$ & $\begin{array}{r}9.24 \\
9.75 \\
9.84 \\
9.29 \\
8.41 \\
8.32 \\
8.52 \\
8.90 \\
9.16 \\
9.64 \\
10.54 \\
11.62 \\
12.40 \\
12.71 \\
12.88 \\
13.07 \\
12.95\end{array}$ & $\begin{array}{l}2,790 \\
3,200 \\
3,270 \\
2,830 \\
2,130 \\
2,060 \\
2,220 \\
2,520 \\
2,730 \\
3,110 \\
3,840 \\
4,870 \\
5,650 \\
5,980 \\
6,170 \\
6,380 \\
6,250\end{array}$ \\
\hline
\end{tabular}


Gage height, in feet, and discharge, in cublc feet per second, at indlcated time, 1964, of Clear Creek at Harrodsburg, Ind.--Continued

\begin{tabular}{|c|c|c|c|c|c|c|c|c|c|c|c|}
\hline Date & Hour & $\begin{array}{c}\text { Gage } \\
\text { he1ght }\end{array}$ & $\begin{array}{l}\text { Dis- } \\
\text { charge }\end{array}$ & Date & Hour & $\begin{array}{c}\text { Cage } \\
\text { he1ght }\end{array}$ & $\begin{array}{l}\text { D1s - } \\
\text { charge }\end{array}$ & Date & Hour & $\begin{array}{c}\text { Crage } \\
\text { hejght }\end{array}$ & $\begin{array}{c}\text { D1s- } \\
\text { charge }\end{array}$ \\
\hline Mar. 9 & 2400 & 12.55 & 5,810 & Mar. 11 & 1200 & 5.85 & 500 & Mar. 15 & 1200 & 4.64 & 139 \\
\hline 10 & 0100 & 11.16 & 4,410 & & $\begin{array}{l}1800 \\
2400\end{array}$ & 5.72 & $\begin{array}{l}448 \\
448\end{array}$ & & & & 126 \\
\hline & $\begin{array}{l}0200 \\
0300 \\
0400\end{array}$ & $\begin{array}{l}9.30 \\
8.56 \\
8.30\end{array}$ & $\begin{array}{l}2,840 \\
2,250 \\
2,040\end{array}$ & 12 & $\begin{array}{l}0400 \\
1200\end{array}$ & $\begin{array}{l}5.75 \\
5.52\end{array}$ & $\begin{array}{l}460 \\
368\end{array}$ & 16 & $\begin{array}{l}1200 \\
2400\end{array}$ & $\begin{array}{l}4.48 \\
4.43\end{array}$ & $\begin{array}{l}112 \\
105\end{array}$ \\
\hline & & $\begin{array}{l}7.76 \\
7.22\end{array}$ & $\begin{array}{l}1,620 \\
1,240\end{array}$ & & 2400 & 5.36 & 318 & 17 & $\begin{array}{l}1200 \\
2400\end{array}$ & $\begin{array}{l}4.35 \\
4.31\end{array}$ & $\begin{array}{l}93 \\
87\end{array}$ \\
\hline & $\begin{array}{l}1200 \\
1600\end{array}$ & $\begin{array}{l}6.89 \\
6.68\end{array}$ & 1,020 & 13 & 1200 & 5.20 & 27 & & & & \\
\hline & 2000 & $\begin{array}{l}6.68 \\
6.49\end{array}$ & 805 & & 2400 & 5.04 & 225 & 18 & $\begin{array}{l}1200 \\
2400\end{array}$ & $\begin{array}{l}4.22 \\
4.23\end{array}$ & $\begin{array}{l}75 \\
76\end{array}$ \\
\hline & & & 705 & 14 & 1200 & 4.89 & 188 & & & & \\
\hline 11 & 0600 & 6.04 & 580 & & 2400 & 4.79 & 168 & & & & \\
\hline
\end{tabular}

(132) 3-3730. Salt Creek near Peerless, Ind.

Location. --Lat $38^{\circ} 56^{\prime} 35^{\prime \prime}$, long $86^{\circ} 30^{\prime} 38^{\prime \prime}$, in NW $\frac{1}{4}$ sec.22, T.6 N., R.I W., on downstream side near center of Monon Ra1lroad bridge, 3,400 ft downstream from Little Salt Creek, 1.5 miles north of Peerless, and 18.6 miles upstream from mouth.

Drainage area. $--582 \mathrm{sq} \mathrm{mi}$.

Gage-height record.--Graph drawn from twice-dally wire-welght-gage readings. Datum of gage is $476.02 \mathrm{ft}$ above mean sea level, datum of 1929.

Discharge record.--Stage-discharge relation defined by current-meter measurements below $22,000 \mathrm{cf}$. Backwater from return of overbank flow or from East Fork wh1te River Mar. 8, 11-20.

Maxima.--March 1964: D1scharge, 10,000 cfs Mar. 10, 1000 hours (gage height, 29.00 f't, from graph based on gage readings).

1939-50, 1957 to February 1964: Discharge, 25,100 cfs May 10, 1961 (gage helght, $35.33 \mathrm{ft}$, from graph based on gage readings).

Remarks.-Flow regulated by Monroe Reservolr (flood control storage capac1ty, 258, 800 acre-ft).

Mean discharge, in cublc feet per second, March 1964

\begin{tabular}{|c|c|c|c|c|c|c|c|c|c|}
\hline Day & Discharge & Day & D1scharge & Day & Discharge & Day & D1scharge & Day & Discharge \\
\hline $\begin{array}{l}1 \ldots \ldots \\
2 \ldots \ldots \\
3 \ldots \ldots \\
4 \ldots \\
5 \ldots \\
6 \ldots\end{array}$ & $\begin{array}{r}67 \\
63 \\
67 \\
362 \\
3,100 \\
2,470\end{array}$ & $\begin{array}{r}7 \ldots \ldots \\
8 \ldots \ldots \\
3 \ldots \ldots \\
10 \ldots \ldots \\
11 \ldots \ldots \\
12 \ldots \ldots\end{array}$ & $\begin{array}{l}2,460 \\
2,200 \\
5,190 \\
8,910 \\
5,700 \\
2,400\end{array}$ & $\begin{array}{l}13 \ldots \ldots \\
14 \ldots \ldots \\
15 \ldots \ldots \\
16 \ldots \ldots \\
17 \ldots \ldots \\
18 \ldots \ldots\end{array}$ & $\begin{array}{r}670 \\
510 \\
460 \\
440 \\
430 \\
1,450\end{array}$ & $\begin{array}{l}19 \ldots \ldots \\
20 \ldots \ldots \\
21 \ldots \ldots \\
22 \ldots \ldots \\
23 \ldots \ldots \\
24 \ldots \ldots\end{array}$ & $\begin{array}{l}2,700 \\
2,900 \\
3,320 \\
3,170 \\
3,080 \\
3,290\end{array}$ & $\mid \begin{array}{l}25 \ldots \ldots \\
26 \ldots \ldots \\
27 \ldots \ldots \\
28 \ldots \ldots \\
29 \ldots \ldots \\
30 \ldots \ldots \\
31 \ldots \ldots\end{array}$ & $\begin{array}{l}3,260 \\
2,820 \\
3,240 \\
3,720 \\
3,770 \\
3,650 \\
3,310\end{array}$ \\
\hline
\end{tabular}


(133) 3-3732. Indian Creek near Springville, Ind.

Location.--Lat $38^{\circ} 57^{\prime} 01^{\prime \prime}$, long $86^{\circ} 40^{\prime} 30^{\prime \prime}$, in SW $\frac{1}{4}$ sec.18, T.6 N., R.2 W., on left bank at downstream side of State Highway 54 bridge, a quarter of a mile downstream from Popcorn Creek, and 4 miles northwest of Springville.

Dra1nage area. $--60.9 \mathrm{sq} \mathrm{ml}$.

Gage-height record.--Water-stage recorder graph and digital recorder tape punched at 15-minute Intervals. Datum of gage is $580.00 \mathrm{ft}$ above mean sea level, datum of 1929 , unadjusted.

Discharge record.--Stage-discharge relation defined by current-meter measurements below 5,000 cfs, and extended to 6,500 cfs by logarithmic plotting.

Maxima.--Given in the following table.

March 1964:

Mar. 4, 1745 hours....................

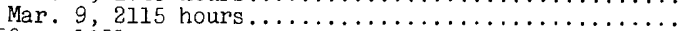

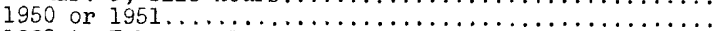

1962 to February 1964:

Mar. 4, 1963 .

$\begin{array}{cc}\text { Discharge } & \text { Gage height } \\ \text { (cfs) } & \text { (feet) } \\ 2,670 & 8.29 \\ 6,450 & 12.95 \\ \text { Unknown } & 18.4\end{array}$

$5,120 \quad 11.60$

Mean discharge, in cub1c feet per second, March 1964

\begin{tabular}{l}
\hline Day \\
\hline $1 \ldots \ldots$
\end{tabular}

(134) 3-3735. East Fork White River at Shoals, Ind.

Location.--Lat $38^{\circ} 40^{\prime} 02^{\prime \prime}$, long $86^{\circ} 47^{\prime} 32^{\prime \prime}$, in sec.30, T.3 N., R.3 W., in first pier from left bank on highway bridge at Shoals, $400 \mathrm{ft}^{\prime}$ upstream from Baltimore and Ohio Railroad bridge, 1 mile upstream from Beaver Creek, and at mile 107.6.

Drainage area. $--4,954 \mathrm{sq} \mathrm{mi}$.

Gage-he1ght record.--Water-stage recorder graph. Datum of gage is $442.25 \mathrm{ft}$ above mean sea level, datum of 1929.

Discharge record.--Stage-discharge relation defined by current-meter measurements below $100,000 \mathrm{cfs}$ and extended above by logarithmic plotting.

Maxima.--March 1964: Discharge, 62,300 cfs Mar. 15, 0030 hours (gage height, $31.02 \mathrm{ft}$ ).

1847 to February 1964: Discharge, 160,000 cfs Mar. 28, 1913 (gage height, $42.2 \mathrm{ft}$ ).

Remarks.--Floodflow affected by storage in Monroe Reservolr (flood-control storage capacity, 258,800 acre-ft).

Mean discharge, in cubic feet per second, March 1964

\begin{tabular}{|c|c|c|c|c|c|c|c|c|c|}
\hline Day & Discharge & Day & Discharge & Day & Discharge & Day & Discharge & Day & Discharge \\
\hline $\begin{array}{l}1 \ldots \ldots \\
2 \ldots \ldots \\
3 \ldots \ldots \\
4 \ldots \ldots \\
5 \ldots \ldots \\
6 \ldots \ldots\end{array}$ & $\begin{array}{r}724 \\
706 \\
697 \\
1,200 \\
10,200 \\
12,500\end{array}$ & $\begin{array}{r}7 \ldots \ldots \\
8 \ldots \ldots \\
9 \ldots \ldots \\
10 \ldots \\
11 \ldots \ldots \\
12 \ldots \ldots\end{array}$ & $\begin{array}{l}10,800 \\
12,400 \\
23,500 \\
39,200 \\
44,500 \\
46,100\end{array}$ & $\begin{array}{l}13 \ldots \ldots \\
14 \ldots \ldots \\
15 \ldots \ldots \\
16 \ldots \ldots \\
17 \ldots \ldots \\
18 \ldots \ldots\end{array}$ & $\begin{array}{l}53,900 \\
61,000 \\
61,400 \\
57,300 \\
51,500 \\
45,500\end{array}$ & $\begin{array}{l}19 \ldots \ldots \\
20 \ldots \ldots \\
21 \ldots \ldots \\
22 \ldots \ldots \\
23 \ldots \ldots \\
24 \ldots \ldots\end{array}$ & $\begin{array}{r}39,000 \\
31,800 \\
23,400 \\
16,500 \\
12,100 \\
9,780\end{array}$ & $\begin{array}{l}25 \ldots \ldots \\
26 \ldots \ldots \\
27 \ldots \ldots \\
28 \ldots \ldots \\
29 \ldots \ldots \\
30 \ldots \ldots \\
31 \ldots \ldots\end{array}$ & $\begin{array}{l}8,900 \\
8,440 \\
8,050 \\
8,660 \\
9,060 \\
8,720 \\
8,050\end{array}$ \\
\hline onthl & an & 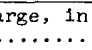 & & & & & & & $\begin{array}{r}23,400 \\
5.44\end{array}$ \\
\hline
\end{tabular}


(135) 3-3740. White River at Petersburg, Ind.

Location.--Lat $38^{\circ} 30^{\prime} 39^{\prime \prime}$, long $87^{\circ} 17^{\prime} 22^{\prime \prime}$, in SW $\frac{1}{4}$ sec.15, T.1 N., R. 8 W., on left bank, $300 \mathrm{ft}$ downstream from bridge on State Highway 61 , three-eighths of a mile upstream from Prides Creek, 1 mile north of Petersburg, and at mile 47.7.

Drainage area. $--11,139 \mathrm{sq} \mathrm{ml}$.

Gage-he1ght record.--Water-stage recorder graph. Datum of gage is $400.00 \mathrm{ft}$ above mean sea level, datum of 1929 .

Discharge record.--Stage-discharge relation defined by current-meter measurements.

Max1ma --March 1964: Discharge, 108,000 cfs Mar. 16, 2100 hours (gage he1ght, $25.13 \mathrm{ft}^{\mathrm{t}}$ )

1907 to February 1964: Discharge, 235,000 cfs (est1mated) Mar. 29, 1913 (gage helght, $29.5 \mathrm{ft}$, from floodmarks, by Corps of Engineers).

Mean discharge, in cublc feet per second, March 1964

\begin{tabular}{|c|c|c|c|c|c|c|c|c|c|}
\hline Day & D1scharge & Day & Discharge & Day & Discharge & Day & Discharge & Day & Discharge \\
\hline $\begin{array}{l}1 \ldots \ldots \\
2 \ldots \ldots \\
3 \ldots \ldots \\
4 \ldots \ldots \\
5 \ldots \ldots \\
6 \ldots \ldots\end{array}$ & $\begin{array}{r}1,710 \\
1,680 \\
1,680 \\
2,160 \\
7,090 \\
14,200\end{array}$ & $\begin{array}{r}7 \ldots \ldots \\
8 \ldots \ldots \\
9 \ldots \\
10 \ldots \\
11 \ldots \ldots \\
12 \ldots \ldots\end{array}$ & $\begin{array}{l}19,000 \\
19,800 \\
29,600 \\
47,200 \\
57,200 \\
68,000\end{array}$ & $\begin{array}{l}13 \ldots \ldots \\
14 \ldots \ldots \\
15 \ldots \ldots \\
16 \ldots \ldots \\
17 \ldots \ldots \\
18 \ldots \ldots\end{array}$ & $\begin{array}{r}78,900 \\
84,200 \\
98,000 \\
108,000 \\
105,000 \\
92,800\end{array}$ & $\begin{array}{l}19 \ldots \ldots \\
20 \ldots \ldots \\
21 \ldots \ldots \\
22 \ldots \ldots \\
23 \ldots \ldots \\
24 \ldots \ldots\end{array}$ & $\begin{array}{l}79,700 \\
68,600 \\
58,300 \\
49,900 \\
39,300 \\
28,200\end{array}$ & $\begin{array}{l}25 \ldots \ldots \\
26 \ldots \ldots \\
27 \ldots \ldots \\
28 \ldots \ldots \\
29 \ldots \ldots \\
30 \ldots \ldots \\
31 \ldots \ldots\end{array}$ & $\begin{array}{l}21,800 \\
19,400 \\
18,200 \\
17,700 \\
18,000 \\
17,800 \\
17,100\end{array}$ \\
\hline
\end{tabular}

(136) 3-3745. Patoka River near Ellsworth, Ind.

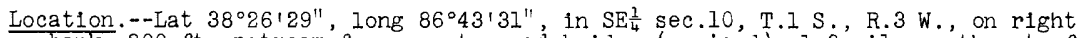
bank, $200 \mathrm{ft}$ upstream from county road bridge (revised), 1.0 mile northwest of Ellsworth, 2.9 miles upstream from Dilion Creek, and 4 miles east of Dubols.

Drainage area. $--171 \mathrm{sq} \mathrm{m}$.

Gage-height record.--Water-stage recorder graph. Datum of gage is $477.00 \mathrm{ft}$ above mean sea level, datum of 1929.

Discharge record.--Stage-discharge relation defined by current-meter measurements below 13,500 cfs and extended above by logarithmic plotting.

Maxima.--Given in the following table.

March 1964:

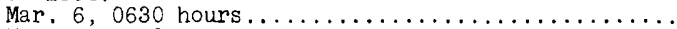

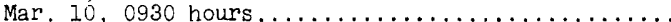

1913 to February 1964: March 1913 .

$\begin{array}{cc}\text { D1scharge } & \text { Gage height } \\ \text { (cfs) } & \text { (feet) } \\ 4,930 & 15.74 \\ 14,700 & 20.02 \\ 12,300 & 19.1\end{array}$

Mean discharge, in cublc feet per second, March 1964

\begin{tabular}{l} 
Day \\
\hline $1 \ldots \ldots$
\end{tabular}

Gage height, in feet, and discharge, in cubic feet per second, at indicated time, 1964

\begin{tabular}{|c|c|c|c|c|c|c|c|c|c|c|c|}
\hline Date & Hour & $\begin{array}{c}\text { Gage } \\
\text { helght }\end{array}$ & $\begin{array}{l}\text { Dis- } \\
\text { charge }\end{array}$ & Date & Hour & $\begin{array}{c}\text { Gage } \\
\text { helght }\end{array}$ & $\begin{array}{l}\text { Dis- } \\
\text { charge }\end{array}$ & Date & Hour & $\begin{array}{c}\text { Gage } \\
\text { he1ght }\end{array}$ & $\begin{array}{l}\text { Dis- } \\
\text { charge }\end{array}$ \\
\hline $\begin{array}{ll}\text { Mar. } & 3 \\
& 4\end{array}$ & $\begin{array}{l}2400 \\
0300 \\
0400 \\
0500 \\
0600 \\
0700\end{array}$ & $\begin{array}{l}2.10 \\
2.15 \\
2.20 \\
2.32 \\
2.40 \\
2.48\end{array}$ & $\begin{array}{l}13 \\
17 \\
22 \\
35 \\
66 \\
81\end{array}$ & Mar. 4 & $\begin{array}{l}0800 \\
0900 \\
1000 \\
1100 \\
1130 \\
1200 \\
1230\end{array}$ & $\begin{array}{l}2.76 \\
3.08 \\
3.46 \\
4.22 \\
5.75 \\
7.84 \\
8.94\end{array}$ & $\begin{array}{r}141 \\
216 \\
298 \\
438 \\
683 \\
932 \\
1,050\end{array}$ & Mar. 4 & $\begin{array}{l}1300 \\
1400 \\
1500 \\
1600 \\
1700 \\
1800 \\
1900\end{array}$ & $\begin{array}{r}9.64 \\
10.60 \\
11.21 \\
11.85 \\
12.54 \\
12.97 \\
13.51\end{array}$ & $\begin{array}{l}1,130 \\
1,230 \\
1,310 \\
1,400 \\
1,700 \\
1,960 \\
2,310\end{array}$ \\
\hline
\end{tabular}

$782-0010-65-11$ 
Gage he1ght, in feet, and discharge, in cub1c feet per second, at ind1cated t1me, 1964, of

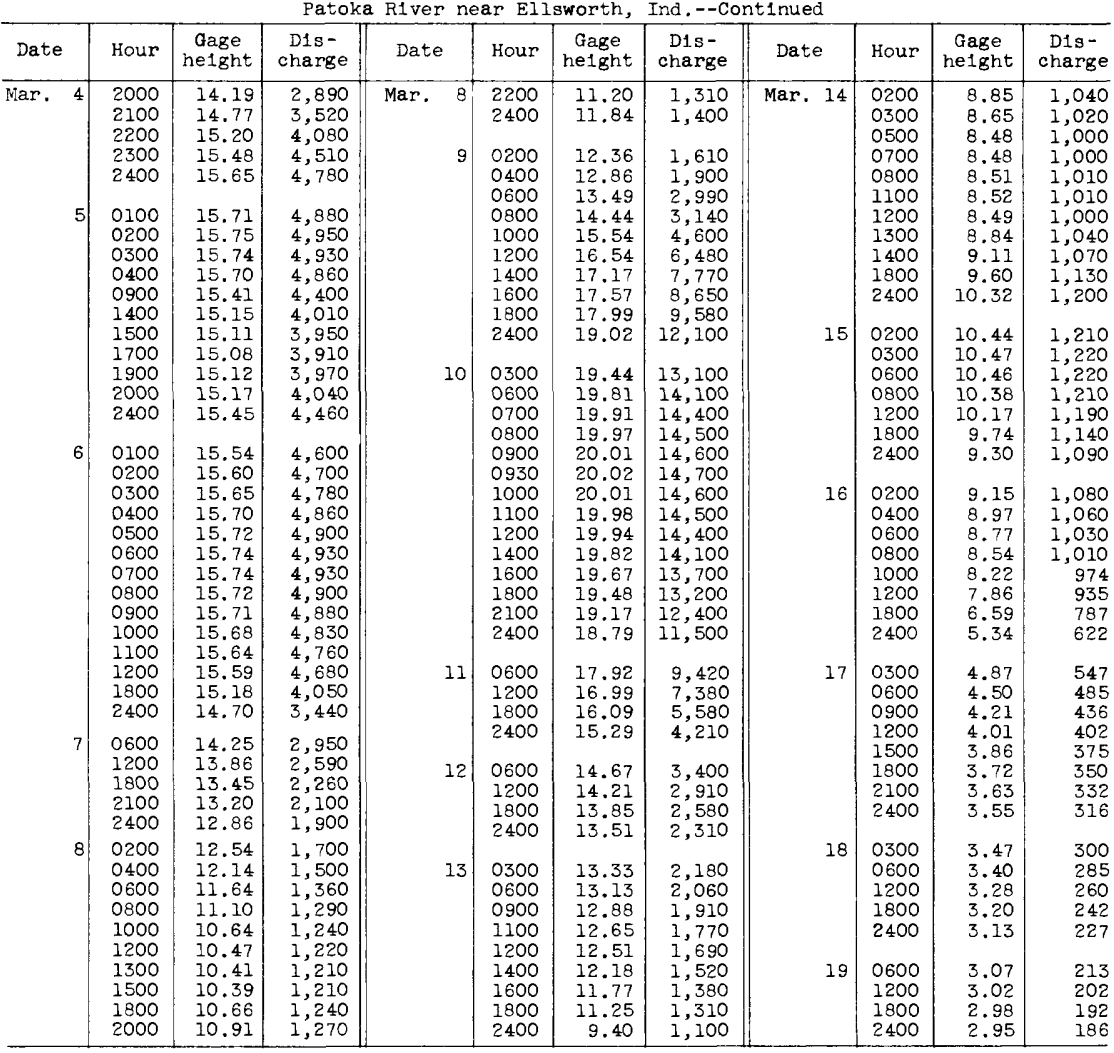

(137) 3-3755. Patoka River at Jasper, Ind.

Location.--Lat $38^{\circ} 24^{\prime} 49^{\prime \prime}$, long $86^{\circ} 52^{\prime} 36^{\prime \prime}$, in SE $\frac{1}{4}$ sec.20, T.1 S., R.4 W., on left bank 0.3 mile upstream from unnamed outlet of Jasper Lake, 1.0 mile downstream from Coon Seitz bridge, 1.2 miles downstream from Beaver Creek, and 3.3 miles northeast of Jasper.

Drainage area. $--257 \mathrm{sq} \mathrm{m} 1$.

Gage-height record.--Water-stage recorder graph except Mar. 6-17 for wh1ch graph was drawn on basis of readings at supplementary gage. Datum of gage $1 \mathrm{~s} 446.19 \mathrm{ft}$ above mean sea level, datum of 1929. Supplementary gage, 5.6 miles downstream, 1s at datum $0.34 \mathrm{ft}$ lower.

Discharge record.--Stage-discharge relation defined by current-meter measurements. Discharge, Mar. 6, 0000-1200 hours and Mar. 15, 1200 hours, to Mar. 17, 2400 hours, adjusted for channel storage between base gage and supplementary gage.

Maxima.--March 1964: D1scharge, 14,100 cfs Mar. 11, 1400 hours (gage he1ght, 15.17 ft at supplementary gage, $20.20 \mathrm{ft}$ at base gage, from floodmark).

1913 to February 1964: Discharge, $16,000 \mathrm{cfs}$ March 1913 (gage height, $15.9 \mathrm{ft}$ at supplementary gage, from floodmark, furnished by local residents). 
Mean discharge, In cublc feet per second, March 1964, of Patoka R1ver at Jasper, Ind.

\begin{tabular}{|c|c|c|c|c|c|c|c|c|c|}
\hline Day & D1scharge & Day & D1scharge & Day & Discharge & Day & Discharge & Day & Discharge \\
\hline $\begin{array}{l}1 \ldots \ldots \\
2 \ldots \\
3 \ldots \\
4 \ldots \\
5 \ldots \\
6 \ldots \\
\end{array}$ & $\begin{array}{r}22 \\
20 \\
20 \\
530 \\
1,290 \\
1,890\end{array}$ & $\begin{array}{r}7 \ldots \ldots \\
8 \ldots \ldots \\
9 \ldots \\
10 \ldots \\
11 \ldots \ldots \\
12 \ldots \ldots\end{array}$ & $\begin{array}{r}3,590 \\
4,330 \\
5,770 \\
9,970 \\
13,600 \\
11,900\end{array}$ & $\begin{array}{l}13 \ldots \ldots \\
14 \ldots \ldots \\
15 \ldots \ldots \\
16 \ldots \ldots \\
17 \ldots \ldots \\
18 \ldots \ldots\end{array}$ & $\begin{array}{l}7,740 \\
4,800 \\
3,500 \\
2,470 \\
1,740 \\
1,220\end{array}$ & $\mid \begin{array}{l}19 \ldots \ldots \\
20 \ldots \ldots \\
21 \ldots \ldots \\
22 \ldots \ldots \\
23 \ldots \ldots \\
24 \ldots \ldots\end{array}$ & $\begin{array}{l}659 \\
371 \\
306 \\
281 \\
240 \\
198\end{array}$ & $\left\{\begin{array}{l}25 \ldots \ldots \\
26 \ldots \ldots \\
27 \ldots \ldots \\
28 \ldots \ldots \\
29 \ldots \ldots \\
30 \ldots \ldots \\
31 \ldots \ldots\end{array}\right.$ & $\begin{array}{l}176 \\
272 \\
624 \\
589 \\
378 \\
256 \\
190\end{array}$ \\
\hline
\end{tabular}

Gage helght. In feet, and discharge, In cuble feet per second, at indicated time, 1964

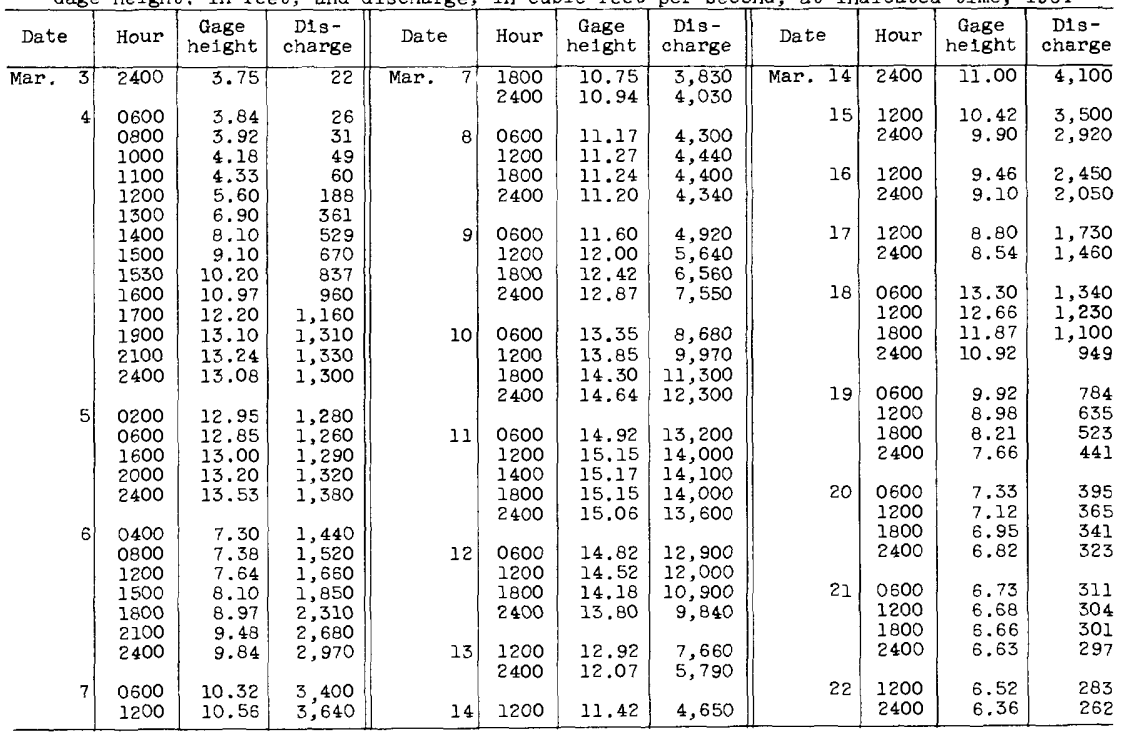

(138) 3-3763. Patoka River at Winslow, Ind.

Location.--Lat $38^{\circ} 22^{\prime} 48^{\prime \prime}$, long $87^{\circ} 13^{100^{\prime \prime}}$, in SW $\frac{1}{4}$ sec.32, T.1 S., R.7 W., on right bank at abandoned bridge abutment, $65^{\circ} \mathrm{ft}$ upstream from State Road 61 bridge, $100 \mathrm{ft}$ downstream from dam of Winslow water Co., and $41.3 \mathrm{miles}$ above mouth.

Drainage area. --603 sq $\mathrm{mi}$.

Gage-helght record.--Water-stage recorder graph, except Mar. 4, 0800 hours, to Mar. 17, 1730 hours, for which graph was drawn on basis of readings of wirewelght gage on downstream side of State Road 61 bridge.

Discharge record.--Stage-discharge relation defined by current-meter measurements .

Maxima.--March 1964: Discharge, 15,500 cfs Mar. 13, 0200 hours (gage height, $28.84 \mathrm{ft}$ ).

1937: Gage height, $28.9 \mathrm{ft}$ (discharge unknown).

1961 to February 1964: D1scharge, 13,700 cfs May 12, 1961 (gage height $28.3 \mathrm{ft}$ ).

\begin{tabular}{|c|c|c|c|c|c|c|c|c|c|}
\hline Day & Discharge & Day & D1 scharge & Day & Discharge & Day & D1scharge & Day & Discharge \\
\hline $\begin{array}{l}1 \ldots \ldots \\
2 \ldots \\
3 \ldots \ldots \\
4 \ldots \ldots \\
5 \ldots \ldots \\
6 \ldots \ldots\end{array}$ & $\begin{array}{r}40 \\
37 \\
37 \\
544 \\
1,960 \\
1,850\end{array}$ & $\begin{array}{r}7 \ldots \ldots \\
8 \ldots \ldots \\
9 \ldots \\
10 \ldots \\
11 \ldots \ldots \\
12 \ldots\end{array}$ & $\begin{array}{r}1,950 \\
2,650 \\
5,650 \\
10,900 \\
13,300 \\
15,100\end{array}$ & $\begin{array}{l}13 \ldots \ldots \\
14 \ldots \ldots \\
15 \ldots \ldots \\
16 \ldots \ldots \\
17 \ldots \ldots \\
18 \ldots \ldots\end{array}$ & $\begin{array}{r}15,200 \\
14,600 \\
13,600 \\
11,900 \\
9,940 \\
7,910\end{array}$ & $\begin{array}{l}19 \ldots \ldots \\
20 \ldots \ldots \\
21 \ldots \ldots \\
22 \ldots \ldots \\
23 \ldots \ldots \\
24 \ldots \ldots\end{array}$ & $\begin{array}{l}6,040 \\
4,740 \\
3,960 \\
3,420 \\
2,930 \\
2,370\end{array}$ & $\mid \begin{array}{l}25 \ldots \ldots \\
26 \ldots \ldots \\
27 \ldots \ldots \\
28 \ldots \ldots \\
29 \ldots \ldots \\
30 \ldots \ldots \\
31 \ldots \ldots\end{array}$ & $\begin{array}{r}1,800 \\
1,480 \\
1,290 \\
1,120 \\
1,010 \\
862 \\
704\end{array}$ \\
\hline $\begin{array}{l}\text { nthly } \\
\text { noff, }\end{array}$ & $\begin{array}{l}\text { mean dis } \\
\text { in inche }\end{array}$ & ge, & $\ldots$ & per : & & & & & $\begin{array}{r}5,126 \\
9.8\end{array}$ \\
\hline
\end{tabular}


Gage helght, In feet, and discharge, in cuble feet per second, at indicated time, 1964, of

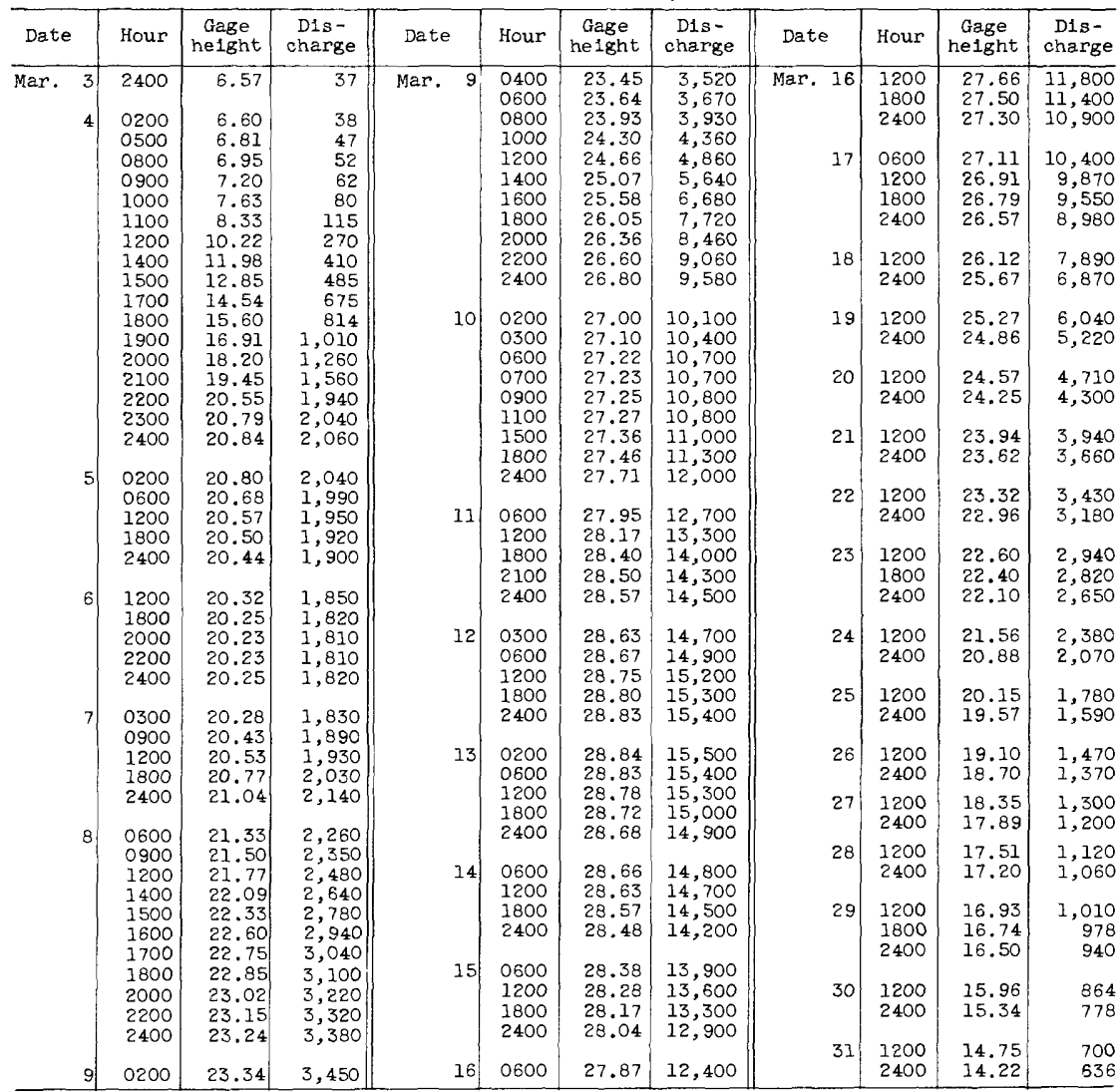


(139) 3-3765. Patoka River near Princeton, Ind.

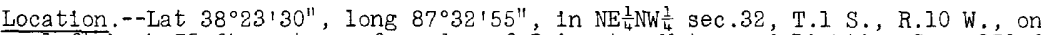
left bank $75 \mathrm{ft}$ upstream from dam of Princeton water and Lighting Co., $270 \mathrm{ft}$ upstream from bridge on State Highway 65 , half a mile downstream from Indian Creek, and 2 miles northeast of Princeton.

Drainage area. $--815 \mathrm{sq} \mathrm{mi}$.

Gage-height record.--Water-stage recorder graph. Datum of gage is $394.138 \mathrm{ft}$ above mean sea level, datum of 1929 .

Discharge record.--Stage-discharge relation defined by current-meter measurements below $12,000 \mathrm{cfs}$, and extended above by logarithmic plotting.

Maxima.--March 1964: Discharge, 15,200 cfs Mar. 16, 0700 hours (gage helght, $21.50 \mathrm{ft})$.

1935 to February 1964: Discharge, 18,700 efs Jan. 26, 1937; gage height, $22.8 \mathrm{ft}$ Jan. 28, 1937.

Mean discharge, in cubic feet per second, March 1964

\begin{tabular}{|c|c|c|c|c|c|c|c|c|c|}
\hline Day & Discharge & Day & Discharge & Day & Discharge & Day & Discharge & Day & Discharge \\
\hline $\begin{array}{l}1 \ldots \ldots \\
2 \ldots \ldots \\
3 \ldots \ldots \\
4 \ldots \ldots \\
5 \ldots \ldots \\
6 \ldots \ldots\end{array}$ & $\begin{array}{r}46 \\
43 \\
41 \\
371 \\
1,120 \\
1,200\end{array}$ & $\begin{array}{r}7 \ldots \ldots \\
8 \ldots \ldots \\
9 \ldots \\
10 \ldots \\
11 \ldots \ldots \\
12 \ldots \ldots\end{array}$ & $\begin{array}{r}1,300 \\
1,570 \\
2,910 \\
4,770 \\
7,670 \\
10,100\end{array}$ & $\begin{array}{l}13 \ldots \ldots \\
14 \ldots \ldots \\
15 \ldots \ldots \\
16 \ldots \ldots \\
17 \ldots \ldots \\
18 \ldots \ldots\end{array}$ & $\begin{array}{l}11,600 \\
13,200 \\
14,700 \\
15,100 \\
14,500 \\
13,600\end{array}$ & $\begin{array}{l}19 \ldots \ldots \\
20 \ldots \ldots \\
21 \ldots \ldots \\
22 \ldots \ldots \\
23 \ldots \ldots \\
24 \ldots \ldots\end{array}$ & $\begin{array}{r}12,300 \\
11,000 \\
9,360 \\
8,100 \\
6,790 \\
5,660\end{array}$ & $\mid \begin{array}{l}25 \ldots \ldots \\
26 \ldots \ldots \\
27 \ldots \ldots \\
28 \ldots \ldots \\
29 \ldots \ldots \\
30 \ldots \ldots \\
31 \ldots \ldots\end{array}$ & $\begin{array}{l}4,840 \\
4,230 \\
3,780 \\
3,360 \\
2,910 \\
2,600 \\
2,360\end{array}$ \\
\hline
\end{tabular}

Gage height, in feet, and discharge, in cubic feet per second, at indicated time, 1964

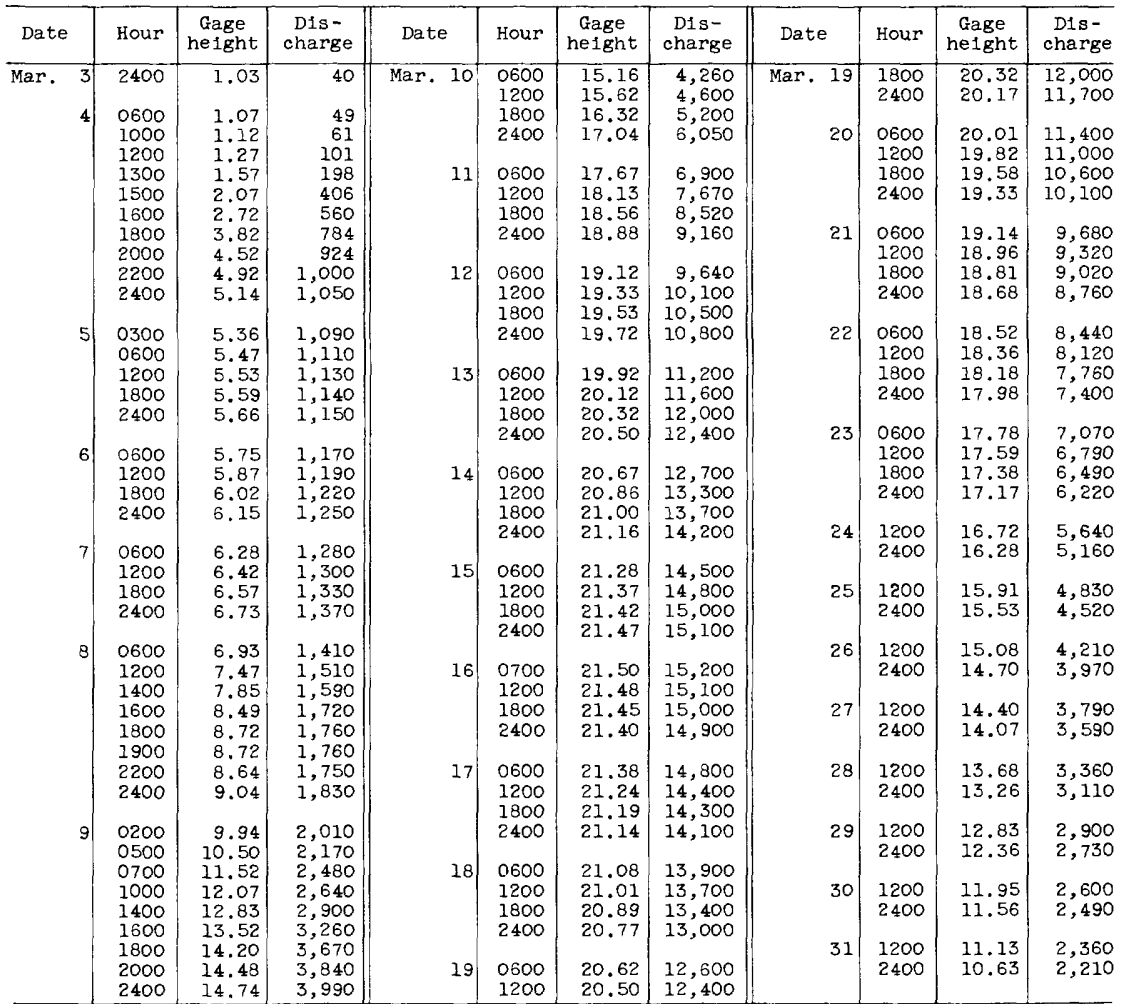


(140) 3-3775. Wabash River at Mount Carmel, I11.

Location.--Lat $38^{\circ} 24^{\prime} 07^{\prime \prime}$, long $87^{\circ} 45^{\prime} 10^{\prime \prime}$, in sec.28, T.1 S., R.12 W., on right bank on downstream side of Southern Railway bridge at Mount Carmel, Wabash County, and 0.1 mile downstream from Patoka River.

Dra1nage area.--28,600 sq $\mathrm{mi}$, approximately.

Gage-height record.--Water-stage recorder graph. Datum of gage is $371.46 \mathrm{ft}$ above mean sea level, datum of 1929.

D1scharge record.--Stage-discharge relation defined by current-meter measurements below 310,000 cfs.

Maxima --March 1964: Discharge, 146,000 cfs Mar. 17, 1900 hours (gage height, $23.97 \mathrm{ft}$ ).

1875 to February 1964: Discharge, 428,000 cf's Mar. 30, 1913 (gage height, $31.0 \mathrm{ft})$.

Mean discharge, in cublc feet per second, March 1964

\begin{tabular}{|c|c|c|c|c|c|c|c|c|c|}
\hline Day & Discharge & Day & Discharge & Day & Discharge & Day & Discharge & Day & D1scharge \\
\hline $\begin{array}{l}1 \ldots \ldots \\
2 \ldots \ldots \\
3 \ldots \ldots \\
4 \ldots \ldots \\
5 \ldots \ldots \\
6 \ldots \ldots\end{array}$ & $\begin{array}{r}4,450 \\
4,270 \\
4,270 \\
5,050 \\
8,290 \\
15,600\end{array}$ & $\begin{array}{r}7 \ldots \ldots \\
8 \ldots \ldots \\
9 \ldots \ldots \\
10 \ldots \\
11 \ldots \ldots \\
12 \ldots \ldots\end{array}$ & $\begin{array}{l}23,000 \\
27,700 \\
38,000 \\
58,200 \\
69,600 \\
79,900\end{array}$ & $\begin{array}{l}13 \ldots \ldots \\
14 \ldots \ldots \\
15 \ldots \ldots \\
16 \ldots \ldots \\
17 \ldots \ldots\end{array}$ & $\begin{array}{r}92,800 \\
110,000 \\
124,000 \\
137,000 \\
146,000 \\
144,000\end{array}$ & $\begin{array}{l}19 \ldots \ldots \\
20 \ldots \ldots \\
21 \ldots \ldots \\
22 \ldots \ldots \\
23 \ldots \ldots \\
24 \ldots \ldots\end{array}$ & $\begin{array}{r}137,000 \\
128,000 \\
115,000 \\
99,600 \\
83,300 \\
69,200\end{array}$ & $\begin{array}{l}25 \ldots \ldots \\
26 \ldots \ldots \\
27 \ldots \ldots \\
28 \ldots \ldots \\
29 \ldots \ldots \\
30 \ldots \ldots \\
31 \ldots \ldots\end{array}$ & $\begin{array}{l}56,300 \\
45,700 \\
40,600 \\
40,500 \\
40,600 \\
41,400 \\
41,000\end{array}$ \\
\hline
\end{tabular}

\section{TRADEWATER RIVER BASIN}

(141) 3-3830. Tradewater River at Olney, Ky .

Location.--Iat $37^{\circ} 13^{\prime} 26^{\prime \prime}$, Iong $87^{\circ} 46^{\prime} 53^{\prime \prime}$, on downstream side of left abutment of highway bridge at 0lney, Hopkins County, 1.1 miles upstream from Cave Creek, 5.1 miles downstream from Flynn Creek, and $9 \frac{1}{2}$ miles northeast of Princeton.

Drainage area. $-255 \mathrm{sq} \mathrm{mi}$, of which about $9 \mathrm{sq} \mathrm{ml}$ does not contribute directly to surface runoff.

Gage-height record.--Digital recorder tape punched at 30-minute intervals. Datum of gage 1s $362.80 \mathrm{ft}$ above mean sea levei, adjustment of 1907 .

Discharge record. --Stage-discharge relation defined by current-meter measurements below $12,000 \mathrm{cfs}$ and by slope-area measurement at 17,000 cfs. Rate of change in stage used as a factor Mar. 4, 2400 hours, to Mar. 9, 0600 hours.

Maxima.--Given in the following table.

March 1964:

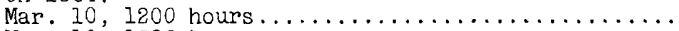

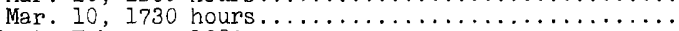

1937 to February 1964:

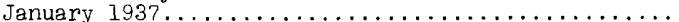

$\begin{array}{cc}\begin{array}{c}\text { Discharge } \\ \text { (cfs) }\end{array} & \text { Gage he lght } \\ 13,600 & - \\ \text { (feet) } & 18.68 \\ 17,000 & 19.27\end{array}$

Mean discharge, in cublc feet per second, March 1964

\begin{tabular}{|c|c|c|c|c|c|c|c|c|c|}
\hline Day & Discharge & Day & Discharge & Day & Discharge & Day & Discharge & Day & D1scharge \\
\hline $\begin{array}{l}1 \ldots \ldots \\
2 \ldots \ldots \\
3 \ldots \ldots \\
4 \ldots \ldots \\
5 \ldots \ldots \\
6 \ldots \ldots\end{array}$ & $\begin{array}{r}8.3 \\
7.8 \\
7.4 \\
407 \\
1,530 \\
1,520\end{array}$ & $\begin{array}{r}7 \ldots \ldots \\
8 \ldots \ldots \\
9 \ldots \ldots \\
10 \ldots \ldots \\
11 \ldots \ldots \\
12 \ldots\end{array}$ & $\begin{array}{r}1,610 \\
2,560 \\
7,150 \\
13,200 \\
12,200 \\
9,340\end{array}$ & $\begin{array}{l}13 \ldots \ldots \\
14 \ldots \ldots \\
15 \ldots \ldots \\
16 \ldots \ldots \\
17 \ldots \ldots \\
18 \ldots \ldots\end{array}$ & $\begin{array}{r}6,550 \\
4,550 \\
3,040 \\
2,090 \\
1,290 \\
754\end{array}$ & $\begin{array}{l}19 \ldots \ldots \\
20 \ldots \ldots \\
21 \ldots \ldots \\
22 \ldots \ldots \\
23 \ldots \ldots \\
24 \ldots \ldots\end{array}$ & $\begin{array}{l}417 \\
258 \\
224 \\
200 \\
179 \\
160\end{array}$ & $\begin{array}{l}25 \ldots \ldots \\
26 \ldots \ldots \\
27 \ldots \ldots \\
28 \ldots \ldots \\
29 \ldots \ldots \\
30 \ldots \ldots \\
31 \ldots \ldots\end{array}$ & $\begin{array}{r}141 \\
133 \\
128 \\
113 \\
102 \\
89 \\
77\end{array}$ \\
\hline
\end{tabular}

Note.--Da1ly mean discharges computed on basis of 30 -minute intervals. 
Gage height, in feet, and discharge, in cubic feet per second, at indicated time, 1964, of

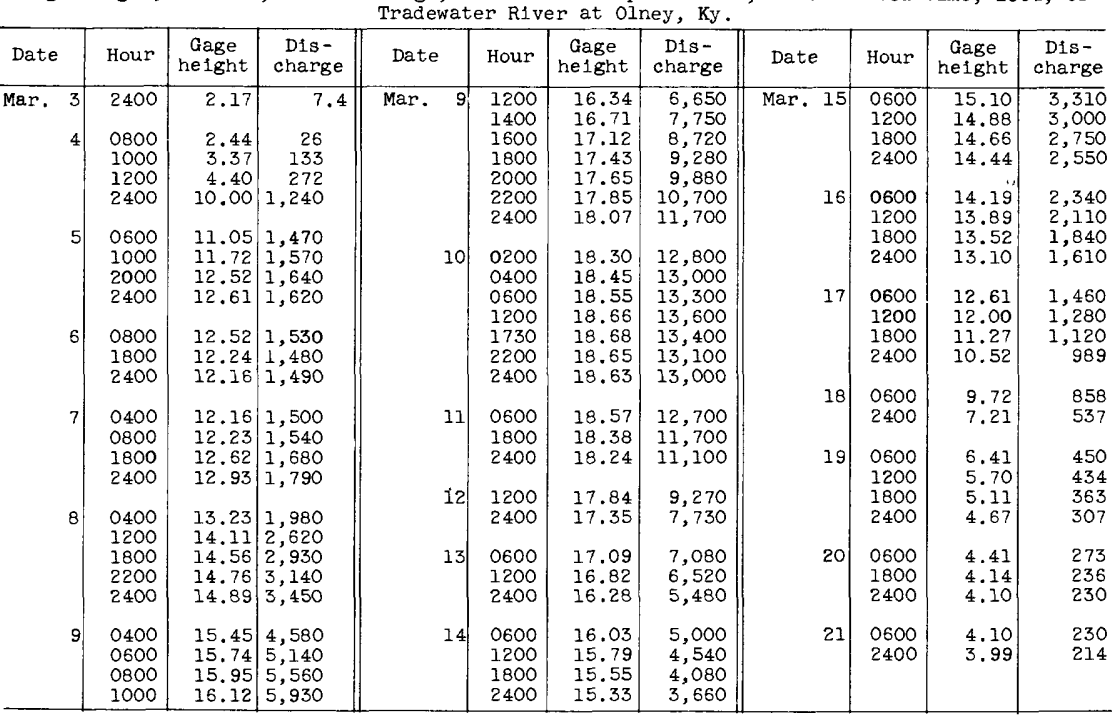

\section{OHIO RIVER MAIN STEM}

(142) 3-3845. Oh1o River at Golconda, III.

Location.--Lat $37^{\circ} 21^{\prime} 28^{\prime \prime}$, long $88^{\circ} 28^{\prime} 57^{\prime \prime}$, on right bank at lock and dam 51, at Golconda, Pope County, 0.5 mile upstream from McGllilgan Creek, 0.7 mile downstream from Lusk Creek, and at mile 903.1.

Drainage area.--143,900 $\mathrm{sq} \mathrm{ml}$, approximately.

Gage-helght record.--Graphs drawn from hourly upper-staff-gage readings. Aux1llary gage 26.3 miles downstream. Datum of gage is $294.6 \mathrm{ft}$ above mean sea level, Oh10 River datum.

Discharge record.--Stage-fall-discharge relation defined by current-meter measurements. Fall used as a factor in computing discharge. Stage-fall-discharge relation indefinite Mar. 1-4; discharge estimated on basis of records for other Oh1o River main-stem stations.

Maxtma.--March 1964: Discharge, 958,000 cfs Mar. 20, 1800 hours; gage helght, $52.40 \mathrm{ft}$ Mar. 20,1200 hours.

1937 to February 1964: Discharge, 1,470,000 cfs Feb. 2, 3, 1937; gage height, 62.6 ft Feb. 1-3, 1937.

Remarks.--Partly regulated by locks, dams, and reservoirs.

Cooperation.--Gage-helght record furnished by Corps of Engineers.

Mean gage he1ght, in feet, and discharge, in cublc feet per second, 1964

\begin{tabular}{|c|c|c|c|c|c|c|c|c|c|}
\hline \multicolumn{2}{|c|}{ Date } & $\begin{array}{c}\text { Gage } \\
\text { he1ght }\end{array}$ & Discharge & Date & $\begin{array}{c}\text { Gage } \\
\text { helght }\end{array}$ & Discharge & Date & $\begin{array}{c}\text { Gage } \\
\text { he1ght }\end{array}$ & Discharge \\
\hline ar. & $\begin{array}{r}1 \ldots \\
2 \ldots \\
3 \ldots \\
4 \ldots \\
5 \ldots \\
6 \ldots \\
7 \ldots \\
8 \ldots \\
9 \ldots \\
10 \ldots \\
11 \ldots \\
12 \ldots\end{array}$ & $\begin{array}{l}15.30 \\
15.50 \\
15.13 \\
15.26 \\
18.36 \\
22.90 \\
29.32 \\
33.30 \\
37.78 \\
40.84 \\
42.67 \\
44.89\end{array}$ & $\begin{array}{r}45,000 \\
50,000 \\
60,000 \\
90,000 \\
140,000 \\
223,000 \\
335,000 \\
396,000 \\
462,000 \\
528,000 \\
598,000 \\
683,000\end{array}$ & Mar. $\begin{array}{l}13 \ldots \\
14 \ldots \\
15 \ldots \\
16 \ldots \\
17 \ldots \\
18 \ldots \\
19 \ldots \\
20 \ldots \\
21 \ldots \\
22 \ldots \\
23 \ldots \\
14 \ldots\end{array}$ & $\begin{array}{l}46.82 \\
48.32 \\
49.45 \\
50.47 \\
51.48 \\
52.04 \\
52.24 \\
52.34 \\
52.30 \\
52.18 \\
51.88 \\
51.43\end{array}$ & $\begin{array}{l}749,000 \\
812,000 \\
854,000 \\
887,000 \\
913,000 \\
923,000 \\
942,000 \\
956,000 \\
954,000 \\
930,000 \\
915,000 \\
883,000\end{array}$ & $\begin{array}{r}25 \ldots \\
26 \ldots \\
27 \ldots \\
28 \ldots \\
29 \ldots \\
30 \ldots \\
31 \ldots \\
1 \ldots \ldots \\
2 \ldots \ldots \\
3 \ldots \ldots \\
4 \ldots \ldots \\
5 \ldots\end{array}$ & $\begin{array}{l}50.79 \\
49.74 \\
48.50 \\
47.00 \\
45.11 \\
43.08 \\
40.74 \\
37.64 \\
34.13 \\
30.93 \\
28.06 \\
26.38\end{array}$ & $\begin{array}{l}837,000 \\
798,000 \\
732,000 \\
681,000 \\
615,000 \\
554,000 \\
492,000 \\
430,000 \\
376,000 \\
327,000 \\
280,000 \\
260,000\end{array}$ \\
\hline
\end{tabular}

Note.--Monthly mean d1scharge for March 1954, $614,100 \mathrm{cfs}$. 


\section{CUMBERLAND RIVER BASIN}

(143) 3-4380. Little River near Cadiz, Ky.

Location.--Lat $36^{\circ} 46^{\prime} 40^{\prime \prime}$, long $87^{\circ} 43^{\prime} 18^{\prime \prime}$, on right bank at upstream side of highway bridge, $50 \mathrm{ft}$ downstream from Casey Creek and $8 \frac{3}{4}$ miles southeast of Cadiz, Trigg County.

Drainage area. $--244 \mathrm{sq} \mathrm{ml}^{\mathrm{l}}$, of which about $94 \mathrm{sq} \mathrm{mi}$ does not contribute directly to surface runoff.

Gage-height record. - Water-stage recorder graph. Datum of gage is 391.45 ft above mean sea level, unadjusted.

Discharge record.--Stage-discharge relation defined by current-meter measurements below 10,000 cfs.

Maxima.--Given in the following table.

March 1964:

Mar. 5, 1100 hours

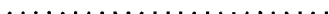

1940 to Fébruary 1964 :

$\begin{array}{cc}\begin{array}{c}\text { Discharge } \\ \text { (cfs) }\end{array} & \begin{array}{c}\text { Gage height } \\ \text { (feet) }\end{array} \\ 3,500 & 11.63 \\ 12,600 & 20.27 \\ 14,200 & 21.00\end{array}$

Mean discharge, in cublc feet per second, March 1964

\begin{tabular}{|c|c|c|c|c|c|c|c|c|c|}
\hline Day & Discharge & Day & D1scharge & Day & D1scharge & Day & D1scharge & Day & D1scharge \\
\hline $\begin{array}{l}1 \ldots \ldots \\
2 \ldots \ldots \\
3 \ldots \ldots \\
4 \ldots \\
5 \ldots \\
6 \ldots\end{array}$ & $\begin{array}{r}58 \\
57 \\
55 \\
1,490 \\
2,960 \\
1,050\end{array}$ & $\begin{array}{r}7 \ldots \ldots \\
8 \ldots \ldots \\
9 \ldots \ldots \\
10 \ldots \ldots \\
11 \ldots \ldots \\
12 \ldots \ldots\end{array}$ & $\begin{array}{r}677 \\
3,830 \\
7,140 \\
7,230 \\
5,380 \\
1,980\end{array}$ & $\left\{\begin{array}{l}13 \ldots \ldots \\
14 \ldots \ldots \\
15 \ldots \ldots \\
16 \ldots \ldots \\
17 \ldots \ldots \\
18 \ldots \ldots\end{array}\right.$ & $\begin{array}{r}1,620 \\
1,290 \\
1,150 \\
986 \\
850 \\
752\end{array}$ & $\mid \begin{array}{l}19 \ldots \ldots \\
20 \ldots \ldots \\
21 \ldots \ldots \\
22 \ldots \ldots \\
23 \ldots \ldots \\
24 \ldots \ldots\end{array}$ & $\begin{array}{l}677 \\
630 \\
605 \\
564 \\
502 \\
494\end{array}$ & $\begin{array}{l}25 \ldots \ldots \\
26 \ldots \ldots \\
27 \ldots \ldots \\
28 \ldots \ldots \\
29 \ldots \ldots \\
30 \ldots \ldots \\
31 \ldots \ldots\end{array}$ & $\begin{array}{l}482 \\
466 \\
418 \\
394 \\
350 \\
330 \\
314\end{array}$ \\
\hline $\begin{array}{l}\text { onthly } \\
\text { anoff, }\end{array}$ & $\begin{array}{l}\text { mean disc } \\
\text { in inches }\end{array}$ & ge, & 1c & per & & & & & $\begin{array}{r}1,445 \\
6.83\end{array}$ \\
\hline
\end{tabular}

(144) 3-4385. Cumberland River at Smithland, Ky.

Location.--Lat $37^{\circ} 08^{\prime} 45^{\prime \prime}$, long $88^{\circ} 24^{\prime} 2^{\prime \prime}$, on downstream side of left center pier of bridge on U.S. Highway 60 at Smithland, Livingston County, 1 mile downstream from McCormick Creek and 2.8 miles upstream from mouth.

Drainage area. $--17,913 \mathrm{sq} \mathrm{mi}$.

Gage-height record.--Water-stage recorder graphs. Auxiliary gage 16.8 miles upstream. Datum of gage is $300.00 \mathrm{ft}$ above mean sea level, Sandy Hook datum.

Discharge record.--Stage-fall-discharge relation defined by current-meter measurements.

Maxima.--Given in the following table.

March 1964

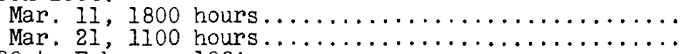

Discharge

(cfs)

115,000

Gage height

939 to February 1964:

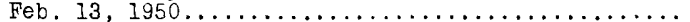

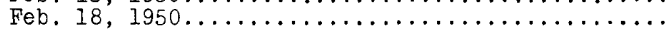

$201 \overline{1,000}$

(feet)

35.07

Maximum stage known, 51.1 ft January to February 1937.

Remarks.--Some regulation by navigation dams on Cumberland River, and by Great Falls Lake, Lake Cumberland, Dale Hollow and Center Hill Reservoirs and Old Hickory Lake.

Mean d1scharge, in cub1c feet per second, March 1964

\begin{tabular}{|c|c|c|c|c|c|c|c|c|c|}
\hline Day & Discharge & Day & D1scharge & Day & D1scharge & Day & Discharge & Day & Discharge \\
\hline $\begin{array}{l}1 \ldots \ldots \\
2 \ldots \ldots \\
3 \ldots \ldots \\
4 \ldots \ldots \\
5 \ldots \ldots\end{array}$ & $\begin{array}{l}21,000 \\
20,400 \\
23,400 \\
32,800 \\
58,000 \\
74,400\end{array}$ & $\begin{array}{r}7 \ldots \ldots \\
8 \ldots \ldots \\
9 \ldots \ldots \\
10 \ldots \ldots \\
12 \ldots \ldots\end{array}$ & $\begin{array}{r}79,300 \\
74,600 \\
97,100 \\
114,000 \\
113,000 \\
106,000\end{array}$ & $\begin{array}{l}13 \ldots \ldots \\
14 \ldots \ldots \\
15 \ldots \ldots \\
16 \ldots \ldots \\
17 \ldots \ldots \\
18 \ldots\end{array}$ & $\begin{array}{l}89,200 \\
67,900 \\
56,500 \\
59,800 \\
70,800 \\
75,400\end{array}$ & $\mid \begin{array}{l}19 \ldots \ldots \\
20 \ldots \ldots \\
21 \ldots \ldots \\
22 \ldots \ldots \\
23 \ldots \\
24 \ldots\end{array}$ & $\begin{array}{l}71,700 \\
63,900 \\
61,100 \\
64,400 \\
67,000 \\
66,400\end{array}$ & $\mid \begin{array}{l}25 \ldots \\
26 \ldots \\
27 \ldots \ldots \\
28 \ldots \\
29 \ldots \\
30 \ldots \ldots \\
31 \ldots \ldots\end{array}$ & $\begin{array}{l}60,800 \\
60,400 \\
69,700 \\
74,100 \\
72,000 \\
66,200 \\
60,500\end{array}$ \\
\hline
\end{tabular}


TENNESSEE RIVER BASIN

(145) 3-6095. Tennessee River near Paducah, Ky .

Location.--Lat $37^{\circ} 01^{\prime} 11^{\prime \prime}$, long $88^{\circ} 16^{\prime} 50^{\prime \prime}$, on left bank at Gilbertsville, Marshall County, 4,000 ft downstream from Kentucky Dam, 2.3 miles upstream from Shadle Creek, 16 miles east of Paducah, McCracken County, and at m1le 21.6.

Drainage area. $--40,200 \mathrm{sq} \mathrm{ml}$, approximately (at Gilbertsville).

Gage-he1ght record.--Water-stage recorder graph. Datum of gage is $286.35 \mathrm{ft}^{t}$ above mean sea level, datum of 1929 .

Discharge record.--Stage-fall-discharge relation defined by current-meter measurements.

Maxima.--March 1964: Discharge, 231,000 cfs Mar. 29, 1800 hours; gage height, $49.23 \mathrm{ft}$ Mar. 17, 1000 hours.

1889 to February 1964: Discharge, 500,000 cfs Feb. 17, 1948; max1mum gage helght, $62.43 \mathrm{ft}$ Feb. 2, 1937, at Gilbertsville, present datum.

Remarks. --Flow regulated by Kentucky Lake.

Mean discharge, in cub1c feet per second, March 1964

\begin{tabular}{|c|c|c|c|c|c|c|c|c|c|}
\hline Day & Discharge & Day & Discharge & Day & D1scharge & Day & Discharge & Day & Discharge \\
\hline $\begin{array}{l}1 \ldots \ldots \\
2 \ldots \ldots \\
3 \ldots \ldots \\
4 \ldots \ldots \\
5 \ldots \ldots \\
6 \ldots \ldots\end{array}$ & $\begin{array}{r}56,300 \\
69,600 \\
101,000 \\
142,000 \\
180,000 \\
196,000\end{array}$ & $\begin{array}{r}7 \ldots \ldots \\
8 \ldots \ldots \\
9 \ldots \ldots \\
10 \ldots \\
11 \ldots \ldots \\
12 \ldots \ldots\end{array}$ & $\begin{array}{l}192,000 \\
199,000 \\
201,000 \\
205,000 \\
165,000 \\
125,000\end{array}$ & $\begin{array}{l}13 \ldots \ldots \\
14 \ldots \ldots \\
15 \ldots \ldots \\
16 \ldots \ldots \\
17 \ldots \ldots \\
18 \ldots \ldots\end{array}$ & $\begin{array}{l}116,000 \\
105,000 \\
107,000 \\
167,000 \\
168,000 \\
130,000\end{array}$ & $\begin{array}{l}19 \ldots \ldots \\
20 \ldots \ldots \\
21 \ldots \ldots \\
22 \ldots \ldots \\
23 \ldots \ldots \\
24 \ldots \ldots\end{array}$ & $\begin{array}{l}106,000 \\
104,000 \\
105,000 \\
102,000 \\
121,000 \\
136,000\end{array}$ & $\begin{array}{l}25 \ldots \ldots \\
26 \ldots \ldots \\
27 \ldots \ldots \\
28 \ldots \ldots \\
29 \ldots \ldots \\
30 \ldots \ldots \\
31 \ldots \ldots\end{array}$ & $\begin{array}{l}150,000 \\
174,000 \\
189,000 \\
205,000 \\
222,000 \\
225,000 \\
204,000\end{array}$ \\
\hline
\end{tabular}

(146) 3-6105. East Fork Clarks River near Benton, Ky.

Location.--Lat $36^{\circ} 52^{\prime} 24^{\prime \prime}$, long $88^{\circ} 20^{\prime} 48^{\prime \prime}$, on downstream side of right pler of bridge on U.S. Highway 641 and State Highway 58, 1 mile north of Benton, Marshall. County, and 6.8 miles upstream from Middle Fork Creek.

Dra1nage area,$--227 \mathrm{sq} \mathrm{ml}$.

Gage-height record.--Digital recorder tape punched at 30-minute intervals. Datum of gage is $344.53 \mathrm{ft}$ above mean sea level, datum of 1929 (Tennessee Vailey Authority bench mark).

D1scharge record.--Stage-discharge relation defined by current-meter measurements below 16,000 cfs.

Maxima.--Given in the following table.

March 1964:

Mar. 5, 0930 hours

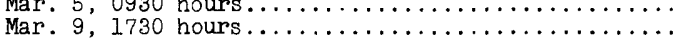

1938 to February 1964 :

Nov. 19, 1957.

D1scharge Gage height

$(\mathrm{cfs})$ (feet)

$6,460 \quad 13.79$

$\begin{array}{rr}6,460 & 13.79 \\ 21,300 & 15.80\end{array}$

Maximum stage known, $17.8 \mathrm{ft}$ in February 1937, from floodmarks.

Mean discharge, in cublc feet per second, March 1964

\begin{tabular}{|c|c|c|c|c|c|c|c|c|c|}
\hline Day & D1scharge & Day & Discharge & Day & D1scharge & Day & D1 scharge & Day & D1scharge \\
\hline $\begin{array}{l}1 \ldots \\
2 \ldots \\
3 \ldots \\
4 \ldots \\
5 \ldots \\
6 \ldots \\
\end{array}$ & $\begin{array}{r}29 \\
29 \\
31 \\
1,460 \\
5,170 \\
4,280\end{array}$ & $\begin{array}{r}7 \ldots \ldots \\
8 \ldots \ldots \\
9 \ldots \\
10 \ldots \\
11 \ldots \ldots \\
12 \ldots \ldots\end{array}$ & $\begin{array}{r}1,270 \\
2,810 \\
13,600 \\
15,100 \\
5,770 \\
1,860\end{array}$ & $\begin{array}{c}13 \ldots \ldots \\
14 \ldots \ldots \\
15 \ldots \ldots \\
16 \ldots \ldots \\
17 \ldots \ldots \\
18 \ldots \ldots\end{array}$ & $\begin{array}{l}430 \\
259 \\
198 \\
164 \\
128 \\
106\end{array}$ & $\begin{array}{l}19 \ldots \ldots \\
20 \ldots \ldots \\
21 \ldots \ldots \\
22 \ldots \ldots \\
23 \ldots \ldots \\
24 \ldots \ldots\end{array}$ & $\begin{array}{r}95 \\
106 \\
102 \\
90 \\
79 \\
73\end{array}$ & $\begin{array}{l}25 \ldots \ldots \\
26 \ldots \ldots \\
27 \ldots \ldots \\
28 \ldots \ldots \\
29 \ldots \ldots \\
30 \ldots \ldots \\
31 \ldots \ldots\end{array}$ & $\begin{array}{l}73 \\
76 \\
71 \\
64 \\
58 \\
52 \\
48\end{array}$ \\
\hline $\begin{array}{l}\text { nthly } \\
\text { inoff, }\end{array}$ & 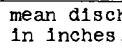 & .. & 010 & per & & & & & $\begin{array}{r}1.732 \\
8.79\end{array}$ \\
\hline
\end{tabular}




\section{OBION CREEK BASIN}

(149) 7-235, Obion Creek at Pryorsburg, Ky,

Location.--Lat $36^{\circ} 41^{\prime} 10^{\prime \prime}$, long $88^{\circ} 43^{\prime} 35^{\prime \prime}$, on right bank at downstream side of bridge on U.S. H1ghway $45,0.5 \mathrm{mlle}$ southwest of Pryorsburg, Graves County, and 3.1 miles ups tream from Cane Creek.

Drainage area. $--36.8 \mathrm{sq} \mathrm{mi}$.

Gage-height record.--Digital recorder tape punched at 15-minute intervals. Datum of gage is $393.55 \mathrm{ft}$ above mean sea level, datum of 1929.

Discharge record.--Stage-d1scharge relation def1ned by current-meter measurements

below 4,560 cis.

Maxima.--Given in the following table.

March 1964:

Mar. 4, 1600 hours ..................... 1949:

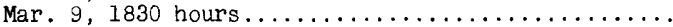

Feb, 14,1949

1951 to February 1964 :

Nov. 18,1957

Discharge Gage height
(cfs)

$4,520 \quad 12.37$

$5,000 \quad 12.85$

Unknown

13.0

5,330

12.60

Mean discharge, in cubic feet per second, March 1964

\begin{tabular}{|c|c|c|c|c|c|c|c|c|c|}
\hline Day & Discharge & Day & Discharge & Day & Discharge & Day & Discharge & Day & D1scharge \\
\hline $\begin{array}{l}1 \ldots \ldots \\
2 \ldots \ldots \\
3 \ldots \ldots \\
4 \ldots \\
5 \ldots \ldots \\
6 \ldots \ldots\end{array}$ & $\begin{array}{r}0.6 \\
.7 \\
2,590 \\
292 \\
30.4\end{array}$ & $\begin{array}{r}7 \ldots \ldots \\
8 \ldots \ldots \\
9 \ldots \\
10 \ldots \\
11 \ldots \ldots \\
12 \ldots \ldots\end{array}$ & $\begin{array}{r}13.1 \\
1,960 \\
3,700 \\
1,210 \\
98 \\
45.6\end{array}$ & $\begin{array}{l}13 \ldots \ldots \\
14 \ldots \ldots \\
15 \ldots \ldots \\
16 \ldots \ldots \\
17 \ldots \ldots \\
18 \ldots \ldots\end{array}$ & $\begin{array}{r}25.9 \\
18.2 \\
12.4 \\
10.2 \\
8.8 \\
7.6\end{array}$ & $\begin{array}{l}19 \ldots \ldots \\
20 \ldots \ldots \\
21 \ldots \ldots \\
22 \ldots \ldots \\
23 \ldots \ldots \\
24 \ldots \ldots\end{array}$ & $\begin{array}{l}7.8 \\
8.4 \\
6.9 \\
6.0 \\
5.4 \\
5.1\end{array}$ & $\mid \begin{array}{l}25 \ldots \ldots \\
26 \ldots \ldots \\
27 \ldots \ldots \\
28 \ldots \ldots \\
29 \ldots \ldots \\
30 \ldots \ldots \\
31 \ldots \ldots\end{array}$ & $\begin{array}{l}5.1 \\
8.2 \\
4.2 \\
3.6 \\
3.2 \\
2.8 \\
2.8\end{array}$ \\
\hline
\end{tabular}

\section{BAYOU DU CHIEN BASIN}

(150) 7-240. Bayou du Chien near Clinton, Ky.

Location.--Lat $36^{\circ} 37^{\prime} 43^{\prime \prime}$, long $88^{\circ} 57^{\prime} 50^{\prime \prime}$, on left bank at upstream side of bridge on U.S. Highway $51,1.1 \mathrm{mlles}$ upstream from Cane Creek, $3 \frac{1}{2}$ miles southeast of clinton, H1ckman County, and $13 \frac{1}{2}$ miles upstream from mouth.

Drainage area. $--68.7 \mathrm{sq} \mathrm{mi}$.

Gage-height record.--Water-stage recorder graph. Datum of gage is $307.71 \mathrm{ft}$ above mean sea level, unadjusted.

Discharge record.--Stage-discharge relation defined by current-meter measurements below $4,000 \mathrm{c}$ ss.

Maxima.--Given in the following table.

March 1964

Mar. 5, 0830 hours.

Mar. 10, 0300 hour

1939 to February 1964:

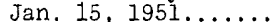

$\begin{array}{cc}\begin{array}{c}\text { Discharge } \\ \text { (cfs) }\end{array} & \text { Gage height } \\ 4,660 & \text { feet) } \\ 6,140 & 14.36 \\ & 14.80\end{array}$

Mean discharge, in cubic feet per second, March 1964

\begin{tabular}{|c|c|c|c|c|c|c|c|c|c|}
\hline Day & Discharge & Day & Discharge & Day & Discharge & Day & Discharge & Day & Discharge \\
\hline $\begin{array}{l}1 \ldots \ldots \\
2 \ldots \ldots \\
3 \ldots \ldots \\
4 \ldots \ldots \\
5 \ldots \ldots \\
6 \ldots \ldots\end{array}$ & $\begin{array}{r}18 \\
18 \\
22 \\
1,320 \\
3,500 \\
579\end{array}$ & $\begin{array}{r}7 \ldots \ldots \\
8 \ldots \ldots \\
9 \ldots \ldots \\
10 \ldots \\
11 \ldots \ldots \\
12 \ldots \ldots\end{array}$ & $\begin{array}{r}35 \\
1,420 \\
5,180 \\
4,500 \\
1,000 \\
168\end{array}$ & $\begin{array}{l}13 \ldots \ldots \\
14 \ldots \ldots \\
15 \ldots \ldots \\
16 \ldots \ldots \\
17 \ldots \ldots \\
18 \ldots \ldots\end{array}$ & $\begin{array}{l}95 \\
71 \\
49 \\
37 \\
32 \\
28\end{array}$ & $\begin{array}{l}19 \ldots \ldots \\
20 \ldots \ldots \\
21 \ldots \ldots \\
22 \ldots \ldots \\
23 \ldots \ldots \\
24 \ldots \ldots\end{array}$ & $\begin{array}{l}32 \\
46 \\
30 \\
26 \\
24 \\
24\end{array}$ & $\mid \begin{array}{l}25 \ldots \ldots \\
26 \ldots \ldots \\
27 \ldots \ldots \\
28 \ldots \ldots \\
29 \ldots \ldots \\
30 \ldots \ldots \\
31 \ldots \ldots\end{array}$ & $\begin{array}{l}26 \\
28 \\
20 \\
20 \\
18 \\
17 \\
18\end{array}$ \\
\hline th1y & ean d & 8 & $1 \mathrm{C}$ & er & & & & & $\begin{array}{r}594 \\
9.96\end{array}$ \\
\hline
\end{tabular}


Allegheny River at Natrona, Pa..... Page Anderson River, Middle Fork, at Bristow, Ind ........

Bacon Creek near Priceville, Ky.... Bayou du Chien near Clinton, Ky ..... Bear Creek near Leitchfield, Ky..... Beargrass Creek, Middle Fork, at Cannons Lane, at Louisvilie, Ky ................... 15,98 South Fork, at Loulsvilie, Ky.... 97 Beargrass Creek basin, flood

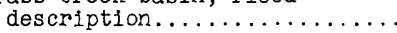

records.....................97-99

Beaver River at Beaver Falls, $\mathrm{Pa} . . .445$

Beech Fork, at Bardstown, Ky........ 111

near Springfield, Ky............. 11$]$

Big Darby Creek, at Darbydale, Ohio. at Darbyville, Ohio............ Big Eagle Creek at Sadievilie, Ky... Big Sandy River at Louisa, Ky........ Blue River near White Cloud, Ind.... Bullskin Creek near Simpsonvilie,

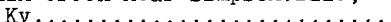

Burr Oak Reservolr at Burr oak,

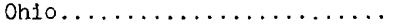

Caesar Creek at Harveysburg, Ohto... Caney Creek near Horse Branch, Ky... Clarks River, East Fork, near Benton, Ky.............

Clear Creek (Hocking River basin) near Rockbridge, Ohio........

Clear Creek (Salt River basin) near Shelbyville, Ky ............

Clear Creek (Wabash River basin) at

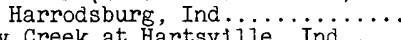

Clifty Creek at Hártsviiie, Ind.....

Cumberland River at Smithland, Ky...

Cumberland River basin, records.....

Damage, estimated...........25,27

Deer Creek, at Pancoastburg, Oh10...

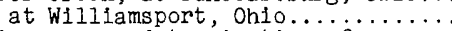

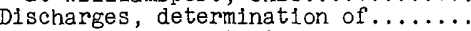
peaks, summary table...........

Eagle Creek at Glencoe, Ky .......15,96 Elkhorn Creek near Frankfort, $\mathrm{Ky}_{\ldots} \ldots . .9$

Flat Creek near Frankfort, Ky...... Floyds Fork, at Fishervilie, Ky.....

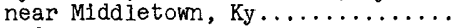

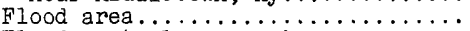
Flood-controi reservolrs..............11,

Flood discharges, determination.....

Flood peaks, summary table........

Great Miami River at Hamilton, Oh1o. Great Miami River basin, records... .88-89 Green River at lock 2, at Calhoun, $\mathrm{Ky} . \ldots \ldots \ldots \ldots \ldots \ldots \ldots \ldots \ldots \ldots$ Green River basin, fiood description records...................118-130 Guyandotte River at Branchiand,

W. $\mathrm{Va} . . . \ldots \ldots \ldots \ldots \ldots$

Hocking River, at Athens, Ohio.....12,57

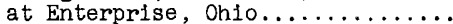
at Lancaster, OhIo...............

Hocking River basin, fiood

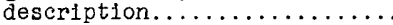
records .....................52-57
Indian Creek (tributary to East Fork White River) near Springvilie, Ind................... Al44

Indian Creek (tributary to Ohio River) near Corydon, Ind....21,115

Kanawha River at Charleston, W. Va. 59

Kentucky River, at lock 2, at Lockport, $\mathrm{Ky} \ldots \ldots \ldots \ldots \ldots \ldots \ldots . \ldots . \ldots 9$

at lock 4, at Frankfort, Ky...... 90

Kentucky River basin, flood description............. 16 records ....................90-97

Kokosing River, at Millwood, Ohio... 48

at Mount Vernon, Oh1o.......... 47

North Branch, near Fredericktown, Oh10.................. 47

6 Laughery Creek near Farmers Retreat, nd.....

Licking River (tributary to Muskingum River) near Newark,

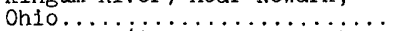

Licking River itributary to onio River) at Blue Lick Springs

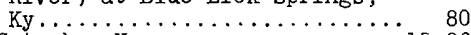

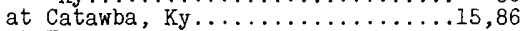
at Farmers, $\mathrm{Ky} \ldots \ldots \ldots \ldots \ldots \ldots . \ldots . \ldots . \ldots$ at McKinneysburg, Ky ............. 82 North Fork, near Lewisburg, Ky.... 81

South Fork, at Cynthiana, Ky...... 85

licking River basin, flood description............. 15

records ...................... $80-86$

Iittle Kanawha River at Palestine, W. Va................. 52

Itttle Miami River, at Mi iford,

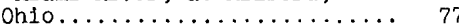

at Spring Valiey, Ohio......... 75

East Fork, at Perintown, ohio.....12,79 at Williamsburg, Oh10............ 78

nean Fort Ancient, Ohio........... 75

Little Miami River basin, flood

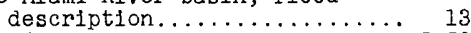

records...................75-79

Iittle Plum Creek, near Waterford, Ky................... 106

near Wilsonvilie, Ky. See Plum Creek subwatershed $15 \ldots \ldots \ldots 105$

Little River near Cadiz, Ky.......... 152

Little Rush Creek near Rushvilie, ohio................... 53

Little Sandy River near Grayson, $\mathrm{Ky} \ldots \ldots \ldots \ldots \ldots \ldots \ldots \ldots \ldots$

31. McDougal Creek near Hodgenville, Ky. 118

34 Mayfleld Creek at Lovelaceville,

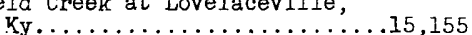

88 M111 Creek at Carthage, ohio........ 88

Monongahela River at Braddock, Pa... 43

Moxahala Creek at Roseville, Ohio... 50

Muscatatuck River near Austin. Ind. 136

Muskingum River at McConnelsville, Oh10................... 50

Muskingum River basin, fiood

description............. 11

records ..................47-50

4 Nolin River, at Kyrock, Ky,....... 122

at White Mills, Ky.............. 120

North Fork, at Hodgenviiie, Ky.... 119

Nolin River Reservolr near Kyrock.

$\mathrm{Ky} \ldots \ldots \ldots \ldots \ldots \ldots \ldots \ldots \ldots$ 
North Elkhorn Creek near Georgetown, Ky......................

Oblon Creek at Pryorsburg, Ky...... Oh10 Brush Creek near west Union,

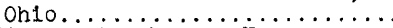

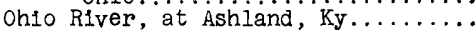

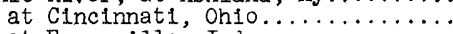

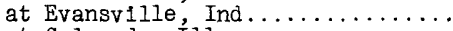

at Golconda, Ili.

at Huntington, $\mathrm{W} . \mathrm{Va} . \ldots \ldots \ldots \ldots$

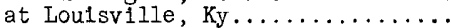

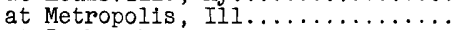

at Parkersburg, W. Va...........

at Point Pleasant, $W$. Va..........

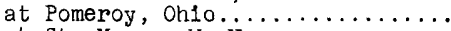

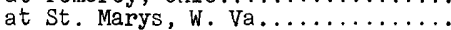

at Sewickley, Pa...............

comparat1ve crest stages..........

discharge hydrographs.

flood peak recurrence intervals for selected stations......... general description of floods at stations...............21-25

near Maysvilie, Ky............ 73

Paint Creek, at damsite near Bainbridge, Ohio.

near Bournevilie.

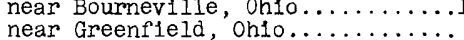

Patoka River, at Jasper, Ind.........

at Winslow, Ind.............. 14

near E1lsworth, Ind ...............

near Princeton, Ind.

Pigeon Creek at Evansvilie, Ind .....

Plum Creek, at waterford, Ky........

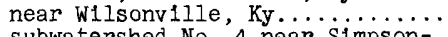
subwatershed No. 4 near Simpsonville, Ky ..... is iitile Pium subwatershed No. 15 (Little Plum subwatershed No. 17 near Waterford,

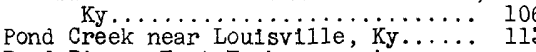

Pond River, East Fork, near Apex,

Ky...................... 129

Precipitation........................

Raccoon Creek at Adamsv1lle, Oh10...

Rock Llck Creek near Glen Dean, Ky..

Rocky Fork near Barretts M11ls,

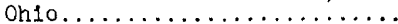

Rocky Fork Lake near Rainsboro, $0 h 10 \ldots \ldots \ldots \ldots \ldots \ldots \ldots \ldots$

Rolling Fork, near Boston, Ky ........... near Lebanon, Ky ............... Rough RIver, at Fails of Rough, Ky.

Page

112

110
127
Rough River, near Dundee, Ky.................... North Fork, near Westview, Ky..... 123 Rough River Reservo1r near Falls of Rough, Ky................ 125

Salt Creek (Scloto River basin) near Londonderry, Oh10..........71

Salt Creek, (Wabash Rlver basin) near Harrodsburg, Ind........ 141

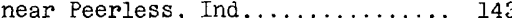

North Fork, at Nashvilie, Ind..... 139

near Belmont, Ind........... 140

South Fork, at Kurtz, Ind........... 138

Salt River, at Shepherdsville, Ky..15,109

at Taylorsville, Ky............ 103

near Harrodsburg, Ky ........... 102

near Van Buren, Ky............... 102

Salt River basin, flood description. 16

records...................102-114

Sand Creek near Brewersville,

Ind ................... 135

Scloto Rlver, at Higby, Ohlo........ 70

at Piketon, Ohio............. 71

Scloto River basin, flood

description............. 13

records.......... neilersburg......65-71

Ind.................... 99

South Elkhorm Creek at Fort Spring, Ky .................. 92

South Hogan Creek near Diilsboro, Ind................... 89

Stoner Creek at Par1s, Ky......... 84

Sunday Creek, at Burr Oak, Ohlo.... 55

at Glouster, Oh10............ 56

Tennessee River near Paducah, Ky.... 153

Tennessee River basin, records...... 153

104 Todd Fork near Roachester, On10..... 76

Tradewater River at O1ney, Ky....... 150

105 Tygarts Creek near Greenup, Ky...... 65

06 Vernon Fork at Vernon, Ind........ 136

Wabash River, at Mount Carmel, I1l.. 150

at Vincennes, Ind ................ 133

Wabash River basin, records ....... 133-150

White River, at Newberry, Ind....... 133

at Petersburg, Ind............ 145

East Fork, at Columbus, Ind ...... 134

at Seymour, Ind.............. 135

at Shoals, Ind.............. 144

near Bedford, Ind.......... 137

68 Whiteoak Creek near Georgetown,

Whitewater River at Brookviiie.

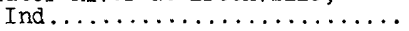

